\title{
CORRECTIVE ACTION DECISION DOCUMENT FOR CORRECTIVE ACTION UNIT 383: AREA 12 E-TUNNEL SITES, NEVADA TEST SITE
}
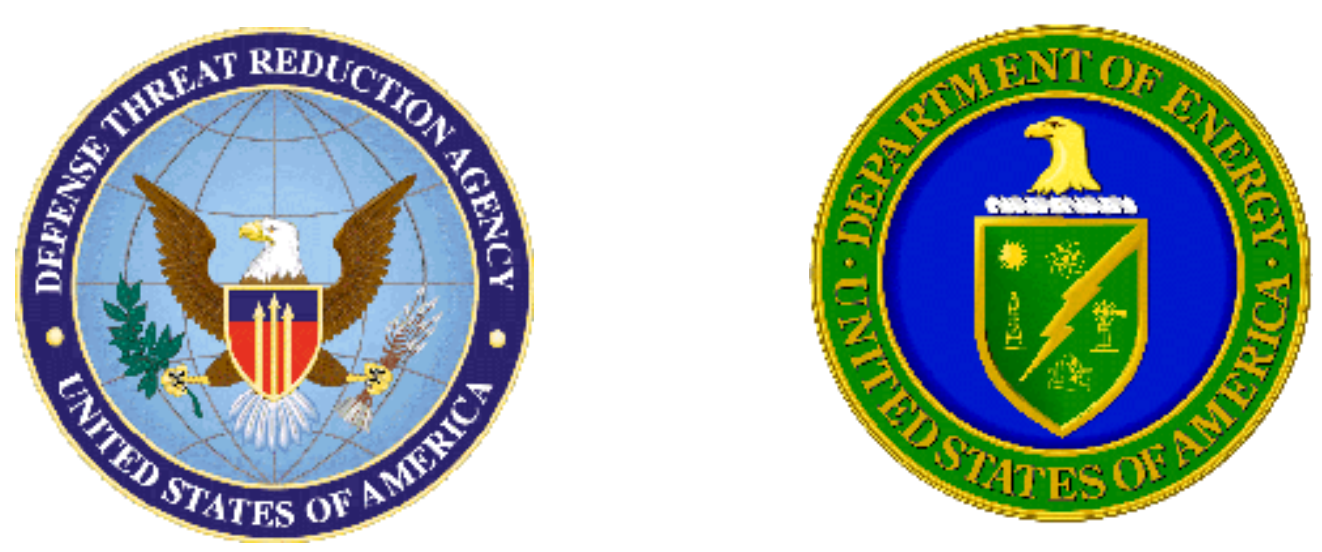

Controlled Copy No:

Revision No.: 0

January 2005

Approved for public release; further dissemination unlimited. 
Available for sale to the public from:

\author{
U.S. Department of Commerce \\ National Technical Information Service \\ 5285 Port Royal Road \\ Springfield, VA 22161 \\ Phone: 800.553 .6847 \\ Fax: 703.605.6900 \\ Email: orders@ntis.gov \\ Online ordering: http://www.osti.gov/ordering.htm
}

Available electronically at $\underline{h t p: / / w w w . o s t i . g o v / b r i d g e}$

Available for a processing fee to U.S. Department of Energy and its contractors, in paper, from:

U.S. Department of Energy

Office of Scientific and Technical Information

P.O. Box 62

Oak Ridge, TN 37831-0062

Phone: 865.576.8401

Fax: 865.576.5728

Email: reports@adonis.osti.gov

Reference herein to any specific commercial product, process, or service by trade name, trademark, manufacturer, or otherwise, does not necessarily constitute or imply its endorsement, recommendation, or favoring by the Unites States Government or any agency thereof or its contractors or subcontractors. 


\title{
CORRECTIVE ACTION DECISION DOCUMENT FOR CORRECTIVE ACTION UNIT 383: AREA 12 E-TUNNEL SITES, NEVADA TEST SITE
}

\author{
Prepared by \\ Defense Threat Reduction Agency \\ Nevada Operations Office \\ Mercury, Nevada \\ and \\ U.S. Department of Energy \\ National Nuclear Security Administration \\ Nevada Site Office \\ Las Vegas, Nevada \\ Controlled Copy No. \\ Revision No.: 0 \\ January 2005
}

Approved for public release; further dissemination unlimited. 


\section{CORRECTIVE ACTION DECISION DOCUMENT \\ FOR CORRECTIVE ACTION UNIT 383: \\ AREA 12 E-TUNNEL SITES, \\ NEVADA TEST SITE}

Approved by: Signature Approved

Date: $1 / 24 / 05$

TRUDY H. CLARK

Maj Gen, USAF

Acting Director

Defense Threat Reduction Agency

\footnotetext{
Approved by: $\frac{\text { Signature Approved }}{\text { Janet Appenzeller-Wing, Acting Division Director }}$

Date: $1 / 24 / 05$

Environmental Restoration Division

U.S. Department of Energy, National Nuclear Security Administration

Nevada Site Office
} 


\section{Table of Contents}

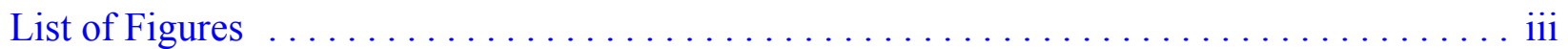

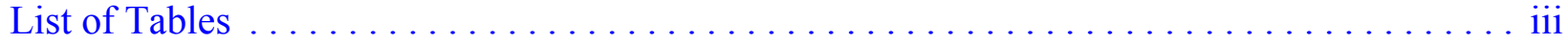

List of Acronyms and Abbreviations $\ldots \ldots \ldots \ldots \ldots \ldots \ldots \ldots \ldots \ldots \ldots \ldots \ldots \ldots \ldots \ldots \ldots$

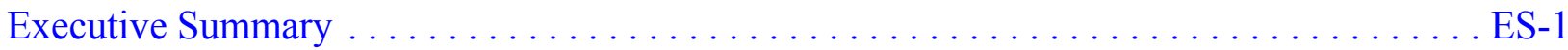

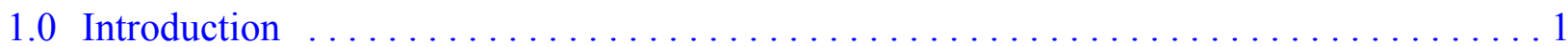

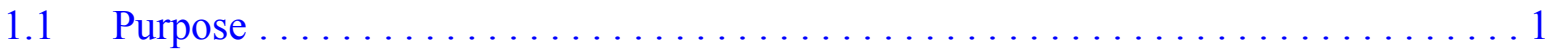

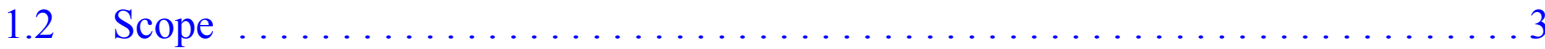

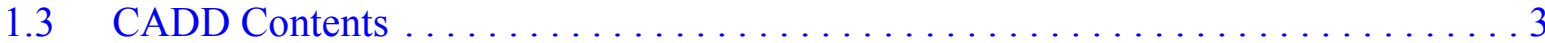

2.0 Corrective Action Investigation Summary $\ldots \ldots \ldots \ldots \ldots \ldots \ldots \ldots \ldots \ldots \ldots \ldots \ldots \ldots \ldots$

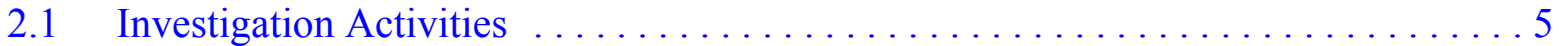

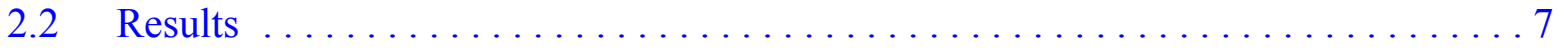

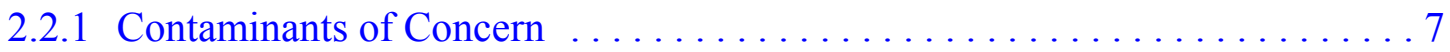

2.2.2 Assessment Summary . . . . . . . . . . . . . . . . . 9

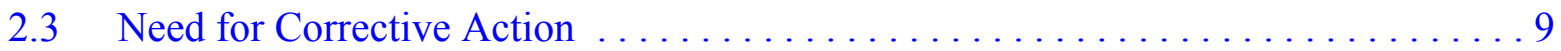

2.3.1 Nevada Administrative Code 445A.227 (2) (a-k) Analysis . . . . . . . . . 13

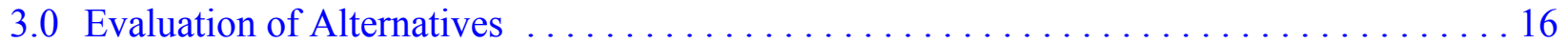

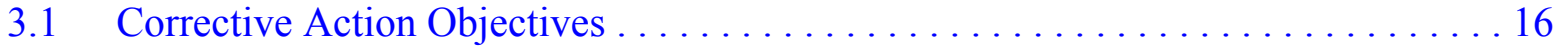

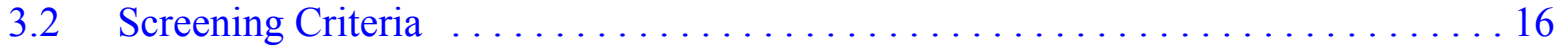

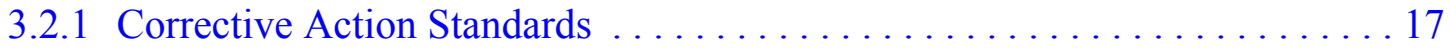

3.2.2 Remedy Selection Decision Factors $\ldots \ldots \ldots \ldots \ldots \ldots \ldots \ldots \ldots$

3.3 Development of Corrective Action Alternatives . . . . . . . . . . . . . 20

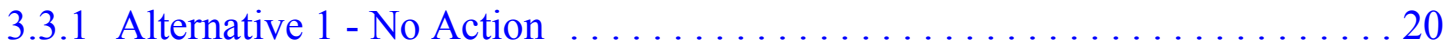

3.3.2 Alternative 2 - Clean Closure by Excavation of the Oil Spill; Radioactive Material Area; Muckpile; Ponds One, Two, and Three; and

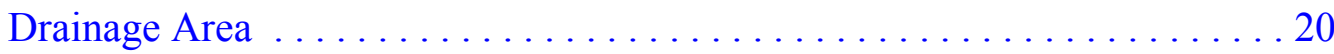




\section{Table of Contents (Continued)}

3.3.3 Alternative 3 - Closure by Excavation of the Oil Spill and Radioactive Material Area, Grading and Capping of the Muckpile, Drainage Controls, and

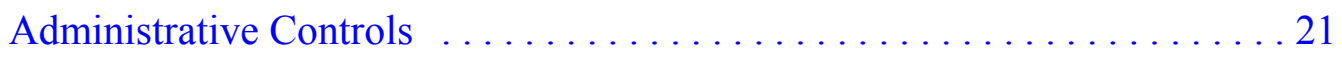

3.4 Evaluation and Comparison of Alternatives $\ldots \ldots \ldots \ldots \ldots \ldots \ldots \ldots \ldots \ldots \ldots \ldots \ldots \ldots \ldots$

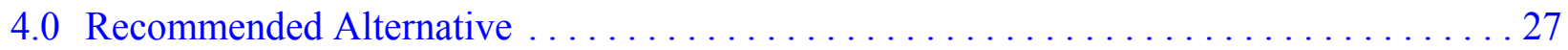

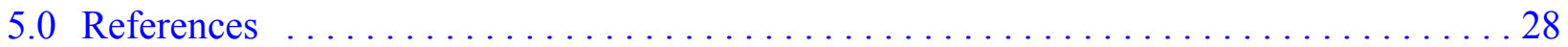

Appendix A - Corrective Action Investigation Summary Report for Corrective Action Unit 383: Area 12, E-Tunnel Sites, Nevada Test Site

Attachment A - Soil Boring Logs

Attachment B - Cross Sections

Attachment C - Analytical Results Table

Appendix B - Data Assessment

Appendix C - Cost Estimates

Appendix D - Assessment of Risk for Evaluated Alternatives CAU 383: E-Tunnel Sites, NTS

Appendix E - Project Organization 


\section{List of Figures}

Number

Title

Page

1-1 E-Tunnel Sites Location Map, Area 12, Nevada Test Site $. . . \ldots \ldots \ldots \ldots \ldots . \ldots 2$

2-1 Location of Structures or Impediments at E-Tunnel Sites . . . . . . . . . . . . . . 15

\section{List of Tables}

Number

Title

Page

2-1 Chemical Sample Results that Exceed Action Levels $\ldots \ldots \ldots \ldots \ldots \ldots$

2-2 Radiological Sample Results that Exceed Action Levels ................... 11

3-1 Detailed Evaluation of Corrective Action Alternatives $\ldots \ldots \ldots \ldots \ldots \ldots \ldots$

3-2 Comparative Evaluation of Alternatives . . . . . . . . . . . . . . . . 26 


\section{List of Acronyms and Abbreviations}

\begin{tabular}{|c|c|}
\hline Am & Americium \\
\hline bgs & Below ground surface \\
\hline CADD & Corrective Action Decision Document \\
\hline CAI & Corrective Action Investigation \\
\hline CAIP & Corrective Action Investigation Plan \\
\hline CAS & Corrective Action Site \\
\hline CAU & Corrective Action Unit \\
\hline CFR & Code of Federal Regulations \\
\hline $\mathrm{COC}$ & Contaminant of Concern \\
\hline COPC & Contaminant of Potential Concern \\
\hline $\mathrm{Cs}$ & Cesium \\
\hline CSM & Conceptual site model \\
\hline DOE & U.S. Department of Energy \\
\hline DQO & Data Quality Objectives \\
\hline DTRA & Defense Threat Reduction Agency \\
\hline EPA & U.S. Environmental Protection Agency \\
\hline FD & Field sample duplicate \\
\hline FFACO & Federal Facility Agreement and Consent Order \\
\hline $\mathrm{ft}$ & Foot \\
\hline in. & Inches \\
\hline LCS & Laboratory control sample \\
\hline LCSD & Laboratory control sample duplicate \\
\hline $\mathrm{mg} / \mathrm{kg}$ & Milligrams per kilogram \\
\hline $\mathrm{mi}$ & Mile \\
\hline $\mathrm{mrem} / \mathrm{yr}$ & Millirem per year \\
\hline MS & Matrix spike \\
\hline MSD & Matrix spike duplicate \\
\hline NAC & Nevada Administrative Code \\
\hline NDEP & Nevada Division of Environmental Protection \\
\hline NNSA/NSO & $\begin{array}{l}\text { U.S. Department of Energy, National Nuclear Security Administration } \\
\text { Nevada Site Office }\end{array}$ \\
\hline NRS & Nevada Revised Statutes \\
\hline NTS & Nevada Test Site \\
\hline NTTR & Nevada Test and Training Range \\
\hline PAHs & Polynuclear aromatic hydrocarbons \\
\hline
\end{tabular}




\section{List of Acronyms and Abbreviations (Continued)}

$\begin{array}{ll}\text { PAL } & \text { Preliminary Action Level } \\ \text { pCi/g } & \text { Picocuries per gram } \\ \text { PRG } & \text { Preliminary remediation goal } \\ \mathrm{Pu} & \text { Plutonium } \\ \text { QC } & \text { Quality control } \\ \text { RCRA } & \text { Resource Conservation and Recovery Act } \\ \text { RMA } & \text { Radioactive material area } \\ \text { RPD } & \text { Relative percent difference } \\ \text { RT } & \text { Regulatory threshold } \\ \text { SAPS } & \text { Small-Area Plastic Scintillator Detector } \\ \text { SDG } & \text { Sample delivery group } \\ \text { SVOC } & \text { Semivolatile organic compound } \\ \text { TPH-D } & \text { Total petroleum hydrocarbon-diesel } \\ \text { VOC } & \text { Volatile organic compound } \\ \% \text { R } & \text { Percent recovery }\end{array}$




\section{Executive Summary}

This Corrective Action Decision Document (CADD) was prepared by the Defense Threat Reduction Agency (DTRA) and the U.S. Department of Energy, National Nuclear Security Administration Nevada Site Office (NNSA/NSO). The recommendations and corrective actions described within this document apply to the future closure of Corrective Action Unit (CAU) 383, Area 12 E-Tunnel Sites, which is a joint DTRA and NNSA/NSO site. The CAU consists of three (3) Corrective Action Sites (CASs): CAS 12-06-06 (Muckpile); CAS 12-25-02 (Oil Spill); and CAS 12-28-02 (Radioactive Material). In addition to these CASs, E-Tunnel Ponds One, Two, and Three, and the Drainage Area above the ponds were included since closure of the Muckpile will impact these areas.

This CADD is consistent with the requirements of the Federal Facility Agreement and Consent Order agreed to by the State of Nevada, the U.S. Department of Energy, and the U.S. Department of Defense. The DTRA point of contact is the Nevada Operations Office, Environmental Project Manager; currently Ms. Tiffany A. Lantow. The NNSA/NSO point of contact is the Environmental Restoration, Industrial Sites Project Manager; currently Ms. Janet Appenzeller-Wing.

The purpose of this CADD is to identify and provide the rationale for the selection of a recommended corrective action alternative for CAU 383. This document presents the recommended corrective action for CAU 383 (E-Tunnel Sites); however, implementation may be affected by the corrective action (to be determined) for CAU 551 (Area 12 Muckpiles) due to the close proximity of B, C, D, and F-Tunnels. The scope of this CADD consists of the following tasks:

- Develop corrective action objectives.

- Identify corrective action alternative screening criteria.

- Develop corrective action alternatives.

- Perform detailed and comparative evaluations of the corrective action alternatives in relation to the corrective action objectives and screening criteria.

- $\quad$ Recommend and justify a preferred corrective action alternative for CAU 383. 
A corrective action investigation was performed in May and June 2004, as set forth in the Corrective Action Investigation Plan for Corrective Action Unit 383: Area 12 E-Tunnel Sites. The contaminants of concern (COCs) identified during the investigation include diesel-range organics, radionuclides, and semivolatile organic compounds. The E-Tunnel Muckpile Corrective Action Site ([CAS] 12-06-06), drainage, and pond sediments were found to be contaminated, as were the Oil Spill (CAS 12-25-02) and the Radioactive Material Area (CAS 12-28-02). Based on the potential exposure pathways, the following corrective action objectives have been identified for CAU 383:

- Prevent or mitigate exposure to near-surface soil containing COCs at concentrations exceeding the action levels defined in the Corrective Action Investigation Plan for Corrective Action Unit 383: Area 12 E-Tunnel Sites.

- Prevent migration of COCs beyond the CAU boundary.

- Prevent adverse impacts to groundwater quality.

Based on the review of existing data, the proposed future use of the E-Tunnel Sites as a nuclear and high explosive test zone, current operations at the Nevada Test Site (NTS), and the determination by the Nevada Division of Environmental Protection (NDEP) that the Muckpiles be closed in accordance with applicable administrative codes for solid waste disposal sites the following alternatives were developed for consideration at CAU 383:

- Alternative 1 - No Action

- Alternative 2 - Clean Closure by Excavation of the Oil Spill; Radioactive Material Area; Muckpile; Ponds One, Two, and Three; and Drainage Area

- Alternative 3 - Closure by Excavation of the Oil Spill and Radioactive Material Area, Grading and Capping of the Muckpile, Drainage Controls, and Administrative Controls

The corrective action alternatives were evaluated based on four general corrective action standards; five remedy selection decision factors; and technical merit, focusing on safety, reliability, feasibility, and performance. Based on the results of this evaluation, the preferred alternative for CAU 383 is Alternative 3 - Closure by Excavation of the Oil Spill and Radioactive Material Area, Grading and Capping of the Muckpile, Drainage Controls, and Administrative Controls. This alternative meets all applicable state and federal regulations for closure of the site, is most protective of human health and the environment, and will eliminate potential future exposure pathways to the contaminated soils at the E-Tunnel Sites. 


\subsection{Introduction}

This Corrective Action Decision Document (CADD) has been developed in accordance with the Federal Facility Agreement and Consent Order (FFACO) agreed to by the State of Nevada, the U.S. Department of Energy (DOE), and the U.S. Department of Defense (DoD). This document covers Corrective Action Unit (CAU) 383, Area 12 E-Tunnel Sites which consist of three Corrective Action Sites (CASs) (12-06-06, Muckpile; 12-25-02, Oil Spill; 12-28-02, Radioactive Material). The Area 12 E-Tunnel Sites are located approximately 42 miles (mi) north of Mercury in Area 12 of the Nevada Test Site (NTS) (Figure 1-1). This CADD describes the corrective action that is selected as a result of investigation activities and the rationale for its selection. The rationale consists of an analysis of the considered alternatives ranging from no action to clean closure (FFACO, 1996). This document presents the recommended corrective action for CAU 383 (E-Tunnel Sites); however, implementation may be affected by the corrective action (to be determined) for CAU 551 (Area 12 Muckpiles) due to the close proximity of B, C, D, and F-Tunnels.

\subsection{Purpose}

The purpose of this CADD is to identify and provide the rationale for the selection of a corrective action alternative based on the corrective action investigation (CAI) of CAU 383: Area 12 E-Tunnel Sites. E-Tunnel was used for nine nuclear weapons tests between 1958 and 1977. The Muckpile contains roughly 355,000 cubic yards of material consisting primarily of mining debris (rock) generated during excavation of the underground facilities and disturbed geologic materials; construction/reentry debris comprise the remainder of the Muckpile.

Corrective Action Site 12-28-02 (Radioactive Material) is located at the E-Tunnel main portal and is part of the Muckpile. Corrective Action Site 12-25-02 (Oil Spill) is located on the blower pad, which is on a bench approximately $100 \mathrm{ft}$ above the E-Tunnel south portal. The Drainage Area and Ponds One, Two, and Three, which were included in this investigation, trend easterly and extend approximately $350 \mathrm{ft}$ down canyon from the Muckpile. The investigation of the sites was conducted in accordance with the Corrective Action Investigation Plan for CAU 383: E-Tunnel Sites (DTRA, 2003). 


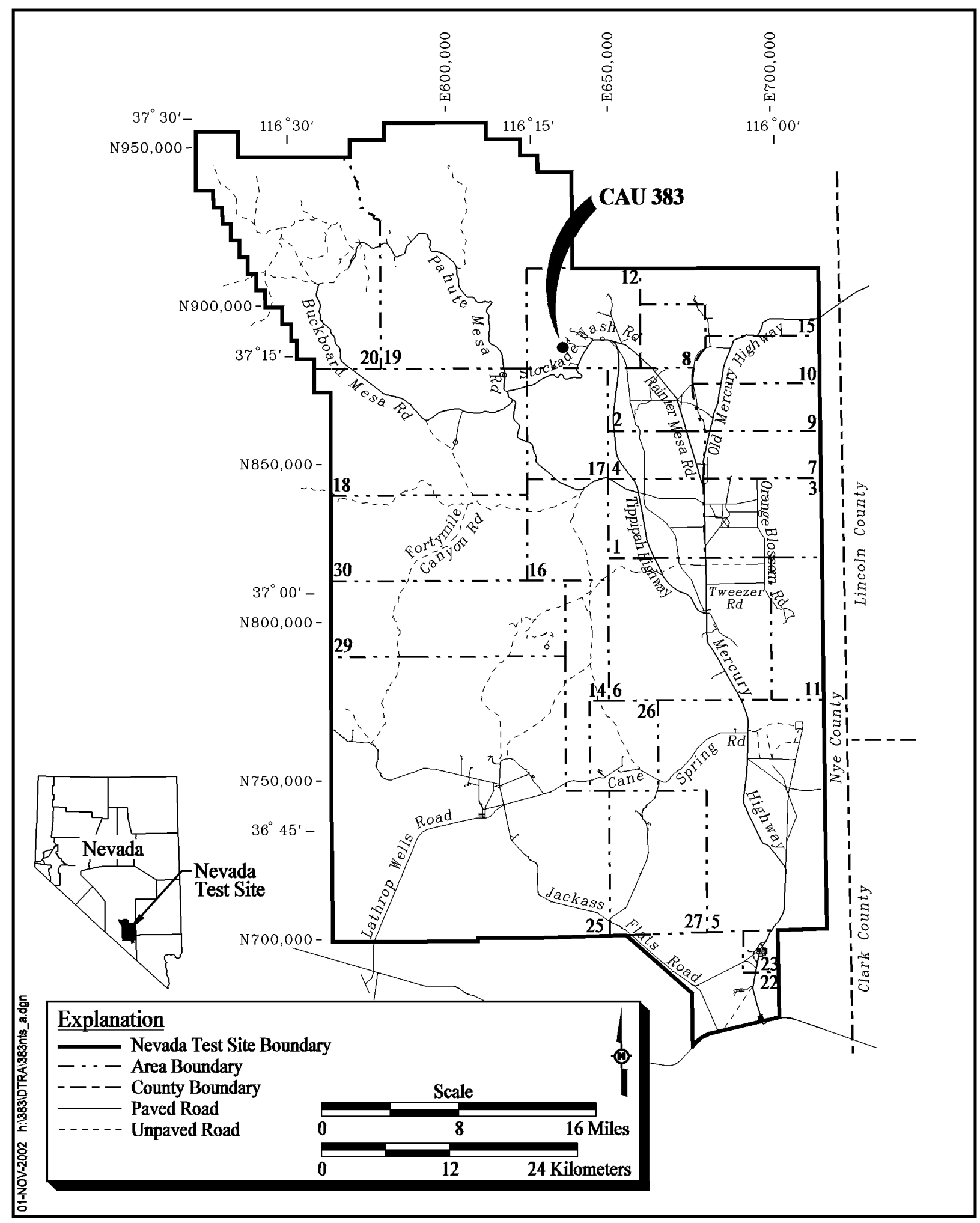

Figure 1-1

E-Tunnel Sites Location Map, Area 12, Nevada Test Site

(Source: Modified from DOE/NV, 1996) 


\subsection{Scope}

The Area 12 E-Tunnel Sites are identified in the FFACO as CAU 383, which consists of three CASs (12-06-06, Muckpile; 12-25-02, Oil Spill; and 12-28-02, Radioactive Material). In addition, the investigation and this CADD include the Drainage Area below the Muckpile and Ponds One, Two, and Three. The Area 12 E-Tunnel Sites are located approximately 42 miles north of Mercury in Area 12 of the Nevada Test Site (Figure 1-1). The site is referred to in this CADD as CAU 383 or the E-Tunnel Sites. The scope of the CAU 383 CADD is to select and justify a remediation alternative for closing the E-Tunnel Sites. To accomplish this, the following activities will be completed:

- Develop corrective action objectives.

- Develop corrective action alternatives based on the results of the corrective action investigation and the corrective action objectives.

- Perform detailed and comparative evaluations of corrective action alternatives in relation to corrective action objectives and screening criteria.

- Recommend and justify a preferred corrective action alternative for the CAU.

\subsection{CADD Contents}

This CADD is divided into the following sections:

- Section 1.0 - Introduction: summary of the purpose, scope, and contents of this CADD.

- Section 2.0 - CAI Summary: summary of the field investigation activities and the analytical results.

- Section 3.0 - Evaluation of Alternatives: objectives and goals of the corrective action, screening criteria, development of corrective action alternatives, and evaluation and comparison of alternatives.

- Section 4.0 - Recommended Alternative: presentation of the preferred corrective action alternative and the rationale for its selection based on the corrective action objectives and alternative screening criteria.

- Section 5.0 - References: a list of all documents referenced in the CADD.

- Appendix A: Corrective Action Investigation Summary Report for CAU 383: E-Tunnel Sites, Nevada Test Site, Nevada 
- Appendix B: Data Assessment

- Appendix C: Cost Estimates

- Appendix D: Assessment of Risk for Evaluated Alternatives CAU 383: E-Tunnel Sites, NTS

- Appendix E: Project Organization

All work was performed in accordance with the following documents:

- Corrective Action Investigation Plan for CAU 383: Area 12 E-Tunnel Sites, Nevada Test Site, Nevada, Rev. 0 (DTRA, 2003)

- Industrial Sites Quality Assurance Project Plan, Rev. 3 (NNSA/NV, 2002)

- Federal Facility Agreement and Consent Order (FFACO, 1996)

- SNJV Site-Specific Health and Safety Plan for Corrective Action Unit 383: E-Tunnel Muckpile, Rev. 0 (SNJV, 2004) 


\subsection{Corrective Action Investigation Summary}

The following sections describe and summarize the results of the Corrective Action Investigation (CAI) activities conducted at CAU 383. For detailed CAI results, please refer to Appendix A.

\subsection{Investigation Activities}

Corrective Action Investigation activities were performed at the E-Tunnel Sites as set forth in the CAIP (DTRA, 2003) between May 19 and June 24, 2004. The purpose of the CAI was to determine whether or not the E-Tunnel Muckpile; Ponds One, Two, and Three; the underlying native soils; the Drainage Area below the Muckpile; the Oil Spill; and/or the Radioactive Material Area have been impacted by contaminants of potential concern (COPCs) at concentrations that exceed regulatory limits and to provide sufficient information and data to develop appropriate corrective action strategies for these areas. In accordance with the CAIP (DTRA, 2003) the following tasks were performed:

- Radiological Surveys - Prior to commencing drilling and sampling, walk-over and drive-over radiological surveys were conducted to identify surface and near-surface areas with elevated readings.

- Sampling of the Muckpile (muck) and underlying native material - Thirty-four locations were drilled or hand sampled on the Muckpile to characterize the muck and the native material under the Muckpile. A total of 1,039.5 ft were drilled in 32 boreholes ranging from 1.5 to $91.5 \mathrm{ft}$ deep. Two locations were sampled by hand to 6-inches deep. Up to three samples were collected from each location. One location was drilled but not sampled. During the characterization, 66 environmental samples were collected. These included 41 muck samples and 25 native material samples. All samples were sent to an approved off-site analytical laboratory for analysis. Analyses performed included volatile organic compounds (VOCs), semivolatile organic compounds (SVOCs), total petroleum hydrocarbons-diesel (TPH-D), total Resource Conservation and Recovery Act (RCRA) metals, beryllium, and radionuclides (including strontium-90 and isotopic plutonium $[\mathrm{Pu}])$.

- Sampling of the drainage - Thirty-five locations were drilled or hand sampled in the drainage to characterize the site. The two boreholes were each drilled to five $\mathrm{ft}$ deep. Thirty-three locations were hand sampled to 6-inches deep. One sample was collected from each location for a total of 35 environmental samples. All samples were sent to an approved off-site analytical laboratory, to be analyzed for the same analytes as the Muckpile samples. 
- Sampling of the Pond sediments and underlying native material - Nine locations were drilled or hand sampled in Ponds One, Two, and Three to characterize the sediment and the native material under the sediments. In Pond One, a total of $80 \mathrm{ft}$ was drilled in five boreholes ranging from depths of 18.0 to $21.0 \mathrm{ft}$. Due to soil wetness in Ponds Two and Three, only two locations were sampled in each pond; all four locations were hand sampled to 6-inches deep. During the characterization 14 environmental samples were collected. These included nine sediment samples and five native material samples. All samples were sent to an approved off-site analytical laboratory to be analyzed for the same analytes as the Muckpile samples.

- Sampling of the Oil Spill (CAS 12-25-02) - Eight locations were hand sampled to 6-inches deep in the Oil Spill to characterize the site. One sample was collected from each location for a total of eight environmental samples. All samples were sent to an approved off-site analytical laboratory to be analyzed for the same analytes as the Muckpile samples.

- Sampling of the Radioactive Material Area (RMA) (CAS 12-28-02) - Four locations were hand sampled to 6-inches deep in the RMA to characterize the site. One sample was collected from each location for a total of four environmental samples. All samples were sent to an approved off-site analytical laboratory to be analyzed for the same analytes as the Muckpile samples.

- Background and preliminary soil samples were collected at eight locations on and around the Muckpile. Three background samples were hand collected at three undisturbed locations around the Muckpile. Five preliminary samples were hand collected at five biased locations on the Muckpile. Seven of the samples were analyzed for beryllium, RCRA metals, and radionuclides; one sample, 383GB0901, was analyzed only for radionuclides (strontium-90, isotopic plutonium, and gamma spec). Analysis was done at an approved off-site analytical laboratory.

The conceptual site model (CSM) postulated the following:

- The majority of the Muckpile does not contain COPCs, and if any are present they are probably isolated.

- The location of buried COPCs cannot be predicted but surface radiological surveys can be used to increase the probability of finding impacted areas.

- It is possible, but unlikely, that the native soil beneath the Muckpile has been impacted by downward migration of COPCs.

- Releases to the Muckpile from surface activities of concern (i.e., equipment maintenance and storage of equipment and petroleum products) may be locally significant. These releases, if present, are expected to have limited lateral and vertical extent. 
Although the first CSM postulate was shown to be incorrect, the other postulates and the assumptions used to develop the CSM (DTRA, 2003) were proven to be correct by the CAI. The fact that the contamination is more wide spread than anticipated does not invalidate the rest of the model. The more important concepts of the model, that there was no leaching into the native material and that migration was taking place from the Muckpile in storm runoff, were shown to be correct.

\subsection{Results}

The following is a summary of the data obtained during the CAI.

\subsubsection{Contaminants of Concern}

Contaminants of potential concern were determined in the Data Quality Objectives (DQO) process as listed in the CAIP (DTRA, 2003). Analytical results obtained from the CAI were evaluated to determine whether COPCs were detected above action levels. If the action levels were exceeded, those constituents became COCs for CAU 383. Based on the results of this evaluation, diesel-range organics, benzo(a)anthracene, benzo(a)pyrene, benzo(b)fluoranthene, dibenzo(a,h)anthracene, indeno(1,2,3-cd)pyrene, and man-made radionuclides were identified as the COCs for the site.

The future use for the Area 12 E-Tunnel Sites, as defined in the Final Environmental Impact Statement for the Nevada Test Site and Off-Site Locations in the State of Nevada (DOE/NV, 1996), is assumed to be similar to its current use (Nuclear and High Explosive Test Zone). The U.S. Environmental Protection Agency (EPA) Region 9 Industrial Preliminary Remediation Goals (PRGs) for evaluating contaminated sites assume exposure pathway scenarios that are most comparable to the activities conducted at similar Defense Threat Reduction Agency (DTRA) sites on the NTS (i.e., training, high explosive tests, and other industrial uses). Under industrial scenarios, the potential exposure pathways are ingestion, inhalation, and dermal contact with contaminated soil (EPA, 2002). Site workers could potentially be exposed to contaminated soil during excavation activities associated with general maintenance or through construction and maintenance of facilities and underground utilities. The results for samples collected in the potential zone of intrusion (less than $10 \mathrm{ft}$ ) indicate the limited presence of diesel-range organics, polynuclear aromatic hydrocarbons, (PAHs) and man-made radionuclides. Therefore, diesel-range organics, benzo(a)anthracene, benzo(a)pyrene, benzo(b)fluoranthene, dibenzo(a,h)anthracene, indeno(1,2,3-cd)pyrene, and man-made 
radionuclides must be considered during evaluation of the identified exposure pathways of ingestion, inhalation, and dermal contact with contaminated soil.

As part of the CAIP (DTRA, 2003), the CSM developed for CAU 383 identified a potential pathway for migration of contaminants by percolation of water through the Muckpile material, pond sediment, and underlying alluvium and into the groundwater. Analytical results from samples in the native material beneath the Muckpile show only a single instance of radiological contamination in the underlying native material. In that case the sample from the muck seven $\mathrm{ft}$ above the native sample contained the same contaminants at a higher concentration. However, due to drill refusal $1.5 \mathrm{ft}$ below the contact it was not possible to get a sample of the native material that was not impacted by the muck. Based on the evidence listed above, the contamination in the native material is most likely the result of slough in the borehole and not migration of contaminants from the muck to the native material.

The CAI analytical results (Appendix A) indicate the following:

- Diesel-range organic concentrations above the action level of 100 milligrams per kilogram (mg/kg) (NAC, 1996b) were detected in nine samples, three from widely spaced locations in the Muckpile, one from Pond One, and five from the Oil Spill. All of the samples were surface samples collected from $0-1 \mathrm{ft}$ in depth. All but one of the samples were entirely muck or sediment. The one exception contained both muck and native material. The muck/native contact for this hole was at a depth of $0.3 \mathrm{ft}$ and there was refusal at $1.5 \mathrm{ft}$. Drilling and sampling of other boreholes showed that the extent of the TPH-d contamination in all of the areas was limited both laterally and vertically as was postulated in the CSM. Based on this information, TPH-d contamination is most likely the result of localized spills and not migration through the Muckpile or native material.

- Seven muck samples had concentrations of a group of SVOCs known as PAHs that exceeded the industrial soil PRGs. The PAHs found [(benzo(a)anthracene, benzo(a)pyrene, benzo(b)fluoranthene, dibenzo(a,h)anthracene, and indent $(1,2,3-\mathrm{cd})$ pyrene] were found in five samples from the Oil Spill and two samples from the muck. One of the muck samples was from near the outer edge of the Muckpile and the other was from the RMA. Both of these samples contained concentrations of benzo(a)pyrene.

- Radionuclide results were compared to the recommended screening limits in the National Council on Radiation Protection and Measurements Report No. 129 (NCRP, 1999) recommended screening limits for construction, commercial, industrial land-use scenarios (NCRP, 1999) scaled from 25 to 15 millirem per year (mrem/yr) dose and the 
generic guidelines for residual concentration of radionuclides in DOE Order 5400.5 (DOE, 1993).

The man-made radionuclide cesium-137 (Cs-137) was found in muck or sediment samples from the Muckpile, the Drainage, the Ponds, the Oil Spill, and the RMA. Cesium (Cs)-137 was also found in a single native soil sample from under the Muckpile as discussed above. Americium (Am)-241 was found in three muck samples from a centralized portion of the Muckpile. Plutonium-238 was found in one muck sample in the same centralized portion of the Muckpile. Plutonium-239 was found in four muck samples, three in the centralized portion and one on the outer edge of the Muckpile. Additionally, Cs-137, $\mathrm{Pu}-238$, and $\mathrm{Pu}-239$ were found in a preliminary muck sample just outside of the RMA.

Based on these results, the nature and extent of contamination at CAU 383 has been adequately characterized.

\subsubsection{Assessment Summary}

Details of the methods used and results found during the CAI are presented in Appendix A. A statistical analysis of the investigative results showed that the 90 percent confidence interval has been exceeded; therefore, CAU 383 has been adequately characterized, (see Appendix B). The results of the CAI meet the DQOs outlined in the CAIP. Based on these results, the nature and extent of the COCs at CAU 383 (Table 2-1 and 2-2) have been adequately identified to develop and evaluate corrective action alternatives.

\subsection{Need for Corrective Action}

Analytes detected during the CAI were evaluated using the action levels outlined in the CAIP (DTRA, 2003). Diesel-range organics, PAHs, and man-made radionuclides were found at CAU 383 at concentrations exceeding the action level. Based on the identification of COCs above the action levels at the E-Tunnel Sites, potential corrective action alternatives are identified and evaluated in this CADD to ensure worker, public, and environmental protection against potential exposure to COCs. 
Table 2-1

Chemical Sample Results that Exceed Action Levels

\begin{tabular}{|c|c|c|c|c|}
\hline Contaminant of Concern & $\begin{array}{c}\text { Samples Exceeding } \\
\text { Action Level }\end{array}$ & $\begin{array}{l}\text { Results } \\
\text { (mg/kg) }\end{array}$ & $\begin{array}{c}\text { Depth of } \\
\text { Sample (ft) }\end{array}$ & $\begin{array}{c}\text { Action Level }{ }^{1} \\
\text { (mg/kg) }\end{array}$ \\
\hline \multicolumn{5}{|c|}{ Muckpile Muck } \\
\hline Benzo(A)Pyrene & $383 \mathrm{MB} 1000.5$ & 1.3 & $0-1$ & 0.21 \\
\hline Diesel-Range Organics & $383 \mathrm{MB} 0500.5$ & 160 & $0-1$ & $100^{* *}$ \\
\hline Diesel-Range Organics & $383 \mathrm{MB} 0800.5$ & 300 & $0-1$ & $100^{* *}$ \\
\hline \multicolumn{5}{|c|}{ Muckpile Native } \\
\hline Diesel-Range Organics & 383MH0600.5 & 700 & $0-1$ & $100^{* *}$ \\
\hline \multicolumn{5}{|c|}{ Pond Sediment } \\
\hline Diesel-Range Organics & 383PH0216.0 & 330 & $16-17$ & $100^{* *}$ \\
\hline \multicolumn{5}{|c|}{ Oil Spill } \\
\hline Benzo(A)Anthracene & 383OB0700.5RR1 & 4.8 & $0-1$ & 2.1 \\
\hline Benzo(A)Anthracene & 383OB0800.5RR1 & 18 & $0-1$ & 2.1 \\
\hline Benzo(A)Pyrene & $3830 B 0300.5$ & 0.23 & $0-1$ & 0.21 \\
\hline Benzo(A)Pyrene & $3830 B 0500.5$ & 0.25 & $0-1$ & 0.21 \\
\hline Benzo(A)Pyrene & 383OB0700.5RR1 & 4.8 & $0-1$ & 0.21 \\
\hline Benzo(A)Pyrene & 383OB0800.5RR1 & 23 & $0-1$ & 0.21 \\
\hline Benzo(B)Fluoranthene & 383OB0200.5RR1 & 2.8 & $0-1$ & 2.1 \\
\hline Benzo(B)Fluoranthene & 383OB0700.5RR1 & 9.6 & $0-1$ & 2.1 \\
\hline Benzo(B)Fluoranthene & 383OB0800.5RR1 & 43 & $0-1$ & 2.1 \\
\hline Dibenzo(A,H)Anthracene & $383 \mathrm{OB} 0700.5$ & 2.1 & $0-1$ & 0.21 \\
\hline Dibenzo(A,H)Anthracene & $3830 B 0800.5$ & 10 & $0-1$ & 0.21 \\
\hline Indeno(1,2,3-Cd)Pyrene & 383OB0800.5RR1 & 12 & $0-1$ & 2.1 \\
\hline Diesel-Range Organics & $3830 B 0700.5$ & 120 & $0-1$ & $100^{* *}$ \\
\hline Diesel-Range Organics & $3830 B 0300.5$ & 330 & $0-1$ & $100^{* *}$ \\
\hline Diesel-Range Organics & $3830 B 0800.5$ & 490 & $0-1$ & $100^{* *}$ \\
\hline Diesel-Range Organics & $3830 B 0200.5$ & 2500 & $0-1$ & $100^{* *}$ \\
\hline Diesel-Range Organics & $3830 B 0100.5$ & 3400 & $0-1$ & $100^{* *}$ \\
\hline \multicolumn{5}{|c|}{ Radioactive Material Area } \\
\hline Benzo(A)Pyrene & 383RB0400.5 & 0.23 & $0-1$ & 0.21 \\
\hline
\end{tabular}

RR1 - Indicates the sample was reanalyzed at a higher dilution due to higher than expected results.

$\mathrm{mg} / \mathrm{kg}$ - Milligram per kilogram

${ }^{1}$ PALs (as per the CAU 383 CAIP)

${ }^{* *}$ Diesel-range organics (i.e., petroleum hydrocarbons) level is defined by NAC 445.2272 (NAC, 1996b). 
Table 2-2

Radiological Sample Results that Exceed Action Levels (Page 1 of 3)

\begin{tabular}{|c|c|c|c|c|}
\hline $\begin{array}{l}\text { Contaminant of } \\
\text { Concern }\end{array}$ & $\begin{array}{c}\text { Samples } \\
\text { Exceeding Action } \\
\text { Level }\end{array}$ & $\begin{array}{l}\text { Results } \\
\text { (pCi/g) }\end{array}$ & $\begin{array}{l}\text { Depth of Sample } \\
\text { (ft) }\end{array}$ & $\begin{array}{l}\text { Action Level }^{a} \\
(\mathrm{pCi} / \mathrm{g})\end{array}$ \\
\hline \multicolumn{5}{|c|}{ Muckpile Muck } \\
\hline Am-241 & 383МH0559.5 & 12.8 & $59-60$ & 7.62 \\
\hline Am-241 & $383 \mathrm{MH} 2 \mathrm{~A} 37.5$ & 23.5 & $37-38$ & 7.62 \\
\hline Am-241 & $383 \mathrm{MB} 0543.5$ & 28.8 & $43-44$ & 7.62 \\
\hline Cs-137 & 383MB1400.5 & 8.5 & $0-1$ & 7.3 \\
\hline Cs-137 & $383 \mathrm{MH} 1407.5$ & 10.7 & $7-8$ & 7.3 \\
\hline Cs-137 & 383MB0200.5 & 18.4 & $0-1$ & 7.3 \\
\hline Cs-137 & 383MB1500.5 & 20.7 & $0-1$ & 7.3 \\
\hline Cs-137 & $383 \mathrm{MH} 1215.0$ & 23.5 & $14.5-15.5$ & 7.3 \\
\hline Cs-137 & $383 \mathrm{MB} 0300.5$ & 24.2 & $0-1$ & 7.3 \\
\hline Cs-137 & $383 \mathrm{MH} 0722.5$ & 26.9 & $22-23$ & 7.3 \\
\hline Cs-137 & 383MB0500.5 & 29.3 & $0-1$ & 7.3 \\
\hline Cs-137 & 383MH1609.5 & 34.5 & $9-10$ & 7.3 \\
\hline Cs-137 & 383MB1114.5 & 37.4 & 14-15 & 7.3 \\
\hline Cs-137 & $383 \mathrm{MH} 1100.5$ & 47.3 & $0-1$ & 7.3 \\
\hline Cs-137 & $383 \mathrm{MH} 0723.5$ & 83.7 & $23-24$ & 7.3 \\
\hline Cs-137 & 383MB0912.5 & 135 & $12-13$ & 7.3 \\
\hline Cs-137 & 383МH0353.5 & 144 & $53-54$ & 7.3 \\
\hline Cs-137 & $383 \mathrm{MH} 2 \mathrm{~A} 37.5$ & 218 & $37-38$ & 7.3 \\
\hline Cs-137 & 383MB1403.5 & 240 & $3-4$ & 7.3 \\
\hline Cs-137 & 383MB0400.5 & 393 & $0-1$ & 7.3 \\
\hline Cs-137 & 383MH0559.5 & 471 & $59-60$ & 7.3 \\
\hline Cs-137 & 383MB0543.5 & 1,060 & $43-44$ & 7.3 \\
\hline Pu-238 & 383MB0543.5 & 15.6 & $43-44$ & 7.78 \\
\hline Pu-239 & 383MB0400.5 & 17.8 & $0-1$ & 7.62 \\
\hline Pu-239 & 383МH0559.5 & 25.3 & $59-60$ & 7.62 \\
\hline Pu-239 & $383 \mathrm{MH} 2 \mathrm{~A} 37.5$ & 80 & $37-38$ & 7.62 \\
\hline Pu-239 & 383MB0543.5 & 84 & $43-44$ & 7.62 \\
\hline \multicolumn{5}{|c|}{ Muckpile Native } \\
\hline Cs-137 & $383 \mathrm{MH} 2 \mathrm{~A} 45.0$ & 9.8 & $44.5-45.5$ & 7.3 \\
\hline \multicolumn{5}{|c|}{ Pond Sediment } \\
\hline Cs-137 & 383РH0101.0 & 19 & $0.5-1.5$ & 7.3 \\
\hline Cs-137 & 383PB0400.5 & 46.9 & $0-1$ & 7.3 \\
\hline Cs-137 & 383PB0300.5 & 56.3 & $0-1$ & 7.3 \\
\hline Cs-137 & 383РB0200.5 & 91 & $0-1$ & 7.3 \\
\hline Cs-137 & $383 \mathrm{PH} 0216.0$ & 142 & $15.5-16.5$ & 7.3 \\
\hline
\end{tabular}


Table 2-2

Radiological Sample Results that Exceed Action Levels (Page 2 of 3)

\begin{tabular}{|c|c|c|c|c|}
\hline $\begin{array}{l}\text { Contaminant of } \\
\text { Concern }\end{array}$ & $\begin{array}{c}\text { Samples } \\
\begin{array}{c}\text { Exceeding Action } \\
\text { Level }\end{array}\end{array}$ & $\begin{array}{c}\text { Results } \\
\text { (pCi/g) }\end{array}$ & $\begin{array}{l}\text { Depth of Sample } \\
\text { (ft) }\end{array}$ & $\begin{array}{l}\text { Action Level }{ }^{\mathrm{a}} \\
(\mathrm{pCi} / \mathrm{g})\end{array}$ \\
\hline \multicolumn{5}{|c|}{ Drainage } \\
\hline Cs-137 & $383 \mathrm{DH} 3300.5$ & 7.5 & $0-1$ & 7.3 \\
\hline Cs-137 & 383DH1100.5 & 7.52 & $0-1$ & 7.3 \\
\hline Cs-137 & 383DH3000.5 & 7.67 & $0-1$ & 7.3 \\
\hline Cs-137 & 383DH3100.5 & 8.4 & $0-1$ & 7.3 \\
\hline Cs-137 & 383DH1500.5 & 11.2 & $0-1$ & 7.3 \\
\hline Cs-137 & $383 \mathrm{DH} 3400.5$ & 11.7 & $0-1$ & 7.3 \\
\hline Cs-137 & 383DH0800.5 & 12.4 & $0-1$ & 7.3 \\
\hline Cs-137 & $383 \mathrm{DH} 3200.5$ & 12.7 & $0-1$ & 7.3 \\
\hline Cs-137 & $383 \mathrm{DH} 2800.5$ & 14 & $0-1$ & 7.3 \\
\hline Cs-137 & 383DH0600.5 & 18.4 & $0-1$ & 7.3 \\
\hline Cs-137 & 383DH1600.5 & 20.5 & $0-1$ & 7.3 \\
\hline Cs-137 & 383DH1800.5 & 23.1 & $0-1$ & 7.3 \\
\hline Cs-137 & 383DH1000.5 & 23.7 & $0-1$ & 7.3 \\
\hline Cs-137 & $383 \mathrm{DH} 2000.5$ & 24.1 & $0-1$ & 7.3 \\
\hline Cs-137 & $383 \mathrm{DH} 2600.5$ & 28.4 & $0-1$ & 7.3 \\
\hline Cs-137 & $383 \mathrm{DH} 2200.5$ & 29.3 & $0-1$ & 7.3 \\
\hline Cs-137 & $383 \mathrm{DH} 2400.5$ & 32 & $0-1$ & 7.3 \\
\hline Cs-137 & $383 \mathrm{DH} 2900.5$ & 33.1 & $0-1$ & 7.3 \\
\hline Cs-137 & 383DH1200.5 & 33.8 & $0-1$ & 7.3 \\
\hline Cs-137 & 383DH0100.5 & 34.4 & $0-1$ & 7.3 \\
\hline Cs-137 & $383 \mathrm{DH} 2300.5$ & 37.9 & $0-1$ & 7.3 \\
\hline Cs-137 & $383 \mathrm{DH} 2700.5$ & 41.7 & $0-1$ & 7.3 \\
\hline Cs-137 & 383DH1900.5 & 42.4 & $0-1$ & 7.3 \\
\hline Cs-137 & 383DH0200.5 & 45.8 & $0-1$ & 7.3 \\
\hline Cs-137 & 383DH0900.5 & 48.7 & $0-1$ & 7.3 \\
\hline Cs-137 & $383 \mathrm{DH} 2500.5$ & 55.6 & $0-1$ & 7.3 \\
\hline Cs-137 & 383DH1700.5 & 68 & $0-1$ & 7.3 \\
\hline Cs-137 & $383 \mathrm{DH} 1400.5$ & 68.8 & $0-1$ & 7.3 \\
\hline Cs-137 & $383 \mathrm{DH} 2100.5$ & 78.1 & $0-1$ & 7.3 \\
\hline \multicolumn{5}{|c|}{ Oil Spill } \\
\hline Cs-137 & $3830 B 0800.5$ & 13.6 & $0-1$ & 7.3 \\
\hline Cs-137 & $3830 B 0700.5$ & 20.5 & $0-1$ & 7.3 \\
\hline \multicolumn{5}{|c|}{ Radioactive Material Area } \\
\hline Cs-137 & 383RB0200.5 & 7.63 & $0-1$ & 7.3 \\
\hline Cs-137 & 383RB0300.5 & 23.4 & $0-1$ & 7.3 \\
\hline Cs-137 & 383RB0100.5 & 114 & $0-1$ & 7.3 \\
\hline
\end{tabular}


Table 2-2

Radiological Sample Results that Exceed Action Levels (Page 3 of 3)

\begin{tabular}{||c|c|c|c|c||}
\hline $\begin{array}{c}\text { Contaminant of } \\
\text { Concern }\end{array}$ & $\begin{array}{c}\text { Samples } \\
\text { Exceeding Action } \\
\text { Level }\end{array}$ & $\begin{array}{c}\text { Results } \\
\text { (pCi/g) }\end{array}$ & $\begin{array}{c}\text { Depth of Sample } \\
\text { (ft) }\end{array}$ & $\begin{array}{c}\text { Action Level } \\
\text { (pCi/g) }\end{array}$ \\
\hline \hline \multicolumn{3}{|c|}{ Preliminary } \\
\hline Cs-137 & $383 G B 0901$ & 180 & $0-1$ & 7.3 \\
\hline Pu-238 & $383 G B 0901$ & 11.3 & $0-1$ & 7.78 \\
\hline Pu-239 & 383GB0901 & 61 & $0-1$ & 7.62 \\
\hline
\end{tabular}

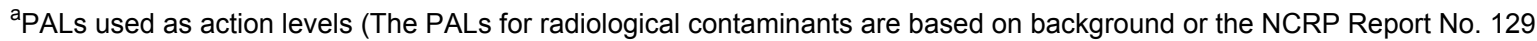
recommended screening limits for construction, commercial, and industrial land-use scenario (NCRP, 1999) scaled from 25 to $15 \mathrm{mrem} / \mathrm{yr}$ dose and the generic guidelines for residual concentration of radionuclides in DOE Order 5400.5 (DOE, 1993).

pCi/g - Picocuries per gram

\subsubsection{Nevada Administrative Code 445A.227 (2) (a-k) Analysis}

The Nevada Administrative Code (NAC) 445A.227 (2) (a-k) (NAC, 1996a) analysis is used to help determine if remediation is needed. The a-k analysis asks the following questions to help determine if a site needs to be remediated:

(a) The depth of any groundwater

(b) The distance to irrigation wells or wells for drinking water

(c) The type of soil that is contaminated

(d) The annual precipitation

(e) The type of waste or substance that was released

(f) The extent of the contamination

(g) The present and potential use for the land

(h) The preferred routes of migration

(i) The location of structures or impediments

(j) The potential for a hazard related to fire, vapor, or an explosion

(k) Any other information specifically related to the site which the director determines is appropriate

The following NAC 445A.227 (2) (a-k) analysis (NAC, 1996a) of the E-Tunnel Sites describes the factors to be considered in determining whether corrective action is required:

(a) Well ER-12-1 is located approximately a quarter mi from CAU 383. The depth to groundwater has been documented as $4,290 \mathrm{ft}$ above mean sea level or approximately $1,527 \mathrm{ft}$ below ground surface bgs (USGS, 2004). 
(b) The distance from CAU 383 to the nearest water-supply well (Well No. 1) is approximately $2.7 \mathrm{mi}$.

(c) The Muckpile and ponds were constructed on a thin veneer of alluvium overlying dolomites and interbedded limestones and dolomites. The Muckpile and RMA consist primarily of mining debris (rock) generated during excavation of the underground facilities, disturbed geologic materials and construction/reentry debris comprise the remainder of the Muckpile. The material in Ponds One, Two, and Three consists of fine-grained sediments that settled out of construction and groundwater from the E-Tunnel workings. The Drainage Area and Oil Spill consist of native fill.

(d) Average annual precipitation is approximately 6 to 8 inches (in.) (Winograd and Thordarson, 1975).

(e) The regulated substances released were diesel-range organics, PAHs, and man-made radionuclides.

(f) It is believed that, based on field screening and analytical data, the lateral extent of contamination encompasses all of CAU 383. The vertical extent of contamination based on field screening and analytical data, is limited to the muck and pond sediments (see Appendix A).

(g) The CAU is located on a government-controlled facility. The NTS is a restricted area that is guarded on a 24-hour, 365-day-per-year basis; unauthorized personnel are not admitted to the facility. Current and future land use is designated for training, high explosive tests, and other industrial uses.

(h) Migration of contaminants might result if storm water run-off suddenly exceeded the infiltration rate of the Muckpile and drainage channel soils.

(i) For the location of structures or impediments see Figure 2-1

(j) The potential for a hazard related to fire, vapor, or explosion is nonexistent for the COCs at the CAU.

(k) The PAHs found were benzo(a)anthracene, benzo(a)pyrene, benzo(b)fluoranthene dibenzo(a,h)anthracene, and indeno(1,2,3-cd)pyrene. Cesium-137 above action levels was found in 62 samples throughout the CAU. Contaminants, above action levels, were found only in the muck or pond sediment samples. No VOCs, RCRA metals or beryllium above action levels were found. 
Date: March 2005

Page 15 of 30

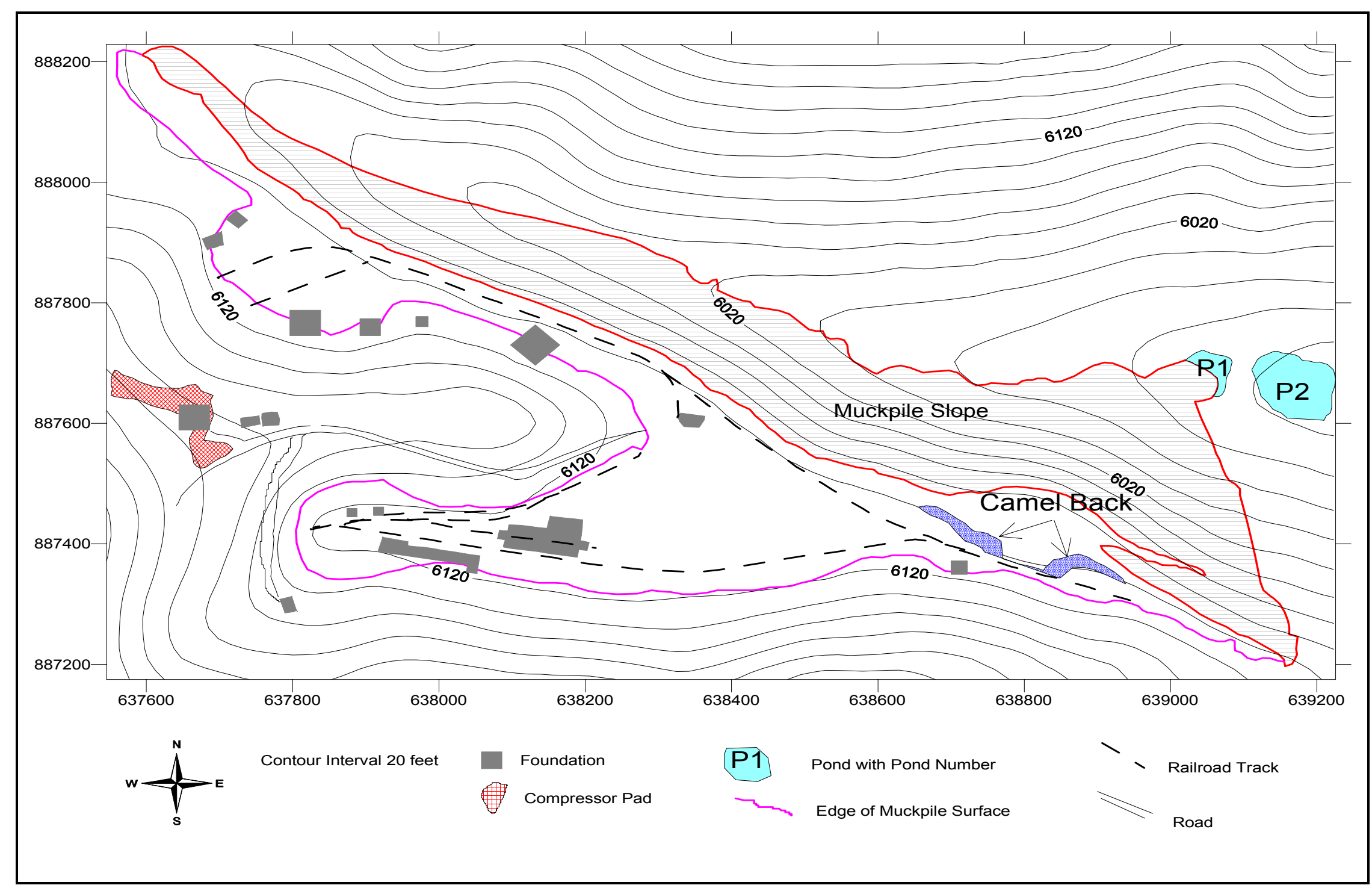

Figure 2-1

Location of Structures or Impediments at E-Tunnel Sites 


\subsection{Evaluation of Alternatives}

At the completion of the first DTRA Muckpile characterization, the Nevada Division of Environmental Protection (NDEP) made the determination that the Muckpiles needed to be closed in accordance with applicable solid waste disposal site administrative codes. The following section presents the corrective action objectives for CAU 383, describes the general standards and decision factors used to screen the corrective action alternatives, and develops and evaluates a set of corrective action alternatives that could be used to meet the corrective action objectives.

\subsection{Corrective Action Objectives}

The corrective action objectives are media-specific goals for protecting human health and the environment. Based on the potential exposure pathways (see Section 2.2.1), the following corrective action objectives have been identified for CAU 383:

- Prevent or mitigate exposure to near-surface soils containing COCs at concentrations exceeding the action levels defined in the CAIP (DTRA, 2003).

- Prevent migration of COCs beyond the CAU boundary.

- Prevent adverse impacts to groundwater quality.

As identified in the CAIP, the future use for the CAU is assumed to be industrial, similar to current use (DOE/NV, 1998). As part of the CAIP (DTRA, 2003), a CSM was developed that identified the potential exposure mechanism as disturbance (excavation) of contaminated soil by site workers. This implies a potential exposure pathway through ingestion, inhalation, and/or dermal contact with contaminated media under industrial scenarios.

\subsection{Screening Criteria}

The screening criteria are used to develop corrective action alternatives, evaluate each alternative, and select the preferred corrective action alternative. These criteria are identified in the EPA Guidance on RCRA Corrective Action Decision Documents (EPA, 1991), the RCRA Corrective Action Plan, Final (EPA, 1994), and NAC 445A.227, "Contamination of soil: Order by Director for corrective action; factors to be considered in determining whether corrective action is required" (NAC, 1996a). 
Corrective action alternatives will be developed and evaluated based on four general corrective action standards and five remedy selection decision factors. All corrective action alternatives must meet the general standards to be selected for evaluation using the remedy selection decision factors. The general corrective action standards are:

- Protection of human health and the environment

- Compliance with media cleanup standards

- Control the source(s) of the release

- Compliance with applicable federal, state, and local standards for waste management

The remedy selection decision factors are:

- Short-term reliability and effectiveness

- Reduction of toxicity, mobility, and/or volume

- Long-term reliability and effectiveness

- Feasibility

- Cost

\subsubsection{Corrective Action Standards}

The following sections describe the corrective action standards used to evaluate each corrective action alternative.

\section{Protection of Human Health and the Environment}

Protection of human health and the environment is a general mandate of the RCRA statute (CFR, 1999). This mandate requires that the corrective action include any necessary protective measures. These measures may or may not be directly related to media cleanup, source control, or management of wastes. Because the corrective action objectives are directed by the goal of protection of human health and the environment, the corrective action alternatives are evaluated for their ability to meet corrective action objectives as defined in Section 3.1.

\section{Compliance with Media Cleanup Standards}

Each corrective action alternative must have the ability to meet the proposed media cleanup standards as set forth in applicable state and federal regulations and as specified in the CAIP (DTRA, 2003). For this CAU, EPA's Region 9 PRGs (EPA, 2002), which are derived from data in the Integrated Risk Information System, and NAC 445A.227 (NAC, 1996a) are the basis for establishing the action levels for chemical contaminants. Isotopic or total activity above the limits listed in NCRP Report No. 129 are the basis for the action levels set for radionuclides. 


\section{Control the Source(s) of the Release}

An objective of a corrective action remedy is to stop further environmental degradation by controlling or eliminating additional releases that may pose a threat to human health and the environment. Unless source control measures are taken, efforts to clean up releases may be ineffective or, at best, will essentially involve a perpetual cleanup. Therefore, each corrective action alternative must use an effective source control program to ensure the long-term effectiveness and protection offered by the corrective action.

\section{Comply with Applicable Federal, State, and Local Standards for Waste Management}

During implementation of any corrective action alternative, all waste management activities must be conducted in accordance with applicable state and federal regulations (e.g., Nevada Revised Statutes [NRS] 459.400 - 459.600, "Disposal of Hazardous Waste" [NRS, 1995]; Title 40 Code of Federal Regulations (CFR) Parts 260 - 282, "RCRA Regulations" [CFR, 1999]; NAC 444, "Solid Waste Disposal" [NAC, 2002]; and NAC 459.9974, "Disposal and Evaluation of Contaminated Soil" [NAC, 1999]). The requirements for management of the waste, if any, derived from the corrective action will be determined based on applicable state and federal regulations, field observations, process knowledge, characterization data, and data collected and analyzed during corrective action implementation. Administrative controls (e.g., decontamination procedures and corrective action strategies) will minimize waste generated during site corrective action activities. Decontamination activities will be performed in accordance with approved procedures and will be determined by the COCs present at the site.

\subsubsection{Remedy Selection Decision Factors}

The following text describes the remedy selection decision factors used to evaluate the corrective action alternatives.

\section{Short-Term Reliability and Effectiveness}

Each corrective action alternative must be evaluated with respect to its effects on human health and the environment during the construction and implementation of the corrective action. The following factors will be addressed for each alternative:

- Protection of the community from potential risks associated with implementation such as fugitive dust, transportation of hazardous materials, and explosion.

- Protection of workers during construction and implementation. 
- Environmental impacts that may result from construction and implementation.

- The amount of time until the corrective action objectives are achieved.

\section{Reduction of Toxicity, Mobility, and/or Volume}

Each corrective action alternative must be evaluated for its ability to reduce the toxicity, mobility, and/or volume of the contaminated medium. Reduction in toxicity, mobility, and/or volume refers to changes in one or more characteristics of the contaminated medium by the use of corrective measures that decrease the inherent threats associated with that medium.

\section{Long-Term Reliability and Effectiveness}

Each corrective action alternative must be evaluated in terms of risk remaining at the CAU after the corrective action alternative has been implemented. The primary focus of this evaluation is on the extent and effectiveness of the controls that may be required to manage risk posed by treatment residuals and/or untreated wastes.

\section{Feasibility}

The feasibility criterion addresses the technical and administrative feasibility of implementing a corrective action alternative and the availability of services and materials needed during implementation. Each corrective action alternative must be evaluated for the following criteria:

- Construction and Operation - refers to the feasibility of implementing a corrective action alternative given the existing set of waste and site-specific conditions.

- Administrative Feasibility - refers to the administrative activities needed to implement the corrective action alternative (e.g., permits, public acceptance, rights-of-way, off-site approval).

- Availability of Services and Materials - refers to the availability of adequate off-site and on-site treatment, storage capacity, disposal services, necessary technical services and materials, and prospective technologies for each corrective action alternative.

\section{Cost}

Rough order of magnitude estimates for each alternative are presented in Appendix $\mathrm{C}$ for comparison purposes only. The cost estimate for each corrective action alternative includes applicable capital and operation and maintenance costs. The following is a brief description of each component: 
- Capital Costs - Direct costs may consist of engineering design, materials, labor, mobilization, demobilization, site preparation, equipment purchase and/or rental, equipment decontamination, sampling and analysis, waste disposal, health and safety measures, permits and/or fees, start-up costs, and any contingency allowances.

- Operation and Maintenance - These costs include labor, training, sampling and analysis, maintenance materials, utilities, and health and safety measures.

- Cost summaries for each alternative are provided in Appendix C.

\subsection{Development of Corrective Action Alternatives}

This section identifies and briefly describes the viable corrective action technologies and the corrective action alternatives considered for the affected media. Based on the review of existing data, future use, and current operations at the NTS, the following alternatives have been developed for consideration at CAU 383:

- Alternative 1 - No Action (developed for the purpose of providing a baseline).

- Alternative 2 - Clean Closure by Excavation of the Oil Spill; RMA; Muckpile; Ponds One, Two, and Three; and Drainage Area.

- Alternative 3 - Closure by Excavation of the Oil Spill and RMA, Grading and Capping of the Muckpile, Drainage Controls, and Administrative Controls.

\subsubsection{Alternative 1 - No Action}

Under the No Action alternative, no corrective action activities would be implemented. This alternative does not comply with the NAC for closure of a solid waste disposal site. This alternative is used as a starting point to establish a baseline for comparison with the other corrective action alternatives.

The estimated cost for Alternative 1 is $\$ 0$.

\subsubsection{Alternative 2 - Clean Closure by Excavation of the Oil Spill; Radioactive Material Area; Muckpile; Ponds One, Two, and Three; and Drainage Area}

Alternative 2 consists of removing muck with COC concentrations greater than the action levels at the Oil Spill (CAS 12-25-02); the RMA (CAS 12-28-02); the E-Tunnel Muckpile (12-06-06); Ponds One, Two, and Three; and the Drainage Area. Due to the wide spread contamination, clean closure requires removing all of the non-native soil in the oil spill, ponds, and Muckpile, which 
includes the RMA, and most of the surface soil in the drainage. Contaminated soils will be removed and disposed of in the Area 3 Radioactive Waste Management Site.

Activities will include excavating, containerizing, hauling and disposing of approximately 390,000 cubic yards of contaminated muck and sediment to the Area 3 Radioactive Waste Management site, confirmation sampling, importing native soil, and revegetation.

The estimated cost for Alternative 2 is $\$ 108,508,000$.

\subsubsection{Alternative 3 - Closure by Excavation of the Oil Spill and Radioactive Material Area, Grading and Capping of the Muckpile, Drainage Controls, and Administrative Controls}

Alternative 3 consists of removing soil with COC concentrations greater than the action levels at the Oil Spill (CAS 12-25-02) and the RMA (CAS 12-28-02), grading and capping the E-Tunnel Muckpile (CAS 12-06-06), installing drainage controls, and installing signs around the Muckpile and drainage to warn of the danger of unauthorized intrusion.

Under this alternative, impacted soils in the Oil Spill, the surface soil on the road leading to the Oil Spill, and the RMA will be removed. The removed soils will be deposited on the Muckpile and/or in E-Tunnel Ponds One, Two, and/or Three. Activities will include excavation and relocation of approximately 2,000 cubic yards of contaminated soil to the Muckpile or ponds, confirmation sampling, and backfilling the oil spill with clean material. The RMA is located near the edge of the Muckpile; therefore, it will be excavated and moved onto the Muckpile or to the ponds where it will be covered by the cap as part of the Muckpile closure.

The Muckpile will be graded using cut and fill design, away from the tunnel portals and over Ponds One, Two, and Three; the Drainage Area; and portions of the access road to achieve a 3 to 1 side slope. Since the graded Muckpile will extend across the drainage area and access road, a new drainage channel will be constructed and/or culvert installed to allow drainage of the upper reaches of the canyon. To accommodate the changes to the drainage a new access road will be constructed.

Capping the Muckpile will consist of covering the Muckpile with native soil. Drainage controls will consist of constructing diversion channels and chutes to direct and control storm water. The entire CAU will be revegetated. 
To avert intrusion into the Muckpile, use restrictions will be recorded and appropriate signage will be installed.

The estimated cost for Alternative 3 is $\$ 14,773,000$.

\subsection{Evaluation and Comparison of Alternatives}

The general corrective action standards, remedy selection decision factors, and a-k analysis described in Section 2.2 were used to conduct detailed and comparative analyses of each corrective action alternative. The advantages and disadvantages of each alternative were assessed to select a preferred alternative for CAU 383. Table 3-1 presents a summary of the detailed analysis of the alternatives. Table 3-2 provides a comparative evaluation of the alternatives. 
Table 3-1

\section{Detailed Evaluation of Corrective Action Alternatives}

(Page 1 of 3 )

\begin{tabular}{|c|c|c|c|}
\hline Evaluation Criteria & $\begin{array}{l}\text { Alternative } 1 \\
\text { No Action }\end{array}$ & $\begin{array}{c}\text { Alternative } 2 \\
\text { Clean Closure by Excavation of the Oil Spill; } \\
\text { Radioactive Material Area; Muckpile; Ponds } \\
\text { One, Two, and Three; and Drainage Area }\end{array}$ & $\begin{array}{c}\text { Alternative } 3 \\
\text { Closure by Excavation of the Oil Spill and } \\
\text { Radioactive Material Area, Grading and } \\
\text { Capping of the Muckpile, Drainage Controls, } \\
\text { and Administrative Controls }\end{array}$ \\
\hline \multicolumn{4}{|c|}{ Closure Standards } \\
\hline $\begin{array}{l}\text { Protection of Human } \\
\text { Health and the } \\
\text { Environment }\end{array}$ & $\begin{array}{l}\text { Does not meet corrective action } \\
\text { objectives for preventing or } \\
\text { controlling inadvertent intrusion into } \\
\text { a contaminated soil zone. } \\
\text { No worker exposure or risk } \\
\text { associated with implementation. } \\
\text { Does not prevent migration of COCs } \\
\text { beyond the CAU. } \\
\text { Long-term risk to the public is low } \\
\text { because of remote location and } \\
\text { controlled access. } \\
\text { NAC } 445 \text { A.227 (2) (a-k) analysis } \\
\text { (Section 2.3) shows groundwater is } \\
\text { protected. }\end{array}$ & $\begin{array}{l}\text { Controls inadvertent intrusion into a contaminated } \\
\text { soil zone. } \\
\text { Increased risk of short-term NTS worker exposure } \\
\text { to contamination during transportation of } \\
\text { contaminated soil. } \\
\text { Increased risk of short-term remediation worker } \\
\text { exposure to contaminated material and } \\
\text { occupational hazards during excavation, } \\
\text { transportation, and construction activities. } \\
\text { Long-term risk to the public is low because of } \\
\text { remote location and controlled access. } \\
\text { NAC } 445 \text { A.227 (2) (a-k) analysis (Section 2.3) } \\
\text { shows groundwater is protected. } \\
\text { Controls further migration of surface contamination. }\end{array}$ & $\begin{array}{l}\text { Controls inadvertent intrusion into a contaminated } \\
\text { soil zone. } \\
\text { Increased risk of short-term remediation worker } \\
\text { exposure to contaminated material and } \\
\text { occupational hazards during excavation, } \\
\text { transportation, and construction activities. } \\
\text { Long-term risk to the public is low because of } \\
\text { remote location and controlled access. } \\
\text { NAC } 445 \text { A. } 227(2) \text { (a-k) analysis (Section } 2.3) \\
\text { shows groundwater is protected } \\
\text { Controls further migration of surface contamination. }\end{array}$ \\
\hline $\begin{array}{l}\text { Compliance with Media } \\
\text { Cleanup Standards }\end{array}$ & $\begin{array}{l}\text { Does not comply with Media Cleanup } \\
\text { Standards. }\end{array}$ & Standards achieved by removal of contaminants. & $\begin{array}{l}\text { Standards achieved by removal, covering, and } \\
\text { limiting pathways for contaminant migration. }\end{array}$ \\
\hline $\begin{array}{l}\text { Control the Source(s) of } \\
\text { Release }\end{array}$ & $\begin{array}{l}\text { Release of contamination from site is } \\
\text { not controlled. }\end{array}$ & $\begin{array}{l}\text { Source is removed and relocated to a permitted } \\
\text { facility. Therefore, the source of release from the } \\
\text { areas is controlled. }\end{array}$ & $\begin{array}{l}\text { Although the release source is not removed, the } \\
\text { mechanisms for transport or release of } \\
\text { contamination are limited by relocating, covering } \\
\text { the contaminated areas, and installing drainage } \\
\text { controls. }\end{array}$ \\
\hline
\end{tabular}


Table 3-1

Detailed Evaluation of Corrective Action Alternatives

\section{(Page 2 of 3 )}

\begin{tabular}{|c|c|c|c|}
\hline Evaluation Criteria & $\begin{array}{l}\text { Alternative } 1 \\
\text { No Action }\end{array}$ & $\begin{array}{c}\text { Alternative } 2 \\
\text { Clean Closure by Excavation of the Oil Spill; } \\
\text { Radioactive Material Area; Muckpile; Ponds } \\
\text { One, Two, and Three; and Drainage Area }\end{array}$ & $\begin{array}{c}\text { Alternative } 3 \\
\text { Closure by Excavation of the Oil Spill and } \\
\text { Radioactive Material Area, Grading and } \\
\text { Capping of the Muckpile, Drainage Controls, } \\
\text { and Administrative Controls } \\
\end{array}$ \\
\hline $\begin{array}{l}\text { Comply with Applicable } \\
\text { Federal, State, and Local } \\
\text { Standards for Waste } \\
\text { Management }\end{array}$ & No waste generated. & $\begin{array}{l}\text { All waste (primarily contaminated soil) will be } \\
\text { handled and disposed of in accordance with } \\
\text { applicable standards. }\end{array}$ & $\begin{array}{l}\text { All waste will be handled and disposed of in } \\
\text { accordance with applicable standards. }\end{array}$ \\
\hline \multicolumn{4}{|c|}{ Selection Decision Factors } \\
\hline $\begin{array}{l}\text { Short-Term Reliability and } \\
\text { Effectiveness }\end{array}$ & $\begin{array}{l}\text { No change from current conditions, } \\
\text { does not protect the environment. }\end{array}$ & $\begin{array}{l}\text { Is reliable but not fully effective for the short term } \\
\text { because of risk of spillage during transport. } \\
\text { Moderate risks associated with excavation and } \\
\text { transport of large volumes of contaminated media. } \\
\text { Increases potential exposure to soils containing } \\
\text { COCs at concentrations exceeding the action levels } \\
\text { defined in the CAIP (DTRA, 2003) in the short term. }\end{array}$ & $\begin{array}{l}\text { Is reliable but not fully effective for the short term } \\
\text { because of risk of spillage during transport. } \\
\text { Increases potential exposure to soils containing } \\
\text { COCs at concentrations exceeding the action levels } \\
\text { defined in the CAIP (DTRA, 2003) in the short term. }\end{array}$ \\
\hline $\begin{array}{l}\text { Reduction of Toxicity, } \\
\text { Mobility, and/or Volume }\end{array}$ & $\begin{array}{l}\text { Does not change any of these } \\
\text { criteria. }\end{array}$ & $\begin{array}{l}\text { The mobility of the contaminants in the soil will be } \\
\text { reduced by removing and relocating them to a } \\
\text { permitted facility. The toxicity and volume will } \\
\text { remain the same but at a permitted facility. }\end{array}$ & $\begin{array}{l}\text { The mobility of the contaminants in the Oil Spill and } \\
\text { RMA will be reduced by disposing of them on the } \\
\text { Muckpile and/or ponds. Mobility of the } \\
\text { contaminants in the Muckpile and ponds will be } \\
\text { reduced by capping and limiting the potential for } \\
\text { intrusion into the contaminated areas. The toxicity } \\
\text { and volume will remain the same. }\end{array}$ \\
\hline $\begin{array}{l}\text { Long-Term Reliability and } \\
\text { Effectiveness }\end{array}$ & $\begin{array}{l}\text { No change from current conditions, } \\
\text { does not protect the environment. } \\
\text { NTS is a restricted access facility, } \\
\text { which minimizes potential risk to the } \\
\text { general public. }\end{array}$ & $\begin{array}{l}\text { NTS is a restricted access facility, which minimizes } \\
\text { potential risk to the general public. } \\
\text { Long-term responsibility for disposition of wastes } \\
\text { remains. }\end{array}$ & $\begin{array}{l}\text { Periodic inspection and maintenance will be } \\
\text { needed to ensure the long-term reliability and } \\
\text { effectiveness. } \\
\text { NTS is a restricted access facility, which minimizes } \\
\text { potential risk to the general public. } \\
\text { Long-term responsibility for disposition of wastes } \\
\text { remains. }\end{array}$ \\
\hline
\end{tabular}


Table 3-1

Detailed Evaluation of Corrective Action Alternatives

(Page 3 of 3 )

\begin{tabular}{|c|c|c|c|}
\hline Evaluation Criteria & $\begin{array}{l}\text { Alternative } 1 \\
\text { No Action }\end{array}$ & $\begin{array}{c}\text { Alternative } 2 \\
\text { Clean Closure by Excavation of the Oil Spill; } \\
\text { Radioactive Material Area; Muckpile; Ponds } \\
\text { One, Two, and Three; and Drainage Area }\end{array}$ & $\begin{array}{c}\text { Alternative } 3 \\
\text { Closure by Excavation of the Oil Spill and } \\
\text { Radioactive Material Area, Grading and } \\
\text { Capping of the Muckpile, Drainage Controls, } \\
\text { and Administrative Controls }\end{array}$ \\
\hline Feasibility & $\begin{array}{l}\text { Community Advisory Board } \\
\text { notification required. }\end{array}$ & $\begin{array}{l}\text { Large volume of impacted media to transport and } \\
\text { dispose of. } \\
\text { Is feasible using existing technologies. }\end{array}$ & $\begin{array}{l}\text { Community Advisory Board notification required. } \\
\text { Appropriate placarding, and signage must be } \\
\text { installed. } \\
\text { Is feasible using existing technologies. }\end{array}$ \\
\hline Cost & $-0-$ & $\$ 108,508,000$ & $\$ 14,773,000$ \\
\hline
\end{tabular}


Table 3-2

Comparative Evaluation of Alternatives

\begin{tabular}{|c|c|}
\hline Evaluation Criteria & $\begin{array}{l}\text { Alternative } 1 \text { - No Action } \\
\text { Alternative } 2 \text { - Clean Closure by Excavation of the Oil Spill; RMA; Muckpile; Ponds One, } \\
\text { Two, and Three; and Drainage area } \\
\text { Alternative } 3 \text { - Closure by Excavation of the Oil Spill and RMA, Grading and Capping of } \\
\text { the Muckpile, Drainage Controls, and Administrative Controls }\end{array}$ \\
\hline \multicolumn{2}{|r|}{ Closure Standards } \\
\hline $\begin{array}{l}\text { Protection of Human } \\
\text { Health and the } \\
\text { Environment }\end{array}$ & $\begin{array}{l}\text { Alternative } 1 \text { is least protective in the long term because it does nothing to prevent the } \\
\text { contamination from migrating. Alternatives } 2 \text { and } 3 \text { meet the corrective action } \\
\text { objectives. Alternative } 2 \text { is most intrusive with greatest risk of exposure and } \\
\text { occupational risks associated with excavation of contaminated media. Alternative } 3 \text { has } \\
\text { lower associated health risks. NAC 445A.227 (2) (a-k) analysis shows the contaminants } \\
\text { are not threatening groundwater in either alternative. In addition, the site resides on } \\
\text { controlled land, which decreases the risk of exposure to the public. }\end{array}$ \\
\hline $\begin{array}{l}\text { Compliance with } \\
\text { Media Cleanup } \\
\text { Standards }\end{array}$ & $\begin{array}{l}\text { Alternative } 1 \text { does not comply because no action is taken. Alternatives } 2 \text { and } 3 \text { achieve } \\
\text { compliance with Media Cleanup Standards, Alternative } 2 \text { by removing the affected } \\
\text { media, Alternative } 3 \text { by moving and covering affected media and controlling pathways for } \\
\text { mobility. Monitoring of seasonal runoff may be required to control migration of shallow } \\
\text { subsurface contaminants for Alternative } 3 \text {. }\end{array}$ \\
\hline $\begin{array}{l}\text { Control the Source(s) } \\
\text { of Release }\end{array}$ & $\begin{array}{l}\text { Alternative } 1 \text { does nothing to control the source. Alternative } 2 \text { removes all contamination } \\
\text { for disposal at a permitted location. Alternative } 3 \text { controls the source by moving the Oil } \\
\text { Spill and RMA soils then covering all the contaminated soils, reducing the permeability of } \\
\text { the surface soils, and installing run-off controls. Administrative controls in Alternative } 3 \\
\text { further mitigate the potential for human exposure to affected media. }\end{array}$ \\
\hline $\begin{array}{l}\text { Comply with } \\
\text { Applicable Federal, } \\
\text { State, and Local } \\
\text { Standards for Waste } \\
\text { Management }\end{array}$ & $\begin{array}{l}\text { Alternative } 1 \text { does not generate any wastes. Waste volumes would be significantly } \\
\text { greater for Alternative } 2 \text { than Alternative } 3 \text {. Any waste generated would be handled in } \\
\text { accordance with applicable standards. }\end{array}$ \\
\hline \multicolumn{2}{|r|}{ Remedy Selection Decision Factors } \\
\hline $\begin{array}{l}\text { Short-Term Reliability } \\
\text { and Effectiveness }\end{array}$ & $\begin{array}{l}\text { Alternative } 1 \text { is not reliable or effective for the short term as nothing is changed from the } \\
\text { current conditions. Alternative } 2 \text { is slightly less reliable and effective in the short term } \\
\text { only in the potential for spills during excavation and transportation of large quantities of } \\
\text { soil. Otherwise Alternatives } 2 \text { and } 3 \text { offer the same degree of short-term reliability and } \\
\text { effectiveness. }\end{array}$ \\
\hline $\begin{array}{l}\text { Reduction of Toxicity, } \\
\text { Mobility, and/or } \\
\text { Volume }\end{array}$ & $\begin{array}{l}\text { Alternative } 1 \text { does nothing to reduce the toxicity, mobility, or volume. Alternative } 2 \\
\text { removes the contamination and reduces the potential for migration of the contaminants, } \\
\text { although additional mobilization of contaminants could occur during handling of affected } \\
\text { media. Alternative } 3 \text { reduces the potential for migration by moving the Oil Spill and RMA } \\
\text { soils then covering all the contaminated soils, compacting the surface to prevent } \\
\text { intrusion by water, and the installation of drainage controls. Alternatives } 2 \text { and } 3 \\
\text { decrease the mobility caused by human intrusion. Neither alternative changes the } \\
\text { toxicity or the volume. }\end{array}$ \\
\hline $\begin{array}{l}\text { Long-Term Reliability } \\
\text { and Effectiveness }\end{array}$ & $\begin{array}{l}\text { Alternative } 1 \text { has no long-term reliability or effectiveness. Alternative } 2 \text { achieves } \\
\text { long-term reliability and effectiveness, but long-term responsibility for disposition of } \\
\text { wastes remains. Alternative } 3 \text { may only achieve long-term effectiveness by periodic } \\
\text { inspection and maintenance of the site. }\end{array}$ \\
\hline Feasibility & All alternatives are feasible using existing resources and technologies. \\
\hline Cost & $\begin{array}{llll}\text { Alternative } 2 & \$ 108,508,000 & \text { Alternative } 3 \quad \$ 14,773,000 \\
\end{array}$ \\
\hline
\end{tabular}




\subsection{Recommended Alternative}

Based on the results of the detailed and comparative analysis of the potential corrective action alternatives presented in this document, the preferred corrective action alternative for implementation at CAU 383 is Alternative 3 - Closure by Excavation of the Oil Spill and RMA, Grading and Capping of the Muckpile, Drainage Controls, and Administrative Controls.

Alternative 3 was chosen for the following reasons:

- Risks associated with excavation and transport of large volumes of contaminated media over long distances is eliminated

- The potential for remediation worker exposure to contaminated media is reduced as compared to Alternative 2 because less material is being excavated.

- The contaminated soil will be covered with clean backfill.

- The drainage controls will adequately reduce the contaminant mobility.

- Waste generated will be minimized.

The corrective action alternatives were evaluated on their technical merits and compliance with state and federal regulations, focusing on safety, reliability, feasibility, and performance.

Alternative 3 was judged to best meet all requirements for the technical components evaluated and compliance with all applicable state and federal regulations for closure of the site and will eliminate potential future exposure pathways to the contaminated soil at the Area 12 E-Tunnel Sites.

Based on the evaluation in this CADD, the preferred closure method for CAU 383 is closure by excavation of the Oil Spill and RMA, grading and capping of the Muckpile, engineered drainage controls, and administrative controls. 


\subsection{References}

BN, see Bechtel Nevada.

Bechtel Nevada. 1995. Nevada Test Site Performance Objective for Certification of Nonradioactive Hazardous Waste. Las Vegas, NV.

CFR, see Code of Federal Regulations.

Code of Federal Regulations. 1999. Title 40 Part 260 - 282, "Protection of the Environment: RCRA Regulations." Washington, DC: Government Printing Office.

DOE, see U.S. Department of Energy.

DOE/NV, see U.S. Department of Energy, Nevada Operations Office.

DTRA, see Defense Threat Reduction Agency.

Defense Threat Reduction Agency. 2003. Corrective Action Investigation Plan for Corrective Action Unit 383: Area 12 E-Tunnel Sites, Nevada Test Site, Nevada, Rev. 0. Prepared by SNJV. Las Vegas, NV.

EPA, see U.S. Environmental Protection Agency.

FFACO, see Federal Facility Agreement and Consent Order.

Federal Facility Agreement and Consent Order. 1996 (as amended). Agreed to by the State of Nevada, the U.S. Department of Energy, and the U.S. Department of Defense.

McArthur, R.D., and F.L. Miller, Jr. 1989. Offsite Radiation Exposure Review Project (ORERP), Phase II Soils Programs, DOE/NV/10384-23. Las Vegas, NV: Desert Research Institute.

NAC, see Nevada Administrative Code.

NBMG, see Nevada Bureau of Mines and Geology.

NCRP, see National Council on Radiation Protection and Measurements.

NNSA/NV, see U.S. Department of Energy, National Nuclear Security Administration Nevada Operations Office.

NRS, see Nevada Revised Statutes. 
National Council on Radiation Protection and Measurements. 1999. Recommended Screening Limits for Contaminated Surface Soil and Review of Factors Relevant to Site-Specific Studies. Report No. 129. Bethesda, MD.

Nevada Administrative Code. 1996a. NAC 445A.227, "Contamination of Soil: Order by Director for Corrective Action Factors to be Considered in Determining Whether Corrective Action is Required." Carson City, NV.

Nevada Administrative Code. 1996b. NAC 445A.2272, "Contamination of Soil: Establishment of Action Levels.” Carson City, NV.

Nevada Administrative Code. 1999. NAC 459.9974, "Disposal and Evaluation of Contaminated Soil." Carson City, NV.

Nevada Administrative Code. 2002. NAC 444, "Solid Waste Disposal." Carson City, NV.

Nevada Bureau of Mines and Geology. 1998. Mineral and Energy Resource Assessment of the Nellis Air Force Range, Open File Report 98-1. Las Vegas, NV.

Nevada Revised Statutes. 1995. NRS 459.400-459-600, “Disposal of Hazardous Waste.” Carson City, NV.

SNJV, see Stoller-Navarro Joint Venture.

Shacklette, H.T., and J.G. Boerngen. 1984. Element Concentrations in Soils and Other Surficial Materials of the Conterminous United States, U.S. Geological Survey Professional Paper 1270. Washington, DC: U.S. Government Printing Office.

Stoller-Navarro Joint Venture. 2004. SNJV Site-Specific Health and Safety Plan, Corrective Action Unit 383: Area 12 E-Tunnel Sites, Nevada Test Site, Rev. 0. Las Vegas, NV.

USGS/DOE, see U.S. Geological Survey and U.S. Department of Energy.

U.S. Department of Energy. 1993. DOE Order 5400.5 Change 2, "Radiation Protection of the Public and Environment." Washington, DC: U.S. Government Printing Office.

U.S. Department of Energy, National Nuclear Security Administration Nevada Operations Office. 2002. Industrial Sites Quality Assurance Project Plan, Nevada Test Site, Nevada, DOE/NV--372, Rev. 3. Las Vegas, NV.

U.S. Department of Energy, Nevada Operations Office. 1996. Final Environmental Impact Statement for the Nevada Test Site and Off-Site Locations in the State of Nevada, DOE/EIS 0243, August. Las Vegas, NV. 
U.S. Department of Energy, Nevada Operations Office. 1998. Resource Management Plan, DOE/NV--518. Las Vegas, NV.

U.S. Environmental Protection Agency. 1991. Guidance on RCRA Corrective Action Decision Documents, EPA/ 540/G-91/011. Washington, DC: Office of Solid Waste and Emergency Response.

U.S. Environmental Protection Agency. 1994. Resource Conservation and Recovery Act Corrective Action Plan, Final EPA/520-R-94-004. Washington, DC: Offices of Research and Development.

U.S. Environmental Protection Agency. 2002. Region 9 Preliminary Remediation Goals. San Francisco, CA.

U.S. Geological Survey. 2004. "USGS/DOE Nevada Water Wells." As accessed at http://nevada.usgs.gov/doe_nv/sitepage_temp.cfm?site_id=371106116110401 on 8 March.

Winograd, I.J., and W. Thordarson. 1975. Hydrologic and Hydrochemical Framework, South Central Great Basin Nevada-California, with Special Reference to the Nevada Test Site, U.S. Geological Survey Professional Paper 712-C. Denver, CO. 


\title{
Appendix A
}

\section{Corrective Action Investigation Summary Report for Corrective Action Unit 383: Area 12, E-Tunnel Sites, Nevada Test Site}

\author{
Prepared by \\ Defense Threat Reduction Agency \\ Nevada Operations Office \\ Mercury, Nevada \\ and \\ U.S. Department of Energy \\ National Nuclear Security Administration \\ Nevada Site Office \\ Las Vegas, Nevada
}

January 2005

Approved for public release; further dissemination unlimited. 


\section{Table of Contents}

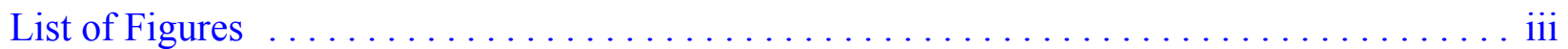

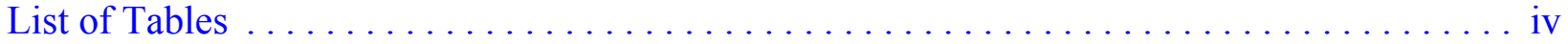

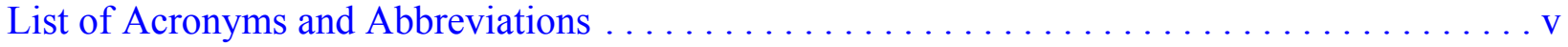

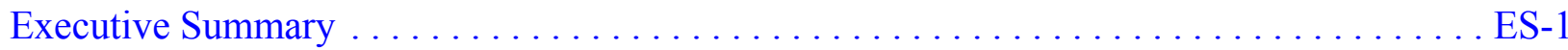

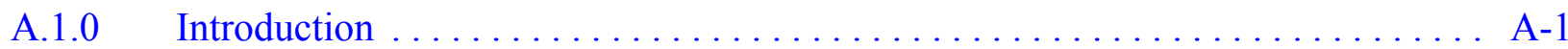

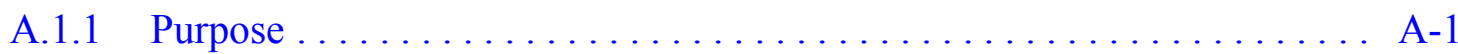

A.1.2 Scope of Work ........................... A-2

A.2.0 Project Description $\ldots \ldots \ldots \ldots \ldots \ldots \ldots \ldots \ldots \ldots \ldots \ldots \ldots \ldots \ldots \ldots \ldots$

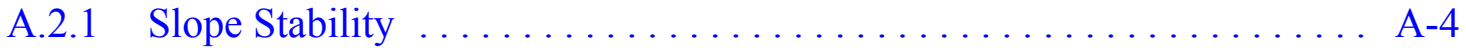

A.2.2 Surface Radiological Surveys $\ldots \ldots \ldots \ldots \ldots \ldots \ldots \ldots \ldots \ldots \ldots$ A

A.2.3 Muckpile (CAS 12-06-06) Sample Locations . . . . . . . . . . . . . . A-4

A.2.4 Drainage Sample Locations . . . . . . . . . . . . . . . . . . A-7

A.2.5 Pond Sample Locations . . . . . . . . . . . . . . . . A-7

A.2.6 Radioactive Materials (CAS 12-28-02) Sample Locations . . . . . . . . . A-7

A.2.7 Oil Spill (CAS 12-25-02) Sample Locations . . . . . . . . . . . . . . . . A-8

A.2.8 Background and Preliminary Sample Locations $\ldots \ldots \ldots \ldots \ldots \ldots$ A $\ldots \ldots$

A.2.9 Checklists . . . . . . . . . . . . . . . . . . . . . . . . . . . A-8

A.3.0 Site Investigation $\ldots \ldots \ldots \ldots \ldots \ldots \ldots \ldots \ldots \ldots \ldots \ldots \ldots \ldots \ldots \ldots \ldots$

A.3.1 Work Packages ......................... A-10

A.3.2 Surface Radiological Surveys $\ldots \ldots \ldots \ldots \ldots \ldots \ldots \ldots \ldots \ldots \ldots \ldots$

A.3.3 Muckpile Samples; CAS 12-06-06 . . . . . . . . . . . . . . . . . A-12

A.3.4 Drainage Soil Samples . . . . . . . . . . . . . . . . . A-13

A.3.5 Pond Soil Samples . . . . . . . . . . . . . . . . . . A-17

A.3.6 Oil Spill Soil Samples, CAS $12-25-02 \ldots \ldots \ldots \ldots \ldots \ldots \ldots$ A-17 


\section{Table of Contents (Continued)}

A.3.7 Radioactive Material Area Soil Samples, CAS 12-28-02 . . . . . . . . A A-20

A.3.8 Background and Preliminary Soil Samples . . . . . . . . . . . . . . A-20

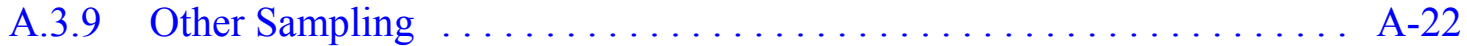

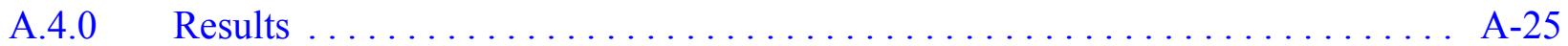

A.5.0 Waste Management . . . . . . . . . . . . . . . . . . . . . A-34

A.5.1 Waste Segregation ......................... A-34

A.5.2 Waste Generation and Accumulation ................. A-34

A.5.2.1 Rinsates . . . . . . . . . . . . . . . . .

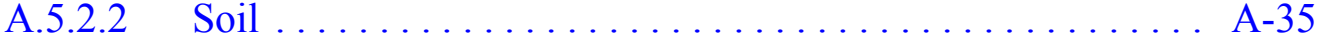

A.5.2.3 PPE, Sampling Equipment, and Plastic Sleeves ........ A-35

A.5.2.4 Plastic Liners . . . . . . . . . . . . . . . A-36

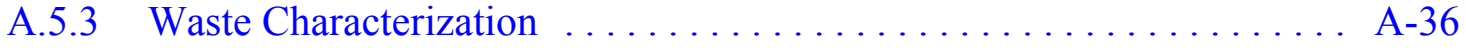

A.5.4 Waste Disposal . . . . . . . . . . . . . . . . . . . A-37

A.6.0 Health and Safety $\ldots \ldots \ldots \ldots \ldots \ldots \ldots \ldots \ldots \ldots \ldots \ldots \ldots$

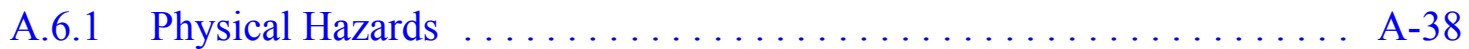

A.6.2 Chemical and Radiological Hazards . . . . . . . . . . . . . . . . A-38

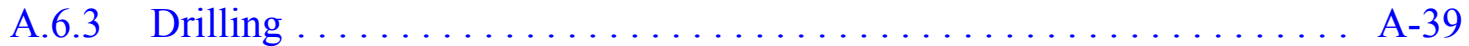

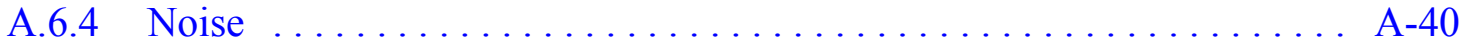

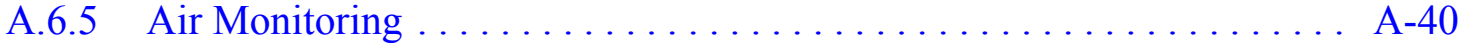

A.6.6 Personnel Monitoring $\ldots \ldots \ldots \ldots \ldots \ldots \ldots \ldots \ldots \ldots \ldots \ldots \ldots$

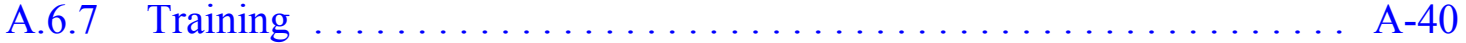

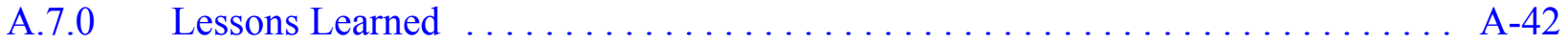

A.8.0 References .................................... A-43

Attachment A - Soil Boring Logs

Attachment B - Cross Sections

Attachment C - Analytical Results Table 


\section{List of Figures}

Number

Title

Page

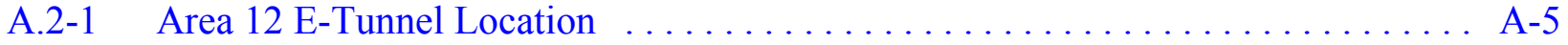

A.2-2 CAU 383 (E-Tunnel Sites) CASs . . . . . . . . . . . . . . . . . . . A

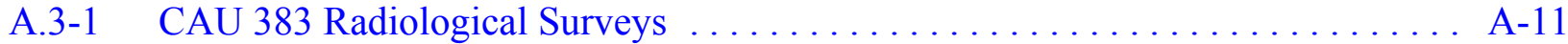

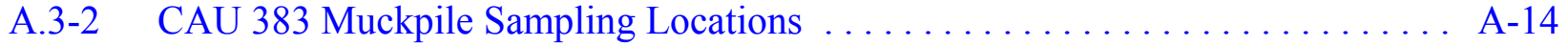

A.3-3 CAU 383 Drainage Sampling Locations $\ldots \ldots \ldots \ldots \ldots \ldots \ldots \ldots \ldots$ A-16

A.3-4 CAU 383 Ponds Sampling Locations $\ldots \ldots \ldots \ldots \ldots \ldots \ldots \ldots \ldots \ldots \ldots \ldots \ldots \ldots \ldots \ldots \ldots \ldots$

A.3-5 CAU 383 Oil Spill and Radioactive Material Area Sampling Locations ....... A A-21

A.3-6 CAU 383 Background and Preliminary Sampling Locations ............. A-23 


\section{List of Tables}

Number

Title

Page

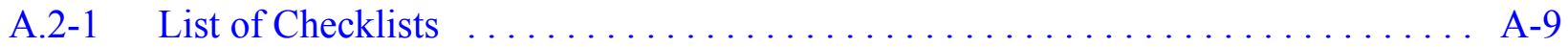

A.3-1 As-Built Muckpile Sampling Locations and Total Depth $\ldots . \ldots \ldots \ldots \ldots$. . . .

A.3-2 As-Built Drainage Sampling Locations and Total Depths . . . . . . . . . . . . . A-18

A.3-3 As-Built Ponds Sampling Locations and Total Depths $\ldots . \ldots \ldots \ldots \ldots \ldots$ A-20

A.3-4 As-Built Oil Spill and Radioactive Material Area

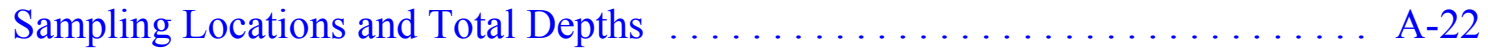

A.3-5 As-Built Background and Preliminary Sampling Locations Total Depths ...... A A-24

A.4-1 Analytical Result Detects for Muckpile and Pond Samples .............. A-26

A.4-2 Analytical Result Detects for the Radioactive Material, Oil Spill , Drainage, and QC Water Samples .................. A-29

A.4-3 Analytical Results for Background / Preliminary Samples . . . . . . . . . . . . A-33

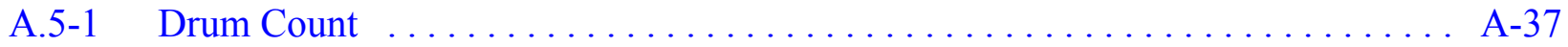




\section{List of Acronyms and Abbreviations}

\begin{tabular}{|c|c|}
\hline $\mathrm{BN}$ & Bechtel Nevada \\
\hline CADD & Corrective Action Decision Document \\
\hline CAI & Corrective Action Investigation \\
\hline CAIP & Corrective Action Investigation Plan \\
\hline CAS & Corrective Action Site \\
\hline CAU & Corrective Action Unit \\
\hline COPC & Contaminant of potential concern \\
\hline cps & Counts per second \\
\hline DLAPS & Dual Large-Area Plastic Scintillation Detector \\
\hline DOE & U.S. Department of Energy \\
\hline DTRA & Defense Threat Reduction Agency \\
\hline EZ & Exclusion Zone \\
\hline FADL & Field Activity Daily Logs \\
\hline FFACO & Federal Facility Agreement and Consent Order \\
\hline FID & Flame ionization detector \\
\hline $\mathrm{ft}$ & Foot \\
\hline GPS & Global Positioning System \\
\hline HWAA & Hazardous waste accumulation area \\
\hline IDW & Investigation-derived waste \\
\hline $\mathrm{mg} / \mathrm{kg}$ & Milligram per kilogram \\
\hline $\mathrm{mg} / \mathrm{L}$ & Milligram per liter \\
\hline ND & Not detected \\
\hline NNSA/NSO & U.S. Department of Energy, National Nuclear Security Administration \\
\hline & Nevada Site Office \\
\hline $\mathrm{pCi} / \mathrm{g}$ & Picocuries per gram \\
\hline $\mathrm{pCi} / \mathrm{L}$ & Picocuries per liter \\
\hline PPE & Personal protective equipment \\
\hline QA & Quality assurance \\
\hline QC & Quality control \\
\hline RCRA & Resource Conservation and Recovery Act \\
\hline
\end{tabular}




\section{List of Acronyms and Abbreviations (Continued)}

$\begin{array}{ll}\text { REOP } & \text { Real Estate/Operations Permit } \\ \text { RMA } & \text { Radioactive material area } \\ \text { SAPS } & \text { Small Area Plastic Scintillation Detector } \\ \text { SCL } & \text { Sample Collection Log } \\ \text { SNJV } & \text { Stoller-Navarro Joint Venture } \\ \text { SS } & \text { Site Supervisor } \\ \text { SSHASP } & \text { Site-Specific Health and Safety Plan } \\ \text { SSO } & \text { Site Safety Officer } \\ \text { SVOC } & \text { Semivolatile organic compound } \\ \text { TID } & \text { Tamper-indicating device } \\ \text { TPH-D } & \text { Total petroleum hydrocarbon-diesel } \\ \text { VOC } & \text { Volatile organic compound } \\ \text { Fg/kg } & \text { Micrograms per kilogram } \\ \text { Fg/L } & \text { Micrograms per liter }\end{array}$




\section{Executive Summary}

The Corrective Action Investigation (CAI) of the E-Tunnel Sites and drainage, identified under the Federal Facility Agreement and Consent Order (1996) classification as Corrective Action Unit 383 (Area 12 E-Tunnel Sites), consists of three Corrective Action Sites (CASs) (12-06-06, Muckpile; 12-25-02, Oil Spill; and 12-28-02, Radioactive Material). Four activities were completed during the CAI, a drive-over radiological survey, a walk-over radiological survey, rotosonic drilling and soil sampling, and hand soil sampling.

In September 2003, Shaw Environmental, Incorporated personnel completed drive-over and walk-over radiological surveys of the E-Tunnel Sites and the drainage below the Muckpile, including the ponds. These surveys identified surface and near-surface areas with elevated readings on the Muckpile, in the drainage and ponds, and near the blower pad.

The next stage of the investigation consisted of rotosonic drilling and hand sampling to collect soil samples from within and beneath the Muckpile and ponds, the drainage, the RMA, and the oil spill (blower pad). This was conducted over 27 workdays between May 19 and June 24, 2004. Ninety locations were drilled or hand sampled to characterize CAU 383. A total of 1,129.5 ft were drilled in 39 boreholes ranging from 1.5 to $91.5 \mathrm{ft}$ deep. Fifty-one locations were sampled by hand. One to three samples were collected from 89 locations and 1 borehole was not sampled.

During the characterization, 127 environmental samples were collected. These included 40 muck samples, 8 oil spill samples, 4 contamination area samples, 37 samples from the drainage, 10 pond sediment samples, and 30 native material samples. Seven duplicate and six laboratory quality control soil samples were collected for quality control purposes. Thirty-seven quality control water samples were collected (7 equipment rinsate samples, 7 field blank samples, 1 source blank, and 22 trip blanks). All soil and water samples were sent to Paragon Analytics, Inc., for analysis. Analyses performed included volatile organic compounds (VOCs), semivolatile organic compounds (SVOCs), total petroleum hydrocarbons-diesel (TPH-D), total Resource Conservation and Recovery Act (RCRA) metals, beryllium, and radionuclides (gamma spec, isotopic plutonium and strontium-90). In addition, three background, five preliminary, one duplicate, and one laboratory quality control soil samples were collected in February and March 2004. These soil samples were also sent to Paragon Analytics, Inc., for analysis. 
Varying amounts of VOCs, SVOCs, TPH-D, beryllium, total RCRA metals, and radionuclides were detected in the muck and or native soil samples from all three CASs included in this investigation.

During the CAI, seven drums of investigation-derived waste were accumulated, four drums of rinsate from decontamination activities, two drums of hydrocarbon contaminated plastic, and one drum of personal protective equipment. The environmental sample analytical results were used to make the waste determination that none of the wastes were considered to be hazardous or radioactive. All of the drums were disposed of as sanitary waste except for the two drums of hydrocarbon contaminated plastic, which were sent to the hydrocarbon landfill.

A lessons learned meeting was not held in conjunction with this field activity. 


\section{A.1.0 Introduction}

The Corrective Action Investigation (CAI) of the Area 12 E-Tunnel Sites and associated drainage was conducted in accordance with the Federal Facility Agreement and Consent Order (FFACO) (1996) that was agreed to by the U.S. Department of Defense, Defense Special Weapons Agency (predecessor to the Defense Threat Reduction Agency [DTRA]); the U.S. Department of Energy (DOE); and the State of Nevada. The investigation was controlled and guided by the Corrective Action Unit (CAU) 383 Corrective Action Investigation Plan (CAIP) (DTRA, 2003); Field Instructions (DTRA, 2004), which referenced the operational checklists; and the site-specific health and safety plan (SSHASP) (SNJV, 2004). The E-Tunnel Muckpile and associated sites are identified under FFACO classification as CAU 383, Area 12 E-Tunnel Sites. Corrective Action Unit 383 is a joint DTRA and U.S. Department of Energy, National Nuclear Security Administration Nevada Site Office (NNSA/NSO) site. The CAU consists of three Corrective Action Sites (CASs) (12-06-06, Muckpile; 12-25-02, Oil Spill; 12-28-02, Radioactive Material). In addition to these sites, the drainage between the Muckpile and the first pond, and Ponds One, Two, and Three were sampled. This report presents a summary of the field activities and the data collected during the field effort.

Section A.1.0 of this report includes a description of the purpose and scope of the project. Section A.2.0 is the project description. Section A.3.0 details the Area 12 E-Tunnel Sites investigation and provides a description of the sample collection activities and locations. Section A.4.0 is a summary of the sample analytical results. Section A.5.0 provides a description of the waste management activities. Section 6.0 covers the health and safety aspects of the project. Section A.7.0 discusses the lessons learned during the project, and Section A.8.0 lists the references.

\section{A.1.1 Purpose}

The purpose of the Area 12 E-Tunnel Sites CAI was to determine whether the Area 12 E-Tunnel Sites and/or the underlying soils have been impacted by contaminants of potential concern (COPCs) at concentrations that exceed regulatory limits. The data collected during the field effort, which are presented in this report, will enable DTRA and NNSA/NSO to make informed decisions about the future use or closure of the site. The Muckpile primarily consists of mining 
debris (rock) generated during tunnel excavation and construction in support of nuclear weapons effects testing. The investigation was conducted to achieve the following goals:

- Determine whether identified COPCs (both chemical and radiological) are present at the surface, within, or beneath the Muckpile, the oil spill, the radioactive material, the drainage, and/or the first three ponds.

- $\quad$ Provide sufficient information and data to develop appropriate corrective action strategies for the Muckpile and associated sites to be evaluated in the Corrective Action Decision Document (CADD).

\section{A.1.2 Scope of Work}

The scope of the Area 12 E-Tunnel Sites investigation included the following:

- Conducted walk-over and drive-over radiological surveys using field-screening instruments to locate areas with elevated radiological levels.

- Collected random and biased Muckpile and native soil samples using the dry rotosonic drilling method or hand tools.

- Collected random and biased soil samples from the drainage below the Muckpile and the ponds using the dry rotosonic drilling method or hand tools.

- Conducted volatile organic compound (VOC) and radiological field screening for health and safety monitoring and to identify the presence or absence of COPCs.

- Logged the drill cores to describe soil characteristics and identify the muck/native material contact.

- Conducted laboratory analysis of the environmental and quality control (QC) samples to determine the presence or absence of COPCs,

- Collected three background samples from undisturbed areas around the Muckpile.

The random sampling locations on the Muckpile and in the drainage were selected based on a simple random sampling method as described in Chapter 4 of Statistical Methods for Environmental Pollution Monitoring (Gilbert, 1987). The random sampling locations in the first pond were selected based on a stratified random sampling method as described in Chapter 5 of the same book (Gilbert, 1987). The biased samples were collected from locations where elevated radiological levels were identified by the drive-over and/or walk-over surveys, process 
CAU 383 CADD

Appendix A

Revision: 0

Date: March 2005

Page A-3 of A-44

knowledge indicates surface operations could have contributed COPCs to the environment, or visual evidence indicates impacted soils. 


\section{A.2.0 Project Description}

The Area 12 E-Tunnel Sites, CAU 383, is located approximately 42 miles north of Mercury in Area 12 of the Nevada Test Site (Figure A.2-1). CAU 383 (Figure A.2-2) consists of three CASs (12-06-06, Muckpile; 12-25-02, Oil Spill; and 12-28-02, Radioactive Material). E-Tunnel was used for nine nuclear weapons effects and weapons related tests between 1958 and 1977. The E-Tunnel Muckpile (CAS 12-06-06) consists of roughly 355,000 cubic yards of material mined out of the tunnel. The surface elevation at the top of the Muckpile is about 6,120 feet (ft) above mean sea level. The Muckpile was deposited in an easterly trend in front of the tunnel portals. The Muckpile is approximately $200 \mathrm{ft}$ across and 1,200 ft long in an east-west direction. The thickness of the Muckpile ranges from less than $5 \mathrm{ft}$ at the west end to approximately $100 \mathrm{ft}$ at the northeast side. The drainage that was included in this investigation trends easterly along the base of the Muckpile.

Corrective Action Site 12-28-02 (Radioactive Material) is located at the E-Tunnel Main portal and is part of the Muckpile.

Corrective Action Site 12-25-02 (Oil Spill) is located on the blower pad, which is on a bench approximately $100 \mathrm{ft}$ above the E-Tunnel south portal. The pad was constructed in the hillside and is about $100 \mathrm{ft}$ long north to south and $50 \mathrm{ft}$ wide. Fill on the pad varies in thickness from less than $1 \mathrm{ft}$ next to the slope to about $10 \mathrm{ft}$ on the north edge of the pad.

\section{A.2.1 Slope Stability}

Given the site conditions and proposed operating parameters, a slope stability analysis was prepared for the E-Tunnel Muckpile. The work restriction, based on the analysis, was that drilling would not be conducted within $20 \mathrm{ft}$ of the edge of the Muckpile.

\section{A.2.2 Surface Radiological Surveys}

Prior to commencing drilling and sampling, walk-over and drive-over radiological surveys were conducted to identify surface and near surface areas with elevated readings (see Figure A.3-1).

\section{A.2.3 Muckpile (CAS 12-06-06) Sample Locations}

A total of 33 Muckpile locations were sampled, 15 biased locations (2 locations were hand sampled) and 18 random locations. The Muckpile random sample locations at CAU 383 were 


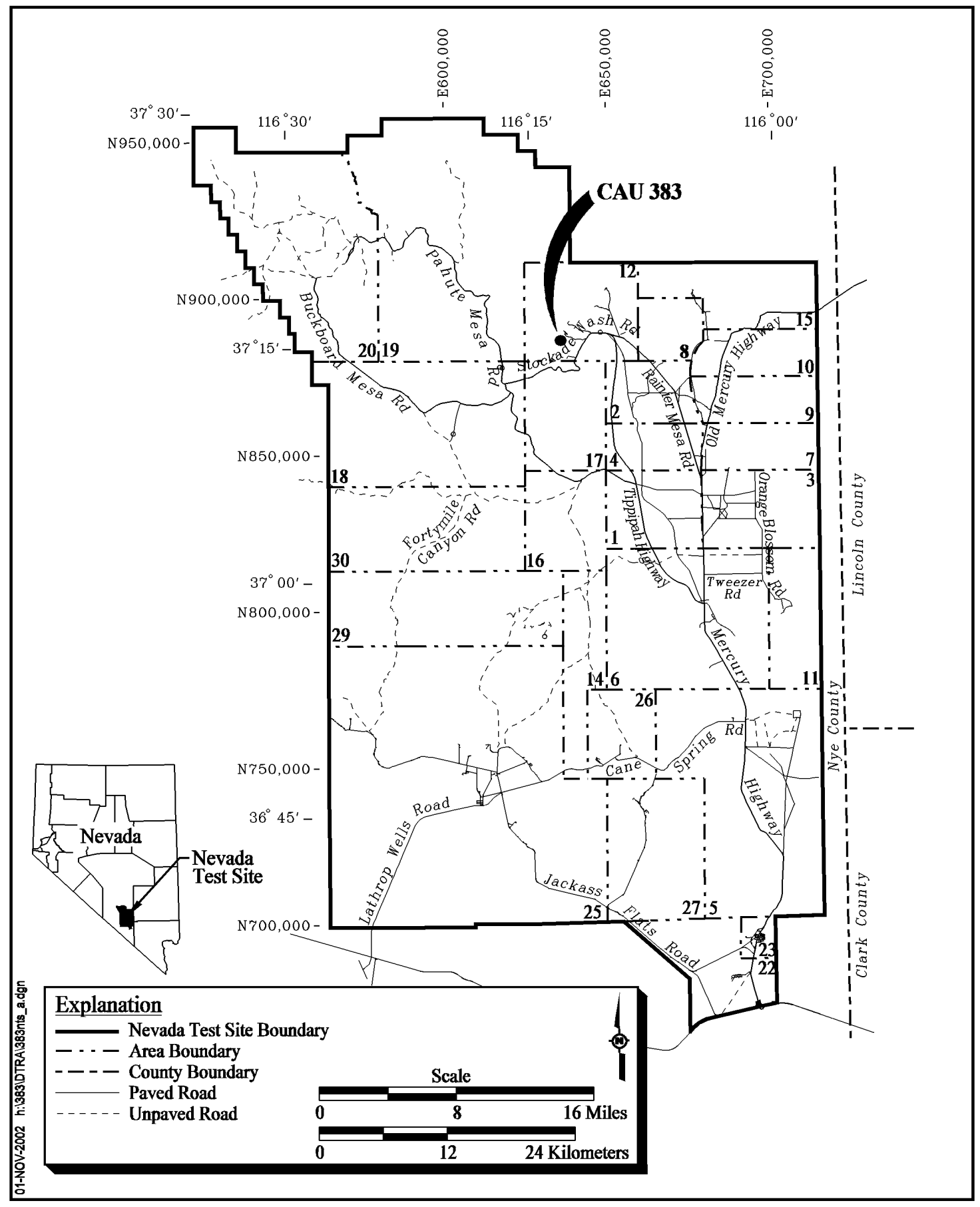

Figure A.2-1

Area 12 E-Tunnel Location

(Source: Modified from DOE/NV, 1996) 
Date: March 2005

Page A-6 of A-44

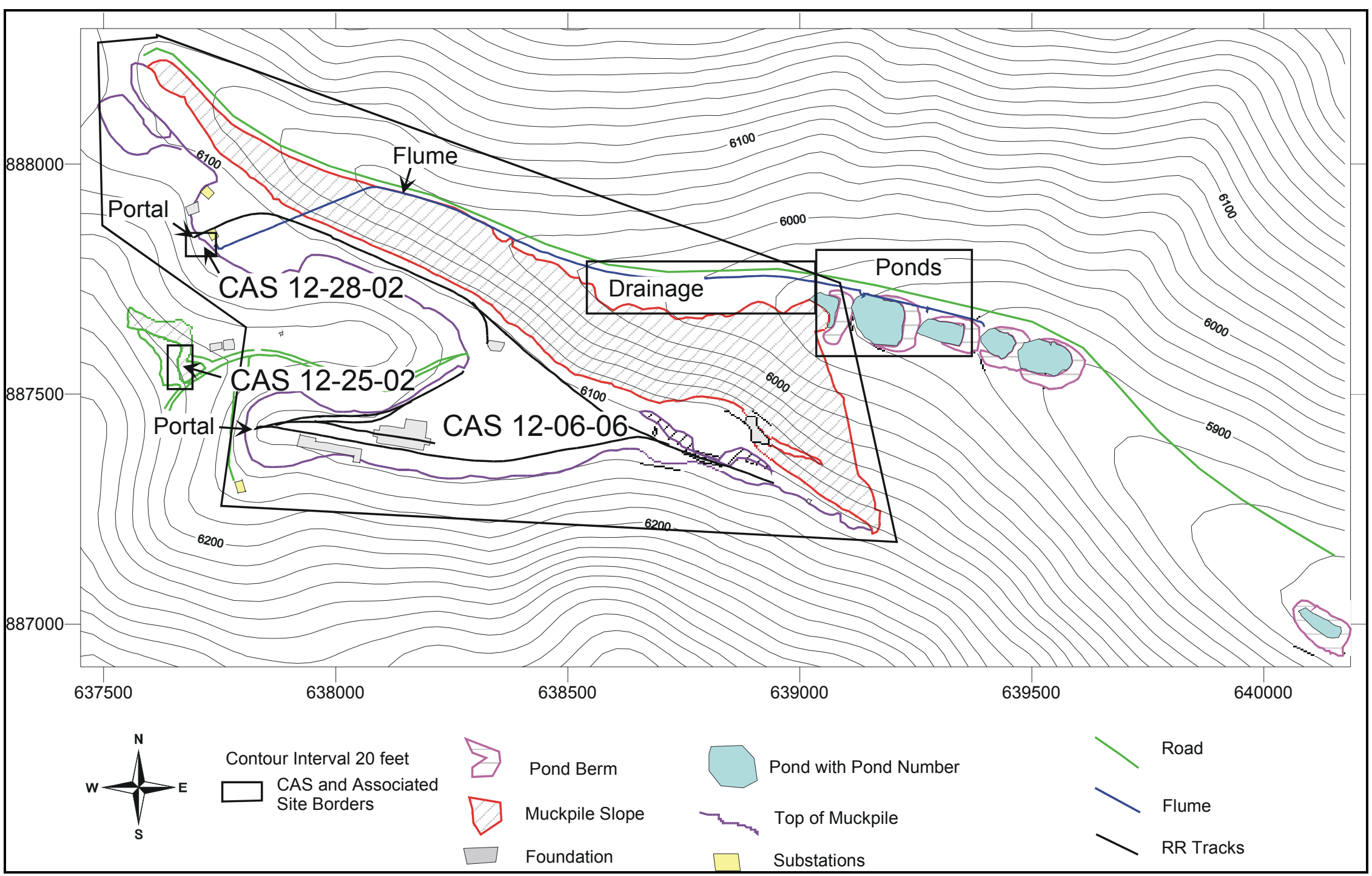

Figure A.2-2

CAU 383 (E-Tunnel Sites) CASs 
identified using a simple random sampling strategy as defined in Gilbert (1987). Prior to commencing the drilling operations, Stoller-Navarro Joint Venture (SNJV) scientists used a Trimble Global Positioning System (GPS) total station surveying instrument to locate and mark each proposed sample location. The CAIP listed six biased sampling locations, the additional nine biased locations were identified and marked by the site supervisor (SS) and the client to provide additional information on the Muckpile close to the outer edge. This information will be needed if the Muckpile is cut down during the remediation. After drilling operations were completed, all sampling locations were surveyed using a GPS to record the as-built locations.

\section{A.2.4 Drainage Sample Locations}

A total of 35 locations were sampled in the drainage, all were random locations and 33 were hand sampled. The drainage random sample locations were identified using a simple random sampling strategy as defined in Gilbert (1987). Prior to the commencement of the sampling operations, SNJV scientists used the Trimble GPS total station surveying instrument to identify and mark the proposed sample locations. After sampling operations were completed, all sampling locations were surveyed using the GPS to record the as-built locations.

\section{A.2.5 Pond Sample Locations}

Nine locations were sampled in the ponds, four random locations and one biased location in Pond One, two biased location in Pond Two, and two biased locations in Pond Three (Ponds Two and Three were hand sampled). Prior to the commencement of the drilling operations, SNJV scientists used the Trimble GPS total station surveying instrument to identify and mark the proposed random sample locations. The biased locations were chosen and marked by the client and site SS during drilling operations. After sampling operations were completed, all sampling locations were surveyed using the GPS to record the as-built locations.

\section{A.2.6 Radioactive Materials (CAS 12-28-02) Sample Locations}

Four biased locations in the Radioactive Materials CAS were sampled, all by hand. Prior to the commencement of the sampling operations, SNJV scientists marked the proposed sample locations. After sampling operations were completed, all sampling locations were surveyed using the GPS to record the as-built locations. 


\section{A.2.7 Oil Spill (CAS 12-25-02) Sample Locations}

Eight biased locations in the Oil Spill CAS were sampled, all by hand. Prior to the commencement of the sampling operations, SNJV scientists marked the proposed sample locations. After sampling operations were completed, all sampling locations were surveyed using a GPS to record the as-built locations.

\section{A.2.8 Background and Preliminary Sample Locations}

Three biased locations were hand sampled in undisturbed areas near the Muckpile for background information. In addition, five biased locations were hand sampled on the Muckpile for preliminary safety and health information. These samples were collected in February and March 2004, prior to start of the drilling activities. The SNJV scientists used the Trimble GPS total station surveying instrument to record the sample locations.

\section{A.2.9 Checklists}

To increase the efficiency and completeness of the field effort, checklists were used during the E-Tunnel Sites characterization to track individual field tasks. Table A.2-1 contains a list of the checklists, as well as detailing when and how often each checklist was used. 
Table A.2-1

List of Checklists

\begin{tabular}{|c|c|c|c|c|}
\hline Checklist & Purpose & $\begin{array}{l}\text { Responsible } \\
\text { Persons } \\
\end{array}$ & Frequency & Timing \\
\hline $\begin{array}{l}\text { Waste } \\
\text { Management Pre- } \\
\text { Field }\end{array}$ & $\begin{array}{l}\text { Ensure that all appropriate } \\
\text { documentation has been prepared and } \\
\text { issued and everything is ready to } \\
\text { properly handle all waste streams } \\
\text { generated at the site. }\end{array}$ & $\begin{array}{l}\text { Waste } \\
\text { Management } \\
\text { Coordinator }\end{array}$ & Once & $\begin{array}{l}\text { Before } \\
\text { mobilization }\end{array}$ \\
\hline $\begin{array}{l}\text { Health \& Safety } \\
\text { Pre-Field and } \\
\text { Mobilization }\end{array}$ & $\begin{array}{l}\text { Ensure all documents and required } \\
\text { forms have been prepared, are } \\
\text { available, that all facilities have been } \\
\text { notified, that all personnel have the } \\
\text { required training and certifications, } \\
\text { that all health and safety monitoring } \\
\text { equipment is available and } \\
\text { operational, and that all health and } \\
\text { safety supplies are available at the } \\
\text { site. This will mitigate potential } \\
\text { exposures to personnel and reduce } \\
\text { the risk of accidents. }\end{array}$ & Site Safety Officer & Once & $\begin{array}{l}\text { Prior to and } \\
\text { during } \\
\text { mobilization }\end{array}$ \\
\hline Mobilization & $\begin{array}{l}\text { Ensure that the site is ready for the } \\
\text { field activities, that all documentation } \\
\text { is in place, and that equipment and } \\
\text { supplies are available on site. }\end{array}$ & Site Supervisor & Once & $\begin{array}{l}\text { Before field } \\
\text { work begins }\end{array}$ \\
\hline Geologist & $\begin{array}{l}\text { Ensure the hole is properly field } \\
\text { screened and logged, all required } \\
\text { information is entered on the borehole } \\
\text { log, and that the hole is properly } \\
\text { abandoned and marked. }\end{array}$ & Rig Geologist & $\begin{array}{l}\text { One for } \\
\text { each } \\
\text { borehole }\end{array}$ & $\begin{array}{l}\text { During and } \\
\text { after drilling }\end{array}$ \\
\hline $\begin{array}{l}\text { Drill Rig Safety } \\
\text { Inspection }\end{array}$ & $\begin{array}{l}\text { Ensure the drill rig meets all required } \\
\text { safety standards to mitigate possible } \\
\text { personnel injury resulting from faulty } \\
\text { or worn equipment, and/or inadequate } \\
\text { safety devices. }\end{array}$ & $\begin{array}{l}\text { Site Supervisor } \\
\text { and/or Rig } \\
\text { Geologist and Site } \\
\text { Safety Officer }\end{array}$ & Once & $\begin{array}{l}\text { Before the } \\
\text { start of field } \\
\text { work }\end{array}$ \\
\hline Sampling & $\begin{array}{l}\text { Ensure all supplies are ready for the } \\
\text { days sampling, that the samples are } \\
\text { collected and handled properly, that } \\
\text { required quality assurance/quality } \\
\text { control samples are collected, and that } \\
\text { all paperwork is completed correctly. }\end{array}$ & Sampler & Daily & $\begin{array}{l}\text { Before, } \\
\text { during, and } \\
\text { after } \\
\text { sampling }\end{array}$ \\
\hline Decontamination & $\begin{array}{l}\text { Ensure adequate decontamination } \\
\text { equipment and supplies are available } \\
\text { for the day, that deconned equipment } \\
\text { is properly handled and screened if } \\
\text { contamination is encountered, and } \\
\text { that the rinsate and any sediment } \\
\text { generated is properly handled. }\end{array}$ & $\begin{array}{l}\text { Rig Geologist } \\
\text { and/or Site Safety } \\
\text { Officer }\end{array}$ & $\begin{array}{l}\text { Daily on } \\
\text { days when } \\
\text { decon is } \\
\text { done }\end{array}$ & $\begin{array}{l}\text { Before, } \\
\text { during, and } \\
\text { after } \\
\text { deconning }\end{array}$ \\
\hline Pressure Washer & $\begin{array}{l}\text { Ensure the pressure washer, is safe } \\
\text { and operational. }\end{array}$ & $\begin{array}{l}\text { Equipment } \\
\text { Operator and/or } \\
\text { Rig Geologist }\end{array}$ & $\begin{array}{l}\text { Initial entry } \\
\text { to site, daily } \\
\text { prior to use }\end{array}$ & $\begin{array}{l}\text { Before use of } \\
\text { the } \\
\text { equipment }\end{array}$ \\
\hline
\end{tabular}




\section{A.3.0 Site Investigation}

Mobilization of equipment and supplies and site set up for the characterizations at E-Tunnel Sites took place May 17 and 18, 2004. The CAI of the E-Tunnel Sites was conducted over 27 work days between May 19 and June 24, 2004. The drive-over and walk-over radiological surveys were completed in September 2003. The drillers mobilized to the site on May 18. Drilling commenced on May 19 and continued to June 14. From June 14 through June 24, hand samples were collected, as-built GPS surveys were conducted, and demobilization of equipment was completed. Days off included May 28 through May 31, June 5 and June 6, and June 18 through June 21.

All visitors coming on site had to check in with the Facility Manager Representative, the SS, or the Site Safety Officer (SSO) to receive a site-specific health and safety briefing and the tailgate safety briefing before being allowed on site.

\section{A.3.1 Work Packages}

DTRA is the primary Real Estate/Operations Permit (REOP) holder (REOP Number DTRA-12) and is responsible for safety at the site. Three work packages were prepared by Shaw/SNJV, and approved by DTRA so that the work could be conducted under the DTRA REOP. Work package Shaw-2003-DTRA-01 covered the drive-over and walk-over surveys. Work package SNJV 04 $E 01$ covered mobilization and demobilization from the site and all activities involved in the characterization. Work package SNJV 04 E 02 covered the waste management activities, which continued after the field work was completed.

Prior to beginning field work, a Nevada Test Site Operations Schedule B form was filled out and sent to the Site Operations Center and Bechtel Nevada (BN) to advise them of the planned work activities, locations, dates, and times.

\section{A.3.2 Surface Radiological Surveys}

The walk-over and drive-over radiological surveys were completed in September 2003. Results from both the walk-over and drive-over surveys are shown in Figure A.3-1. 
Date: March 2005

Page A-11 of A-44

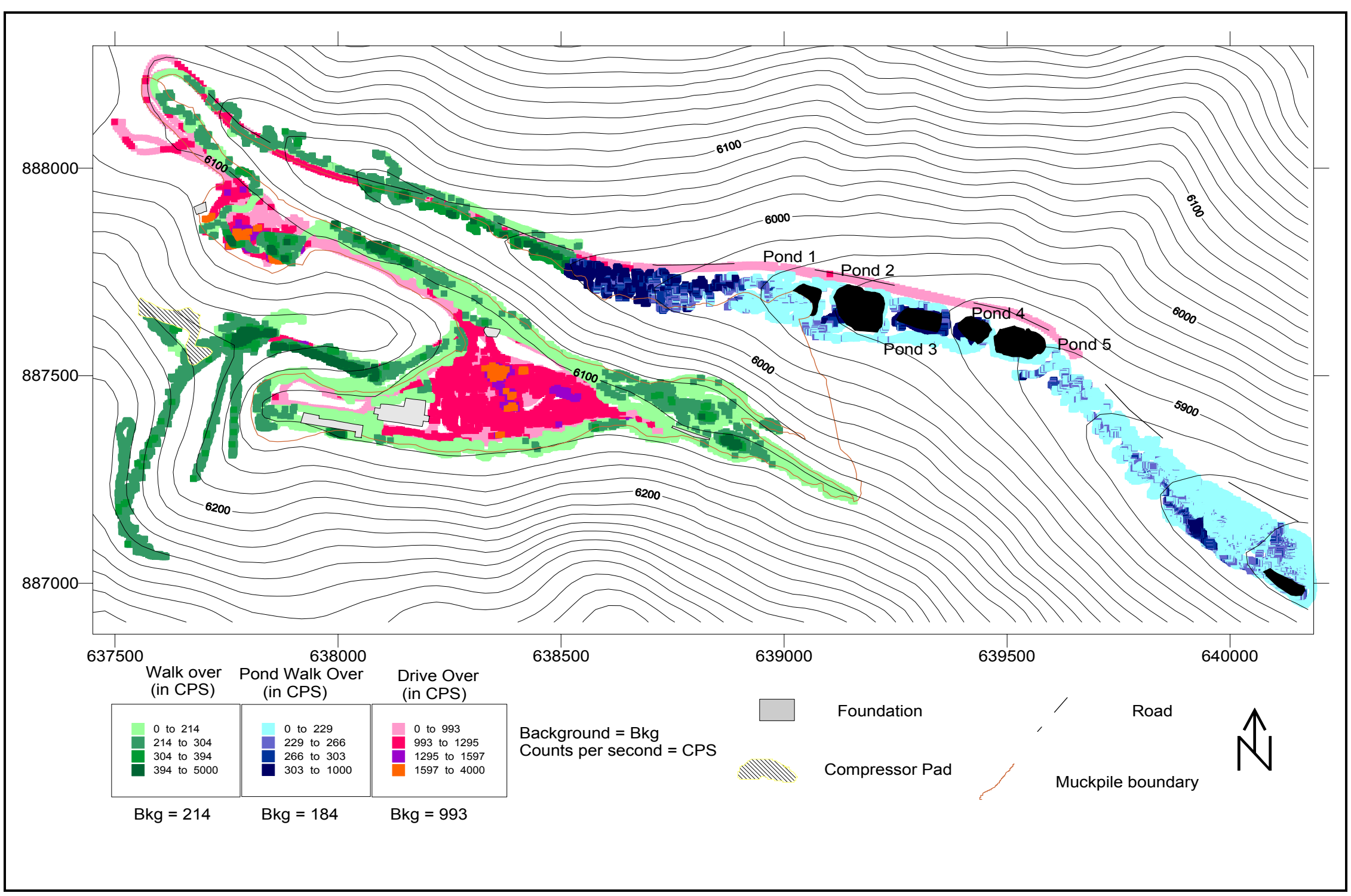

Figure A.3-1

CAU 383 Radiological Surveys 
The drive-over radiological survey of the Muckpile was conducted by towing a Dual Large-Area Plastic Scintillation (DLAPS) Detector Model VRM-3, with a TSA Model SC-755 Controller, and a Trimble Pathfinder Pro XRS ${ }^{\mathrm{TM}}$ GPS receiver with a $\mathrm{TSC}^{\mathrm{TM}}$ data logger behind a four-wheel drive vehicle. Each radiological measurement was taken with the DLAPS detector and recorded on the TSC1 data logger. Each point was then stored with its related GPS location measurement in a combined file. Approximately 6 acres were surveyed and a total of 6,782 beta/gamma measurements were recorded. The highest beta/gamma measurement detected was 3,959 counts per second (cps) at Nevada State Plane coordinates E 637,788.0, N 887,586.0. This high level is 4 times greater than the background radiation emission rate of $993 \mathrm{cps}$. A map showing the results of the drive-over radiological soil survey of E-Tunnel is provided in Figure A.3-1.

The walk-over radiological survey was conducted by walking over the drainage carrying a Small Area Plastic Scintillation (SAPS) Detector Model 8204, with a TSA Model SC-755 Controller, and a Trimble Pathfinder Pro XRS ${ }^{\mathrm{TM}}$ GPS receiver with a TSC1 ${ }^{\mathrm{TM}}$ data logger. Each radiological measurement was taken with the SAPS detector and recorded on the TSC1 data logger and stored with its related GPS measurement in a combined file. Approximately 2 acres were surveyed and over 25,000 beta/gamma measurements were recorded. The highest beta/gamma measurement detected was 1,160 cps at Nevada State Plane coordinates E 638,076.6, $\mathrm{N} 887,817.5$. This high level is 5.2 times greater than the background radiation emission rate of 214 cps. A map showing the results of the walk-over radiological soil survey of the Muckpile, drainage and ponds is provided in Figure A.3-1.

\section{A.3.3 Muckpile Samples; CAS 12-06-06}

Soil sampling was accomplished by either drilling or hand sampling. Drilling consisted of drilling boreholes through the Muckpile into the native material underneath using the rotosonic drilling method. If the native material was alluvial in nature, the borehole was advanced $5 \mathrm{ft}$ into the native material or until refusal. If the native material was bedrock, the borehole was only advanced $2 \mathrm{ft}$ into the native material or until refusal. Copies of the soil boring logs for all boreholes are in Attachment A. Hand sampling consisted of using hand tools to collect a sample from a depth of 0 to $0.5 \mathrm{ft}$ or until refusal. A total of $1,039.5 \mathrm{ft}$ of drilling was completed in 32 boreholes. Samples were collected to characterize the Muckpile and/or the native material under the Muckpile in 31 of the boreholes (borehole EMH02 was not sampled, borehole EMH2A was drilled and sampled as the alternate). Two locations were not accessible to the drill rig and 
were sampled by hand. Cross sections of the Muckpile are in Attachment B. The random locations were designated as EMH01 through EMH18 and the biased locations were designated EMB01 through EMB15 (Figure A.3-2). The boreholes were drilled to depths ranging from 1.5 to $91.5 \mathrm{ft}$.

Up to three soil samples were collected from each random borehole (Table 3-1). In each borehole one sample was collected at a randomly selected depth (the z-depth) and one from the bottom of the borehole. In addition, a sample could be collected from sections of core where field screening indicated elevated alpha, beta, gamma, or flame ionization detector (FID) levels. Three soil samples could have been collected from each biased borehole, one from the top of the borehole and additional samples from sections of core where field screening indicated elevated readings. One sample was collected from each of the hand sample locations. If the field screening identified elevated readings for any hand sample, additional samples could be taken. No additional samples were taken from the hand sample locations due to refusal. A total of 41 environmental soil samples were collected to characterize the Muckpile, and 25 environmental soil samples were collected to characterize the native material underneath the Muckpile. All of the soil samples were sent to an off-site laboratory to be analyzed for VOCs, semivolatile organic compounds (SVOCs), total petroleum hydrocarbon-diesel (TPH-D), beryllium, total Resource Conservation and Recovery Act (RCRA) metals, and radionuclides.

\section{A.3.4 Drainage Soil Samples}

There were 35 locations sampled in the drainage, all were random locations. Due to the inaccessibility of most of the drainage to the drill rig, only two locations were drilled (Figure A.3-3). The other 33 locations were sampled using hand tools. At the locations sampled with the rotosonic drill rig, a hole was drilled to a depth of $5 \mathrm{ft}$ and one sample was collected. At the hand sampled locations, a hole was dug to $0.5 \mathrm{ft}$ deep and one sample was collected. A total of $10.0 \mathrm{ft}$ of rotosonic drilling and $16.5 \mathrm{ft}$ of hand digging were completed in 2 boreholes and 33 hand sample locations to characterize the drainage. Once the drill core was recovered, it was field screened and sampled. If the field screening did not identify elevated readings for any sections of core, a sample was collected between 0 and $1 \mathrm{ft}$ and no other samples were collected. No sections of drill core were found that had elevated radiological readings. The hand samples were collected between 0 and $0.5 \mathrm{ft}$. If the field screening identify elevated readings for any hand sample, additional samples could be taken. No additional samples were taken from the hand sample locations due to refusal. A total of 35 environmental soil samples were collected from 
Date: March 2005

Page A-14 of A-44

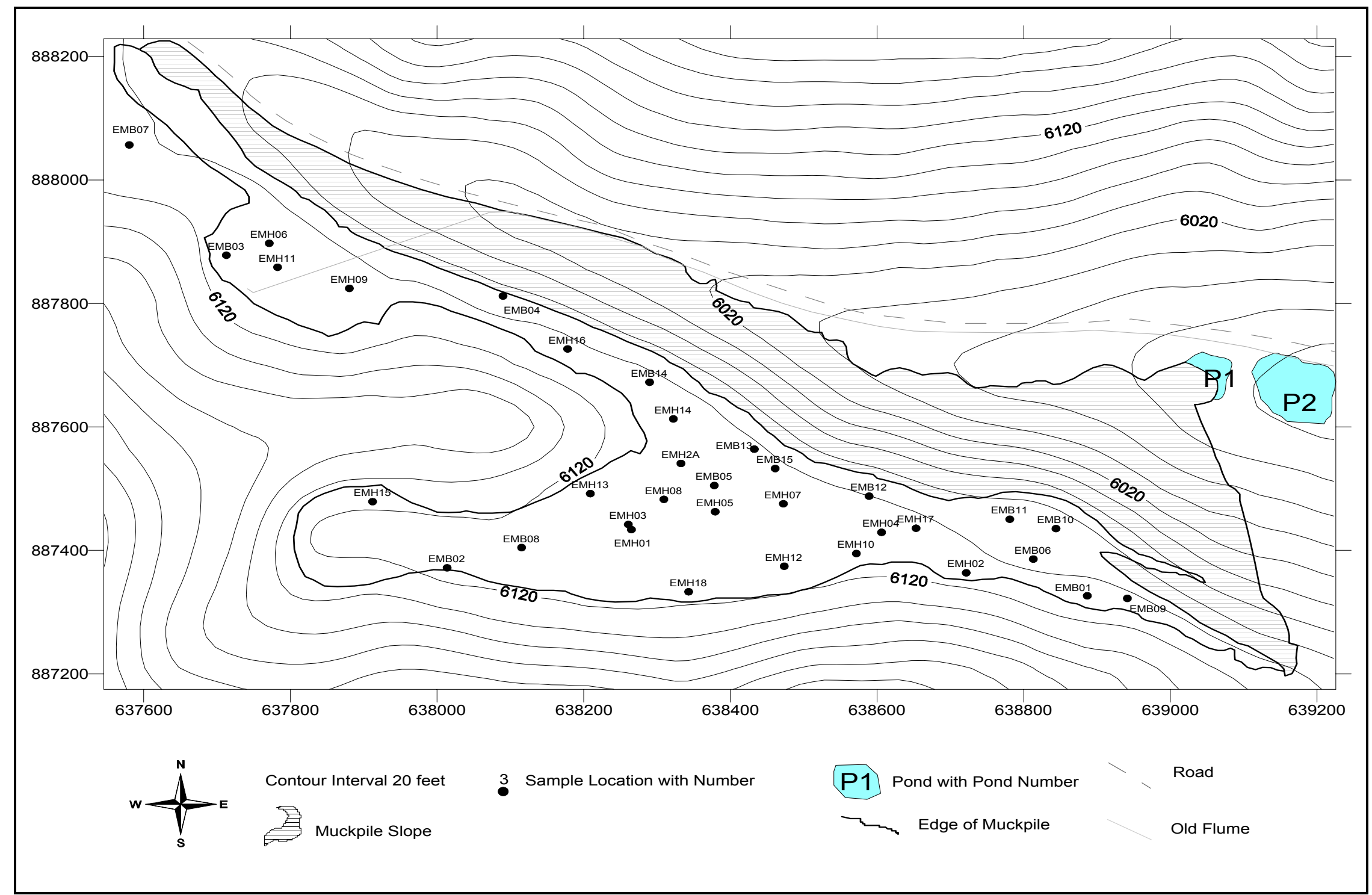

Figure A.3-2

CAU 383 Muckpile Sampling Locations 
Table A.3-1

As-Built Muckpile Sampling Locations and Total Depth

\begin{tabular}{|c|c|c|c|c|c|c|c|}
\hline Hole \# & North & $\overline{\text { East }}$ & Elevation & $\begin{array}{l}\text { Sampling } \\
\text { Method }\end{array}$ & $\begin{array}{c}\text { Drill TD in } \\
\mathrm{ft}\end{array}$ & $\begin{array}{c}\text { Hand TD } \\
\text { in } \mathrm{ft}\end{array}$ & $\begin{array}{c}\text { \# of } \\
\text { Samples }\end{array}$ \\
\hline \multicolumn{8}{|c|}{ Biased Muckpile Locations } \\
\hline EMB01 & 887326.5 & 638886.7 & 6123.5 & drill & 12 & & 1 \\
\hline EMB02 & 887371.8 & 638013.7 & 6136.0 & drill & 3.5 & & 1 \\
\hline EMB03 & 887878.1 & 637712.6 & 6121.5 & drill & 9.5 & & 2 \\
\hline EMB04 & 887812.1 & 638089.9 & 6119.3 & hand & & 0.5 & 1 \\
\hline EMB05 & 887505.2 & 638378.0 & 6117.8 & drill & 86.5 & & 3 \\
\hline EMB06 & 887386.0 & 638813.1 & 6105.0 & drill & 7.5 & & 1 \\
\hline EMB07 & 888056.7 & 637580.3 & 6123.1 & drill & 5 & & 1 \\
\hline EMB08 & 887404.7 & 638115.3 & 6132.5 & drill & 31.5 & & 2 \\
\hline EMB09 & 887322.3 & 638941.3 & 6122.3 & drill & 14 & & 3 \\
\hline EMB10 & 887435.4 & 638844.0 & 6102.2 & drill & 37 & & 1 \\
\hline EMB11 & 887450.5 & 638781.0 & 6107.2 & drill & 25.5 & & 3 \\
\hline EMB12 & 887487.9 & 638589.1 & 6123.1 & drill & 75 & & 1 \\
\hline EMB13 & 887564.0 & 638432.6 & 6127.8 & drill & 91.5 & & 2 \\
\hline EMB14 & 887672.4 & 638289.8 & 6121.6 & drill & 29 & & 3 \\
\hline EMB15 & 887532.7 & 638461.1 & 6119.0 & hand & & 0.5 & 1 \\
\hline & & & & Total & 427.5 & 1.0 & 26 \\
\hline \multicolumn{8}{|c|}{ Random Muckpile Locations } \\
\hline EMH01 & 887433.7 & 638265.0 & 6128.6 & drill & 69 & & 2 \\
\hline EMH02 & 887363.7 & 638721.6 & 6118.8 & drill & 5 & & 0 \\
\hline EMH2A & 887540.8 & 638332.6 & 6123.4 & drill & 45.5 & & 3 \\
\hline EMH03 & 887442.1 & 638260.8 & 6131.4 & drill & 61.5 & & 3 \\
\hline EMH04 & 887429.3 & 638606.1 & 6115.4 & drill & 42 & & 2 \\
\hline EMH05 & 887462.7 & 638379.3 & 6123.6 & drill & 70 & & 3 \\
\hline EMH06 & 887897.5 & 637771.1 & 6119.0 & drill & 1.5 & & 1 \\
\hline EMH07 & 887475.6 & 638472.2 & 6123.5 & drill & 68 & & 3 \\
\hline EMH08 & 887482.7 & 638309.3 & 6125.9 & drill & 59 & & 2 \\
\hline EMH09 & 887824.4 & 637880.4 & 6121.9 & drill & 25 & & 2 \\
\hline EMH10 & 887395.0 & 638571.8 & 6120.8 & drill & 20 & & 2 \\
\hline EMH11 & 887858.6 & 637782.5 & 6116.5 & drill & 4.5 & & 2 \\
\hline EMH12 & 887374.4 & 638473.4 & 6124.1 & drill & 25.5 & & 2 \\
\hline EMH13 & 887492.0 & 638209.0 & 6136.5 & drill & 21 & & 2 \\
\hline EMH14 & 887613.0 & 638322.2 & 6129.7 & drill & 22.5 & & 2 \\
\hline EMH15 & 887479.0 & 637912.1 & 6133.6 & drill & 4 & & 2 \\
\hline EMH16 & 887726.3 & 638178.0 & 6127.8 & drill & 19.5 & & 3 \\
\hline EMH17 & 887435.9 & 638653.1 & 6122.8 & drill & 36.5 & & 2 \\
\hline \multirow[t]{2}{*}{ EMH18 } & 887333.0 & 638343.0 & 6129.9 & drill & 12 & & 2 \\
\hline & & & & Total & 612.0 & 0 & 40 \\
\hline
\end{tabular}




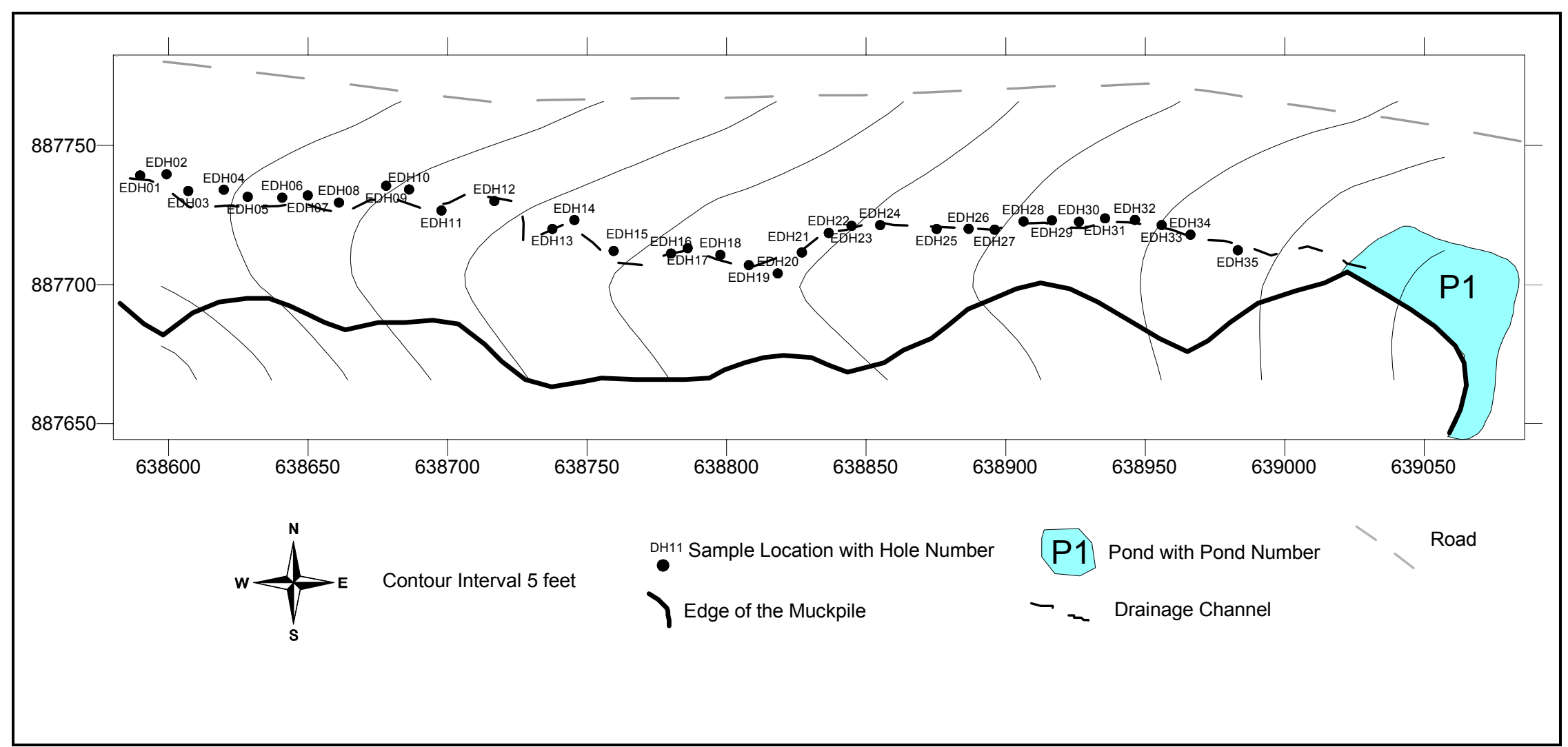

Figure A.3-3

CAU 383 Drainage Sampling Locations 
35 locations to characterize the soil in the drainage. Sample locations in the drainage were designated EDH01 through EDH35. All of the soil samples were sent to an off-site laboratory to be analyzed for VOCs, SVOCs, TPH-D, beryllium, total RCRA metals, and radionuclides. The drainage sampling locations and total depths are shown in Table A.3-2.

\section{A.3.5 Pond Soil Samples}

Fourteen pond soil samples were collected from nine pond locations. Four random and one biased sampling locations were drilled in Pond One. Ponds Two and Three had two biased hand sampling locations each (Figure A.3-4). At the random locations, a hole was drilled to depths of 18.0 to $21.0 \mathrm{ft}$ and two samples were collected. One sample was collected at a randomly selected depth (the z-depth) and one from the bottom of the borehole. Two soil samples were collected from the biased borehole as well, one from above the native material contact and one from the bottom of the borehole. This borehole was five $\mathrm{ft}$ deep. At the hand sampled locations (Ponds Two and Three), a hole was dug to $0.5 \mathrm{ft}$ deep and one sample was collected. Pond One had five sample locations drilled. Due to the wetness of the soil in Ponds Two and Three, only two locations were hand sampled in each pond. If the field screening identified elevated readings for any hand sample, additional samples could be taken. No additional samples were taken from the hand sample locations due to refusal. A total of $80.0 \mathrm{ft}$ of rotosonic drilling and $2.0 \mathrm{ft}$ of hand digging were completed in five boreholes and four hand sample locations. Drilling consisted of drilling boreholes through the pond sediments (muck) into the native material underneath using the rotosonic drill rig. Once the core was recovered, it was field screened and sampled. A total of 14 environmental soil samples, nine Muckpile material and five native material were collected from nine locations to characterize the ponds.

In the ponds, random sample locations were designated EPH01 through EPH04 and the biased samples location were designated as EPB01 through EPB05. All of the soil samples were sent to an off-site laboratory to be analyzed for VOCs, SVOCs, TPH-D, beryllium, total RCRA metals, and radionuclides. The ponds sampling locations and total depths are shown in Table A.3-3.

\section{A.3.6 Oil Spill Soil Samples, CAS 12-25-02}

Eight soil samples were collected from eight oil spill locations (Figure A.3-5). All of the locations were biased locations and all were hand sampled. At each location, a hole was dug to $0.5 \mathrm{ft}$ deep and one sample was collected. Four feet of hand digging was completed at eight hand sample locations. If the field screening identified elevated readings for any hand sample, additional samples could be taken. No additional samples were taken from the hand sample 
Table A.3-2

As-Built Drainage Sampling Locations and Total Depths

\begin{tabular}{|c|c|c|c|c|c|c|c|}
\hline Hole \# & North & East & Elevation & $\begin{array}{c}\text { Sampling } \\
\text { Method }\end{array}$ & \begin{tabular}{|c|}
$\begin{array}{c}\text { Drill TD } \\
\text { in } \mathrm{ft}\end{array}$ \\
\end{tabular} & $\begin{array}{c}\text { Hand TD in } \\
\mathrm{ft}\end{array}$ & $\begin{array}{c}\text { \# of } \\
\text { Samples }\end{array}$ \\
\hline \multicolumn{8}{|c|}{ Random Drainage Locations } \\
\hline EDH01 & 887739.2 & 638589.8 & 6007.8 & hand & & 0.5 & 1 \\
\hline $\mathrm{EDH02}$ & 887739.6 & 638599.3 & 6006.8 & hand & & 0.5 & 1 \\
\hline EDH03 & 887733.6 & 638607.1 & 6004.1 & hand & & 0.5 & 1 \\
\hline $\mathrm{EDH} 04$ & 887734.1 & 638619.8 & 6003.0 & hand & & 0.5 & 1 \\
\hline EDH05 & 887731.5 & 638628.4 & 6003.1 & hand & & 0.5 & 1 \\
\hline EDH06 & 887731.2 & 638640.7 & 5998.9 & hand & & 0.5 & 1 \\
\hline EDH07 & 887732.0 & 638649.9 & 5997.1 & hand & & 0.5 & 1 \\
\hline EDH08 & 887729.5 & 638661.1 & 5999.9 & hand & & 0.5 & 1 \\
\hline EDH09 & 887735.5 & 638678.0 & 5997.2 & hand & & 0.5 & 1 \\
\hline EDH10 & 887734.2 & 638686.2 & 5995.1 & hand & & 0.5 & 1 \\
\hline EDH11 & 887726.6 & 638697.8 & 5984.3 & hand & & 0.5 & 1 \\
\hline EDH12 & 887730.0 & 638716.7 & 5983.9 & hand & & 0.5 & 1 \\
\hline EDH13 & 887720.0 & 638737.5 & 5970.7 & hand & & 0.5 & 1 \\
\hline EDH14 & 887723.2 & 638745.4 & 5982.0 & hand & & 0.5 & 1 \\
\hline EDH15 & 887712.1 & 638759.5 & 5976.8 & hand & & 0.5 & 1 \\
\hline EDH16 & 887711.1 & 638780.1 & 5983.1 & hand & & 0.5 & 1 \\
\hline EDH17 & 887713.1 & 638786.0 & 5978.8 & hand & & 0.5 & 1 \\
\hline EDH18 & 887710.6 & 638797.7 & 5977.6 & hand & & 0.5 & 1 \\
\hline EDH19 & 887707.0 & 638808.0 & 5975.1 & hand & & 0.5 & 1 \\
\hline EDH20 & 887704.0 & 638818.3 & 5972.7 & hand & & 0.5 & 1 \\
\hline $\mathrm{EDH} 21$ & 887711.5 & 638827.0 & 5976.5 & hand & & 0.5 & 1 \\
\hline EDH22 & 887718.5 & 638836.6 & 5972.0 & hand & & 0.5 & 1 \\
\hline EDH23 & 887721.1 & 638844.8 & 5976.1 & hand & & 0.5 & 1 \\
\hline EDH24 & 887721.4 & 638855.0 & 5972.1 & hand & & 0.5 & 1 \\
\hline EDH25 & 887720.0 & 638875.3 & 5968.0 & hand & & 0.5 & 1 \\
\hline EDH26 & 887720.0 & 638886.7 & 5971.4 & hand & & 0.5 & 1 \\
\hline EDH27 & 887719.7 & 638896.0 & 5970.9 & hand & & 0.5 & 1 \\
\hline EDH28 & 887722.7 & 638906.4 & 5972.1 & hand & & 0.5 & 1 \\
\hline EDH29 & 887723.1 & 638916.6 & 5969.8 & hand & & 0.5 & 1 \\
\hline EDH30 & 887722.5 & 638926.3 & 5971.0 & hand & & 0.5 & 1 \\
\hline EDH31 & 887723.8 & 638935.6 & 5969.6 & hand & & 0.5 & 1 \\
\hline EDH32 & 887723.2 & 638946.3 & 5968.2 & hand & & 0.5 & 1 \\
\hline EDH33 & 887721.4 & 638955.9 & 5963.7 & hand & & 0.5 & 1 \\
\hline EDH34 & 887717.9 & 638966.2 & 5964.4 & drill & 5.0 & & 1 \\
\hline \multirow[t]{2}{*}{ EDH35 } & 887712.3 & 638983.2 & 5961.7 & drill & 5.0 & & 1 \\
\hline & & & & Total & 10.0 & 16.5 & 35 \\
\hline
\end{tabular}


Date: March 2005

Page A-19 of A-44

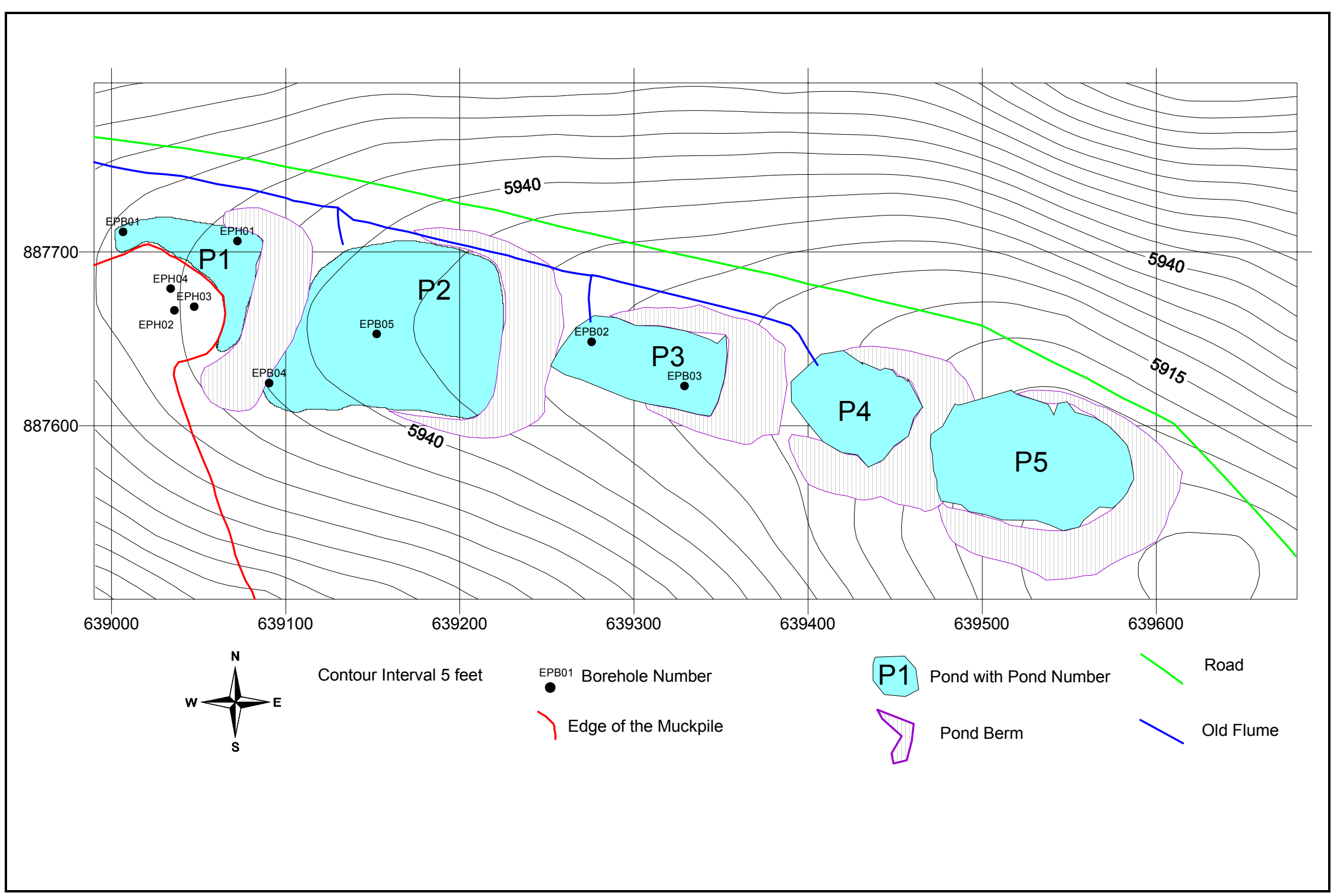

Figure A.3-4

CAU 383 Ponds Sampling Locations 
Table A.3-3

As-Built Ponds Sampling Locations and Total Depths

\begin{tabular}{||c|c|c|c|c|c|c|c||}
\hline Hole \# & North & East & Elevation & $\begin{array}{c}\text { Sampling } \\
\text { Method }\end{array}$ & $\begin{array}{c}\text { Drill TD in } \\
\text { ft }\end{array}$ & $\begin{array}{c}\text { Hand TD in } \\
\text { ft }\end{array}$ & $\begin{array}{c}\text { \# of } \\
\text { Samples }\end{array}$ \\
\hline \hline Biased Pond Locations \\
\hline \hline EPB01 & 887711.6 & 639006.6 & 5959.0 & drill & 5.0 & & 2 \\
\hline EPB02 & 887648.3 & 639275.7 & 5917.5 & hand & & 0.5 & 1 \\
\hline EPB03 & 887622.8 & 639329.0 & 5927.1 & hand & & 0.5 & 1 \\
\hline EPB04 & 887624.5 & 639090.5 & 5942.3 & hand & & 0.5 & 1 \\
\hline EPB05 & 887652.8 & 639152.3 & 5935.2 & hand & & 0.5 & 1 \\
\hline & \multicolumn{7}{|c|}{ Random Pond Location } \\
\hline \hline
\end{tabular}

locations due to refusal. In this CAS, the biased sample locations were designated as EOB01 through EOB08. All of the soil samples were sent to an off-site laboratory to be analyzed for VOCs, SVOCs, TPH-D, beryllium, total RCRA metals, and radionuclides. The oil spill sampling locations and total depths are shown in Table A.3-4.

\section{A.3.7 Radioactive Material Area Soil Samples, CAS 12-28-02}

Four soil samples were collected from four Radioactive Material Area (RMA) locations (Figure A.3-5). All of the locations were biased locations and all were hand sampled. At each location, a hole was dug to $0.5 \mathrm{ft}$ deep and one sample was collected. Two feet of hand digging was completed at four hand sample locations. If the field screening identified elevated readings for any hand sample additional samples could be taken; however, no additional samples were taken due to refusal. In this CAS, the biased sample locations were designated as ERB01 through ERB04. All of the soil samples were sent to an off-site laboratory to be analyzed for VOCs, SVOCs, TPH-D, beryllium, total RCRA metals, and radionuclides. The RMA sampling locations and total depths are shown in Table A.3-4.

\section{A.3.8 Background and Preliminary Soil Samples}

The client and SS selected three undisturbed locations adjacent to the E-Tunnel Sites for collection of background native soil samples and four locations from the surface of the Muckpile for the collection of preliminary samples. All the sampling locations were hand sampled biased 


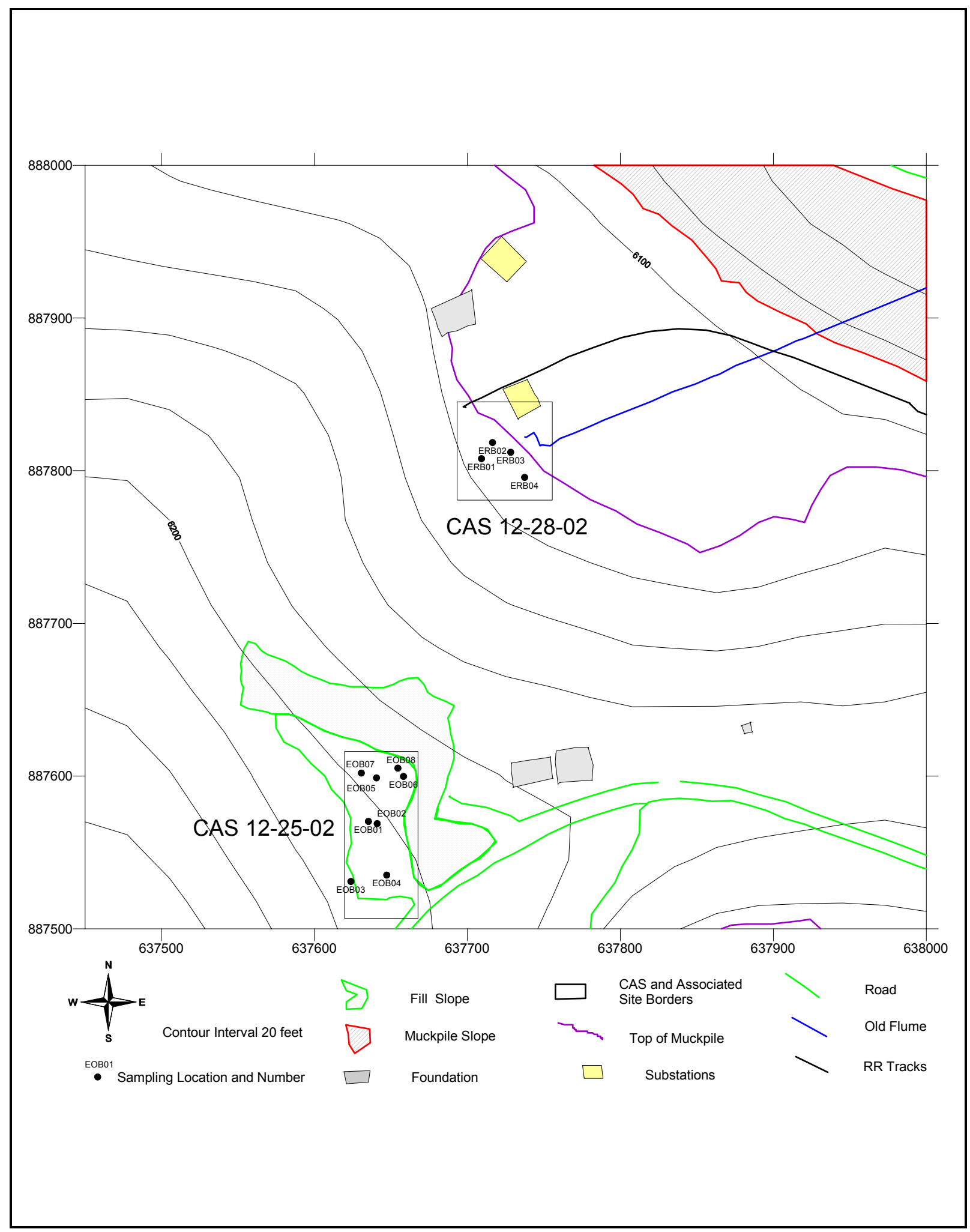

Figure A.3-5

CAU 383 Oil Spill and Radioactive Material Area Sampling Locations 
Table A.3-4

As-Built Oil Spill and Radioactive Material Area Sampling Locations and Total Depths

\begin{tabular}{|c|c|c|c|c|c|c|c|}
\hline Hole \# & North & East & Elevation & $\begin{array}{c}\text { Sampling } \\
\text { Method }\end{array}$ & \begin{tabular}{|c|} 
Drill TD in \\
$\mathrm{ft}$
\end{tabular} & \begin{tabular}{|c|} 
Hand TD in \\
$\mathrm{ft}$
\end{tabular} & $\begin{array}{c}\text { \# of } \\
\text { Samples }\end{array}$ \\
\hline \multicolumn{8}{|c|}{ Biased Oil Spill Locations } \\
\hline EOB01 & 637635.3 & 887570.3 & 6217.6 & hand & & 0.5 & 1 \\
\hline EOB02 & 637641.1 & 887568.9 & 6218.0 & hand & & 0.5 & 1 \\
\hline EOB03 & 637623.9 & 887531.0 & 6213.9 & hand & & 0.5 & 1 \\
\hline EOB04 & 637647.3 & 887535.2 & 6220.8 & hand & & 0.5 & 1 \\
\hline EOB05 & 637630.7 & 887602.0 & 6218.3 & hand & & 0.5 & 1 \\
\hline EOB06 & 637658.3 & 887599.8 & 6213.0 & hand & & 0.5 & 1 \\
\hline EOB07 & 637640.6 & 887598.8 & 6207.4 & hand & & 0.5 & 1 \\
\hline \multirow[t]{2}{*}{ EOB08 } & 637654.6 & 887605.2 & 6220.9 & hand & & 0.5 & 1 \\
\hline & & & & Total & 0 & 4.0 & 8 \\
\hline \multicolumn{8}{|c|}{ Biased Radiation Material Area Locations } \\
\hline ERB01 & 637709.2 & 887807.9 & 6122.6 & hand & & 0.5 & 1 \\
\hline ERB02 & 637716.4 & 887818.4 & 6119.5 & hand & & 0.5 & 1 \\
\hline ERB03 & 637728.3 & 887812.1 & 6121.3 & hand & & 0.5 & 1 \\
\hline \multirow[t]{2}{*}{ ERB04 } & 637737.4 & 887795.6 & 6118.7 & hand & & 0.5 & 1 \\
\hline & & & & Total & 0 & 2.0 & 4 \\
\hline
\end{tabular}

locations and all the samples were collected at $0.5 \mathrm{ft}$ below ground surface in February 2004 (Figure A.3-6). Both sets of samples were sent to an off-site laboratory and analyzed for beryllium, lead, arsenic, and radionuclides. In March 2004, a biased hand sample was collected from a contaminated layer, approximately six inches below the surface in front of the E-Tunnel main portal (location EGB09). The sample was sent to an off-site laboratory, and analyzed for strontium-90, isotopic plutonium, and gamma spec. These sample locations were designated as EGB01 through EGB09. Locations EGB05, EGB06, and EGB07 are the background sampling locations and EGB01, EGB02, EGB03, EGB04, and EGB09 are the preliminary sampling locations (there is no location EGB08). The sampling location coordinates and total depths are shown in Table A.3-5.

\section{A.3.9 Other Sampling}

In addition to the environmental samples, 52 quality assurance (QA)/QC and other samples were collected during the site characterization. The QA/QC samples included eight blind duplicate samples, collected and analyzed to check on the laboratory's precision; 


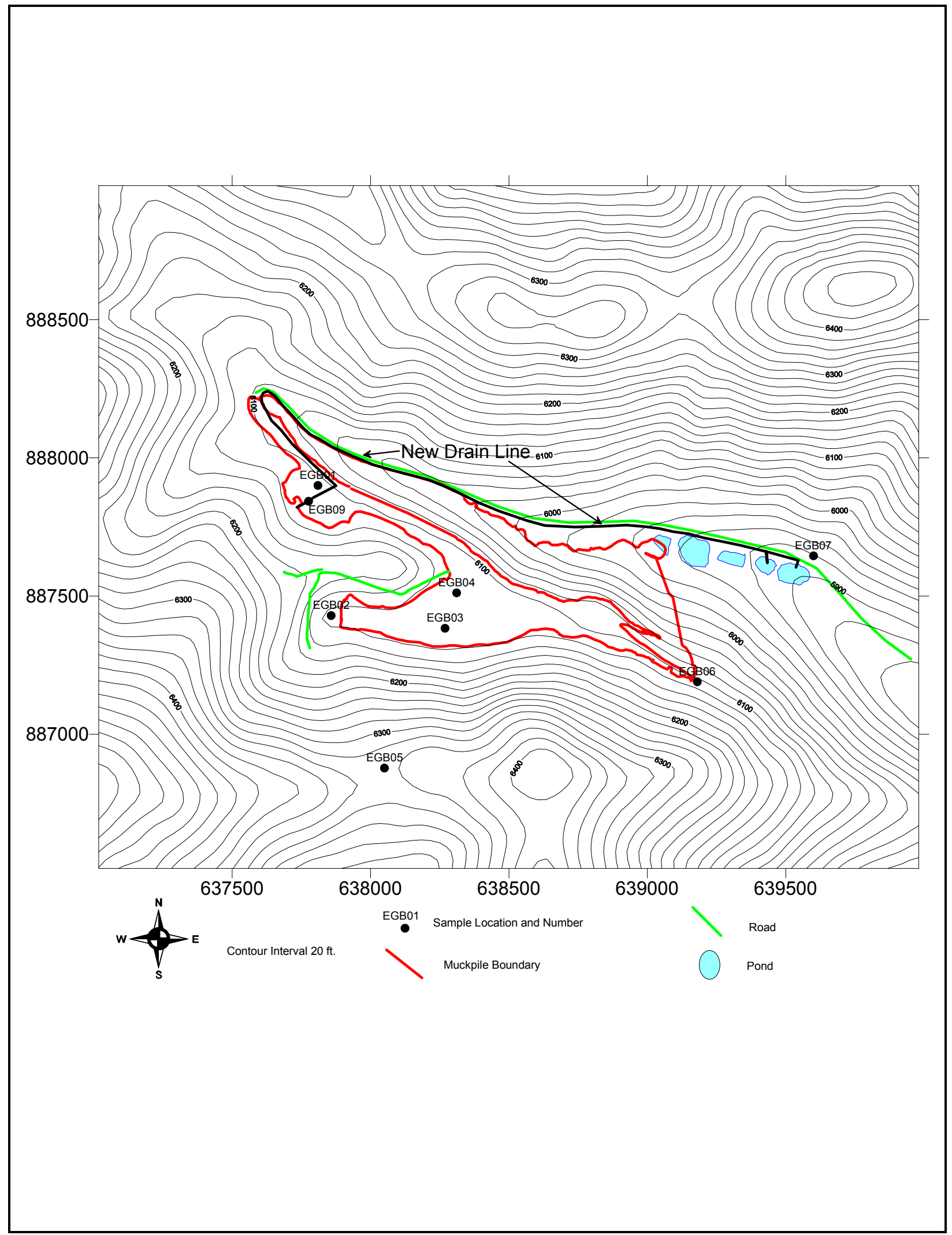


Table A.3-5

As-Built Background and Preliminary Sampling Locations Total Depths

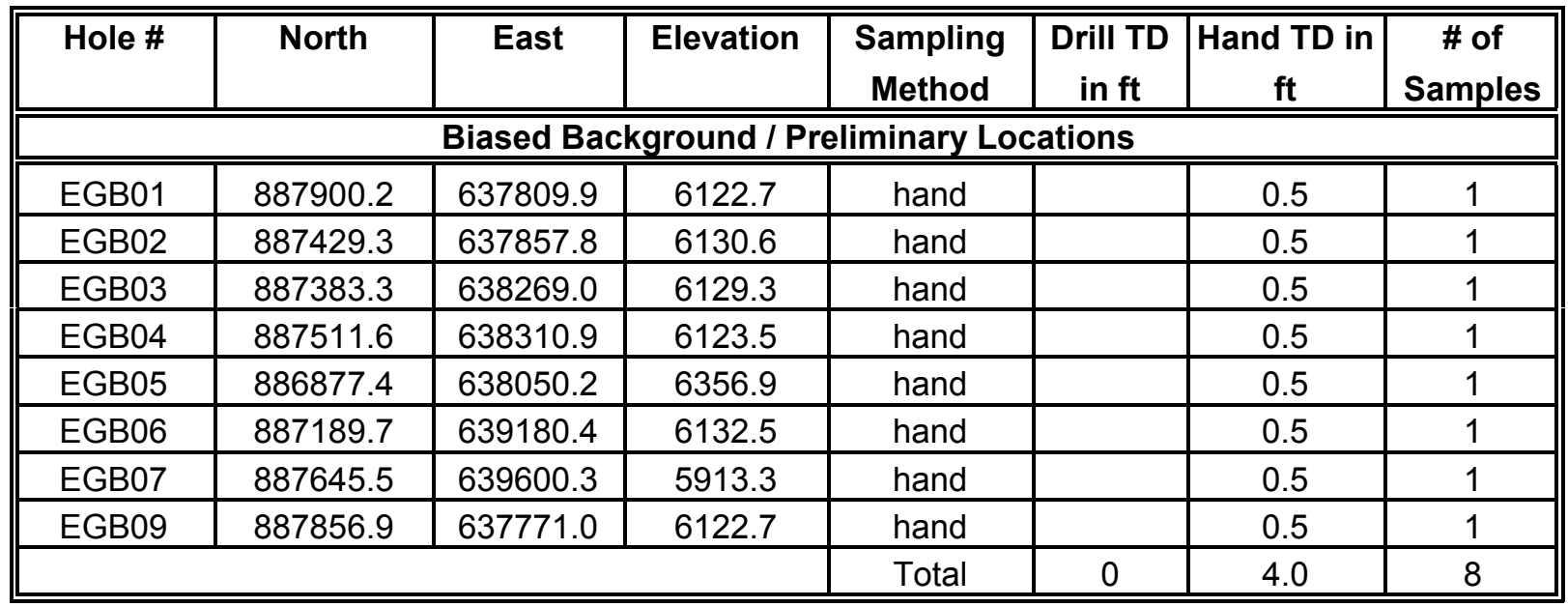

seven laboratory QC samples, collected to check for matrix interference; seven rinsate samples, collected to check on the effectiveness of the decontamination procedures; seven field blanks, collected to check on possible environmental interferences; and 22 trip blanks, sent with the VOC samples. Other sampling consisted of collecting a water sample from the driller's water truck (a source blank). All of the QC samples were sent to the off-site laboratory for analysis. 


\section{A.4.0 Results}

A spreadsheet of the analytical results from the CAU 383 E-Tunnel Sites investigation is provided in Attachment C. The spreadsheet includes only those analytical results where COPCs were detected, even if the detect was qualified as estimated ( $\mathrm{J}$ qualifier) or the radionuclide identification was tentative (TI qualifier). A detect merely indicates that the parameter was identified in the sample and does not have any reference to action levels or regulatory limits. The analytical results from the investigation are summarized in the following tables. Results from duplicate samples are not included. If a sample was reanalyzed by the laboratory, the reanalysis results are included in the table, not the original results. Table A.4-1 is a summary of the detects from the Muckpile and ponds, muck and native soil samples, which were analyzed for VOCs, SVOCs, TPH-D, beryllium, total metals, and radionuclides. Table A.4-2 is a summary of the detects from the RMA, oil spill, drainage, and QC water samples which were analyzed for VOCs, SVOCs, TPH-D, beryllium, total metals, and radionuclides. All the QC water sample results were included. Table A.4-3 is a summary of the detects from the background/preliminary soil samples, collected prior to the start of the characterization, which were analyzed for beryllium, total metals, and radionuclides. An analysis of the data, including comparisons to action levels and regulatory limits, will be included in the CADD. 
Table A.4-1

Analytical Result Detects for Muckpile and Pond Samples

(Page 1 of 3 )

\begin{tabular}{|c|c|c|c|c|c|c|c|c|}
\hline & \multicolumn{4}{|c|}{ Muckpile } & \multicolumn{4}{|c|}{ Ponds } \\
\hline & \multicolumn{2}{|c|}{ Native } & \multicolumn{2}{|c|}{ Muck } & \multicolumn{2}{|c|}{ Native } & \multicolumn{2}{|c|}{ Muck } \\
\hline & Range & $\begin{array}{c}\text { No. of } \\
\text { detects }\end{array}$ & Range & $\begin{array}{c}\text { No. of } \\
\text { detects }\end{array}$ & Range & $\begin{array}{l}\text { No. of } \\
\text { detects }\end{array}$ & Range & $\begin{array}{c}\text { No. of } \\
\text { detects }\end{array}$ \\
\hline Volatile Compounds & $\mu \mathrm{g} / \mathrm{kg}$ & & $\mu \mathrm{g} / \mathrm{kg}$ & & $\mu \mathrm{g} / \mathrm{kg}$ & & $\mu \mathrm{g} / \mathrm{kg}$ & \\
\hline 1,2,4-Trimethylbenzene & ND & 0 & 7.2 & 1 & ND & 0 & ND & 0 \\
\hline 1,3,5-Trimethylbenzene & ND & 0 & 7.2 & 1 & ND & 0 & ND & 0 \\
\hline Acetone & $5.7-18$ & 11 & $7.2-10.0$ & 9 & $6.7-25$ & 5 & $7.2-14.0$ & 3 \\
\hline Ethylbenzene & ND & 0 & 80 & 1 & ND & 0 & 1.6 & 1 \\
\hline Hexachlorobutadiene & 1.5 & 1 & ND & 0 & 1.7 & 1 & ND & 0 \\
\hline M+P-Xylene & ND & 0 & 1200 & 1 & ND & 0 & 8.3 & 1 \\
\hline Methylene Chloride & $0.78-1.6$ & 13 & $0.83-9.5$ & 23 & ND & 0 & $0.95-1.2$ & 3 \\
\hline N-Propylbenzene & ND & 0 & 5.9 & 1 & ND & 0 & ND & 0 \\
\hline O-Xylene & ND & 0 & $1.2-780.0$ & 2 & ND & 0 & 3.3 & 1 \\
\hline P-Isopropyltoluene & ND & 0 & $1.3-13.0$ & 2 & ND & 0 & ND & 0 \\
\hline Semivolatile Compounds & $\mu \mathrm{g} / \mathrm{kg}$ & & $\mu \mathrm{g} / \mathrm{kg}$ & & $\mu \mathrm{g} / \mathrm{kg}$ & & $\mu \mathrm{g} / \mathrm{kg}$ & \\
\hline 2-Methylnaphthalene & ND & 0 & 68 & 1 & ND & 0 & ND & 0 \\
\hline Acenaphthene & ND & 0 & 22 & 1 & ND & 0 & ND & 0 \\
\hline Acenaphthylene & ND & 0 & 14 & 1 & ND & 0 & ND & 0 \\
\hline Anthracene & ND & 0 & 170 & 1 & ND & 0 & ND & 0 \\
\hline Benzo(A)Anthracene & 22 & 1 & $18-1200$ & 10 & ND & 0 & $21-26$ & 2 \\
\hline Benzo(A)Pyrene & 16 & 1 & $15-1300$ & 7 & ND & 0 & $18-28$ & 2 \\
\hline Benzo(B)Fluoranthene & 28 & 1 & $18-1700$ & 8 & ND & 0 & $31-59$ & 2 \\
\hline Benzo $(\mathrm{G}, \mathrm{H}, \mathrm{I})$ Perylene & ND & 0 & $68-760$ & 4 & ND & 0 & ND & 0 \\
\hline Benzo(K)Fluoranthene & ND & 0 & $34-650$ & 6 & ND & 0 & 31 & 1 \\
\hline Bis(2-Ethylhexyl)Phthalate & $84-220$ & 2 & $72-170$ & 6 & ND & 0 & $85-330$ & 2 \\
\hline
\end{tabular}


Table A.4-1

Analytical Result Detects for Muckpile and Pond Samples

(Page 2 of 3 )

\begin{tabular}{|c|c|c|c|c|c|c|c|c|}
\hline & \multicolumn{4}{|c|}{ Muckpile } & \multicolumn{4}{|c|}{ Ponds } \\
\hline & \multicolumn{2}{|c|}{ Native } & \multicolumn{2}{|c|}{ Muck } & \multicolumn{2}{|c|}{ Native } & \multicolumn{2}{|c|}{ Muck } \\
\hline & Range & $\begin{array}{l}\text { No. of } \\
\text { detects }\end{array}$ & Range & $\begin{array}{c}\text { No. of } \\
\text { detects }\end{array}$ & Range & $\begin{array}{c}\text { No. of } \\
\text { detects }\end{array}$ & Range & $\begin{array}{l}\text { No. of } \\
\text { detects }\end{array}$ \\
\hline Butyl Benzyl Phthalate & ND & 0 & 35 & 1 & ND & 0 & ND & 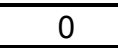 \\
\hline Carbazole & ND & 0 & 57 & 1 & ND & 0 & ND & 0 \\
\hline Chrysene & 23 & 1 & $21-1000$ & 8 & ND & 0 & $30-66$ & 2 \\
\hline Dibenzofuran & ND & 0 & 65 & 1 & ND & 0 & ND & 0 \\
\hline Di-N-Butyl Phthalate & ND & 0 & 26 & 2 & ND & 0 & ND & 0 \\
\hline Fluoranthene & 23 & 1 & $21-1500$ & 8 & ND & 0 & $20-31$ & 3 \\
\hline Fluorene & ND & 0 & 16 & 1 & ND & 0 & ND & 0 \\
\hline Indeno(1,2,3-Cd)Pyrene & ND & 0 & $27-630$ & 5 & ND & 0 & ND & 0 \\
\hline Naphthalene & ND & 0 & 19 & 1 & ND & 0 & ND & 0 \\
\hline Phenanthrene & ND & 0 & $30-350$ & 6 & ND & 0 & ND & 0 \\
\hline Pyrene & ND & 0 & $91-1600$ & 6 & ND & 0 & $50-63$ & 2 \\
\hline Petroleum Hydrocarbons & $\mathrm{mg} / \mathrm{kg}$ & & $\mathrm{mg} / \mathrm{kg}$ & & $\mathrm{mg} / \mathrm{kg}$ & & $\mathrm{mg} / \mathrm{kg}$ & \\
\hline Diesel-Range Organics & 700 & 1 & $3.2-300$ & 16 & ND & 0 & $1.9-330$ & 7 \\
\hline Metals & $\mathrm{mg} / \mathrm{kg}$ & & $\mathrm{mg} / \mathrm{kg}$ & & $\mathrm{mg} / \mathrm{kg}$ & & $\mathrm{mg} / \mathrm{kg}$ & \\
\hline Arsenic & $0.31-3.8$ & 23 & $0.7-6.0$ & 41 & $1.7-2.9$ & 5 & $1.0-14.0$ & 9 \\
\hline Barium & $2.6-370$ & 25 & $22.0-7000$ & 41 & $71.0-3200$ & 5 & $320-6300$ & 9 \\
\hline Beryllium & $0.15-1.0$ & 25 & $0.42-2.4$ & 41 & $0.65-1.0$ & 5 & $0.19-2.0$ & 9 \\
\hline Cadmium & $0.019-1.0$ & 20 & $0.03-15.0$ & 28 & $0.092-0.17$ & 5 & $0.052-0.39$ & 9 \\
\hline Chromium & $0.91-5.8$ & 25 & $0.49-33.0$ & 41 & $1.7-3.4$ & 5 & $1.4-12.0$ & 9 \\
\hline Lead & $1.1-160$ & 16 & $3.4-120$ & 41 & $5.7-12.0$ & 5 & 5.3-38 & 9 \\
\hline Mercury & $0.008-0.33$ & 25 & $0.0012-0.51$ & 40 & $0.011-0.12$ & 5 & $0.0016-0.048$ & 9 \\
\hline Selenium & $0.26-0.29$ & 2 & $0.24-0.99$ & 12 & ND & 0 & $0.31-0.65$ & 4 \\
\hline Silver & $0.071-2.8$ & 20 & $0.1-1.1$ & 5 & 0.48 & 1 & 0.077 & 1 \\
\hline
\end{tabular}


Table A.4-1

Analytical Result Detects for Muckpile and Pond Samples

(Page 3 of 3 )

\begin{tabular}{|c|c|c|c|c|c|c|c|c|}
\hline & \multicolumn{4}{|c|}{ Muckpile } & \multicolumn{4}{|c|}{ Ponds } \\
\hline & \multicolumn{2}{|c|}{ Native } & \multicolumn{2}{|c|}{ Muck } & \multicolumn{2}{|c|}{ Native } & \multicolumn{2}{|c|}{ Muck } \\
\hline & Range & $\begin{array}{c}\text { No. of } \\
\text { detects }\end{array}$ & Range & $\begin{array}{c}\text { No. of } \\
\text { detects }\end{array}$ & Range & $\begin{array}{c}\text { No. of } \\
\text { detects }\end{array}$ & Range & $\begin{array}{c}\text { No. of } \\
\text { detects }\end{array}$ \\
\hline Radionuclides & $\mathrm{pCi} / \mathrm{g}$ & & $\mathrm{pCi} / \mathrm{g}$ & & $\mathrm{pCi} / \mathrm{g}$ & & $\mathrm{pCi} / \mathrm{g}$ & \\
\hline Actinium-228 & $0.42-2.14$ & 5 & $0.5-2.89$ & 39 & $1.67-2.4$ & 5 & $1.48-2.31$ & 7 \\
\hline Aluminum-26 & ND & 0 & 0.108 & 1 & ND & 0 & ND & 0 \\
\hline Americium-241 & 1.1 & 1 & $0.52-28.8$ & 9 & ND & 0 & 0.72 & 1 \\
\hline Bismuth-212 & ND & 0 & $1.77-3.7$ & 6 & ND & 0 & ND & 0 \\
\hline Bismuth-214 & $0.26-0.92$ & 13 & $0.39-2.08$ & 36 & $0.96-1.49$ & 5 & $0.95-1.72$ & 8 \\
\hline Cesium-137 & $0.121-9.8$ & 9 & $0.15-1060$ & 39 & $0.33-4.34$ & 2 & $0.78-142$ & 8 \\
\hline Europium-152 & ND & 0 & 2.64 & 1 & ND & 0 & ND & 0 \\
\hline Europium-155 & 0.32 & 1 & ND & 0 & ND & 0 & ND & 0 \\
\hline Potassium-40 & $2.05-31.7$ & 11 & $3.7-43.8$ & 41 & 26.2-33.2 & 5 & 8.1-33.7 & 9 \\
\hline Lead-212 & $0.212-2.27$ & 10 & $0.35-3.37$ & 39 & $1.87-2.76$ & 5 & $0.27-2.73$ & 9 \\
\hline Lead-214 & $0.238-0.91$ & 18 & $0.3-2.08$ & 34 & $1.08-1.61$ & 5 & $1.09-1.74$ & 7 \\
\hline Plutonium-238 & $0.02-0.213$ & 5 & 0.017-15.6 & 21 & ND & 0 & $0.271-0.74$ & 4 \\
\hline Plutonium-239 & $0.03-4.55$ & 7 & $0.016-84.0$ & 28 & 0.154 & 1 & $0.305-5.37$ & 6 \\
\hline Strontium-90 & $0.048-0.33$ & 2 & $0.21-54.0$ & 16 & ND & 0 & $0.76-3.58$ & 5 \\
\hline Thorium-234 & 1.8 & 1 & 2.7 & 1 & ND & 0 & ND & 0 \\
\hline Thorium-208 & $0.093-0.76$ & 7 & 0.131-1.02 & 34 & $0.59-1.05$ & 5 & $0.51-0.8$ & 8 \\
\hline Tota & & 25 & & 41 & & 5 & & 9 \\
\hline
\end{tabular}

$\mu \mathrm{g} / \mathrm{kg}$ - Micrograms per kilogram

$\mathrm{mg} / \mathrm{kg}$ - Milligrams per kilogram

$\mathrm{pCi} / \mathrm{g}$ - Picocuries per gram

ND - Not detected 
Table A.4-2

Analytical Result Detects for the Radioactive Material, Oil Spill , Drainage, and QC Water Samples

(Page 1 of 4 )

\begin{tabular}{|c|c|c|c|c|c|c|c|c|}
\hline & \multicolumn{2}{|c|}{ "Radioactive Material } & \multicolumn{2}{|c|}{ Oil Spill } & \multicolumn{2}{|c|}{ Drainage } & \multicolumn{2}{|c|}{ QC Water } \\
\hline & Range & $\begin{array}{c}\text { No. of } \\
\text { detects }\end{array}$ & Range & $\begin{array}{c}\text { No. of } \\
\text { detects }\end{array}$ & Range & $\begin{array}{l}\text { No. of } \\
\text { detects }\end{array}$ & Range & $\begin{array}{l}\text { No. of } \\
\text { detects }\end{array}$ \\
\hline Volatile Compounds & $\mu \mathrm{g} / \mathrm{kg}$ & & $\mu \mathrm{g} / \mathrm{kg}$ & & $\mu \mathrm{g} / \mathrm{kg}$ & & $\mu g / L$ & \\
\hline 2-Butanone & ND & 0 & $11.0-17.0$ & 2 & 10 & 1 & ND & 0 \\
\hline Acetone & ND & 0 & $7.2-72.0$ & 3 & $6.2-39.0$ & 12 & $6.9-25.0$ & 9 \\
\hline Chloromethane & ND & 0 & ND & 0 & ND & 0 & $0.58-0.71$ & 2 \\
\hline Ethylbenzene & ND & 0 & ND & 0 & $1.2-11.0$ & 6 & ND & 0 \\
\hline Hexachlorobutadiene & ND & 0 & ND & 0 & 1.6 & 1 & ND & 0 \\
\hline M+P-Xylene & ND & 0 & ND & 0 & $2.1-57.0$ & 11 & ND & 0 \\
\hline Methylene Chloride & ND & 0 & ND & 0 & $0.82-4.0$ & 28 & $0.75-15.0$ & 18 \\
\hline O-Xylene & ND & 0 & ND & 0 & $1.0-23.0$ & 7 & ND & 0 \\
\hline Styrene & ND & 0 & ND & 0 & $0.99-3.6$ & 4 & ND & 0 \\
\hline Toluene & ND & 0 & ND & 0 & $1.0-1.6$ & 4 & $0.61-1.3$ & 4 \\
\hline Semivolatile Compounds & $\mu \mathbf{g} / \mathbf{k g}$ & & $\mu \mathbf{g} / \mathbf{k g}$ & & $\mu \mathrm{g} / \mathbf{k g}$ & & $\mu \mathrm{g} / \mathrm{L}$ & \\
\hline Acenaphthene & ND & 0 & $39-93$ & 2 & ND & 0 & ND & 0 \\
\hline Anthracene & ND & 0 & $86-470$ & 3 & ND & 0 & ND & 0 \\
\hline Benzo(A)Anthracene & $56-170$ & 4 & $14-18000$ & 8 & $26-66$ & 3 & ND & 0 \\
\hline Benzo(A)Pyrene & $39-230$ & 4 & $64-23000$ & 6 & $37-64$ & 3 & ND & 0 \\
\hline Benzo(B)Fluoranthene & $84-620$ & 4 & $140-43000$ & 6 & $51-62$ & 3 & ND & 0 \\
\hline Benzo(G,H,I)Perylene & 90 & 1 & $59-16000$ & 4 & 46 & 1 & ND & 0 \\
\hline Benzo(K)Fluoranthene & $41-230$ & 4 & $45-19000$ & 6 & $25-57$ & 3 & ND & 0 \\
\hline Bis(2-Ethylhexyl)Phthalate & $76-1200$ & 4 & $710-3900$ & 2 & $82-2400$ & 8 & $5.9-42.0$ & 14 \\
\hline
\end{tabular}


Table A.4-2

Analytical Result Detects for the Radioactive Material, Oil Spill , Drainage, and QC Water Samples

(Page 2 of 4 )

\begin{tabular}{|c|c|c|c|c|c|c|c|c|}
\hline & \multicolumn{2}{|c|}{ Radioactive Material } & \multicolumn{2}{|c|}{ Oil Spill } & \multicolumn{2}{|c|}{ Drainage } & \multicolumn{2}{|c|}{ QQ Water } \\
\hline & Range & $\begin{array}{c}\text { No. of } \\
\text { detects }\end{array}$ & Range & $\begin{array}{c}\text { No. of } \\
\text { detects }\end{array}$ & Range & $\begin{array}{c}\text { No. of } \\
\text { detects }\end{array}$ & Range & $\begin{array}{l}\text { No. of } \\
\text { detects }\end{array}$ \\
\hline Butyl Benzyl Phthalate & $23-630$ & 2 & ND & 0 & 33-40 & 2 & ND & $\overline{0}$ \\
\hline Carbazole & 23 & 1 & $160-790$ & 3 & ND & 0 & ND & 0 \\
\hline Chrysene & $63-480$ & 4 & $110-23000$ & 8 & $55-100$ & 3 & ND & 0 \\
\hline Dibenzo $(\mathrm{A}, \mathrm{H})$ Anthracene & 27 & 1 & $2100-10000$ & 2 & ND & 0 & ND & 0 \\
\hline Dimethyl Phthalate & ND & 0 & ND & 0 & 14 & 1 & ND & 0 \\
\hline Di-N-Butyl Phthalate & $45-5100$ & 4 & ND & 0 & 23 & 1 & ND & 0 \\
\hline Di-N-Octyl Phthalate & ND & 0 & ND & 0 & 59 & 1 & ND & 0 \\
\hline Fluoranthene & $86-510$ & 4 & $93-13000$ & 8 & $13-120$ & 4 & ND & 0 \\
\hline Fluorene & ND & 0 & $31-68$ & 2 & ND & 0 & ND & 0 \\
\hline Indeno(1,2,3-Cd)Pyrene & 80 & 1 & $54-12000$ & 3 & ND & 0 & ND & 0 \\
\hline Naphthalene & 18 & 1 & $15-56$ & 4 & ND & 0 & ND & 0 \\
\hline Phenanthrene & $38-210$ & 4 & $32-2500$ & 8 & 49 & 1 & ND & 0 \\
\hline Pyrene & $73-430$ & 4 & $75-64000$ & 8 & $41-190$ & 3 & ND & 0 \\
\hline Petroleum Hydrocarbons & $\mathrm{mg} / \mathrm{kg}$ & & $\mathrm{mg} / \mathrm{kg}$ & & $\mathrm{mg} / \mathrm{kg}$ & & $\overline{\mathrm{mg} / \mathrm{L}}$ & \\
\hline Diesel-Range Organics & $3.0-15$ & 3 & 63-3400 & 6 & $1.8-6.8$ & 8 & ND & 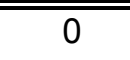 \\
\hline Metals & $\mathrm{mg} / \mathrm{kg}$ & & $\mathrm{mg} / \mathrm{kg}$ & & $\mathrm{mg} / \mathrm{kg}$ & & $\mu \mathrm{g} / \mathrm{L}$ & \\
\hline Arsenic & $4.5-7.1$ & 4 & $2.9-7.1$ & 8 & $2.2-5.3$ & 35 & $3.7-19.0$ & 2 \\
\hline Barium & $140-260$ & 4 & $140-300$ & 8 & $750-6600$ & 35 & $0.49-13.0$ & 6 \\
\hline Beryllium & $0.84-1.7$ & 4 & $0.64-1.2$ & 8 & $0.77-2.1$ & 35 & $0.06-0.09$ & 2 \\
\hline Cadmium & $0.56-0.57$ & 2 & $0.58-1.4$ & 4 & $0.11-0.37$ & 35 & 0.18 & 1 \\
\hline
\end{tabular}


Table A.4-2

Analytical Result Detects for the Radioactive Material, Oil Spill , Drainage, and QC Water Samples

(Page 3 of 4 )

\begin{tabular}{|c|c|c|c|c|c|c|c|c|}
\hline & \multicolumn{2}{|c|}{ Radioactive Material } & \multicolumn{2}{|c|}{ Oil Spill } & \multicolumn{2}{|c|}{ Drainage } & \multicolumn{2}{|c|}{ QC Water } \\
\hline & Range & $\begin{array}{l}\text { No. of } \\
\text { detects }\end{array}$ & Range & $\begin{array}{c}\text { No. of } \\
\text { detects }\end{array}$ & Range & $\begin{array}{l}\text { No. of } \\
\text { detects }\end{array}$ & Range & $\begin{array}{l}\text { No. of } \\
\text { detects }\end{array}$ \\
\hline Chromium & $3.2-9.1$ & 4 & $2.5-10.0$ & 8 & $1.1-9.2$ & 35 & 1.7 & 1 \\
\hline Lead & $18.0-61.0$ & 4 & $12.0-31.0$ & 8 & $14.0-52.0$ & 35 & ND & 0 \\
\hline Mercury & 0.16 & 1 & ND & 0 & $0.0088-0.083$ & 35 & $0.0066-0.03$ & 10 \\
\hline Selenium & ND & 0 & 1.2 & 1 & $0.29-1.1$ & 9 & 2.4 & 1 \\
\hline Silver & ND & 0 & ND & 0 & ND & 0 & $0.66-1.7$ & 7 \\
\hline Radionucides & $\overline{\mathrm{pCi} / \mathrm{g}}$ & & $\overline{p C i / g}$ & & $\overline{p C i / g}$ & & $\overline{p C i / L}$ & \\
\hline Actinium-228 & $1.26-2.9$ & 4 & $1.27-2.21$ & 8 & $0.7-2.26$ & 35 & ND & 0 \\
\hline Aluminum-26 & ND & 0 & ND & 0 & 0.105 & 1 & ND & 0 \\
\hline Americium-241 & ND & 0 & ND & 0 & 1.88 & 1 & ND & 0 \\
\hline Bismuth-212 & 3.2 & 1 & ND & 0 & ND & 0 & ND & 0 \\
\hline Bismuth-214 & $0.7-1.12$ & 3 & $0.58-1.1$ & 8 & $0.76-1.48$ & 35 & ND & 0 \\
\hline Cesium-137 & $7.06-114$ & 4 & $0.53-20.5$ & 8 & $2.72-78.1$ & 35 & ND & 0 \\
\hline Potassium-40 & $21.4-27.8$ & 4 & $19.8-25.0$ & 8 & $17.0-34.5$ & 35 & ND & 0 \\
\hline Lead-212 & $1.52-2.96$ & 4 & $1.83-3.1$ & 8 & $0.74-2.54$ & 35 & ND & 0 \\
\hline Lead-214 & $0.93-1.04$ & 3 & $0.86-1.06$ & 7 & $0.8-1.68$ & 34 & ND & 0 \\
\hline Plutonium-238 & $0.076-0.232$ & 3 & ND & 0 & $0.04-0.73$ & 32 & ND & 0 \\
\hline Plutonium-239 & $0.166-2.09$ & 4 & $0.051-1.04$ & 6 & $0.071-4.21$ & 35 & ND & 0 \\
\hline Strontium-90 & ND & 0 & ND & 0 & $0.45-1.88$ & 25 & ND & 0 \\
\hline Thorium-227 & 4.1 & 1 & ND & 0 & ND & 0 & ND & 0 \\
\hline Thorium-234 & 7.2 & 1 & ND & 0 & 2.4 & 1 & ND & 0 \\
\hline
\end{tabular}


Table A.4-2

Analytical Result Detects for the Radioactive Material, Oil Spill , Drainage, and QC Water Samples

(Page 4 of 4)

\begin{tabular}{|c|c|c|c|c|c|c|c|c|}
\hline & \multicolumn{2}{|c|}{ Radioactive Material } & \multicolumn{2}{|c|}{ Oil Spill } & \multicolumn{2}{|c|}{ Drainage } & \multicolumn{2}{|c|}{ QC Water } \\
\hline & Range & $\begin{array}{c}\text { No. of } \\
\text { detects }\end{array}$ & Range & $\begin{array}{c}\text { No. of } \\
\text { detects }\end{array}$ & Range & $\begin{array}{c}\text { No. of } \\
\text { detects }\end{array}$ & Range & $\begin{array}{l}\text { No. of } \\
\text { detects }\end{array}$ \\
\hline Thallium-208 & $0.47-0.85$ & 4 & $0.54-0.97$ & 8 & $0.3-0.87$ & 34 & ND & 0 \\
\hline \multicolumn{2}{|c|}{ Total No. of Samples Collected } & 4 & & 8 & & 35 & & 37 \\
\hline
\end{tabular}

$\mu \mathrm{g} / \mathrm{kg}$ - Micrograms per kilogram

$\mathrm{mg} / \mathrm{kg}$ - Milligrams per kilogram

$\mathrm{pCi} / \mathrm{g}$ - Picocuries per gram

$\mu \mathrm{g} / \mathrm{L}$ - Micrograms per liter

$\mathrm{mg} / \mathrm{L}$ - Milligrams per liter

ND - Not detected

pCi/l - Picocuries per liter 
Table A.4-3

Analytical Results for Background / Preliminary Samples

\begin{tabular}{||l||c|c||}
\hline & \multicolumn{2}{|c||}{ Background/Preliminary } \\
\hline & Range & No. of Detects \\
\hline Radionuclide & pCi/g & \\
\hline \hline Actinium-228 & $1.44-2.27$ & 6 \\
\hline Americium-241 & 2.83 & 1 \\
\hline Bismuth-214 & $0.45-1.41$ & 4 \\
\hline Cesium-137 & $0.46-180$ & 7 \\
\hline Potassium-40 & $8.8-30.7$ & 8 \\
\hline Lead-212 & $0.72-2.47$ & 8 \\
\hline Lead-214 & $0.6-1.07$ & 4 \\
\hline Plutonium-238 & $0.032-11.3$ & 5 \\
\hline Plutonium-239 & $0.093-61.0$ & 8 \\
\hline Strontium-90 & 18.8 & 1 \\
\hline Thallium-208 & $0.247-0.83$ & 5 \\
\hline \hline Total No. of Samples Collected & 8 \\
\hline
\end{tabular}

\begin{tabular}{||l|c|c||}
\hline & \multicolumn{2}{|c||}{ Background/Preliminary } \\
\hline & Range & No. of Detects \\
\hline \multicolumn{1}{|c|}{ Metals } & mg/kg & \\
\hline \hline Arsenic & $2.6-5.3$ & 7 \\
\hline Barium & $130-290$ & 3 \\
\hline Beryllium & $0.72-1.3$ & 6 \\
\hline Cadmium & $0.11-0.32$ & 2 \\
\hline Chromium & $5.0-8.4$ & 3 \\
\hline Lead & $12.0-75.0$ & 7 \\
\hline Mercury & $0.032-0.065$ & 3 \\
\hline Selenium & $0.52-0.71$ & 2 \\
\hline \hline \multicolumn{2}{|c|}{ Total No. of Samples Collected } & $7^{*}$ \\
\hline
\end{tabular}

$\mathrm{pCi} / \mathrm{g}$ - Picocuries per gram

$\mathrm{mg} / \mathrm{kg}$ - Milligrams per kilogram

* Sample 383GB901 was not analyzed for Metals 


\section{A.5.0 Waste Management}

Management of the investigation-derived waste (IDW) was guided by the CAU 383 CAIP (DTRA, 2003) requirements, field observations, and the results of the laboratory analysis of the E-Tunnel Sites characterization samples. Administrative controls were used to minimize the amount of waste generated during the investigation.

\section{A.5.1 Waste Segregation}

All wastes generated on the site were divided into either sanitary waste or IDW. The sanitary waste was disposed of in the dumpster located by Building 153. The IDW was segregated into solid (personal protective equipment [PPE] and plastic) and liquid (rinsate) waste streams to limit the amount of potentially contaminated waste.

\section{A.5.2 Waste Generation and Accumulation}

Waste generated during the field work was either sanitary waste or IDW. The sanitary waste consisted mostly of paper and kitchen debris generated in the field office and, in accordance with the waste minimization strategy, all PPE that was not visibly contaminated and/or did not have elevated field-screening results. The IDW consisted of potentially contaminated personal protective equipment (PPE), plastic, and all rinsates. This waste was dealt with as described in the rest of this section.

All IDW was put in U.S. Department of Transportation compliant, 55-gallon, steel drums. For drums receiving solid IDW, an absorbent $\left(\right.$ Stergo $^{\circledR}$ ) pad was placed in the bottom, and a plastic liner was inserted. After receiving waste, the drums were stored on plastic spill pallets in the fenced hazardous waste accumulation area (HWAA). After a drum received waste, it was marked with a unique identification number, labeled with a "Hazardous Waste Pending Analysis" sticker, a "Radioactive Material Pending Analysis" sticker, and was secured with a tamper-indicating device (TID). A waste management logbook was used to track the drums used, the contents of each drum, where the material was used, and an estimate of how much material was in each drum. The volume of material recorded for each drum is an estimate of what was put in the drum. 


\section{A.5.2.1 Rinsates}

The drill rig, tools, core barrels, drill bits, and sampling equipment (stainless-steel bowls) were decontaminated at the E-Tunnel decon pad before and after the work at E-Tunnel. After being decontaminated in the decon tub, the stainless-steel bowls, core barrels, and drill bits were cased in plastic sleeves to prevent them from getting contaminated prior to being used. Each hole was drilled using the same core barrel and drill bit for the entire hole. When the hole was completed they were decontaminated and sealed in plastic again. A clean stainless-steel bowl was used for each sample, after which it was deconned and sealed in plastic again. All liquid from the decontamination tub was containerized as rinsate.

Rinsate from the decon tub was transferred into an unlined 55-gallon drum staged next to the decontamination pad. Once used, each drum was marked and controlled (as stated above) and stored on a plastic spill pallet in the fenced HWAA. Waste was added to the drum until it was full (leaving some ullage). It was then secured (as stated before) and moved back to the HWAA. The waste management log book was used to track the drums used, where the equipment decontaminated with the rinsate was used, and an estimate of how much rinsate was in each drum.

\section{A.5.2.2 Soil}

No significant quantity of soil accumulated in the bottom of the decontamination tub, nor was any soil contaminated by spills, so no soil required disposal. The drill core was returned to the borehole it came from.

\section{A.5.2.3 PPE, Sampling Equipment, and Plastic Sleeves}

The PPE used in the exclusion zone consisted of Tyvek ${ }^{\circledR}$ anticontamination clothing, boot covers, cotton gloves, and nitrile or latex gloves. At the end of each shift, the PPE was evaluated with respect to the radiation emissions and the presence of visible contamination. If there were no elevated radiation emissions and there was no visible contamination, the PPE was determined to be sanitary waste and was disposed of in the roll-off bin at Building 153. During the ETunnel Sites and drainage characterization no PPE was determined to be IDW (it was all disposed of as sanitary waste).

The disposable sampling equipment was collected in a plastic bag inside the exclusion zone as the sampling was conducted. The wastes were accumulated until the bag was filled, and then it was taped closed. If there were no elevated radiation emissions and there was no visible 
contamination, the waste was disposed of as sanitary waste at Building 153. None of the sampling wastes were determined to be IDW.

Plastic sleeves were used to keep the decontaminated core barrels clean prior to use and to containerize slough and core as it was removed from the boreholes. After sampling, the core and slough material was returned to the borehole. The empty sleeves were containerized in a clear plastic bag and handled in the same manner as the PPE.

\section{A.5.2.4 Plastic Liners}

Plastic liners were used under the drill rig and the support truck to prevent hydrocarbon leaks from getting on the ground. To minimize waste generation, each liner was used at several locations unless it was damaged, leaked on, or field screening of the liner indicated the presence of contamination, in which case the liner was replaced at the completion of the hole. When the plastic liners became damaged, leaked on, or contaminated, the liner was containerized in a clear plastic bag, a waste tracking tag was attached, and the bag was put into a lined drum and treated as solid IDW (as described above). The samples collected while the plastic liner was in use were entered into the waste management logbook. The waste management log book was updated each time additional liners were added to the drum. No liners were containerized due to the presence of contamination other than minor hydrocarbon leaks.

\section{A.5.3 Waste Characterization}

The IDW was considered potentially contaminated waste by virtue of contact with potentially contaminated media or elevated radiological emissions. No IDW was containerized due to elevated radiological emissions. Therefore, additional sampling and analysis of the IDW, separate from the borehole soil sampling and analysis, was not required unless the sample results showed the potential for the IDW to be contaminated. The data generated as a result of the site characterization were used to make waste determinations of the IDW.

The IDW was characterized by identifying the borehole samples associated with each waste container and then comparing the analytical results from those samples to various standards. For the hazardous waste determination, the borehole sample results were compared to the RCRA waste characterization levels for hazardous waste. For total petroleum hydrocarbon wastes, the sample results were compared to the Nevada Administrative Code for TPH-D determination (NAC, 2002). For the low-level radioactive waste determination, the sample results were compared to the BN 2000 Application for Permit to Operate a Class II Solid Waste Disposal Site 
at the Nevada Test Site, and to the National Council on Radiation Protection and Measurements report entitled, Recommended Screening Limits for Contaminated Surface Soil and Review of Factors Relevant to Site-Specific Studies (NCRP, 1999) and DOE Order 5400.5 Change 2, "Radiation Protection of the Public and Environment" (DOE, 1993).

\section{A.5.4 Waste Disposal}

The hazardous waste determination did not identify any drums that were associated with samples that contained a high enough concentration of hazardous constituents to be considered hazardous. However, the two drums that contained hydrocarbon-stained plastic were shipped to the hydrocarbon landfill for disposal as hydrocarbon waste.

The preliminary radiological waste determination identified several samples that had radionuclide levels above the preliminary action level. However, when comparing the volume of soil in the drums required to be present to be regulated to the most conservative theoretical maximum amount of soil actually present in the drums, the action level was not exceeded. Therefore, the drums were nonradioactive.

Table 5-1 provides a tally of the number of drums used, what they contained, and their final disposition. Five of the drums of IDW were disposed of as sanitary waste, two drums (\#383M04 and \#383M05) were disposed of as hydrocarbon waste.

Table A.5-1

Drum Count

\begin{tabular}{||c|c|c|c||}
\hline Number of Drums & $\begin{array}{c}\text { Volume } \\
\text { (Gallons) }\end{array}$ & Waste Stream & Disposition \\
\hline \hline 4 & 182 & Rinsate & Sanitary \\
\hline 1 & 10 & PPE & Sanitary \\
\hline 2 & 60 & Plastic & Hydrocarbon Landfill \\
\hline \hline Total Number: 7 & & & \\
\hline
\end{tabular}




\section{A.6.0 Health and Safety}

At the start of the project, a preliminary hazard assessment was prepared and used to guide the preparation of the SSHASP (SNJV, 2004), the Radiation Work Permit (SN2004-CAU383-01), and the CAU 383 CAIP (DTRA, 2003). The work was then performed in accordance with these documents.

Prefield health and safety preparation was accomplished using a project-specific health and safety checklist. The checklist was developed to ensure that all required items were verified and/or available before mobilization to the field.

\section{A.6.1 Physical Hazards}

The primary physical hazards fell into three general categories: fatigue; heat stress; and slip, trip, and fall. The sources of these hazards were the long drive to and from the work site, the general hazards of working around moving machinery, the high ambient air temperatures on the test site at that time of year, and working on uneven ground and around the slope of the Muckpile.

The hazards associated with fatigue; heat stress; and slip, trip, and fall were dealt with by discussing the long drive; the hazards involved with the machinery; heat stress symptoms; and site-specific slip, trip, and fall hazards during the morning tailgate safety meetings. The length of the work day was limited and workers were observed by the SSO and SS for fatigue and heat stress and were encouraged to drink water. This ensured that workers did not become overly fatigued and could safely drive back to Mercury at the end of the day.

The hazard posed by the slope of the Muckpile was addressed by applying a restriction to drill no closer than $20 \mathrm{ft}$ from the edge of the Muckpile. This conservative restriction ensured personnel did not work too close to the edge of the Muckpile and ensured that the vibrations and weight of the rotosonic drill rig would not cause the slope to fail.

\section{A.6.2 Chemical and Radiological Hazards}

Chemical hazards believed to be present included mostly hydrocarbon products, epoxies, and chemical grouts that may have been disposed of in the Muckpile. These products were used for fuels, ground support, and various other applications during mining operations. Radiological 
hazards could possibly have come from the rock and construction waste deposited in the Muckpile during reentry mining operations following nuclear testing.

Drive-over and walk-over radiological surveys were conducted at the E-Tunnel Muckpile and the drainage associated with the Muckpile. These surveys identified surface and near-surface areas with elevated readings on the Muckpile, in the drainage and ponds, and near the compressor pad. The highest level was approximately 5.2 times greater than the background radiation emission rate. To ensure radiological safety, additional precautions were taken during drilling and sampling activities, including high volume air sampling. No airborne radioactive particles were detected in the high-volume air sample filters during field activities. All personnel wore PPE in the exclusion zone. All the PPE was determined to be sanitary waste. After each shift, all personnel in the exclusion zone (EZ) were monitored for elevated radiation emission as they left the EZ. No elevated emissions were detected.

Products brought on site to assist with the characterization investigation were required to have a material safety data sheet prior to receiving approval for use. These items generally consisted of fuels, lubricants, and cooling and cleaning agents.

Action levels for the chemical constituents and radiological hazards were posted on the tailgate safety briefing as noted in the SSHASP. All intrusive work required real-time monitoring be conducted and the results documented on the sample collection logs (SCLs) and the Soil Characterization Log. Field-screening results ensured action limits for occupational threats to personnel during the drilling and sampling operations were not exceeded.

As a precaution, all personnel wore gloves, boot covers, and Tyvek ${ }^{\circledR}$ anticontamination clothing in the EZ.

\section{A.6.3 Drilling}

An equipment inspection of each Boart-Longyear drill rig was performed by SNJV personnel upon their arrival on site and prior to any drilling activity. These inspections were documented on the Drill Rig Inspection form as described in the Field Instruction (DTRA, 2004). All safety devices were present and operational. In addition, the drill rig was inspected daily by the driller. This was recorded in the geologist's Field Activity Daily Log (FADL). The rotosonic drill rigs were operated safely throughout the project. 


\section{A.6.4 Noise}

During rotosonic drilling operations personnel exposure to noise is always a concern. Noiselevel monitoring was conducted prior to startup and during actual drilling, with a sound-level meter set on the ' $A$ ' scale with a slow response. Measurements indicated that average noise levels of 85 to 110 decibels were common within $35 \mathrm{ft}$ of the drill rig. With this knowledge, all personnel working or observing operations within this distance were required to wear hearing protection. Personnel were also diligent in watching out for each other to make certain that hearing protection was available and in use prior to equipment startup.

\section{A.6.5 Air Monitoring}

A Photovac Micro FID was used for real-time air monitoring for VOCs during all intrusive work. The FID measures concentrations of photoionizable gases and records the values. The FID cannot distinguish between individual pollutants; it merely lets the operator know that something is present. During drilling activities at the Area 12 E-Tunnel Sites, the FID was used to measure concentrations in a worker's breathing zone. All concentrations logged during field activities were below the established action level of one part per million sustained in the breathing zone.

In addition to the VOC monitoring, radiation monitoring was conducted daily through highvolume air sampling. One air sample was collected in the exclusion zone side of the sample van and another sample was collected in the clean side of the sample van. Two more air samplers were used to collect air samples (one at the exclusion zone hot line and one on the drill rig). These samples were collected by continuously drawing air through the two filters during work hours. Once work ceased for the day, the filters were removed and counted on a Tennelec series 5XLB, low-background alpha/beta counter. No airborne radioactive particles were detected in the high-volume air sample filters during field activities.

\section{A.6.6 Personnel Monitoring}

All personnel were required to wear a thermal luminescent dosimeter while working at the E-Tunnel site.

\section{A.6.7 Training}

Prior to assignment, all necessary training documentation for specific tasks was reviewed and verified as to current status. By verifying all documentation prior to personnel arriving on site, delays to track down missing or incomplete training documentation were minimized. 
Training requirements for this project were outlined in the SSHASP and included 40-hour Hazardous Waste Operations and Emergency Response, annual 8-hour refresher, medical surveillance, and Radiological Worker II. Additional training for specific personnel included 8-hour supervisory training, first aid and cardiopulmonary resuscitation, blood-borne pathogens training, and classroom and field waste management training. 


\section{A.7.0 Lessons Learned}

A lessons learned meeting was not held following the field work. The work all went well and since this was the sixth mudpit/Muckpile field effort, it was decided that there was little that could be gained by holding a lessons learned meeting. 


\section{A.8.0 References}

BN, see Bechtel Nevada.

Bechtel Nevada. 2000. Application for Permit to Operate a Class II Solid Waste Disposal Site at the Nevada Test Site. Las Vegas, NV.

CFR, see Code of Federal Regulations.

Code of Federal Regulations. 2002. Title 40 Part 260 - 282, "Protection of the Environment: RCRA Regulations." Washington, DC: Government Printing Office.

DTRA, see Defense Threat Reduction Agency.

Defense Threat Reduction Agency. 2003. Corrective Action Investigation Plan for Corrective Action Unit 383: Area 12 E-Tunnel Sites, Nevada Test Site, Rev. 0. Prepared by SNJV. Las Vegas, NV.

Defense Threat Reduction Agency. 2004. Field Instructions for CAU 383: Area 12 E-Tunnel Sites, Revision 0. Prepared by SNJV Las Vegas, NV.

FFACO, see Federal Facility Agreement and Consent Order.

Federal Facility Agreement and Consent Order. 1996 (as amended). Agreed to by the State of Nevada, the U.S. Department of Energy, and the U.S. Department of Defense. Las Vegas, NV.

Gilbert, R.O. 1987. Statistical Methods for Environmental Pollution Monitoring, Chapter 4. New York, NY: Van Nostrand Reinhold.

NAC, see Nevada Administrative Code.

National Council on Radiation Protection and Measurements. 1999. Recommended Screening Limits for Contaminated Surface Soil and Review of Factors Relevant to Site-Specific Studies. NCRP Report No. 129. Bethesda MD.

Nevada Administrative Code. 2002. NAC 445A, "Water Controls.” Carson City, NV.

Stoller-Navarro Joint Venture. 2004a. SNJV Site-Specific Health and Safety Plan, CAU 383 : Area 12 E-Tunnel Sites, Nevada Test Site, Revision 0. Las Vegas, NV.

Stoller-Navarro Joint Venture. 2004b. SNJV Waste Characterization Report, Investigation Derived Waste (IDW), Corrective Action Unit (CAU) 383, Area 12, E-Tunnel Sites, Nevada Test Site, Revision 0. Las Vegas, NV. 
U.S. Department of Energy. 1993. DOE Order 5400.5 Change 2, "Radiation Protection of the Public and Environment." Washington, DC.

U.S. Department of Energy, Nevada Operations Office. 1996. Final Environmental Impact Statement for the Nevada Test Site and Off-Site Locations in the State of Nevada, DOE/EIS 0243, August. Las Vegas, NV. 
Attachment A

Soil Boring Logs 
Project Name: CAU 383 E-Tunnel sites

Project Humber: DT04-320

Borehole Number: EDHO1

Logged By: $M$. McLane

Drilled By: M. McLane

Drilling Method: Hand Sampled
Date Started: $6 / 07 / 04$

Date completed: $6 / 07 / 04$

Elevation: 6007.8

Horthing : 887739.2

Easting: 638589.8

Total Depth: 0.5 ft.

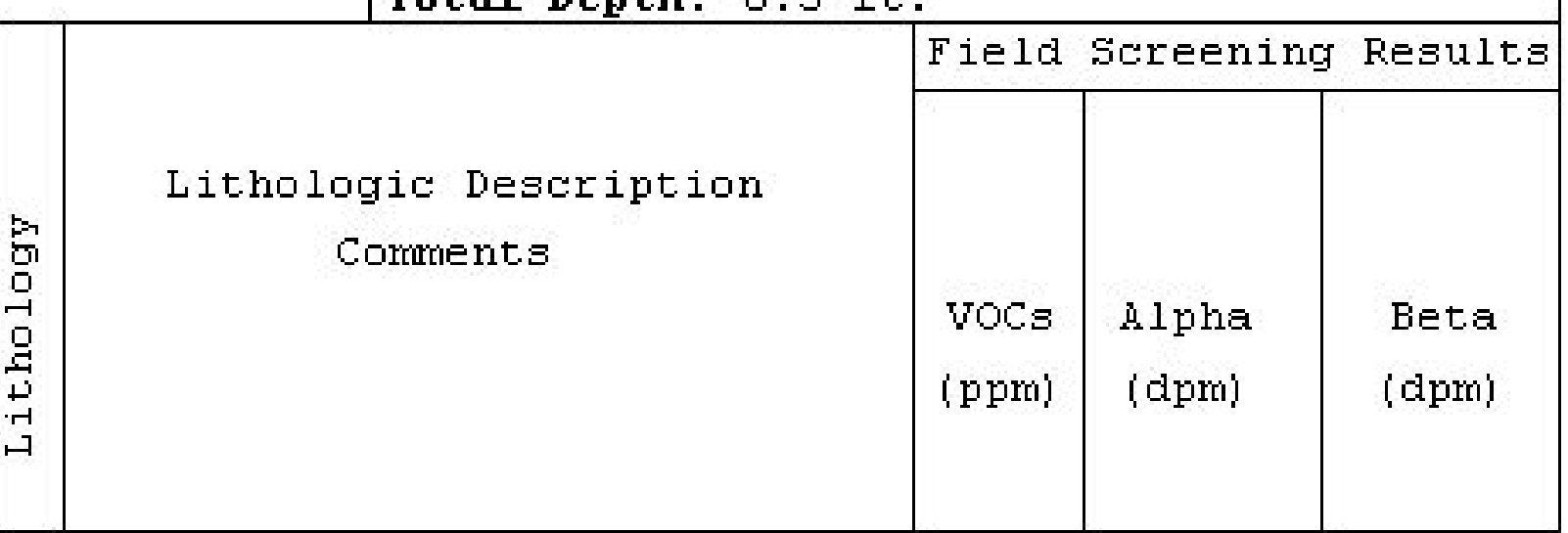

Contact o N/A, Sample 383DH0100.5.

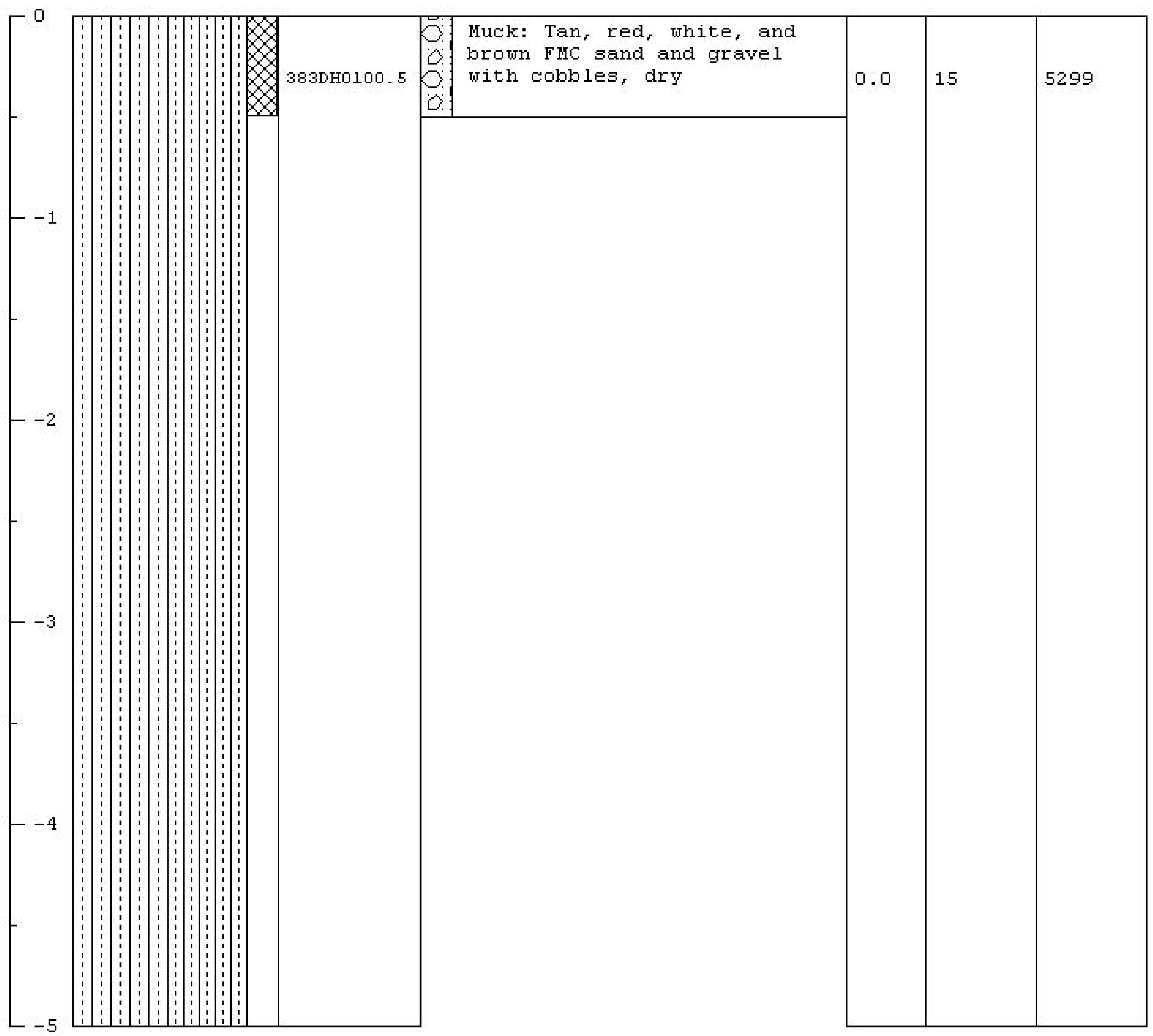


Project Name: CAU 383 E-Tunnel sites

Project Humber: DT04-320

Borehole Number: EDHO2

Logged By: $M$. McLane

Drilled By: M. McLane

Drilling Method: Hand Sampled
Date Started: $6 / 07 / 04$

Date completed: $6 / 07 / 04$

Elevation: 6006.8

Horthing: 887739.7

Easting: 638599.3

Total Depth: 0.5 ft.

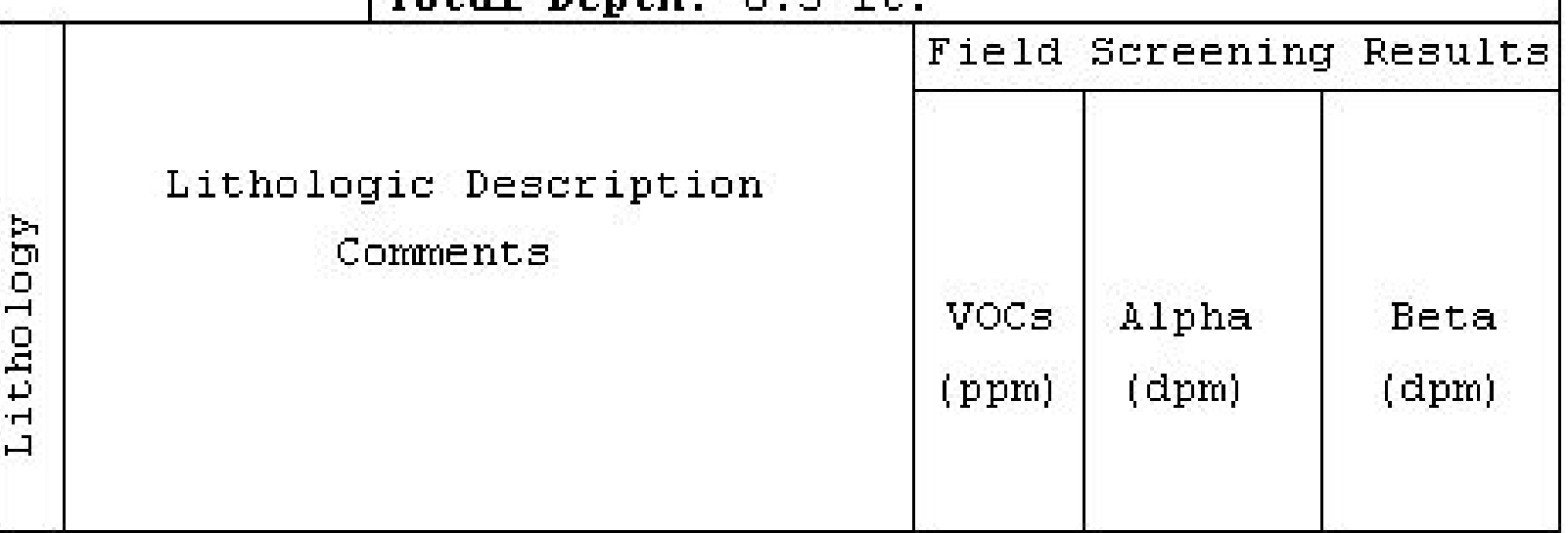

Contact o N/A, Sample 383DH0200.5.

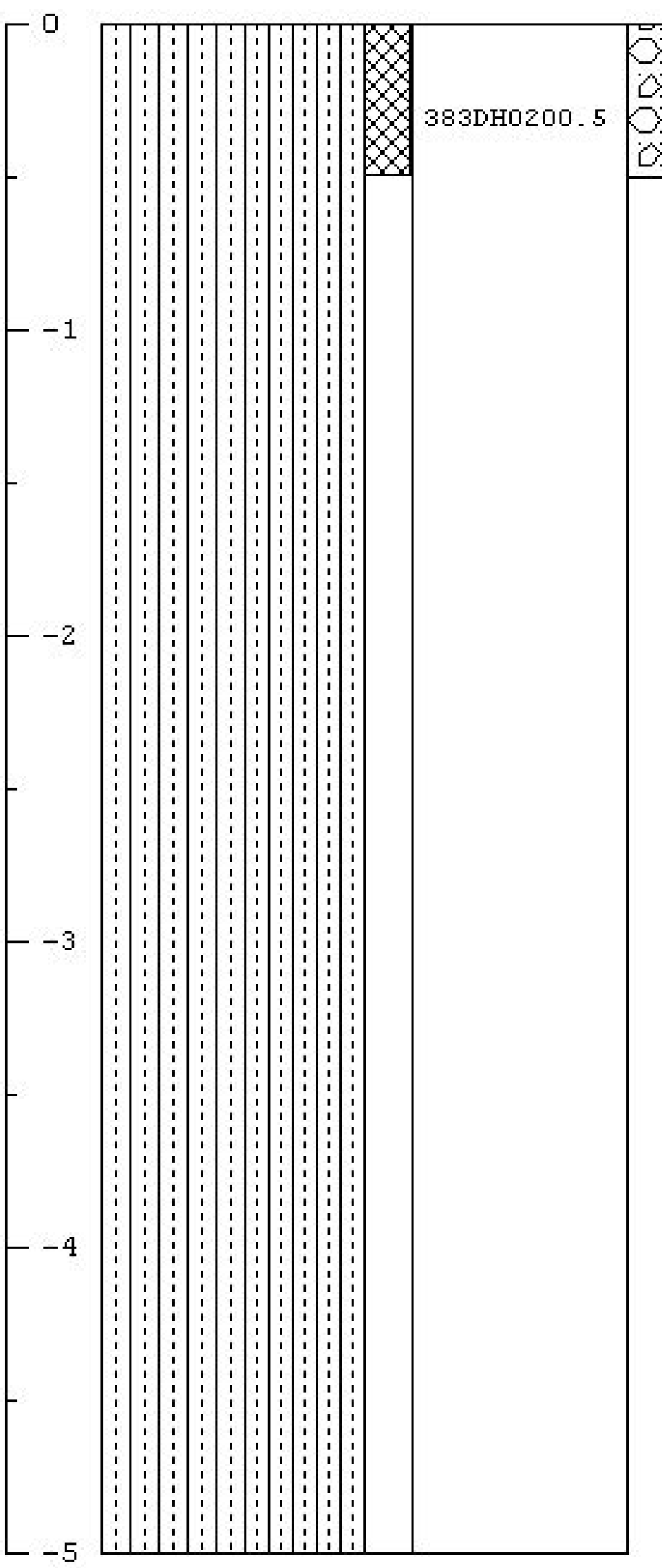

Muck: Tan, red, white, and brown FMC sand and gravel with cobbles, dry

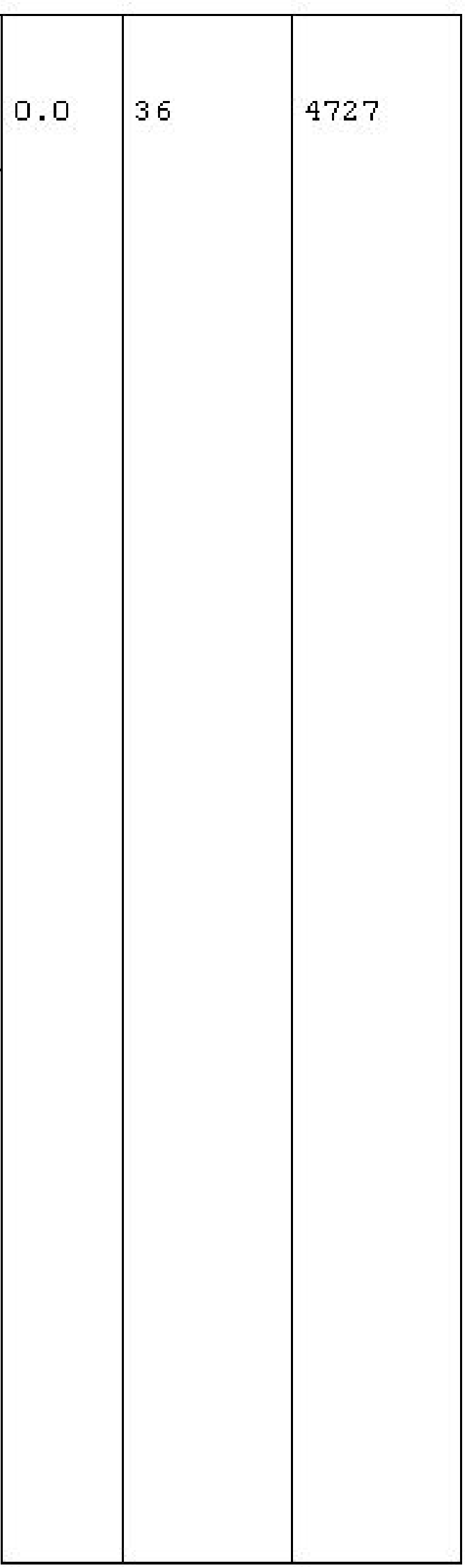


Project Name: CAU 383 E-Tunnel sites

Project Humber: DT04-320

Borehole Number: EDHO3

Logged By: $M$. McLane

Drilled By: M. McLane

Drilling Method: Hand Sampled
Date Started: $6 / 07 / 04$

Date completed: $6 / 07 / 04$

Elevation: 6004.1

Horthing: 887733.6

Easting: 638607.1

Total Depth: 0.5 ft.

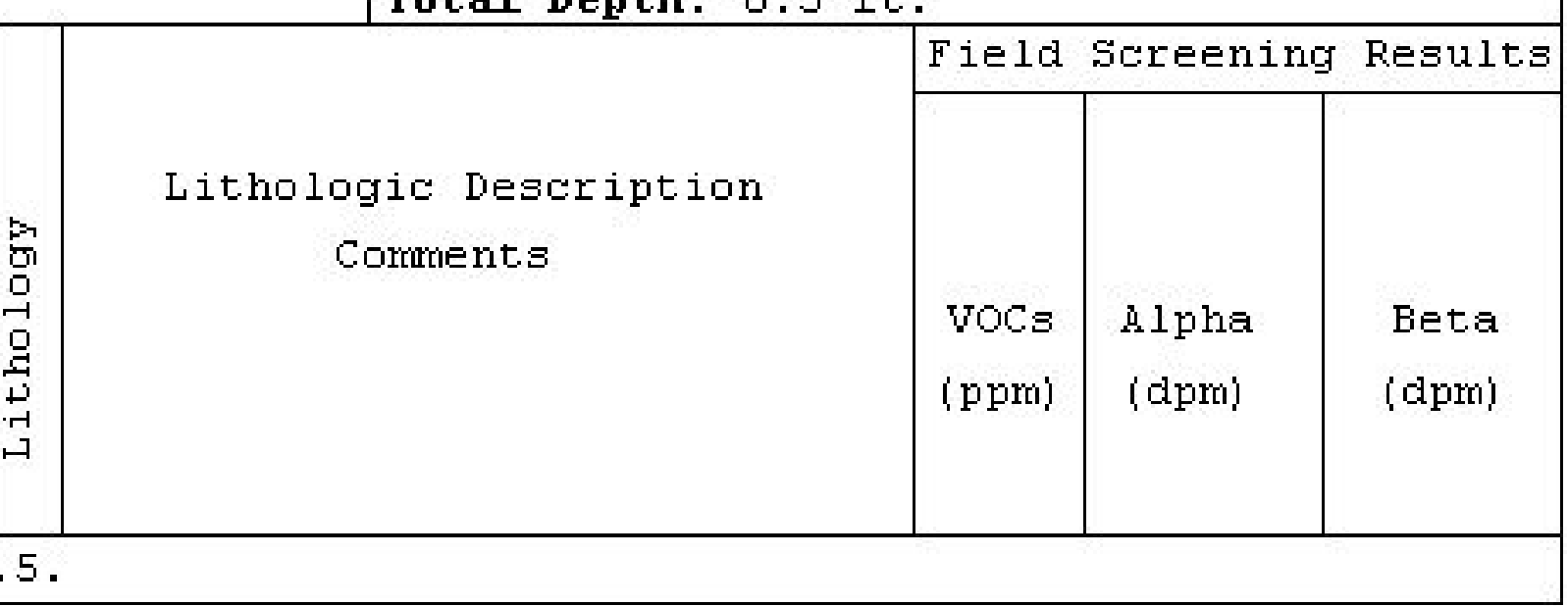

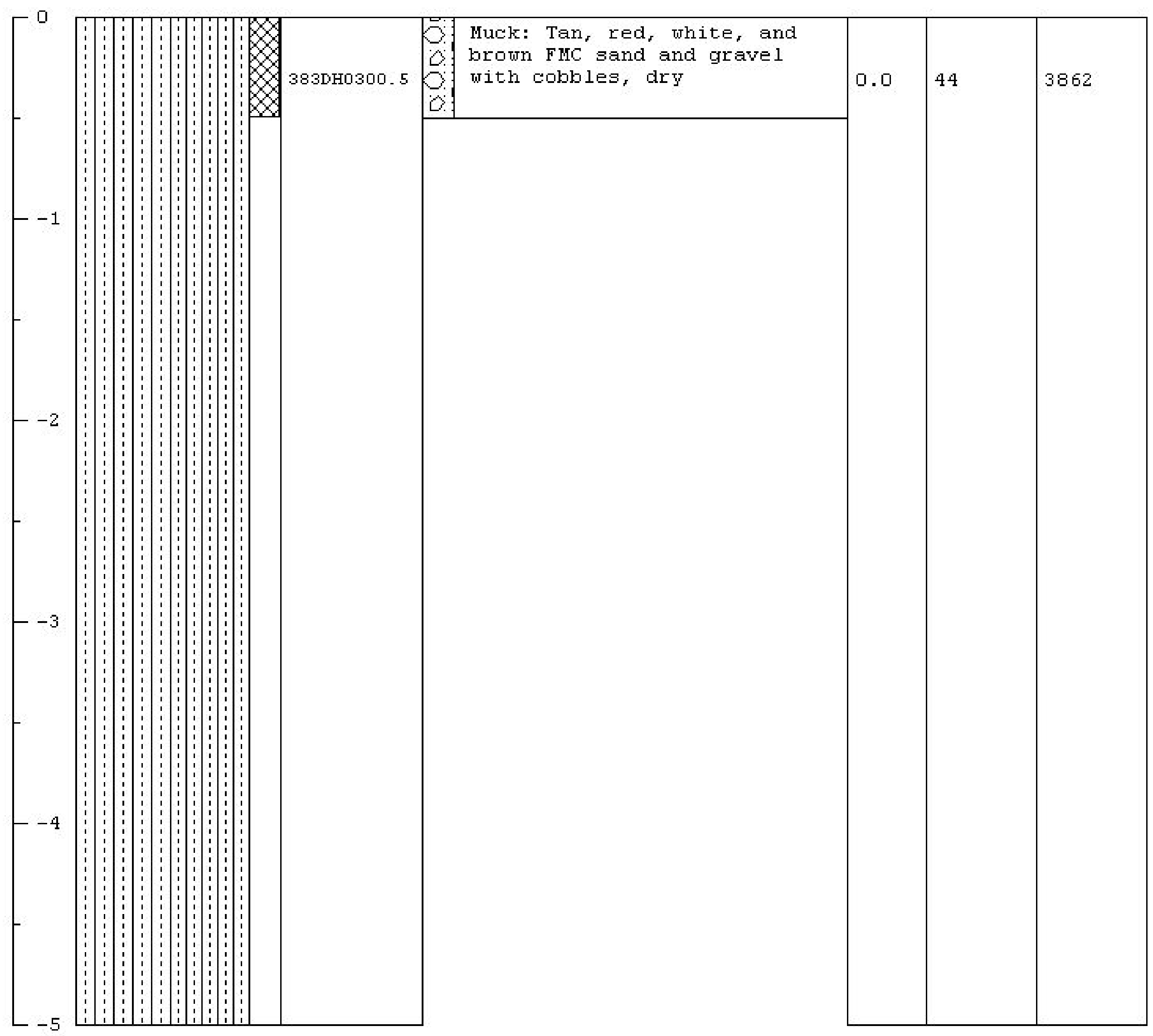


Project Name: CAU 383 E-Tunnel sites

Project Humber: DT04-320

Borehole Number: EDHO4

Logged By: $M$. McLane

Drilled By: M. McLane

Drilling Method: Hand Sampled
Date Started: $6 / 07 / 04$

Date completed: $6 / 07 / 04$

Elevation: 6003.0

Horthing : 887734.1

Easting: 638619.8

Total Depth: 0.5 ft.

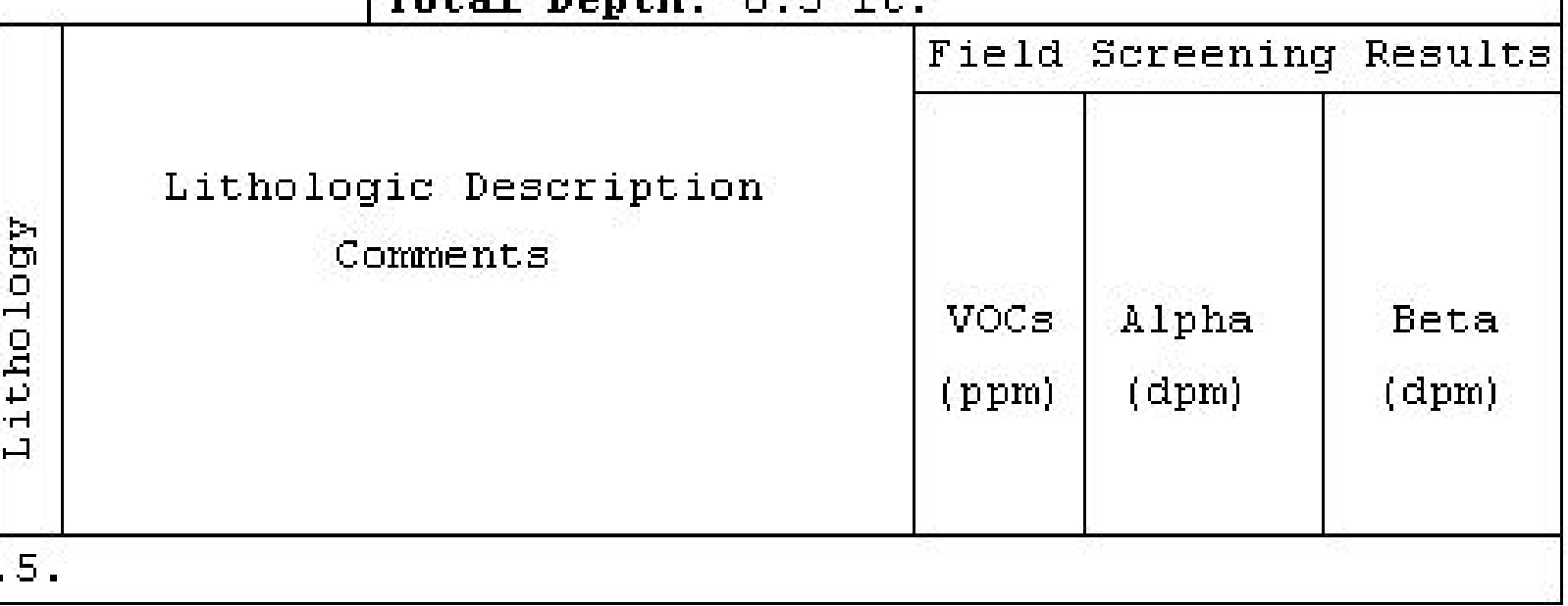

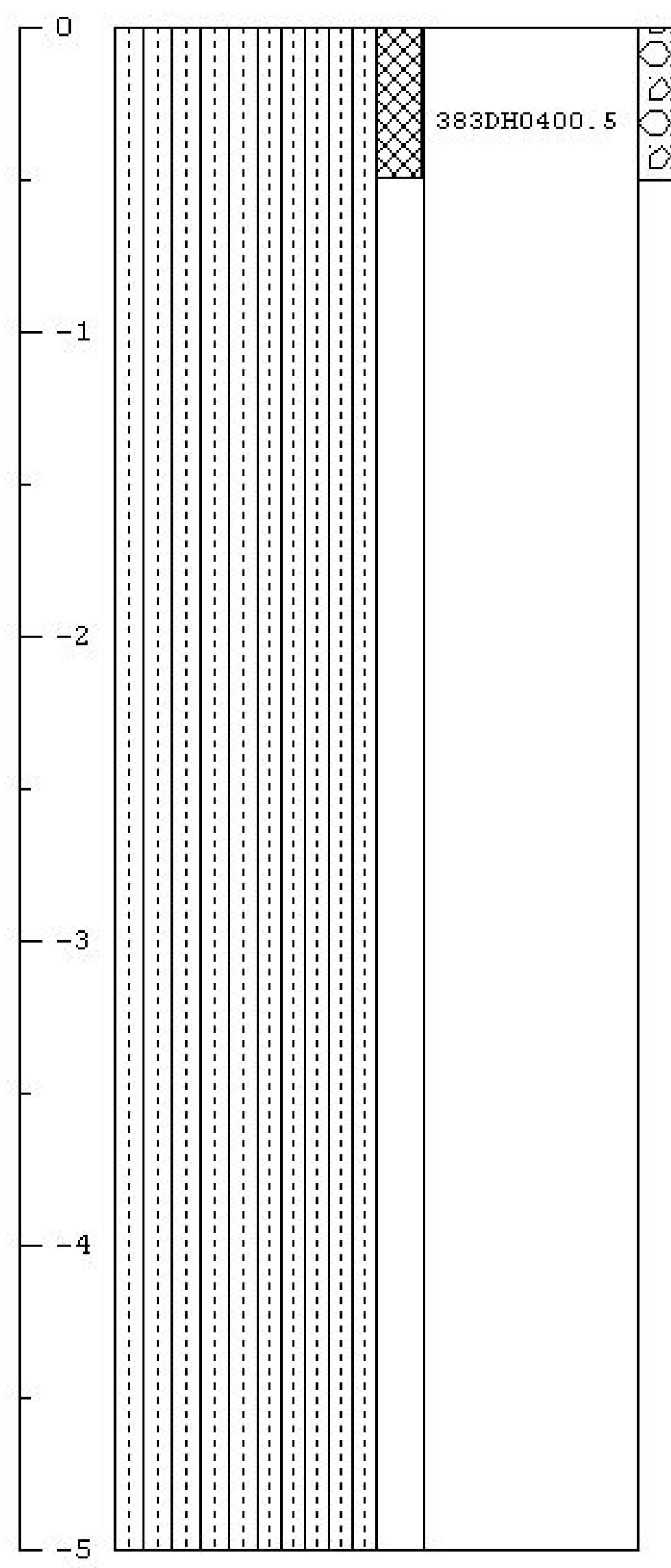

Muck: Tan, red, white, and brown FMC sand and gravel with cobbles, dry

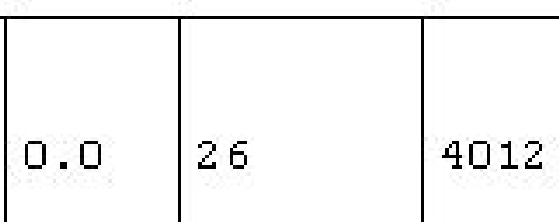


Project Name: CAU 383 E-Tunnel sites

Project Humber: DT04-320

Borehole Number: EDHO5

Logged By: $M$. McLane

Drilled By: M. McLane

Drilling Method: Hand Sampled
Date Started: $6 / 07 / 04$

Date completed: $6 / 07 / 04$

Elevation: 6003.1

Horthing: 887731.5

Easting: 638628.4

Total Depth: 0.5 ft.

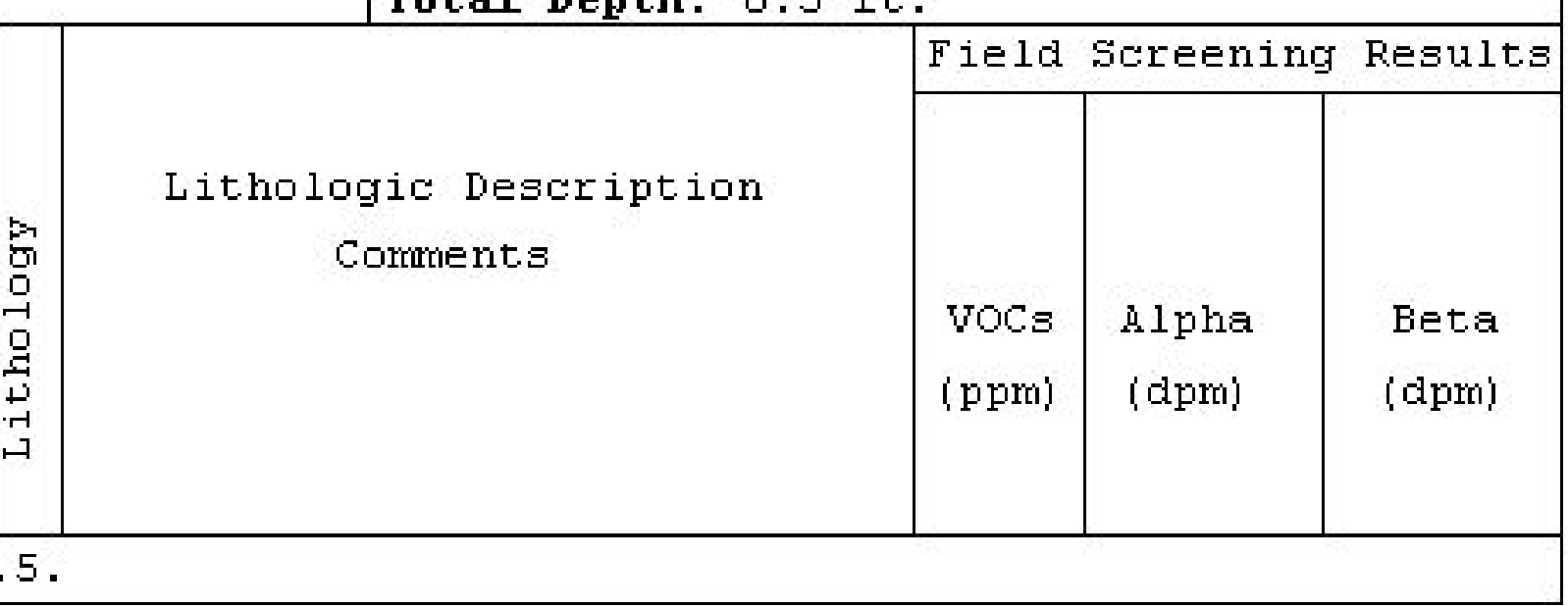

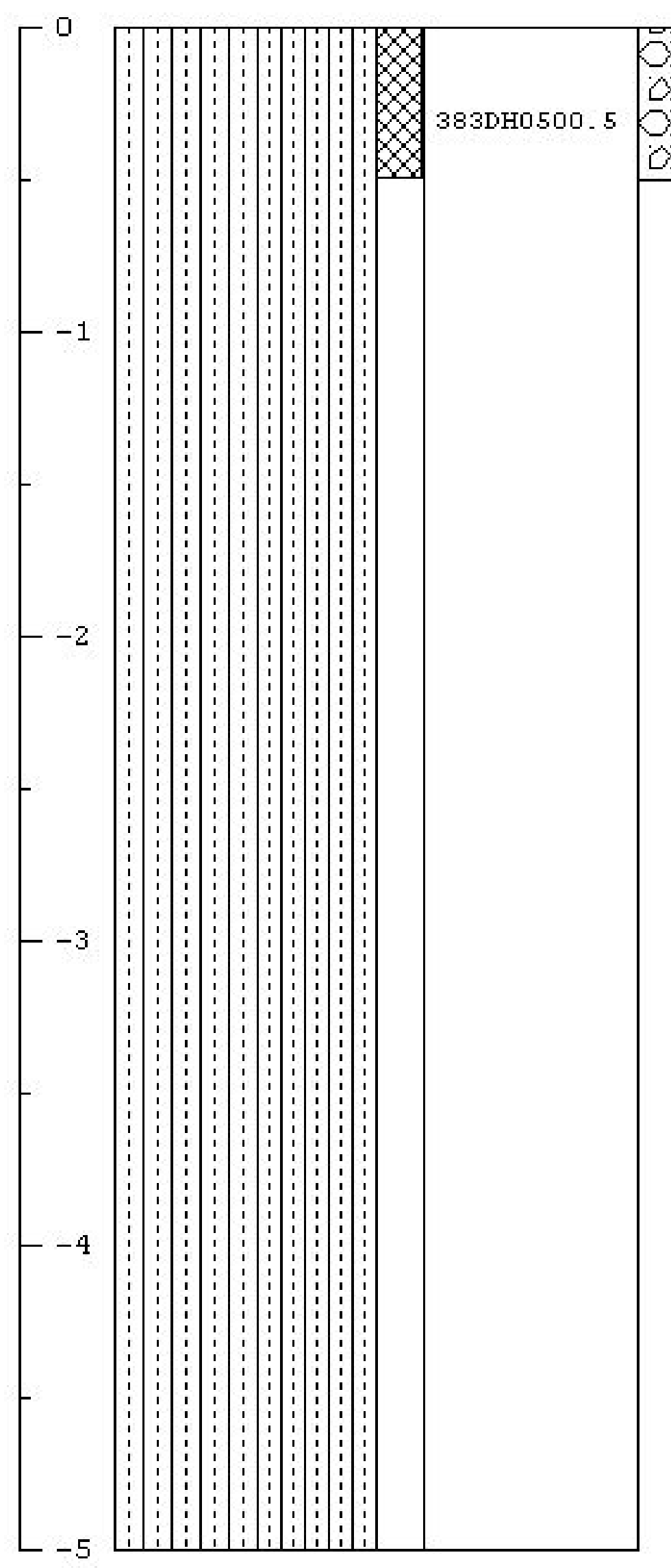

Muck: Tan, red, white, and brown FMC sand and gravel with cobbles, dry

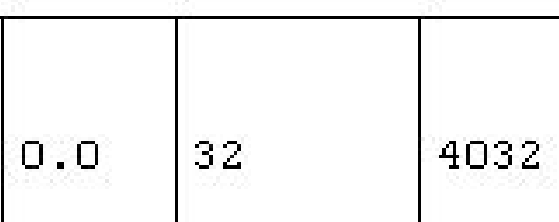


Project Name: CAU 383 E-Tunnel sites

Project Humber: DT04-320

Borehole Number: EDHO 6

Logged By: $M$. McLane

Drilled By: M. McLane

Drilling Method: Hand Sampled
Date Started: $6 / 07 / 04$

Date completed: $6 / 07 / 04$

Elevation : 5998.9

Horthing: 887731.2

Easting: 638640.7

Total Depth: 0.5 ft.

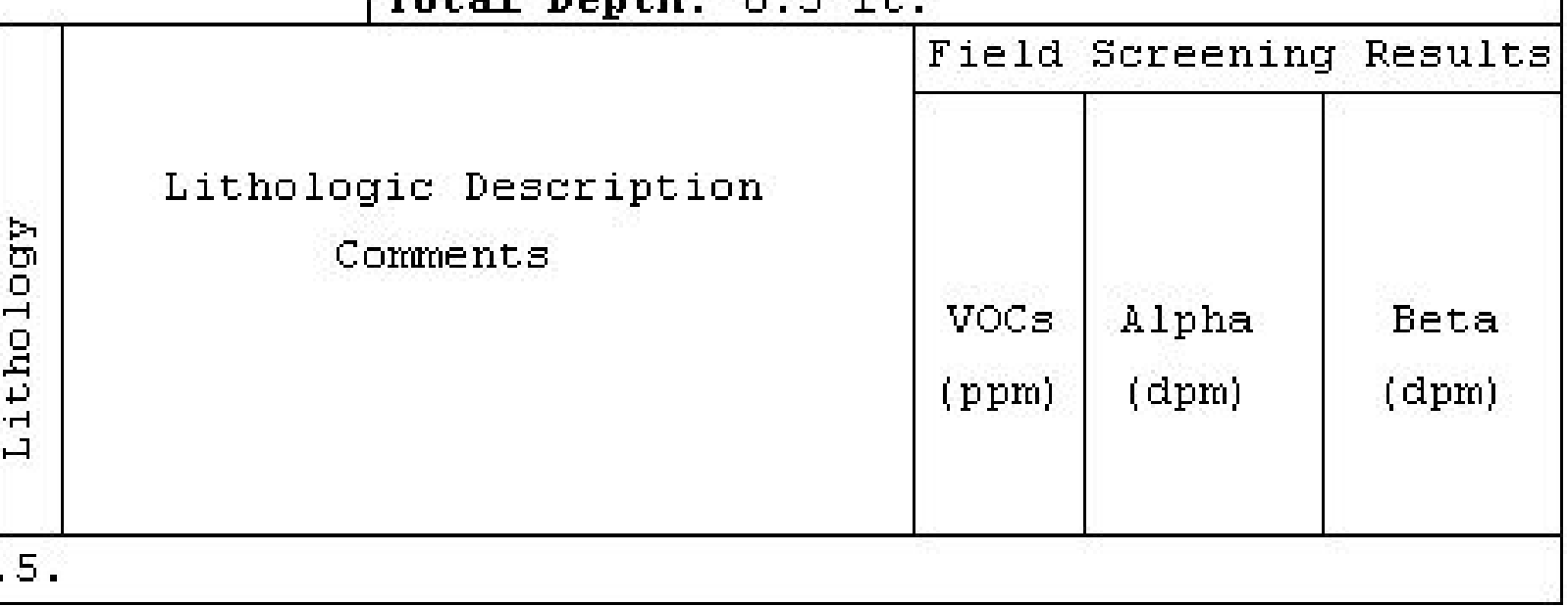

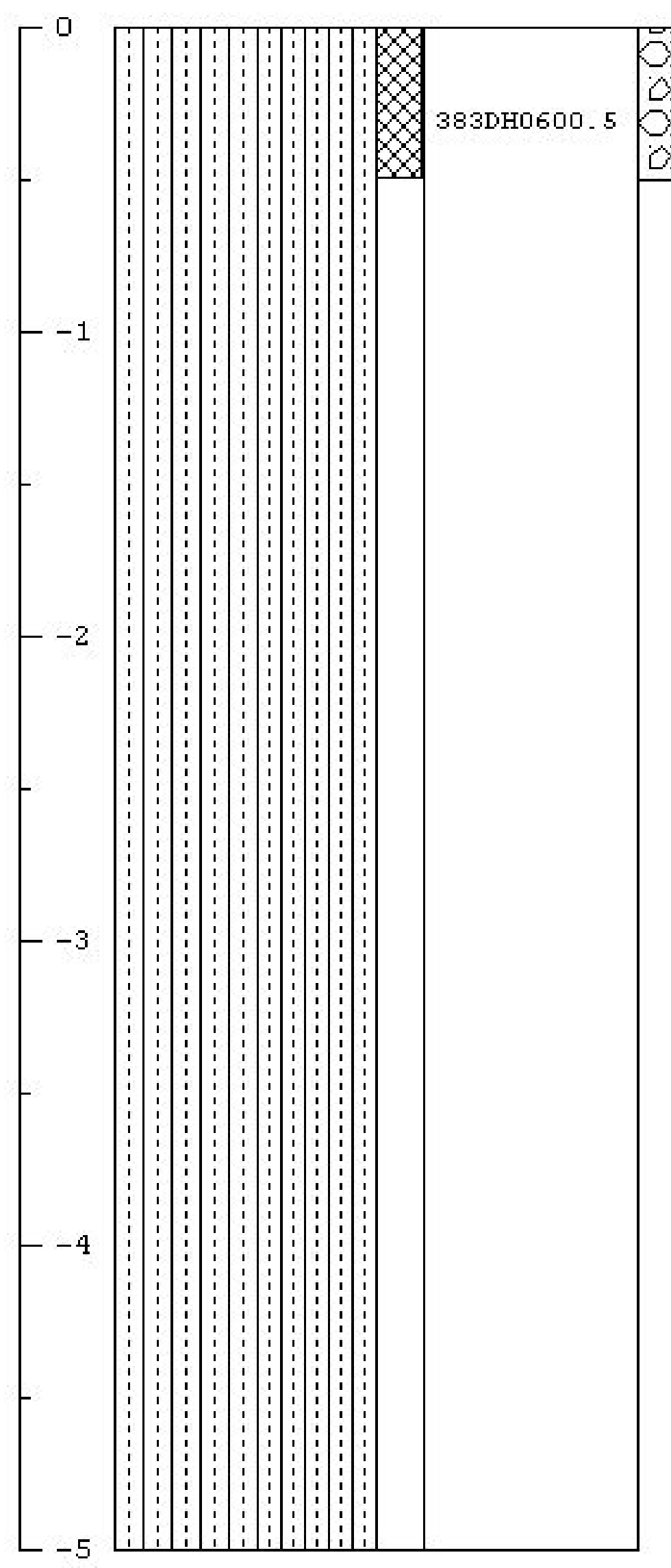

Muck: Tan, red, white, and brown FMC sand and gravel with cobbles, dry

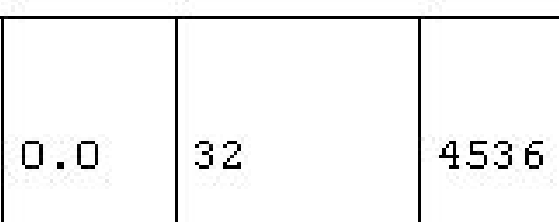


Project Name: CAU 383 E-Tunnel sites

Project Humber: DT04-320

Borehole Number: EDHO7

Logged By: $M$. McLane

Drilled By: M. McLane

Drilling Method: Hand Sampled
Date Started: $6 / 07 / 04$

Date completed: $6 / 07 / 04$

Elevation: 5997.1

Horthing: 887732.0

Easting: 638649.9

Total Depth: 0.5 ft.

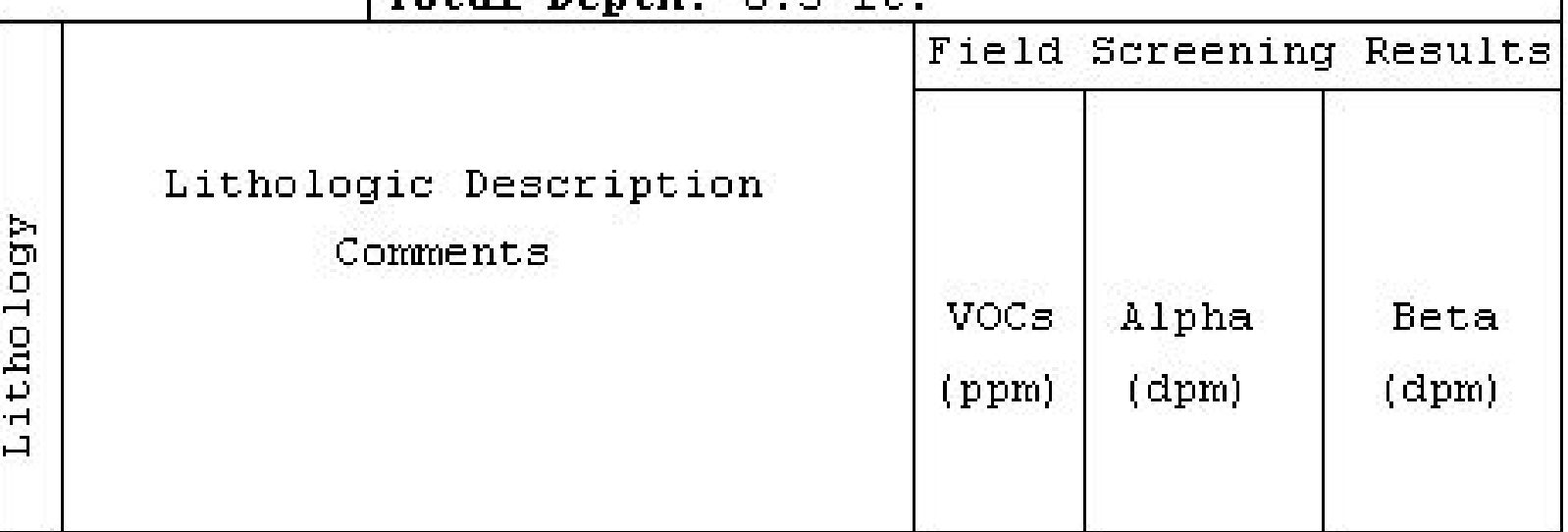

383 DH0700. $5 \mathrm{X}$.

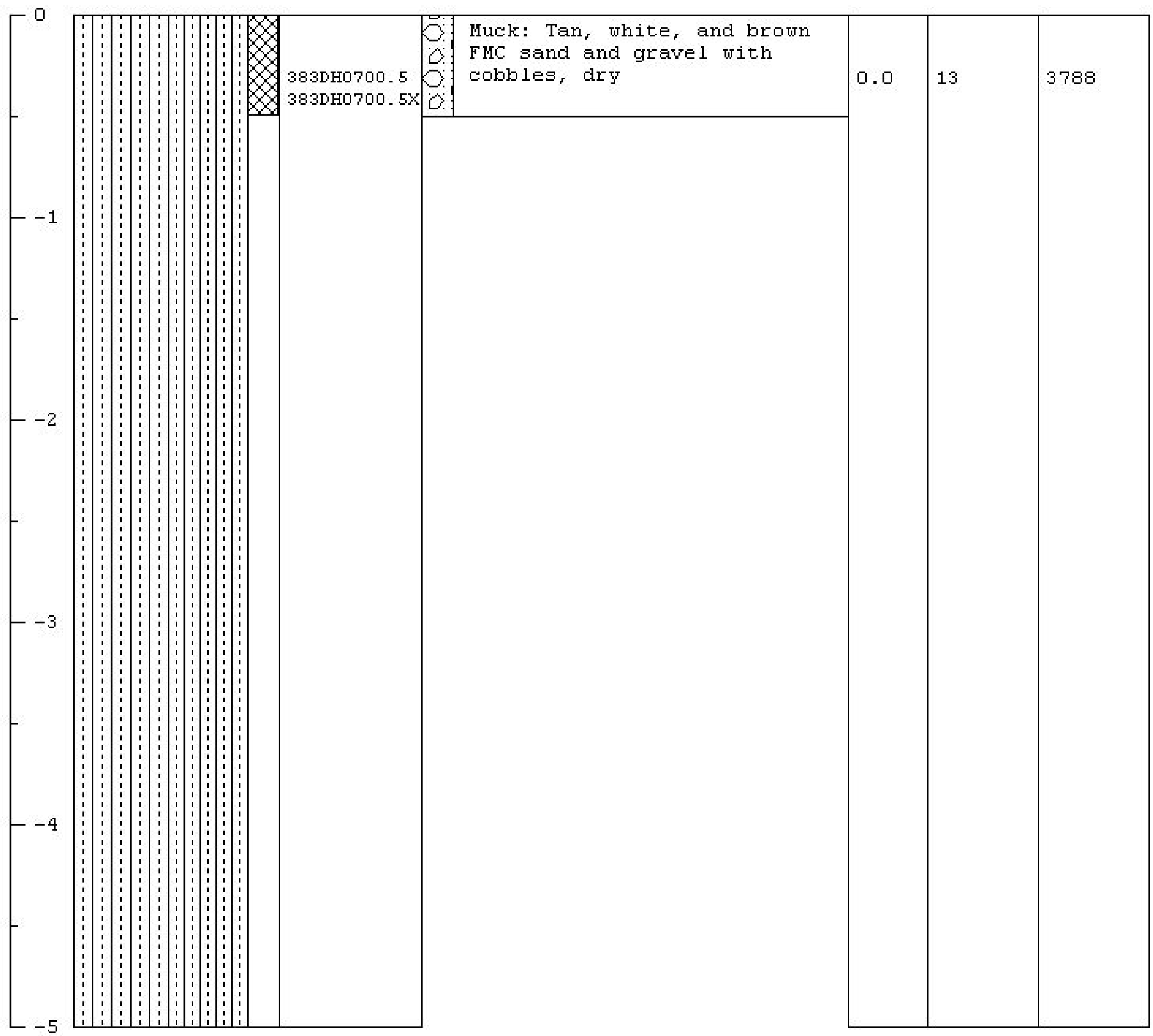


Project Name: CAU 383 E-Tunnel sites

Project Humber: DT04-320

Borehole Number: EDHOB

Logged By: $M$. McLane

Drilled By: M. McLane

Drilling Method: Hand Sampled
Date Started: $6 / 07 / 04$

Date completed: $6 / 07 / 04$

Elevation : 5999.9

Horthing: 887729.5

Easting: 638661.1

Total Depth: 0.5 ft.

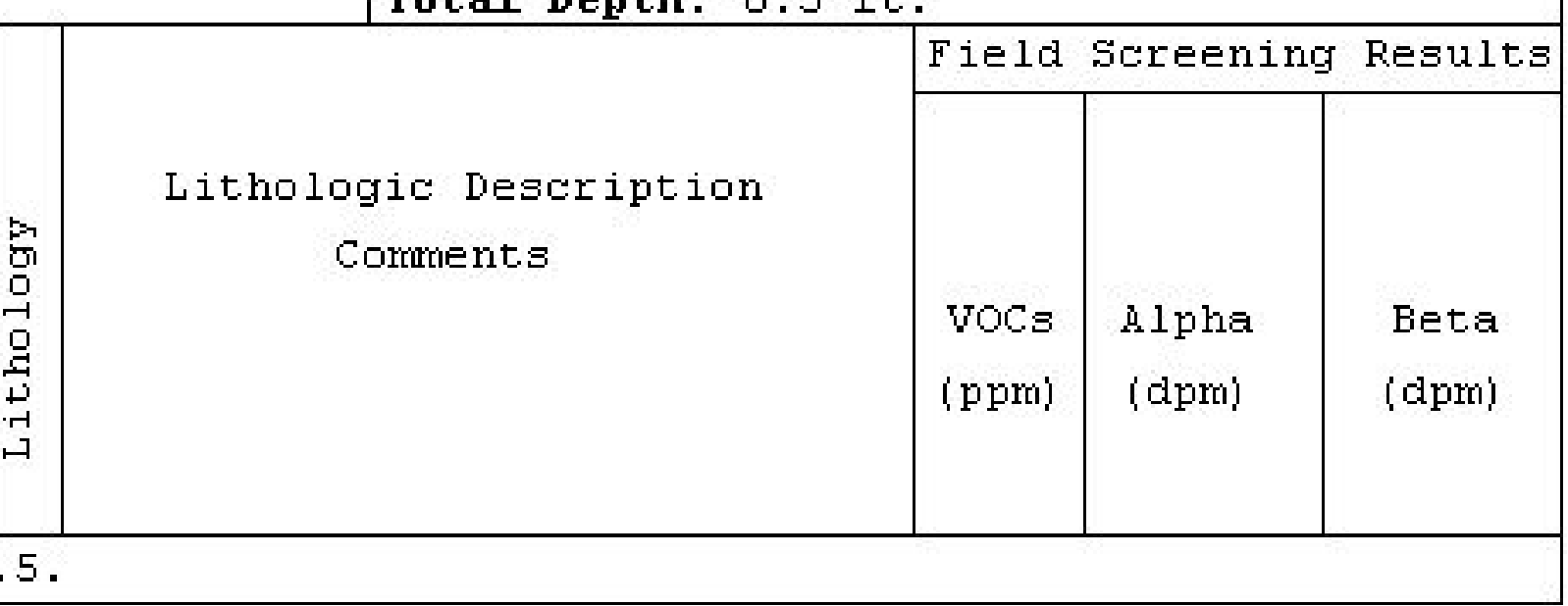

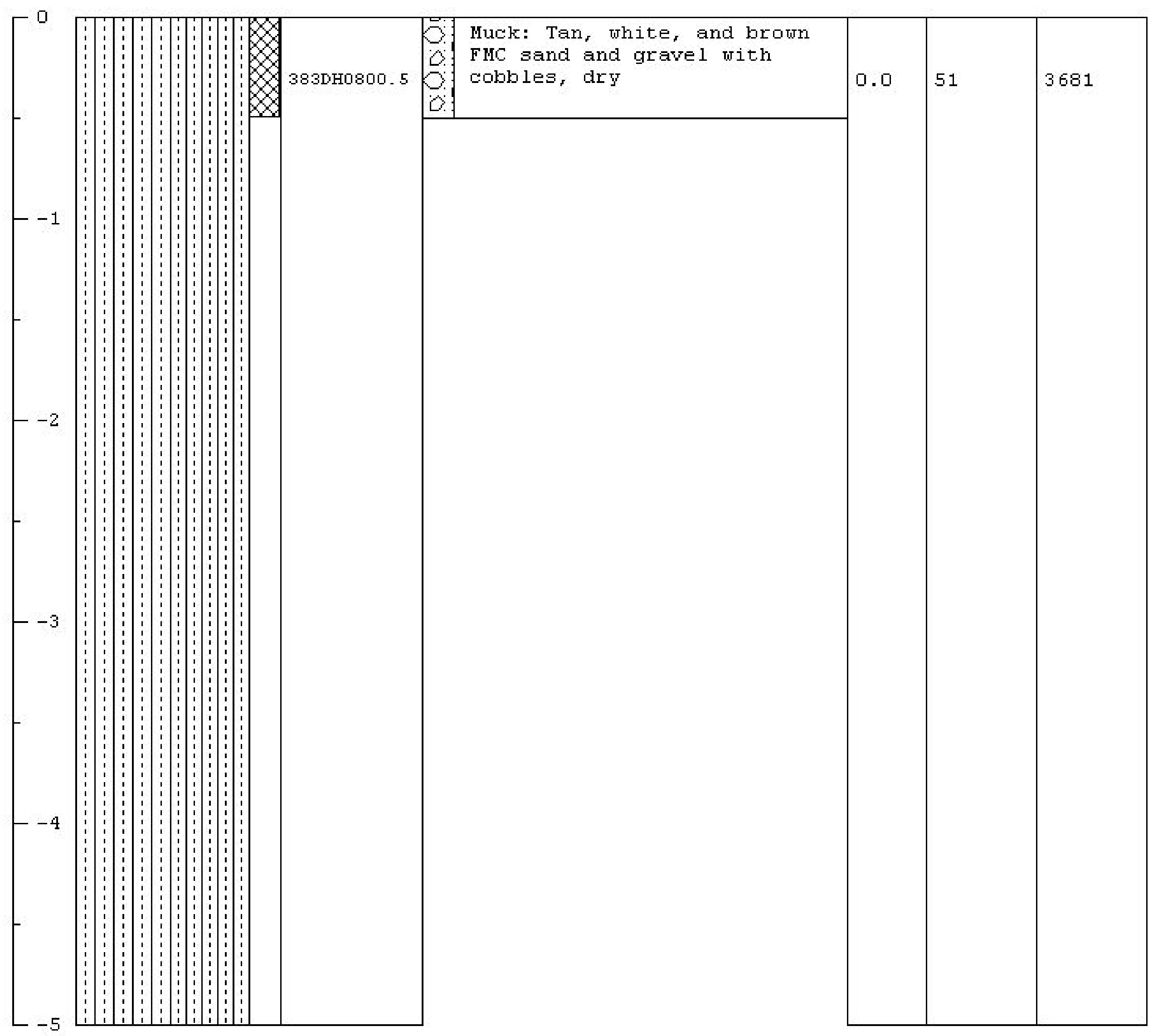


Project Name: CAU 383 E-Tunnel sites

Project Humber: DT04-320

Borehole Number: EDHOg

Logged By: $M$. McLane

Drilled By: M. McLane

Drilling Method: Hand Sampled
Date Started: $6 / 07 / 04$

Date completed: $6 / 07 / 04$

Elevation : 5997.2

Horthing: 887735.5

Easting: 638678.0

Total Depth: 0.5 ft.

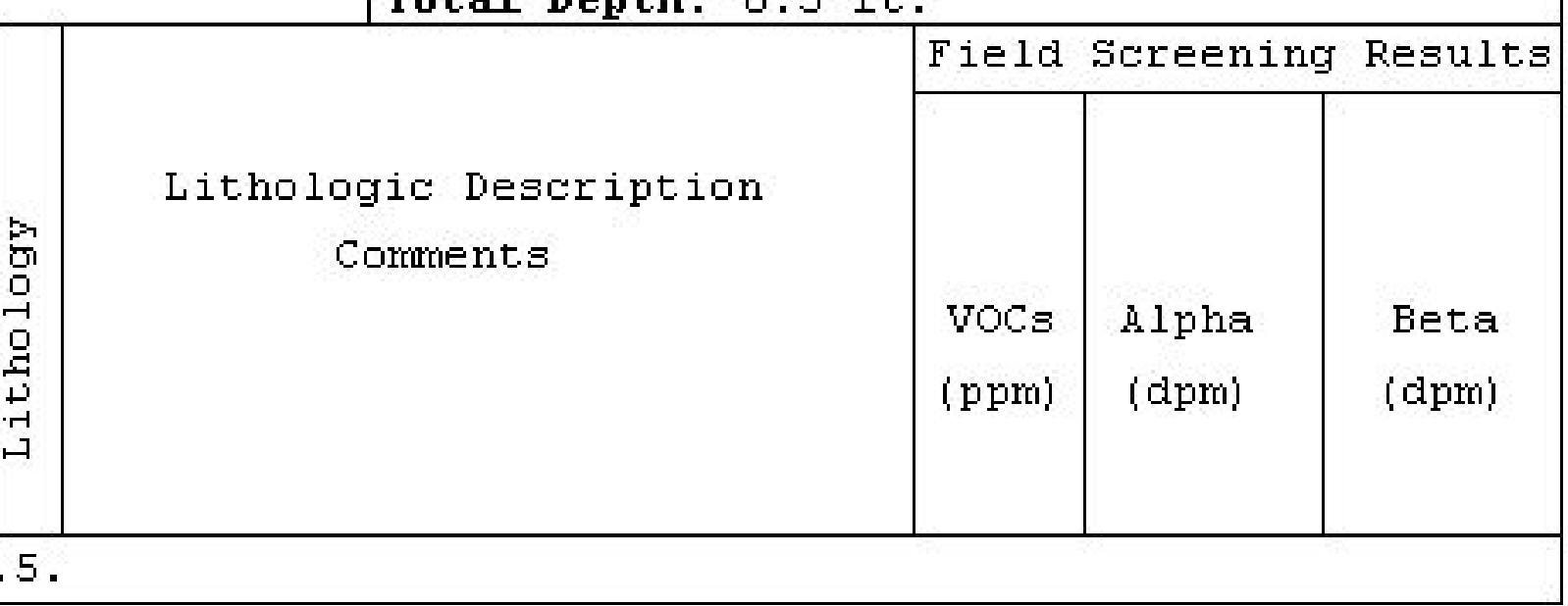

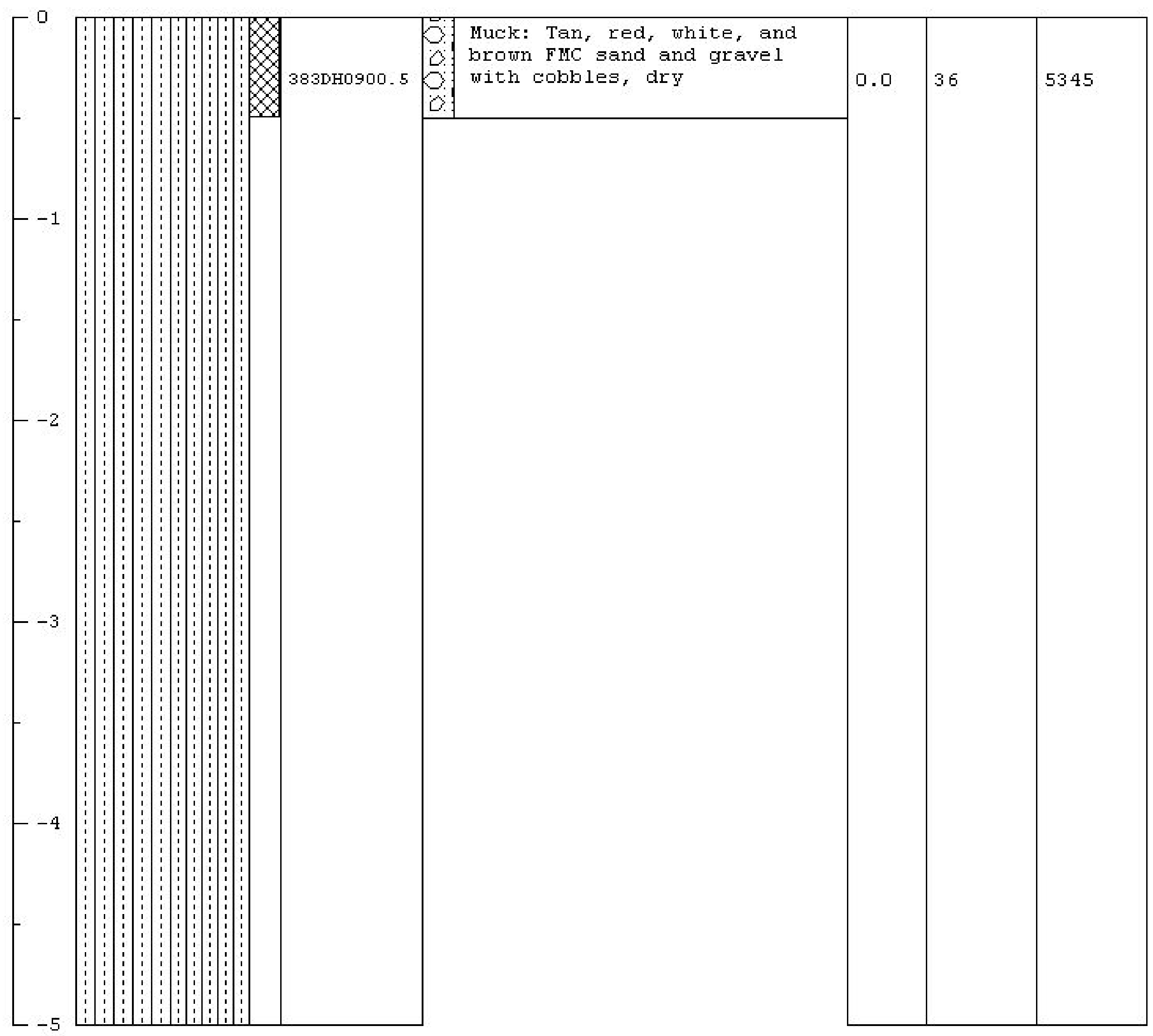


Project Name: CAU 383 E-Tunnel sites

Project Humber: DT04-320

Borehole Number: EDH10

Logged By: $M$. McLane

Drilled By: M. McLane

Drilling Method: Hand Sampled
Date Started: $6 / 07 / 04$

Date completed: $6 / 07 / 04$

Elevation : 5995.1

Horthing: 887734.2

Easting: 638686.2

Total Depth: 0.5 ft.

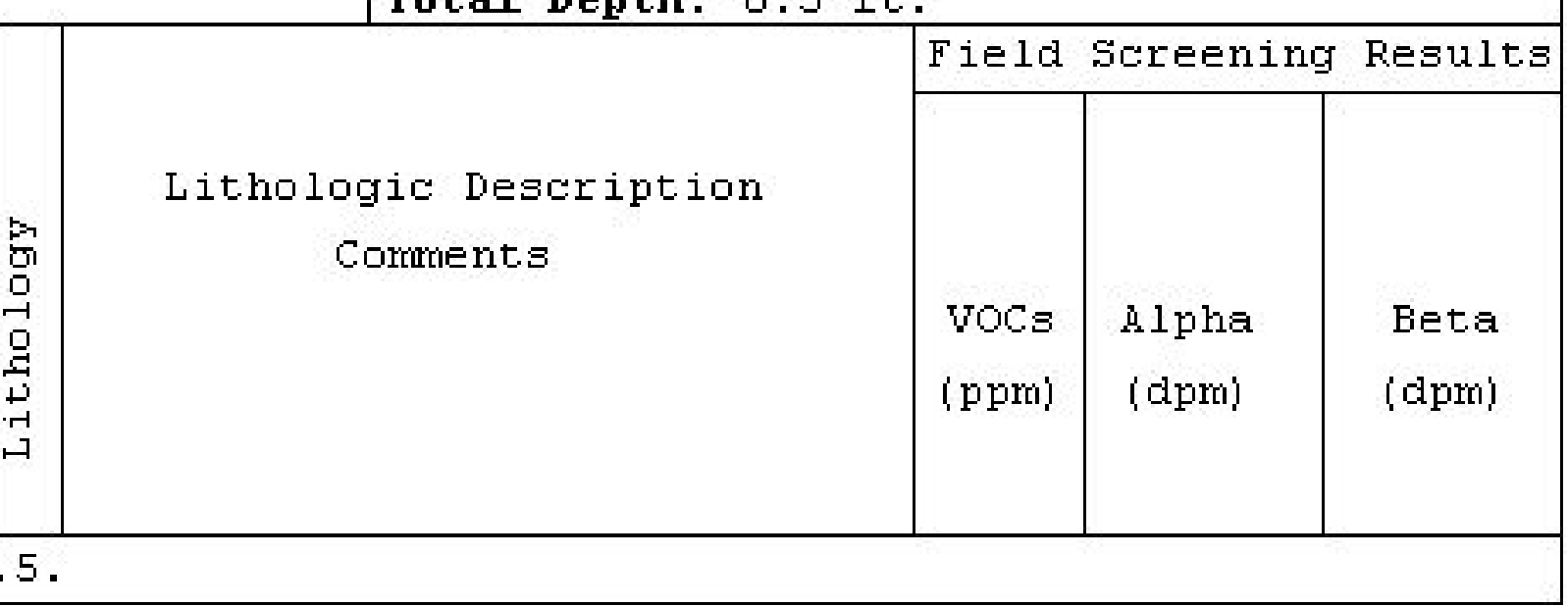

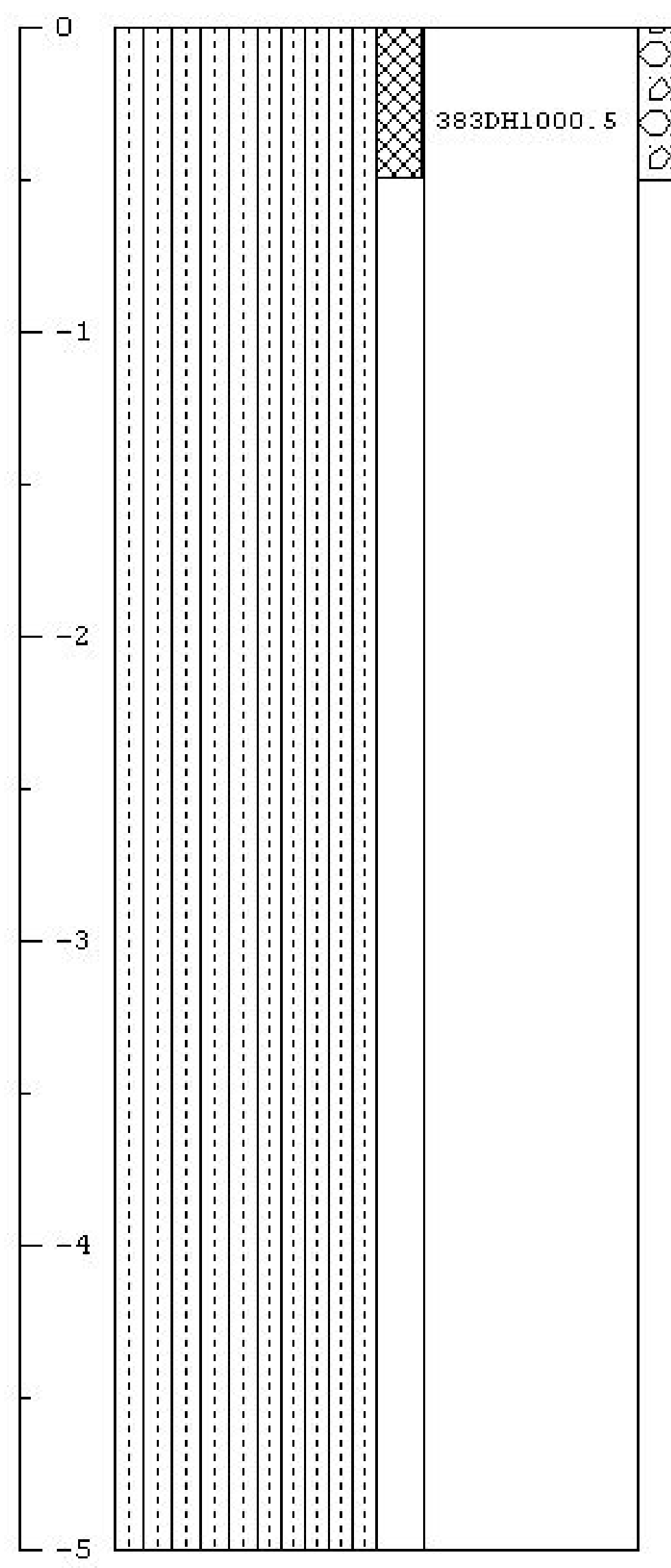

Muck: Tan, white, and brown FMC sand and gravel with cobbles, dry

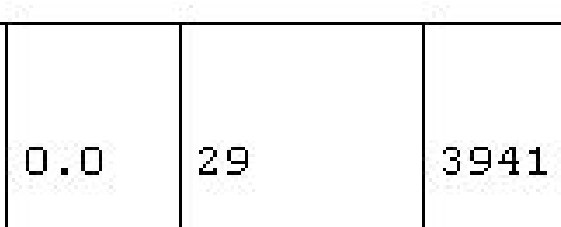


Project Name: CAU 383 E-Tunnel sites

Project Humber: DT04-320

Borehole Number: EDH11

Logged By: $M$. McLane

Drilled By: M. McLane

Drilling Method: Hand Sampled

\begin{tabular}{|c|c|c|c|c|c|}
\hline 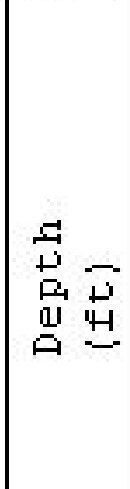 & 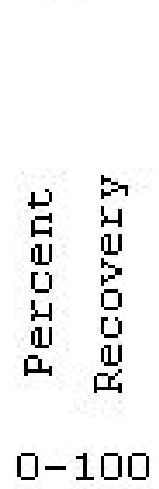 & 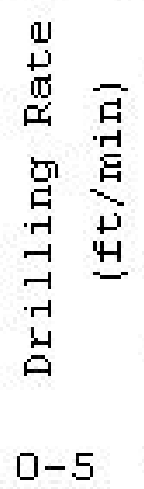 & 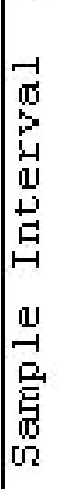 & 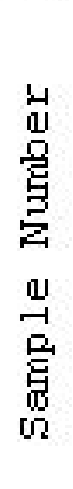 & 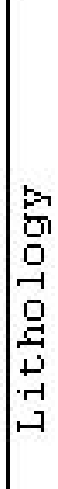 \\
\hline
\end{tabular}

Contact o N/A, Sample 383DH1100.5.
Date Started: $6 / 07 / 04$
Date Completed: $6 / 07 / 04$

Elevation: 5984.3

Horthing: 887726.6

Easting: 638697.8

Total Depth: 0.5 ft.

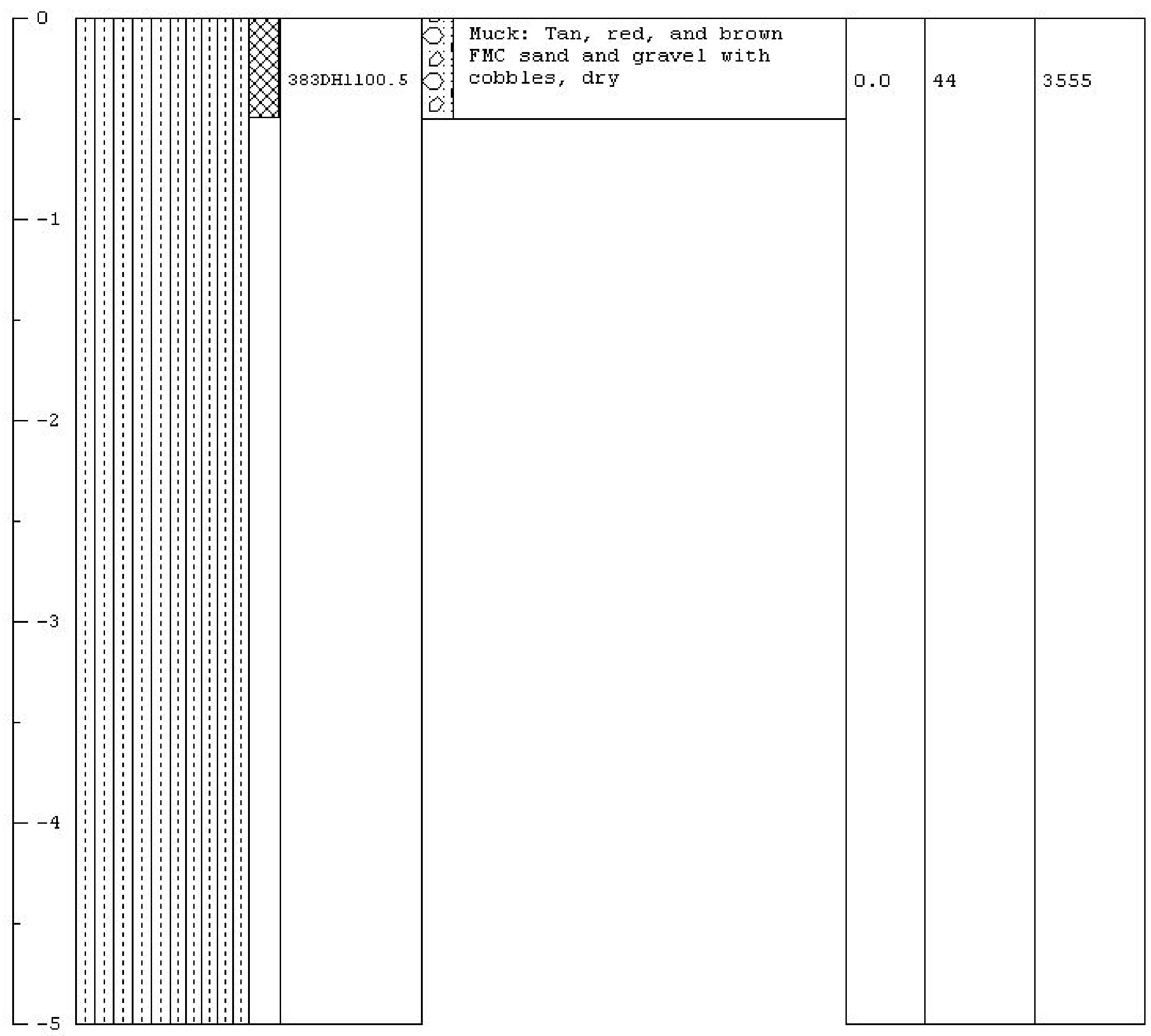


Project Name: CAU 383 E-Tunnel sites

Project Humber: DT04-320

Borehole Number: EDH12

Logged By: $M$. McLane

Drilled By: M. McLane

Drilling Method: Hand Sampled
Date Started: $6 / 07 / 04$

Date completed: $6 / 07 / 04$

Elevation: 5983.9

Horthing: 887730.0

Easting: 638716.7

Total Depth: 0.5 ft.

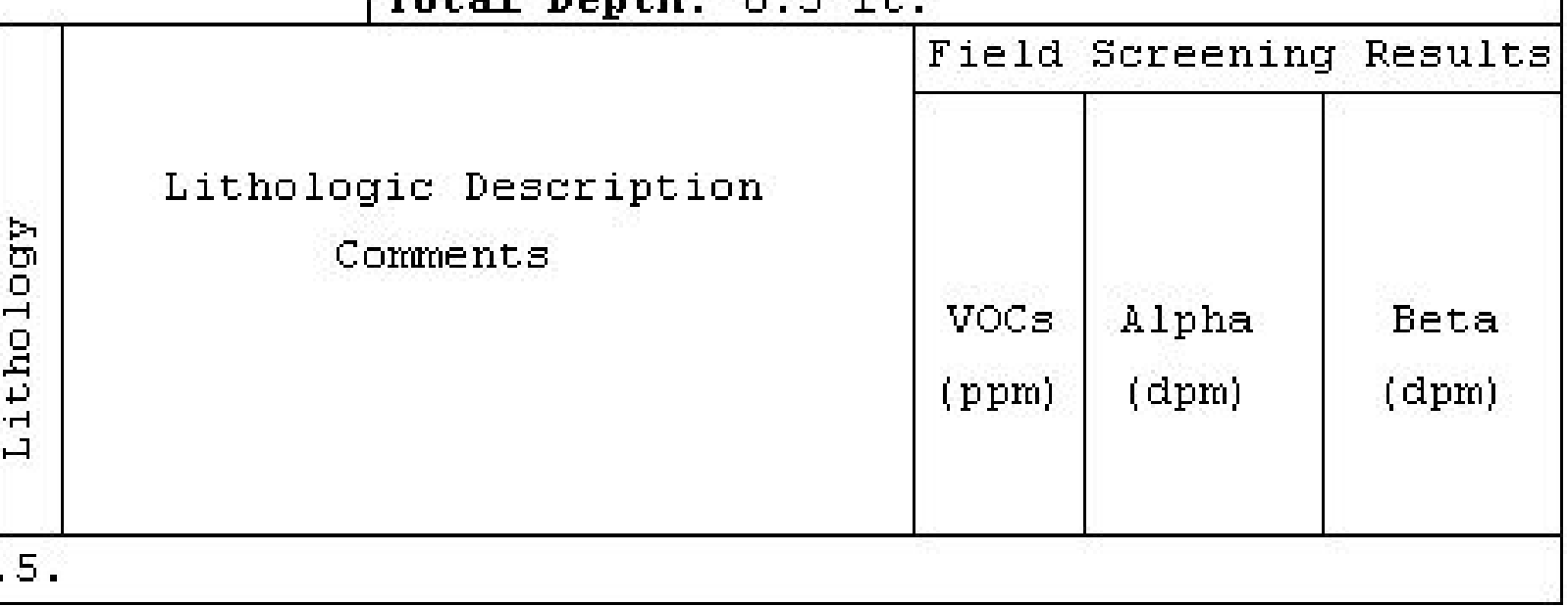

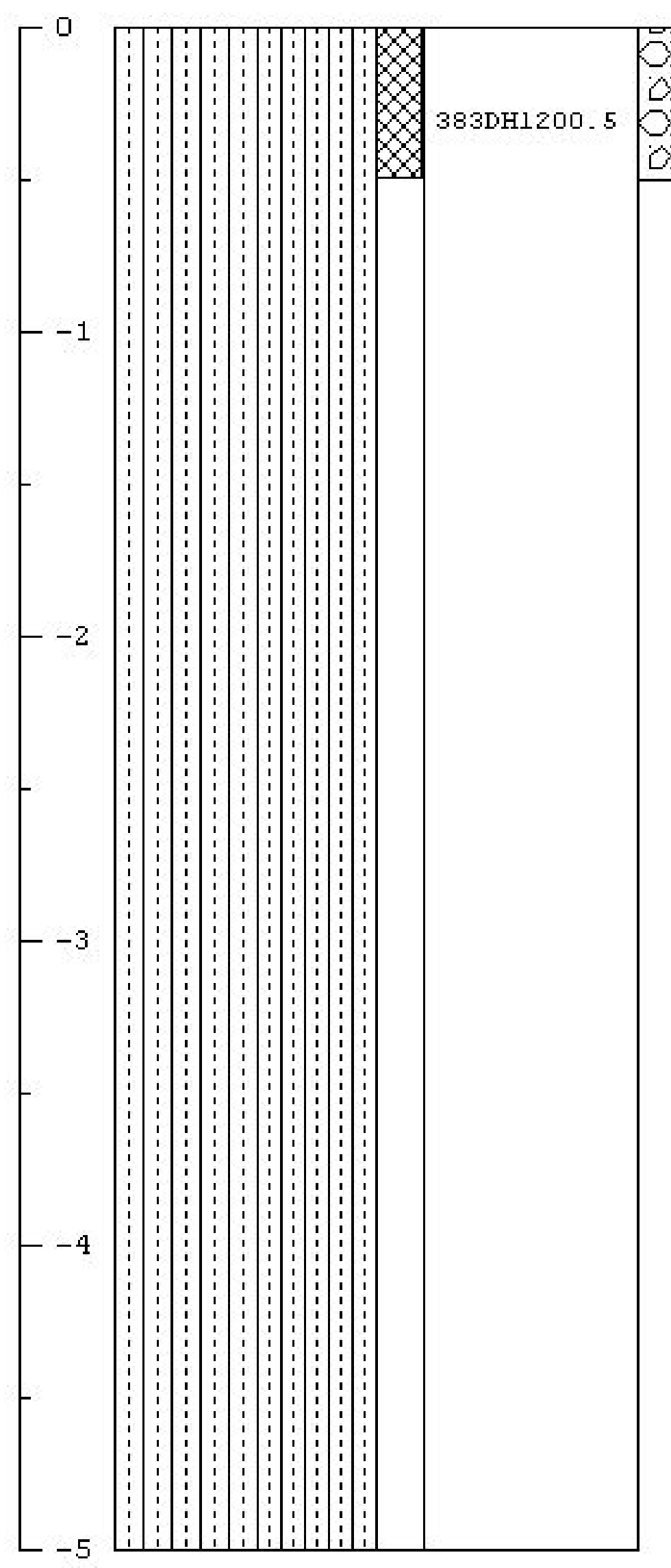

Muck: Tan and brown FMC sand and gravel with cobbles, dry 
Project Name: CAU 383 E-Tunnel sites

Project Humber: DT04-320

Borehole Number: EDH13

Logged By: $M$. McLane

Drilled By: M. McLane

Drilling Method: Hand Sampled
Date Started: $6 / 07 / 04$

Date completed: $6 / 07 / 04$

Elevation : 5970.7

Horthing: 887720.0

Easting: 638737.5

Total Depth: 0.5 ft.

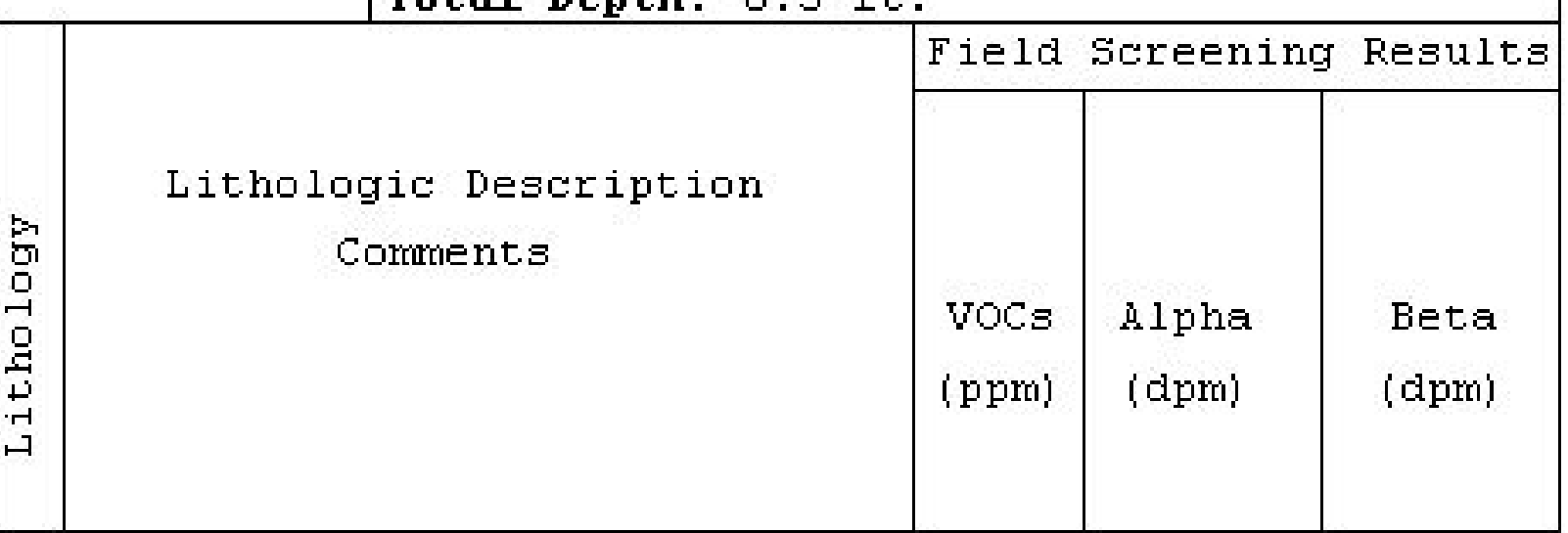

Contact o N/A, Sample 383DH1300.5.

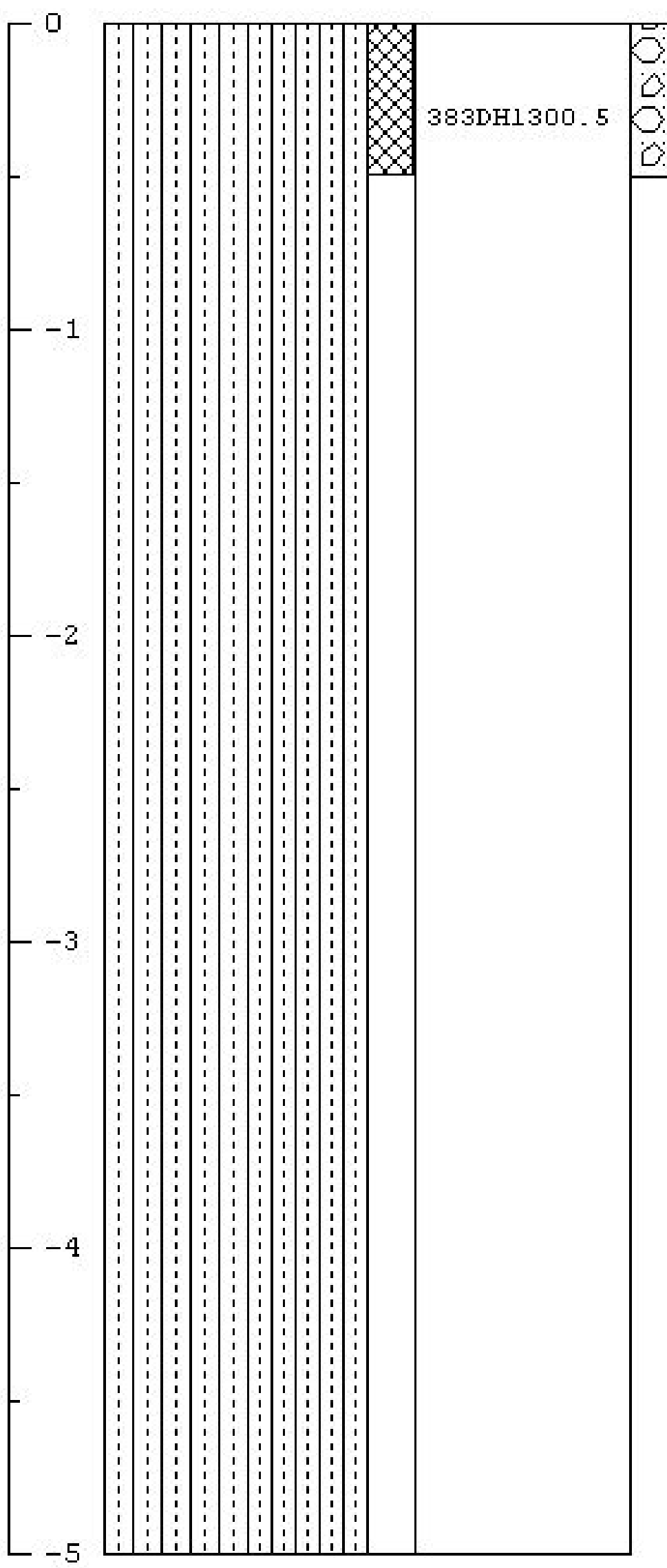

Muck: Brown and grey FMC sand and gravel with cobbles, dry

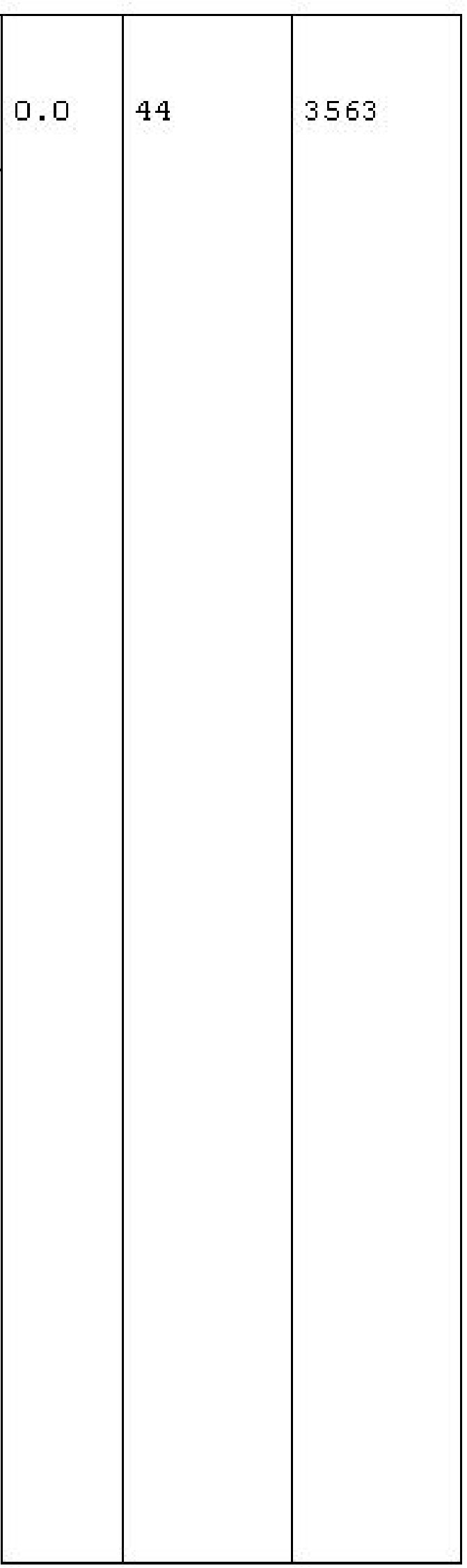


Project Name: CAU 383 E-Tunnel sites

Project Humber: DT04-320

Borehole Number: EDH14

Logged By: $M$. McLane

Drilled By: M. McLane

Drilling Method: Hand Sampled

\begin{tabular}{|c|c|c|c|c|c|}
\hline 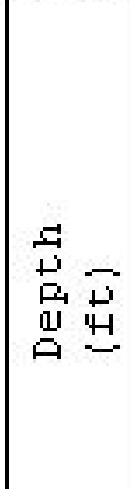 & 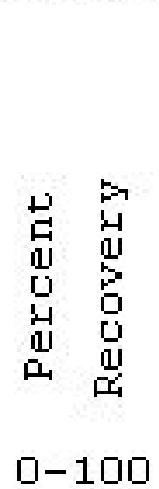 & 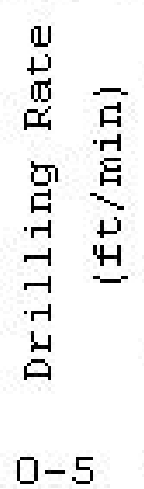 & 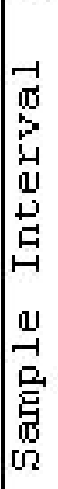 & 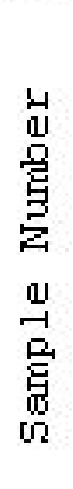 & 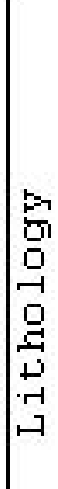 \\
\hline
\end{tabular}

Contact o N/A, Sample 383DH1400.5.

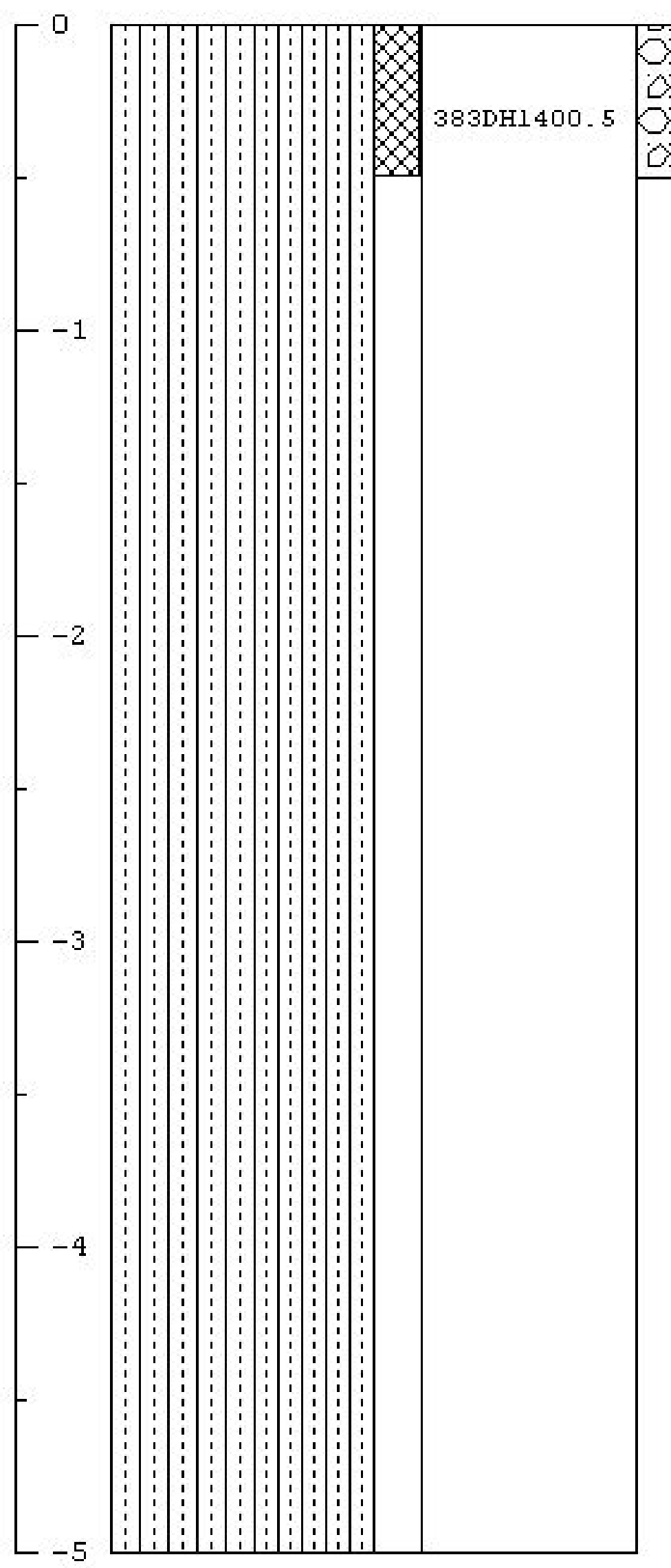

i.

Date Started: $6 / 07 / 04$

Date Completed: $6 / 07 / 04$

Elevation: 5982.0

Horthing: 887723.2

Easting: 638745.4

Total Depth: $0.5 \mathrm{ft}$.

Lithologic Description

Comments

\begin{tabular}{|c|c|c|}
\hline \multicolumn{3}{|c|}{ Field Screening Results } \\
\hline & & \\
& & \\
VoCs & Alpha & Beta \\
(ppm) & (dpm) & (dpm) \\
& & \\
\end{tabular}

Muck: Brown FMC sand and gravel with cobbles, dry 
Project Name: CAU 383 E-Tunnel sites

Project Humber: DT04-320

Borehole Number: EDH15

Logged By: $M$. McLane

Drilled By: M. McLane

Drilling Method: Hand Sampled

\begin{tabular}{|c|c|c|c|c|c|}
\hline 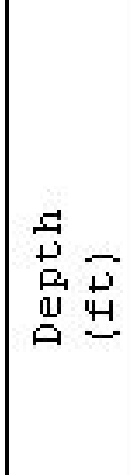 & 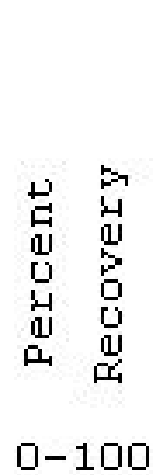 & 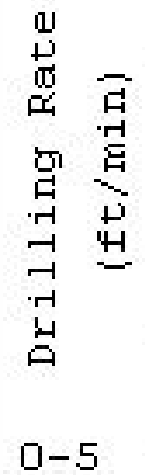 & 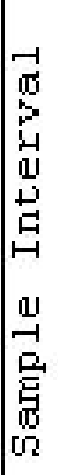 & 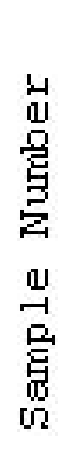 & 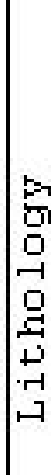 \\
\hline
\end{tabular}

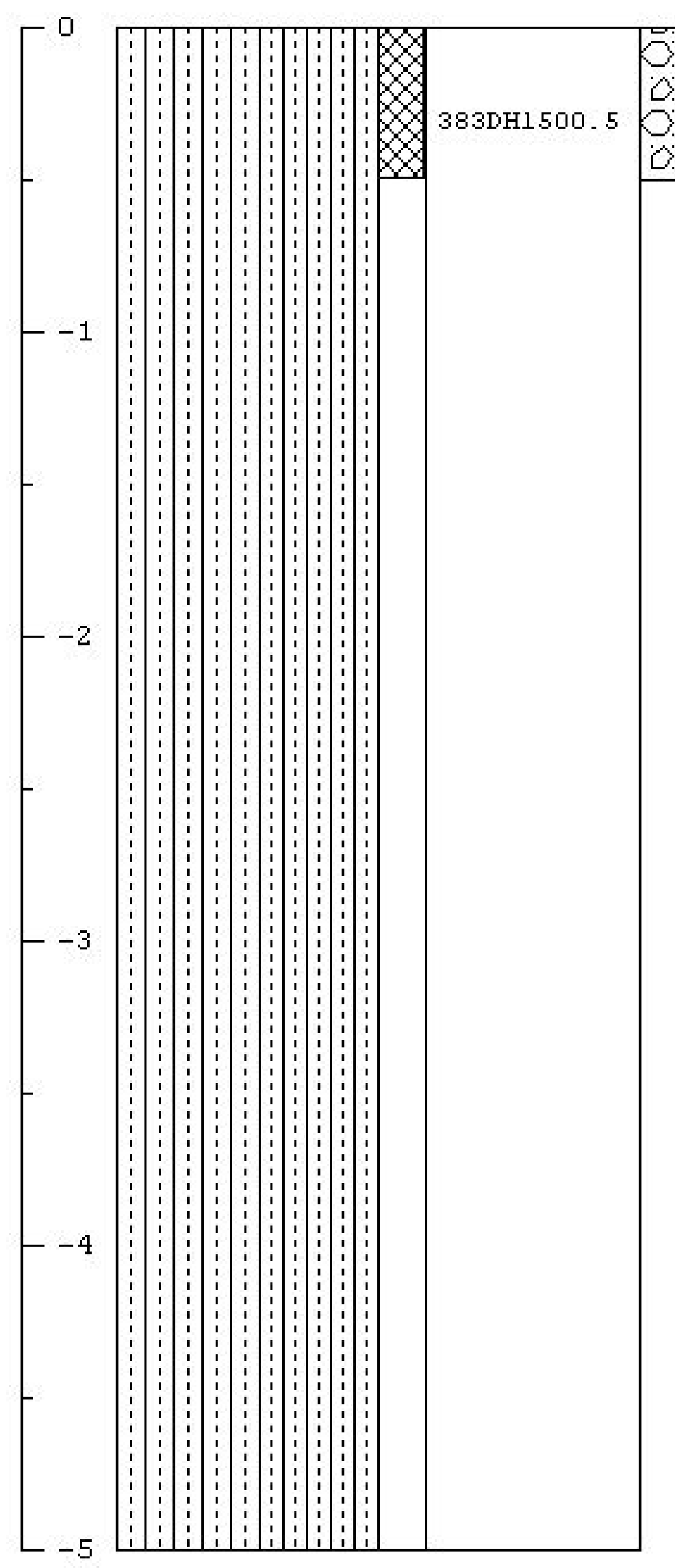

c.

Date Started: $6 / 07 / 04$

Date completed: $6 / 07 / 04$

Elevation: 5976.8

Horthing: 887712.1

Easting: 638759.5

Total Depth: 0.5 ft.

Lithologic Description

Comments

\begin{tabular}{|c|c|c|}
\hline \multicolumn{2}{|c|}{ Field Screening Results } \\
\hline & & \\
vocs & Alpha & Beta \\
(ppm) & (dpm) & (dpm) \\
& & \\
\end{tabular}

Muck: Brown FMC sand and gravel with cobbles, dry

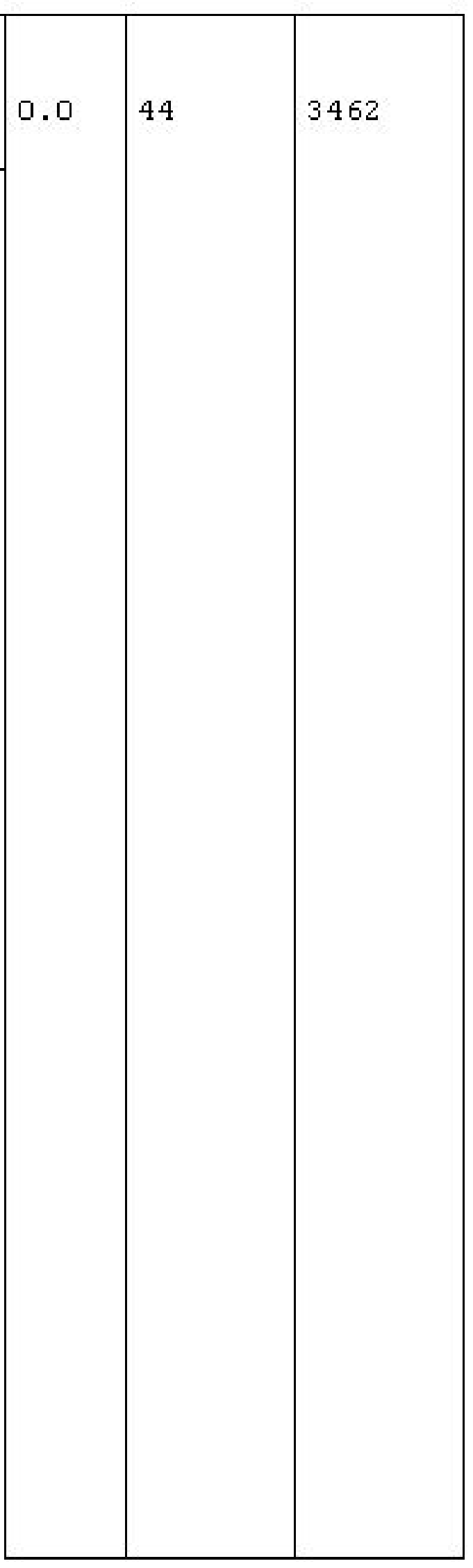


Project Name: CAU 383 E-Tunnel sites

Project Humber: DT04-320

Borehole Number: EDH16

Logged By: $M$. McLane

Drilled By: M. McLane

Drilling Method: Hand Sampled
Date Started: $6 / 07 / 04$

Date completed: $6 / 07 / 04$

Elevation : 5983.1

Horthing : 887711.1

Easting: 638780.1

Total Depth: 0.5 ft.

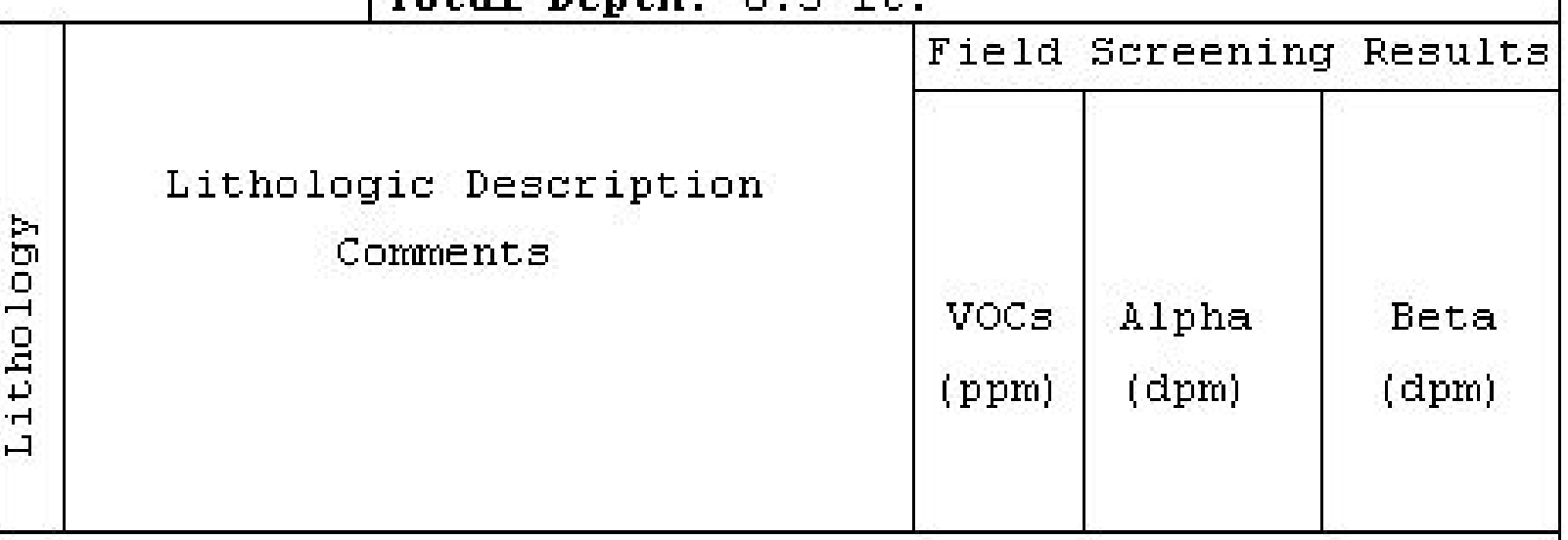

Contact o N/A, Sample 383DH1600.5.

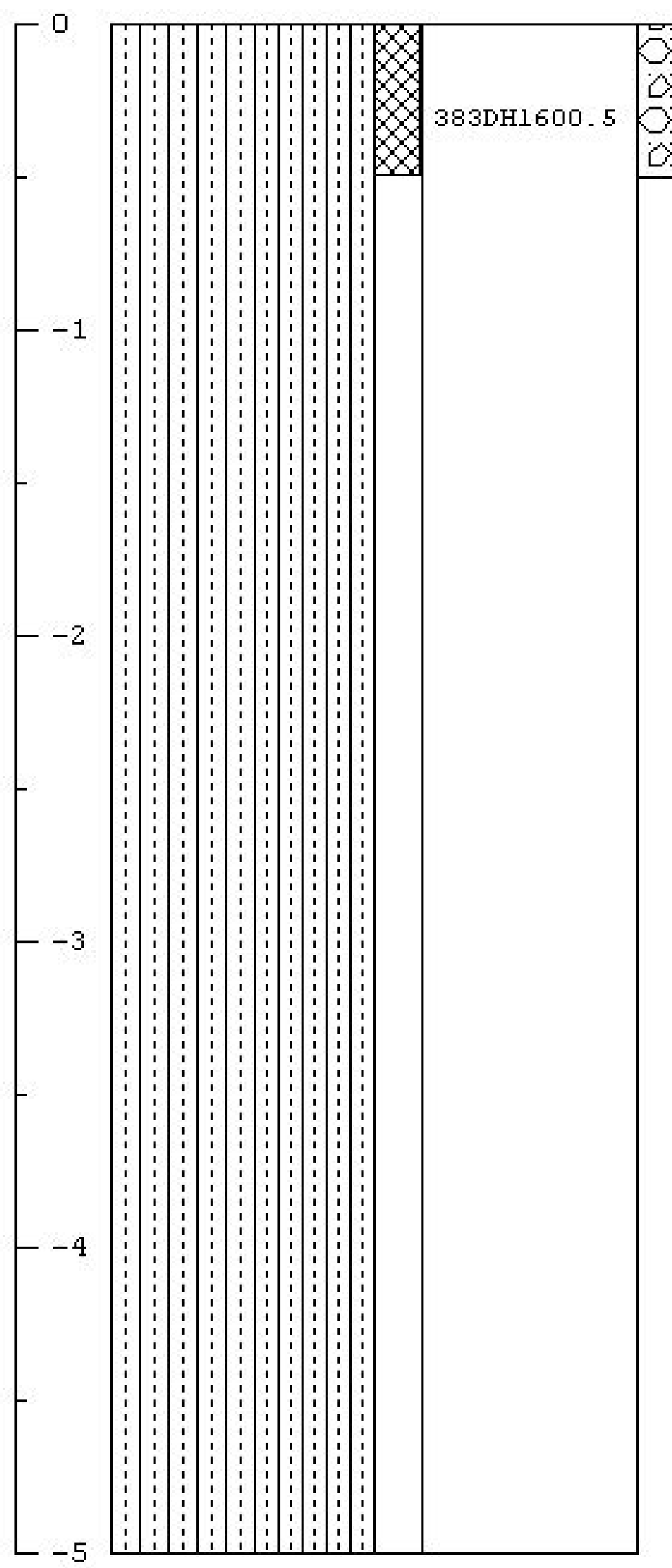

Muck: Brown and red FMC sand and gravel with cobbles, dry 
Project Name: CAU 383 E-Tunnel sites

Project Humber: DT04-320

Borehole Number: EDH17

Logged By: $M$. McLane

Drilled By: M. McLane

Drilling Method: Hand Sampled
Date Started: $6 / 07 / 04$

Date completed: $6 / 07 / 04$

Elevation: 5978.8

Horthing: 887713.1

Easting: 638786.0

Total Depth: 0.5 ft.

\begin{tabular}{|c|c|c|c|c|c|c|c|c|c|}
\hline \multirow{2}{*}{ 哭出 } & \multirow[b]{2}{*}{ 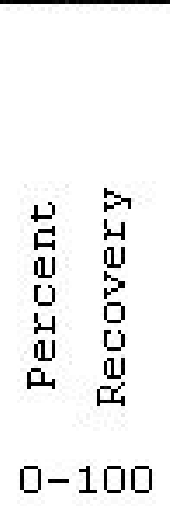 } & \multirow[b]{2}{*}{ 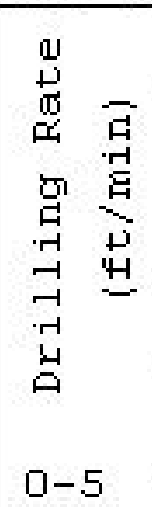 } & \multirow[b]{2}{*}{ 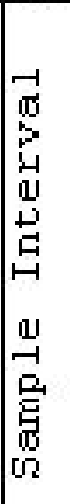 } & \multirow[b]{2}{*}{ 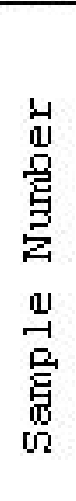 } & \multirow[b]{2}{*}{ 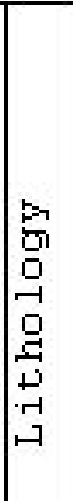 } & \multirow[b]{2}{*}{$\begin{array}{c}\text { Lithologic Description } \\
\text { Comments }\end{array}$} & \multicolumn{3}{|c|}{ Field Screening Results } \\
\hline & & & & & & & $\begin{array}{l}\text { VOCs } \\
\text { (ppm) }\end{array}$ & $\begin{array}{l}\text { Alpha } \\
\text { (dpm) }\end{array}$ & $\begin{array}{l}\text { Beta } \\
\text { (dpri) }\end{array}$ \\
\hline
\end{tabular}

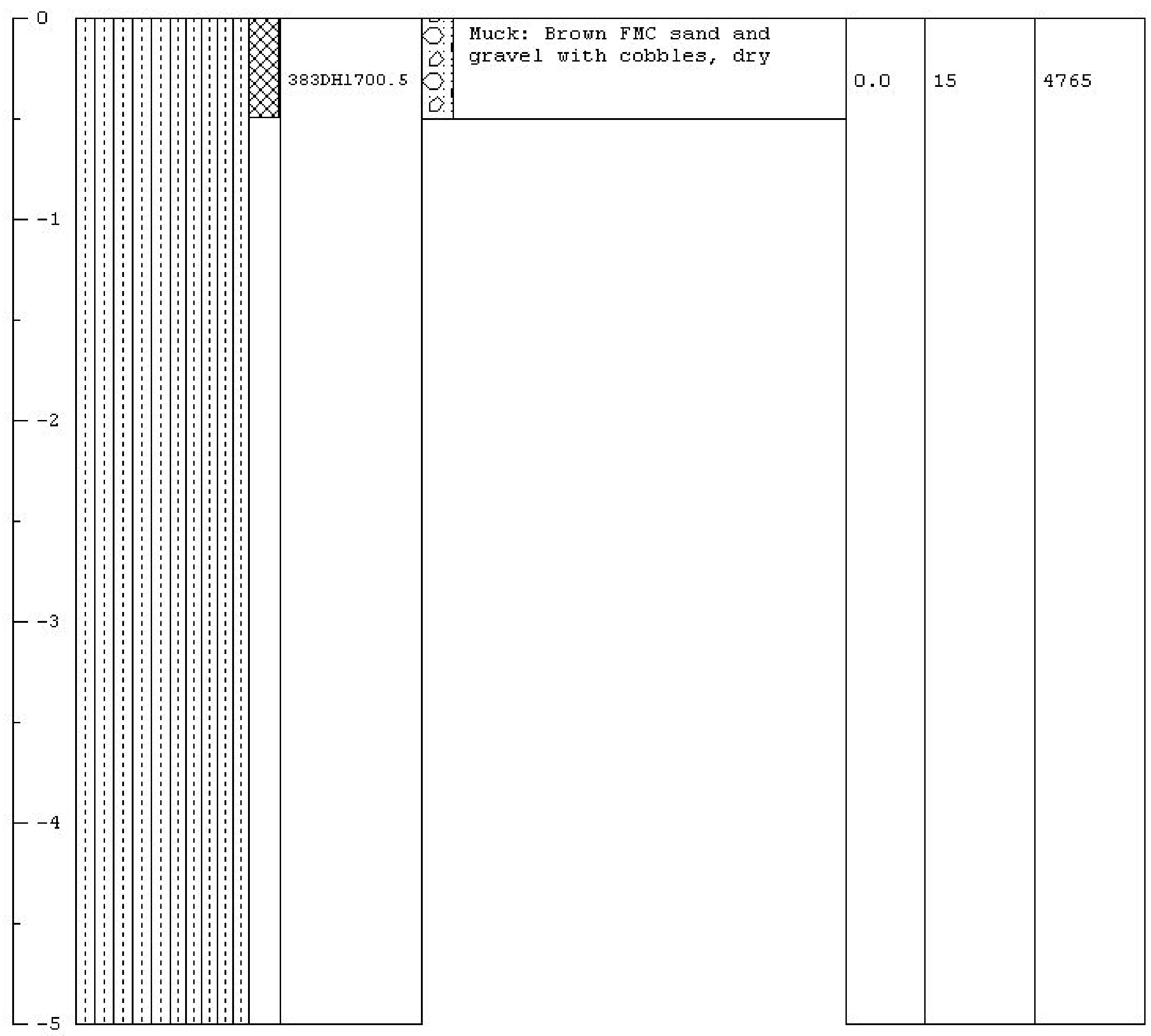


Project Name: CAU 383 E-Tunnel sites

Project Humber: DT04-320

Borehole Number: EDH18

Logged By: $M$. McLane

Drilled By: M. McLane

Drilling Method: Hand Sampled
Date Started: $6 / 07 / 04$

Date completed: $6 / 07 / 04$

Elevation: 5977.6

Horthing : 887710.6

Easting: 638797.7

Total Depth: 0.5 ft.

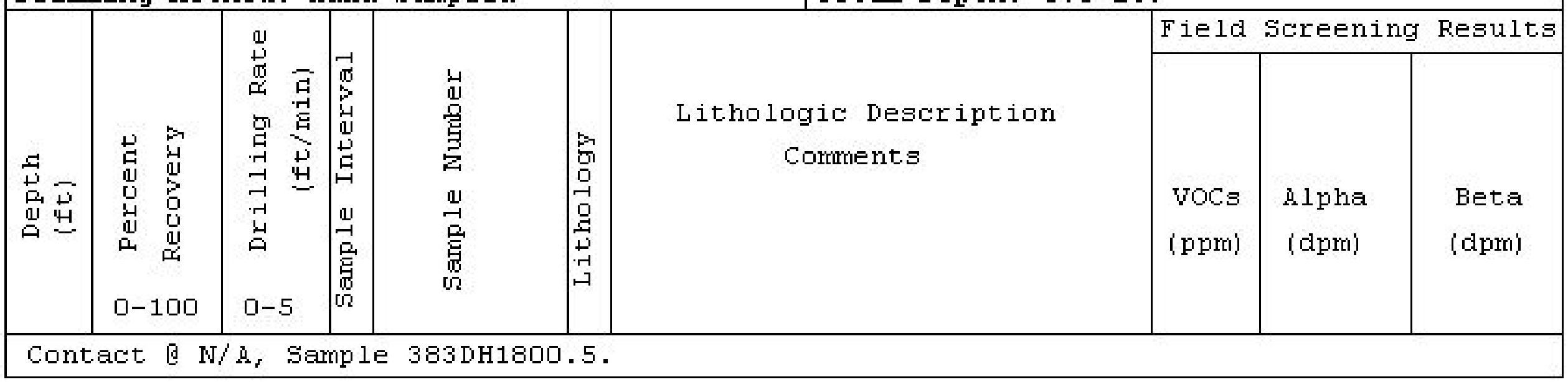

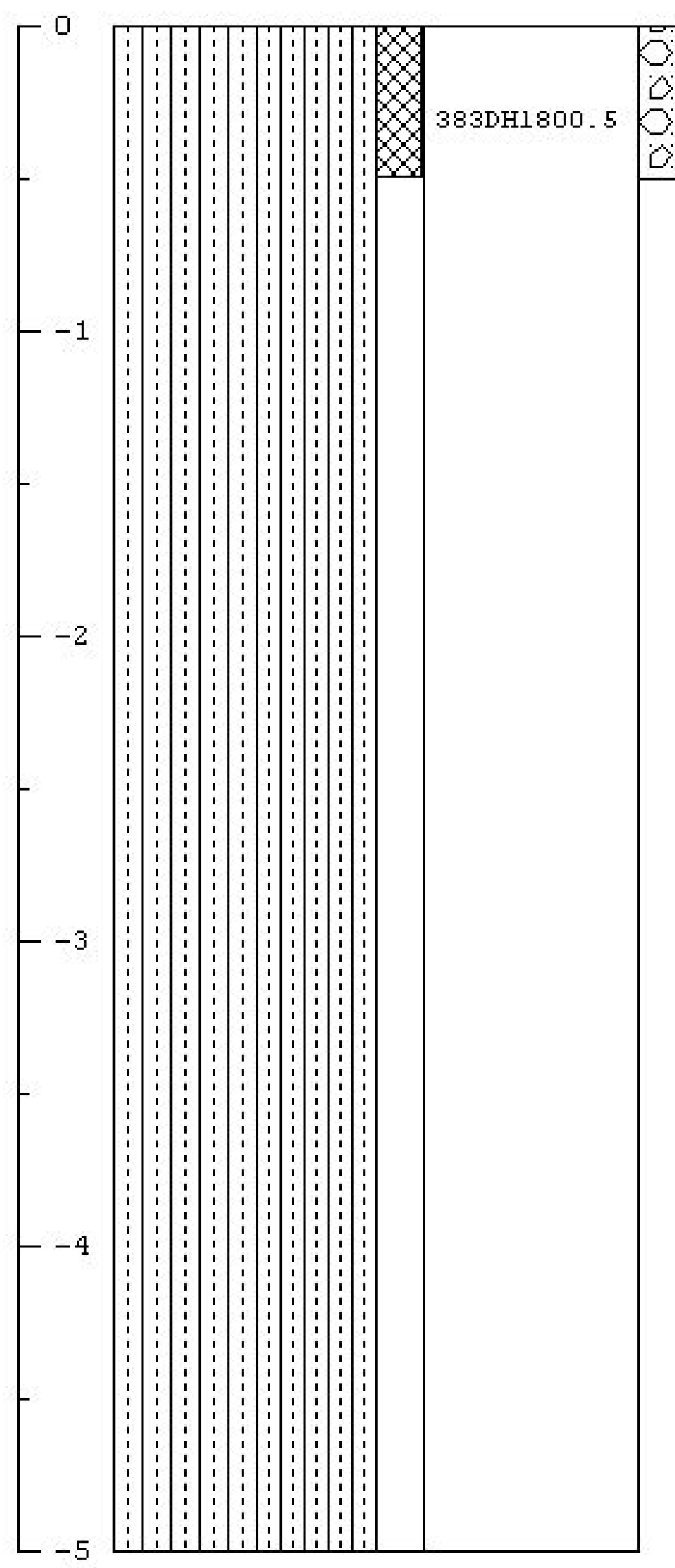

Muck: Brown FMC sand and gravel with cobbles, dry

ci.


Project Name: CAU 383 E-Tunnel sites

Project Humber: DT04-320

Borehole Number: EDH19

Logged By: $M$. McLane

Drilled By: M. McLane

Drilling Method: Hand Sampled

\begin{tabular}{|c|c|c|c|c|c|}
\hline $\begin{array}{l}\stackrel{s}{D} \\
\stackrel{5}{口} \\
\stackrel{+1}{口}\end{array}$ & 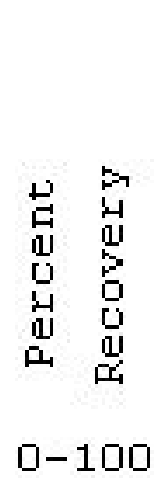 & 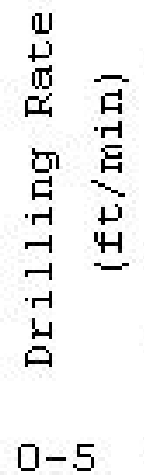 & 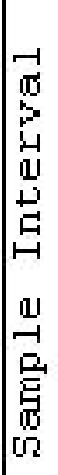 & 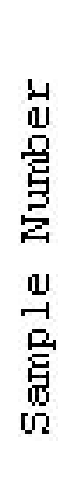 & 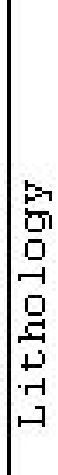 \\
\hline
\end{tabular}

Contact o N/A, Sample 383DH1900.5.
Date Started: $6 / 07 / 04$
Date Completed: $6 / 07 / 04$

Elevation: 5975.1

Horthing: 887707.0

Easting: 638808.0

Total Depth: 0.5 ft.

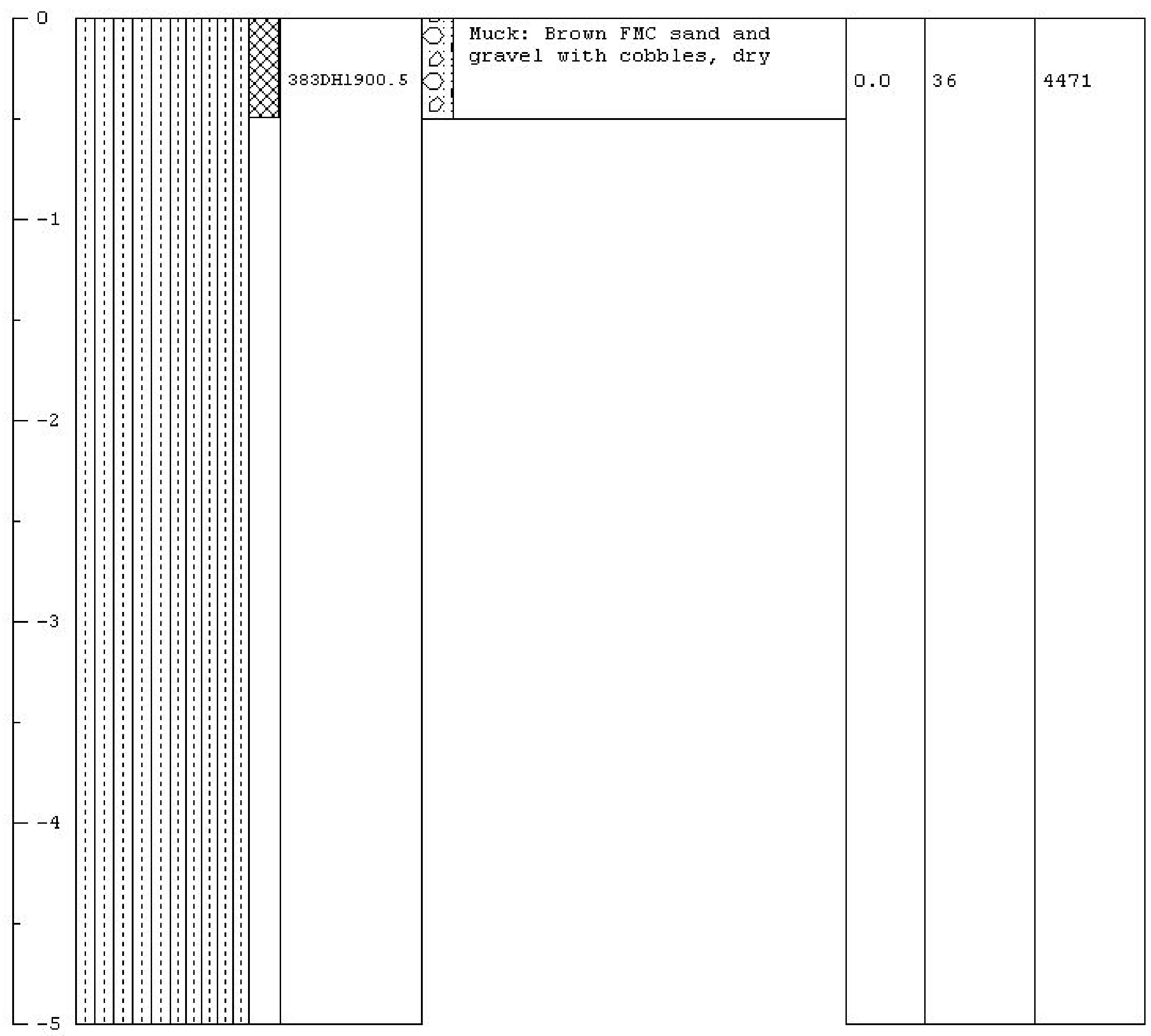


Project Name: CAU 383 E-Tunnel sites

Project Humber: DT04-320

Borehole Number: EDH20

Logged By: $M$. McLane

Drilled By: M. McLane

Drilling Method: Hand Sampled
Date Started: $6 / 07 / 04$

Date completed: $6 / 07 / 04$

Elevation: 5972.7

Horthing: 887704.0

Easting: 638818.3

Total Depth: 0.5 ft.

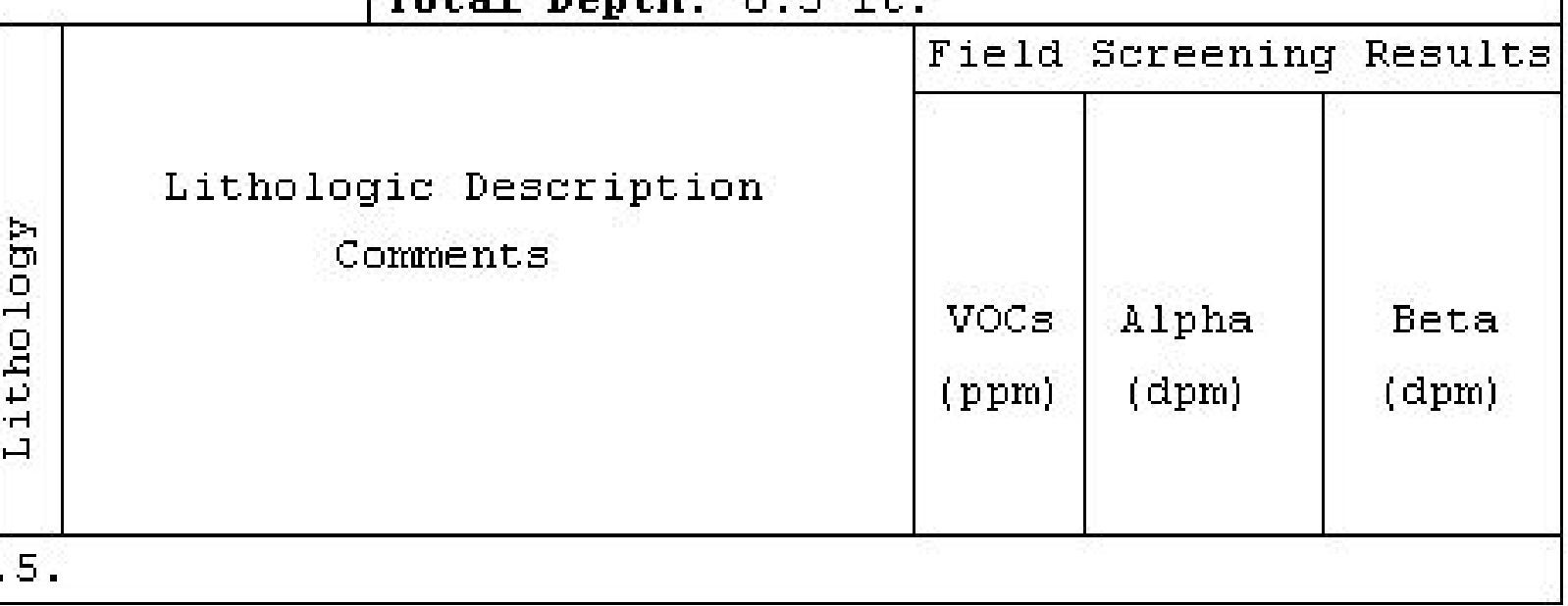

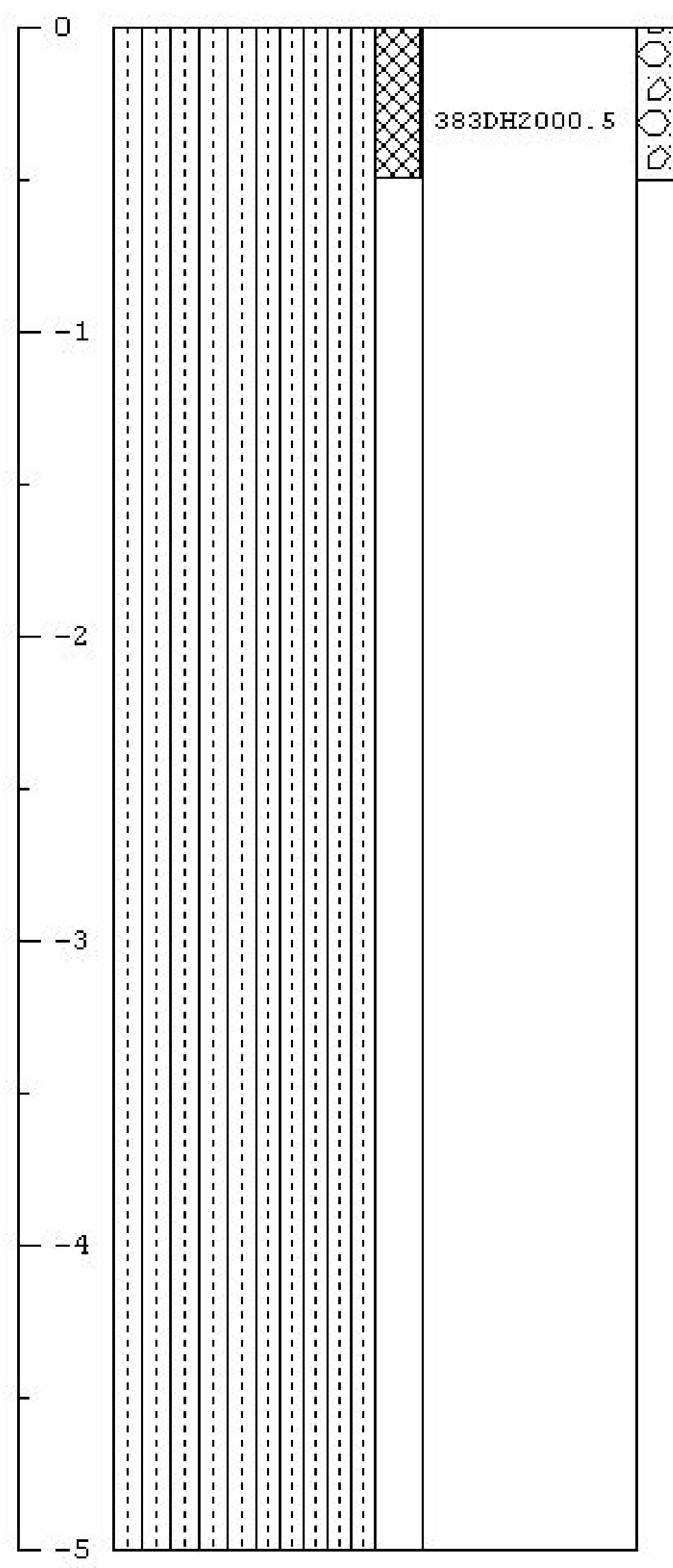

Muck: Brown FMC sand and gravel with cobbles, dry

ci.


Project Name: CAU 383 E-Tunnel sites

Project Humber: DT04-320

Borehole Number: EDH21

Logged By: $M$. McLane

Drilled By: M. McLane

Drilling Method: Hand Sampled

\begin{tabular}{|c|c|c|c|c|c|}
\hline 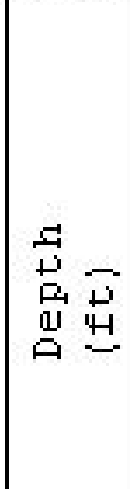 & 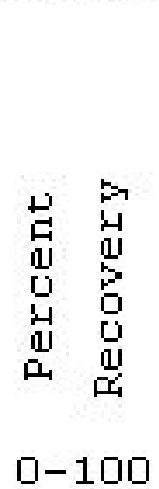 & 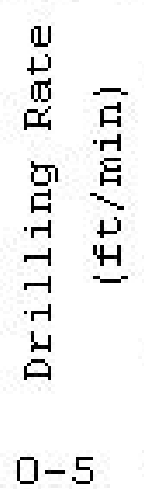 & 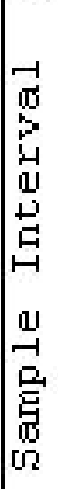 & 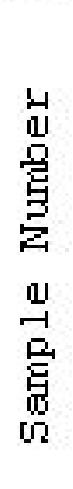 & 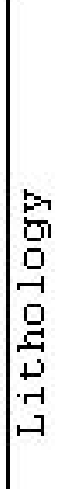 \\
\hline
\end{tabular}

Contact o N/A, Sample 383DH2100.5.

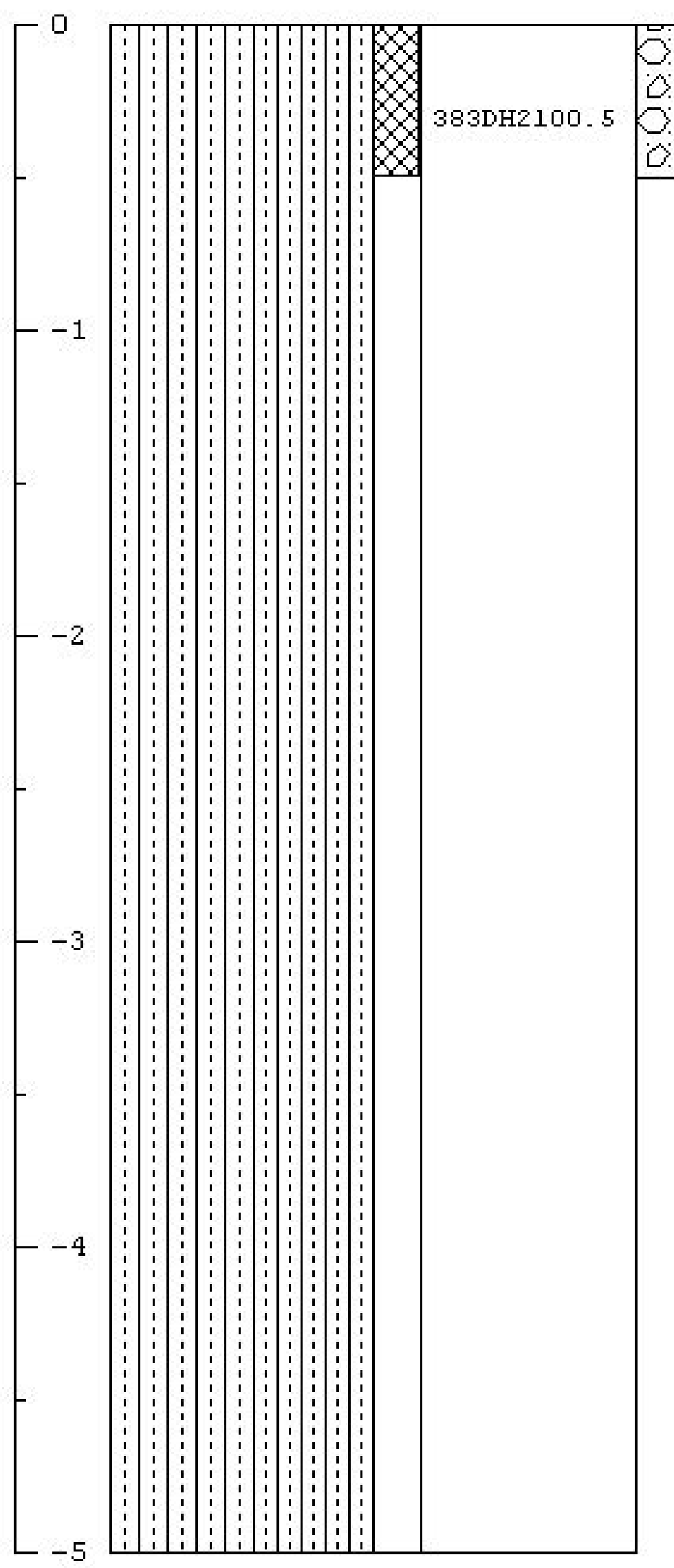

c.

Date Started: $6 / 15 / 04$

Date completed: $6 / 15 / 04$

Elevation : 5976.5

Horthing: 887711.5

Easting: 638827.0

Total Depth: 0.5 ft.

Lithologic Description

Comments

\begin{tabular}{|c|c|c|}
\hline \multicolumn{2}{|c|}{ Field Screening Results } \\
\hline & & \\
vocs & Alpha & Beta \\
(ppm) & (dpm) & (dpm) \\
& & \\
\end{tabular}

Muck: Brown FMC sand and gravel with cobbles, dry 
Project Name: CAU 383 E-Tunnel sites

Project Humber: DT04-320

Borehole Number: EDH 22

Logged By: $M$. McLane

Drilled By: M. McLane

Drilling Method: Hand Sampled
Date Started: $6 / 15 / 04$

Date completed: $6 / 15 / 04$

Elevation: 5972.0

Horthing: 887718.5

Easting: 638836.6

Total Depth: 0.5 ft.

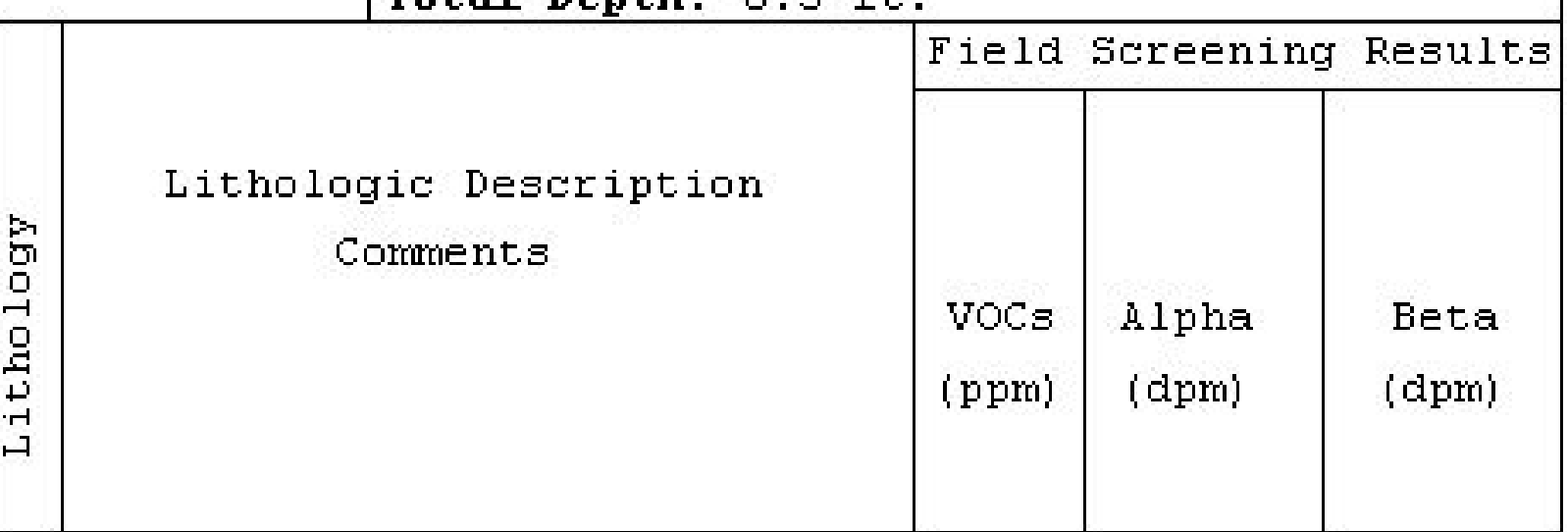

383 DH2200. $5 \mathrm{X}$.

Muck: Brown FMC sand and gravel with cobbles, dry 
Project Name: CAU 383 E-Tunnel sites

Project Humber: DT04-320

Borehole Number: EDH23

Logged By: $M$. McLane

Drilled By: M. McLane

Drilling Method: Hand Sampled

\begin{tabular}{|c|c|c|c|c|c|}
\hline 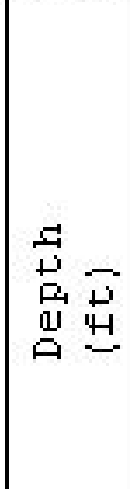 & 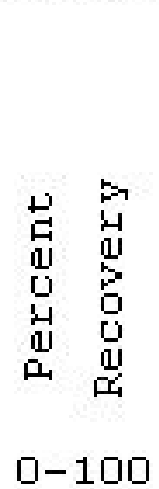 & 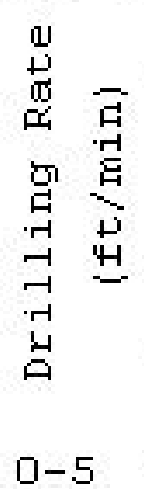 & 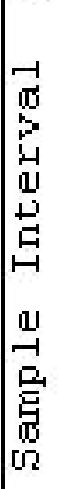 & 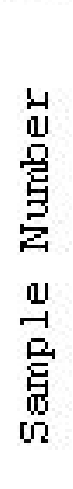 & 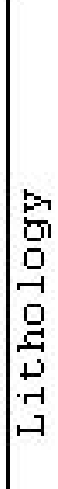 \\
\hline
\end{tabular}

Contact o N/A, Sample 383DH2300.5.

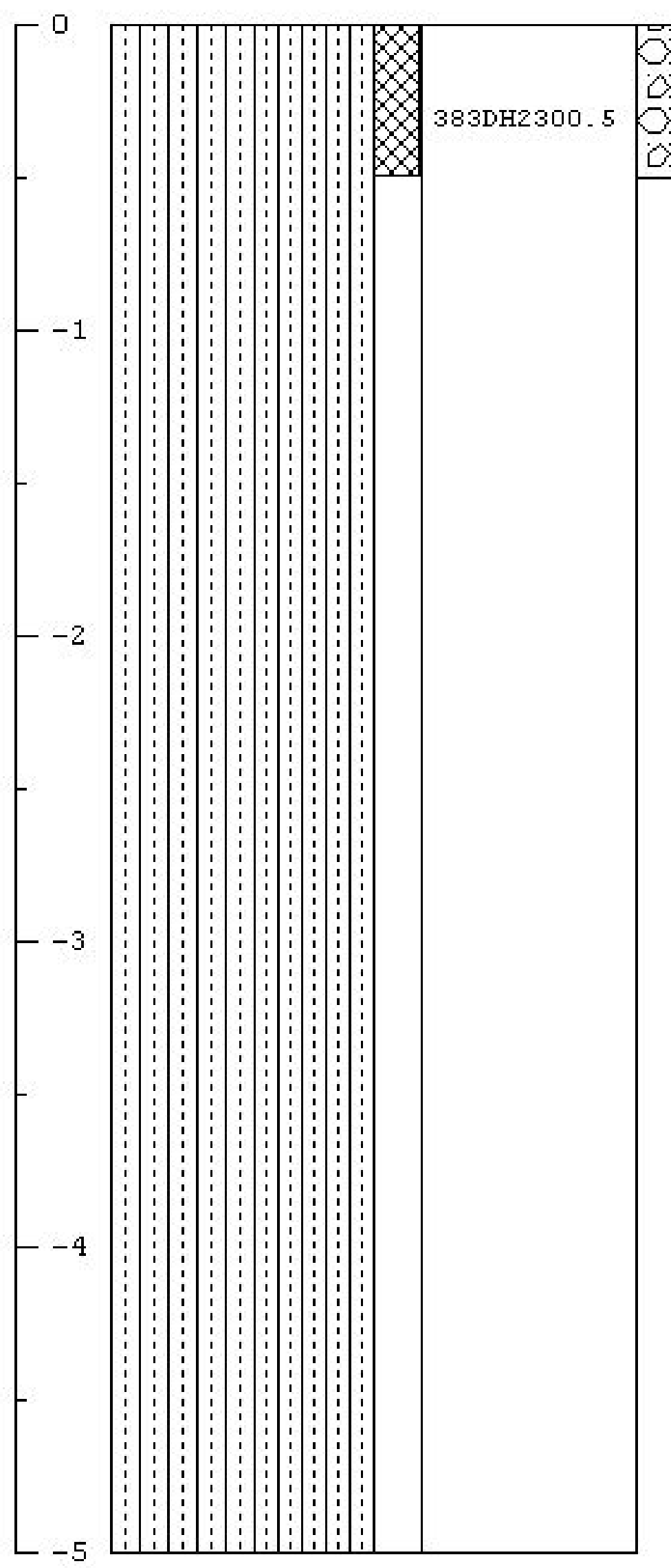

c.

Date Started: $6 / 15 / 04$

Date completed: $6 / 15 / 04$

Elevation : 5976.1

Horthing: 887721.1

Easting: 638844.8

Total Depth: 0.5 ft.

Lithologic Description

Comments

\begin{tabular}{|c|c|c|}
\hline \multicolumn{2}{|c|}{ Field Screening Results } \\
\hline & & \\
vocs & Alpha & Beta \\
(ppm) & (dpm) & (dpm) \\
& & \\
\end{tabular}

Muck: Brown FMC sand and gravel with cobbles, dry 
Project Name: CAU 383 E-Tunnel sites

Project Humber: DT04-320

Borehole Number: EDH24

Logged By: $M$. McLane

Drilled By: M. McLane

Drilling Method: Hand Sampled

\begin{tabular}{|c|c|c|c|c|c|}
\hline 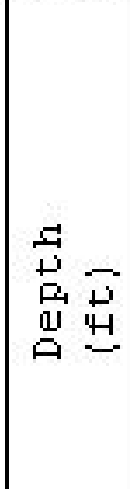 & 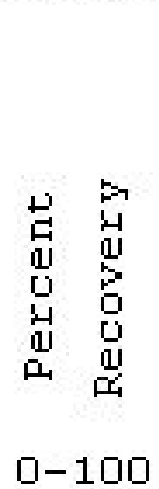 & 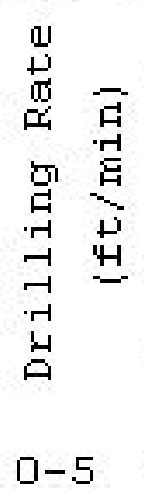 & 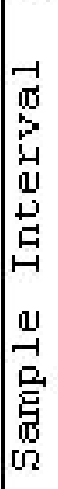 & 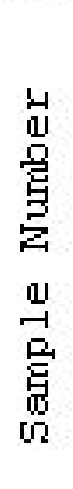 & 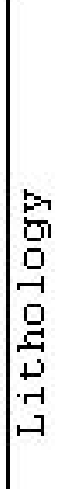 \\
\hline
\end{tabular}

Contact o N/A, Sample 383 DH2400.5.

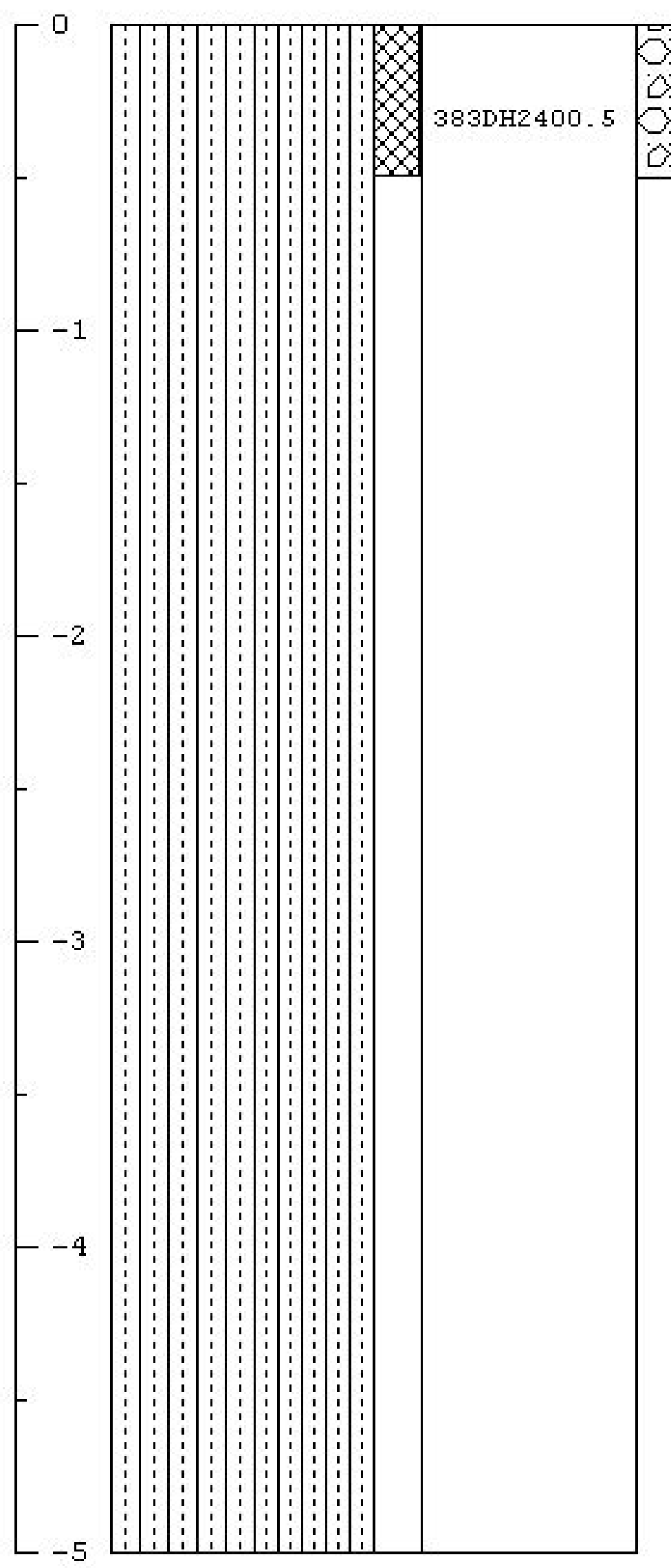

c.

Date Started: $6 / 15 / 04$

Date completed: $6 / 15 / 04$

Elevation : 5972.1

Horthing: 887721.4

Easting: 638855.0

Total Depth: 0.5 ft.

Lithologic Description

Comments

\begin{tabular}{|c|c|c|}
\hline \multicolumn{2}{|c|}{ Field Screening Results } \\
\hline & & \\
vocs & Alpha & Beta \\
(ppm) & (dpm) & (dpm) \\
& & \\
\end{tabular}

Muck: Brown FMC sand and gravel with cobbles, dry 
Project Name: CAU 383 E-Tunnel sites

Project Humber: DT04-320

Borehole Number: EDH25

Logged By: $M$. McLane

Drilled By: M. McLane

Drilling Method: Hand Sampled

\begin{tabular}{|c|c|c|c|c|c|}
\hline 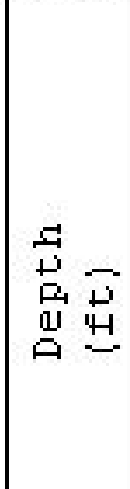 & 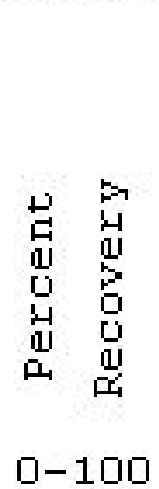 & 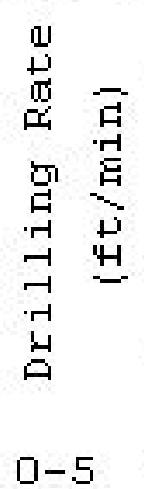 & 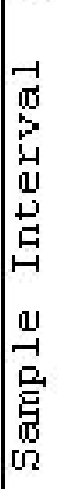 & 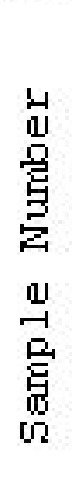 & 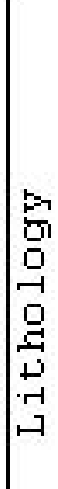 \\
\hline
\end{tabular}

Contact o N/A, Sample 383 DH2500.5.

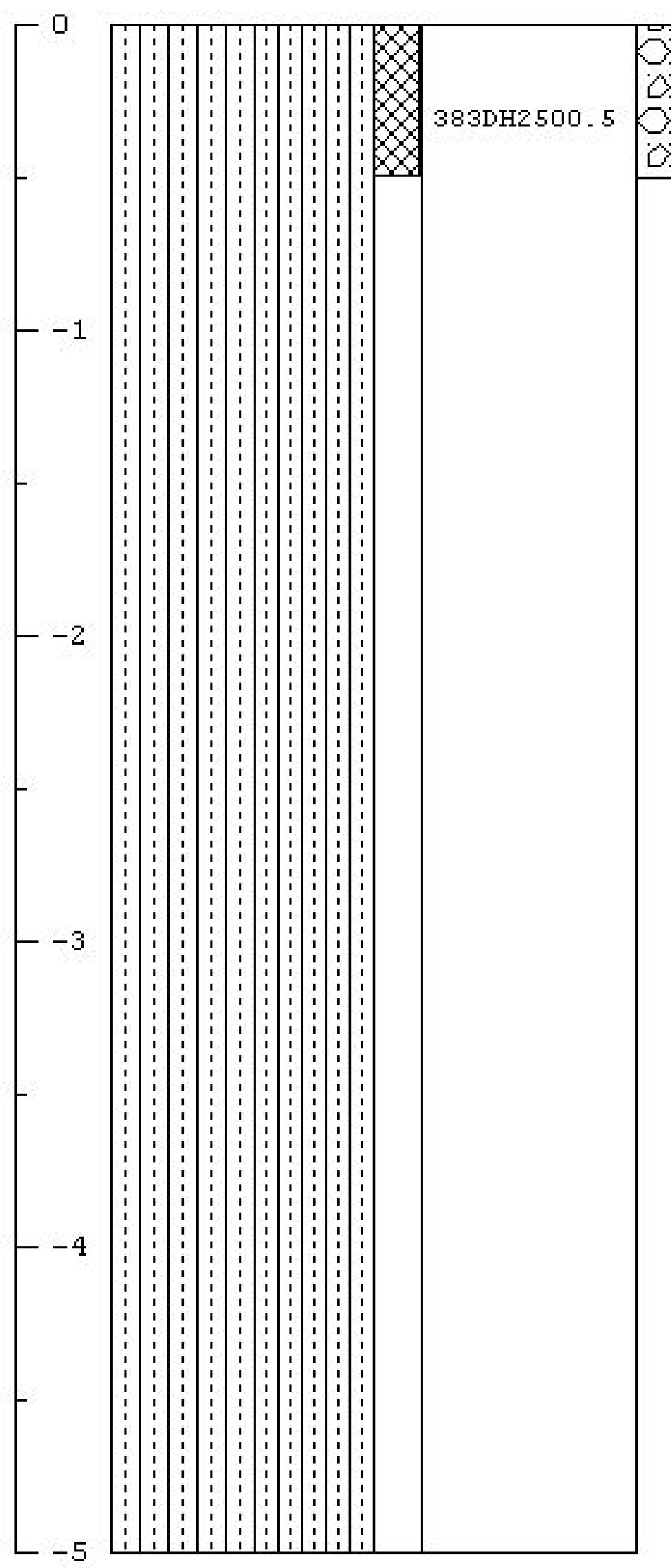

c.

Date Started: $6 / 15 / 04$

Date completed: $6 / 15 / 04$

Elevation: 5968.0

Horthing: 887720.0

Easting: 638875.3

Total Depth: 0.5 ft.

Lithologic Description

Comments

\begin{tabular}{|c|c|c|}
\hline \multicolumn{3}{|c|}{ Field Screening Results } \\
\hline & & \\
& & \\
VoCs & Slpha & Beta \\
(ppri) & (dpm) & (dpm) \\
& & \\
& &
\end{tabular}

Muck: Brown FMC sand and gravel with cobbles, dry 
Project Name: CAU 383 E-Tunnel sites

Project Humber: DT04-320

Borehole Number: EDH2 6

Logged By: $M$. McLane

Drilled By: M. McLane

Drilling Method: Hand Sampled
Date Started: $6 / 15 / 04$

Date completed: $6 / 15 / 04$

Elevation: 5971.4

Horthing: 887720.0

Easting: 638886.7

Total Depth: 0.5 ft.

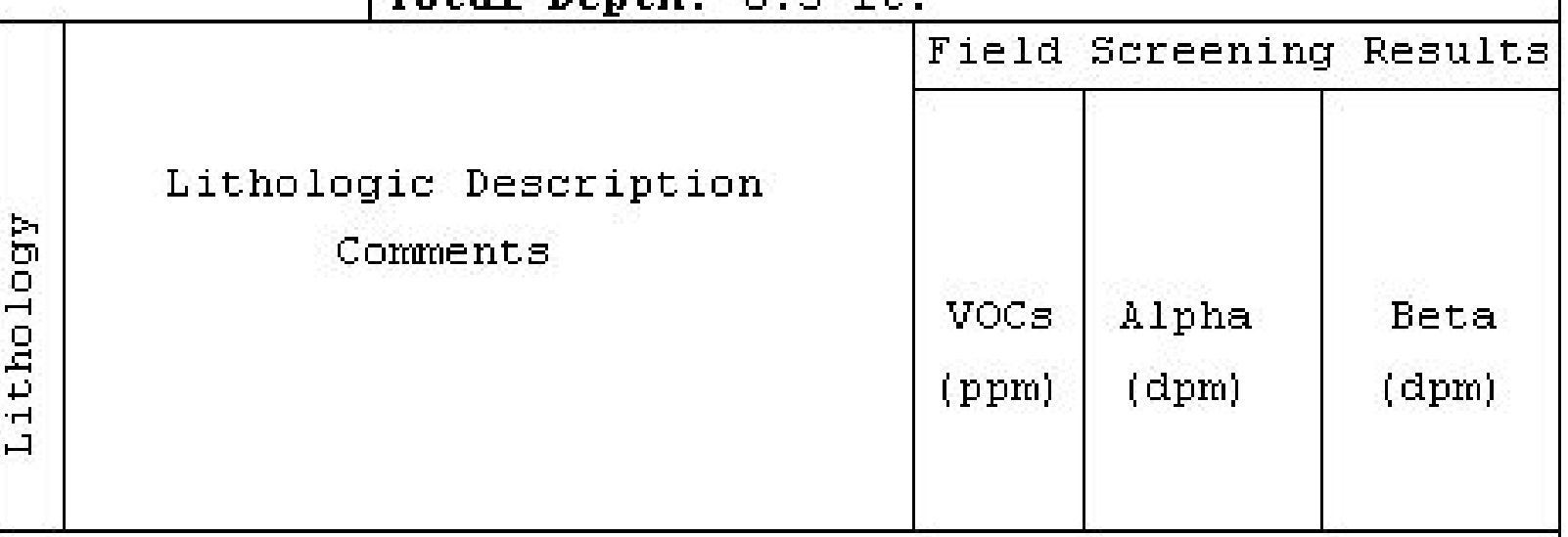

Contact o N/A, Sample 383DH2600.5.

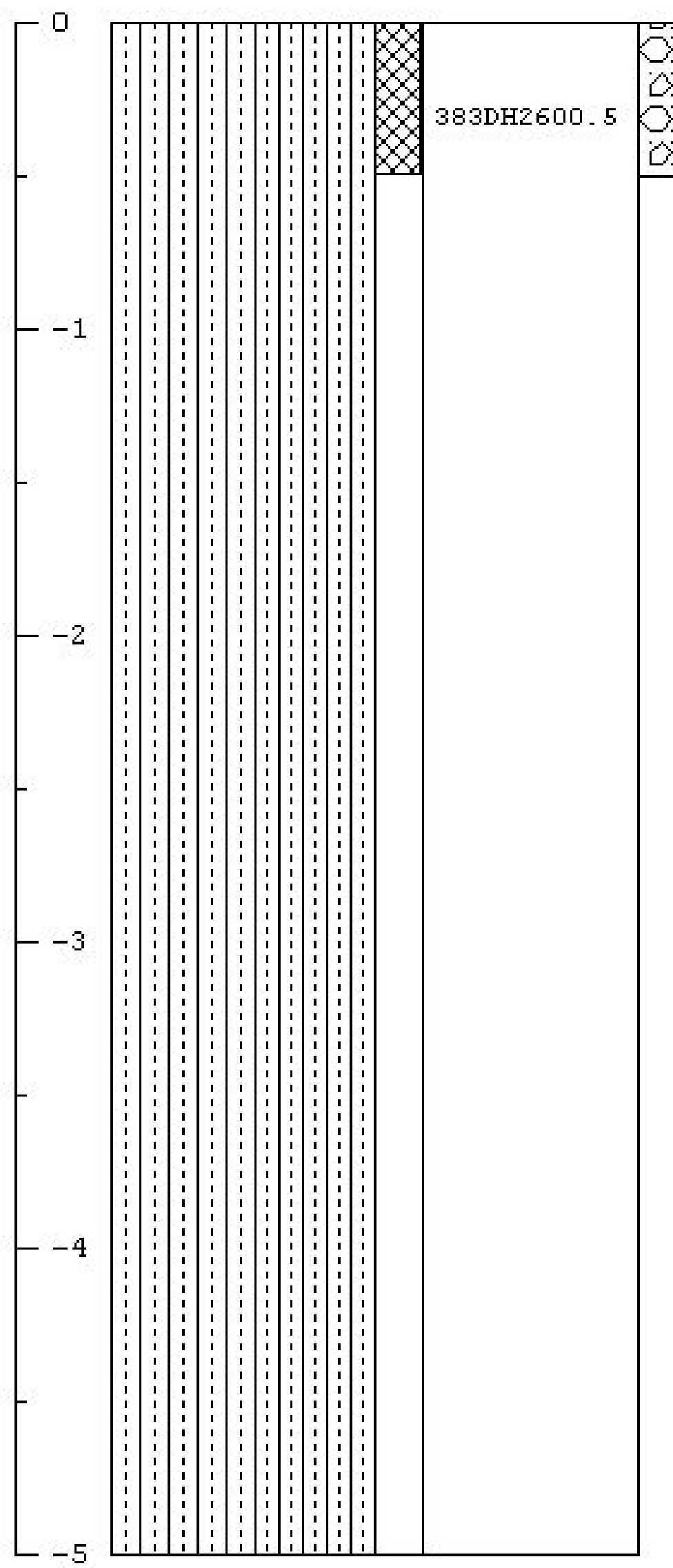

Muck: Brown FMC sand and gravel with cobbles, dry

ci.


Project Name: CAU 383 E-Tunnel sites

Project Humber: DT04-320

Borehole Number: EDH27

Logged By: $M$. McLane

Drilled By: M. McLane

Drilling Method: Hand Sampled
Date Started: $6 / 16 / 04$

Date completed: $6 / 16 / 04$

Elevation : 5970.9

Horthing: 887719.7

Easting: 638896.0

Total Depth: 0.5 ft.

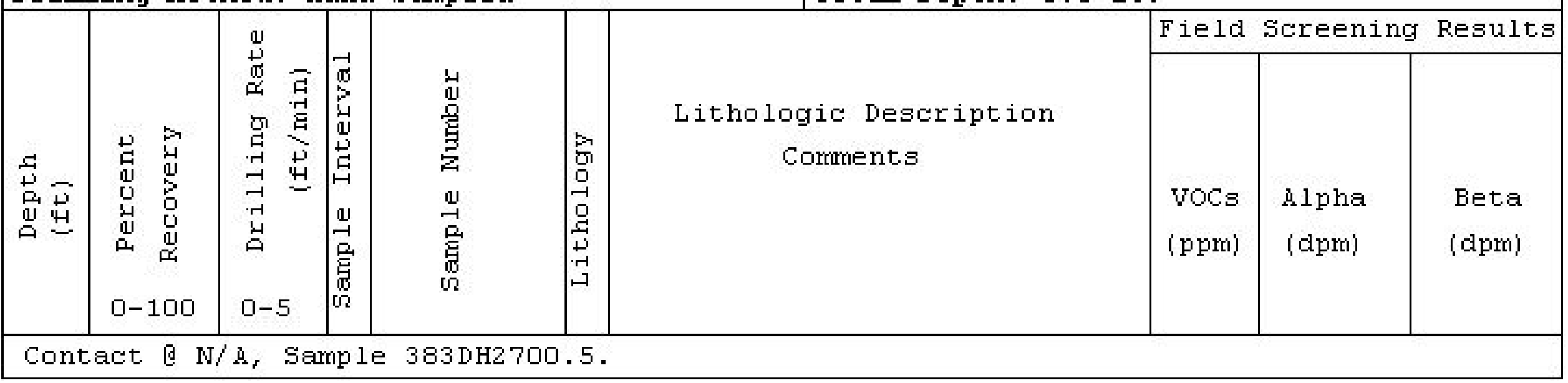

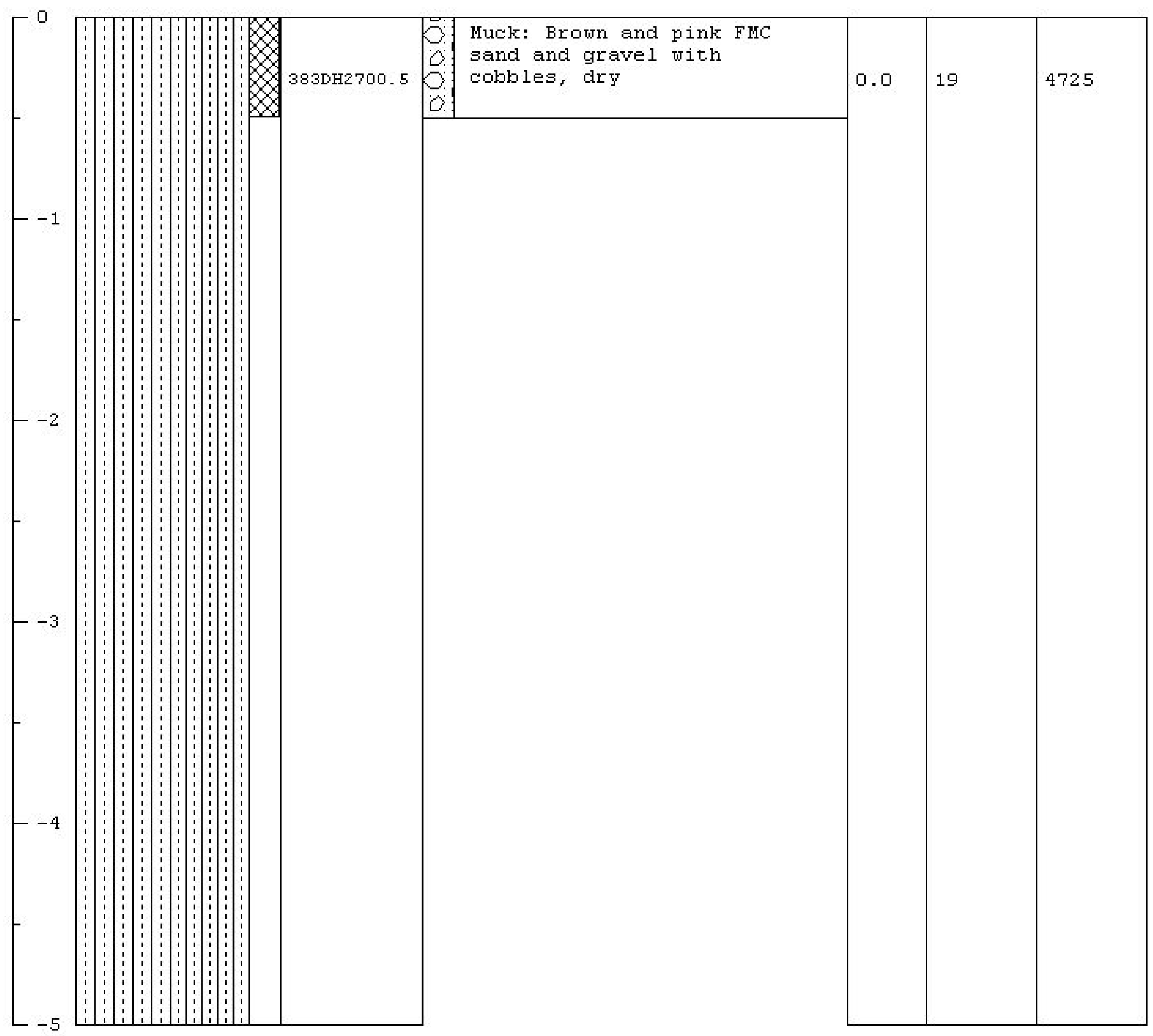


Project Name: CAU 383 E-Tunnel sites

Project Humber: DT04-320

Borehole Number: EDH28

Logged By: $M$. McLane

Drilled By: M. McLane

Drilling Method: Hand Sampled
Date Started: $6 / 16 / 04$

Date completed: $6 / 16 / 04$

Elevation: 5972.0

Horthing: 887722.7

Easting: 638906.4

Total Depth: 0.5 ft.

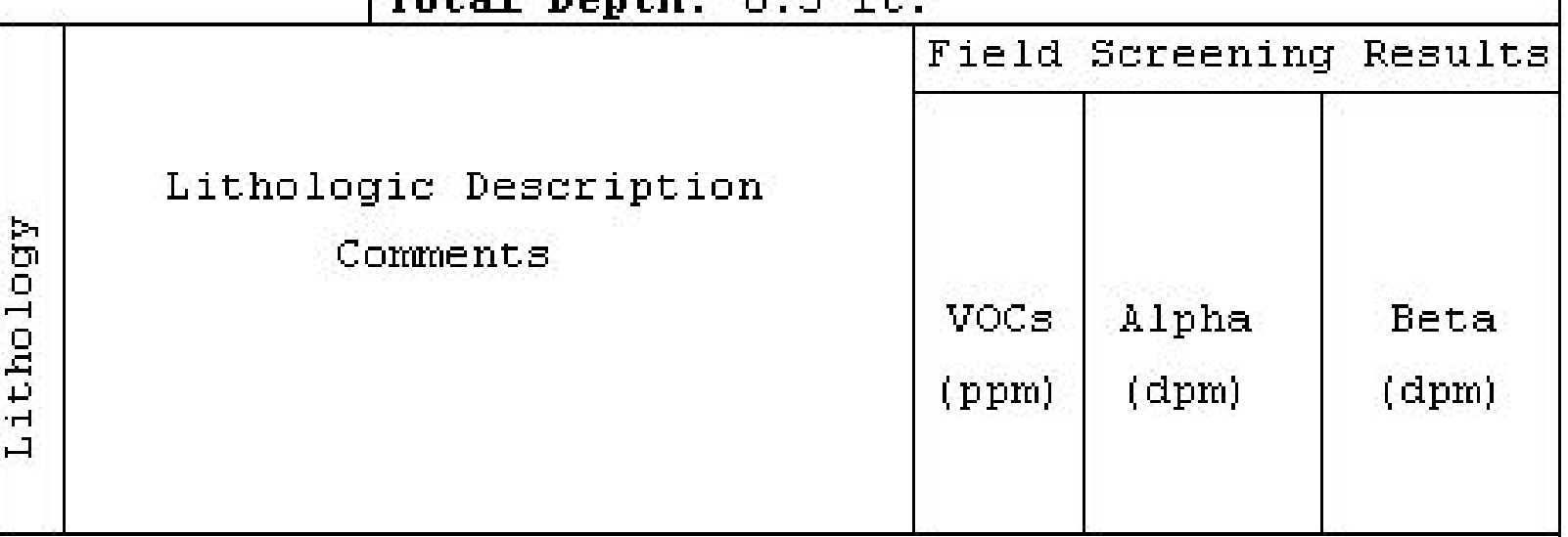

Contact o N/A, Sample 383DH2800.5.

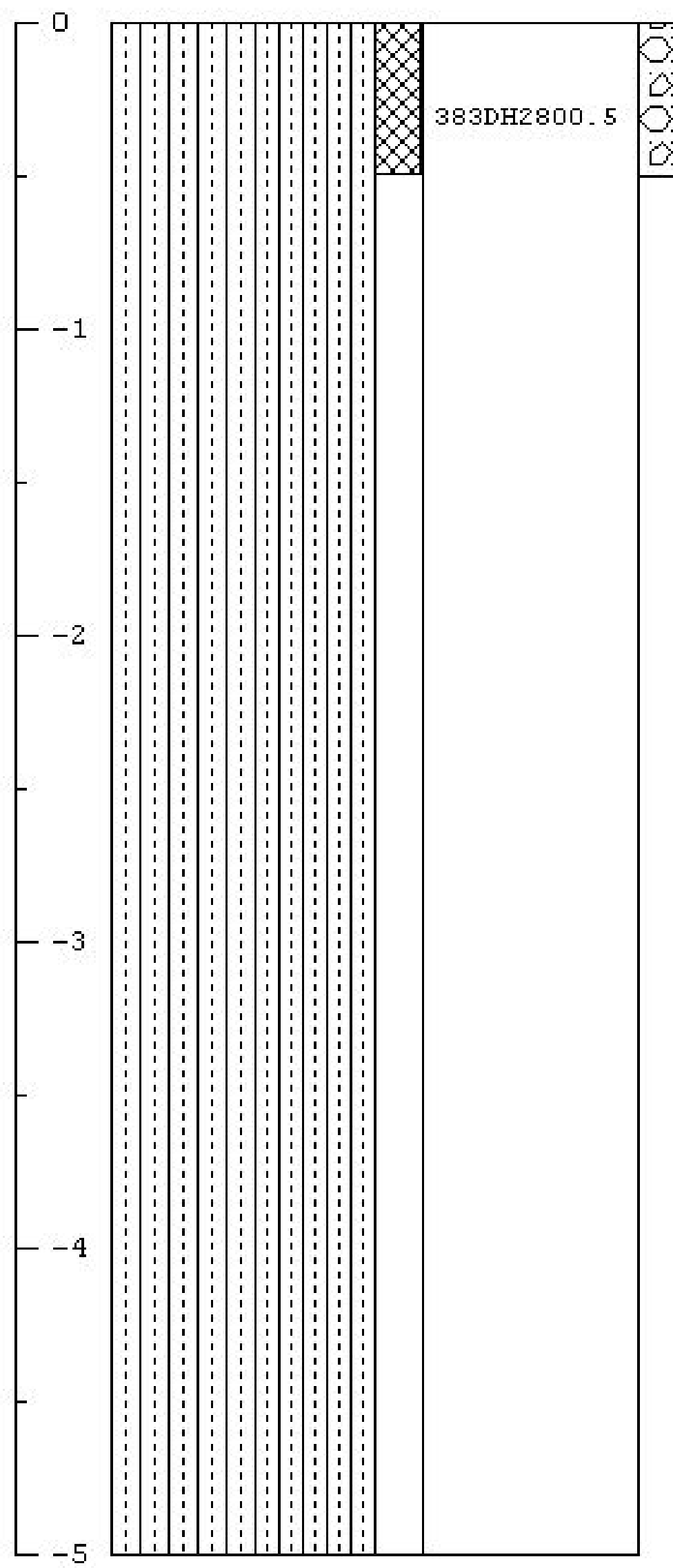

Muck: Brown and pink FMC sand and gravel with cobbles, dry
3721 
Project Name: CAU 383 E-Tunnel sites

Project Humber: DT04-320

Borehole Number: EDH29

Logged By: $M$. McLane

Drilled By: M. McLane

Drilling Method: Hand Sampled
Date Started: $6 / 16 / 04$

Date completed: $6 / 16 / 04$

Elevation : 5969.8

Horthing: 887723.1

Easting: 638916.6

Total Depth: 0.5 ft.

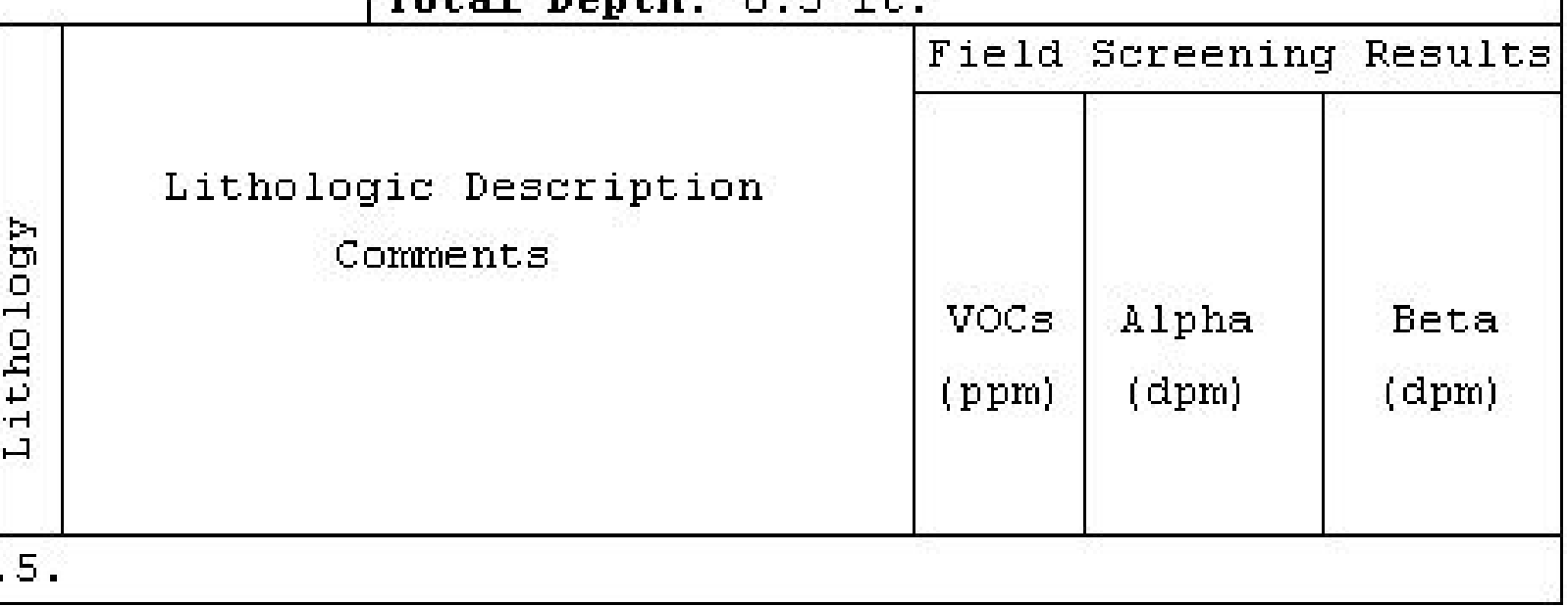

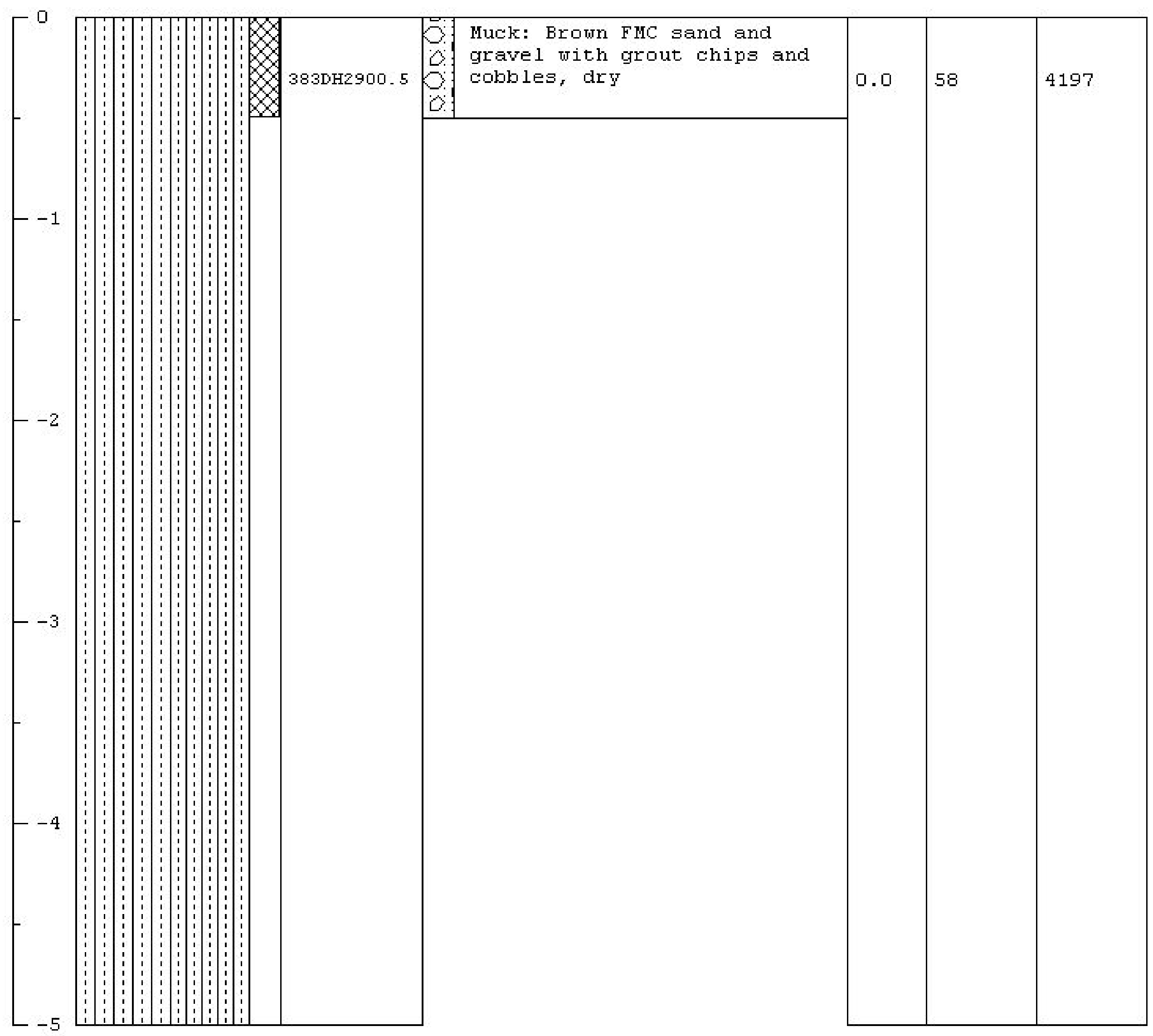


Project Name: CAU 383 E-Tunnel sites

Project Humber: DT04-320

Borehole Number: EDH30

Logged By: $M$. McLane

Drilled By: M. McLane

Drilling Method: Hand Sampled
Date Started: $6 / 16 / 04$

Date completed: $6 / 16 / 04$

Elevation: 5971.0

Horthing: 887722.5

Easting: 638926.3

Total Depth: 0.5 ft.

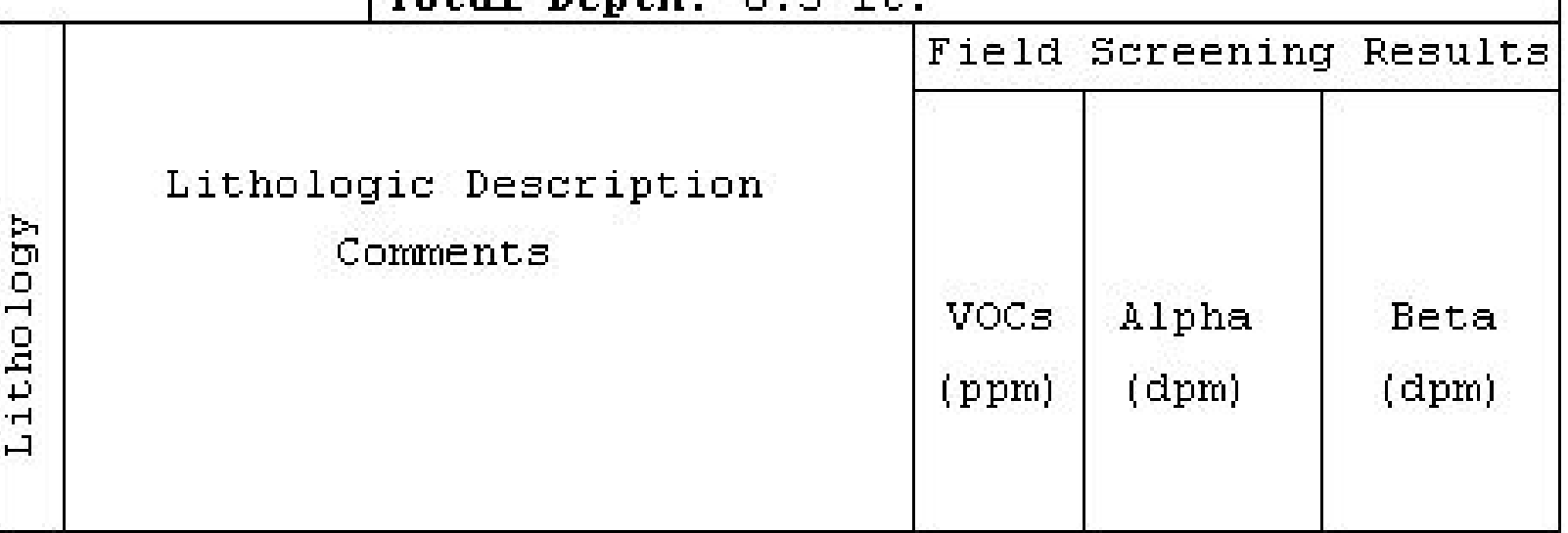

Contact o N/A, Sample 383DH3000.5.

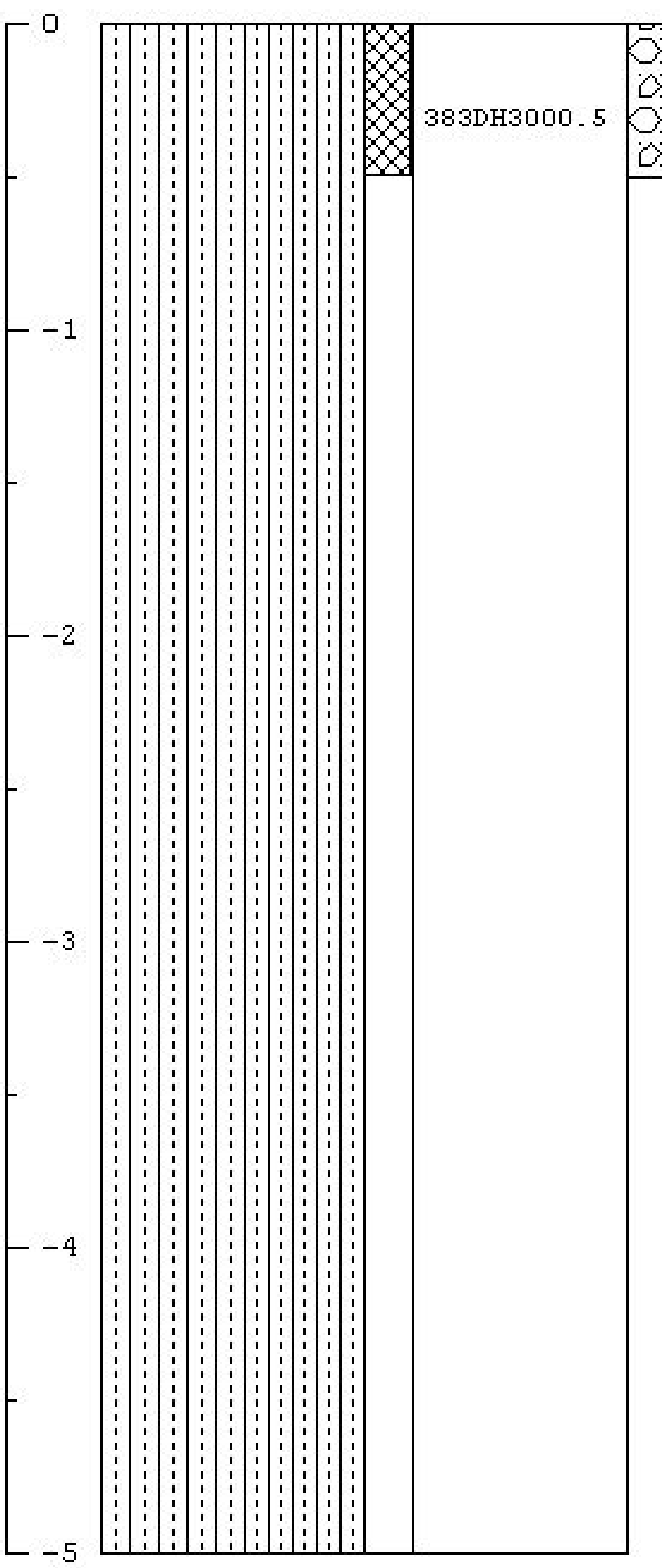

Muck: Brown FMC sand and gravel with grout chips and cobbles, dry

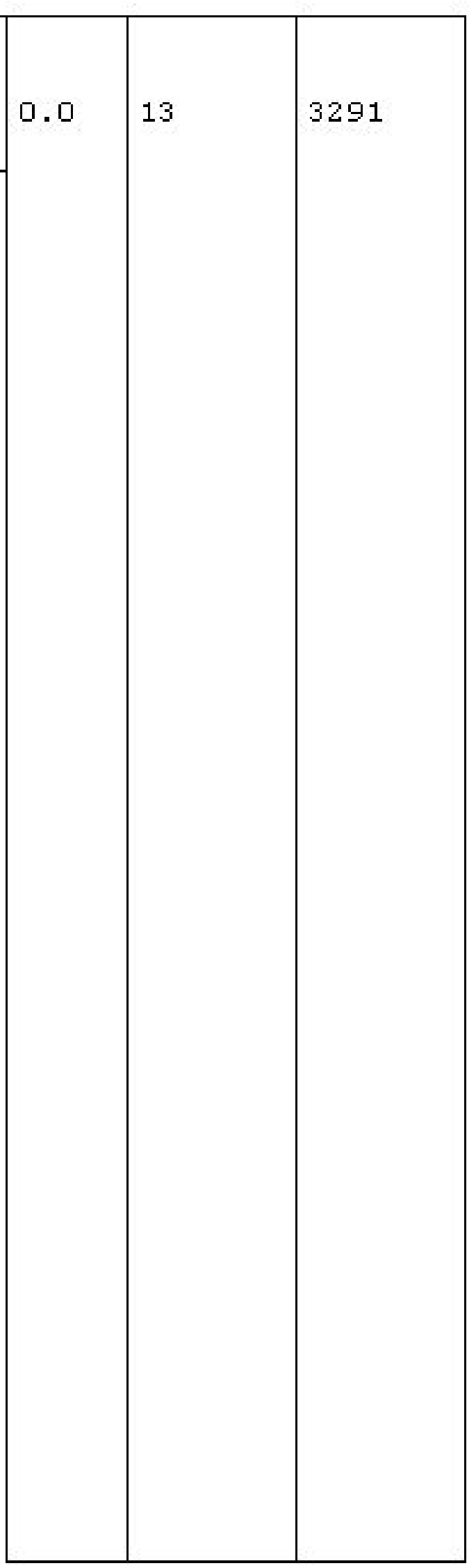


Project Name: CAU 383 E-Tunnel sites

Project Humber: DT04-320

Borehole Number: EDH31

Logged By: $M$. McLane

Drilled By: M. McLane

Drilling Method: Hand Sampled
Date Started: $6 / 16 / 04$

Date completed: $6 / 16 / 04$

Elevation : 5969.6

Horthing: 887723.8

Easting: 638935.6

Total Depth: 0.5 ft.

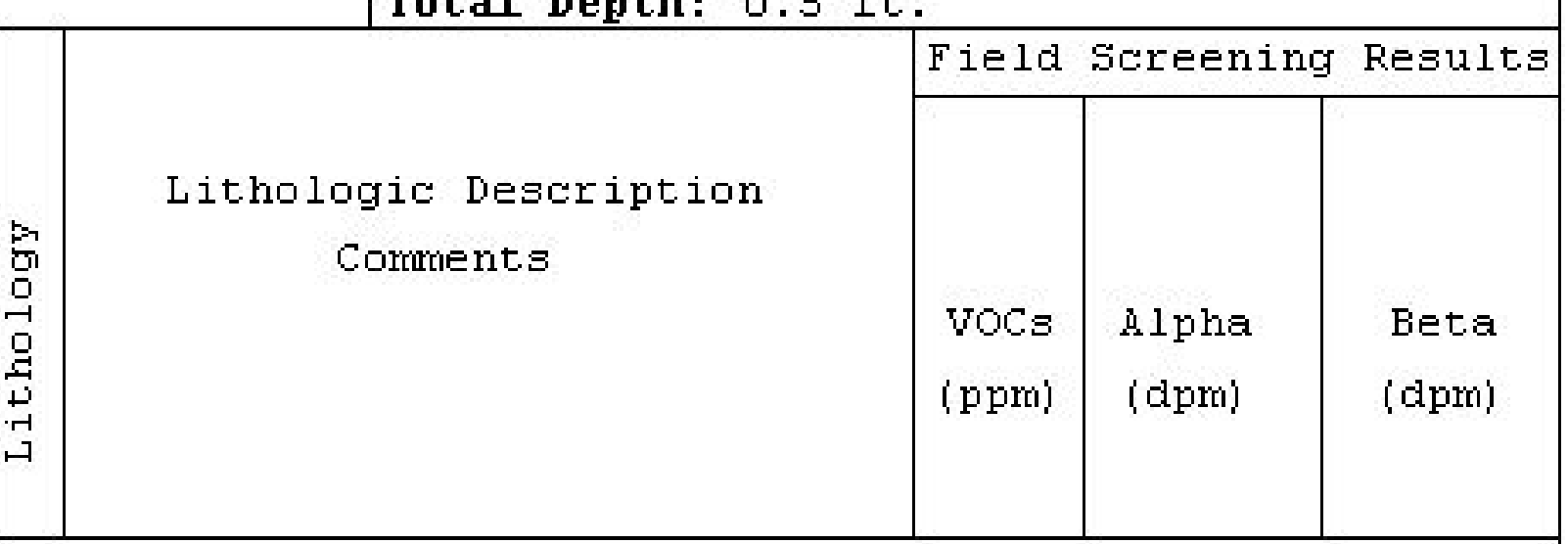

Contact o N/A, Sample 383DH3100.5.

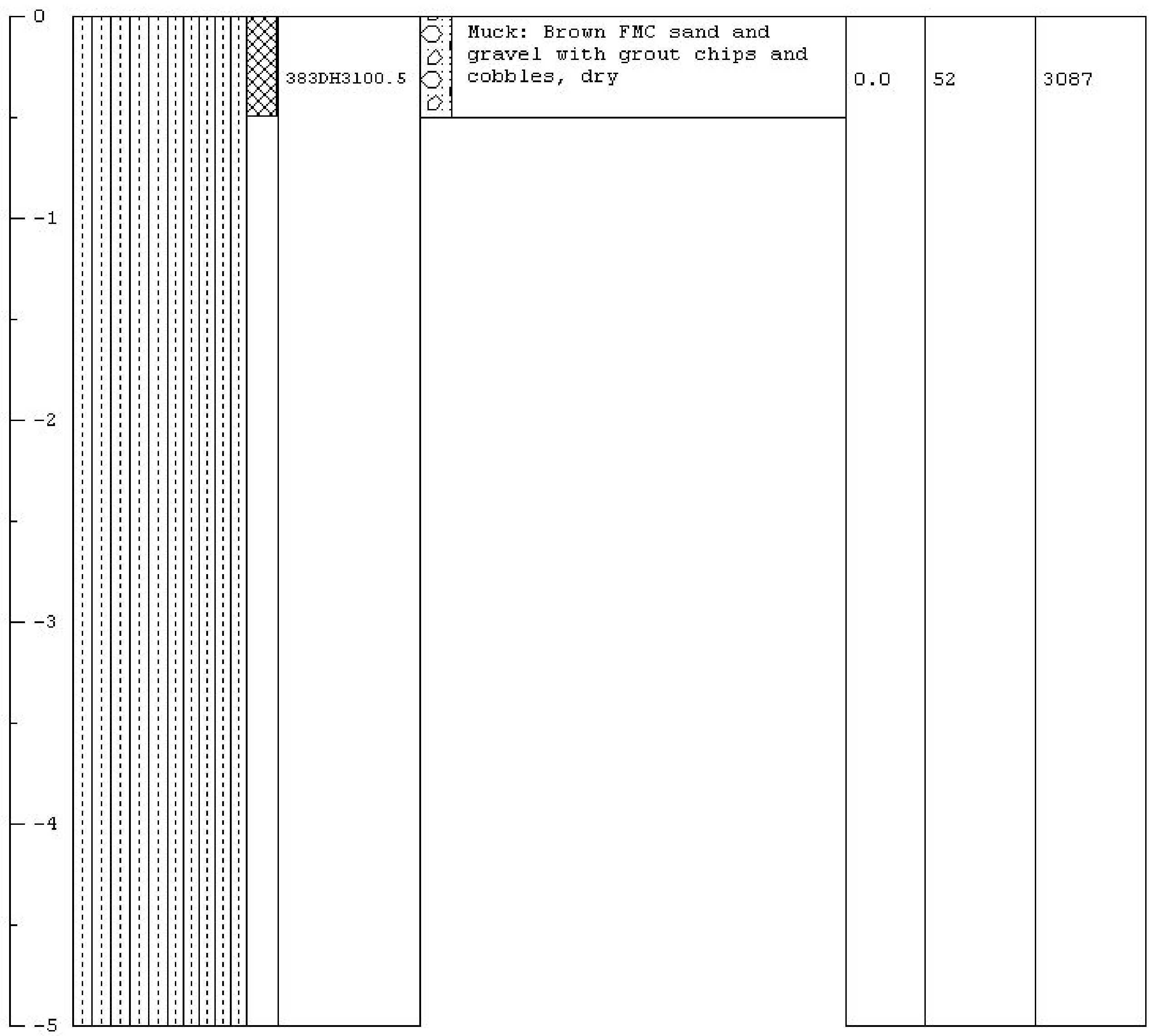


Project Name: CAU 383 E-Tunnel sites

Project Humber: DT04-320

Borehole Number: EDH 32

Logged By: $M$. McLane

Drilled By: M. McLane

Drilling Method: Hand Sampled
Date Started: $6 / 16 / 04$

Date completed: $6 / 16 / 04$

Elevation : 5968.2

Horthing: 887723.2

Easting: 638946.3

Total Depth: 0.5 ft.

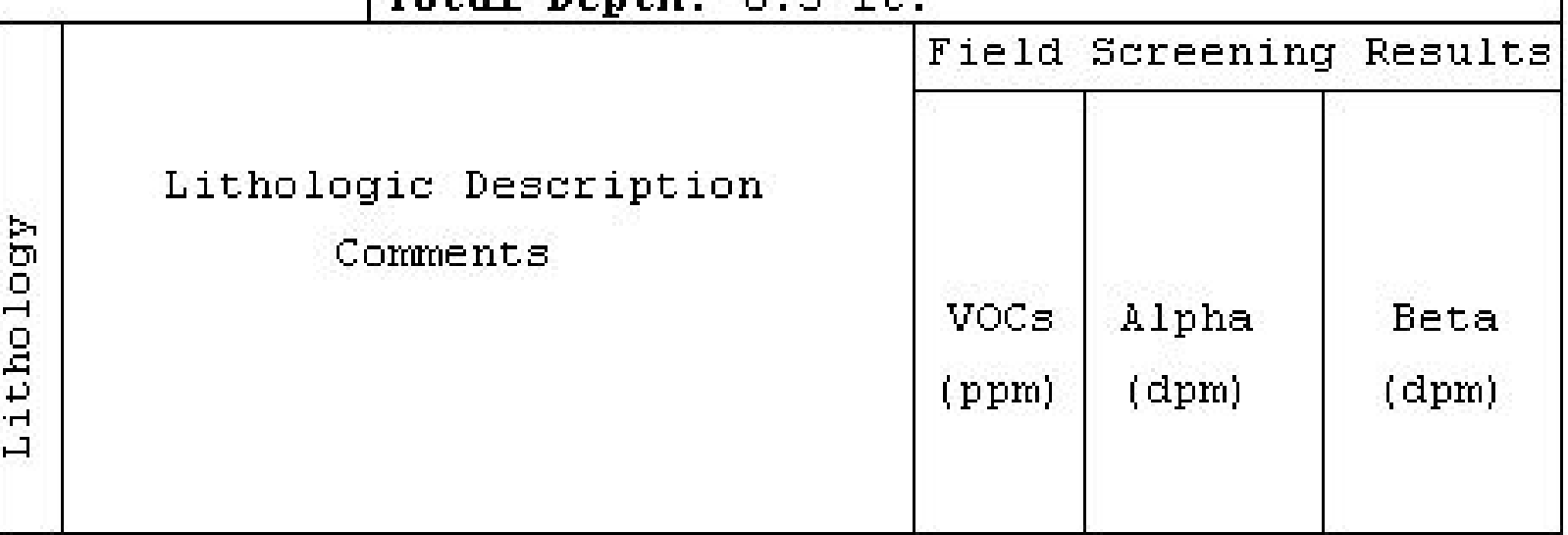

Contact o N/A, Sample 383DH3200.5.

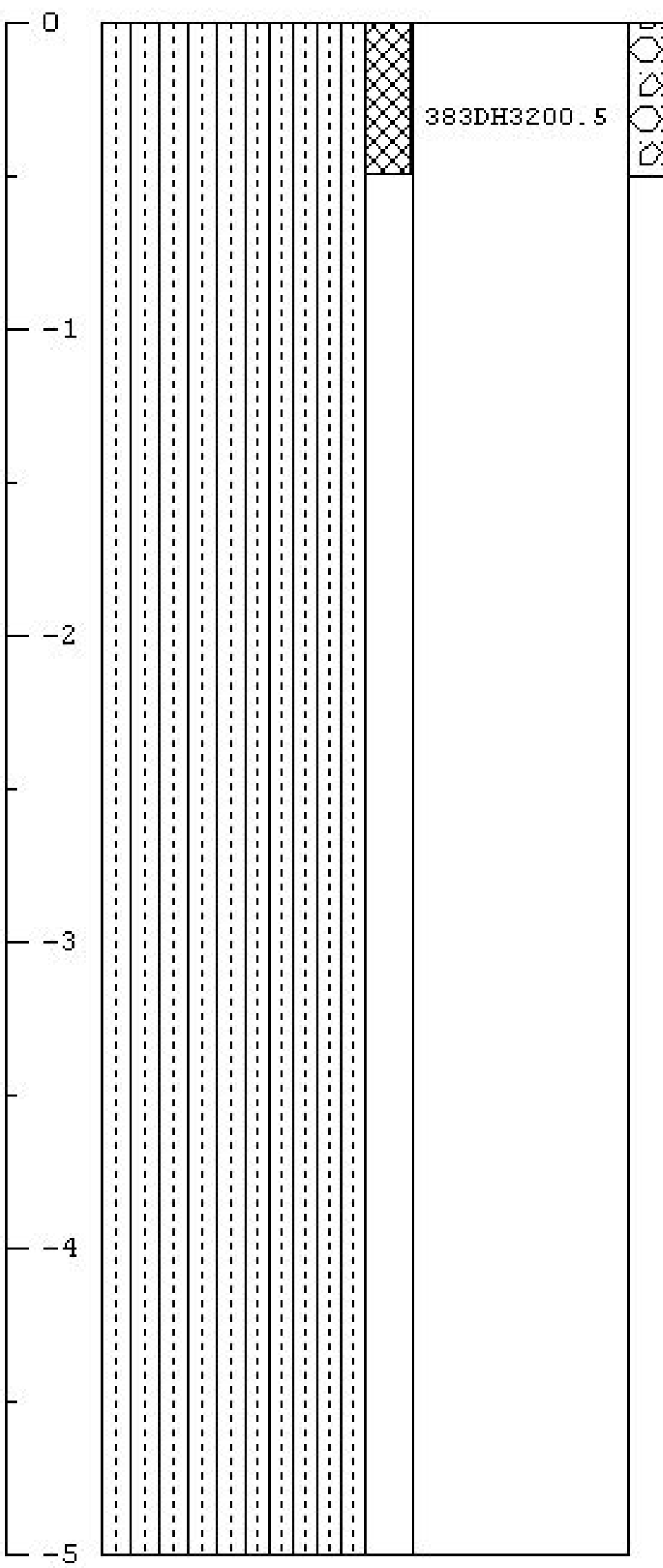

Muck: Brown FMC sand and gravel with grout chips and cobbles, dry

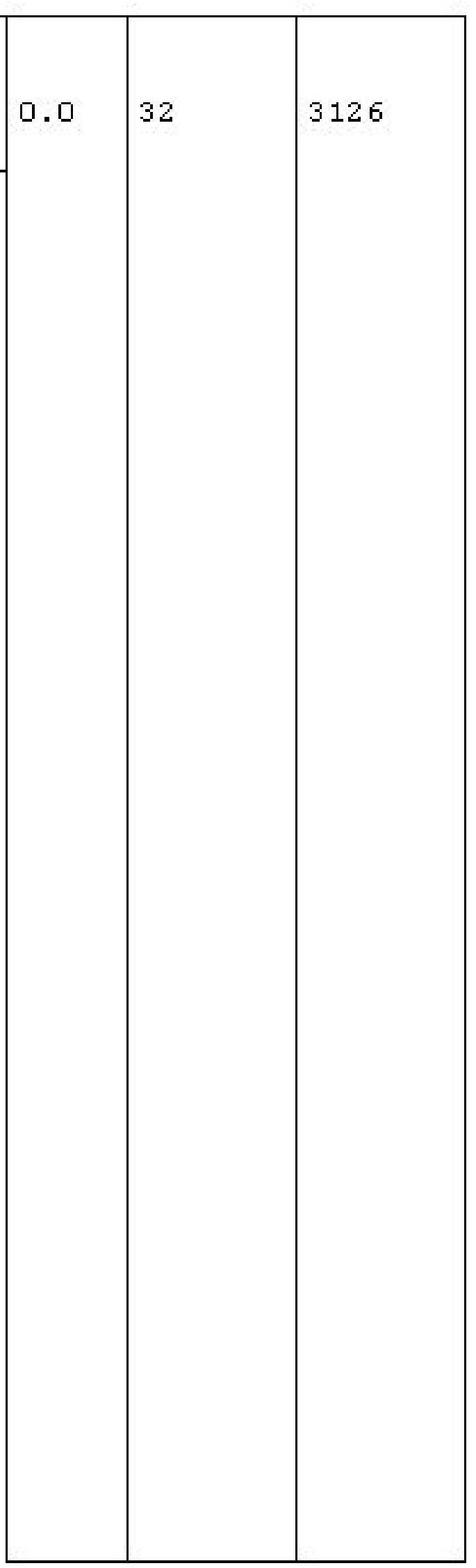


Project Name: CAU 383 E-Tunnel sites

Project Humber: DT04-320

Borehole Number: EDH33

Logged By: $M$. McLane

Drilled By: M. McLane

Drilling Method: Hand Sampled

\begin{tabular}{|c|c|c|c|c|c|}
\hline 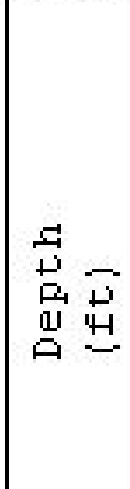 & 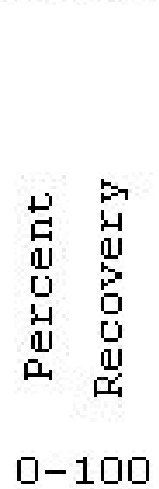 & 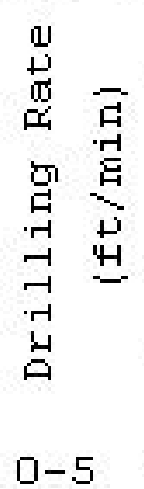 & 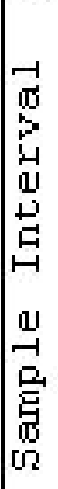 & 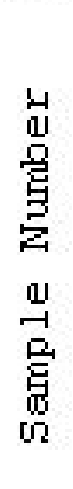 & 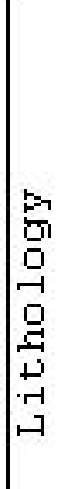 \\
\hline
\end{tabular}

Contact o N/A, Sample 383DH3300.5.

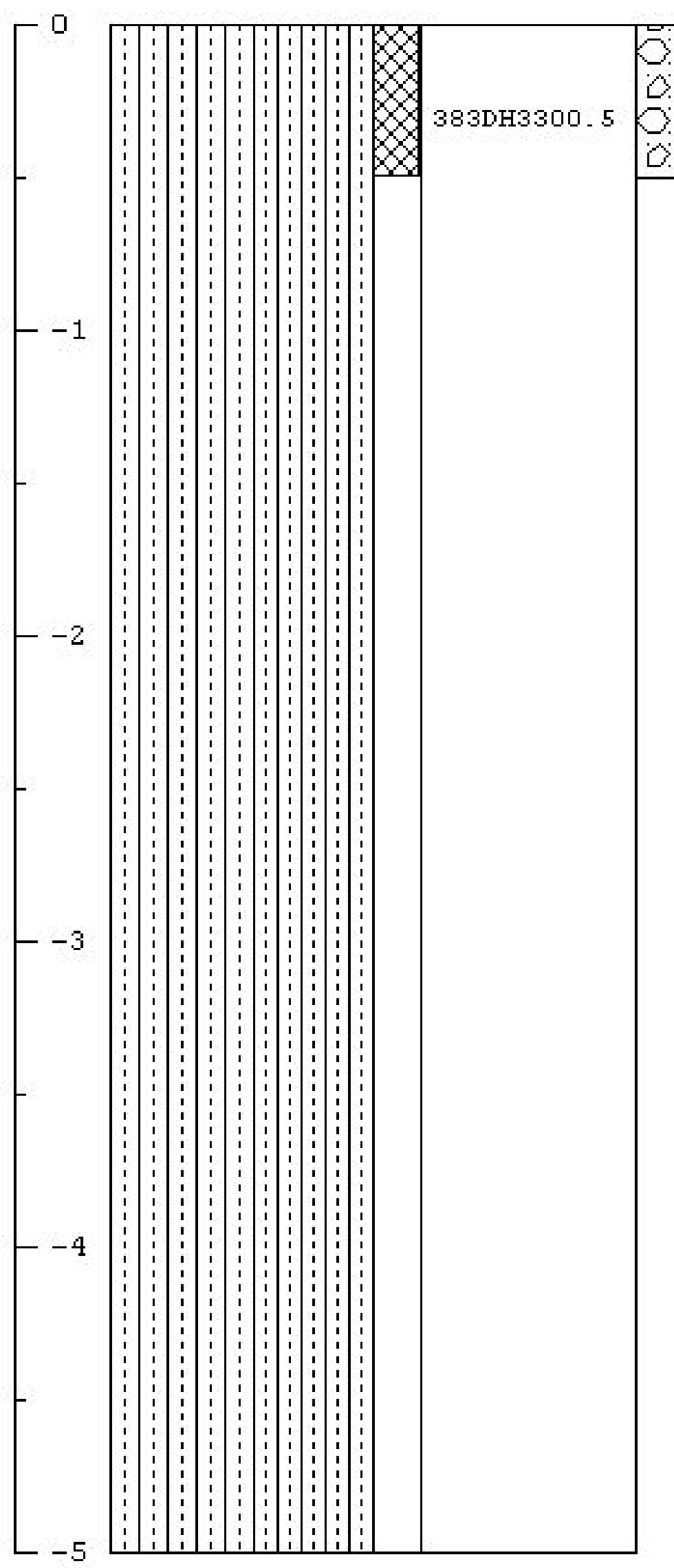

c.

Date Started: $6 / 16 / 04$

Date completed: $6 / 16 / 04$

Elevation: 5963.7

Horthing: 887721.4

Easting: 638955.9

Total Depth: 0.5 ft.

Lithologic Description

Comments

\begin{tabular}{|c|c|c|}
\hline \multicolumn{2}{|c|}{ Field Screening Results } \\
\hline & & \\
vocs & Alpha & Beta \\
(ppm) & (dpm) & (dpm) \\
& & \\
\end{tabular}

Muck: Brown FMC sand and gravel with cobbles, dry 
Project Hame: CAU 383 E-Tunnel sites

Project Humber: DTO4-320

Borehole Humber: EDH34

Logged By: M. MeLane

Drilled By: Boart-Longyear

Drilling Method: Rotosonic
Date Started: $6 / 10 / 04$

Date Completed: $6 / 10 / 04$

Elevation: 5964.4

Horthing: 887717.9

Easting: 638966.2

Total Depth: 5.0 ft.

\begin{tabular}{|c|c|c|c|c|}
\hline & & Field & Screening & Results \\
\hline 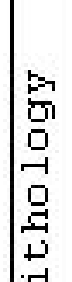 & $\begin{array}{c}\text { Lithologic Description } \\
\text { Comments }\end{array}$ & vOCs & $\begin{array}{l}\text { Alpha } \\
\text { (dpm) }\end{array}$ & $\begin{array}{l}\text { Beta } \\
\text { (dpm) }\end{array}$ \\
\hline
\end{tabular}

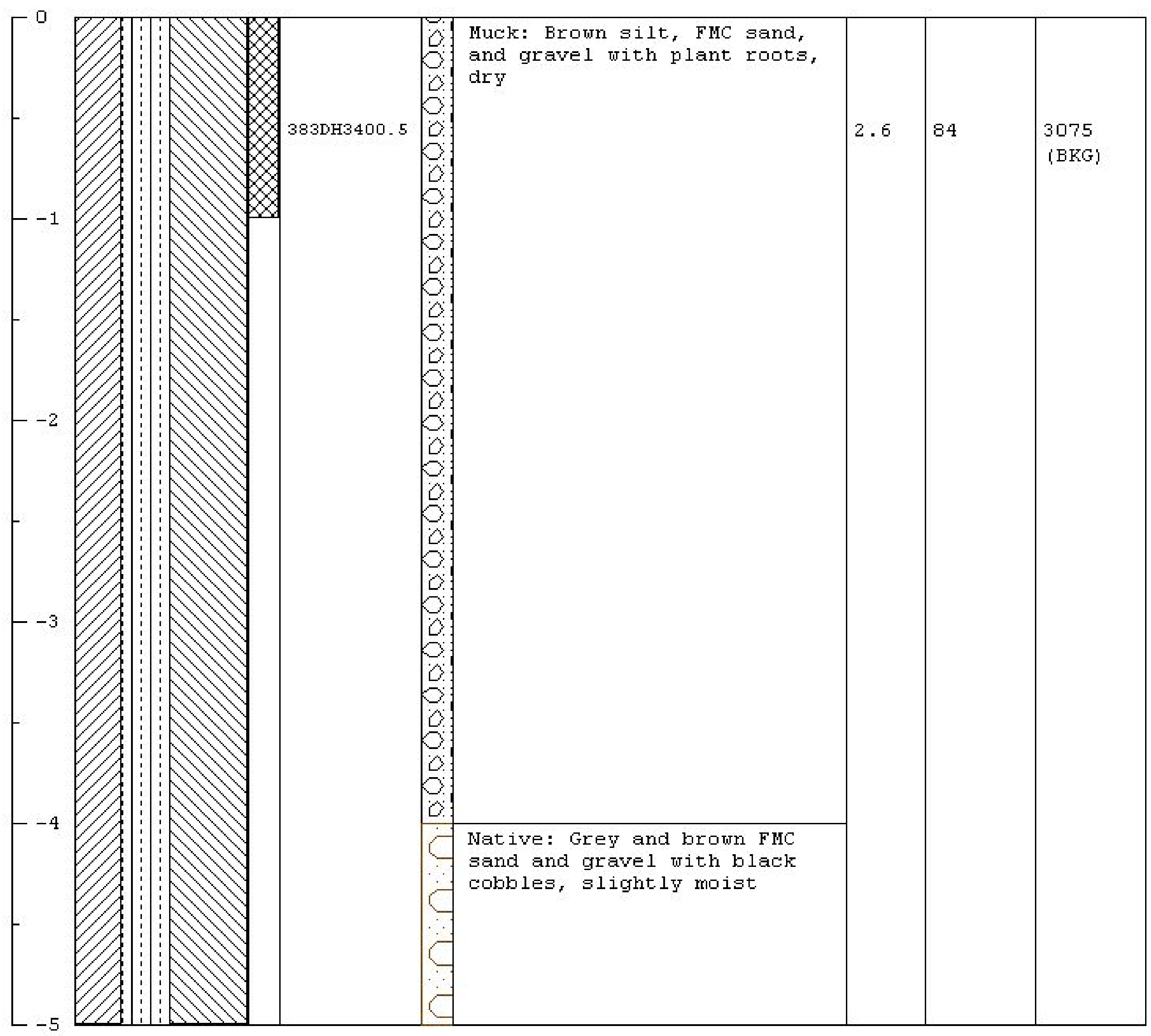


Project Hame: CAU 383 E-Tunnel sites

Project Humber: DTO4-320

Borehole Number: EDH3 5

Logged By: M. MeLane

Drilled By: Boart-Longyear

Drilling Method: Rotosonic
Date Started: $6 / 10 / 04$

Date completed: $6 / 10 / 04$

Elevation: 5961.7

Horthing: 887712.3

Easting: 638983.2

Total Depth: 5.0 ft.

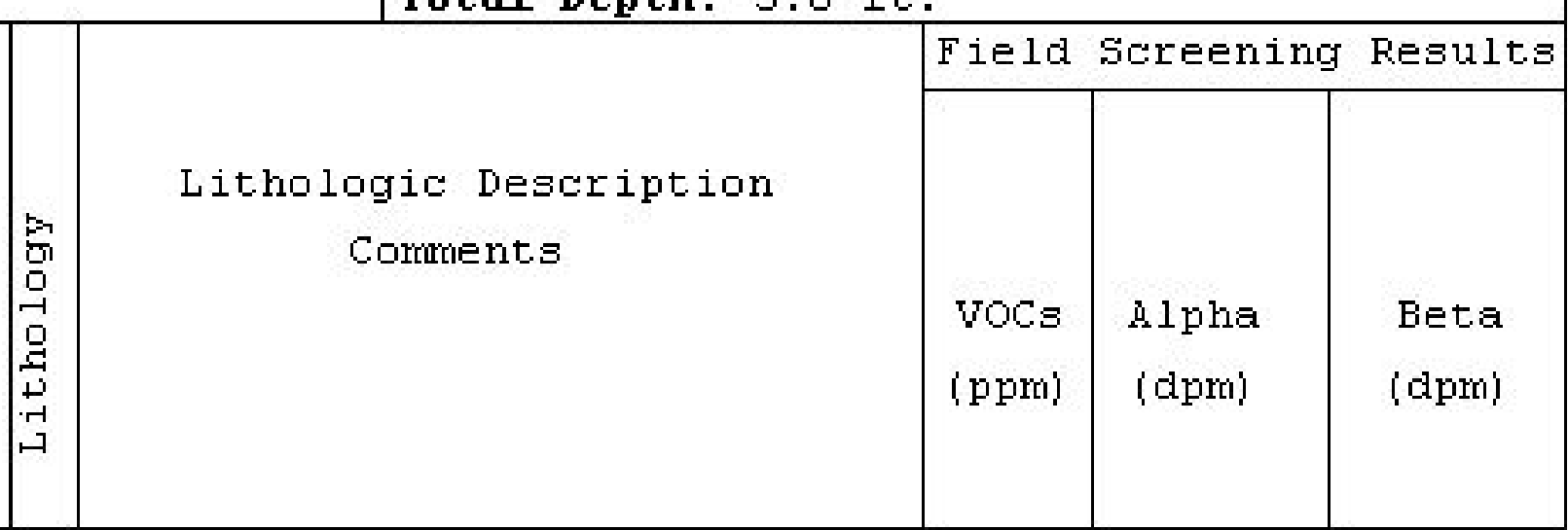

Sample 383DH3500.5.

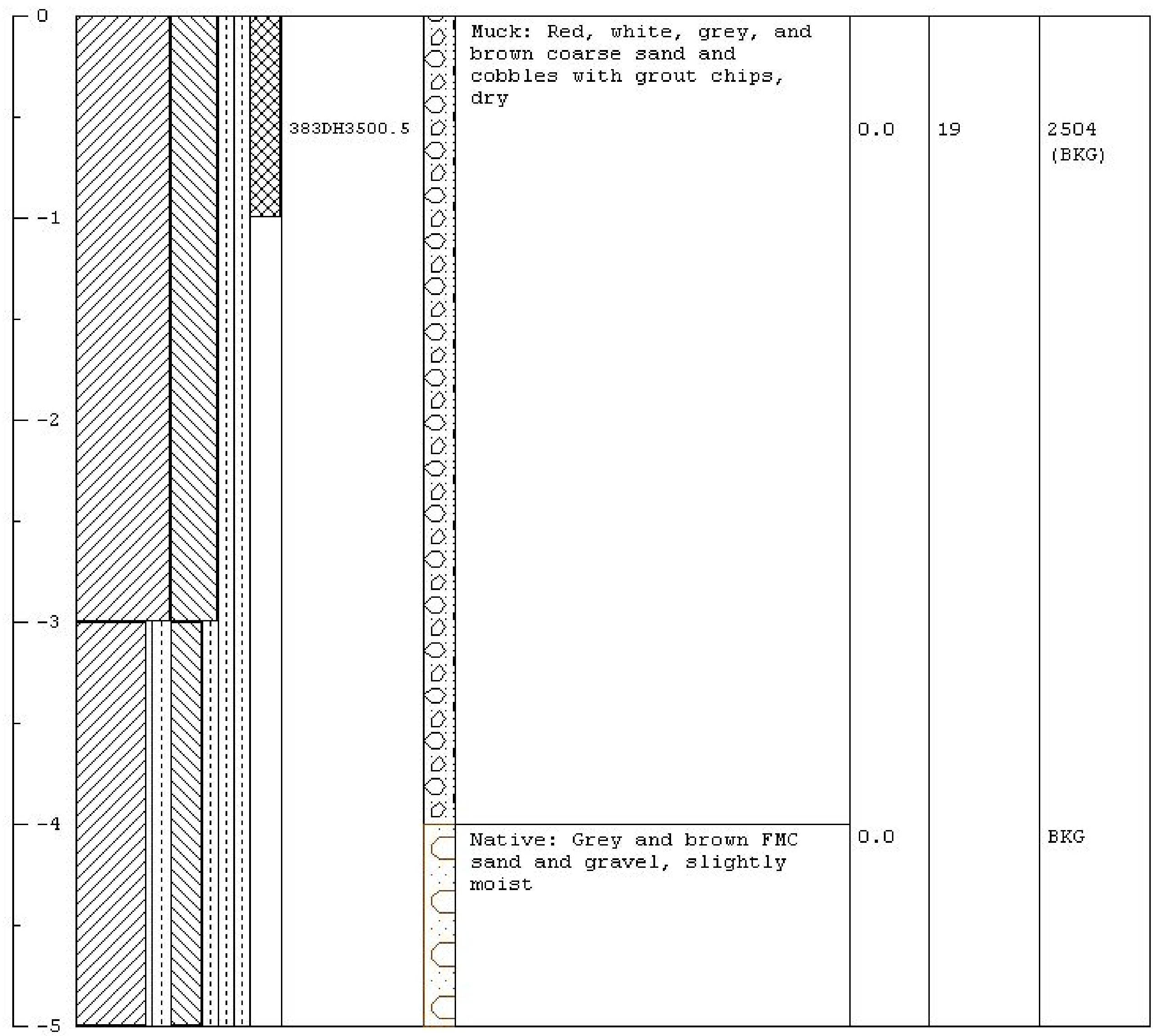


Project Name: CAU 383 E-Tunnel sites

Project Humber: DT04-320

Borehole Number: EMBO1

Logged By: $M$. McLane

Drilled By: Boart-Longyear

Drilling Method: Rotosonic
Date Started: $6 / 12 / 04$

Date completed: $6 / 12 / 04$

Elevation: 6123.5

Horthing : 887326.5

Easting: 638886.7

Total Depth: 12.0 ft.

\begin{tabular}{|c|c|c|c|c|}
\hline \multirow[b]{2}{*}{ 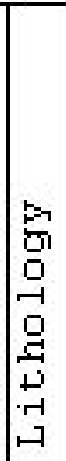 } & \multirow[b]{2}{*}{$\begin{array}{c}\text { Lithologic Description } \\
\text { Comments }\end{array}$} & \multicolumn{3}{|c|}{ Field Screening Results } \\
\hline & & $\begin{array}{l}\text { VOCs } \\
\text { (ppm) }\end{array}$ & $\begin{array}{l}\text { Alpha } \\
\text { (dpri) }\end{array}$ & $\begin{array}{l}\text { Beta } \\
\text { (dprn) }\end{array}$ \\
\hline
\end{tabular}

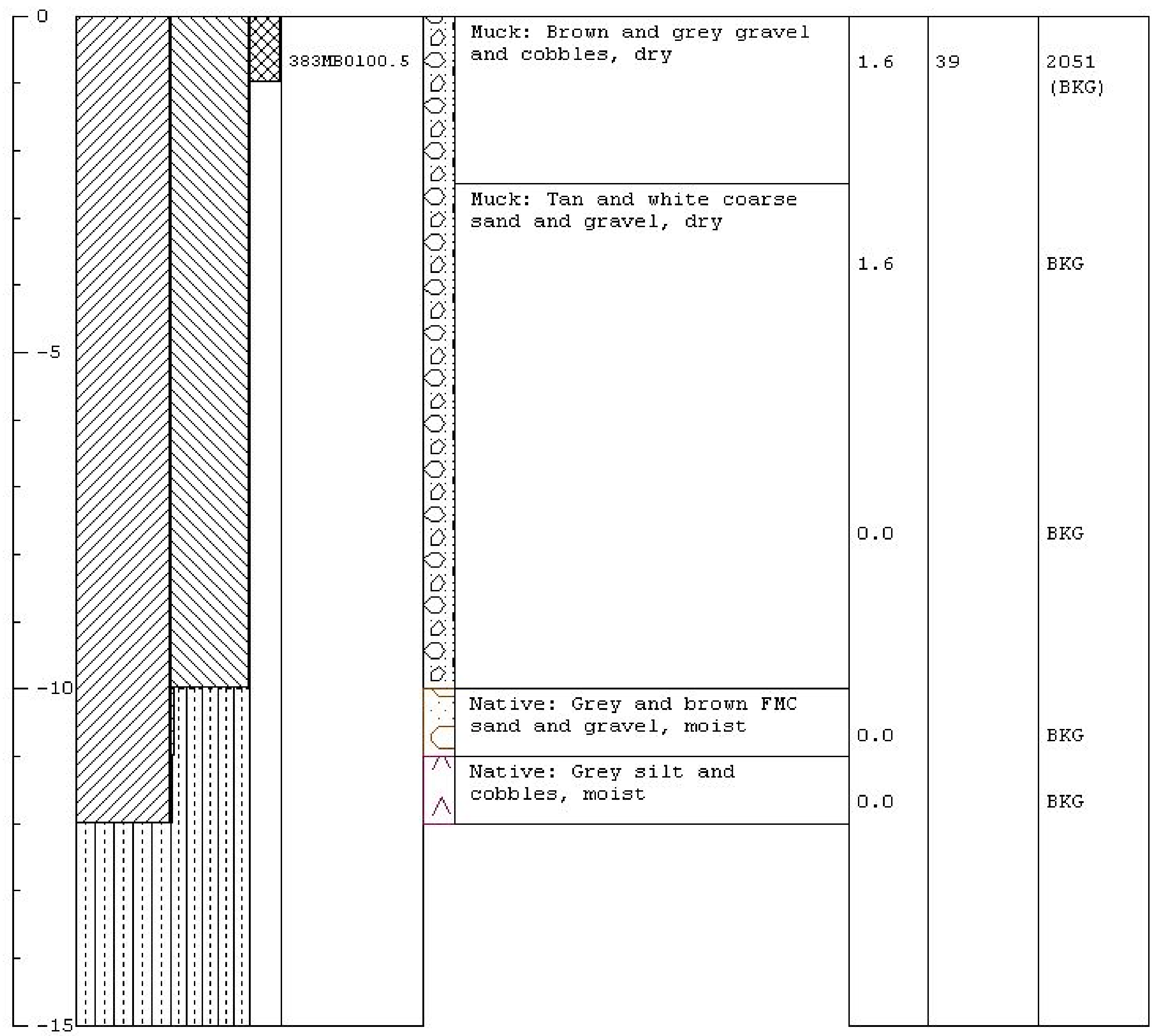


Project Name: CAU 383 E-Tunnel sites

Project Humber: DT04-320

Borehole Number: EMBO2

Logged By: $M$. McLane

Drilled By: Boart-Longyear

Drilling Method: Rotosonic

\begin{tabular}{|c|c|c|c|c|c|}
\hline$\stackrel{\stackrel{s}{D}}{\stackrel{5}{口}}$ & 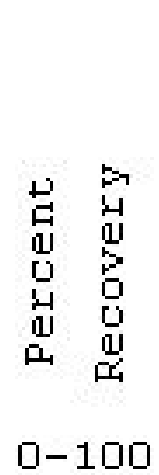 & 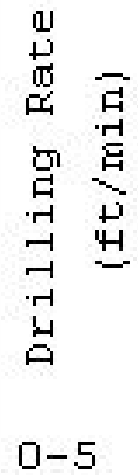 & 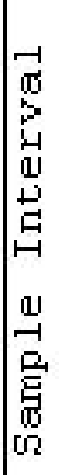 & 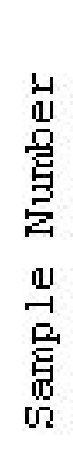 & 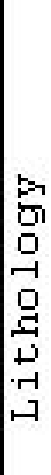 \\
\hline
\end{tabular}

Date Started: $5 / 24 / 04$

Date completed: $5 / 24 / 04$

Elevation: 6136.0

Horthing : 887371.8

Easting: 638013.7

Total Depth: 3.5 ft.

Lithologic Description

Corments

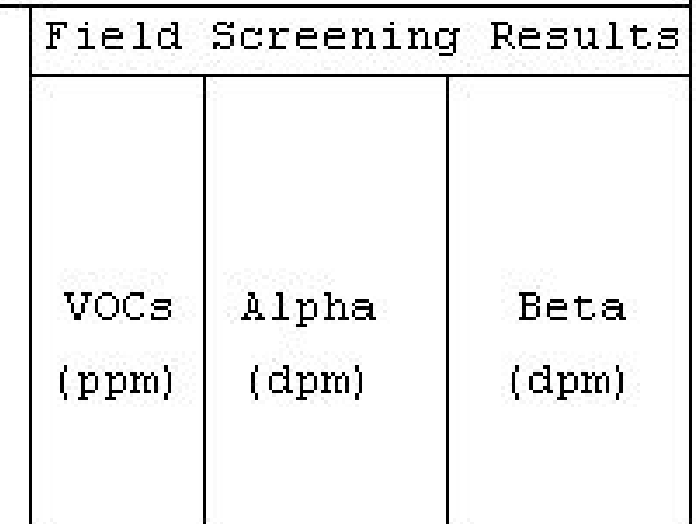

Contact $1.5 \mathrm{ft}$, Sample $383 \mathrm{MB0} 200.5$.
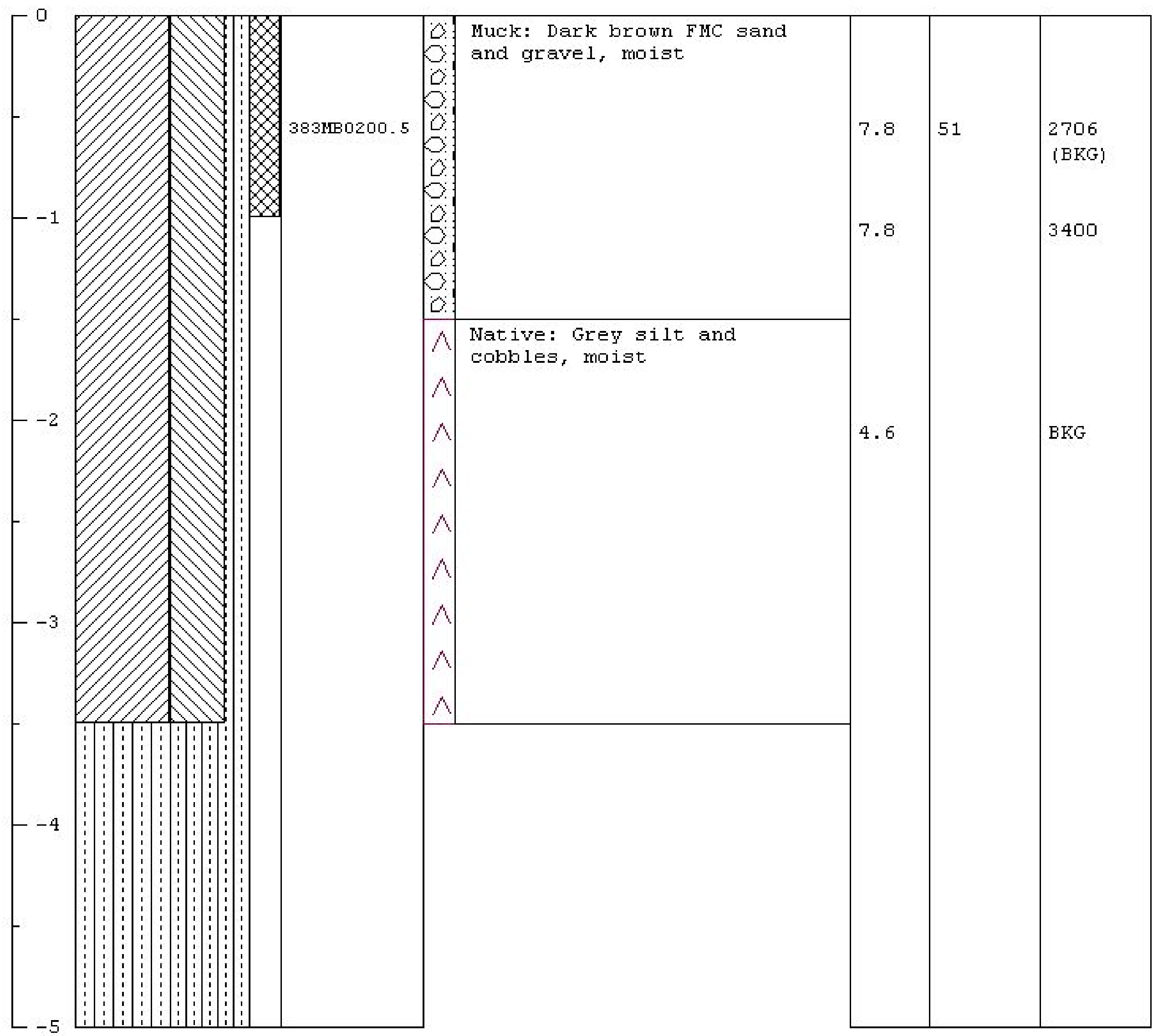
Project Hame: CAU 383 E-Tunnel sites

Project Number: DT04-320

Borehole Number: EMBO3

Logged By: M. MeLane

Drilled By: Boart-Longyear

Drilling Method: Rotosonic
Date Started: $6 / 11 / 04$

Date Completed: $6 / 11 / 04$

Elevation: 6121.5

Horthing: 887878.1

Easting: 637712.6

Total Depth: 9.5 ft.

Lithologic Description

Comments

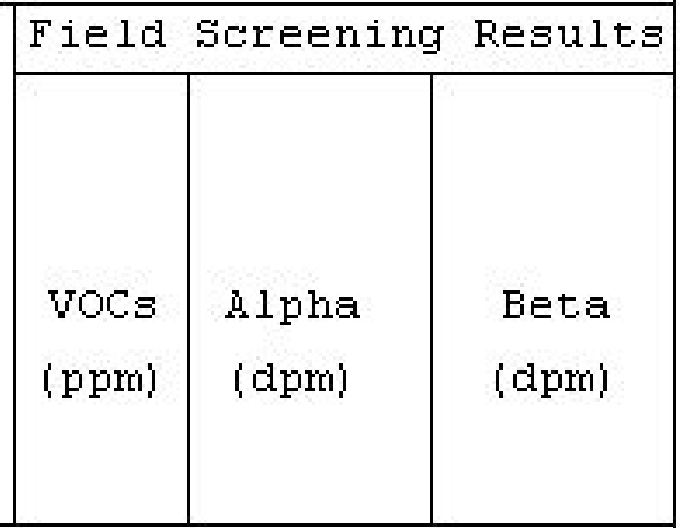

Contact $1.0 \mathrm{ft}$, Samples 383 MB0300.5, 383 MB0305.5.

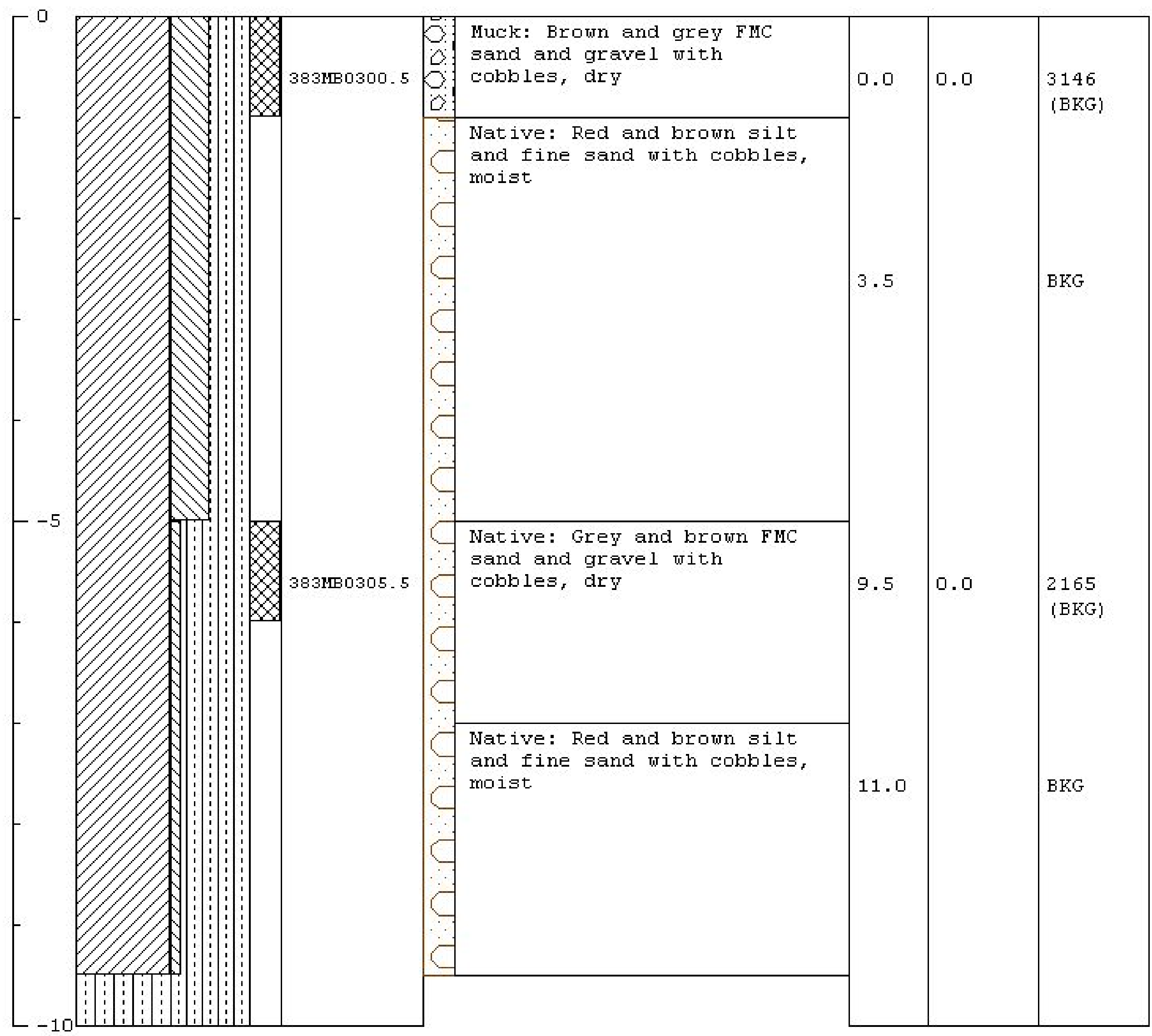


Project Name: CAU 383 E-Tunnel sites

Project Humber: DT04-320

Borehole Number: EMBO4

Logged By: $M$. McLane

Drilled By: M. McLane

Drilling Method: Hand Sampled
Date Started: $6 / 01 / 04$

Date completed: $6 / 01 / 04$

Elevation: 6119.3

Horthing : 887812.1

Easting: 638089.9

Total Depth: 0.5 ft.

Lithologic Description

Corments
Field Screening Results

\begin{tabular}{|c|c|c|}
\hline & & \\
VOCs & s̀lpha & Beta \\
(ppm) & (dpm) & (dpm) \\
& & \\
\hline
\end{tabular}

Contact o N/A, Sample 383 MB0400.5.

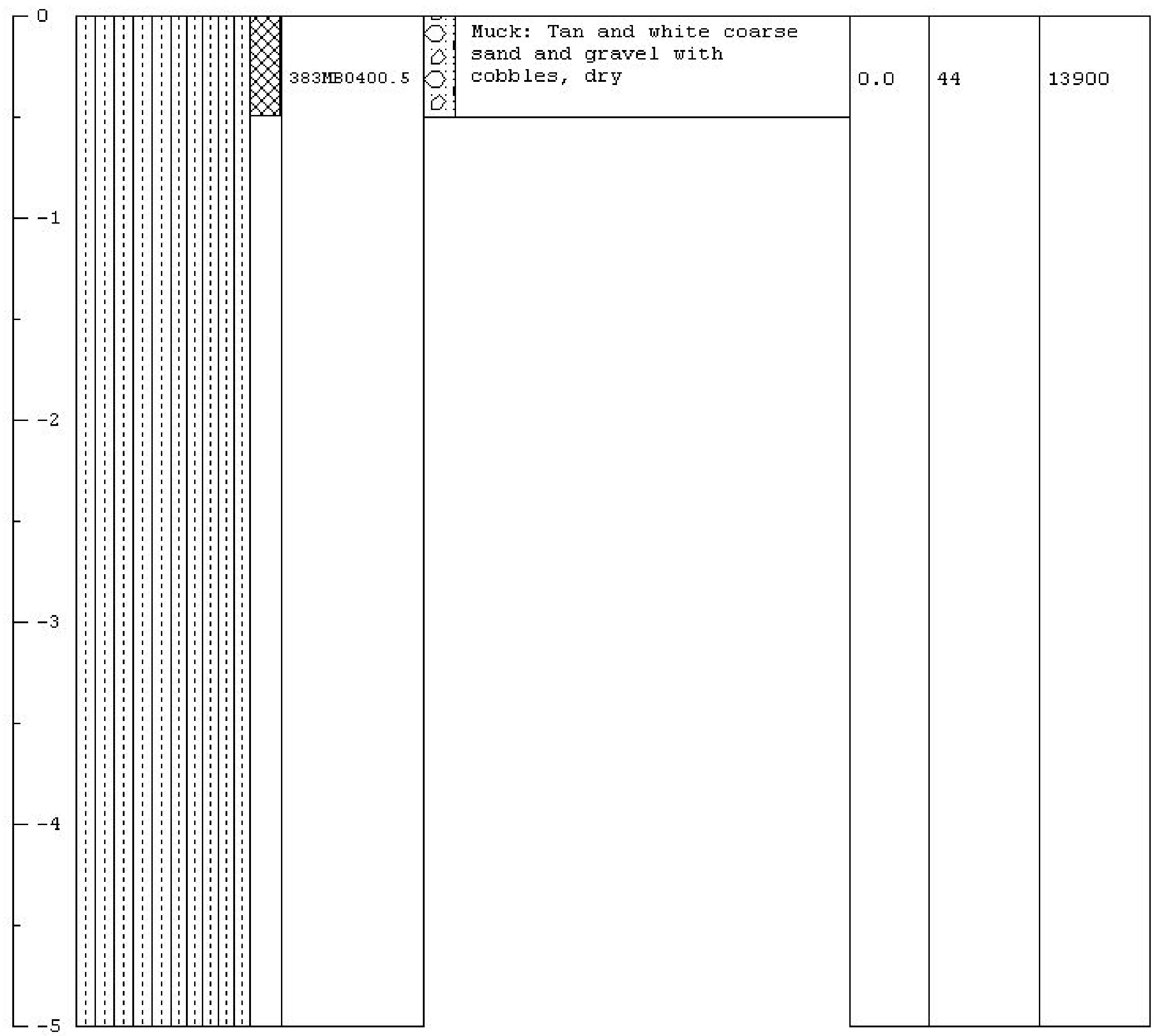


Project Name: CAU 383 E-Tunnel sites

Project Humber: DT04-320

Borehole Number: EMBO5

Logged By: $M$. McLane

Drilled By: Boart-Longyear

Drilling Method: Rotosonic
Date Started: $6 / 02 / 04$

Date completed: $6 / 03 / 04$

Elevation: 6117.8

Horthing: 887505.2

Easting: 638378.0

Total Depth: 86.5 ft.

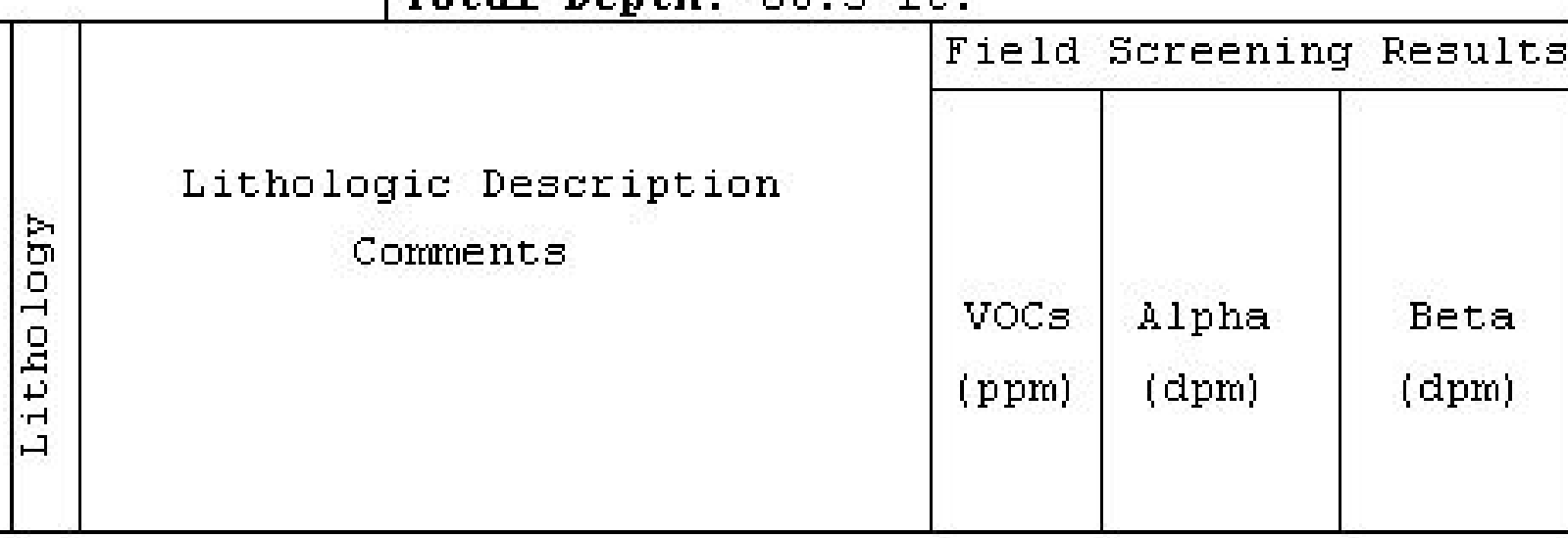

Samples $383 \mathrm{MBO} 500.5,383 \mathrm{MB0} 543.5,383 \mathrm{MB0} 586.0$.

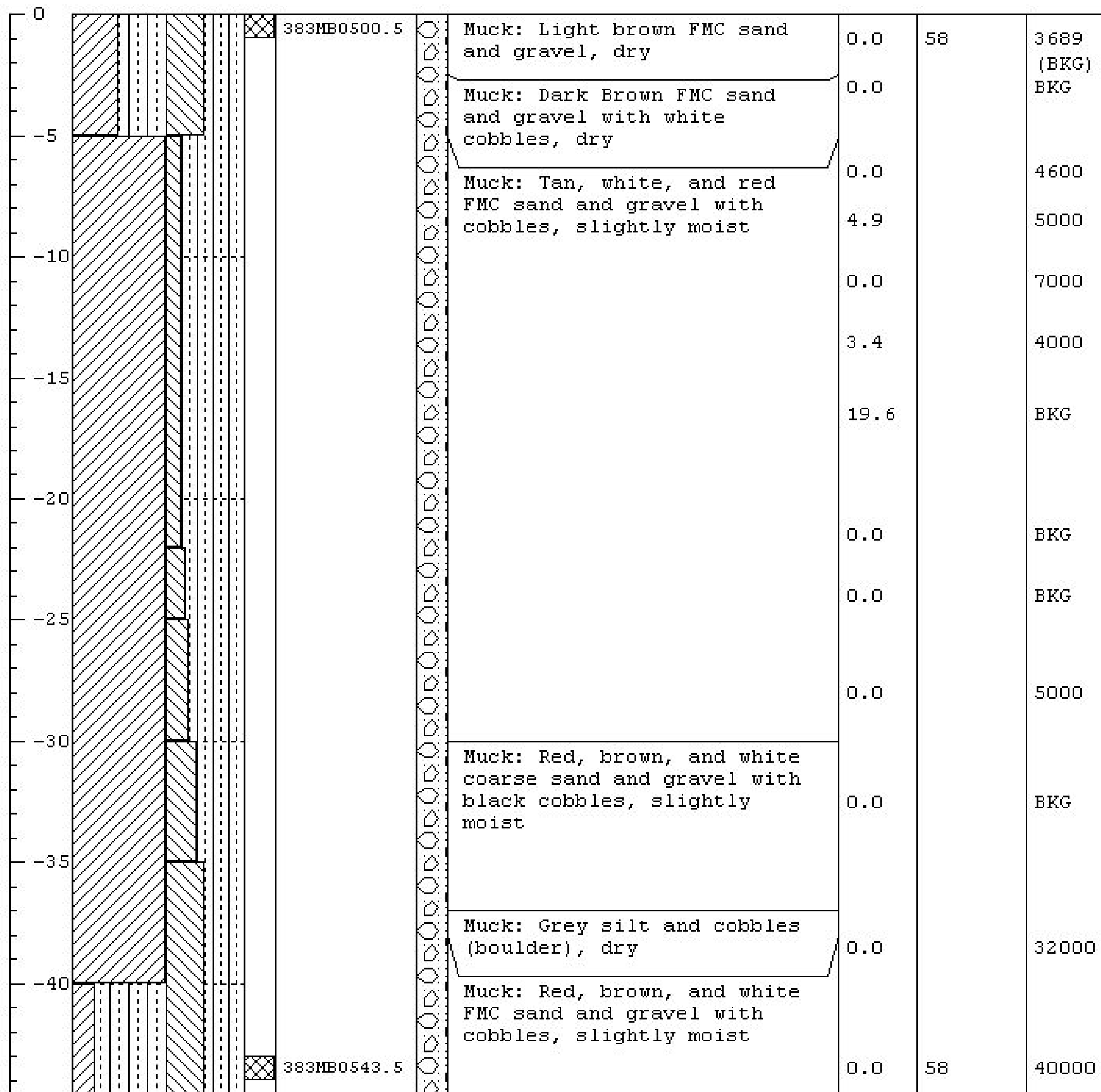


Page 2 of 2

\begin{tabular}{|c|c|c|c|c|c|c|c|c|c|}
\hline \multicolumn{3}{|c|}{ Borehole Number: } & \multicolumn{7}{|c|}{ EMBO5 } \\
\hline \multirow[b]{2}{*}{ 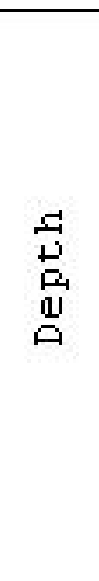 } & \multirow[b]{2}{*}{ 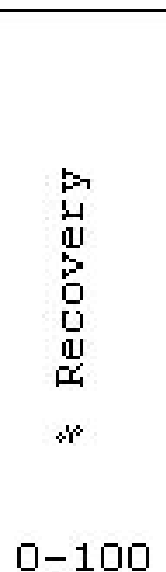 } & \multirow[b]{2}{*}{ 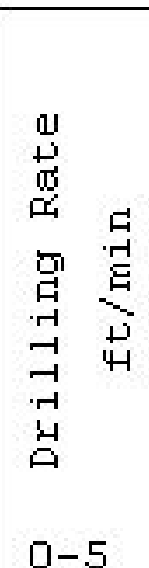 } & \multirow[b]{2}{*}{ 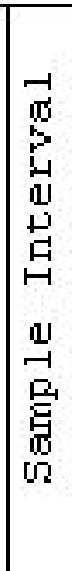 } & \multirow[b]{2}{*}{ 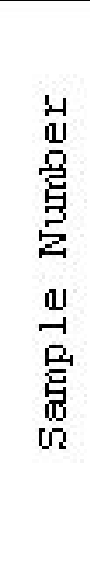 } & \multirow[b]{2}{*}{ 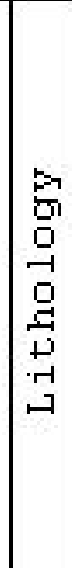 } & \multirow[b]{2}{*}{$\begin{array}{c}\text { Lithologic Description } \\
\text { Comments }\end{array}$} & \multicolumn{3}{|c|}{ Field Screening Results } \\
\hline & & & & & & & $\begin{array}{l}\text { VOCs } \\
\text { (ppro) }\end{array}$ & $\begin{array}{l}\text { Alpha } \\
\text { (dpm) }\end{array}$ & $\begin{array}{l}\text { Beta } \\
\text { (dpm) }\end{array}$ \\
\hline
\end{tabular}
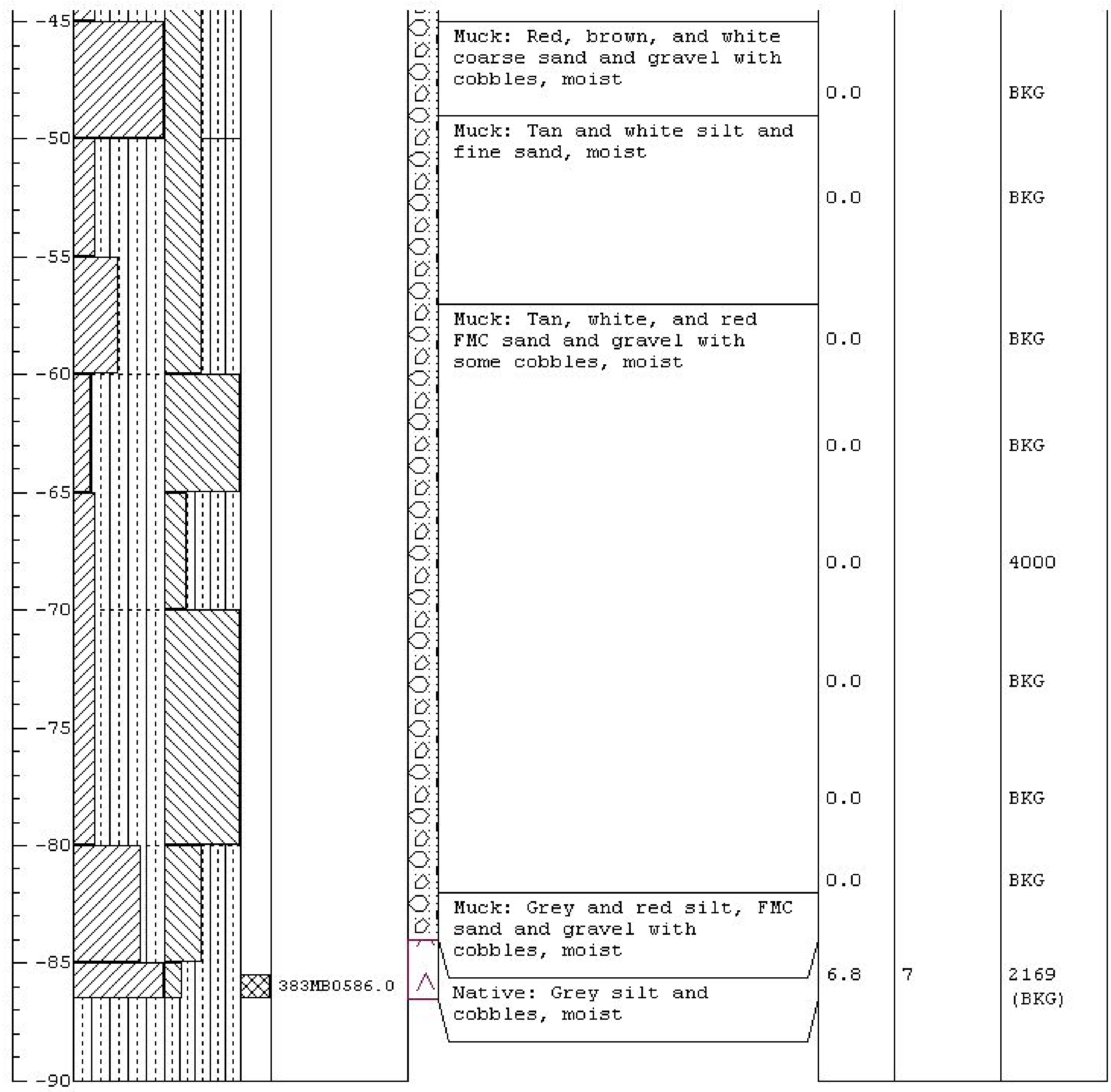
Project Hame: CAU 383 E-Tunnel sites

Project Number: DT04-320

Borehole Number: EMBO6

Logged By: M. MeLane

Drilled By: Boart-Longyear

Drilling Method: Rotosonic
Date Started: $6 / 12 / 04$

Date Completed: $6 / 12 / 04$

Elevation: 6105.0

Horthing: 887386.0

Easting: 638813.1

Total Depth: 7.5 ft.

Lithologic Description

Comments

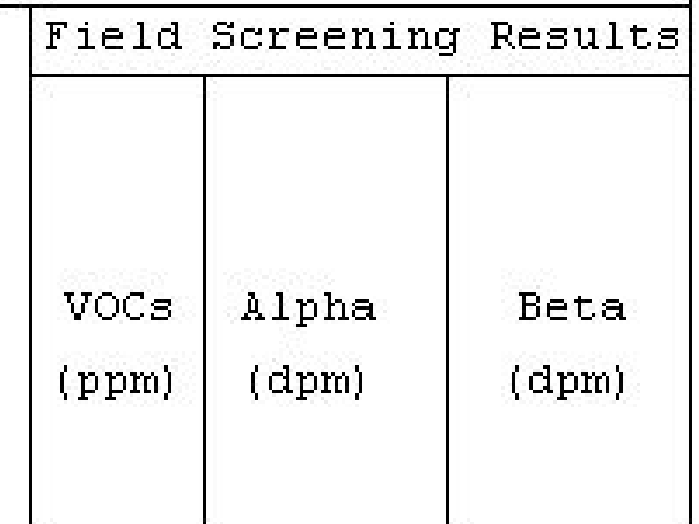

Contact $6.0 \mathrm{ft}$, Samples $383 \mathrm{MB0} 600.5,383 \mathrm{MB0} 600.5 \mathrm{x}$.

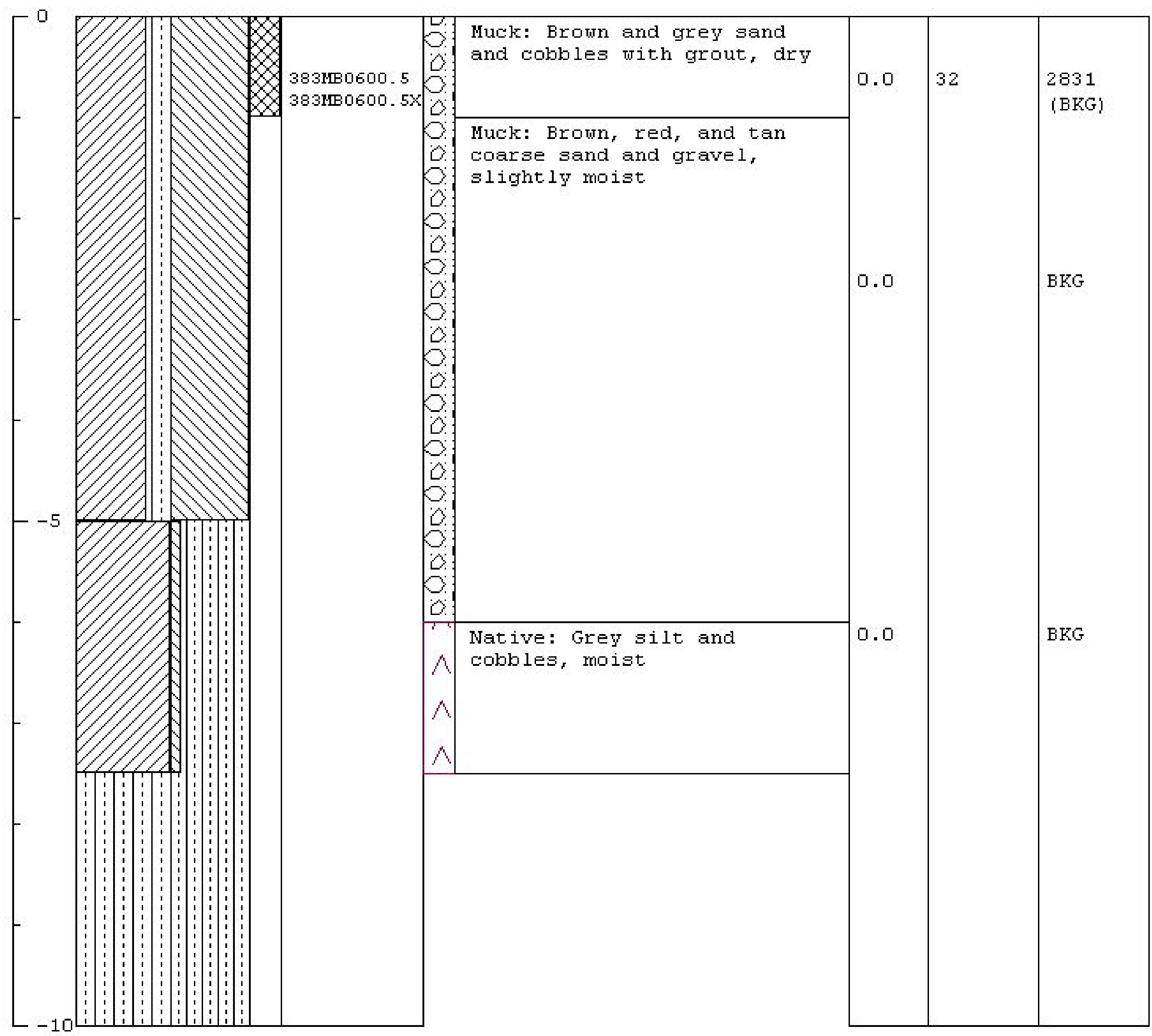


Project Hame: CAU 383 E-Tunnel sites

Project Number: DT04-320

Borehole Number: EMBO7

Logged By: M. MeLane

Drilled By: Boart-Longyear

Drilling Method: Rotosonic

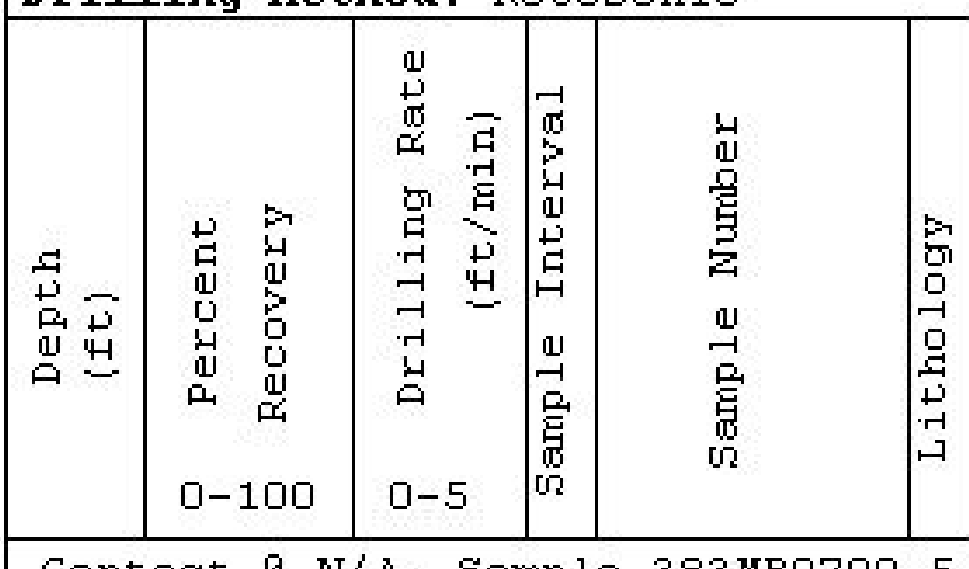

Date Started: $5 / 19 / 04$

Date Completed: $5 / 19 / 04$

Elevation: 6123.1

Horthing: 888056.7

Easting: 637580.3

Total Depth: 5.0 ft.

Lithologic Description

Comments

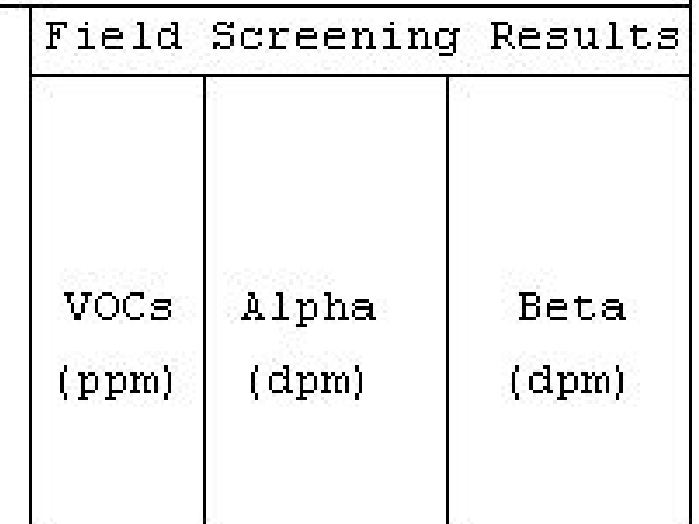

Contact $1 \mathrm{~N} / \mathrm{A}$, Sample $383 \mathrm{MB0} 00.5$.

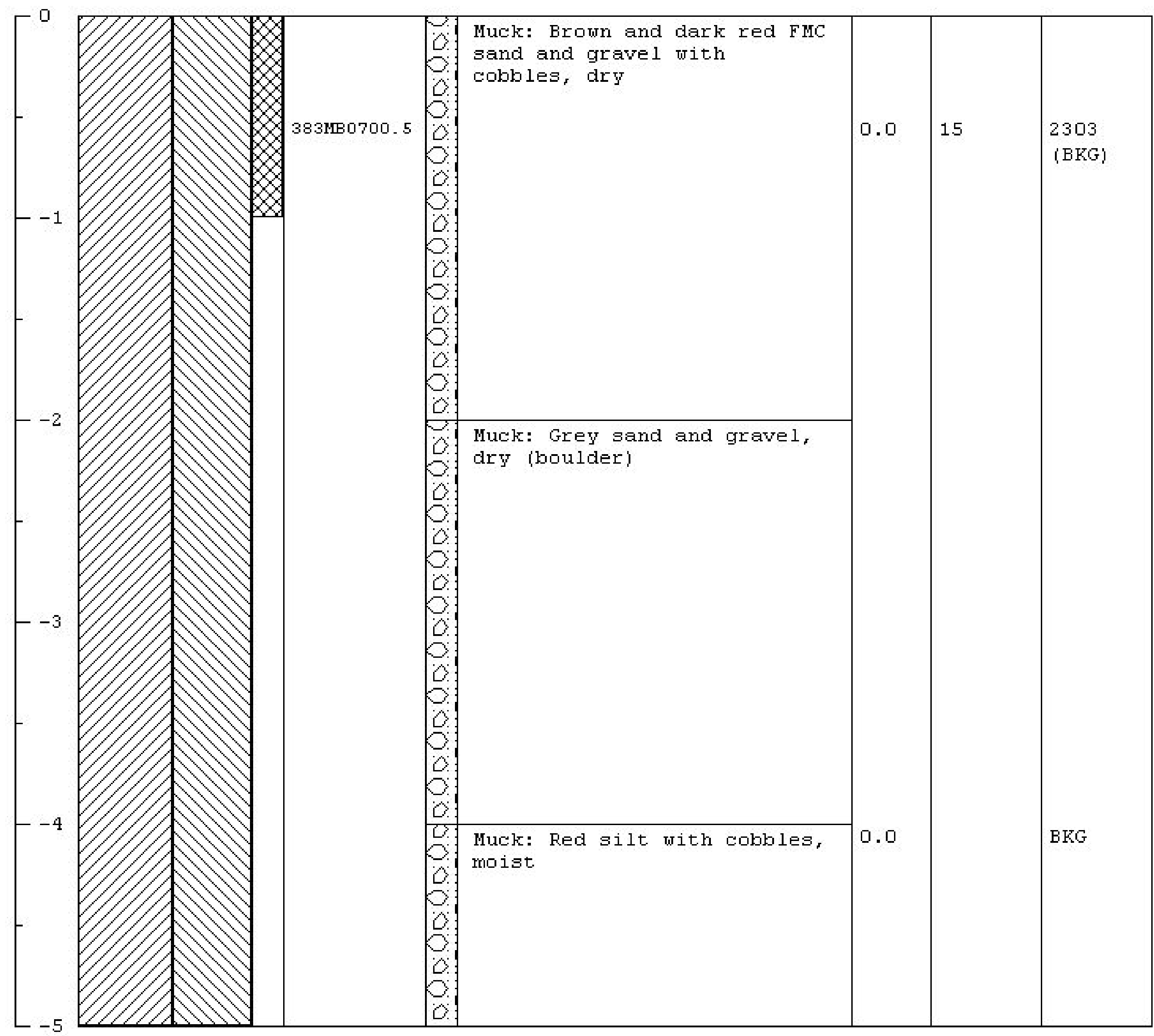


Project Hame: CAU 383 E-Tunnel sites

Project Number: DT04-320

Borehole Number: EMBO8

Logged By: M. MeLane

Drilled By: Boart-Longyear

Drilling Method: Rotosonic
Date Started: $5 / 24 / 04$

Date Completed: $5 / 24 / 04$

Elevation: 6132.5

Horthing: 887404.7

Easting: 638115.3

Total Depth: $31.5 \mathrm{ft}$.

\begin{tabular}{|c|c|c|c|c|}
\hline & & Field & Screen & Results \\
\hline $\begin{array}{l}8 \\
0 \\
9 \\
9 \\
\end{array}$ & $\begin{array}{c}\text { Lithologic Description } \\
\text { Comments }\end{array}$ & vOCs & $\begin{array}{l}\text { Alpha } \\
\text { (dpm) }\end{array}$ & $\begin{array}{l}\text { Beta } \\
\text { (dpm) }\end{array}$ \\
\hline
\end{tabular}

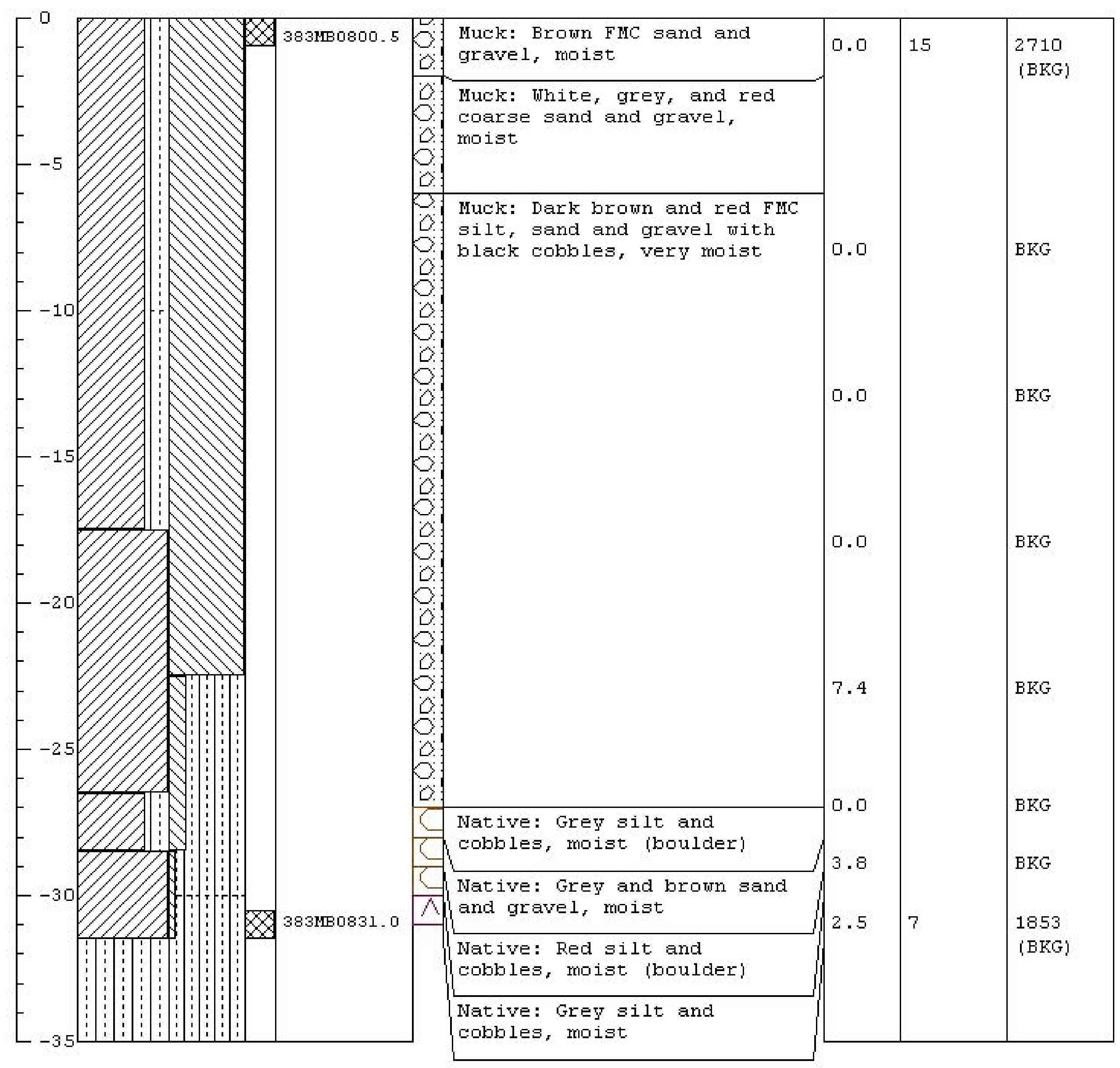


Project Hame: CAU 383 E-Tunnel sites

Project Number: DT04-320

Borehole Number: EMBO9

Logged By: M. MeLane

Drilled By: Boart-Longyear

Drilling Method: Rotosonic
Date Started: $6 / 12 / 04$

Date Completed: $6 / 12 / 04$

Elevation: 6122.3

Horthing: 887322.3

Easting: 638941.3

Total Depth: $14.0 \mathrm{ft}$.

\begin{tabular}{|c|c|c|c|c|}
\hline & & Field & Screen? & Results \\
\hline 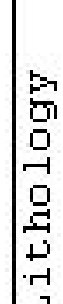 & $\begin{array}{c}\text { Lithologic Description } \\
\text { Comments }\end{array}$ & $\begin{array}{l}\text { voCs } \\
\text { (ppm) }\end{array}$ & $\begin{array}{l}\text { Alpha } \\
\text { (dpm) }\end{array}$ & $\begin{array}{l}\text { Beta } \\
\text { (dpm) }\end{array}$ \\
\hline
\end{tabular}

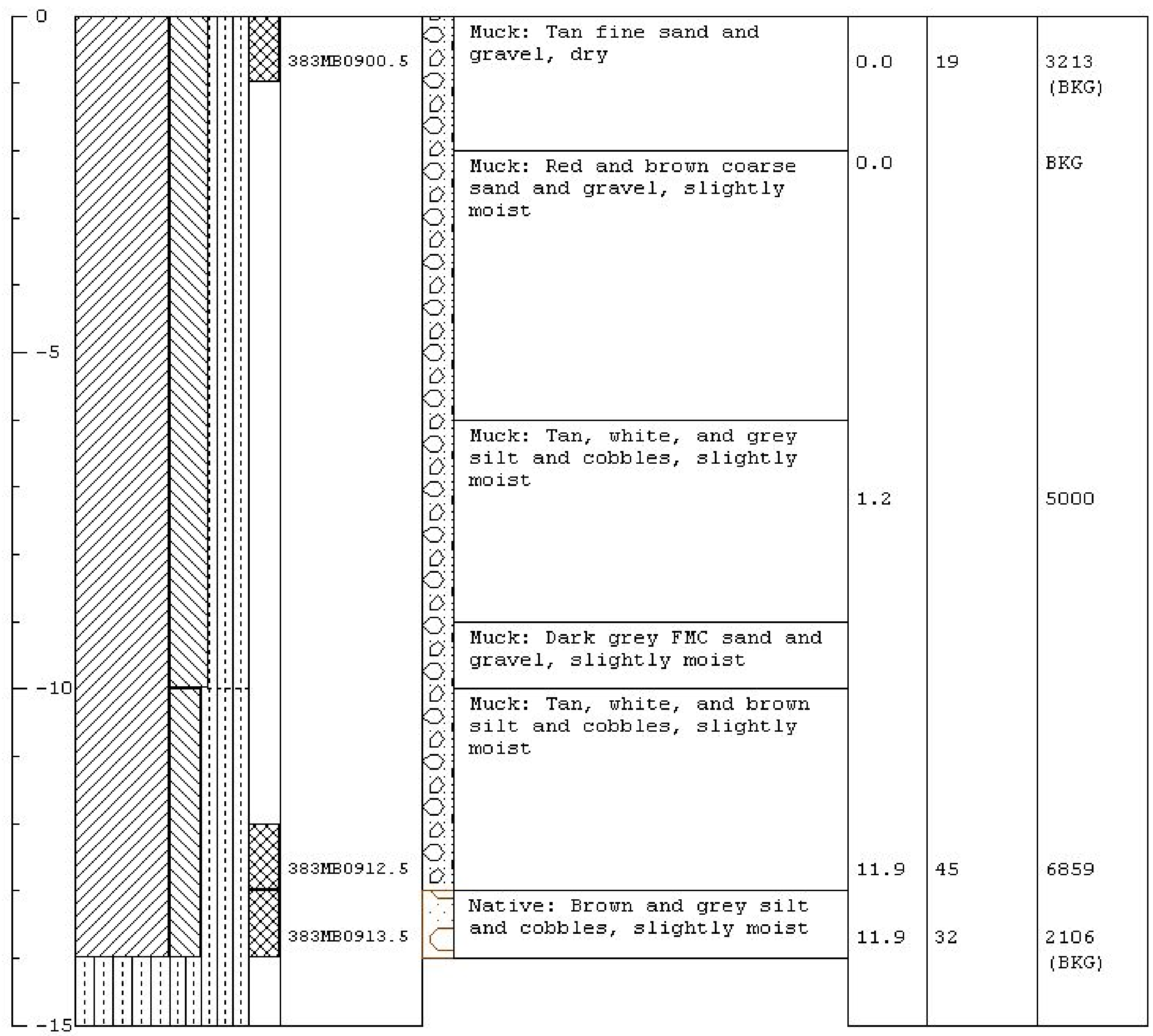


Project Hame: CAU 383 E-Tunnel sites

Project Number: DT04-320

Borehole Number: EMB10

Logged By: M. MeLane

Drilled By: Boart-Longyear

Drilling Method: Rotosonic
Date Started: $6 / 12 / 04$

Date Completed: $6 / 12 / 04$

Elevation: 6102.2

Horthing: 887435.4

Easting: 638844.0

Total Depth: $37.0 \mathrm{ft}$.

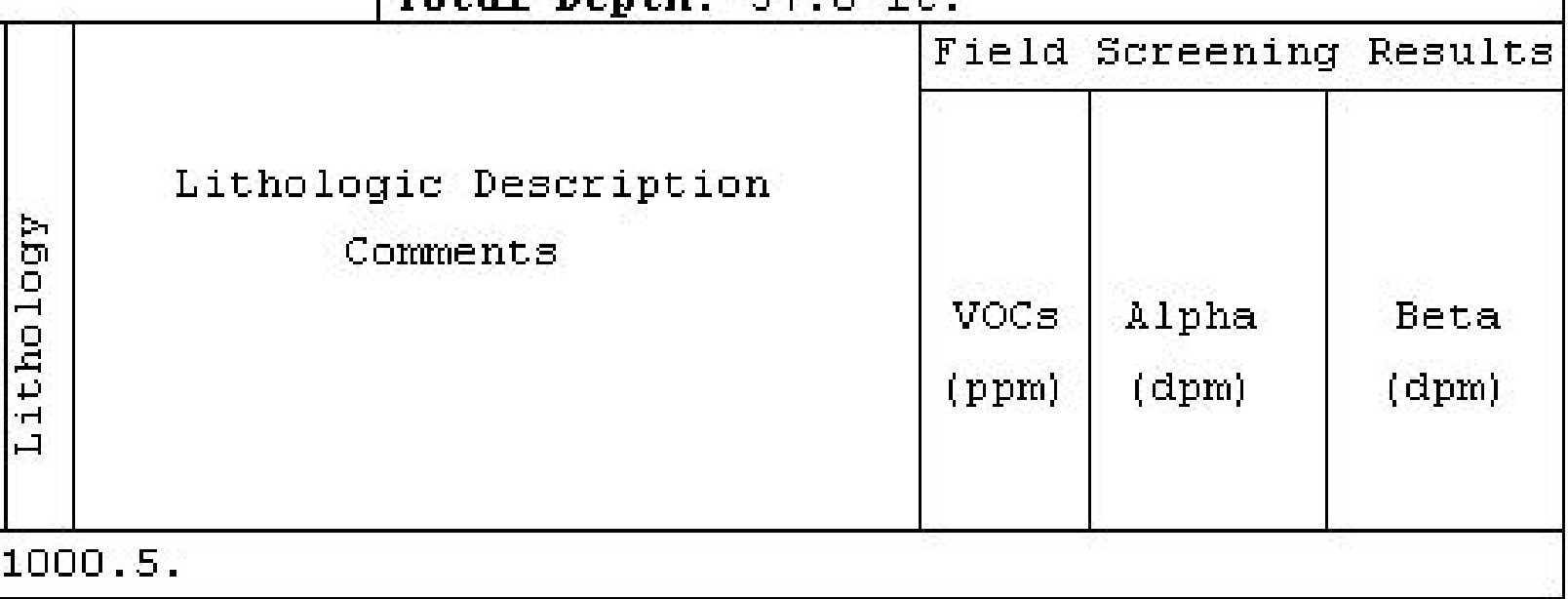

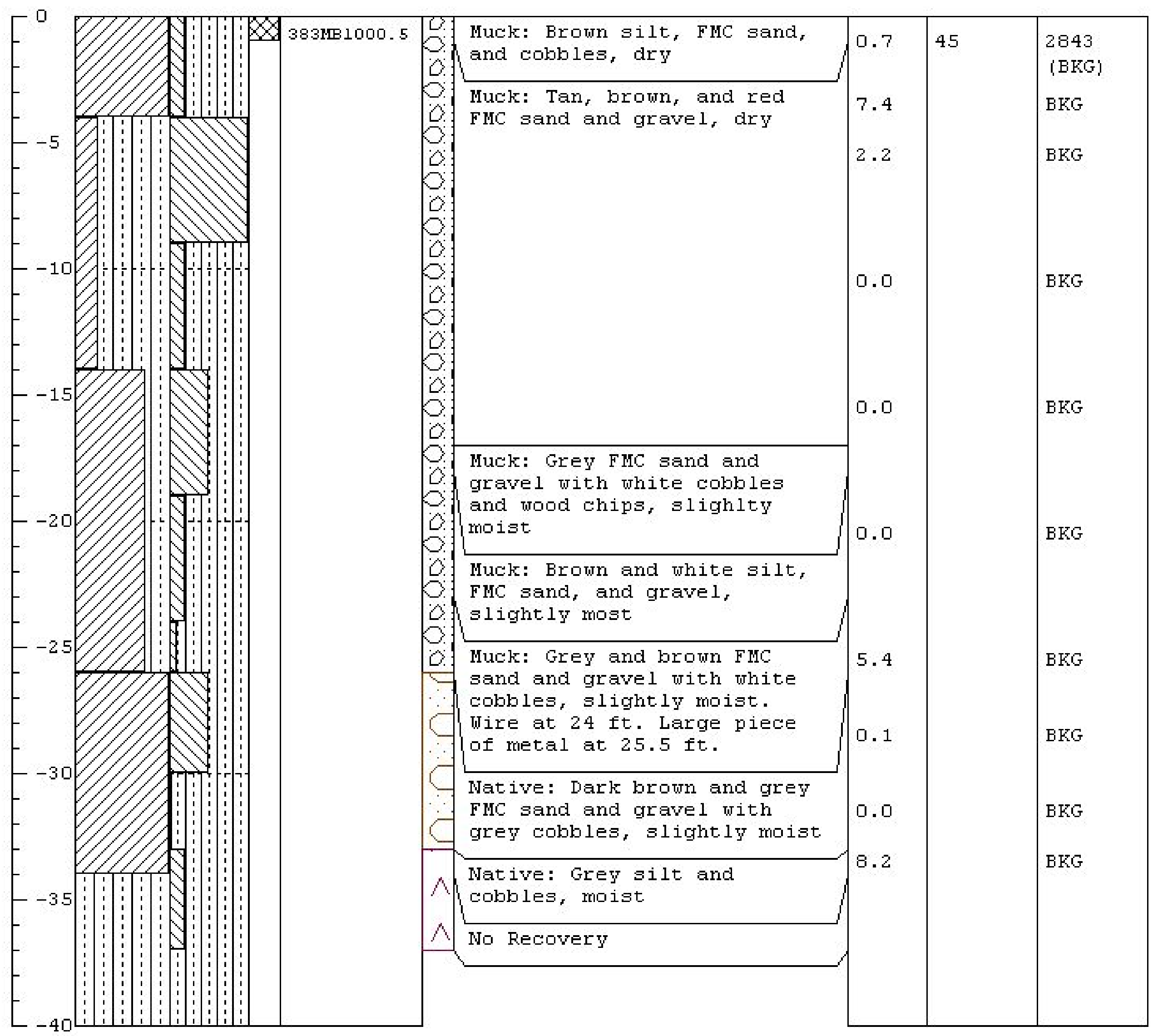


Project Hame: CAU 383 E-Tunnel sites

Project Number: DT04-320

Borehole Number: EMB11

Logged By: M. MeLane

Drilled By: Boart-Longyear

Drilling Method: Rotosonic
Date Started: $6 / 12 / 04$

Date Completed: $6 / 12 / 04$

Elevation: 6107.2

Horthing: 887450.5

Easting: 638781.0

Total Depth: $25.5 \mathrm{ft}$.

\begin{tabular}{|c|c|c|c|c|}
\hline & \multirow[b]{2}{*}{$\begin{array}{c}\text { Lithologic Description } \\
\text { Comments }\end{array}$} & \multicolumn{3}{|c|}{ Field Screening Results } \\
\hline$\frac{91}{9}$ & & $\begin{array}{l}\text { VoCs } \\
\text { (ppm) }\end{array}$ & $\begin{array}{l}\text { Alpha } \\
\text { (dpm) }\end{array}$ & $\begin{array}{l}\text { Beta } \\
\text { (dpm) }\end{array}$ \\
\hline
\end{tabular}

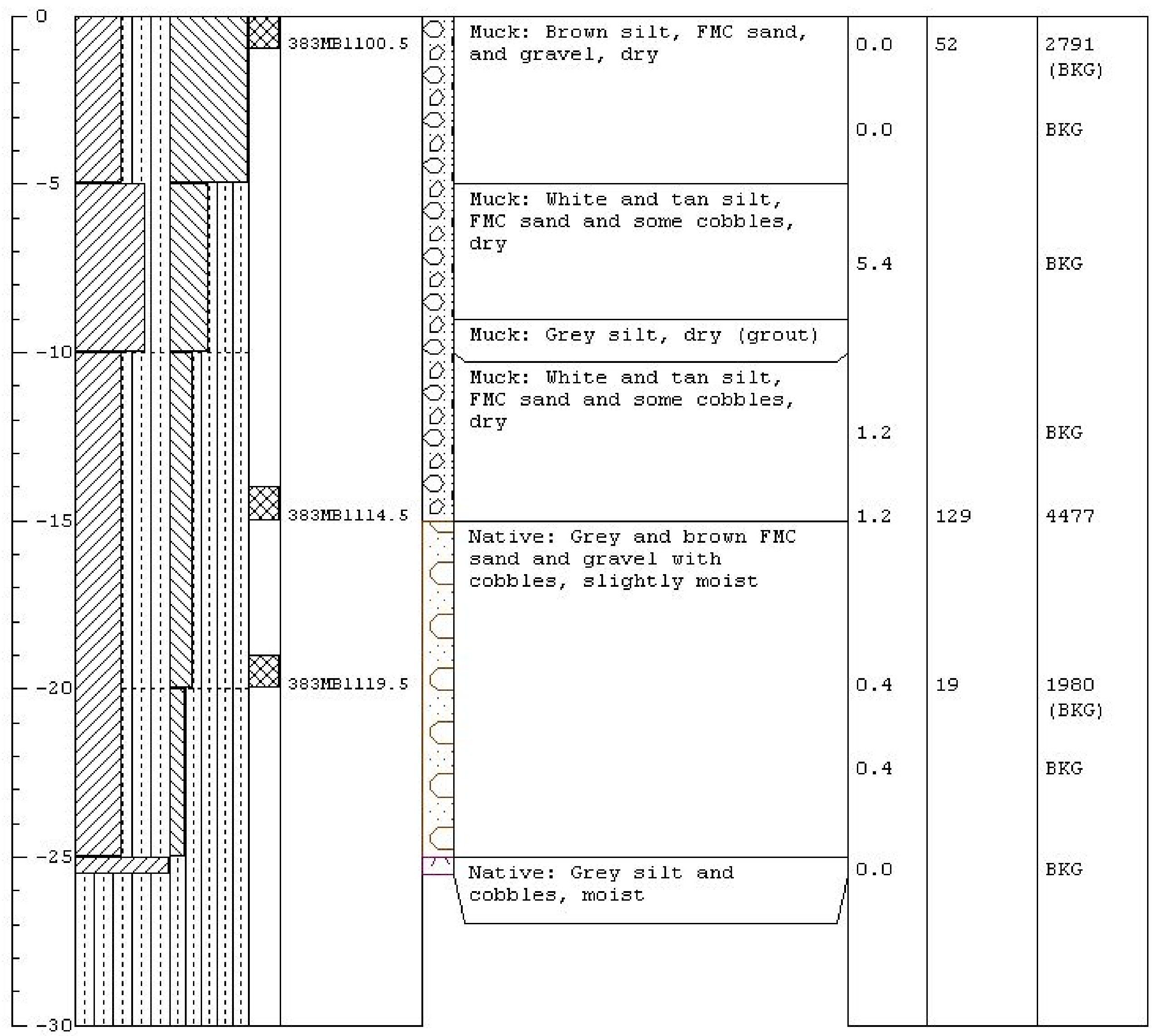


Project Name: CAU 383 E-Tunnel sites

Project Humber: DT04-320

Borehole Number: EMB 12

Logged By: $M$. McLane

Drilled By: Boart-Longyear

Drilling Method: Rotosonic
Date Started: $6 / 13 / 04$

Date Completed: $6 / 13 / 04$

Elevation: 6123.1

Horthing: 887487.9

Easting: 638589.1

Total Depth: 75 ft.

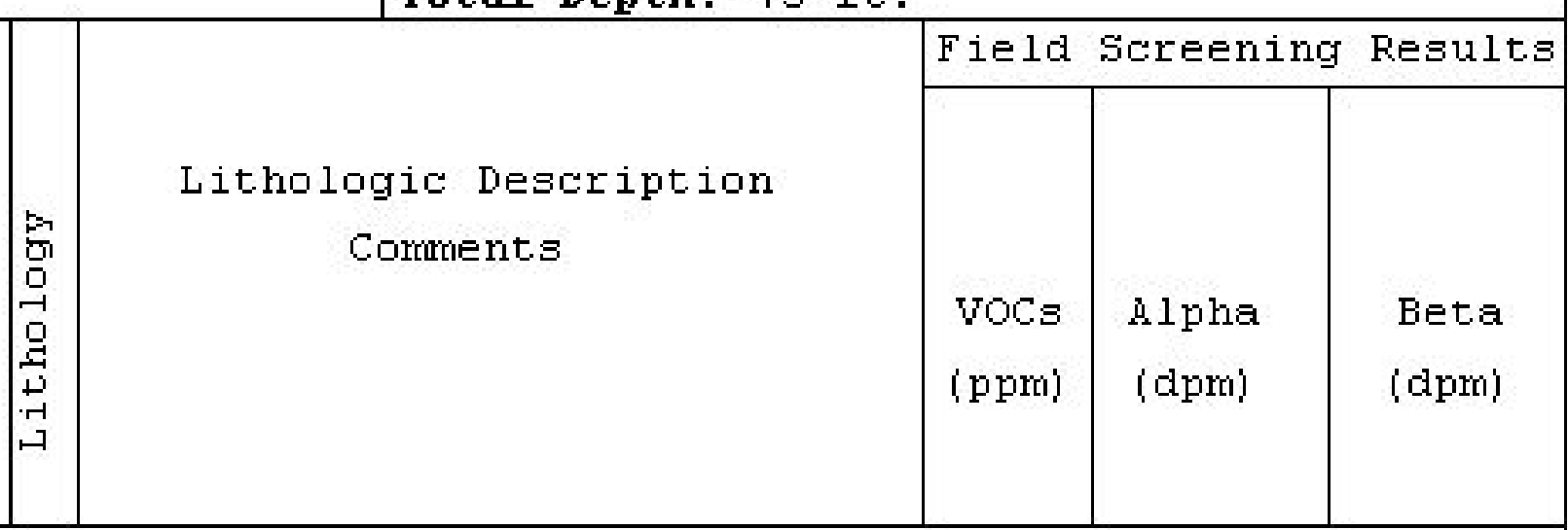

Sample 383 MB1200.5.

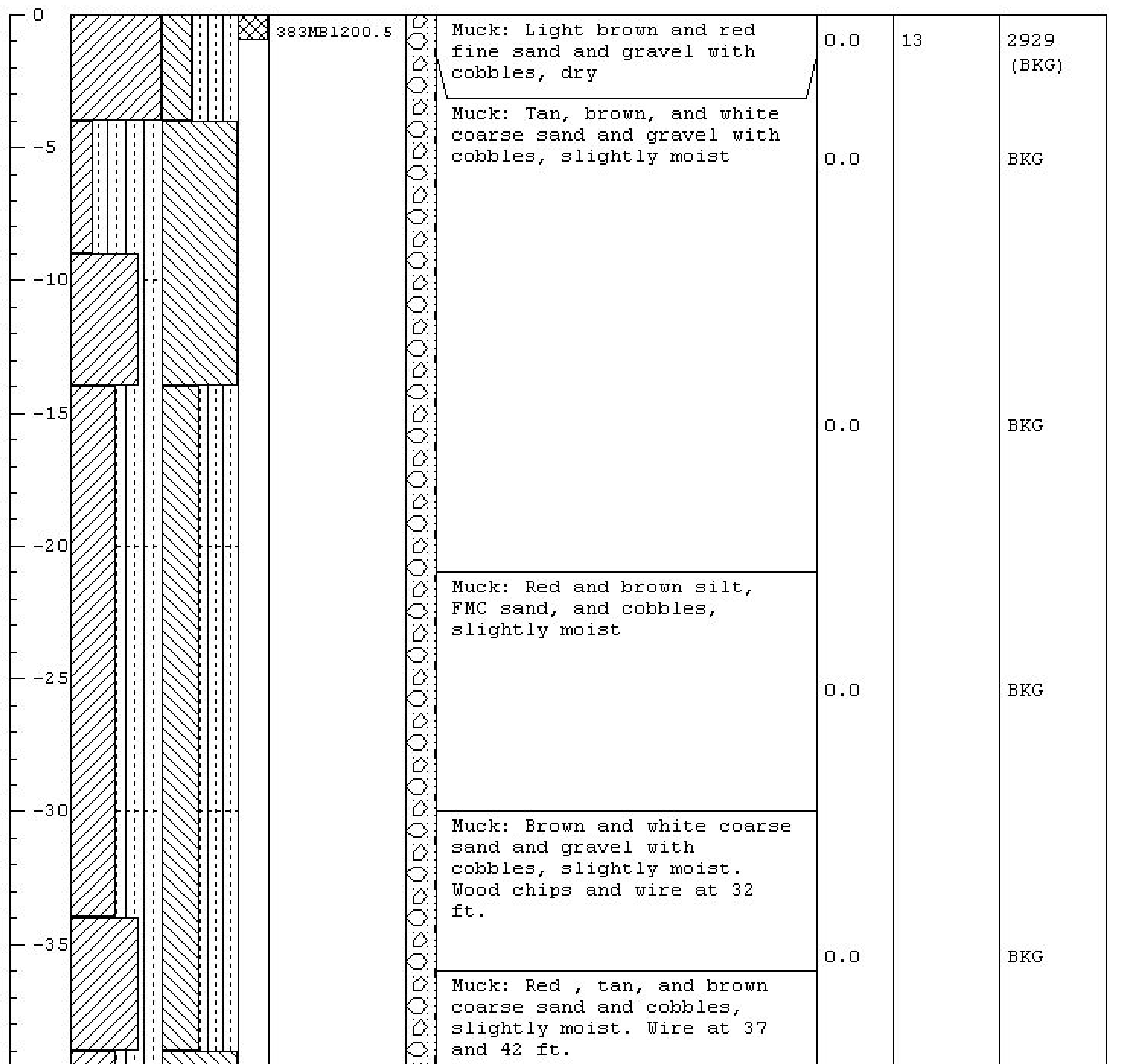


Page 2 of 2

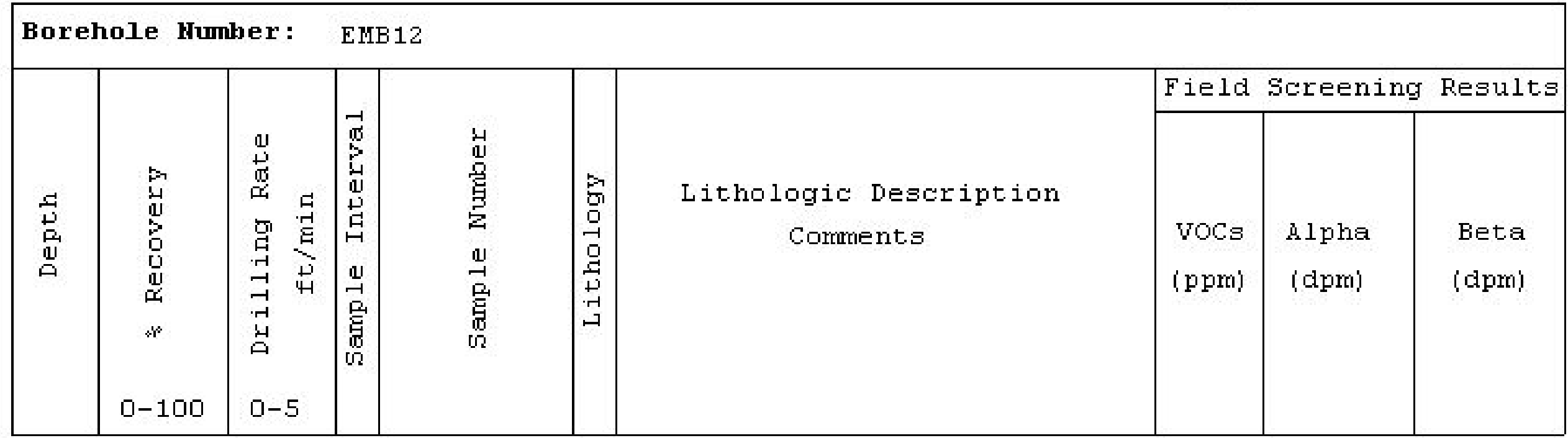

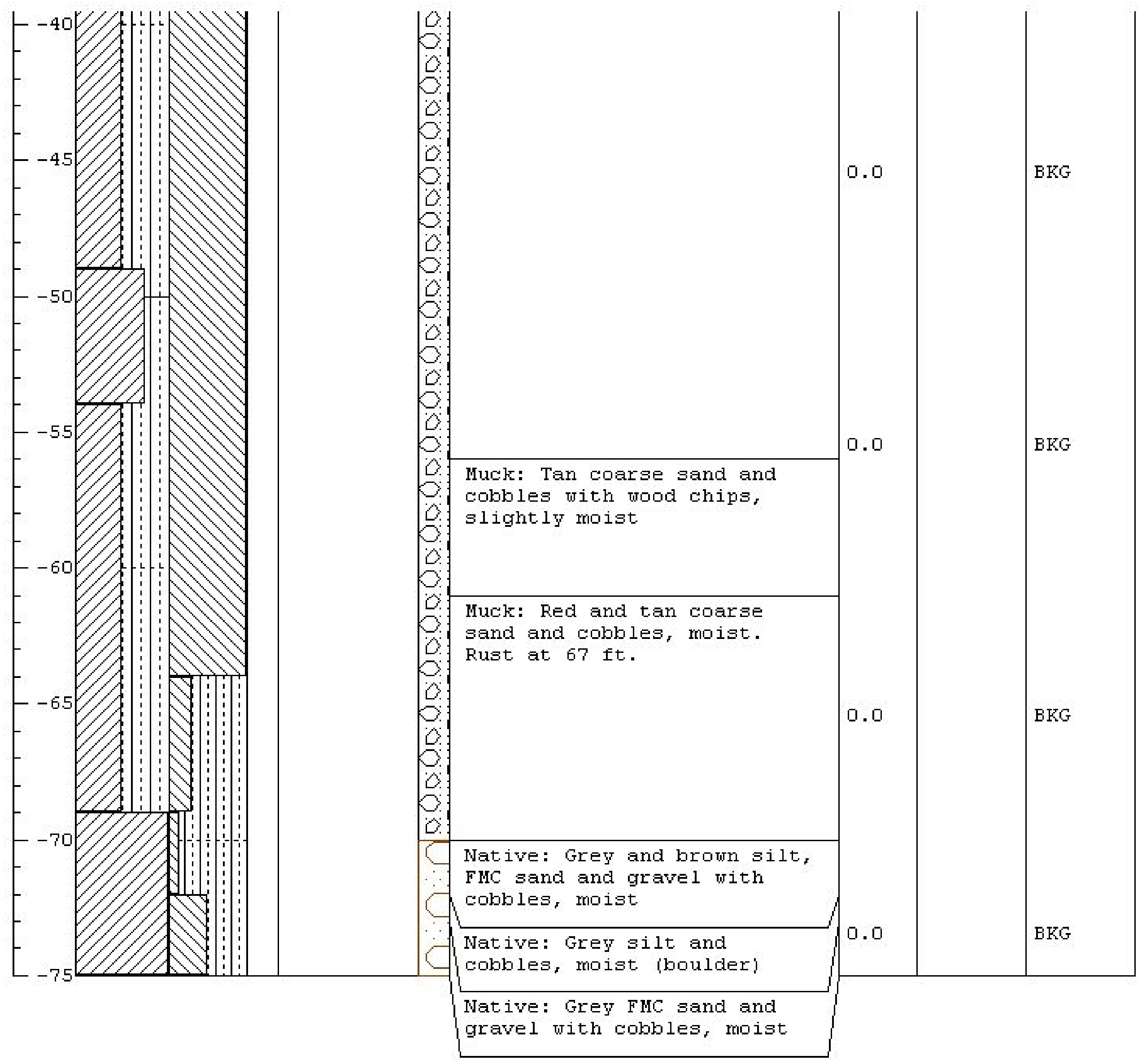


Project Hame: CAU 383 E-Tunnel sites

Project Number: DT04-320

Borehole Number: EMB 13

Logged By: M. MeLane

Drilled By: Boart-Longyear

Drilling Method: Rotosonic
Date Started: $6 / 13 / 04$

Date Completed: $6 / 13 / 04$

Elevation: 6127.8

Horthing: 887564.0

Easting: 638432.6

Total Depth: 91.5 ft.

\begin{tabular}{|c|c|c|c|c|}
\hline & & Field & Screen & Results \\
\hline $\begin{array}{l}8 \\
0 \\
9 \\
9 \\
\end{array}$ & $\begin{array}{c}\text { Lithologic Description } \\
\text { Comments }\end{array}$ & vOCs & $\begin{array}{l}\text { Alpha } \\
\text { (dpm) }\end{array}$ & $\begin{array}{l}\text { Beta } \\
\text { (dpm) }\end{array}$ \\
\hline
\end{tabular}

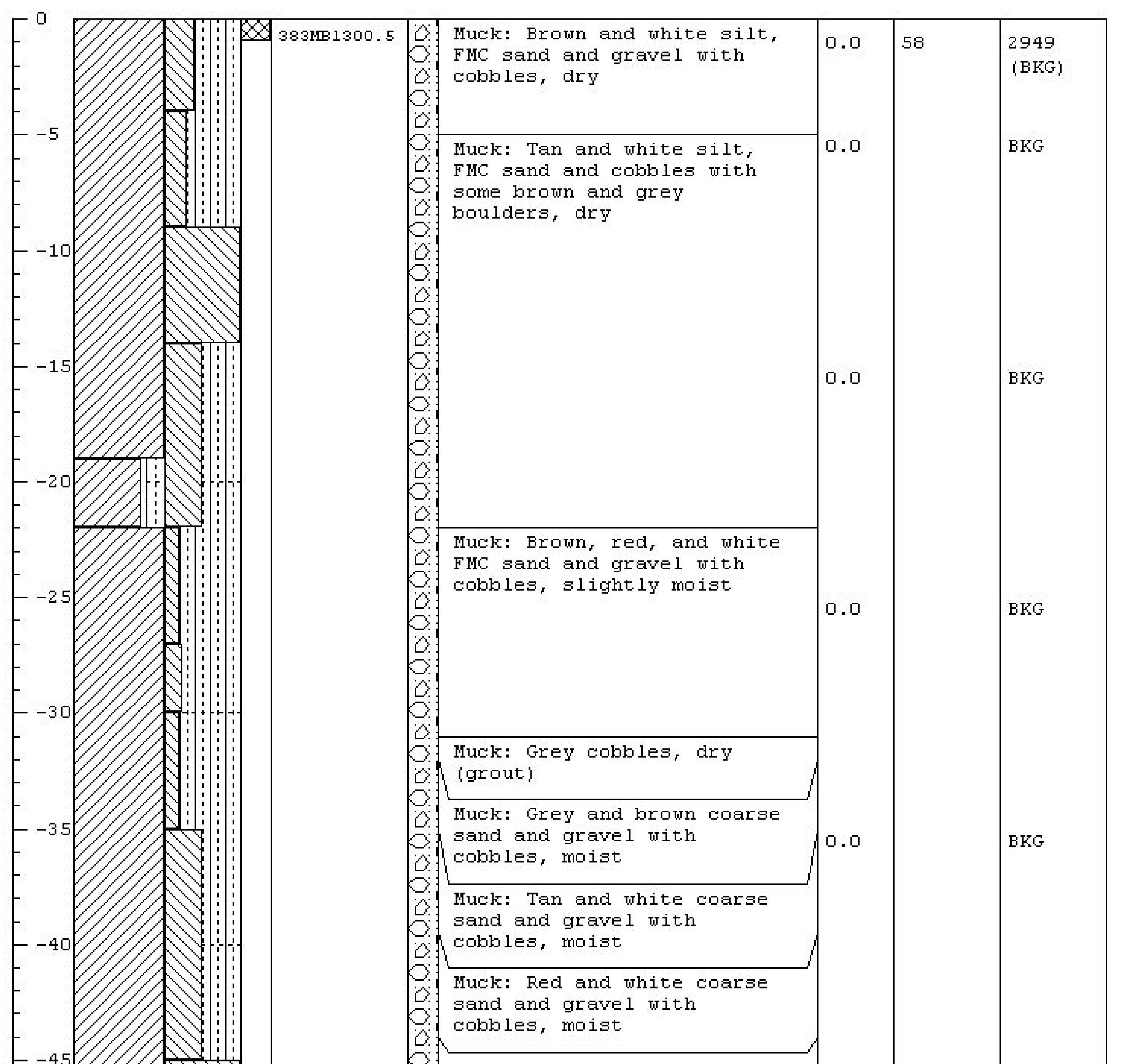


Page 2 of 2

\begin{tabular}{|c|c|c|c|c|c|c|c|c|c|c|}
\hline \multicolumn{3}{|c|}{ Borehole Number: } & \multicolumn{8}{|c|}{ EMB 13} \\
\hline \multirow[b]{2}{*}{$\begin{array}{l}\stackrel{s}{L} \\
\stackrel{5}{\circ} \\
\stackrel{1}{口}\end{array}$} & \multirow[b]{2}{*}{ 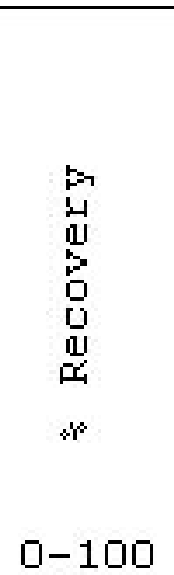 } & \multirow[b]{2}{*}{ 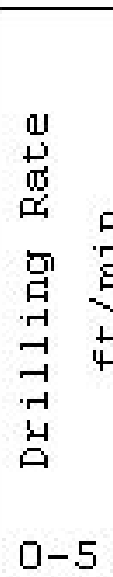 } & \multirow{2}{*}{\multicolumn{2}{|c|}{ 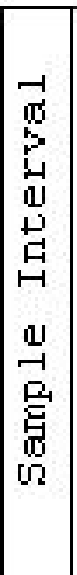 }} & \multirow[b]{2}{*}{ 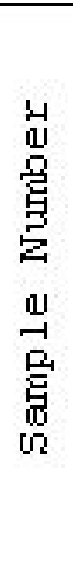 } & \multirow[b]{2}{*}{ 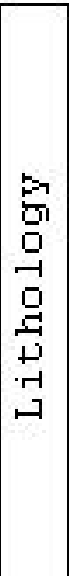 } & \multirow[b]{2}{*}{$\begin{array}{c}\text { Lithologic Description } \\
\text { Comments }\end{array}$} & \multicolumn{3}{|c|}{ Field Screening Results } \\
\hline & & & & & & & & $\begin{array}{l}\text { VOCs } \\
\text { (ppm) }\end{array}$ & $\begin{array}{l}\text { Àlpha } \\
\text { (dpm) }\end{array}$ & $\begin{array}{l}\text { Beta } \\
\text { (dpm) }\end{array}$ \\
\hline
\end{tabular}
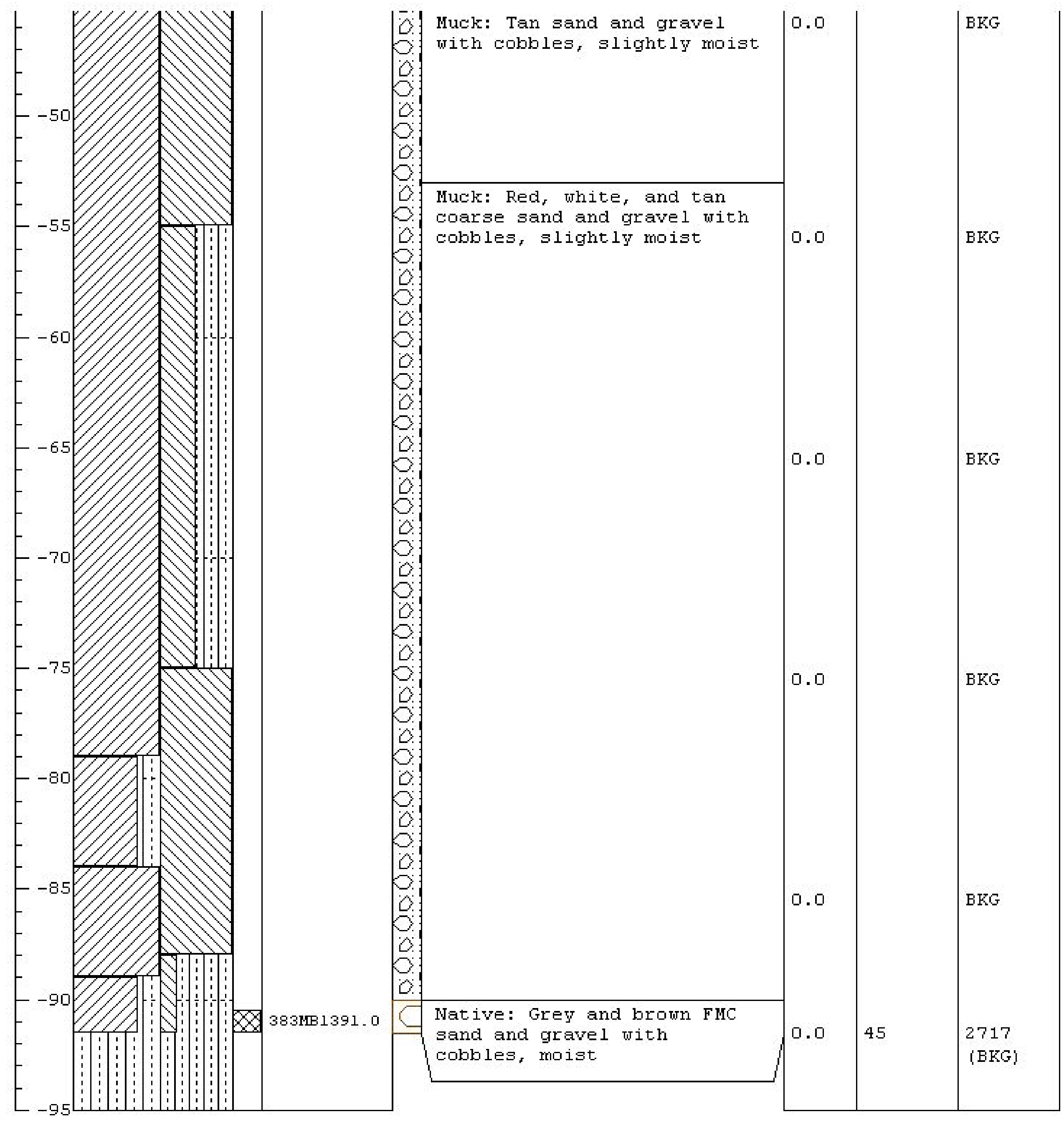
Project Hame: CAU 383 E-Tunnel sites

Project Number: DT04-320

Borehole Number: EMB 14

Logged By: M. MeLane

Drilled By: Boart-Longyear

Drilling Method: Rotosonic
Date Started: $6 / 14 / 04$

Date Completed: $6 / 14 / 04$

Elevation: 6121.6

Horthing: 887672.4

Easting: 638289.8

Total Depth: $29.0 \mathrm{ft}$.

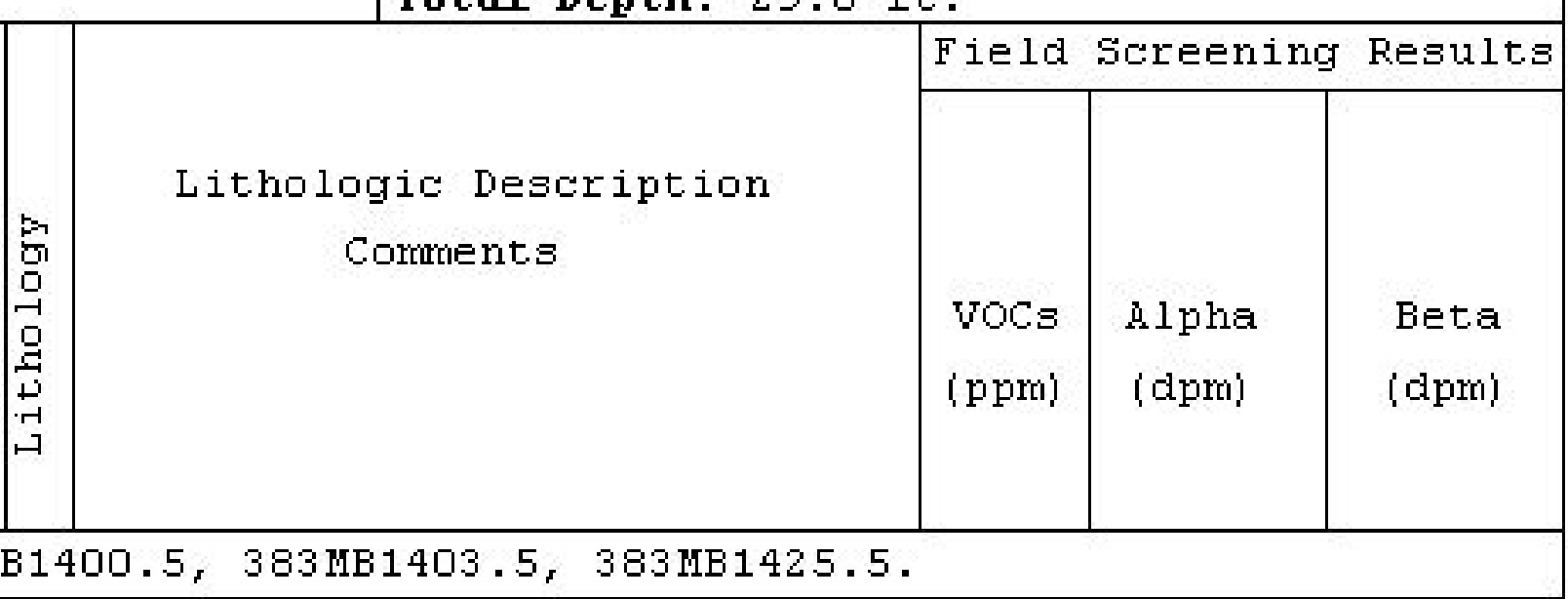

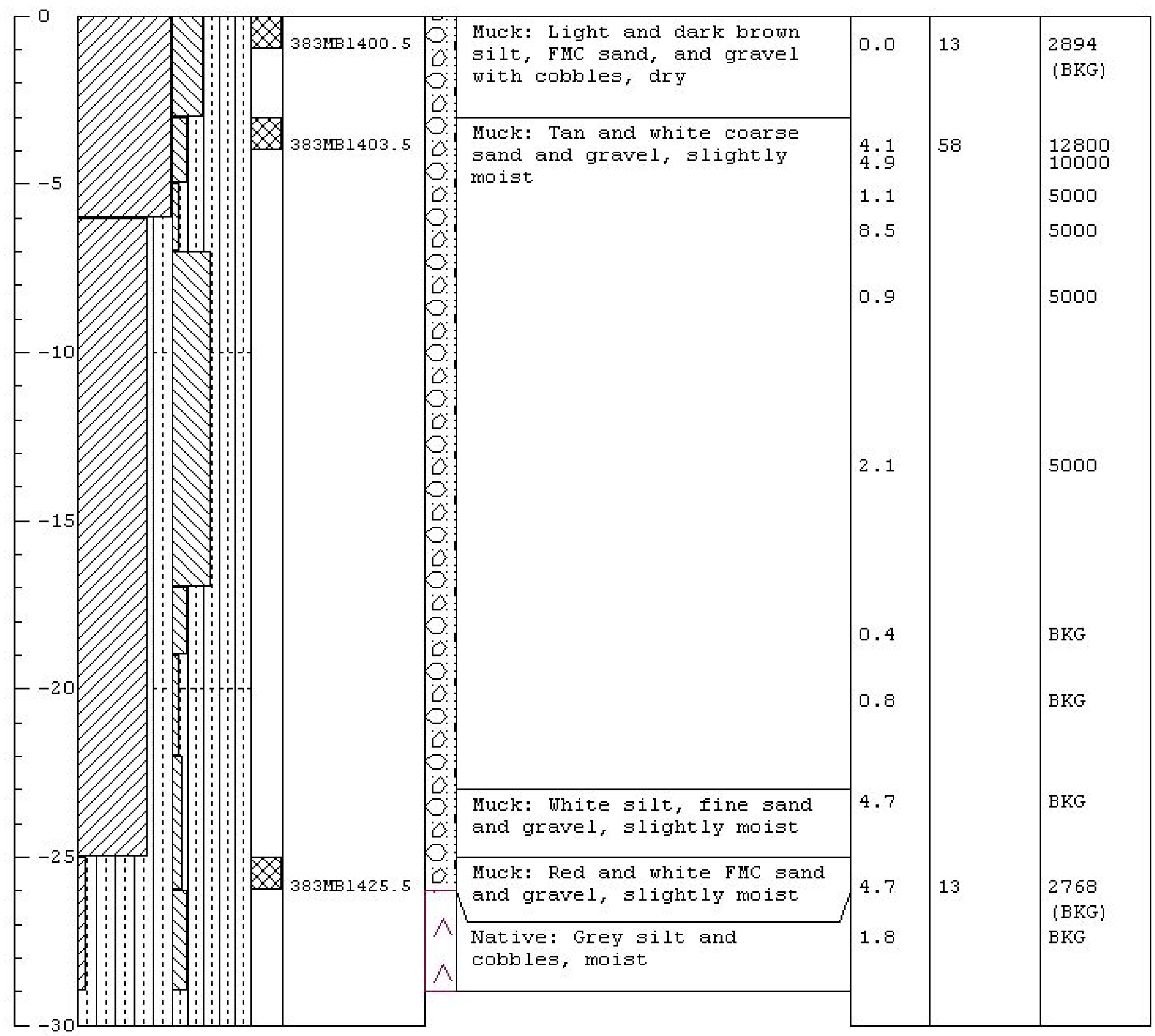


Project Name: CAU 383 E-Tunnel sites

Project Humber: DT04-320

Borehole Number: EMB15

Logged By: $M$. McLane

Drilled By: M. McLane

Drilling Method: Hand Sampled

\begin{tabular}{|c|c|c|c|c|c|}
\hline 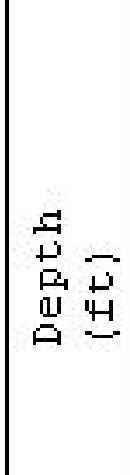 & 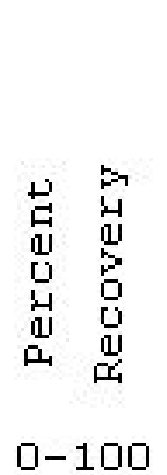 & 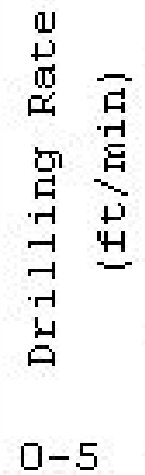 & 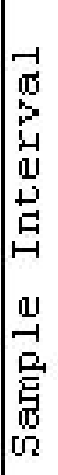 & 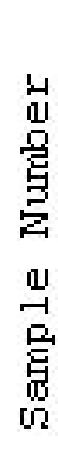 & 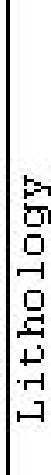 \\
\hline
\end{tabular}

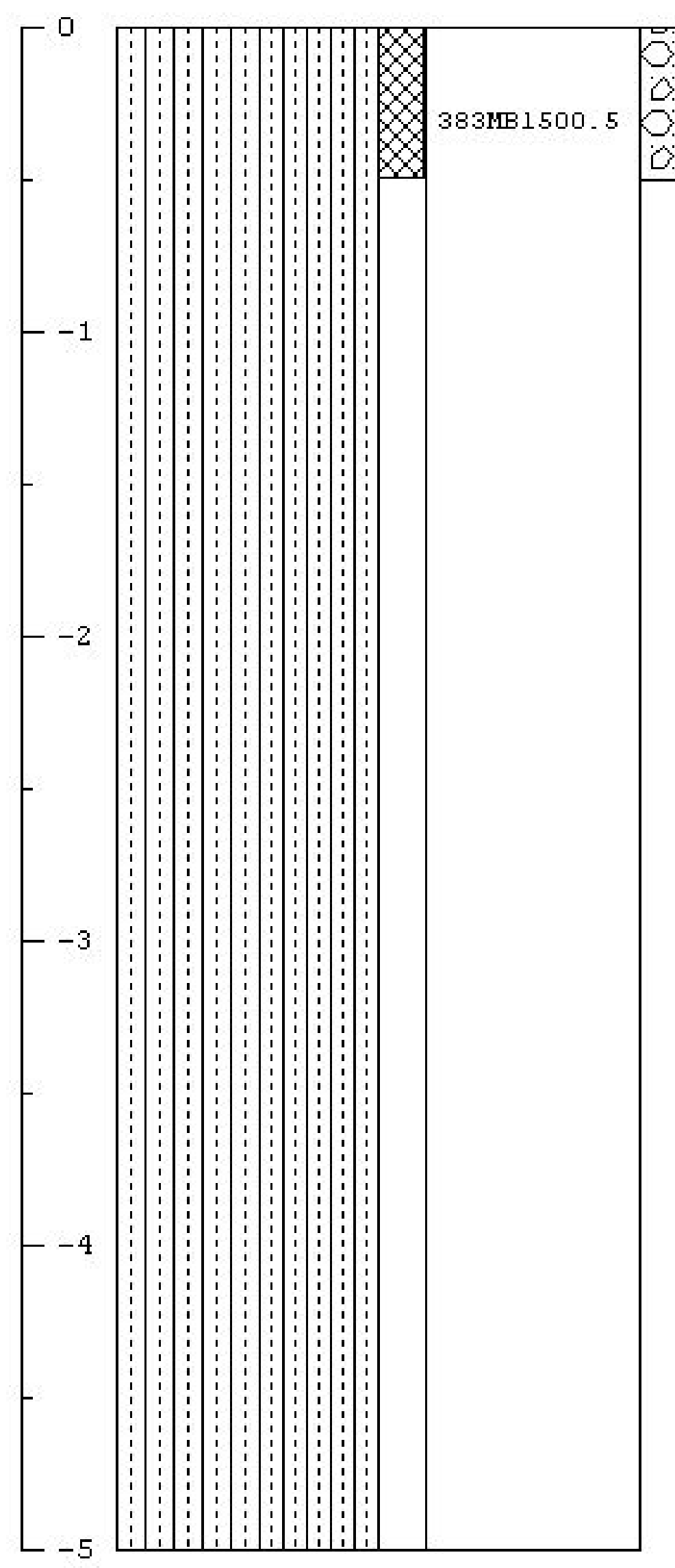

Date Started: $6 / 17 / 04$

Date Completed: $6 / 17 / 04$

Elevation: 6119.0

Horthing: 887532.7

Easting: 638461.1

Total Depth: 0.5 ft.

Lithologic Description

Comments

\begin{tabular}{|c|c|c|}
\hline \multicolumn{2}{|c|}{ Field Screening Results } \\
\hline & & \\
VoCs & Alpha & Beta \\
(ppm) & (dpm) & (dpr) \\
& & \\
\end{tabular}

Muck: Brown FMC sand and gravel with grout cobbles, dry

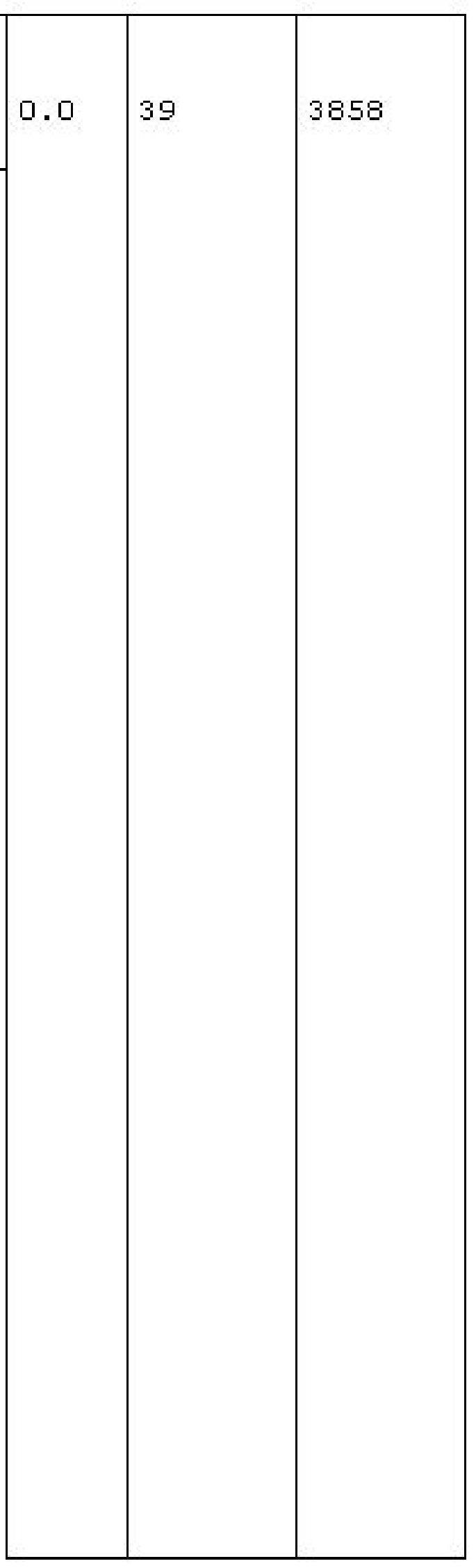


Project Name: CAU 383 E-Tunnel sites

Project Humber: DT04-320

Borehole Number: EMHO1

Logged By: $M$. McLane

Drilled By: Boart-Longyear

Drilling Method: Rotosonic

\begin{tabular}{|c|c|c|c|c|c|}
\hline 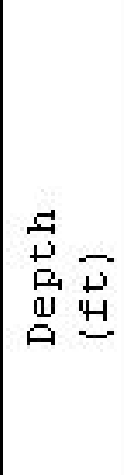 & 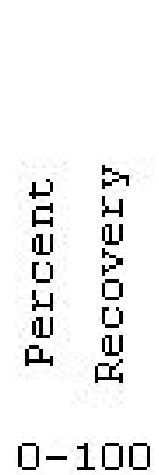 & 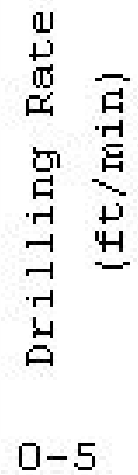 & 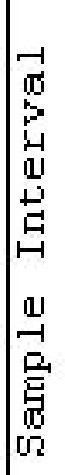 & 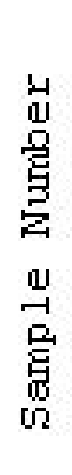 & 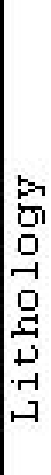 \\
\hline
\end{tabular}

Date Started: $5 / 25 / 04$

Date completed: $5 / 25 / 04$

Elevation: 6128.6

Horthing : 887433.7

Easting: 638265.0

Total Depth: 69.0 ft.

Lithologic Description

Comments
Field Screening Results

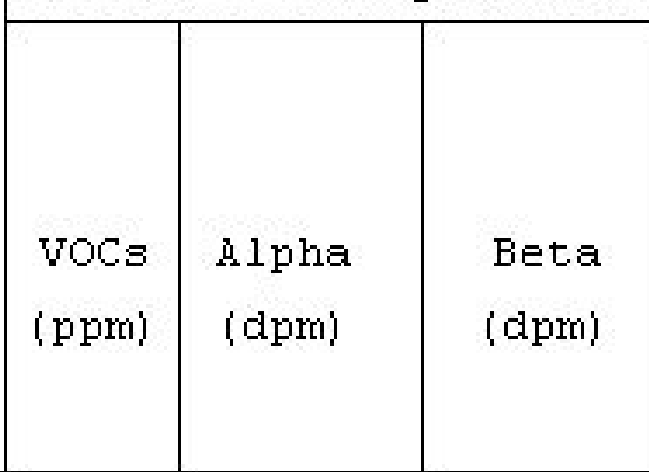

Contact o 6.5.0 ft, Samples 383 MH0136.5, 383 MHO168.5.

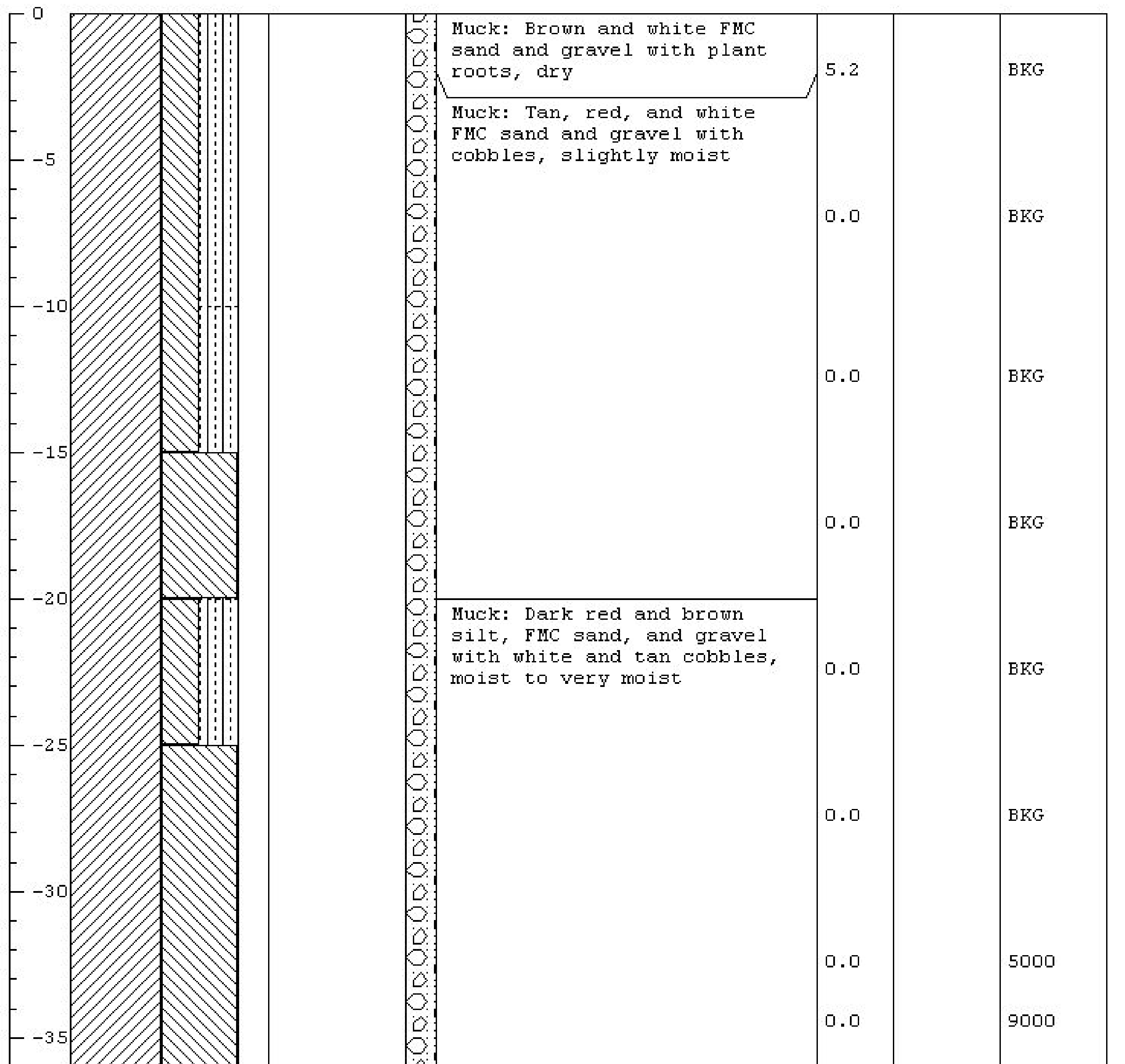


Page 2 of 2

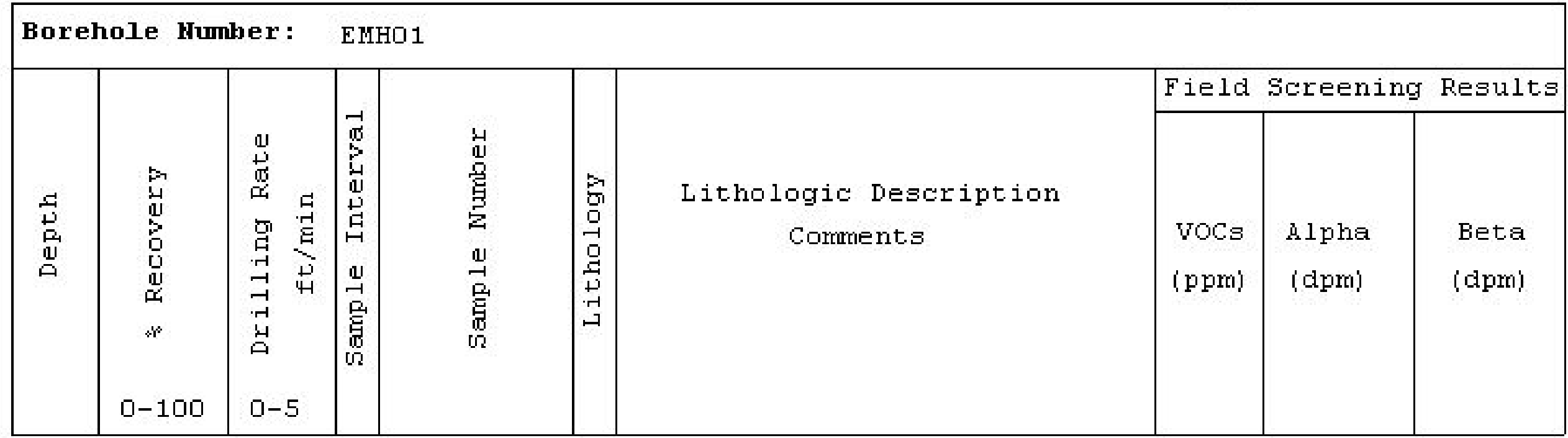
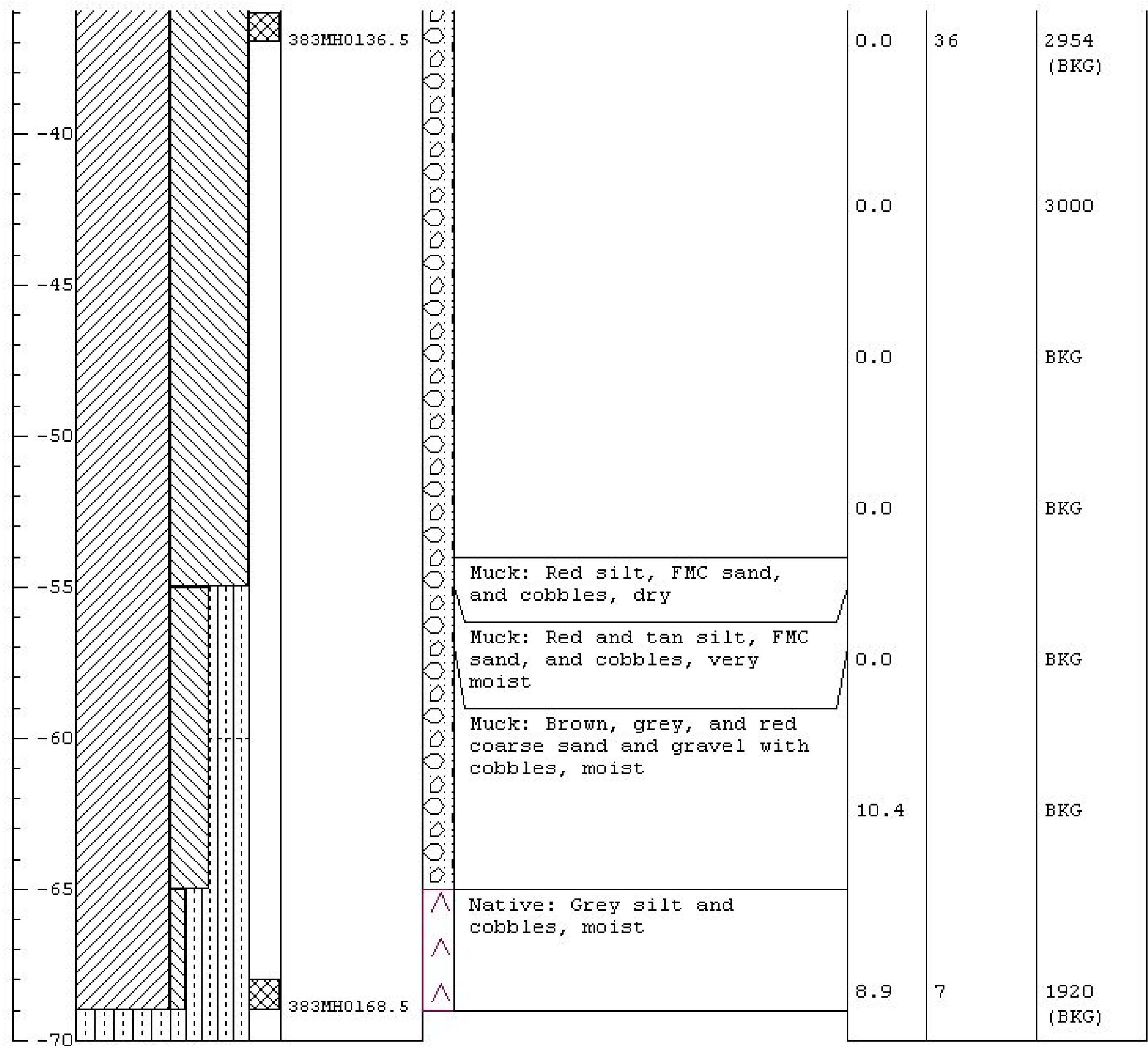
Project Name: CAU 383 E-Tunnel sites

Project Humber: DT04-320

Borehole Number: EMHO2

Logged By: $M$. McLane

Drilled By: Boart-Longyear

Drilling Method: Rotosonic

\begin{tabular}{|c|c|c|c|c|c|}
\hline 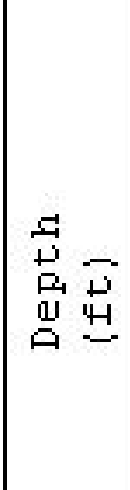 & 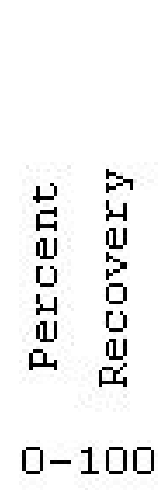 & 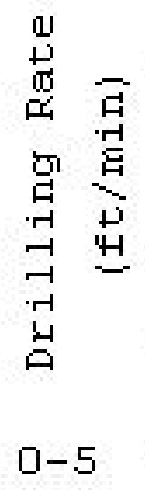 & 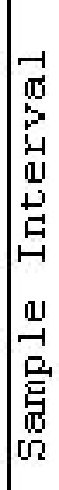 & 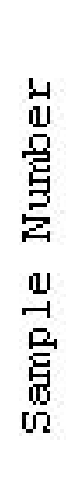 & ge \\
\hline
\end{tabular}

Date Started: $5 / 22 / 04$

Date completed: $5 / 22 / 04$

Elevation: 6118.8

Horthing : 887363.7

Easting: 638721.6

Total Depth: 5.0 ft.

Lithologic Description

Corments

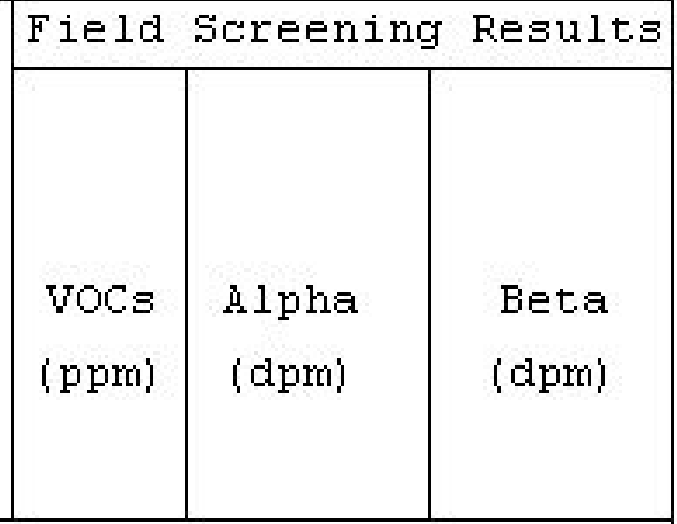

Contact $\mathrm{N} / \mathrm{A}$, No Samples Taken

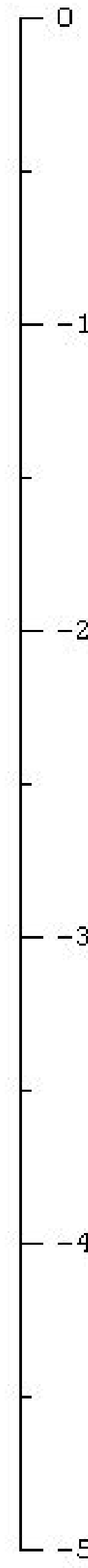

by PFi:i

(2)
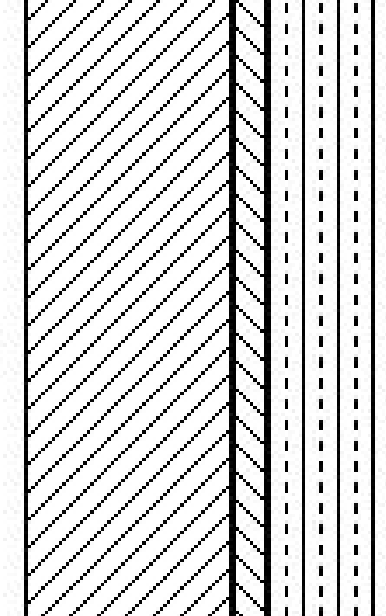

Y

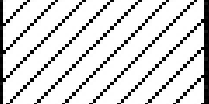

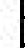
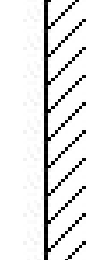

3

$-$

西
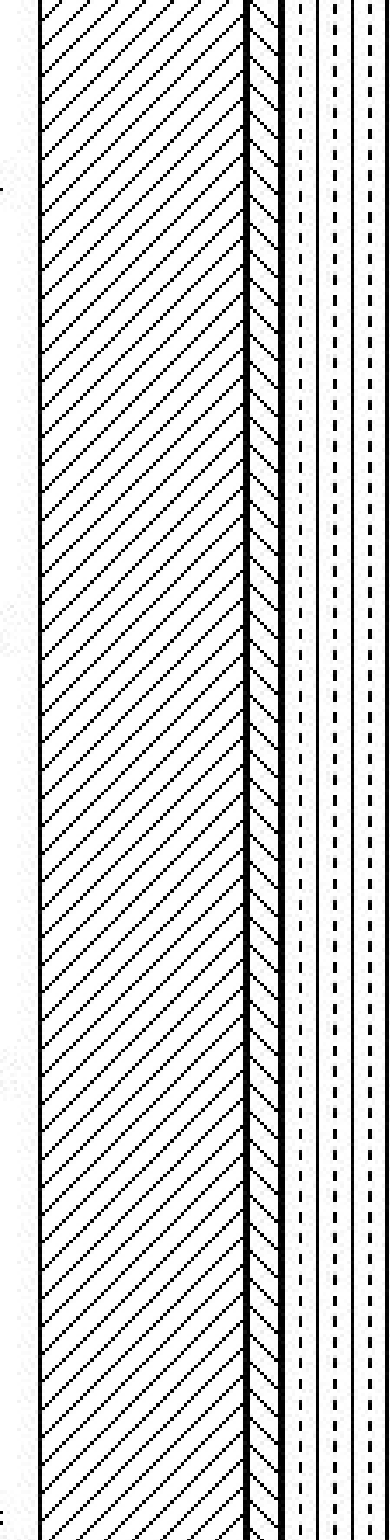

tist
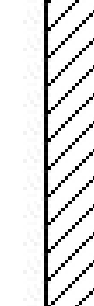

$-5$

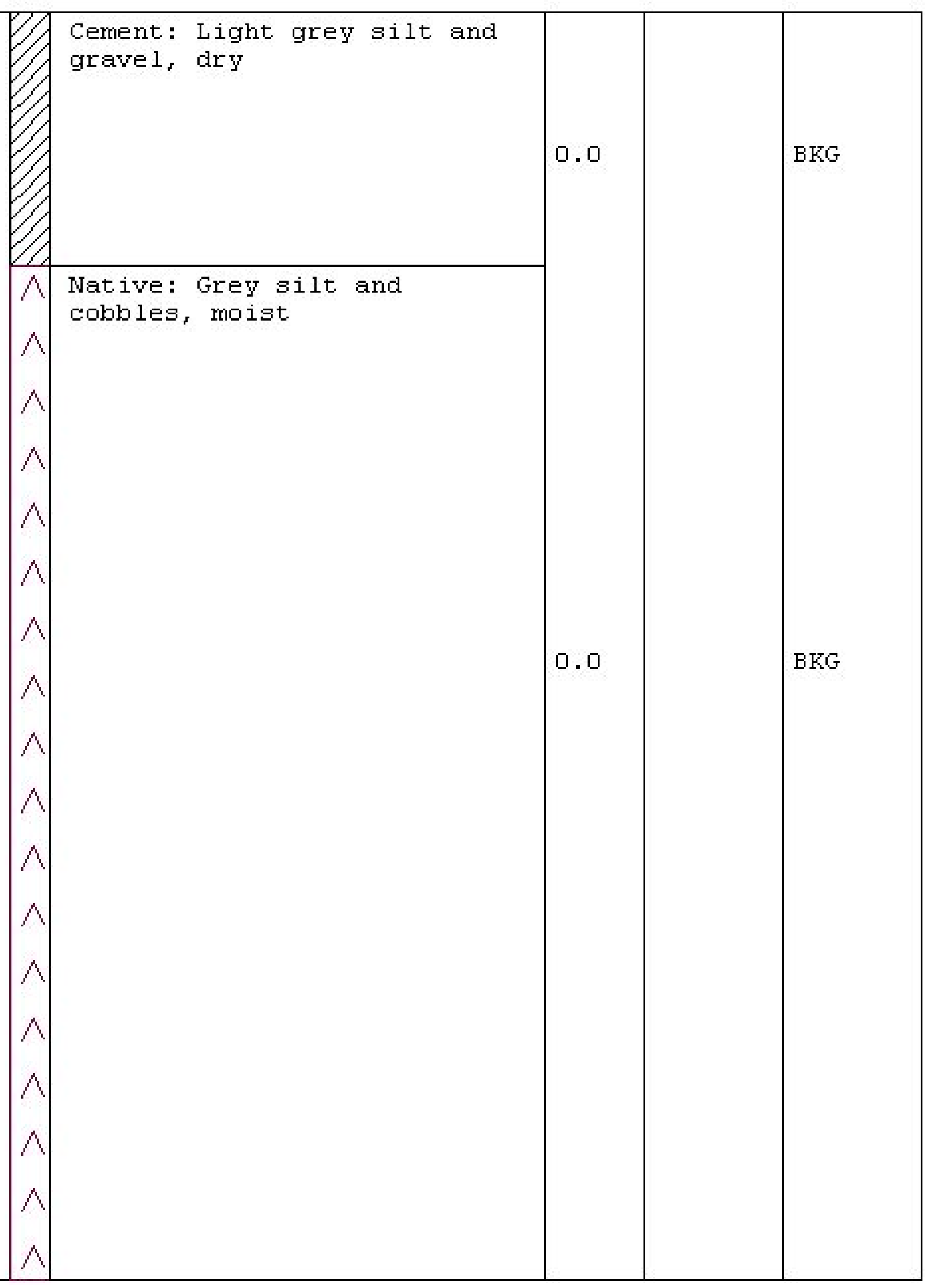


Project Hame: CAU 383 E-Tunnel sites

Project Number: DT04-320

Borehole Humber: EMH2A

Logged By: M. MeLane

Drilled By: Boart-Longyear

Drilling Method: Rotosonic
Date Started: $6 / 03 / 04$

Date Completed: $6 / 08 / 04$

Elevation: 6123.4

Horthing: 887540.8

Easting: 638332.6

Total Depth: $45.5 \mathrm{ft}$.

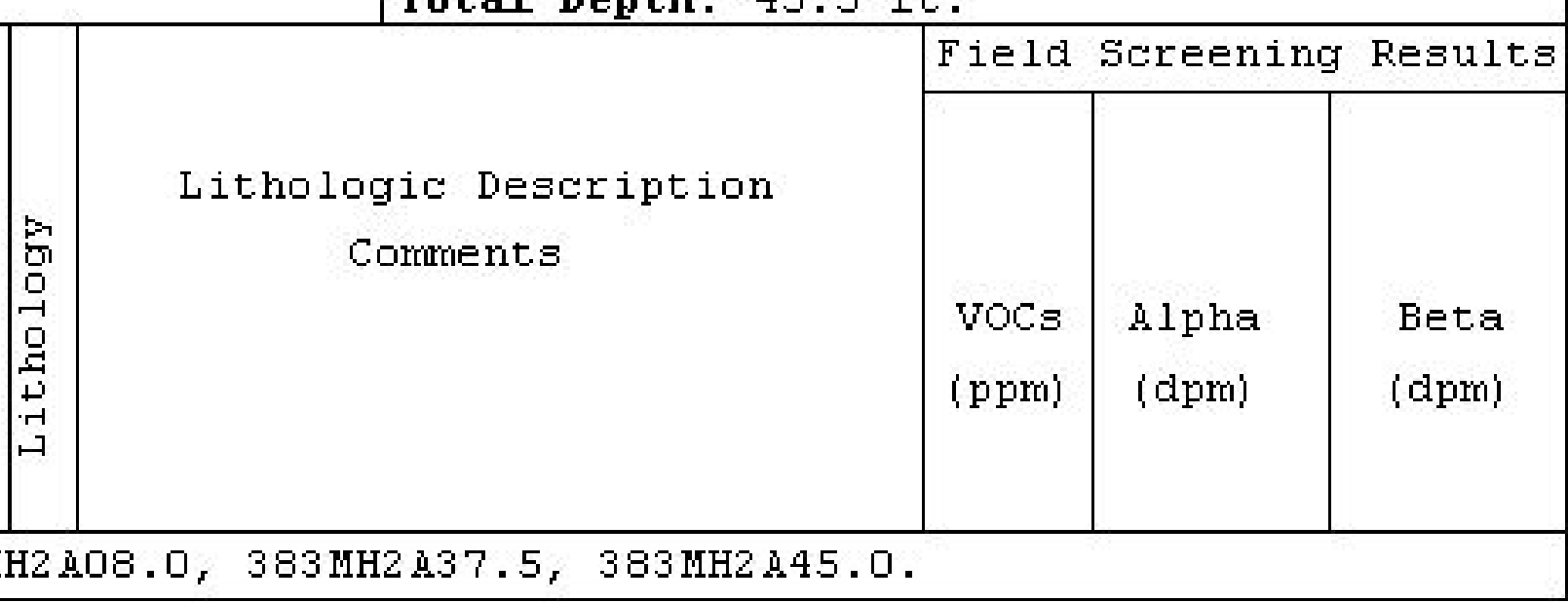

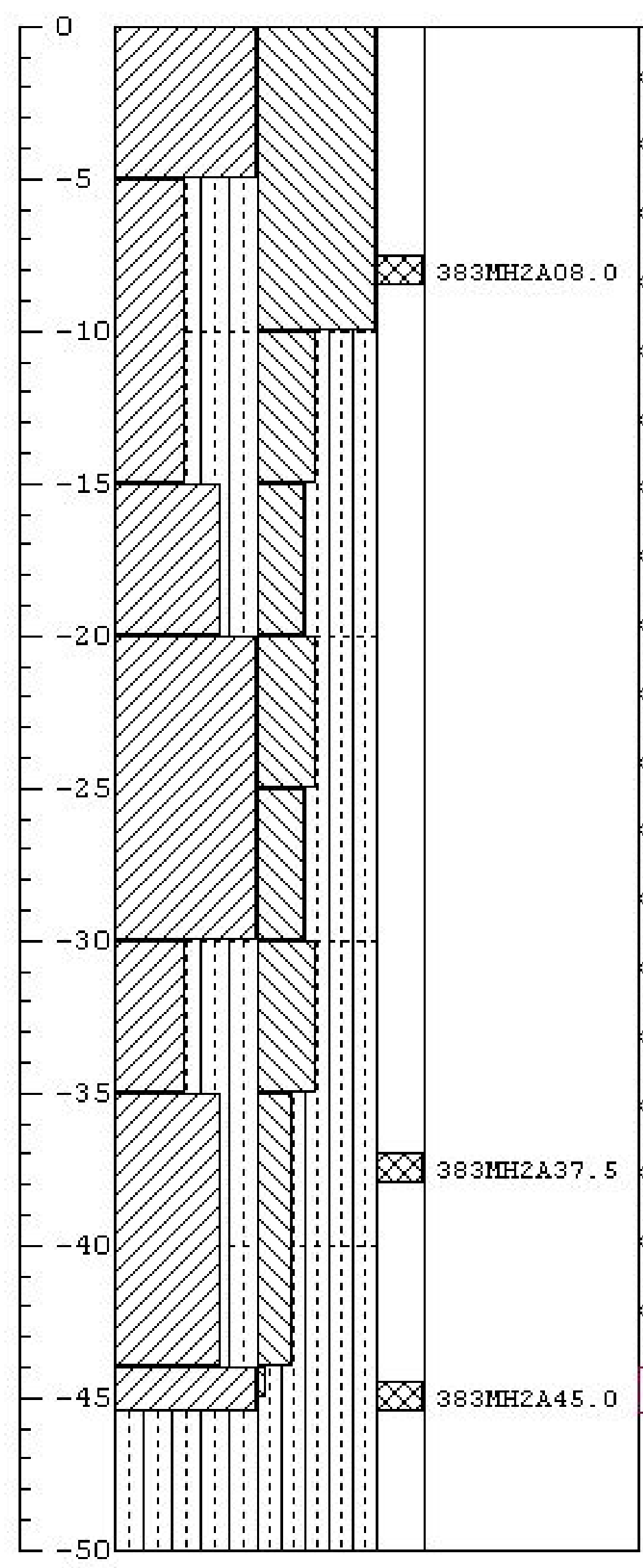

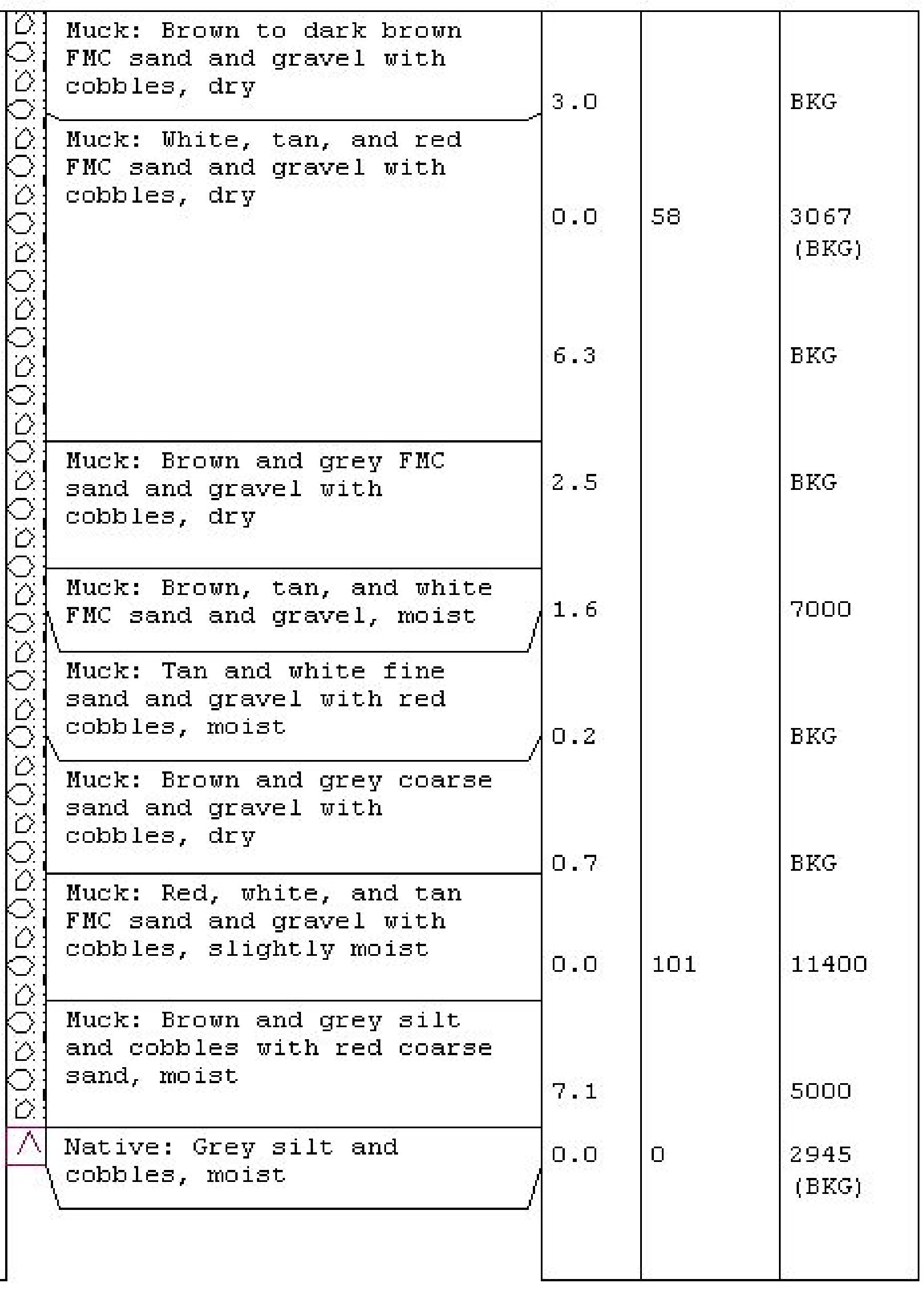


Project Hame: CAU 383 E-Tunnel sites

Project Number: DT04-320

Borehole Number: EMHO3

Logged By: M. MeLane

Drilled By: Boart-Longyear

Drilling Method: Rotosonic
Date Started: $5 / 25 / 04$

Date Completed: $5 / 25 / 04$

Elevation: 6131.4

Horthing: 887442.1

Easting: 638260.8

Total Depth: $61.5 \mathrm{ft}$.

\begin{tabular}{|c|c|c|c|c|}
\hline \multirow[b]{2}{*}{ 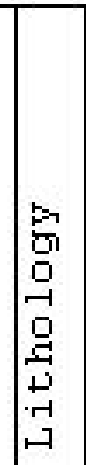 } & \multirow[b]{2}{*}{$\begin{array}{c}\text { Lithologic Description } \\
\text { Comments }\end{array}$} & \multicolumn{3}{|c|}{ Field Screening Results } \\
\hline & & $\begin{array}{l}\text { voCs } \\
\text { (ppm) }\end{array}$ & $\begin{array}{l}\text { Alpha } \\
\text { (dpm) }\end{array}$ & $\begin{array}{l}\text { Beta } \\
\text { (dpm) }\end{array}$ \\
\hline
\end{tabular}
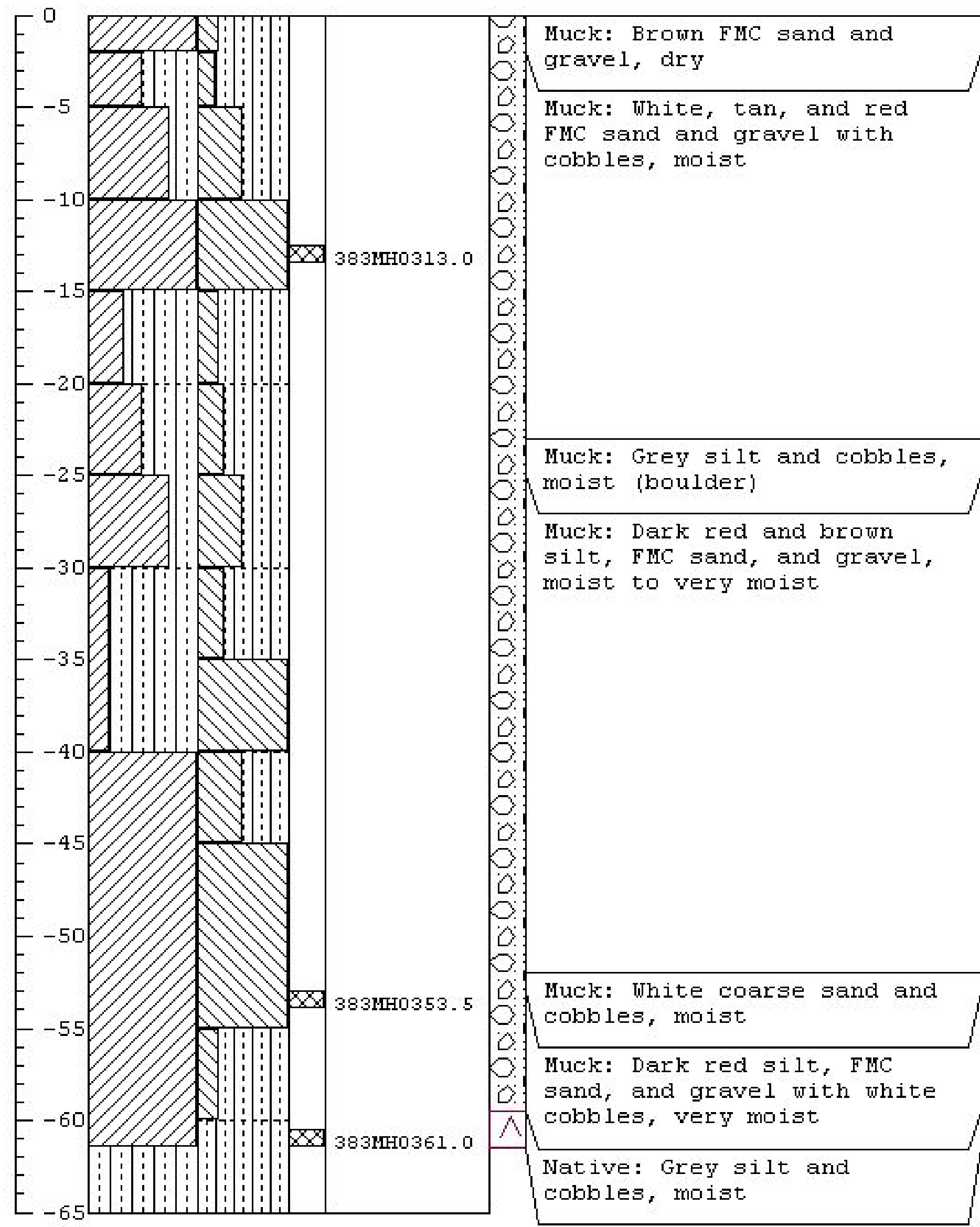

\begin{tabular}{|c|c|c|}
\hline 0.0 & & BKG \\
\hline 0.0 & & BKG \\
\hline 0.0 & & $\mathrm{BKG}$ \\
\hline 0.0 & 22 & $\begin{array}{l}3151 \\
\text { (BKG) }\end{array}$ \\
\hline 1.6 & & $\mathrm{BKG}$ \\
\hline 9.4 & & $\mathrm{BKG}$ \\
\hline 0.5 & & $\mathrm{BKG}$ \\
\hline 0.0 & & $\mathrm{BKG}$ \\
\hline 0.0 & & $\mathrm{BKG}$ \\
\hline 10.1 & & BKG \\
\hline 0.0 & & $\mathrm{BKG}$ \\
\hline 0.0 & 51 & 7129 \\
\hline 14.2 & & BKG \\
\hline 4.8 & 15 & $\begin{array}{l}1866 \\
\text { (BKG) }\end{array}$ \\
\hline
\end{tabular}


Project Hame: CAU 383 E-Tunnel sites

Project Humber: DT04-320

Borehole Humber: EMHO4

Logged By: M. MeLane

Drilled By: Boart-Longyear

Drilling Method: Rotosonic

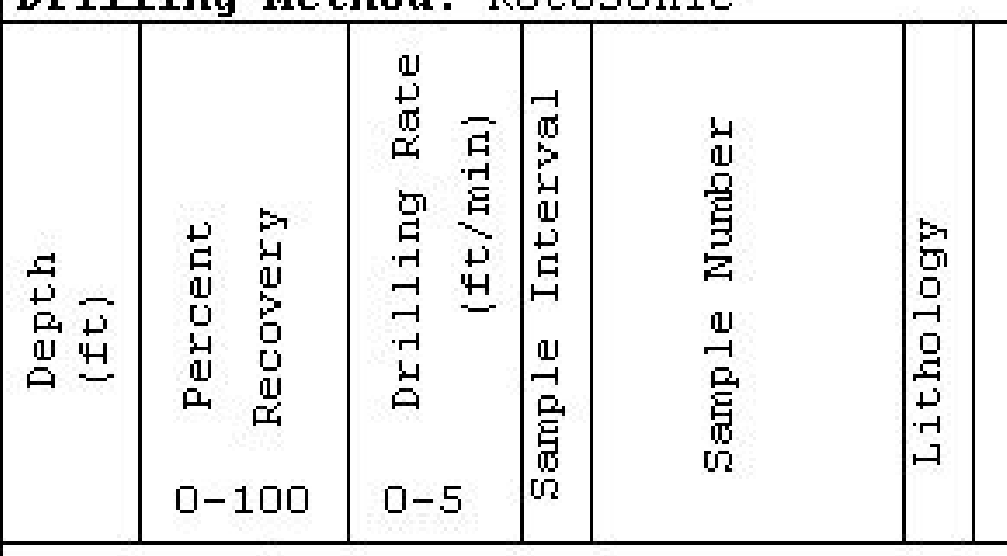

Contact $38.0 \mathrm{ft}$, Samples $383 \mathrm{MHO} 432.0,383 \mathrm{MHO} 441.5$.
Date Started: $5 / 22 / 04$

Date Completed: $5 / 22 / 04$

Elevation: 6115.4

Horthing: 887429.3

Easting: 638606.1

Total Depth: 42.0 ft.

Lithologic Description

Comments
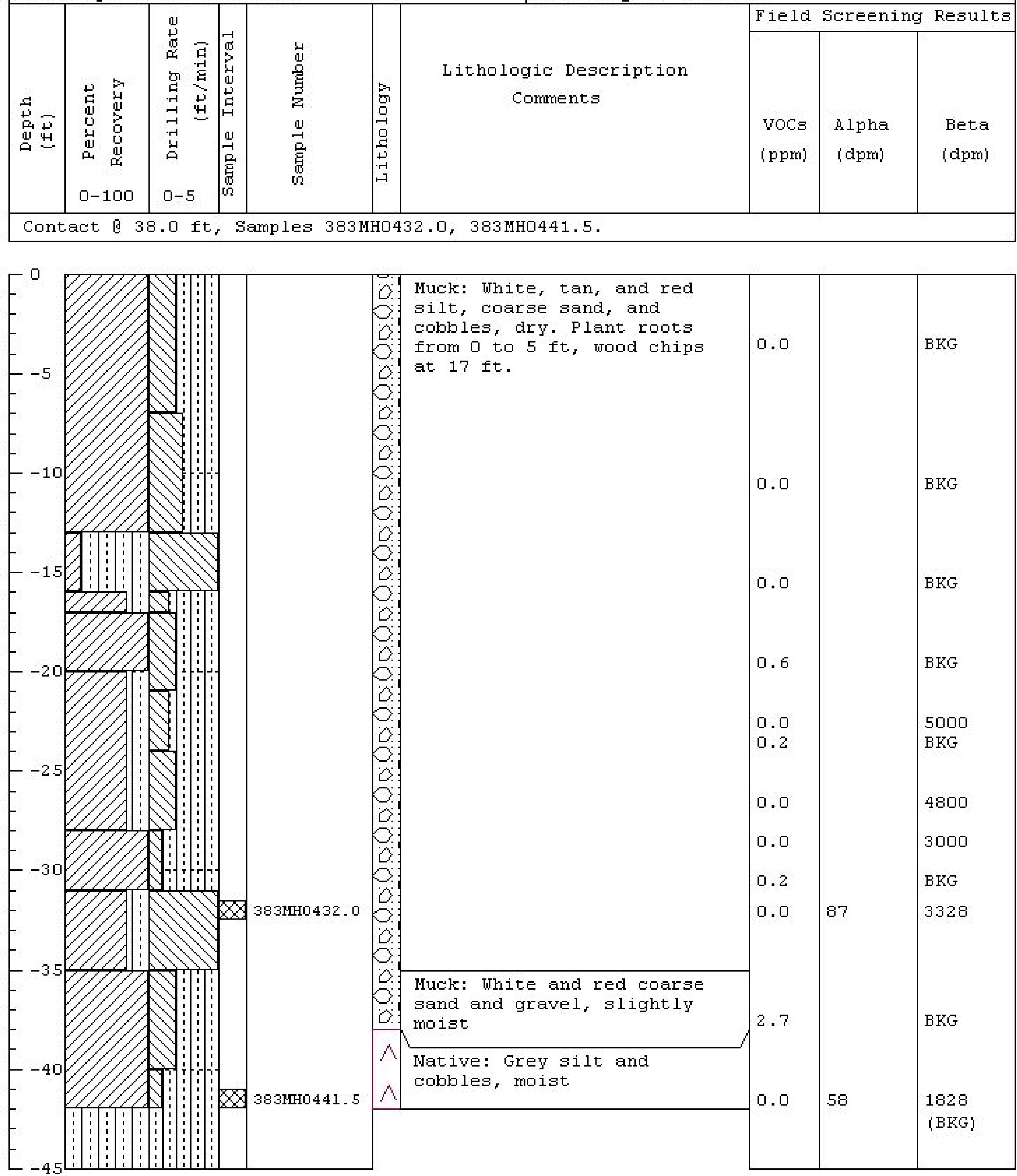
Project Name: CAU 383 E-Tunnel sites

Project Humber: DT04-320

Borehole Number: EMHO5

Logged By: $M$. McLane

Drilled By: Boart-Longyear

Drilling Method: Rotosonic
Date Started: $5 / 26 / 04$

Date completed: $5 / 27 / 04$

Elevation: 6123.6

Horthing : 887462.7

Easting: 638379.3

Total Depth: 70.0 ft.

Lithologic Description Corments

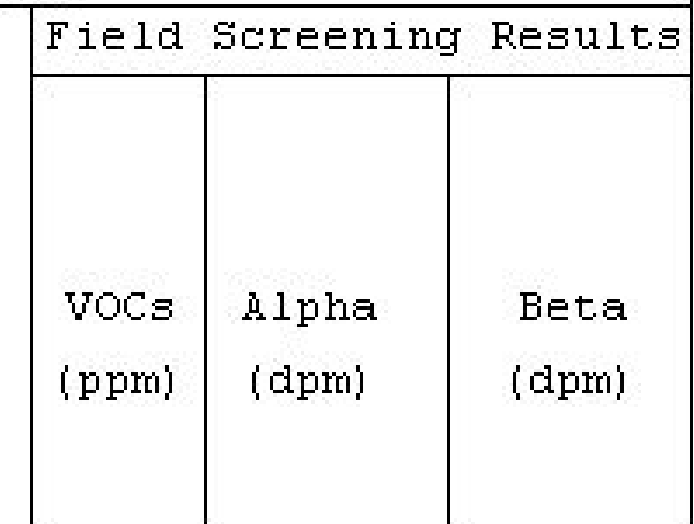

Contact o $64.5 \mathrm{ft}$, Samples $383 \mathrm{MHO} 20.5,383 \mathrm{MH0} 5.59 .5,383 \mathrm{MH0568.5.}$

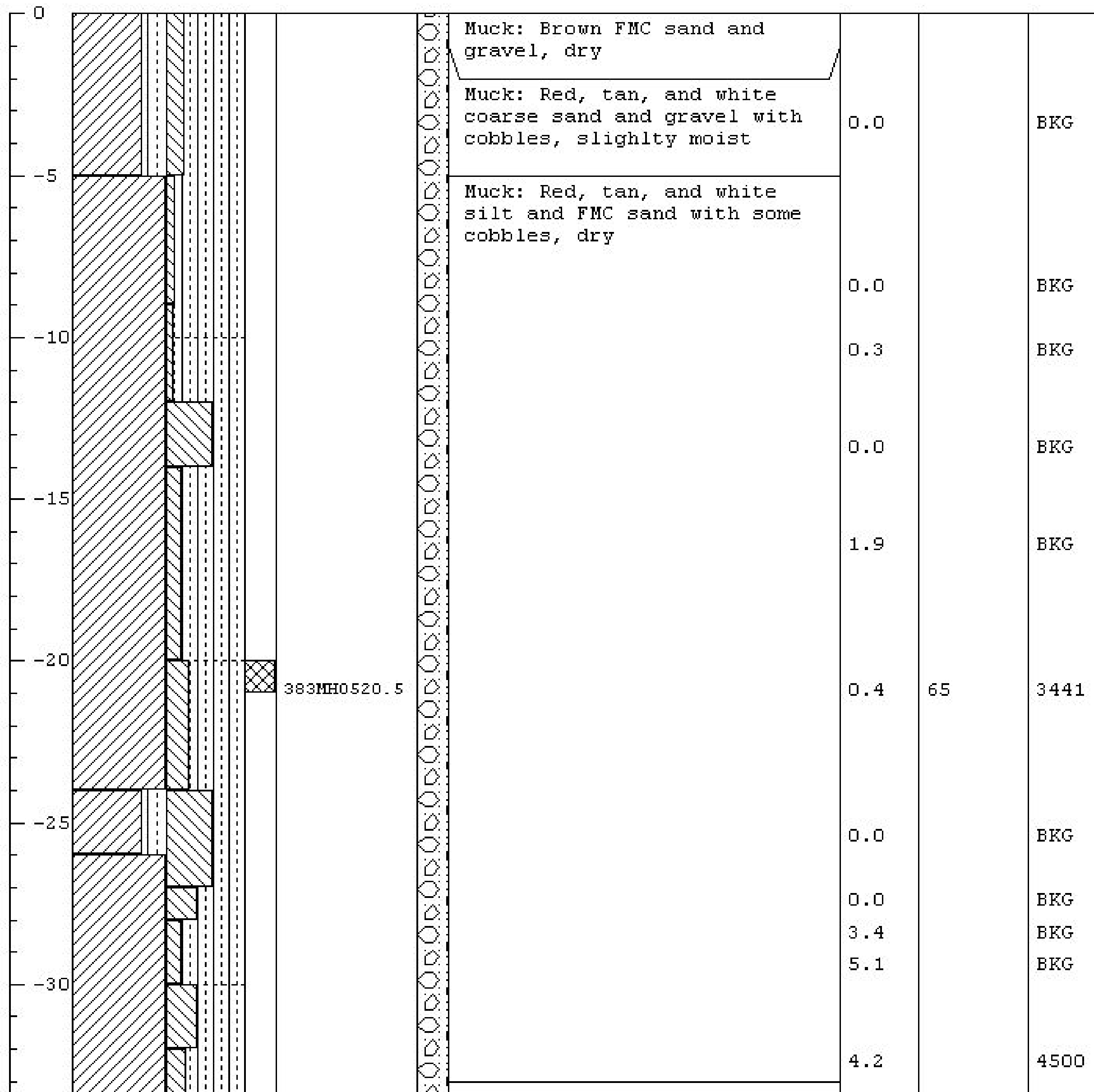


Page 2 of 2

\begin{tabular}{|c|c|c|c|c|c|c|c|c|c|}
\hline \multicolumn{3}{|c|}{ Borehole Number: } & \multicolumn{7}{|c|}{ EMHOS } \\
\hline \multirow{3}{*}{ 空 } & \multirow[b]{2}{*}{ 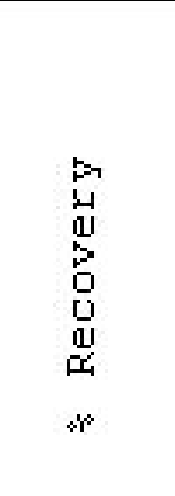 } & \multirow[b]{2}{*}{ 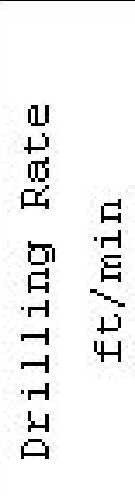 } & \multirow[b]{2}{*}{ 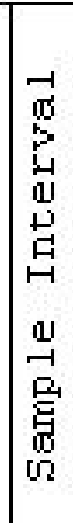 } & \multirow{3}{*}{ 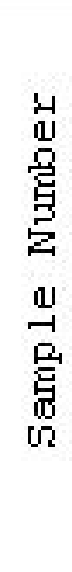 } & \multirow{3}{*}{ 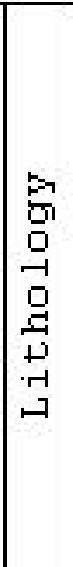 } & \multirow{3}{*}{$\begin{array}{c}\text { Lithologic Description } \\
\text { Comments }\end{array}$} & \multicolumn{3}{|c|}{ Field Screening Results } \\
\hline & & & & & & & $\begin{array}{l}\text { VOCs } \\
\text { (ppm) }\end{array}$ & $\begin{array}{l}\text { Alpha } \\
\text { (dpm) }\end{array}$ & $\begin{array}{l}\text { Beta } \\
\text { (dpm) }\end{array}$ \\
\hline & $0-100$ & $0-5$ & & & & & & & \\
\hline
\end{tabular}

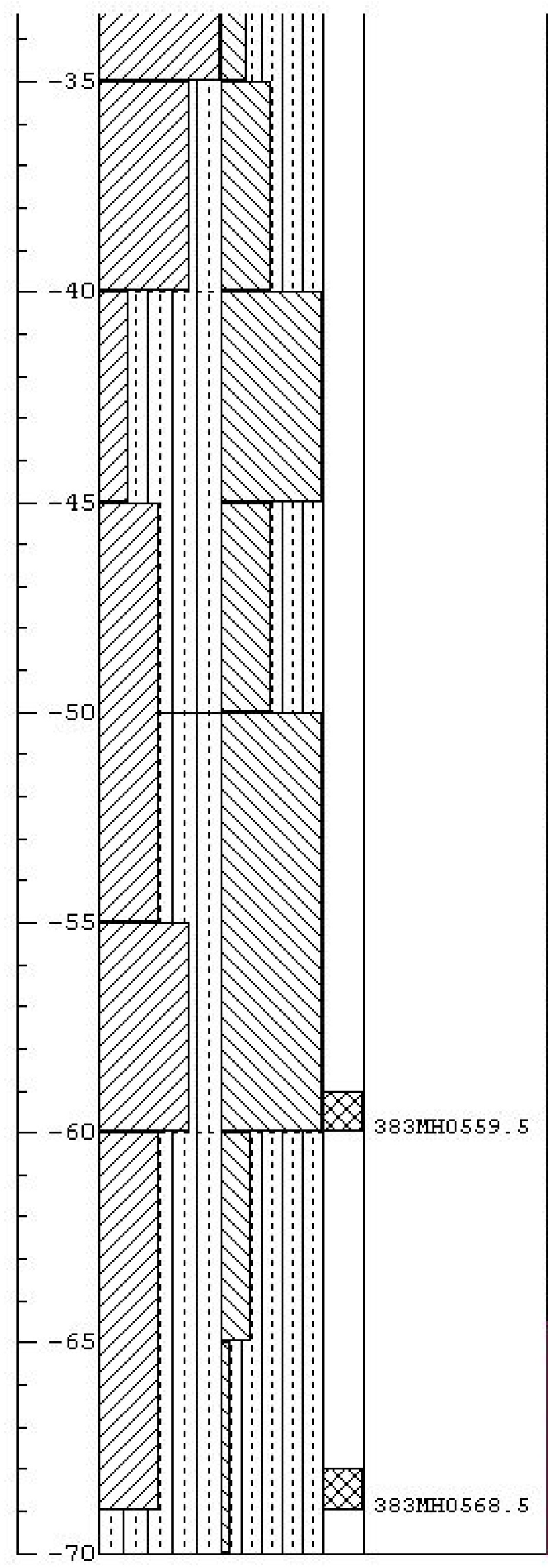

L 5 : Muck: Red, tan, and white

. coarse sand and gravel with cobbles, moist. Wood chips at $35 \mathrm{ft}$.

ㅇ.

Muck: Dark red coarse sand and gravel, slighlty moist . : C. Muck: Dark red, brown and black coarse sand and gravel with black cobbles, wood chips, and sheet metal, moist

Muck: Red, brown, and white coarse sand and gravel with cobbles, very moist

Native: Grey cobbles and gravel, moist 
Project Name: CAU 383 E-Tunnel sites

Project Humber: DT04-320

Borehole Number: EMHO 6

Logged By: $M$. McLane

Drilled By: Boart-Longyear

Drilling Method: Rotosonic
Date Started: $6 / 11 / 04$

Date completed: $6 / 11 / 04$

Elevation: 6119.0

Horthing : 887897.5

Easting: 637771.1

Total Depth: 1.5 ft.

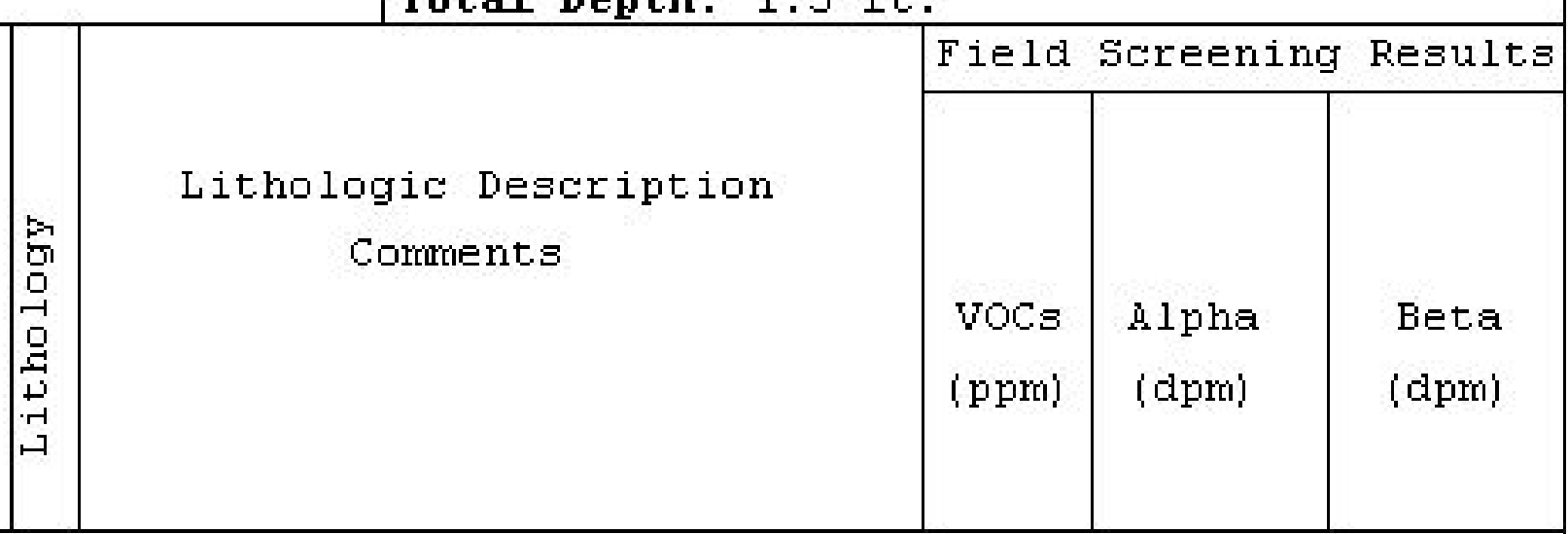

Sample 383MHO600.5.

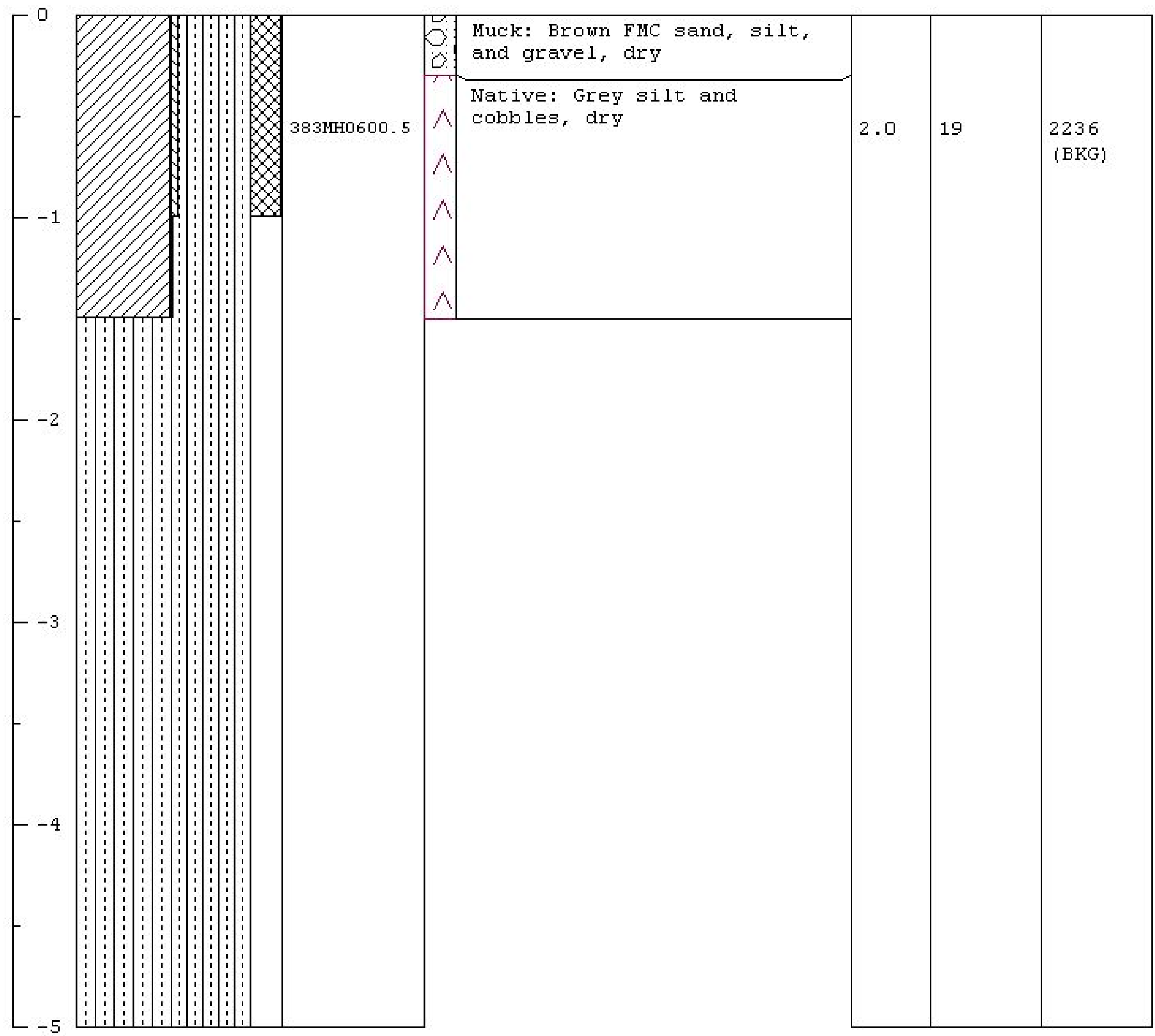


Project Name: CAU 383 E-Tunnel sites

Project Humber: DT04-320

Borehole Number: EMHO7

Logged By: $M$. McLane

Drilled By: Boart-Longyear

Drilling Method: Rotosonic
Date Started: $5 / 20 / 04$

Date completed: $5 / 21 / 04$

Elevation: 6123.5

Horthing: 887475.6

Easting: 638472.2

Total Depth: 68 ft.

Lithologic Description

Comments

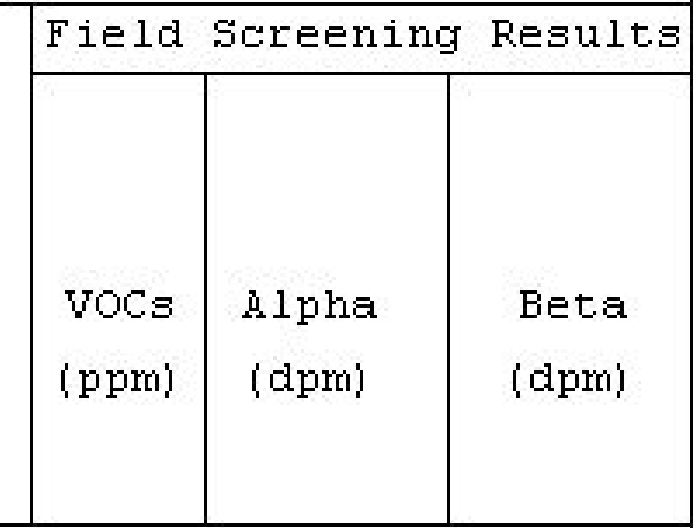

Contact $059.5 \mathrm{ft}$, Samples $383 \mathrm{MHO} 222.5,383 \mathrm{MHO} 223.5,383 \mathrm{MHO} 67.5$.

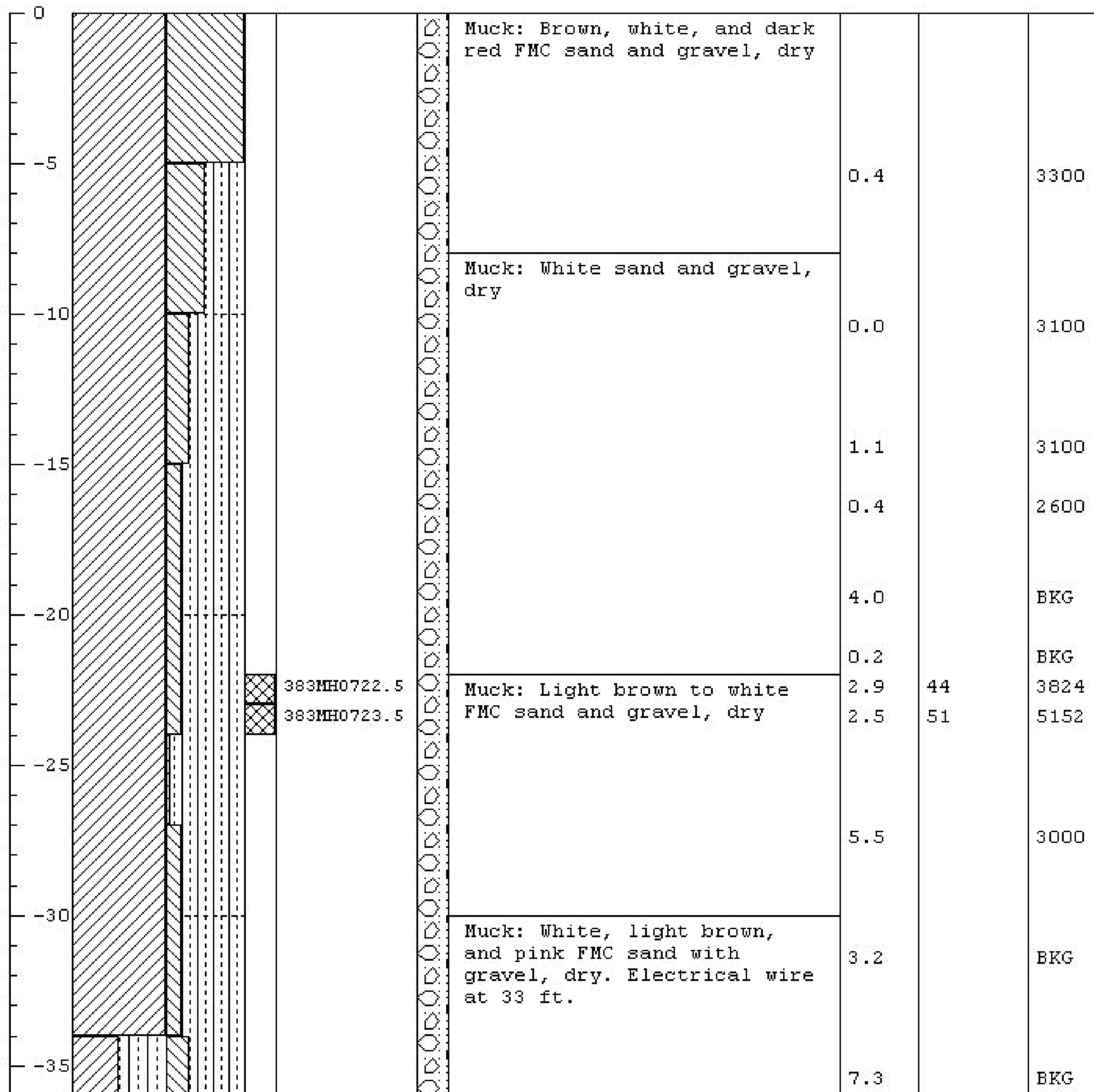


Page 2 of 2

\begin{tabular}{|c|c|c|c|c|c|c|c|c|c|}
\hline \multicolumn{3}{|c|}{ Borehole Number: } & \multicolumn{7}{|c|}{ EMHO7 } \\
\hline \multirow[b]{2}{*}{ 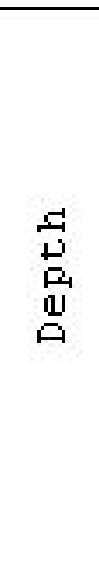 } & \multirow[b]{2}{*}{ 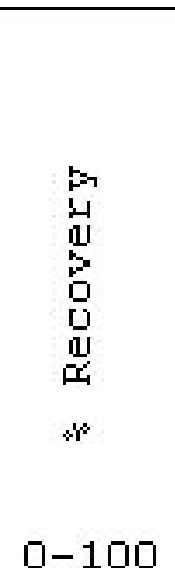 } & \multirow[b]{2}{*}{ 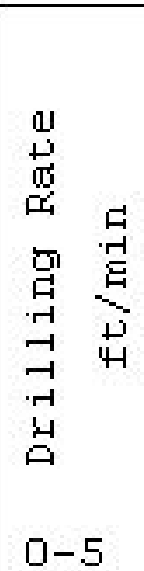 } & \multirow[b]{2}{*}{ 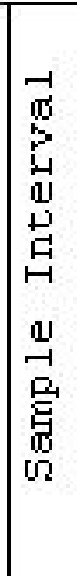 } & \multirow[b]{2}{*}{ 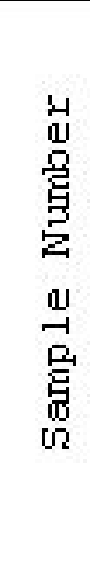 } & \multirow[b]{2}{*}{ 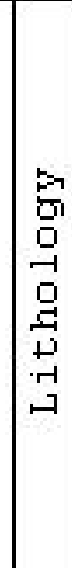 } & \multirow[b]{2}{*}{$\begin{array}{c}\text { Lithologic Description } \\
\text { Comments }\end{array}$} & \multicolumn{3}{|c|}{ Field Screening Results } \\
\hline & & & & & & & $\begin{array}{l}\text { VOCs } \\
\text { (ppm) }\end{array}$ & $\begin{array}{l}\text { Alpha } \\
\text { (dpm) }\end{array}$ & $\begin{array}{l}\text { Beta } \\
\text { (dpm) }\end{array}$ \\
\hline
\end{tabular}
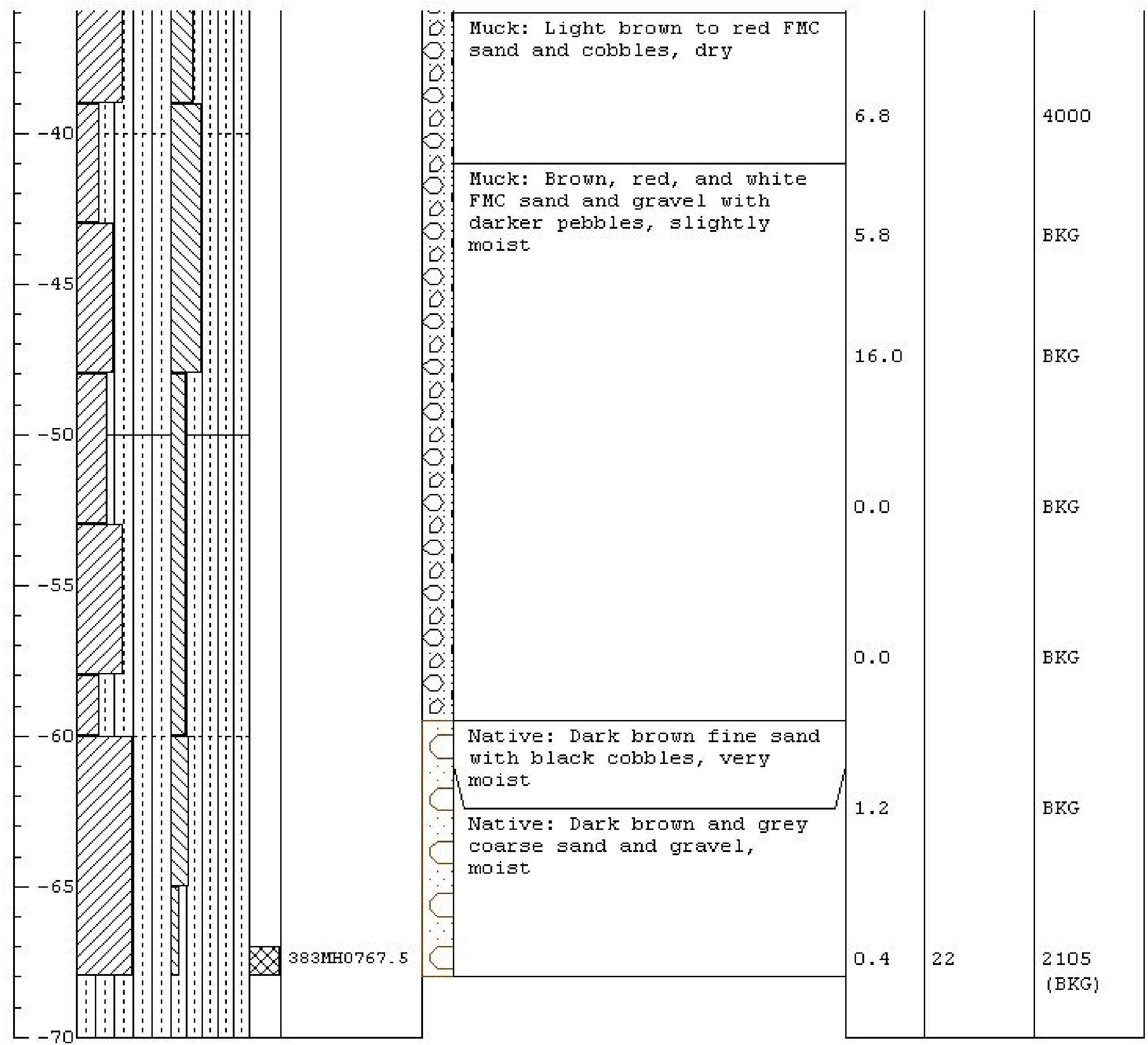
Project Name: CAU 383 E-Tunnel sites

Project Humber: DT04-320

Borehole Number: EMHOB

Logged By: $M$. McLane

Drilled By: Boart-Longyear

Drilling Method: Rotosonic
Date Started: $5 / 26 / 04$

Date completed: $5 / 26 / 04$

Elevation: 6125.9

Horthing : 887482.7

Easting: 638309.3

Total Depth: 59.0 ft.

\begin{tabular}{|c|c|c|c|c|}
\hline 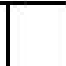 & 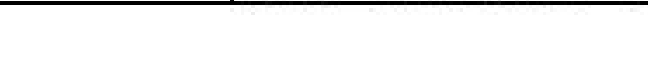 & Field & Screening & Results \\
\hline 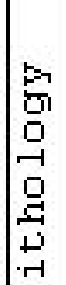 & $\begin{array}{c}\text { Lithologic Description } \\
\text { Comments }\end{array}$ & $\begin{array}{l}\text { VOCs } \\
\text { (ppri) }\end{array}$ & $\begin{array}{l}\text { Alpha } \\
\text { (dpri) }\end{array}$ & $\begin{array}{l}\text { Beta } \\
\text { (dapm) }\end{array}$ \\
\hline
\end{tabular}

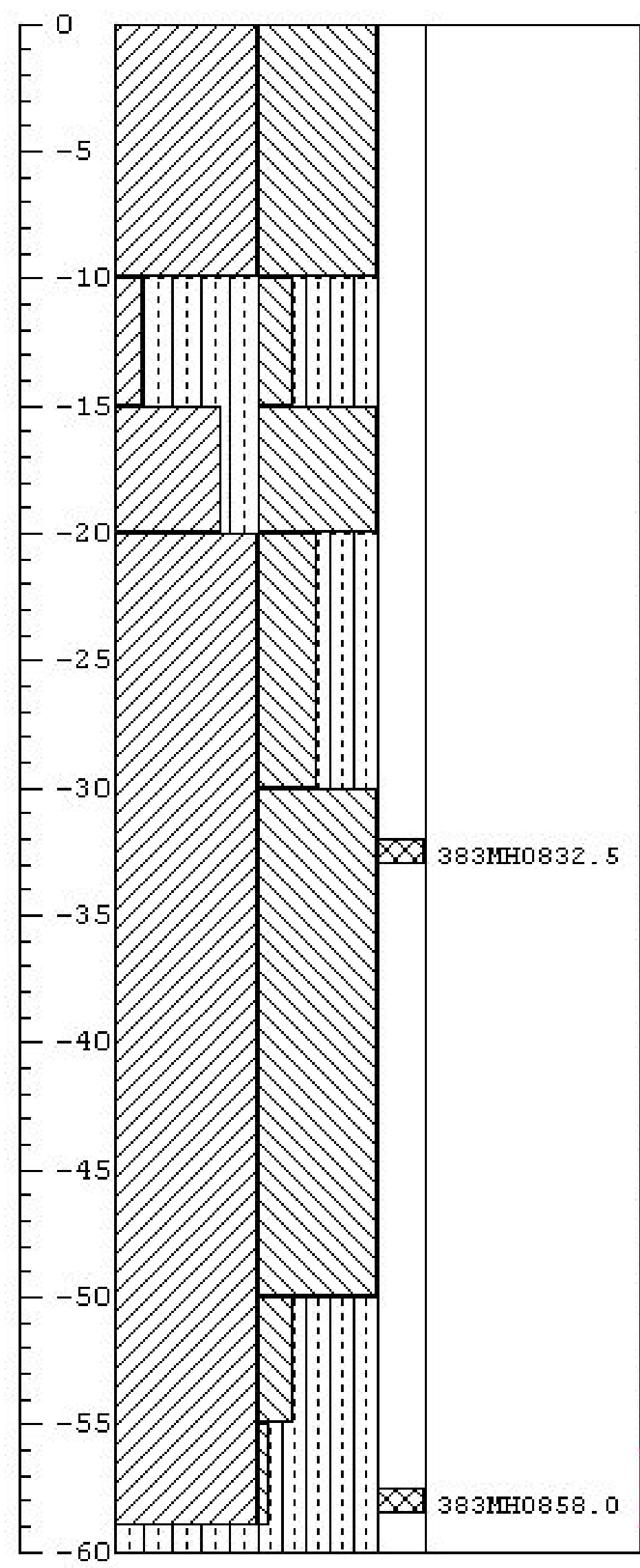

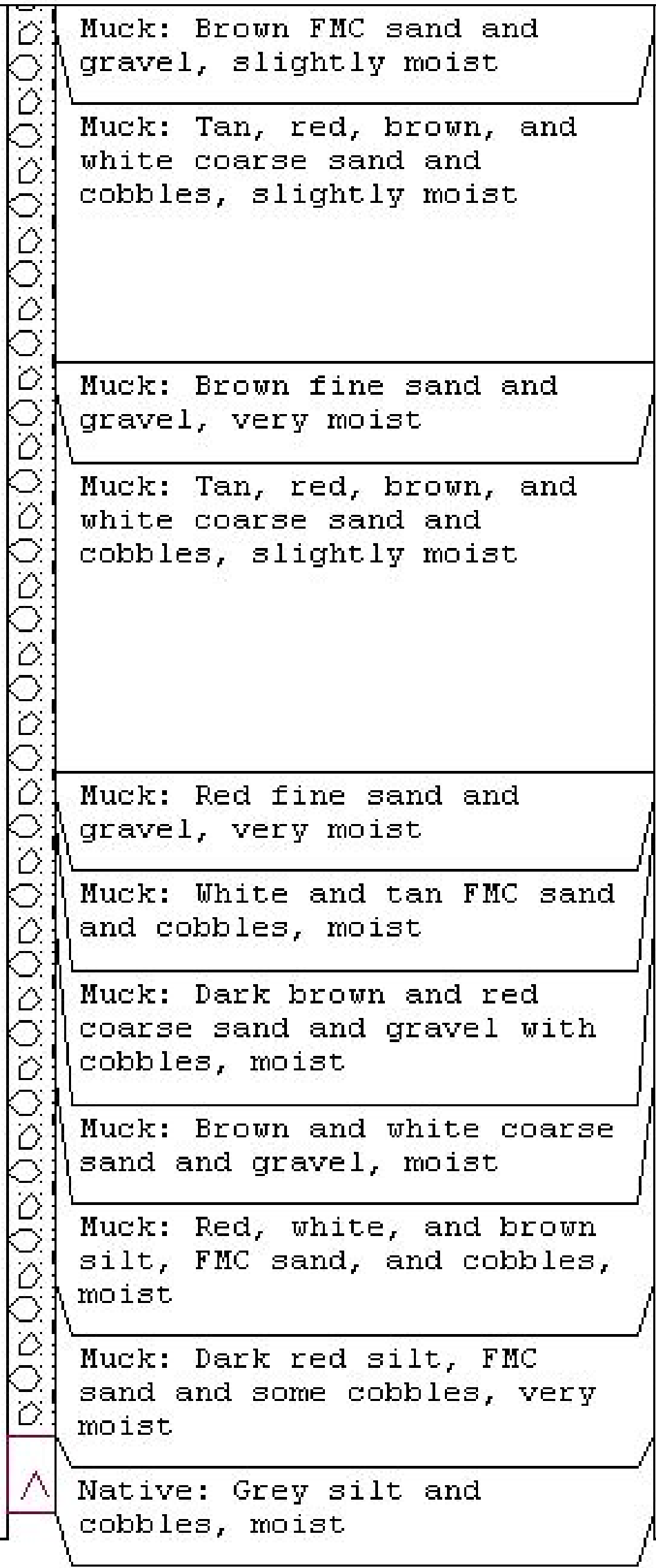

\begin{tabular}{|c|c|c|}
\hline 2.9 & & $\mathrm{BKG}$ \\
\hline 0.0 & & $\mathrm{BKG}$ \\
\hline 0.0 & & $\mathrm{BKG}$ \\
\hline 0.0 & & $\mathrm{BKG}$ \\
\hline 0.0 & 7 & $\begin{array}{l}2071 \\
(\mathrm{BKG})\end{array}$ \\
\hline 0.0 & & $\mathrm{BKG}$ \\
\hline 1.8 & & $\mathrm{BKG}$ \\
\hline 0.0 & & $\mathrm{BKG}$ \\
\hline 1.7 & & $\mathrm{BKG}$ \\
\hline 6.2 & 0 & $\begin{array}{l}194.5 \\
\text { (BKG) }\end{array}$ \\
\hline
\end{tabular}


Project Hame: CAU 383 E-Tunnel sites

Project Humber: DT04-320

Borehole Number: EMHOg

Logged By: M. MeLane

Drilled By: Boart-Longyear

Drilling Method: Rotosonic
Date Started: $6 / 11 / 04$

Date Completed: $6 / 11 / 04$

Elevation: 6121.9

Horthing: 887824.4

Easting: 637880.4

Total Depth: $25.0 \mathrm{ft}$.

\begin{tabular}{|c|c|c|c|c|}
\hline \multirow{2}{*}{ 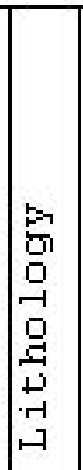 } & \multirow[b]{2}{*}{$\begin{array}{c}\text { Lithologic Description } \\
\text { Comments }\end{array}$} & Field & Screening & Results \\
\hline & & $\begin{array}{l}\text { VOCs } \\
\text { (ppm) }\end{array}$ & $\begin{array}{l}\text { Alpha } \\
\text { (dpm) }\end{array}$ & $\begin{array}{l}\text { Beta } \\
\text { (dpm) }\end{array}$ \\
\hline
\end{tabular}

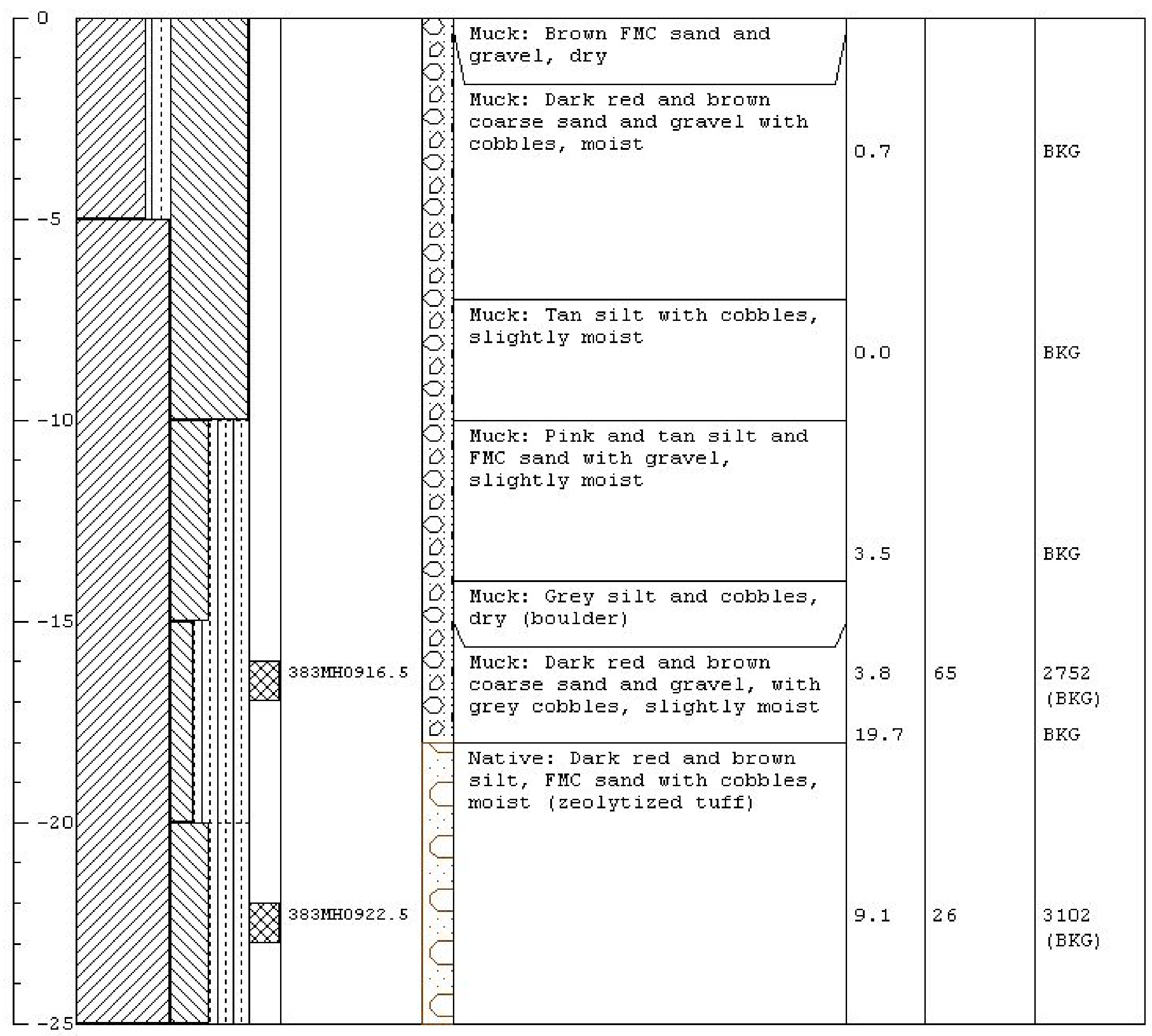


Project Hame: CAU 383 E-Tunnel sites

Project Number: DT04-320

Borehole Number: EMH10

Logged By: M. MeLane

Drilled By: Boart-Longyear

Drilling Method: Rotosonic
Date Started: $5 / 22 / 04$

Date Completed: $5 / 22 / 04$

Elevation: 6120.8

Horthing: 887395.0

Easting: 638571.8

Total Depth: $20.0 \mathrm{ft}$.

\begin{tabular}{|c|c|c|c|c|}
\hline \multirow{2}{*}{ 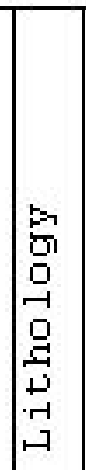 } & \multirow[b]{2}{*}{$\begin{array}{c}\text { Lithologic Description } \\
\text { Comments }\end{array}$} & Field & Screening & Results \\
\hline & & $\begin{array}{l}\text { VOCs } \\
\text { (ppri) }\end{array}$ & $\begin{array}{l}\text { Alpha } \\
\text { (dpm) }\end{array}$ & $\begin{array}{l}\text { Beta } \\
\text { (dpm) }\end{array}$ \\
\hline
\end{tabular}

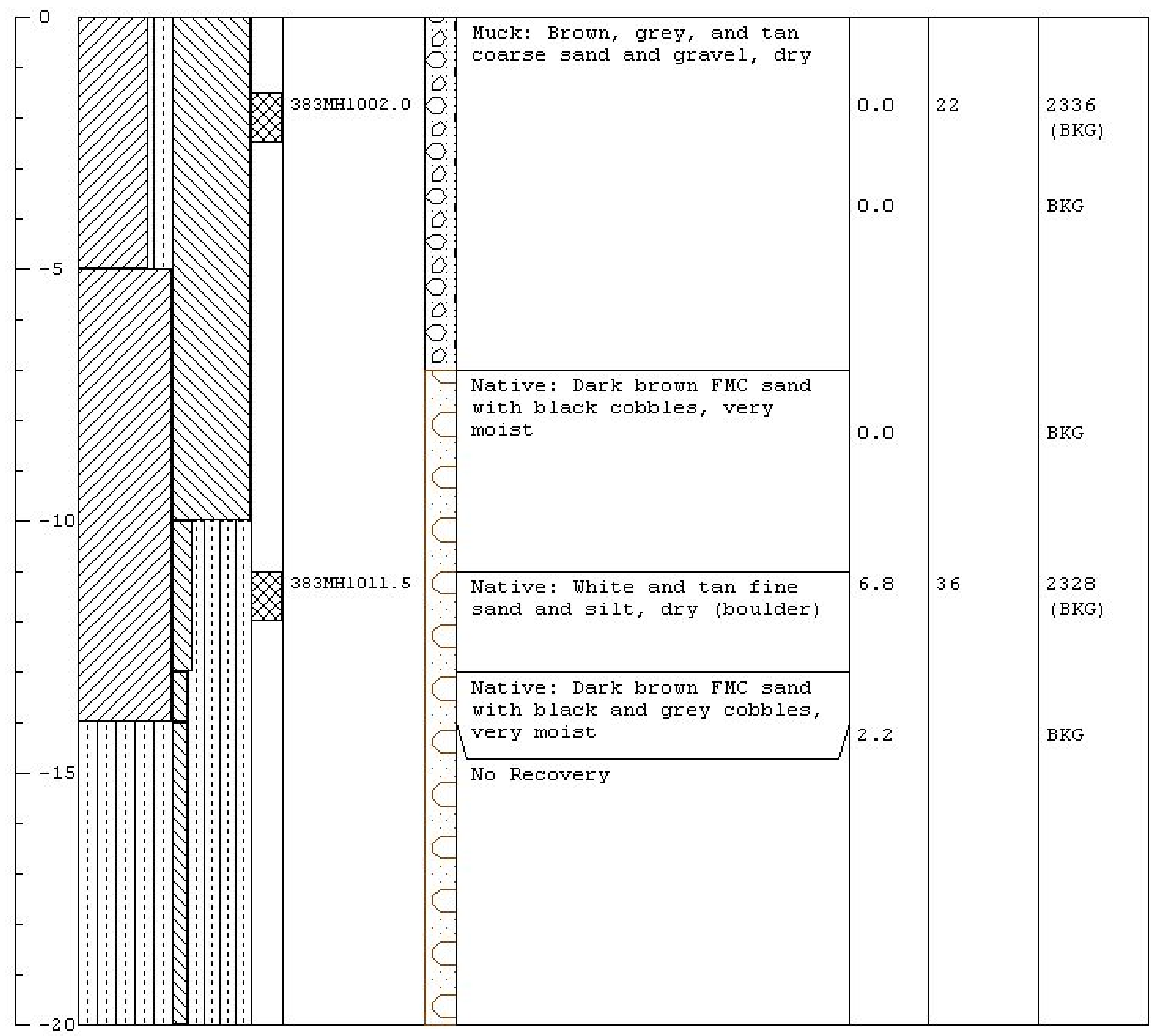


Project Hame: CAU 383 E-Tunnel sites

Project Number: DT04-320

Borehole Number: EMH11

Logged By: M. MeLane

Drilled By: Boart-Longyear

Drilling Method: Rotosonic
Date Started: $6 / 11 / 04$

Date Completed: $6 / 11 / 04$

Elevation: 6116.5

Horthing: 887858.6

Easting: 637782.5

Total Depth: $4.5 \mathrm{ft}$.

\begin{tabular}{|c|c|c|c|c|c|c|c|c|c|}
\hline \multirow[b]{2}{*}{ 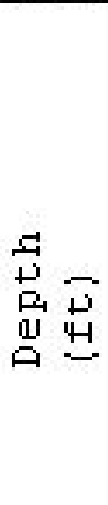 } & \multirow[b]{2}{*}{ 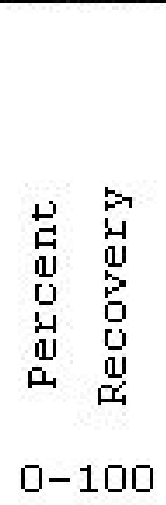 } & \multirow{2}{*}{\multicolumn{2}{|c|}{ 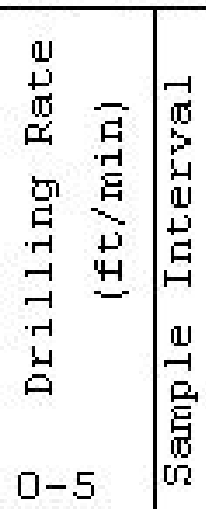 }} & \multirow[b]{2}{*}{ 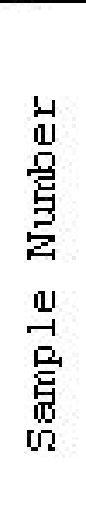 } & \multirow[b]{2}{*}{ 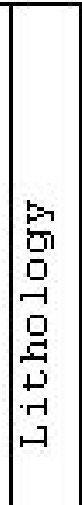 } & \multirow[b]{2}{*}{$\begin{array}{c}\text { Lithologic Description } \\
\text { Comments }\end{array}$} & \multicolumn{3}{|c|}{ Field Screening Results } \\
\hline & & & & & & & $\begin{array}{l}\text { VOCs } \\
\text { (ppm) }\end{array}$ & $\begin{array}{l}\text { Alpha } \\
\text { (dpm) }\end{array}$ & $\begin{array}{l}\text { Beta } \\
\text { (dpm) }\end{array}$ \\
\hline
\end{tabular}

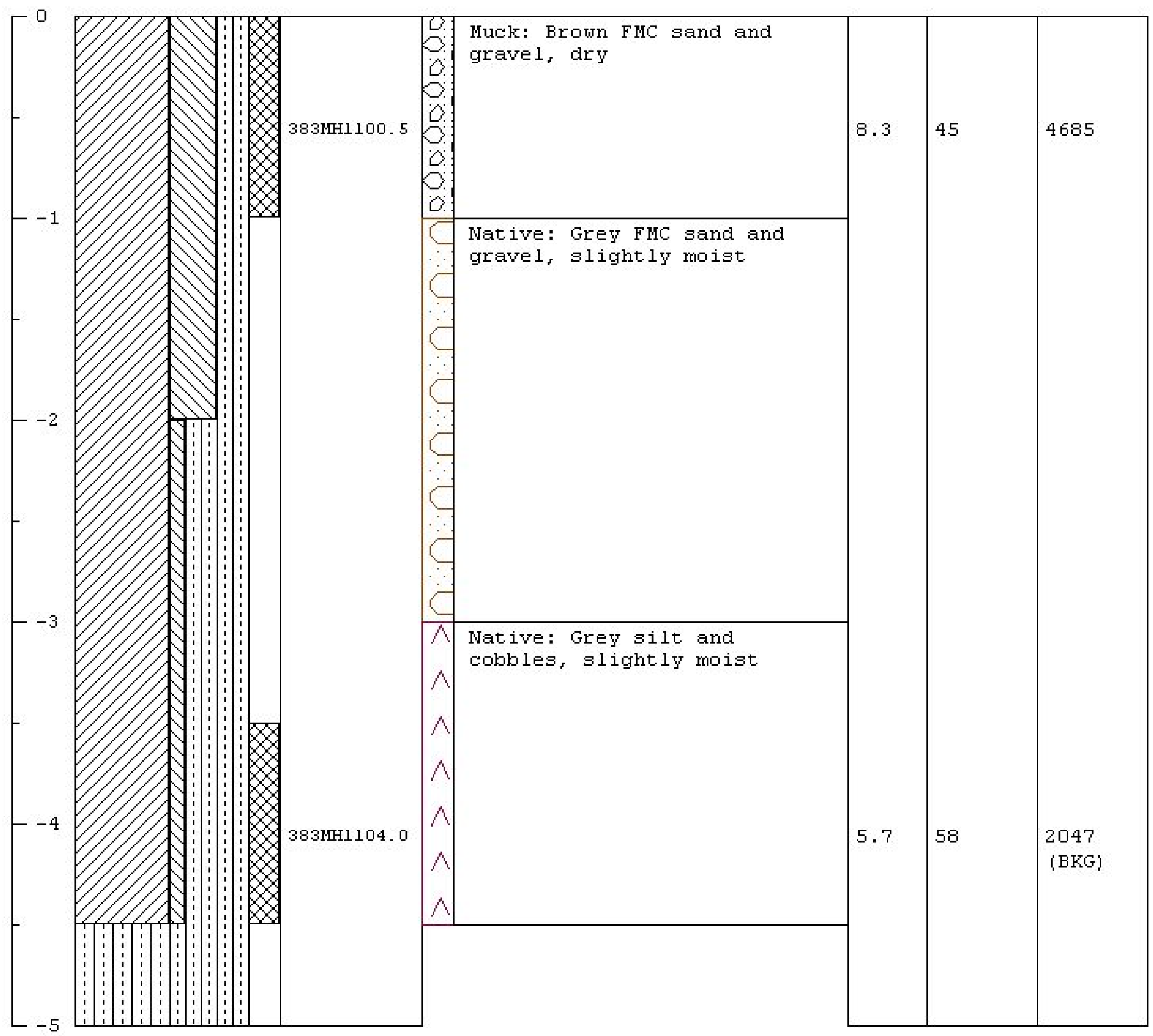


Project Hame: CAU 383 E-Tunnel sites

Project Number: DT04-320

Borehole Number: EMH12

Logged By: M. MeLane

Drilled By: Boart-Longyear

Drilling Method: Rotosonic
Date Started: $5 / 23 / 04$

Date completed: $5 / 23 / 04$

Elevation: 6124.1

Horthing: 887374.4

Easting: 638473.4

Total Depth: $25.5 \mathrm{ft}$.

\begin{tabular}{|c|c|c|c|c|}
\hline \multirow[b]{2}{*}{ 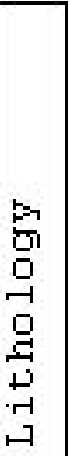 } & \multirow[b]{2}{*}{$\begin{array}{c}\text { Lithologic Description } \\
\text { Comments }\end{array}$} & \multicolumn{3}{|c|}{ Field Screening Results } \\
\hline & & $\begin{array}{l}\text { VOCs } \\
\text { (ppm) }\end{array}$ & $\begin{array}{l}\text { Alpha } \\
\text { (dpm) }\end{array}$ & $\begin{array}{l}\text { Beta } \\
\text { (dpm) }\end{array}$ \\
\hline
\end{tabular}

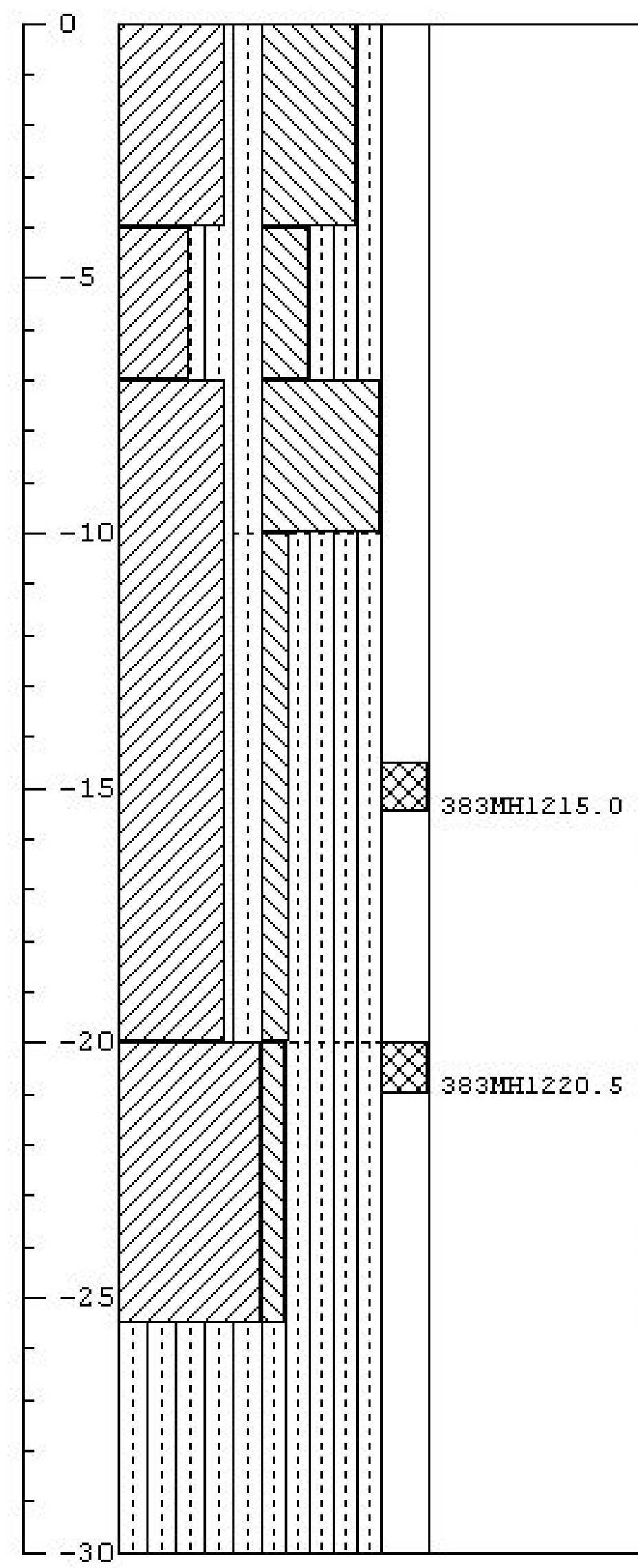

Muck: Light brown FMC sand and gravel, plant roots at $0.1 \mathrm{ft}$, dry<smiles>C1=C[GeH]=C1</smiles>

C. Muck: Dark brown and black

$D$ cobbles with wood chips,

C.). moist

$\rightarrow$

2.). Muck: Light brown silt and C. cobbles, dry (boulder)

Muck: Dark brown and black

c. cobbles with wood chips,

b.) moist

C. Muck: Red, brown, and white

i. coarse sand and gravel, dry

ㄴ.?.

.
Native: Dark grey silt and cobbles, moist
19

BKG

BKG

BKG

0.0 
Project Hame: CAU 383 E-Tunnel sites

Project Number: DT04-320

Borehole Number: EMH13

Logged By: M. MeLane

Drilled By: Boart-Longyear

Drilling Method: Rotosonic
Date Started: $5 / 25 / 04$

Date Completed: 5/25/04

Elevation: 6136.5

Horthing: 887492.0

Easting: 638209.0

Total Depth: $21.0 \mathrm{ft}$.

Lithologic Description

Comments

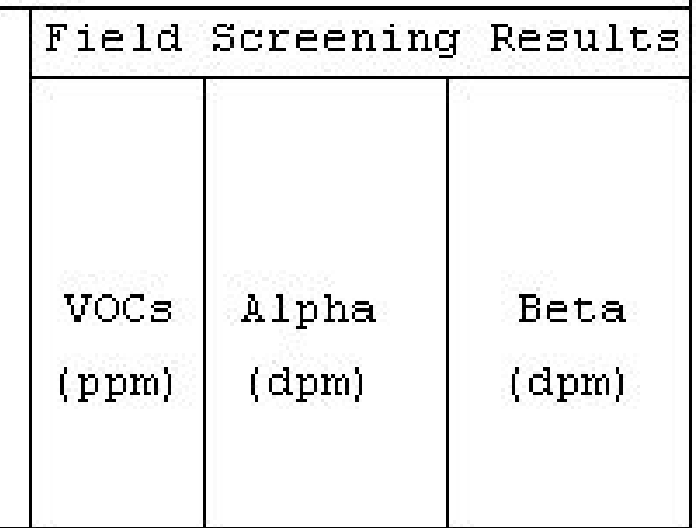

Contact $20.0 \mathrm{ft}$, Samples $383 \mathrm{MH} 1306.5,383 \mathrm{MH} 1320.5$.

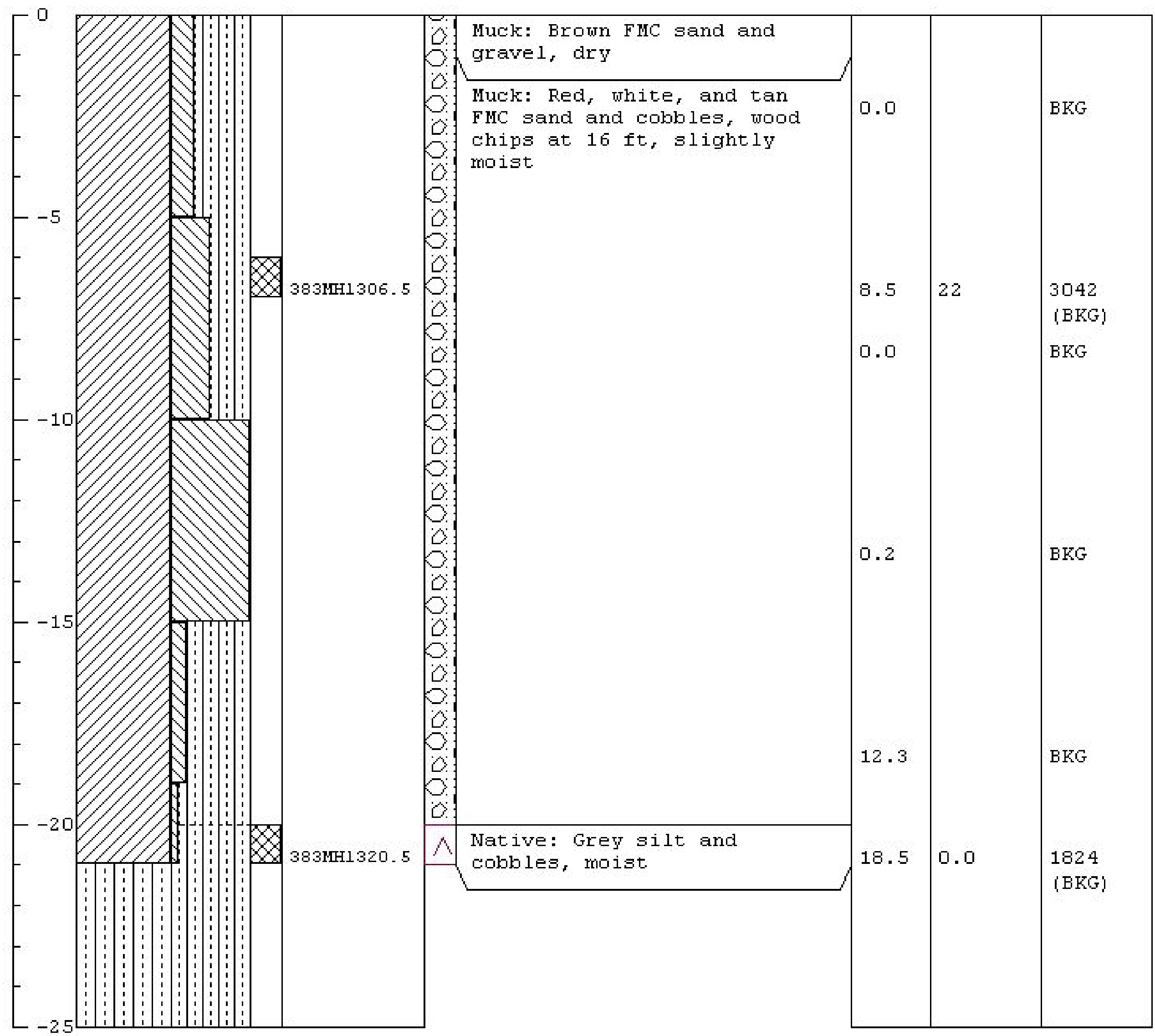


Project Hame: CAU 383 E-Tunnel sites

Project Number: DT04-320

Borehole Number: EMH14

Logged By: M. MeLane

Drilled By: Boart-Longyear

Drilling Method: Rotosonic
Date Started: $5 / 20 / 04$

Date Completed: $5 / 20 / 04$

Elevation: 6129.7

Horthing: 887613.0

Easting: 638322.2

Total Depth: $22.5 \mathrm{ft}$.

\begin{tabular}{|c|c|c|c|c|}
\hline \multirow{2}{*}{ 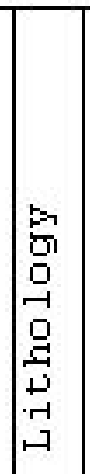 } & \multirow[b]{2}{*}{$\begin{array}{c}\text { Lithologic Description } \\
\text { Comments }\end{array}$} & \multicolumn{3}{|c|}{ Field Screening Results } \\
\hline & & $\begin{array}{l}\text { VOCs } \\
\text { (ppm) }\end{array}$ & $\begin{array}{l}\text { Alpha } \\
\text { (dpm) }\end{array}$ & $\begin{array}{l}\text { Beta } \\
\text { (dpm) }\end{array}$ \\
\hline
\end{tabular}
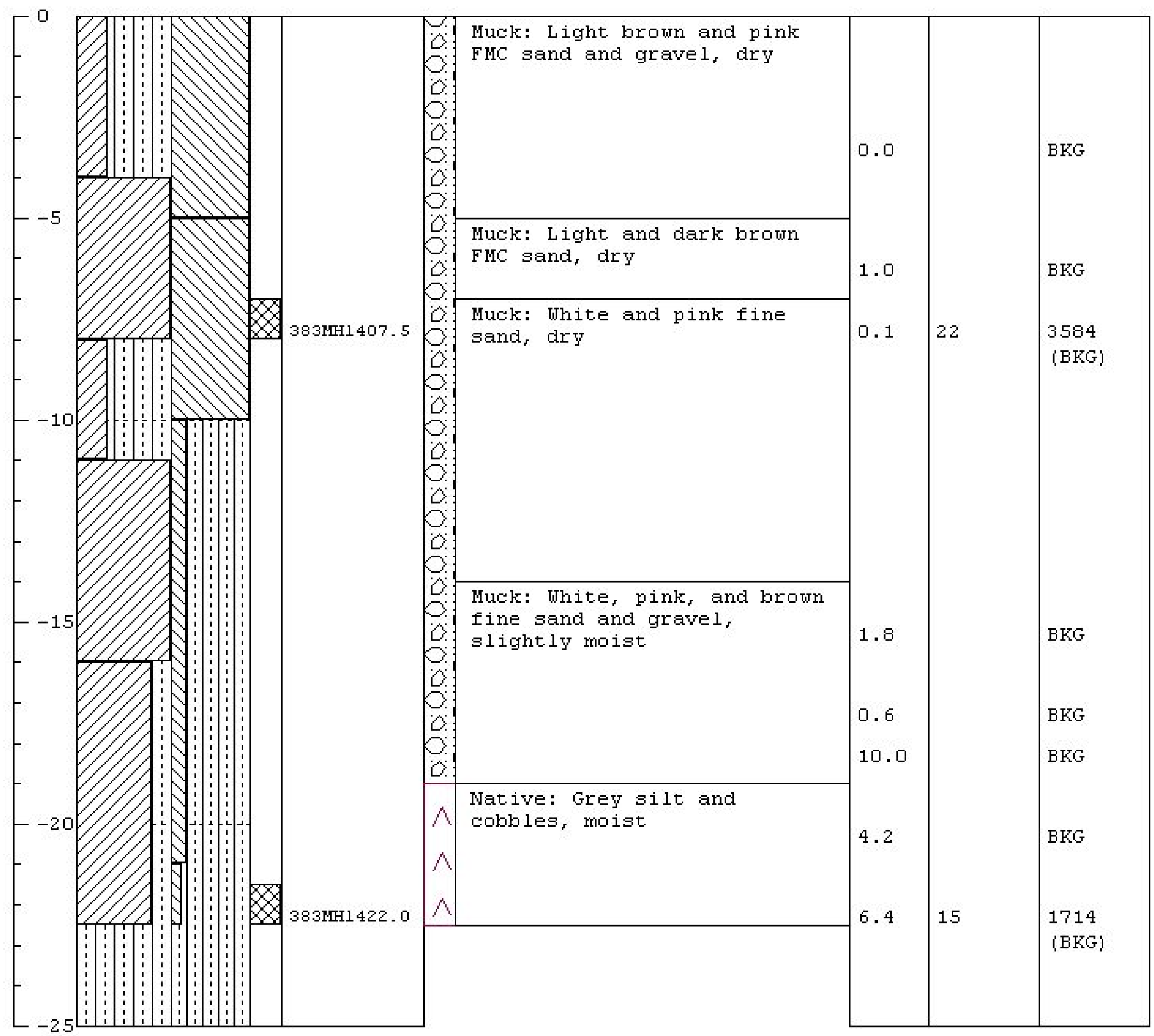
Project Hame: CAU 383 E-Tunnel sites

Project Humber: DT04-320

Borehole Number: EMH15

Logged By: M. MeLane

Drilled By: Boart-Longyear

Drilling Method: Rotosonic
Date Started: $5 / 24 / 04$

Date Completed: 5/24/04

Elevation: 6133.6

Horthing: 887479.0

Easting: 637912.1

Total Depth: 4.0 ft.

Lithologic Description

Comments

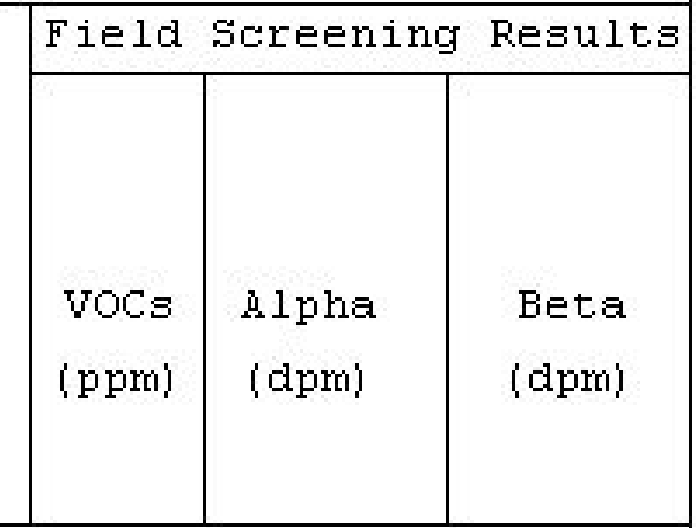

Contact g $2.0 \mathrm{ft}$, Samples $383 \mathrm{MH} 1501.0,383 \mathrm{MH1} 03.5,383 \mathrm{MH} 1503.5 \mathrm{X}$.
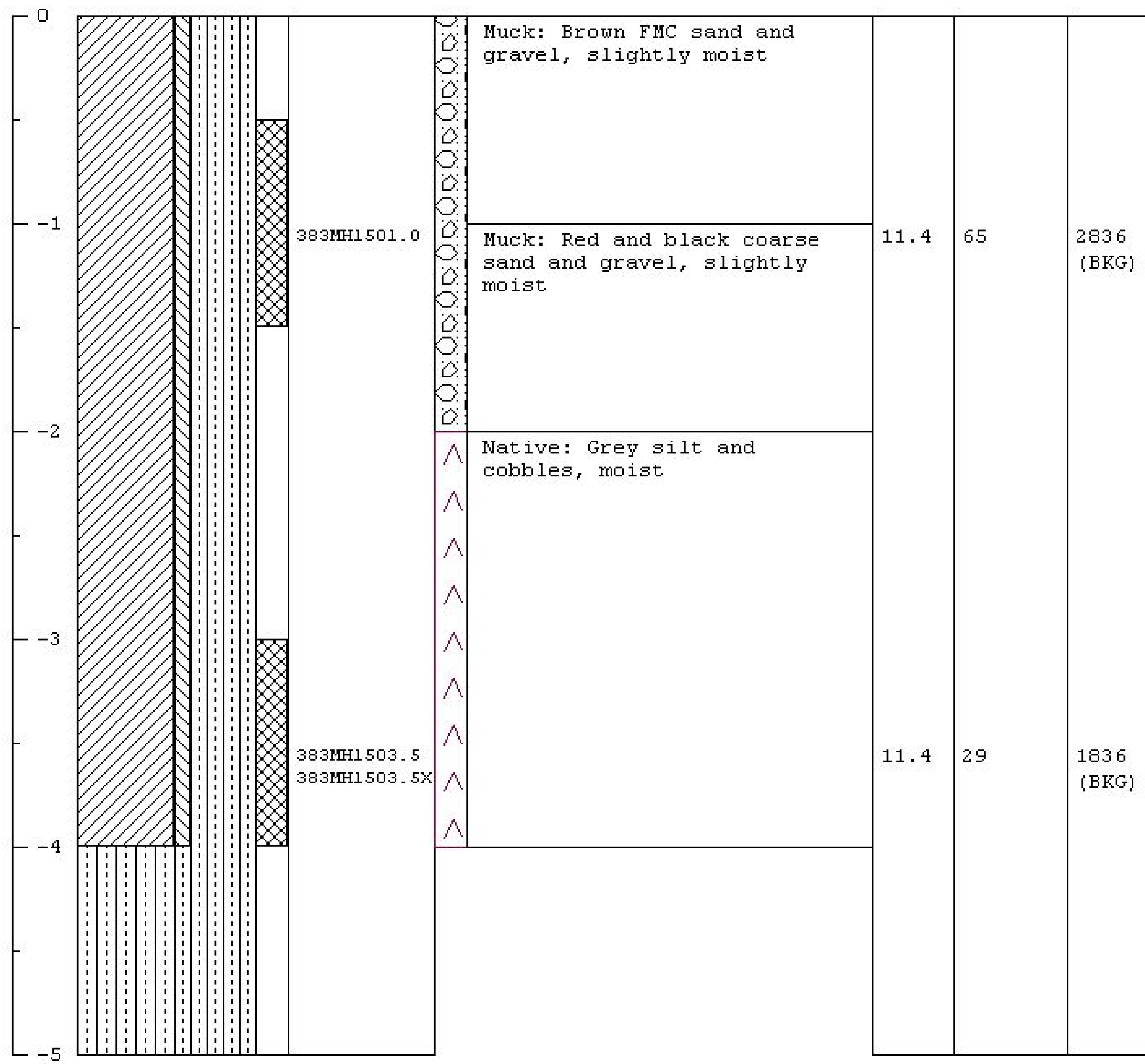
Project Hame: CAU 383 E-Tunnel sites

Project Number: DT04-320

Borehole Number: EMH16

Logged By: M. MeLane

Drilled By: Boart-Longyear

Drilling Method: Rotosonic
Date Started: $6 / 11 / 04$

Date Completed: $6 / 11 / 04$

Elevation: 6127.8

Horthing: 887726.3

Easting: 638178.0

Total Depth: 19.5 ft.

\begin{tabular}{|c|c|c|c|c|}
\hline \multirow{2}{*}{ 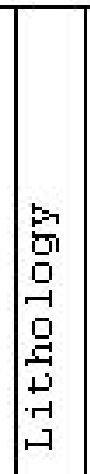 } & \multirow[b]{2}{*}{$\begin{array}{c}\text { Lithologic Description } \\
\text { Comments }\end{array}$} & \multicolumn{3}{|c|}{ Field Screening Results } \\
\hline & & $\begin{array}{l}\text { VOCs } \\
\text { (ppri) }\end{array}$ & $\begin{array}{l}\text { Alpha } \\
\text { (dpm) }\end{array}$ & $\begin{array}{l}\text { Beta } \\
\text { (dpm) }\end{array}$ \\
\hline
\end{tabular}
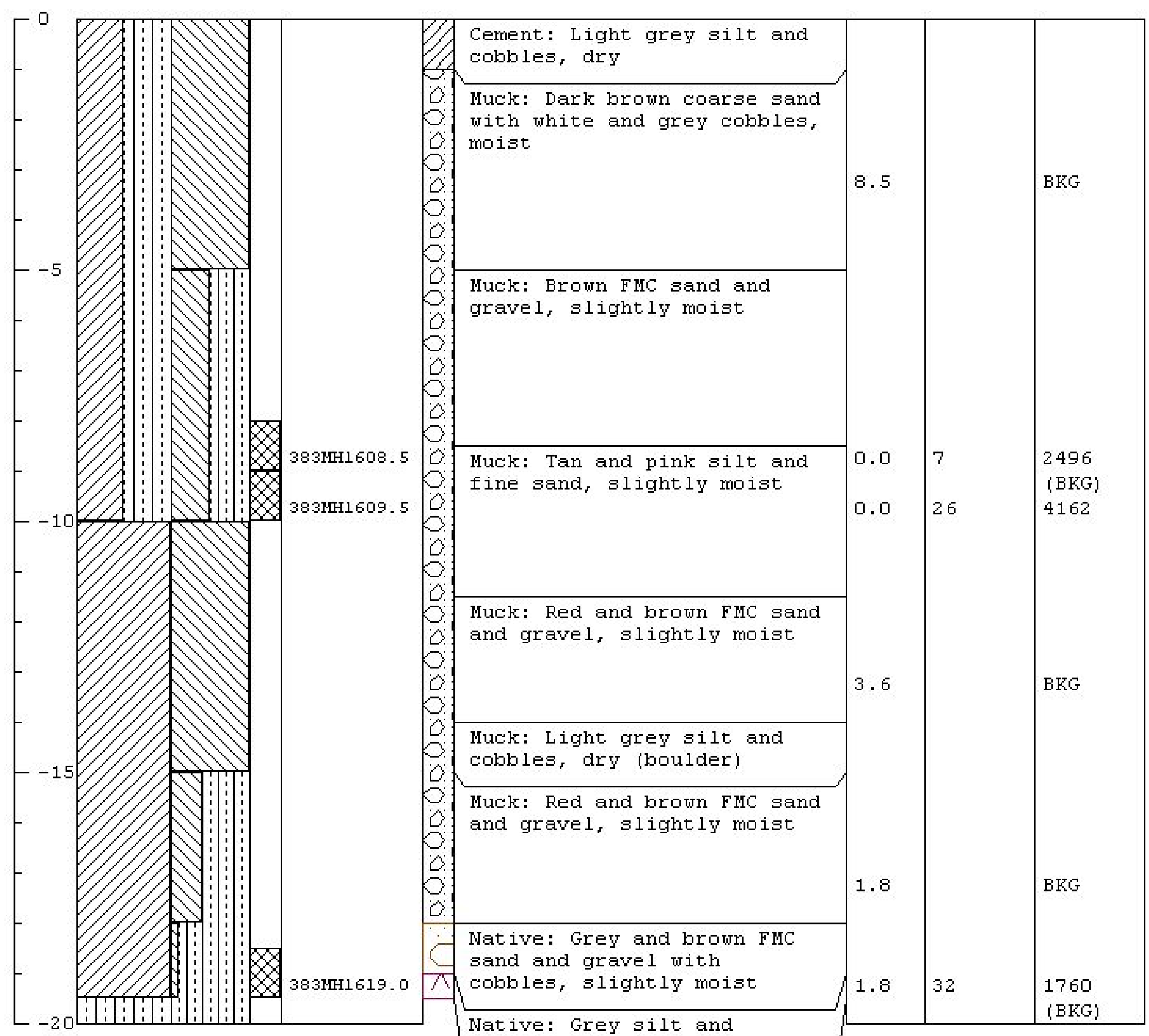
Project Hame: CAU 383 E-Tunnel sites

Project Humber: DTO4-320

Borehole Number: EMH17

Logged By: M. MeLane

Drilled By: Boart-Longyear

Drilling Method: Rotosonic
Date Started: $5 / 21 / 04$

Date Completed: $5 / 22 / 04$

Elevation: 6122.8

Horthing: 887435.9

Easting: 638653.1

Total Depth: 36.5 ft.

\begin{tabular}{|c|c|c|c|c|}
\hline \multirow{2}{*}{ 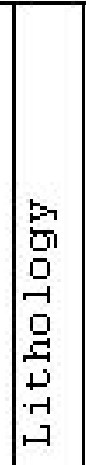 } & \multirow[b]{2}{*}{$\begin{array}{c}\text { Lithologic Description } \\
\text { Comments }\end{array}$} & Field & Screening & Results \\
\hline & & $\begin{array}{l}\text { VOCs } \\
\text { (ppri) }\end{array}$ & $\begin{array}{l}\text { Alpha } \\
\text { (dpm) }\end{array}$ & $\begin{array}{l}\text { Beta } \\
\text { (dpm) }\end{array}$ \\
\hline
\end{tabular}

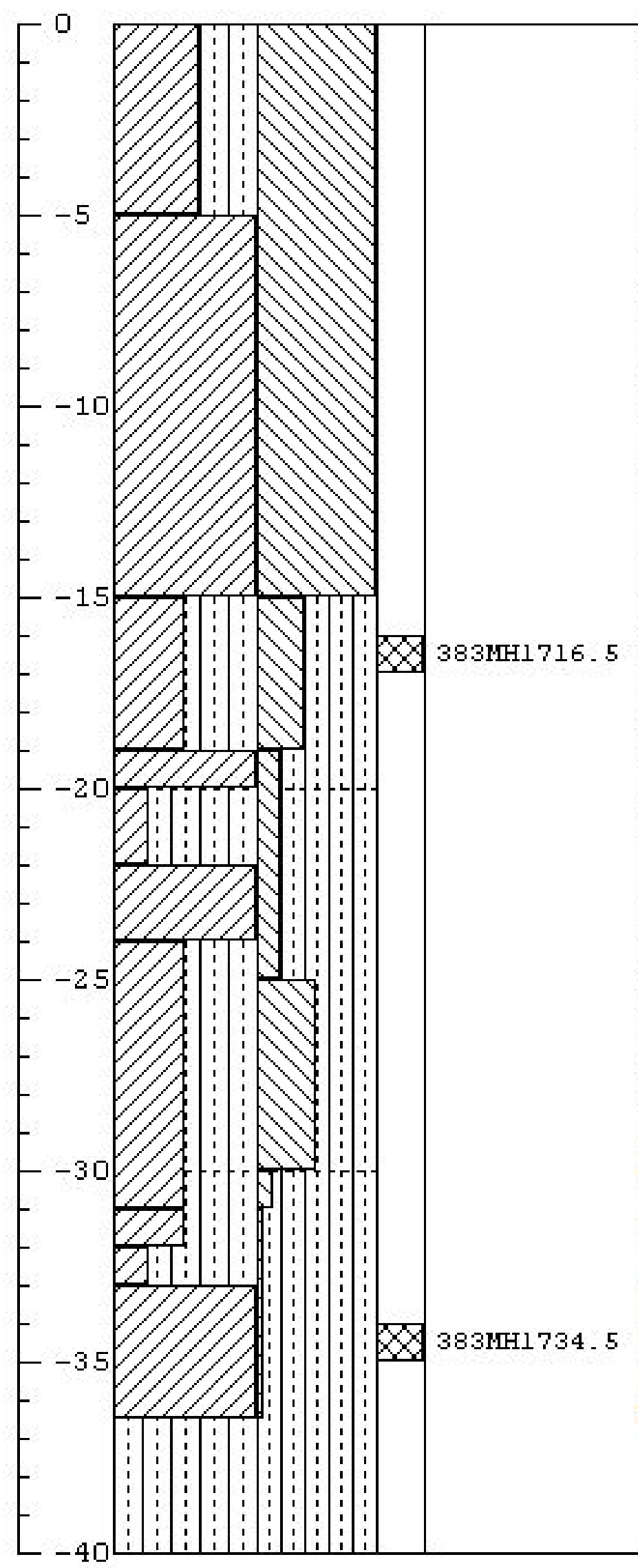

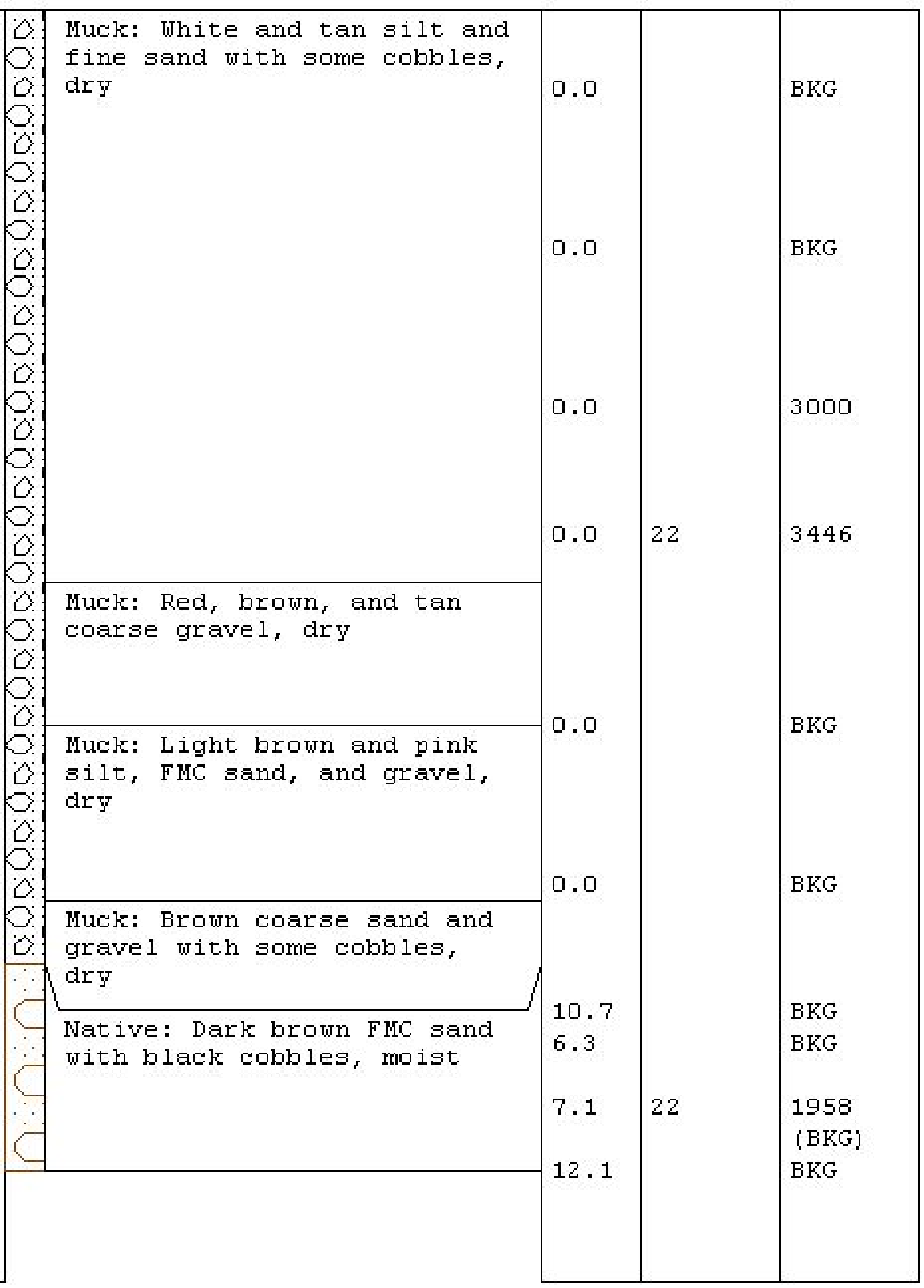


Project Hame: CAU 383 E-Tunnel sites

Project Humber: DTO4-320

Borehole Number: EMH18

Logged By: M. MeLane

Drilled By: Boart-Longyear

Drilling Method: Rotosonic
Date Started: $5 / 23 / 04$

Date Completed: $5 / 23 / 04$

Elevation: 6129.9

Horthing: 887333.0

Easting: 638343.0

Total Depth: $12.0 \mathrm{ft}$.

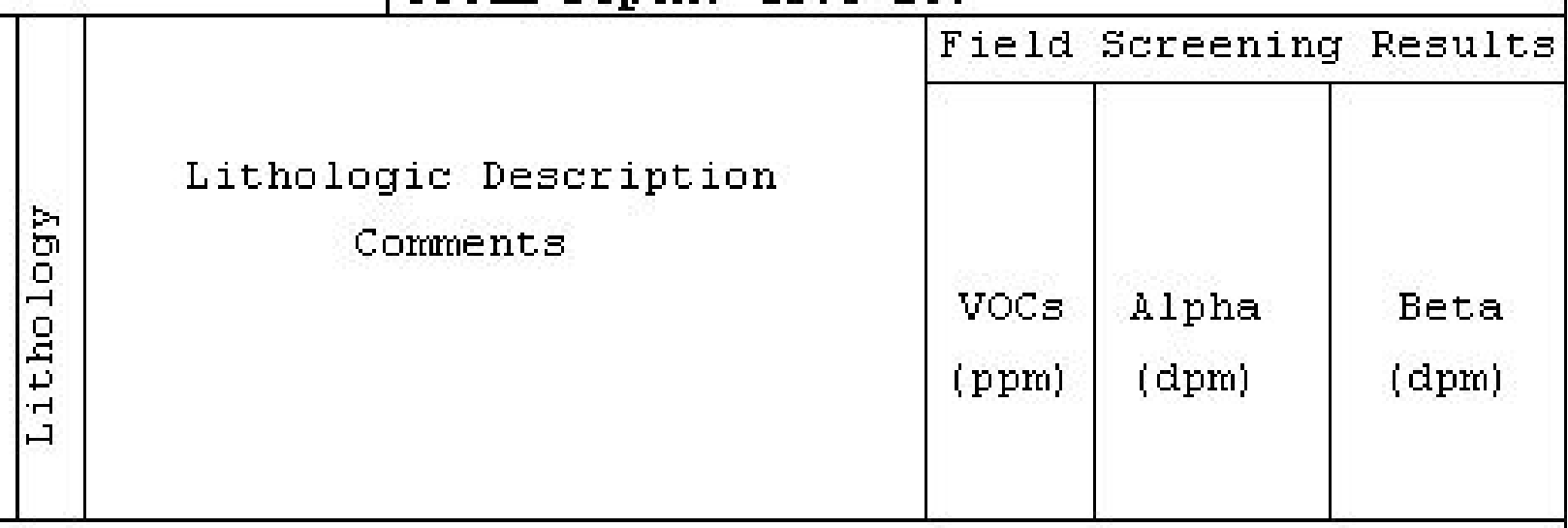

$383 \mathrm{MH} 1803.5,383 \mathrm{MH} 1811.5$.

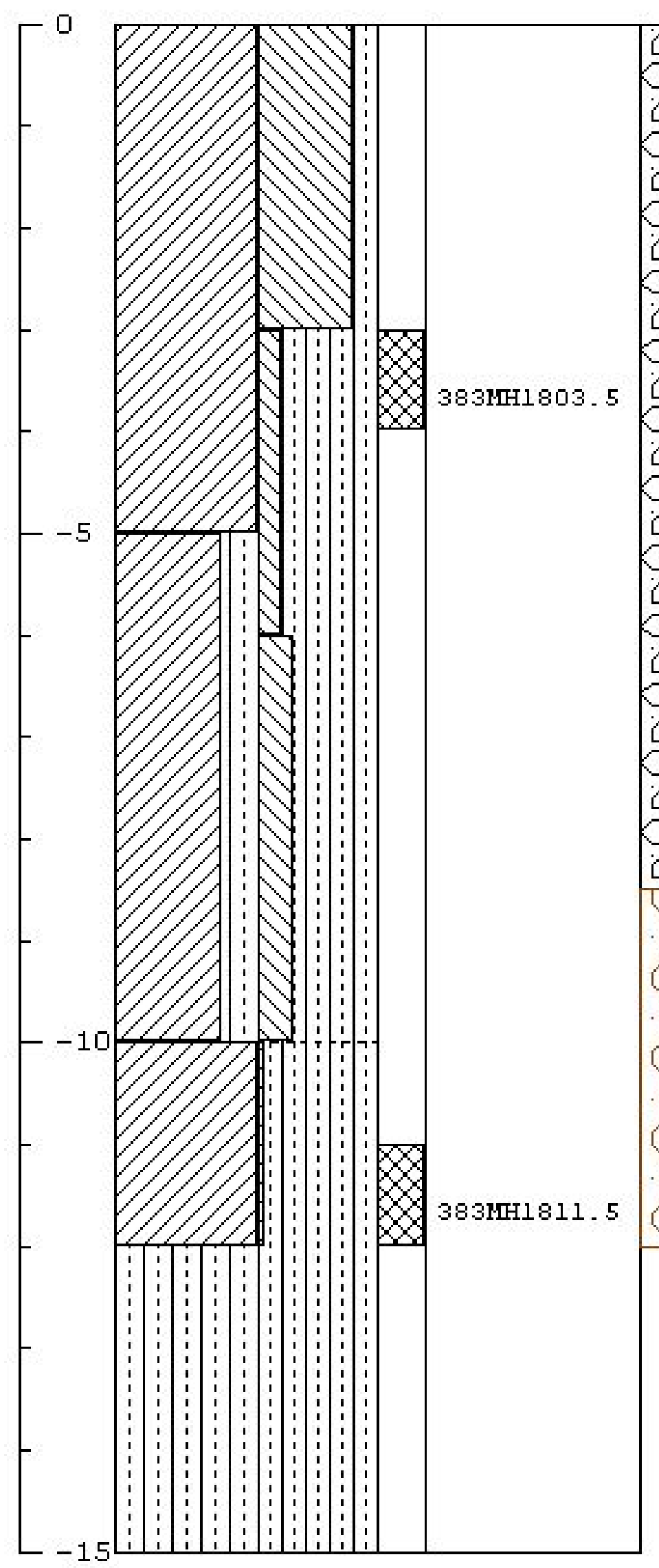

BKG

Muck: Tan, red, brown, and white FMC sand and gravel, dry

Native: Dark brown and grey FMC sand with black and white cobbles, moist 
Project Name: CAU 383 E-Tunnel sites

Project Humber: DT04-320

Borehole Number: EOBO1

Logged By: $M$. McLane

Drilled By: M. McLane

Drilling Method: Hand Sampled
Date Started: $6 / 01 / 04$

Date completed: $6 / 01 / 04$

Elevation: 6217.6

Horthing : 887570.3

Easting: 637635.3

Total Depth: 0.5 ft.

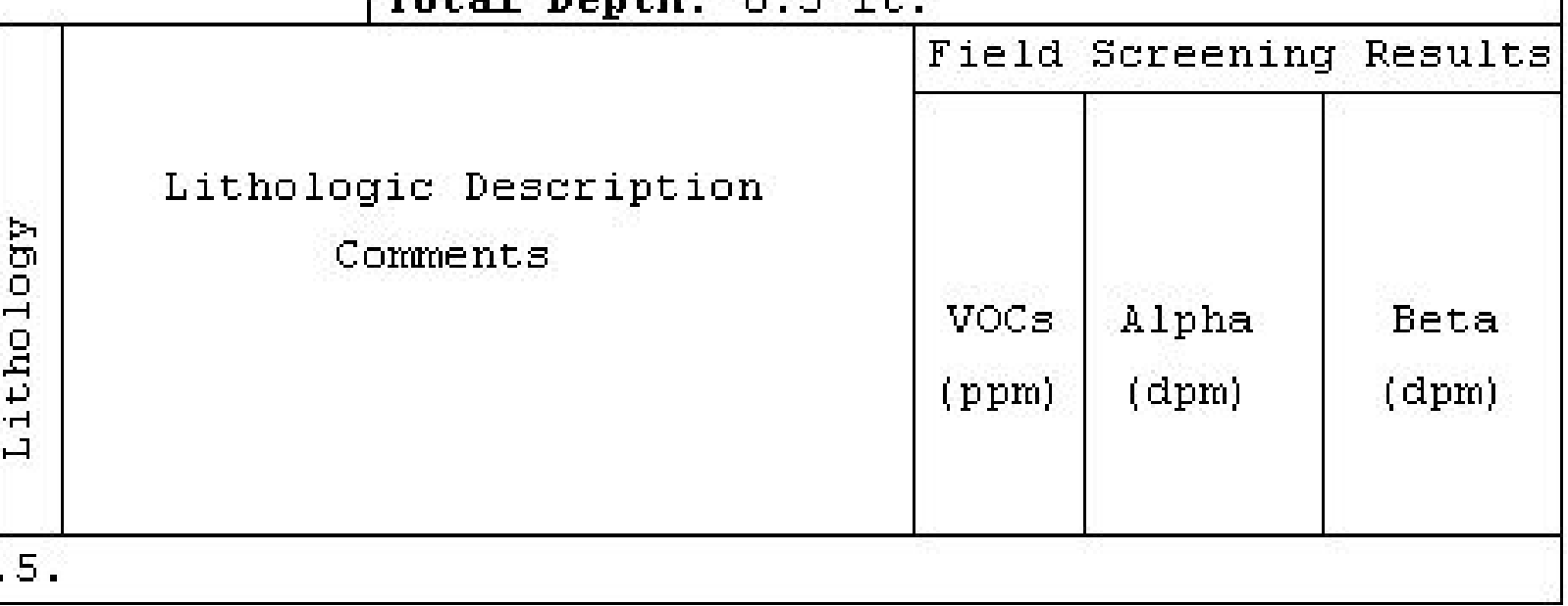

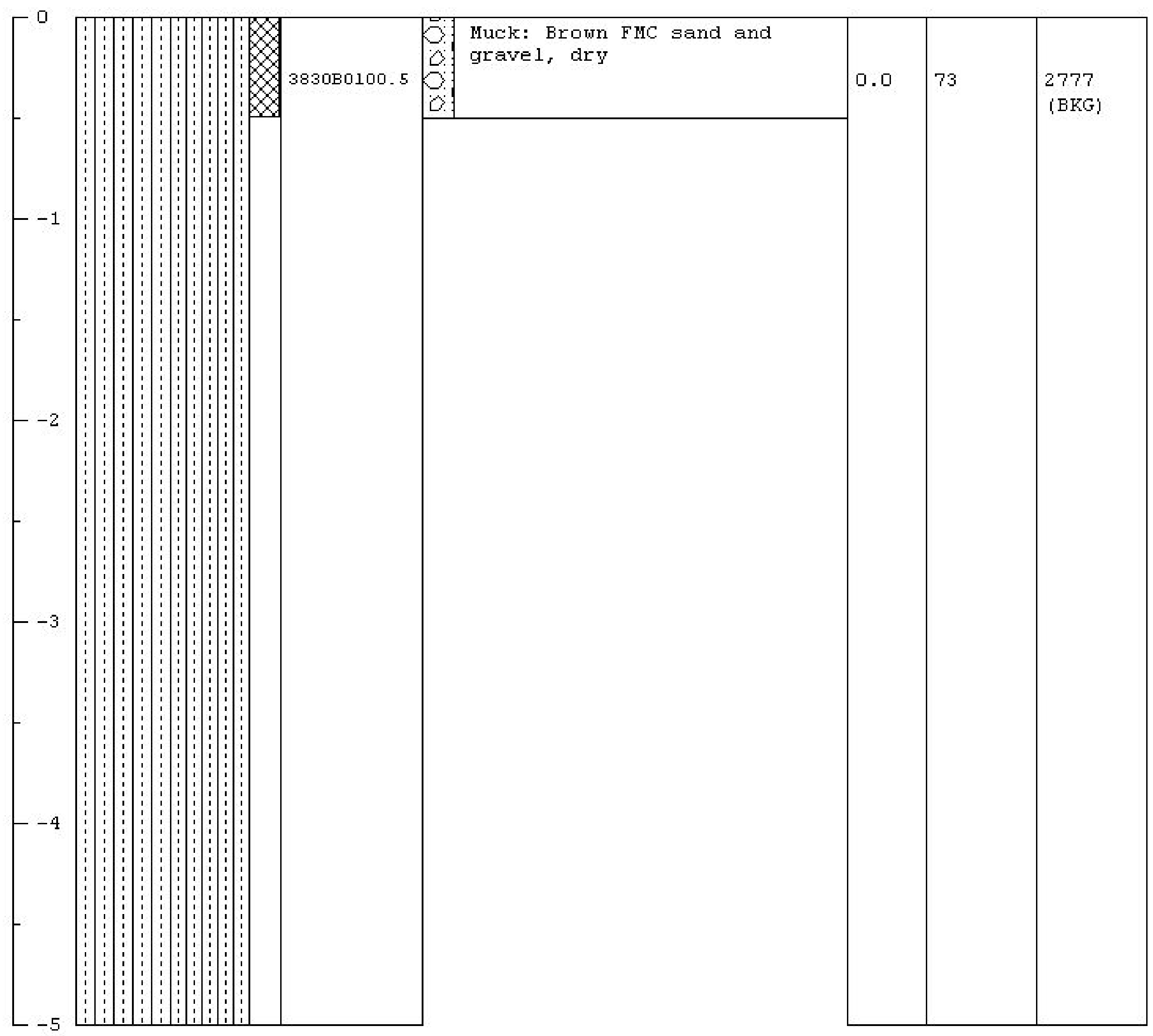


Project Name: CAU 383 E-Tunnel sites

Project Humber: DT04-320

Borehole Number: EOBO2

Logged By: $M$. McLane

Drilled By: M. McLane

Drilling Method: Hand Sampled
Date Started: $6 / 01 / 04$

Date completed: $6 / 01 / 04$

Elevation: 6218.0

Horthing: 887568.9

Easting: 637641.1

Total Depth: 0.5 ft.

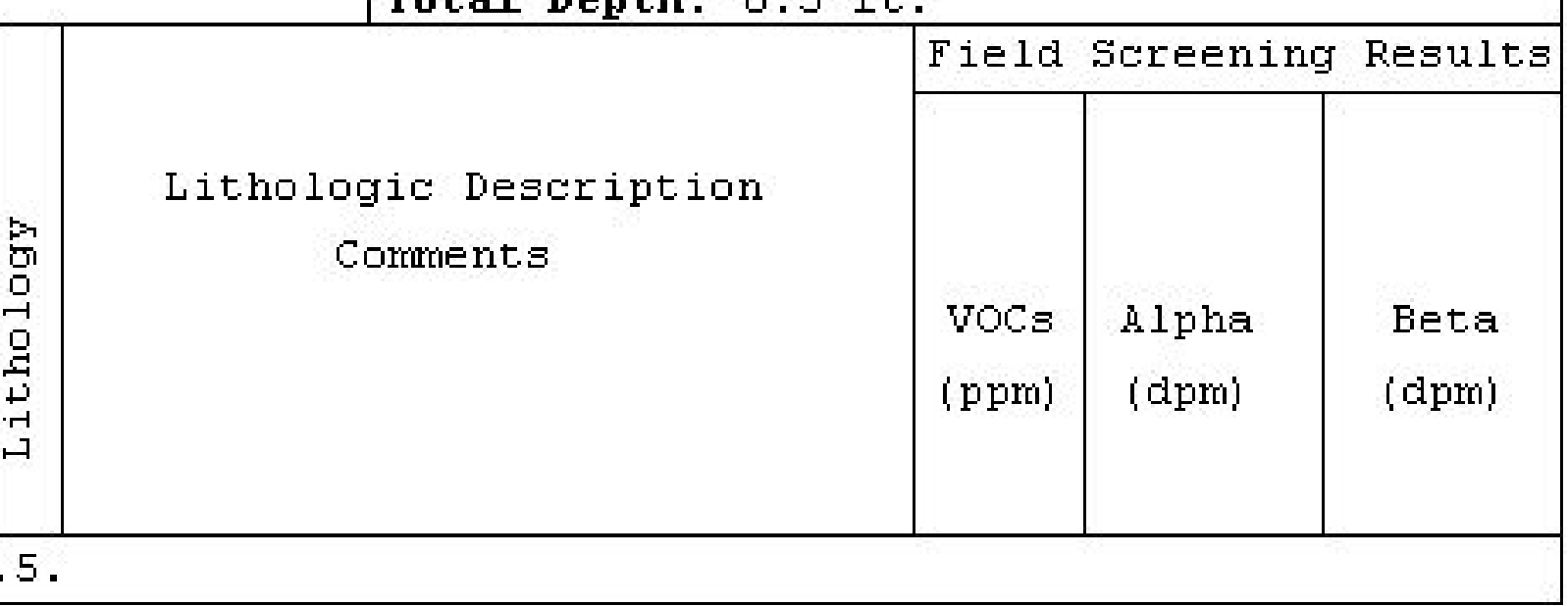

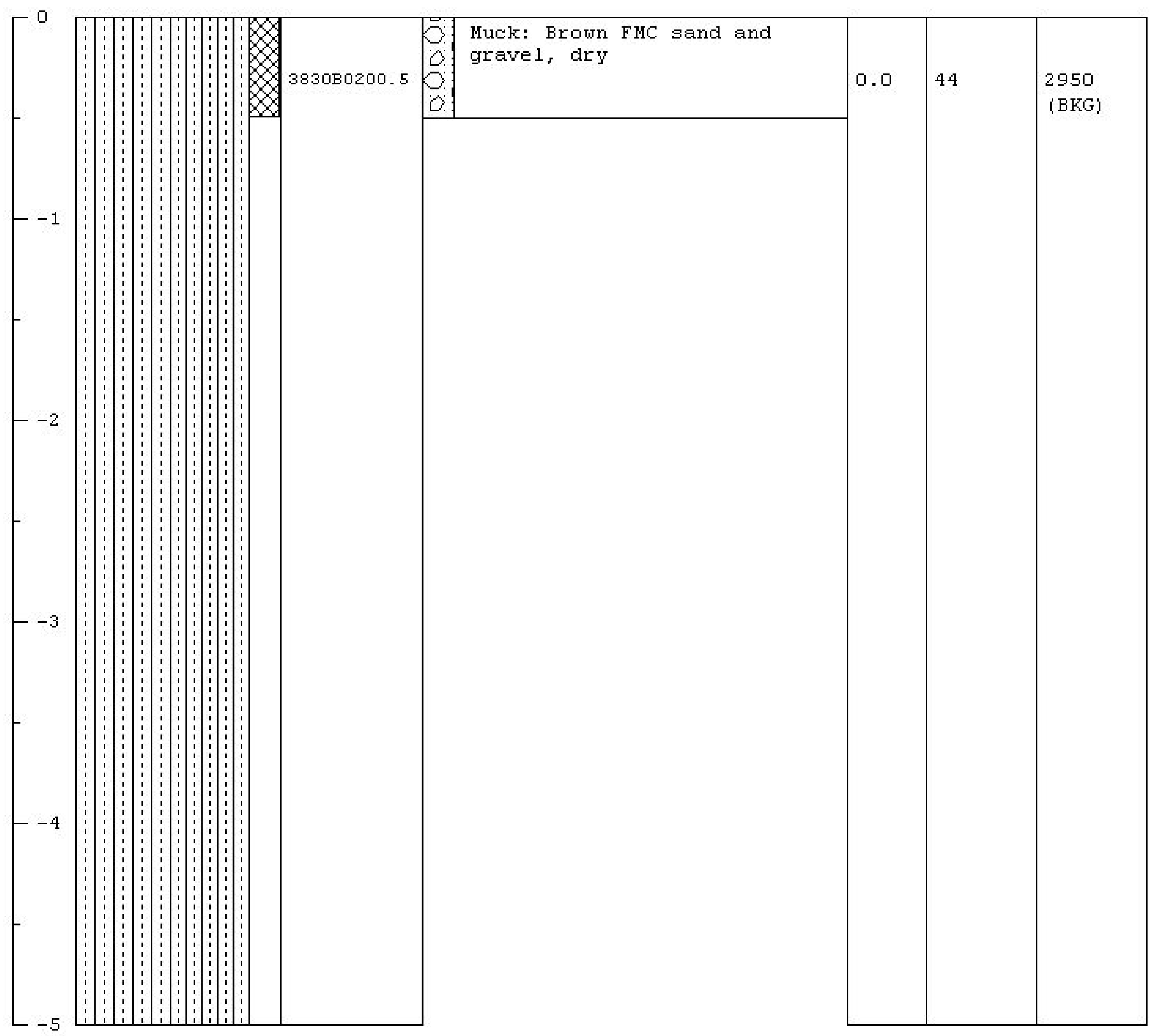


Project Name: CAU 383 E-Tunnel sites

Project Humber: DT04-320

Borehole Number: EOBO3

Logged By: $M$. McLane

Drilled By: M. McLane

Drilling Method: Hand Sampled
Date Started: $6 / 01 / 04$

Date completed: $6 / 01 / 04$

Elevation: 6213.9

Horthing: 887531.0

Easting: 637623.9

Total Depth: 0.5 ft.

Lithologic Description

Comments

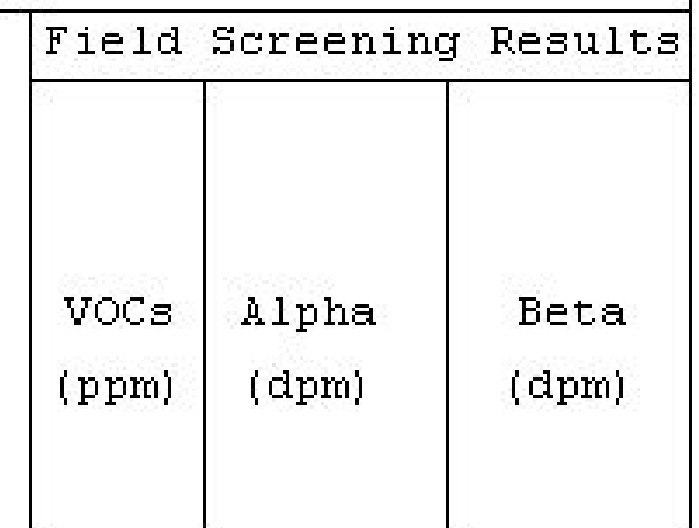

Contact o N/A, Samples 3830B0300.5.

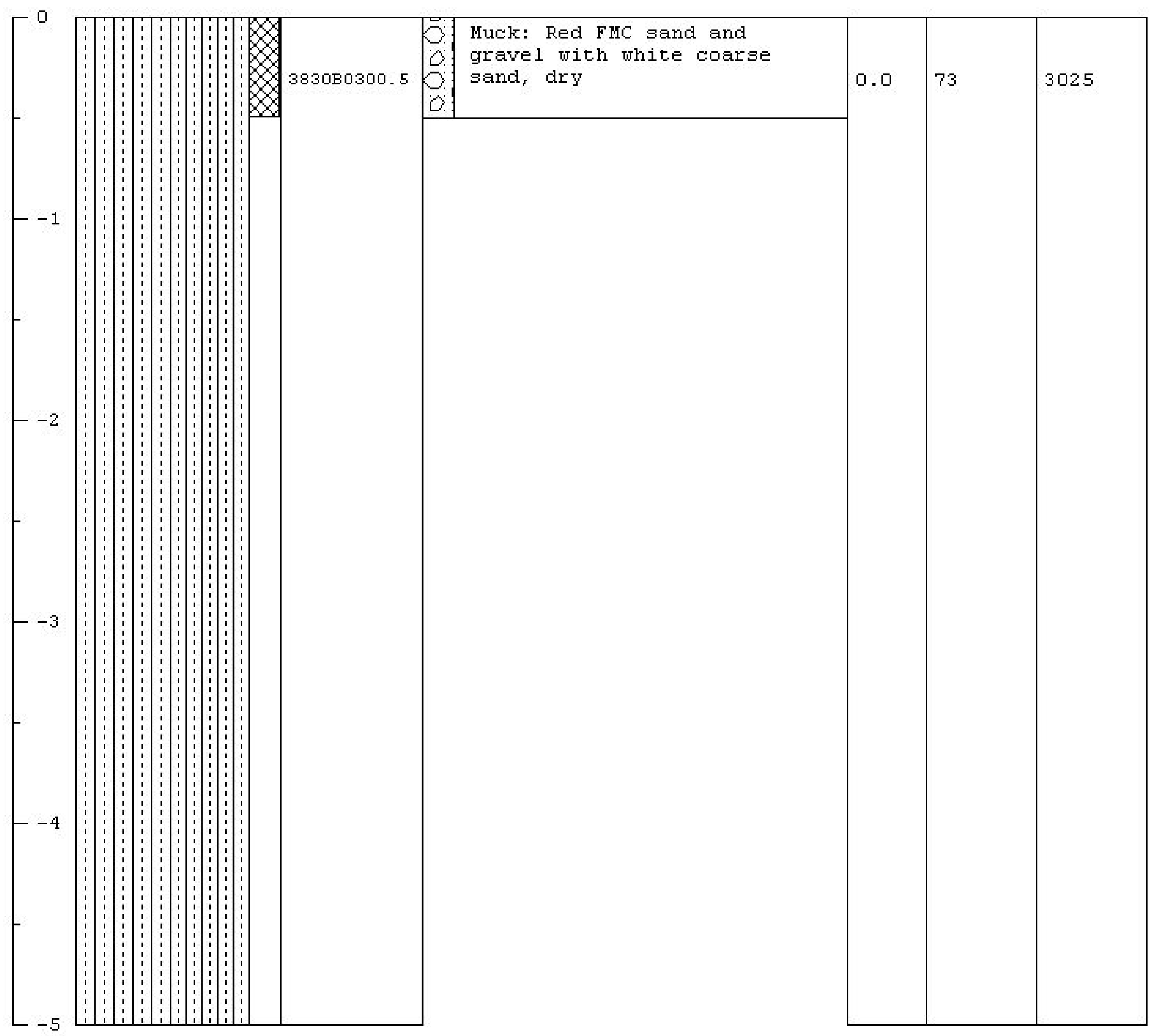


Project Name: CAU 383 E-Tunnel sites

Project Humber: DT04-320

Borehole Number: EOBO4

Logged By: $M$. McLane

Drilled By: M. McLane

Drilling Method: Hand Sampled
Date Started: $6 / 01 / 04$

Date completed: $6 / 01 / 04$

Elevation: 6220.9

Horthing: 887535.2

Easting: 637647.3

Total Depth: 0.5 ft.

Lithologic Description

Corments
Field Screening Results

\begin{tabular}{|c|c|c|}
\hline & & \\
voCs & slpha & Beta \\
(ppri) & (dpr) & (dpr) \\
& & \\
\hline
\end{tabular}

Contact o N/A, Sample 383080400.5 .

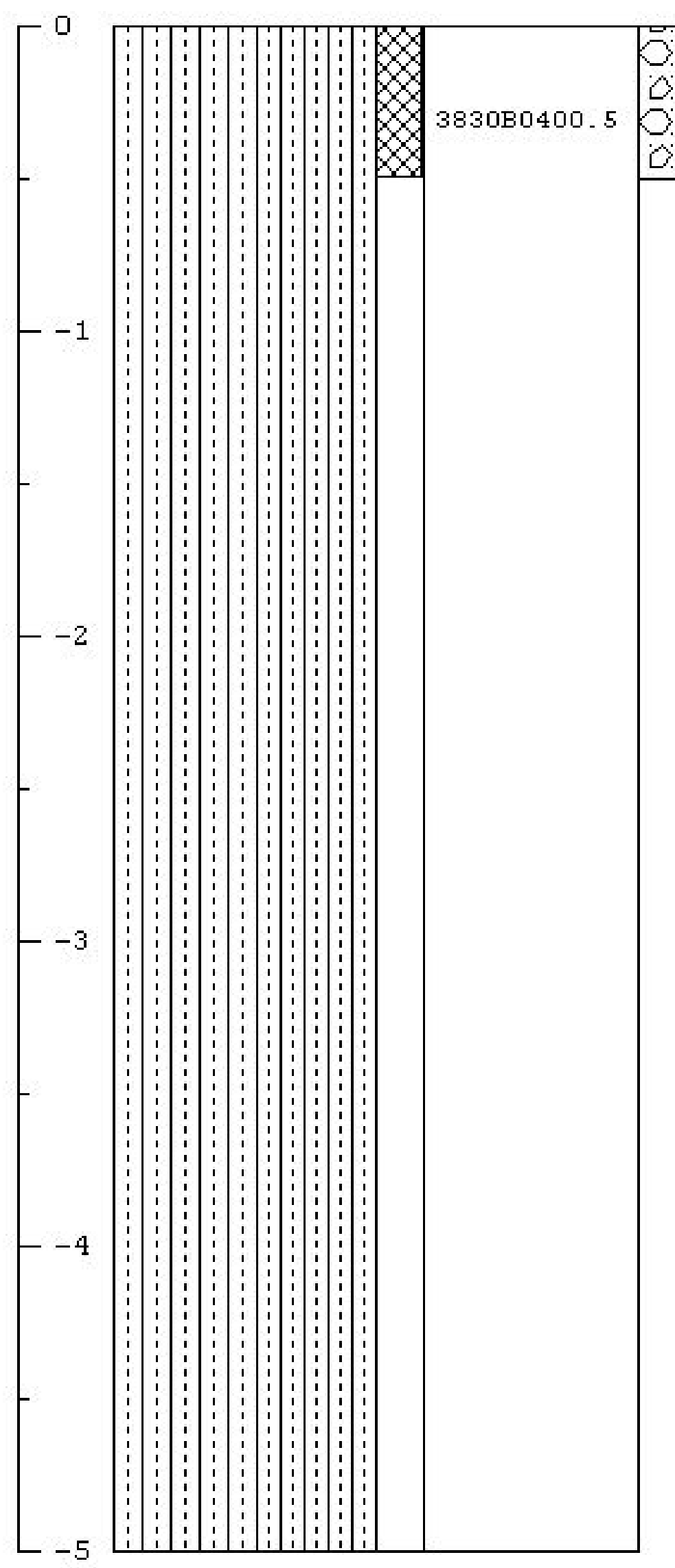

Muck: Brown FMC sand and gravel with white coarse sand, dry

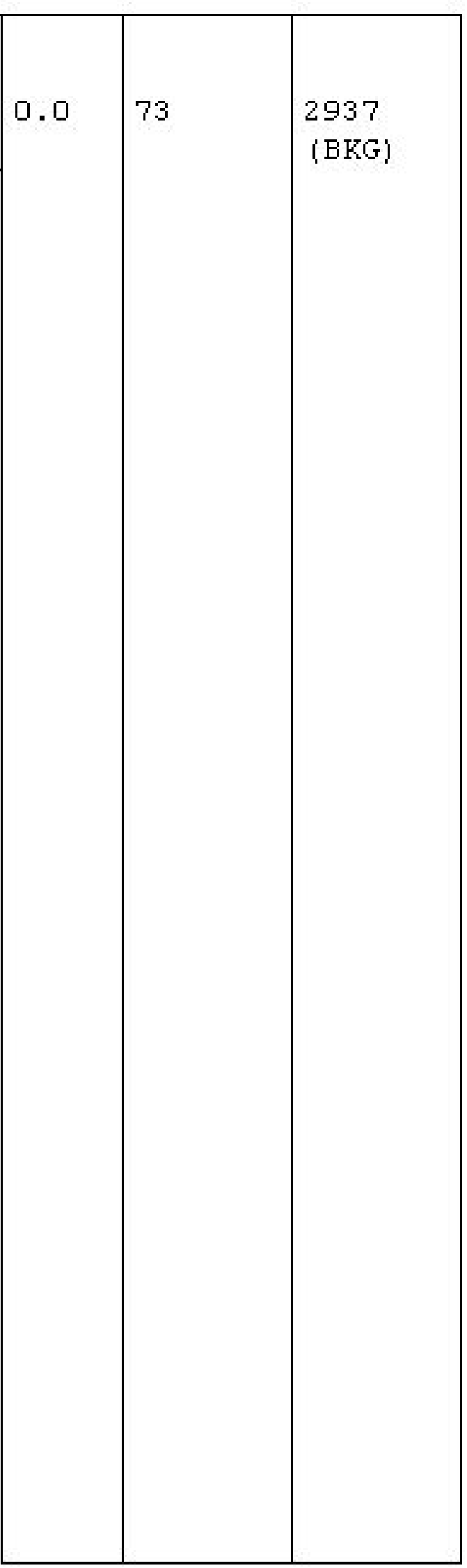


Project Name: CAU 383 E-Tunnel sites

Project Humber: DT04-320

Borehole Number: EOBO5

Logged By: $M$. McLane

Drilled By: M. McLane

Drilling Method: Hand Sampled
Date Started: $6 / 01 / 04$

Date completed: $6 / 01 / 04$

Elevation: 6218.2

Horthing: 887602.0

Easting: 637630.7

Total Depth: 0.5 ft.

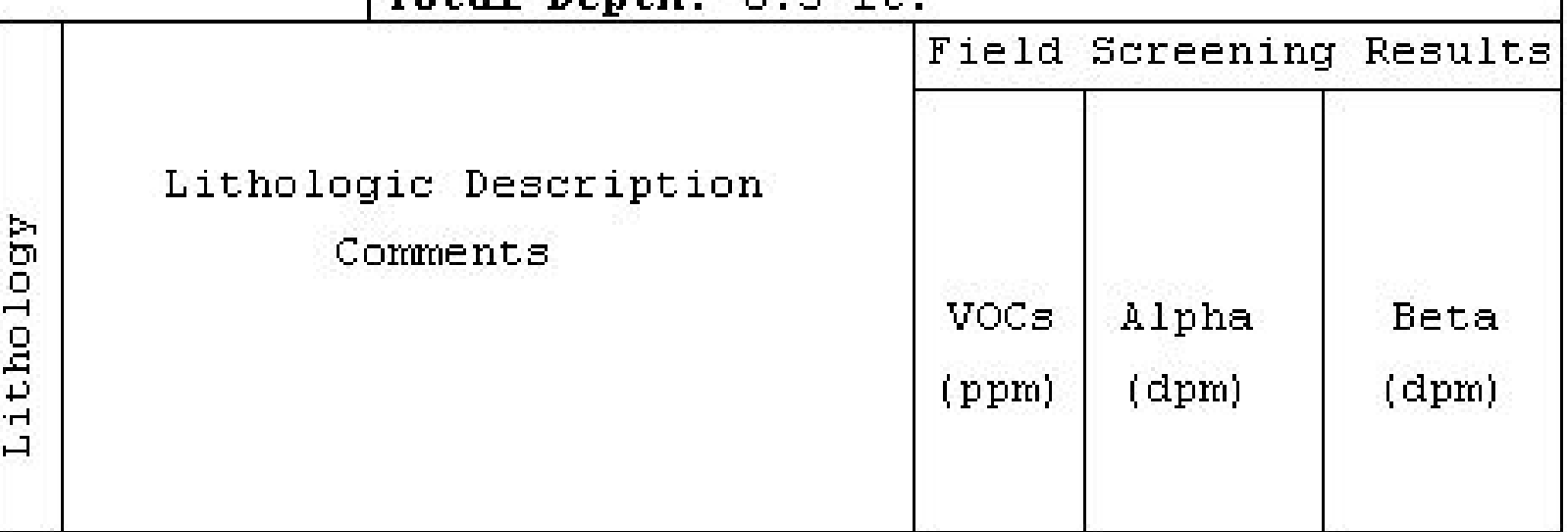

$3830 \mathrm{B0} 500.5 \mathrm{X}$.

Muck: Red FMC sand and gravel, dry 
Project Name: CAU 383 E-Tunnel sites

Project Humber: DT04-320

Borehole Number: EOBO6

Logged By: $M$. McLane

Drilled By: M. McLane

Drilling Method: Hand Sampled
Date Started: $6 / 01 / 04$

Date completed: $6 / 01 / 04$

Elevation: 6212.9

Horthing: 887599.8

Easting: 637658.3

Total Depth: 0.5 ft.

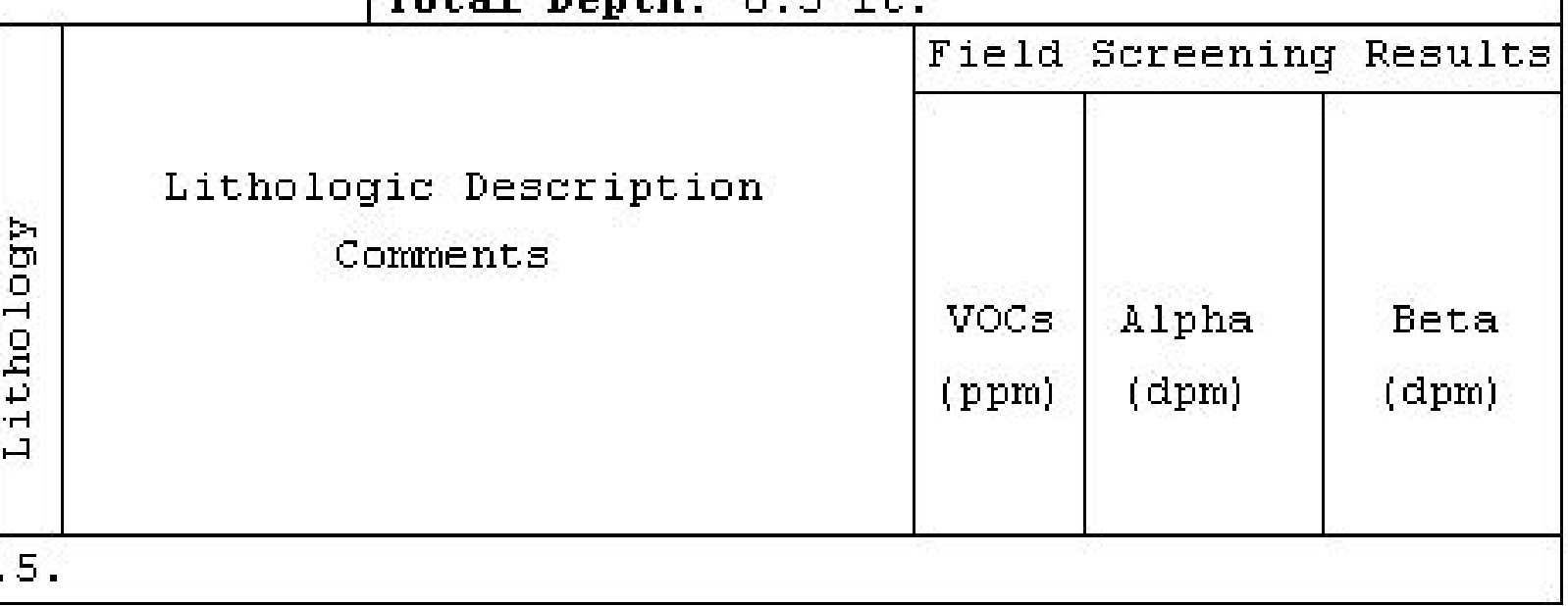

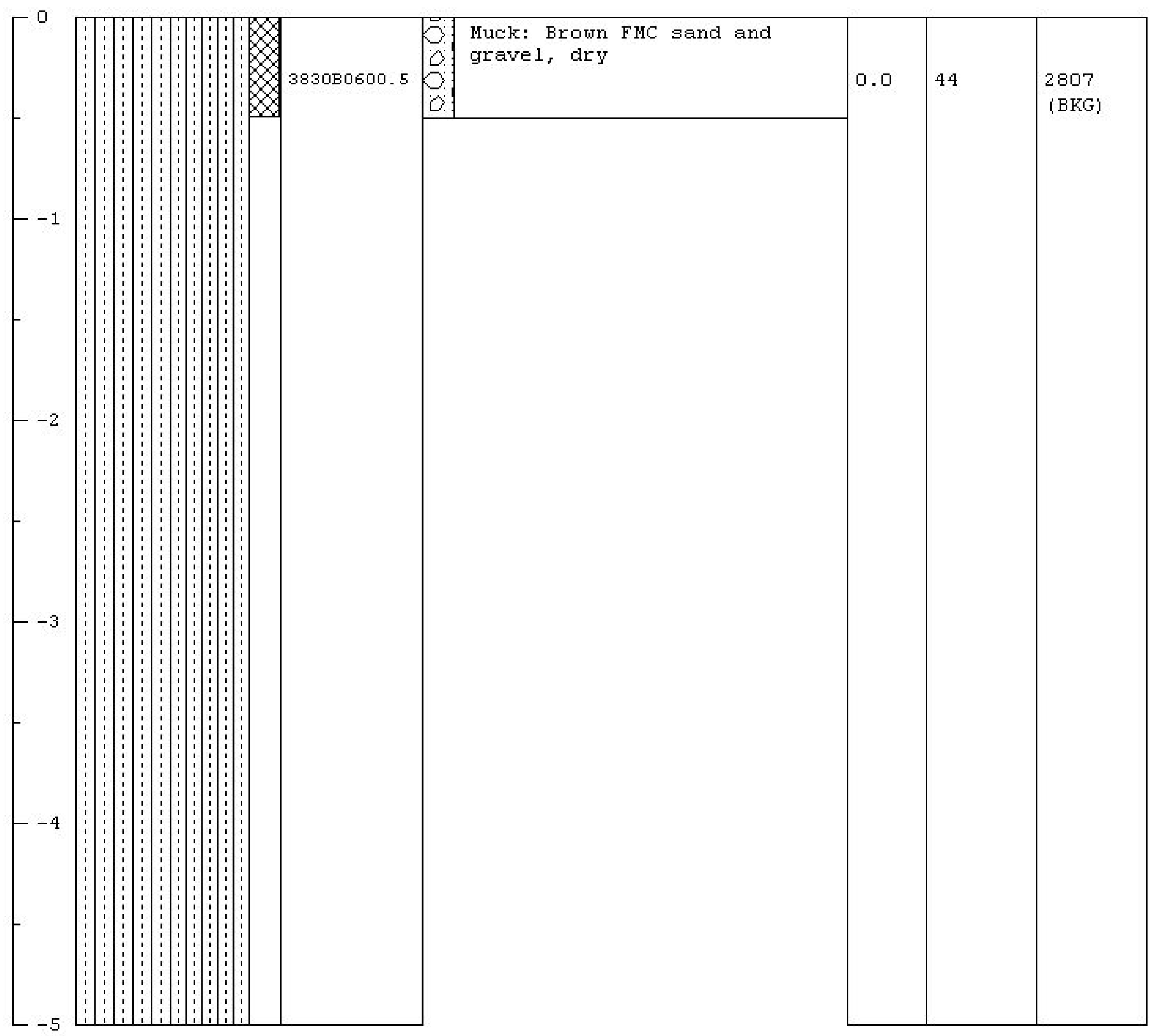


Project Name: CAU 383 E-Tunnel sites

Project Humber: DT04-320

Borehole Number: EOBO7

Logged By: $M$. McLane

Drilled By: M. McLane

Drilling Method: Hand Sampled
Date Started: $6 / 01 / 04$

Date completed: $6 / 01 / 04$

Elevation: 6207.4

Horthing : 887598.9

Easting: 637640.6

Total Depth: 0.5 ft.

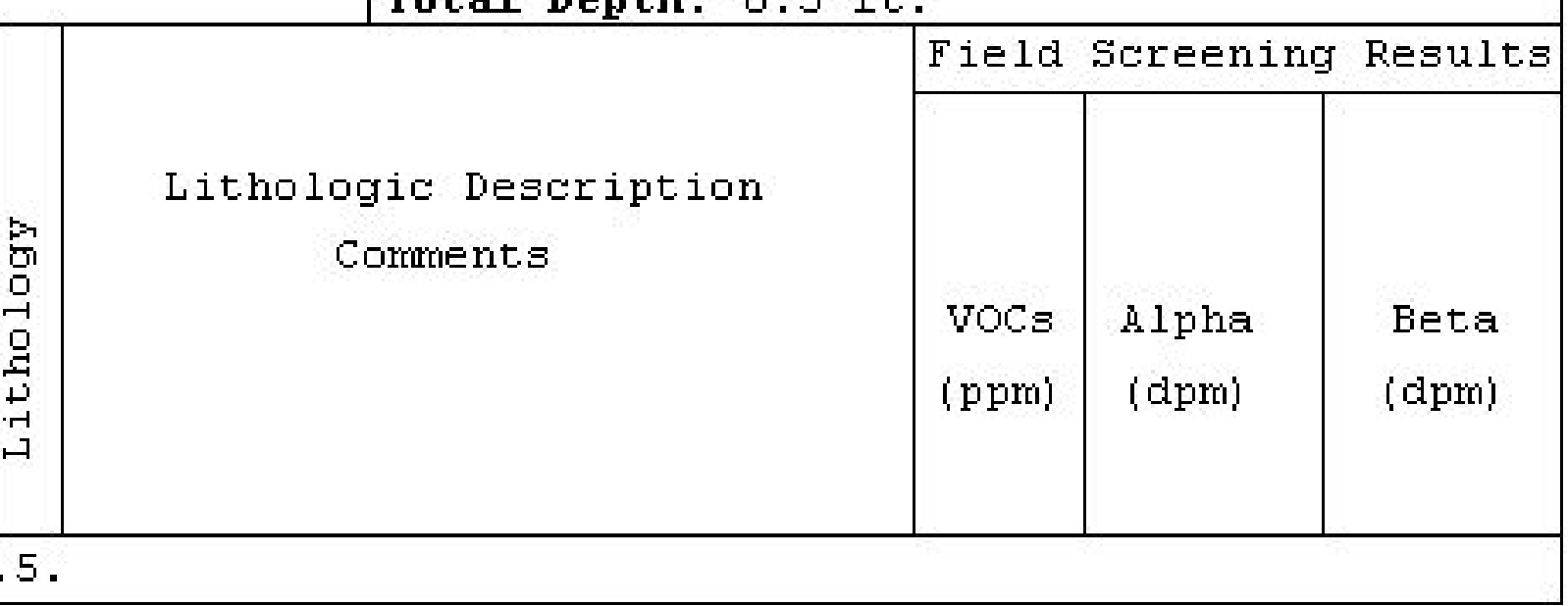

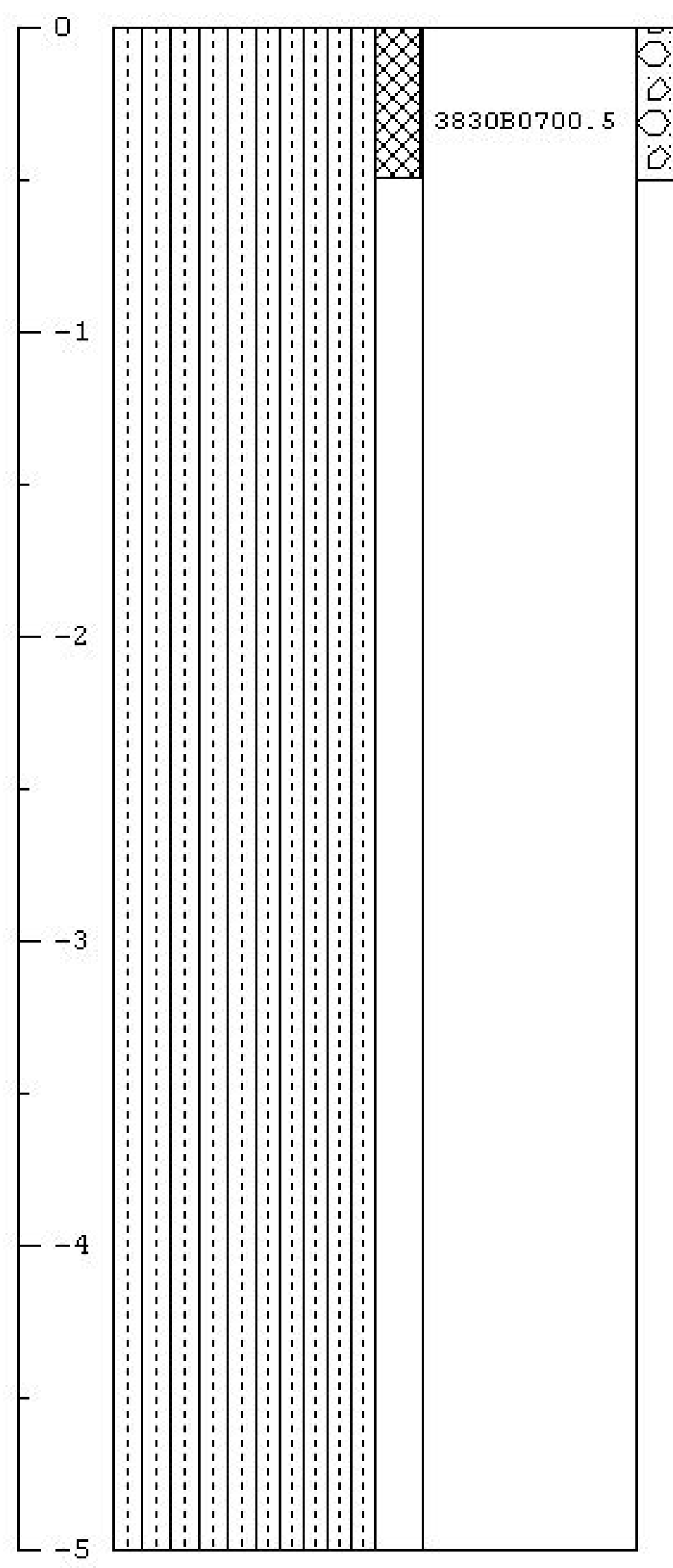

Muck: Brown and grey FMC sand and gravel, dry 
Project Name: CAU 383 E-Tunnel sites

Project Humber: DT04-320

Borehole Number: EOBO8

Logged By: $M$. McLane

Drilled By: M. McLane

Drilling Method: Hand Sampled
Date Started: $6 / 01 / 04$

Date completed: $6 / 01 / 04$

Elevation: 6220.9

Horthing: 887605.2

Easting: 637654.6

Total Depth: 0.5 ft.

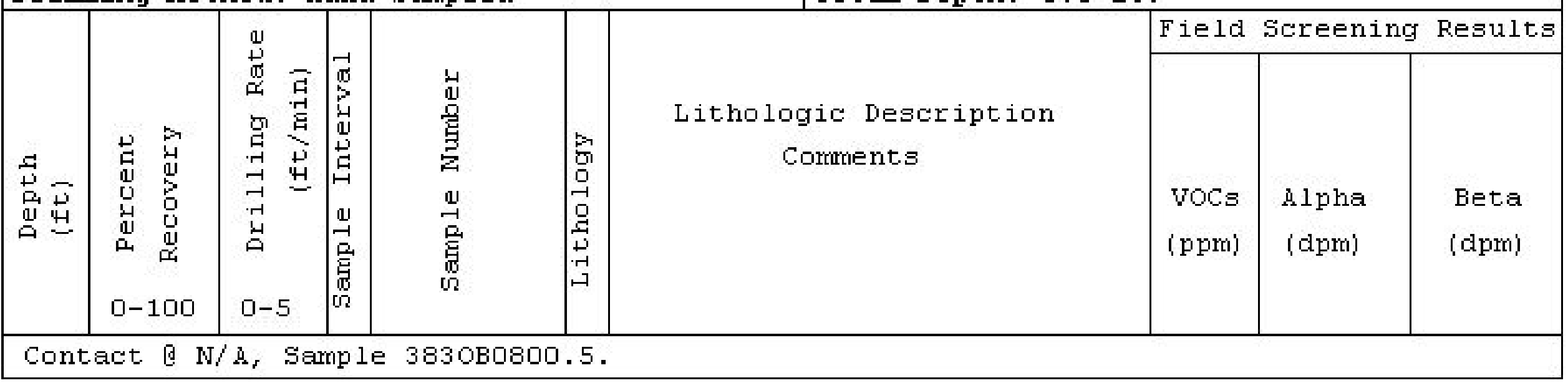

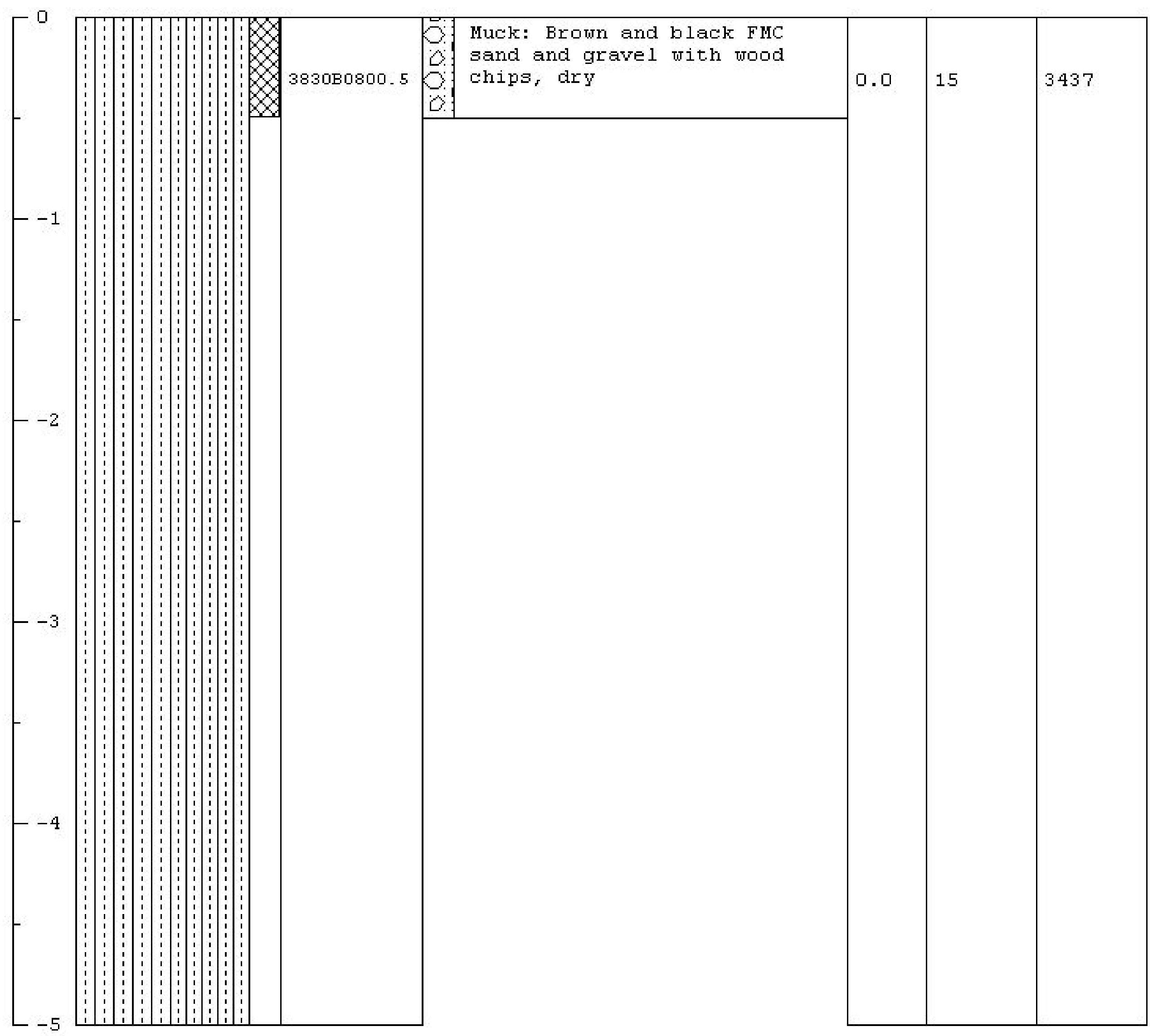


Project Hame: CAU 383 E-Tunnel sites

Project Number: DT04-320

Borehole Number: EPBO1

Logged By: M. MeLane

Drilled By: Boart-Longyear

Drilling Method: Rotosonic
Date Started: $6 / 10 / 04$

Date Completed: $6 / 10 / 04$

Elevation: 5959.0

Horthing: 887711.6

Easting: 639006.6

Total Depth: 5.0 ft.

\begin{tabular}{|c|c|c|c|c|c|c|c|c|c|}
\hline \multirow[b]{2}{*}{ 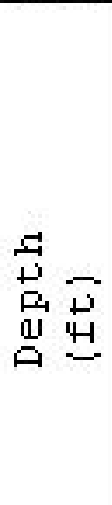 } & \multirow[b]{2}{*}{ 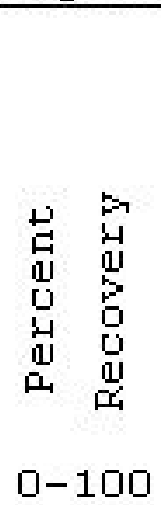 } & \multirow{2}{*}{\multicolumn{2}{|c|}{ 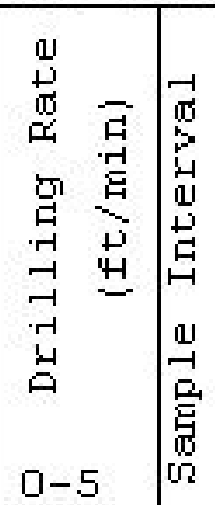 }} & \multirow[b]{2}{*}{ 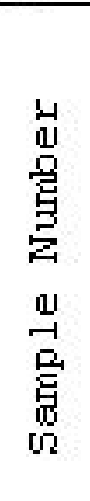 } & \multirow[b]{2}{*}{ 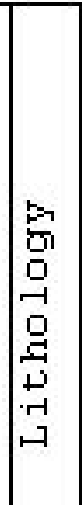 } & \multirow[b]{2}{*}{$\begin{array}{c}\text { Lithologic Description } \\
\text { Comments }\end{array}$} & \multicolumn{3}{|c|}{ Field Screening Results } \\
\hline & & & & & & & $\begin{array}{l}\text { VOCs } \\
\text { (ppm) }\end{array}$ & $\begin{array}{l}\text { Alpha } \\
\text { (dpm) }\end{array}$ & $\begin{array}{l}\text { Beta } \\
\text { (dpm) }\end{array}$ \\
\hline
\end{tabular}
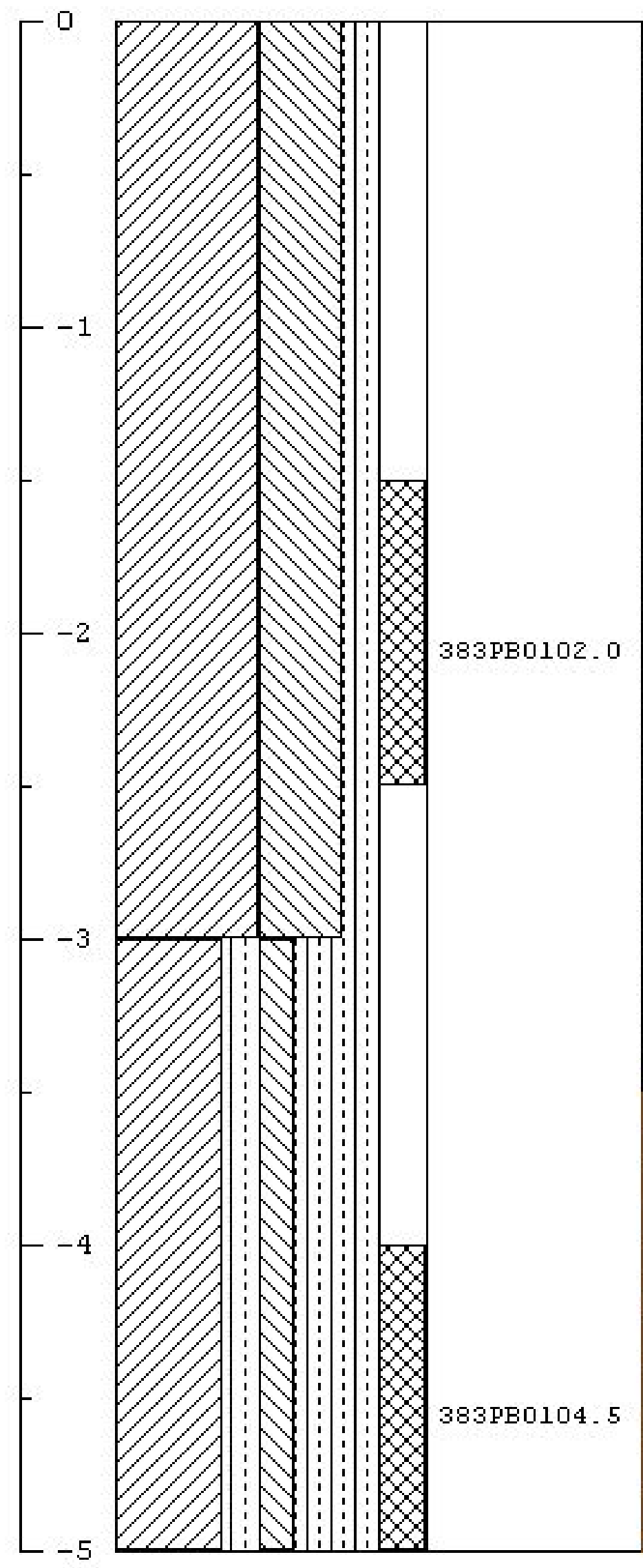

C.: Muck: Brown and grey coarse

383PB0102.0

: sand and cobbles with grout chips, dry

0 .

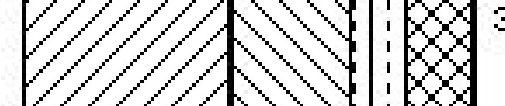

.

3

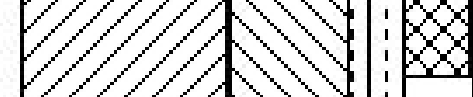

:

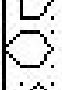

…

0

:.:

:

:

응

(a)

Native: Brown and grey FMC

sand and gravel with black

cobbles, slightly moist 
Project Name: CAU 383 E-Tunnel sites

Project Humber: DT04-320

Borehole Number: EPBO2

Logged By: $M$. McLane

Drilled By: M. McLane

Drilling Method: Hand Sampled
Date Started: $6 / 17 / 04$

Date completed: $6 / 17 / 04$

Elevation: 5917.5

Horthing: 887648.3

Easting: 639275.7

Total Depth: 0.5 ft.

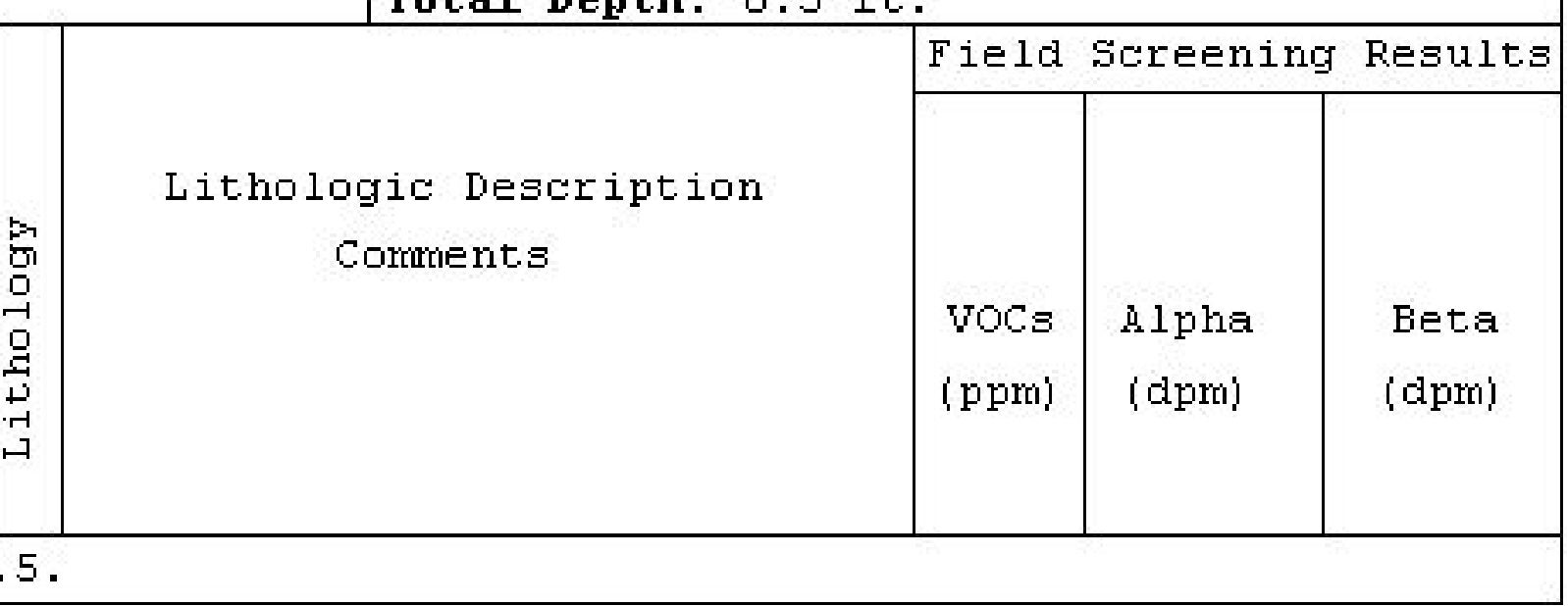

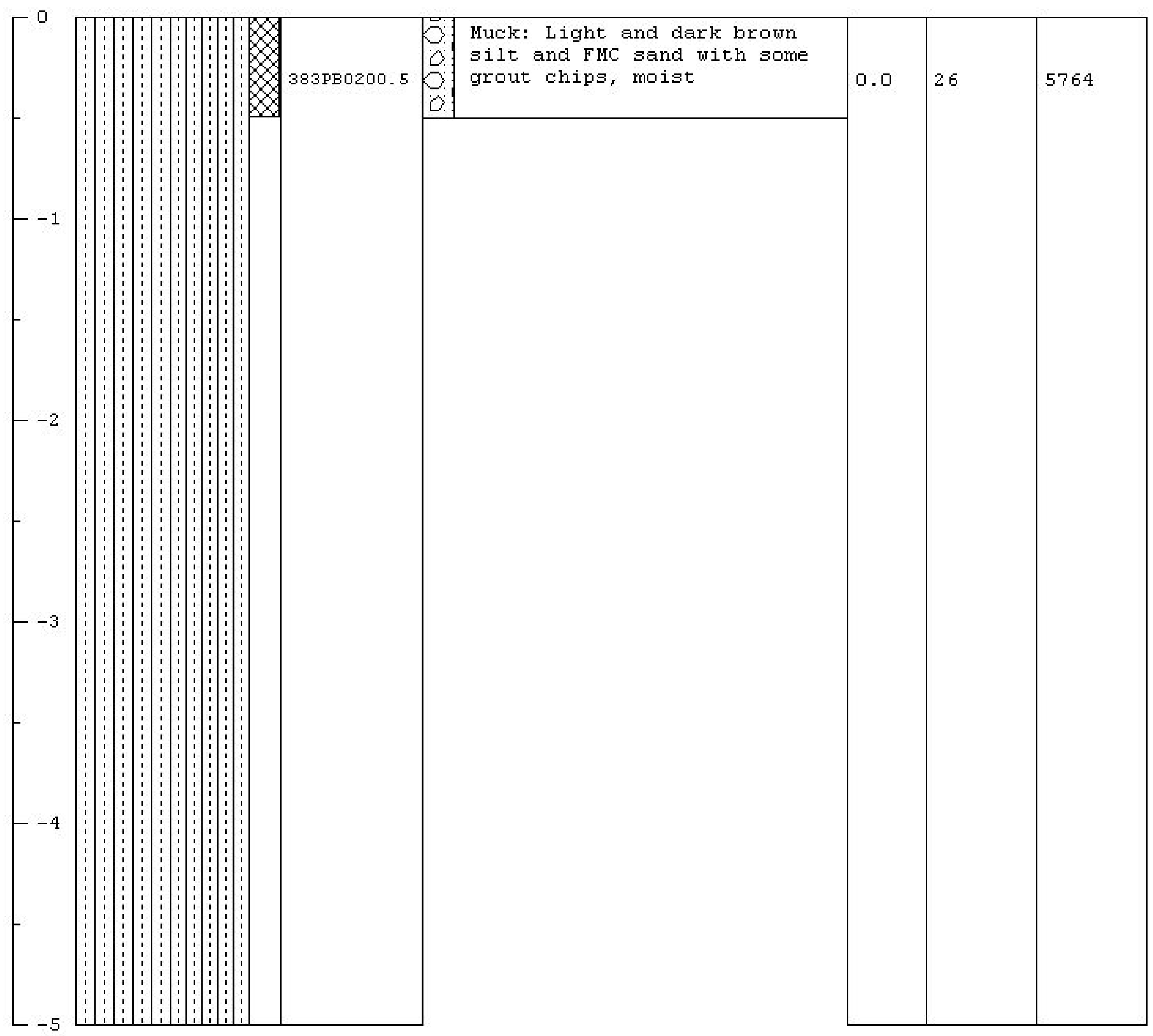


Project Name: CAU 383 E-Tunnel sites

Project Humber: DT04-320

Borehole Number: EPBO3

Logged By: $M$. McLane

Drilled By: M. McLane

Drilling Method: Hand Sampled
Date Started: $6 / 17 / 04$

Date completed: $6 / 17 / 04$

Elevation: 5927.1

Horthing: 887622.8

Easting: 639329.0

Total Depth: 0.5 ft.

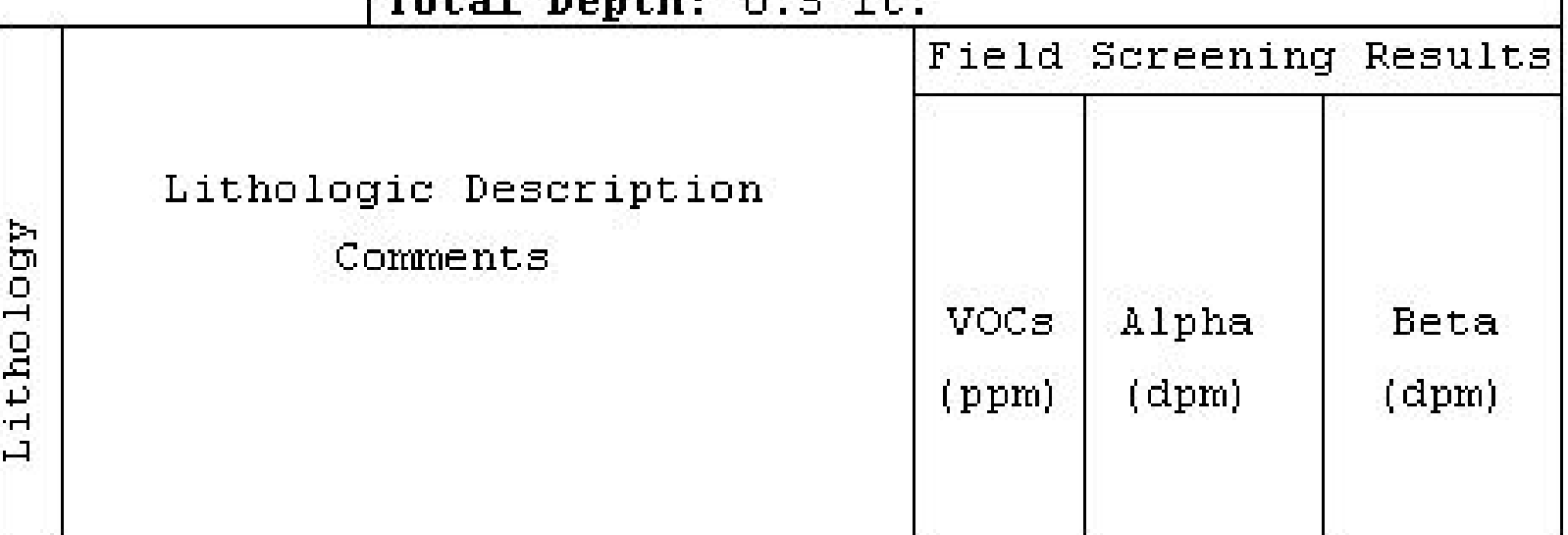

383 PBO300.5X.

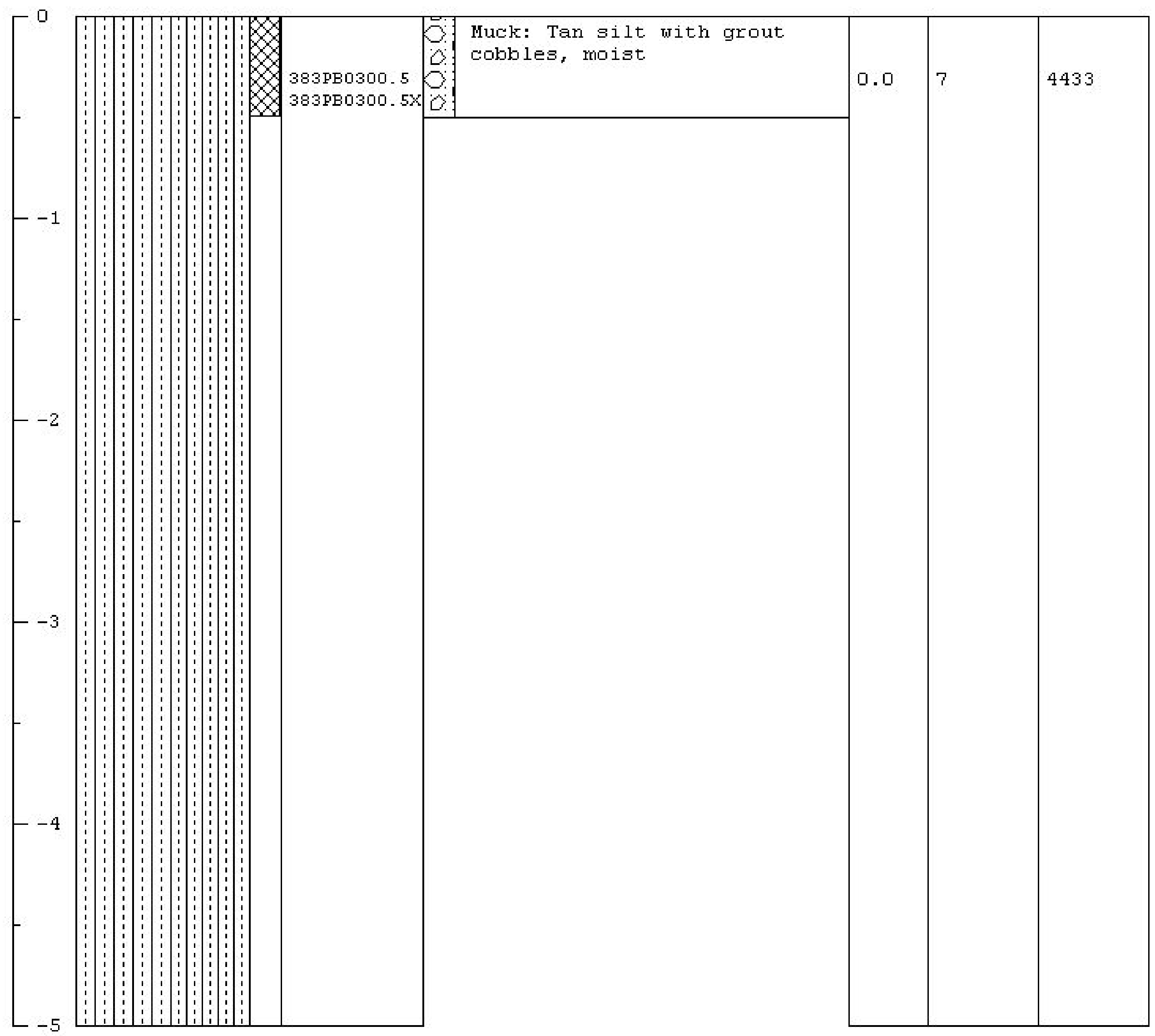


Project Name: CAU 383 E-Tunnel sites

Project Humber: DT04-320

Borehole Number: EPBO4

Logged By: $M$. McLane

Drilled By: M. McLane

Drilling Method: Hand Sampled
Date Started: $6 / 16 / 04$

Date completed: $6 / 16 / 04$

Elevation: 5942.3

Horthing : 887624.5

Easting: 639090.5

Total Depth: 0.5 ft.

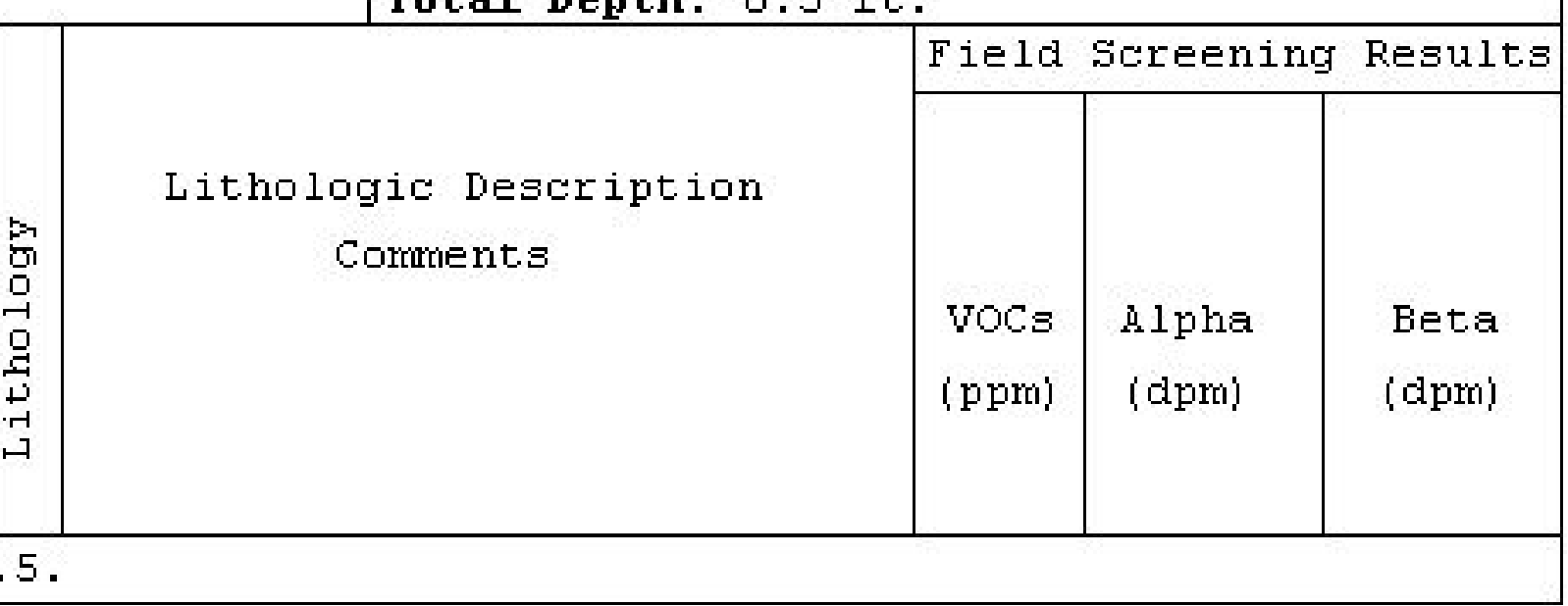

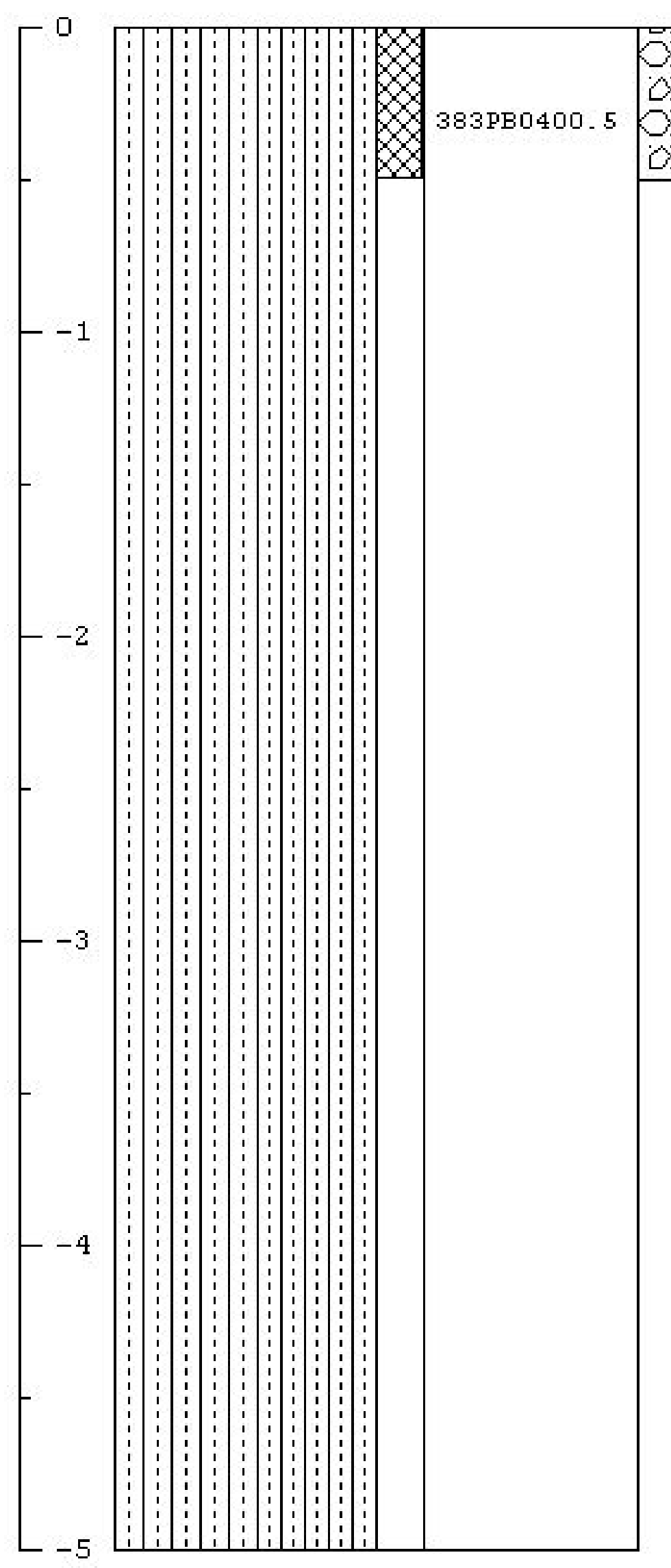

Muck: Light brown FMC sand and gravel, dry 
Project Name: CAU 383 E-Tunnel sites

Project Humber: DT04-320

Borehole Number: EPBO5

Logged By: $M$. McLane

Drilled By: M. McLane

Drilling Method: Hand Sampled
Date Started: $6 / 16 / 04$

Date completed: $6 / 16 / 04$

Elevation : 5935.2

Horthing: 887652.8

Easting: 639152.3

Total Depth: 0.5 ft.

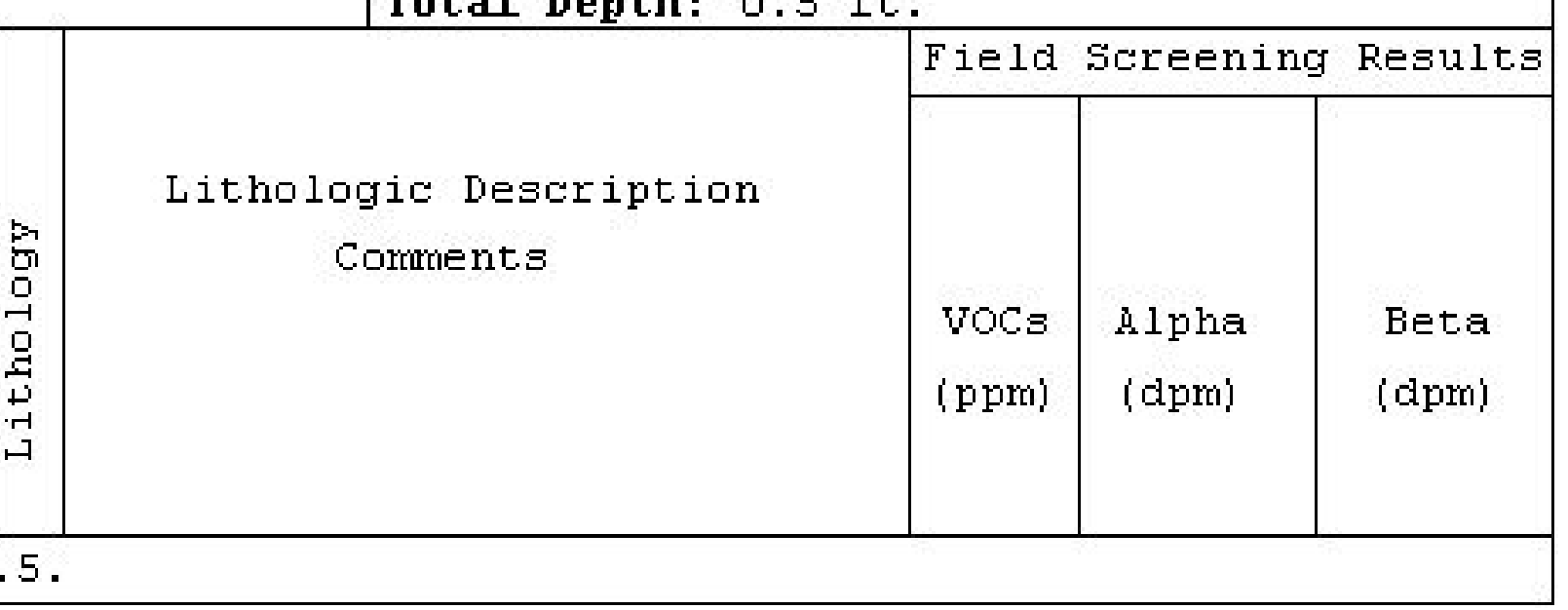

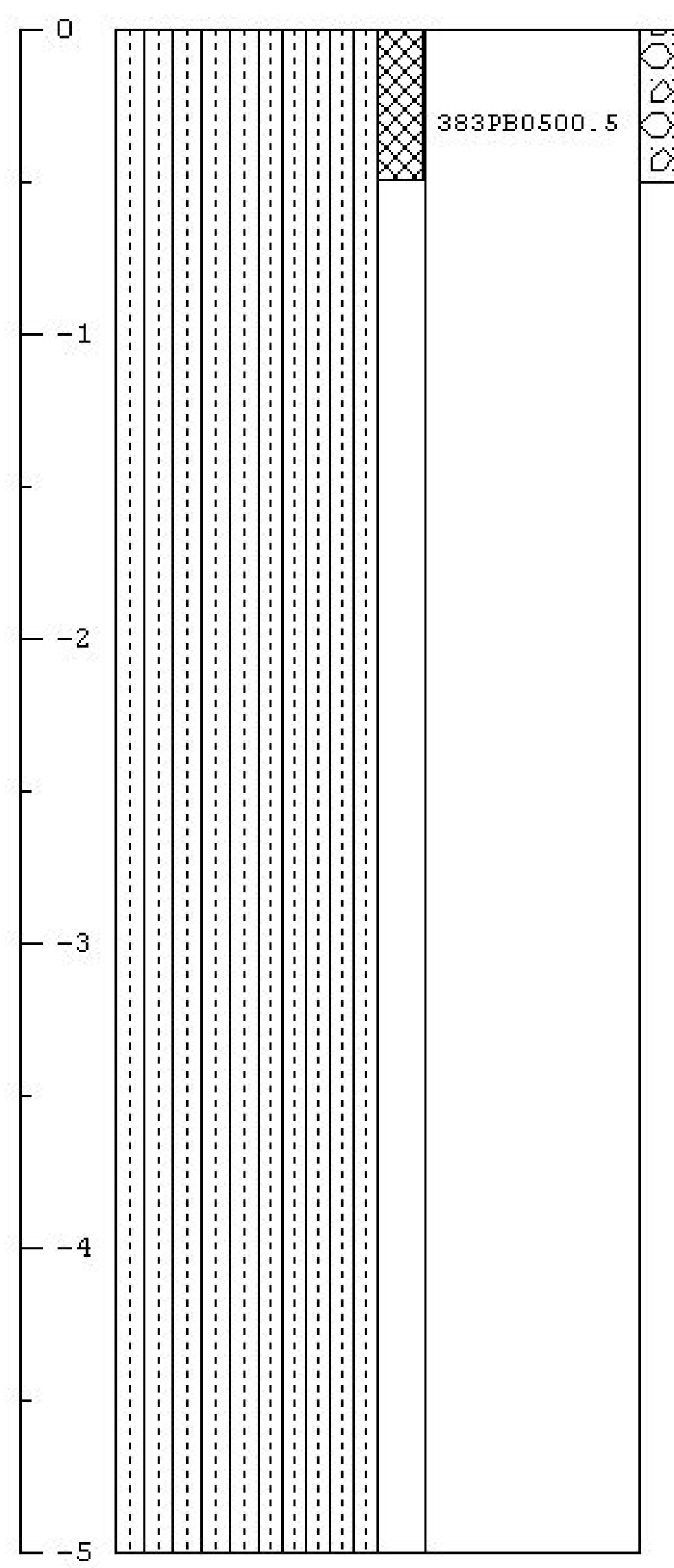

Muck: Brown and dark brown silt and FMC sand, dry 
Project Hame: CAU 383 E-Tunnel sites

Project Number: DT04-320

Borehole Number: EPHO1

Logged By: M. MeLane

Drilled By: Boart-Longyear

Drilling Method: Rotosonic
Date Started: $6 / 09 / 04$

Date Completed: $6 / 09 / 04$

Elevation: 5954.9

Horthing: 887706.3

Easting: 639072.3

Total Depth: $17.0 \mathrm{ft}$.

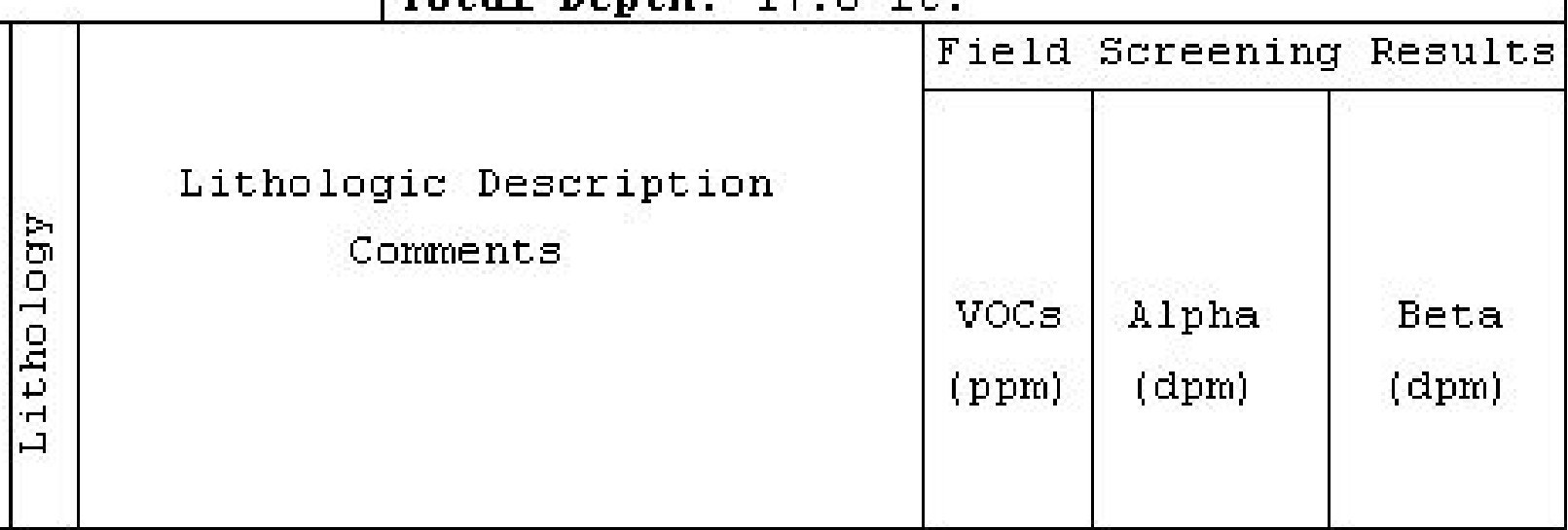

383PHO116.5.

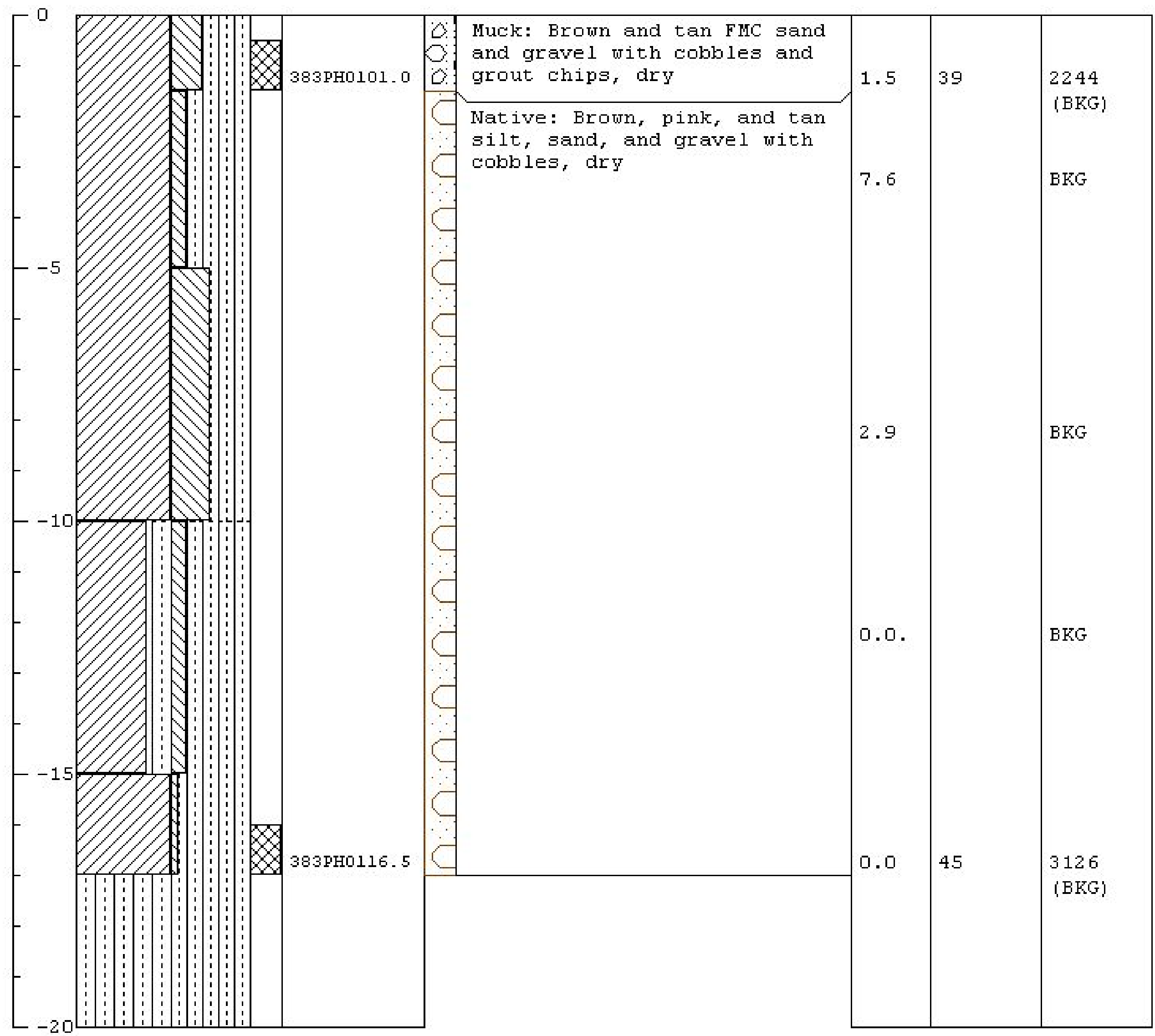


Project Hame: CAU 383 E-Tunnel sites

Project Humber: DTO4-320

Borehole Number: EPHO2

Logged By: M. MeLane

Drilled By: Boart-Longyear

Drilling Method: Rotosonic
Date Started: $6 / 09 / 04$

Date Completed: $6 / 09 / 04$

Elevation : 5954.3

Horthing: 887666.4

Easting: 639036.1

Total Depth: $18.0 \mathrm{ft}$.

\begin{tabular}{|c|c|c|c|c|}
\hline \multirow[b]{2}{*}{ 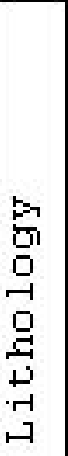 } & \multirow[b]{2}{*}{$\begin{array}{c}\text { Lithologic Description } \\
\text { Comments }\end{array}$} & \multicolumn{3}{|c|}{ Field Screening Results } \\
\hline & & $\begin{array}{l}\text { VoCs } \\
\text { (ppm) }\end{array}$ & $\begin{array}{l}\text { Alpha } \\
\text { (dpm) }\end{array}$ & $\begin{array}{l}\text { Beta } \\
\text { (dpm) }\end{array}$ \\
\hline
\end{tabular}
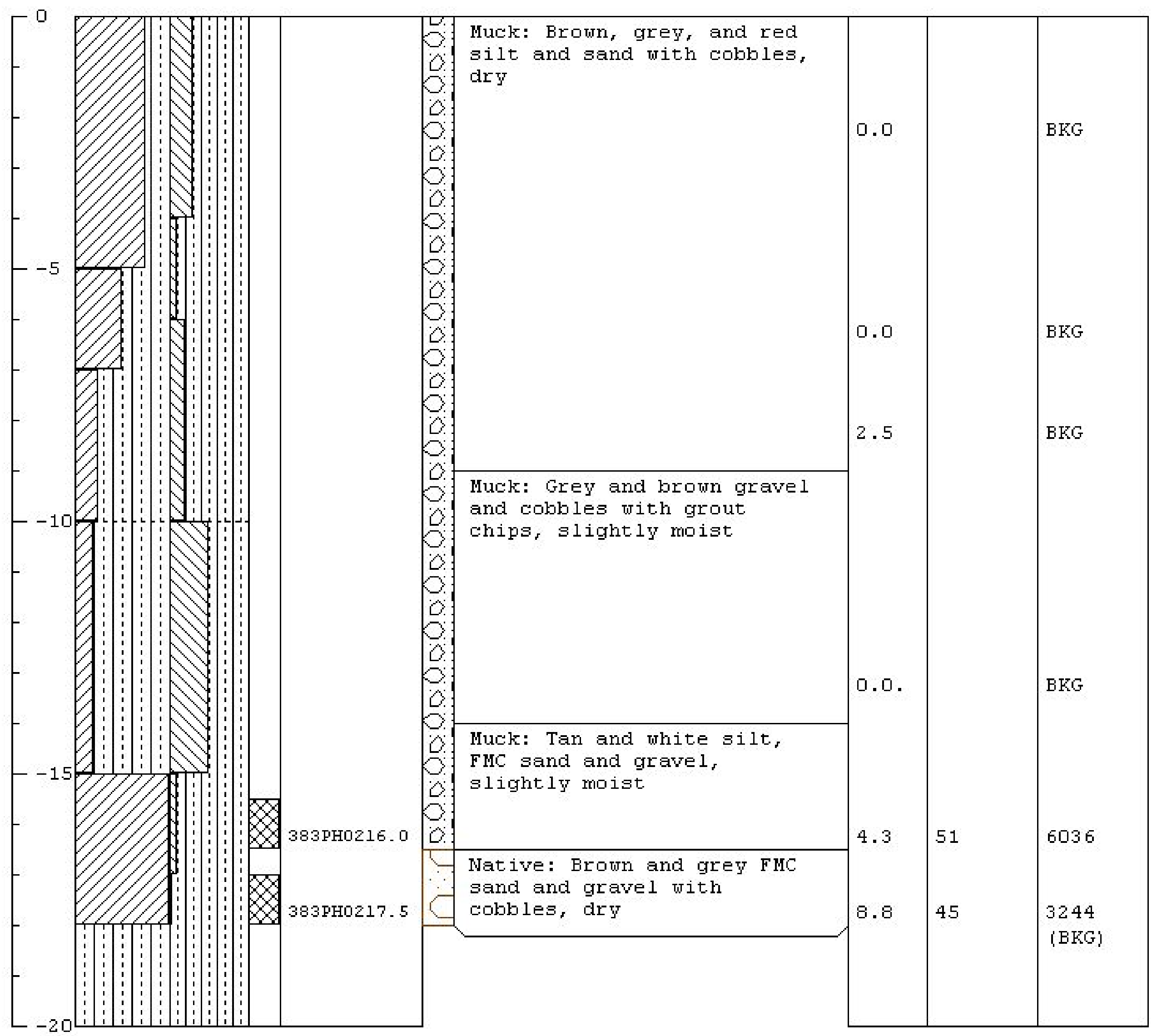
Project Hame: CAU 383 E-Tunnel sites

Project Humber: DT04-320

Borehole Number: EPHO3

Logged By: M. MeLane

Drilled By: Boart-Longyear

Drilling Method: Rotosonic

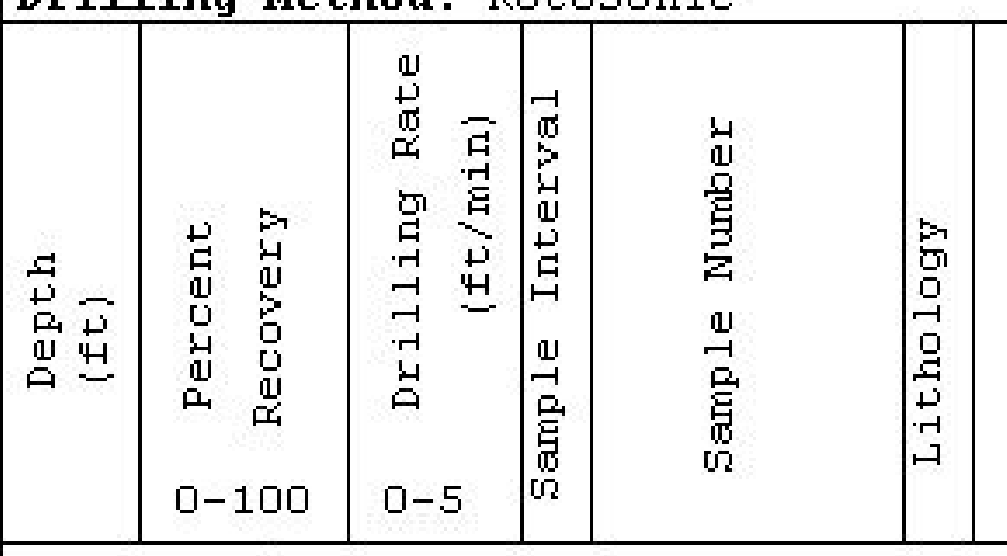

Contact $14.5 \mathrm{ft}$, Samples 383PH0306.5, 383PH0320.5.

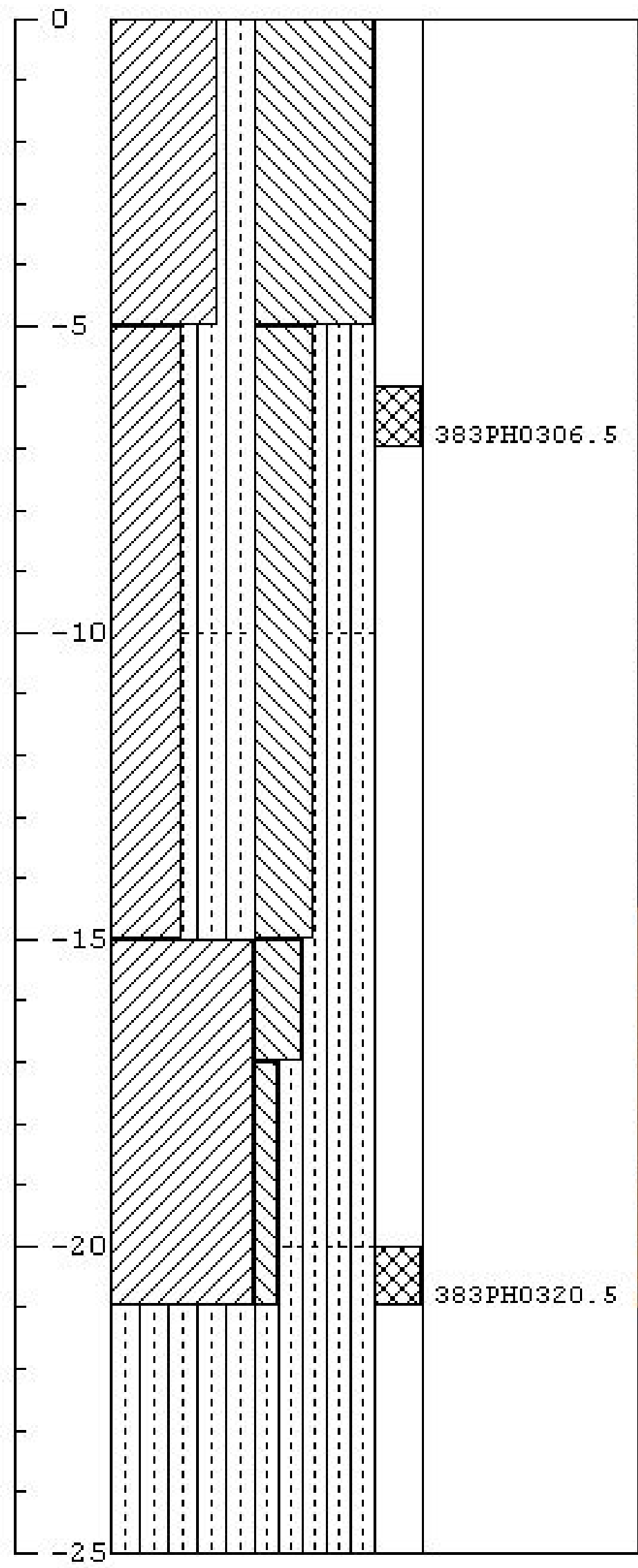

Date Started: $6 / 09 / 04$

Date Completed: $6 / 09 / 04$

Elevation: 5964.2

Horthing: 887668.5

Easting: 639047.4

Total Depth: $21.0 \mathrm{ft}$.

Lithologic Description

Comments

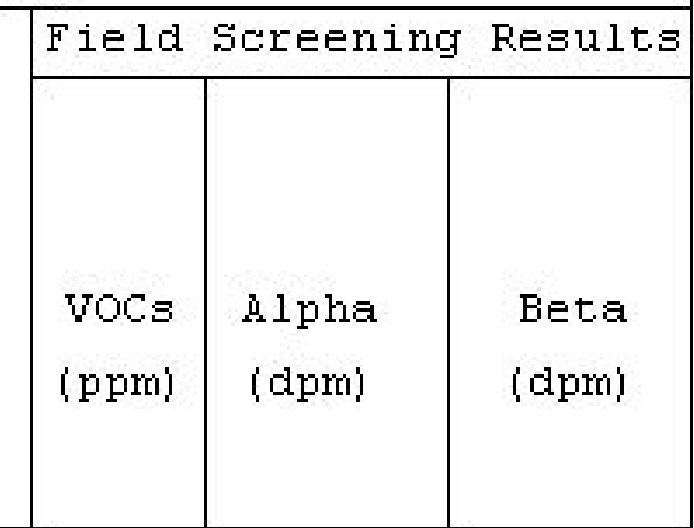

Muck: Pink, tan, and grey

2.) silt and FMC sand with

gravel, dry

.

Muck: Red, white, and grey

gravel and cobbles, dry

$\rightarrow$

i.:

․:

8

Native: Brown and grey silt

and sand with gravel, dry
1.0

BKG
2878
(BKG)
BKG
BKG
BKG

BKG
2878
(BKG)
BKG
BKG
BKG

BKG
2878
(BKG)
BKG
BKG
BKG

BKG
(B)78
BKG
BKG
BKG

BKG
(B)78
BKG
BKG
BKG

0.0 .

BKG
(B)78
BKG
BKG
BKG

1.7 
Project Hame: CAU 383 E-Tunnel sites

Project Humber: DTO4-320

Borehole Humber: EPHO4

Logged By: M. MeLane

Drilled By: Boart-Longyear

Drilling Method: Rotosonic
Date Started: $6 / 10 / 04$

Date Completed: $6 / 10 / 04$

Elevation: 5943.2

Horthing: 887678.9

Easting: 639033.9

Total Depth: $19.0 \mathrm{ft}$.

\begin{tabular}{|c|c|c|c|c|}
\hline & \multirow[b]{2}{*}{$\begin{array}{c}\text { Lithologic Description } \\
\text { Comments }\end{array}$} & \multicolumn{3}{|c|}{ Field Screening Results } \\
\hline 票 & & $\begin{array}{l}\text { VoCs } \\
\text { (ppm) }\end{array}$ & $\begin{array}{l}\text { Alpha } \\
\text { (dpm) }\end{array}$ & $\begin{array}{l}\text { Beta } \\
\text { (dpm) }\end{array}$ \\
\hline
\end{tabular}

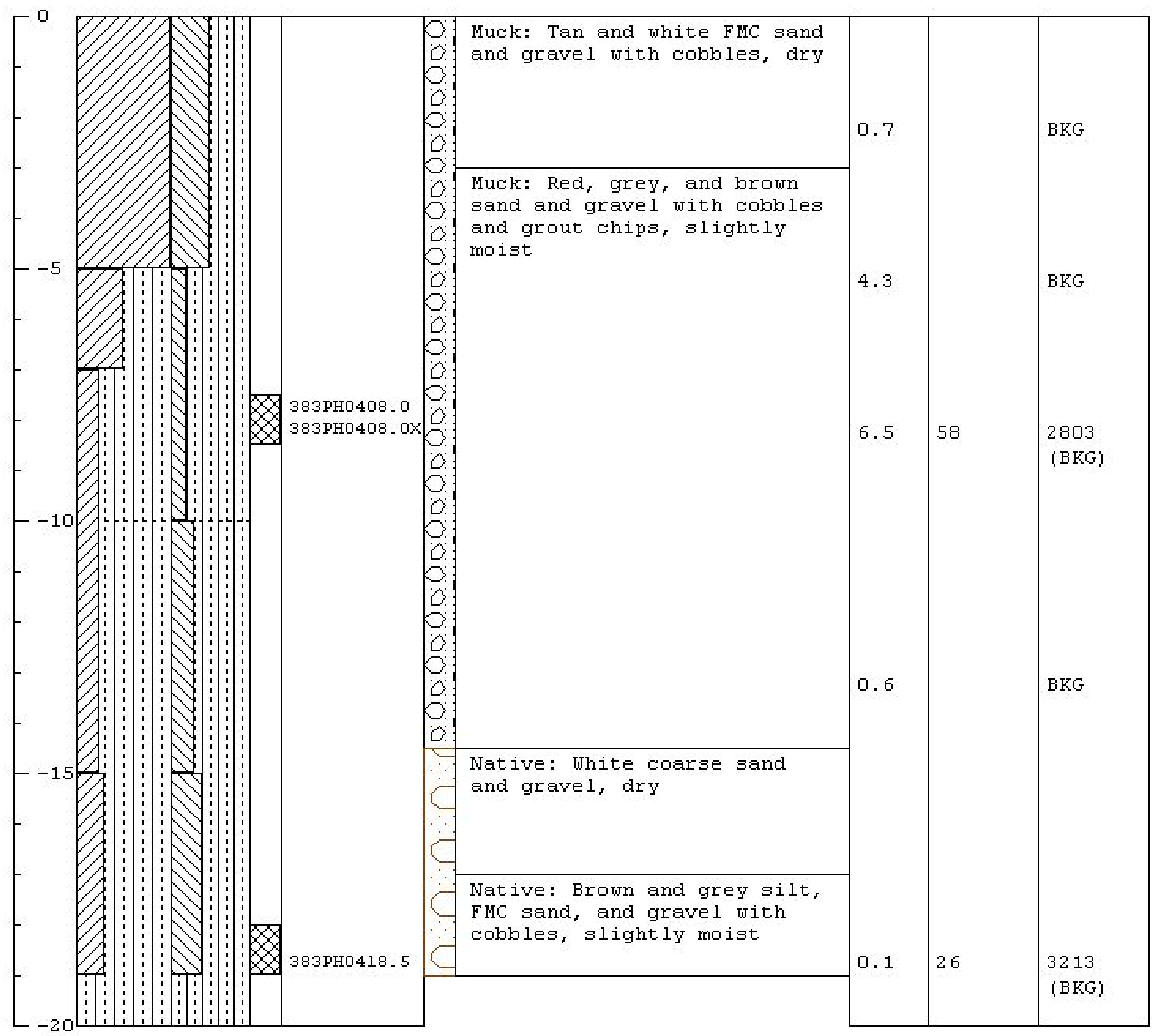


Project Name: CAU 383 E-Tunnel sites

Project Humber: DT04-320

Borehole Number: ERBO1

Logged By: $M$. McLane

Drilled By: M. McLane

Drilling Method: Hand Sampled

\begin{tabular}{|c|c|c|c|c|c|}
\hline 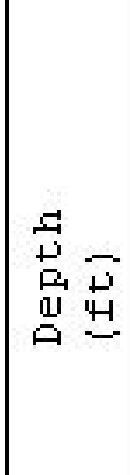 & 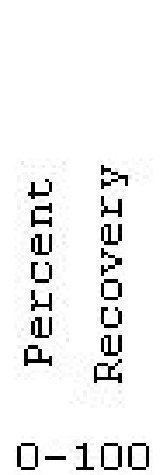 & 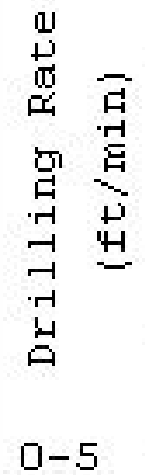 & 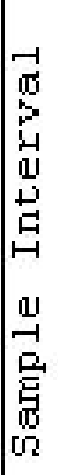 & 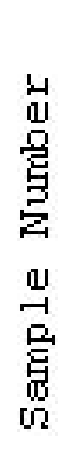 & 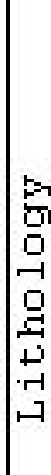 \\
\hline
\end{tabular}

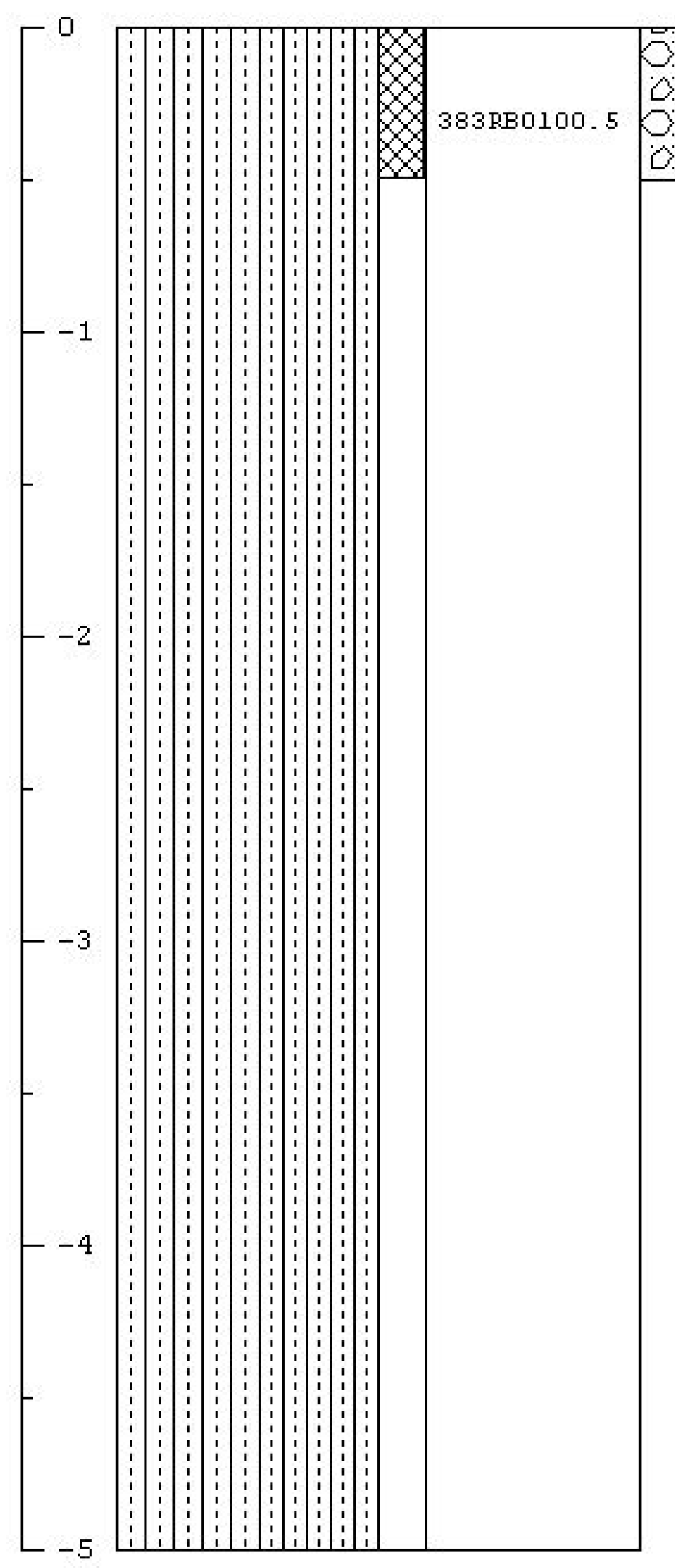

C.

Date Started: $6 / 01 / 04$

Date completed: $6 / 01 / 04$

Elevation: 6122.6

Horthing: 887807.9

Easting: 637709.2

Total Depth: 0.5 ft.

Lithologic Description

Comments

\begin{tabular}{|c|c|c|}
\hline \multicolumn{2}{|c|}{ Field Screening Results } \\
\hline & & \\
voCs & Alpha & Beta \\
(ppri) & (dpm) & (dpm) \\
& & \\
\end{tabular}

Muck: Brown FMC sand and gravel, dry 
Project Name: CAU 383 E-Tunnel sites

Project Humber: DT04-320

Borehole Number: ERBO2

Logged By: $M$. McLane

Drilled By: M. McLane

Drilling Method: Hand Sampled
Date Started: $6 / 01 / 04$

Date completed: $6 / 01 / 04$

Elevation: 6119.5

Horthing: 887818.4

Easting: 637716.4

Total Depth: 0.5 ft.

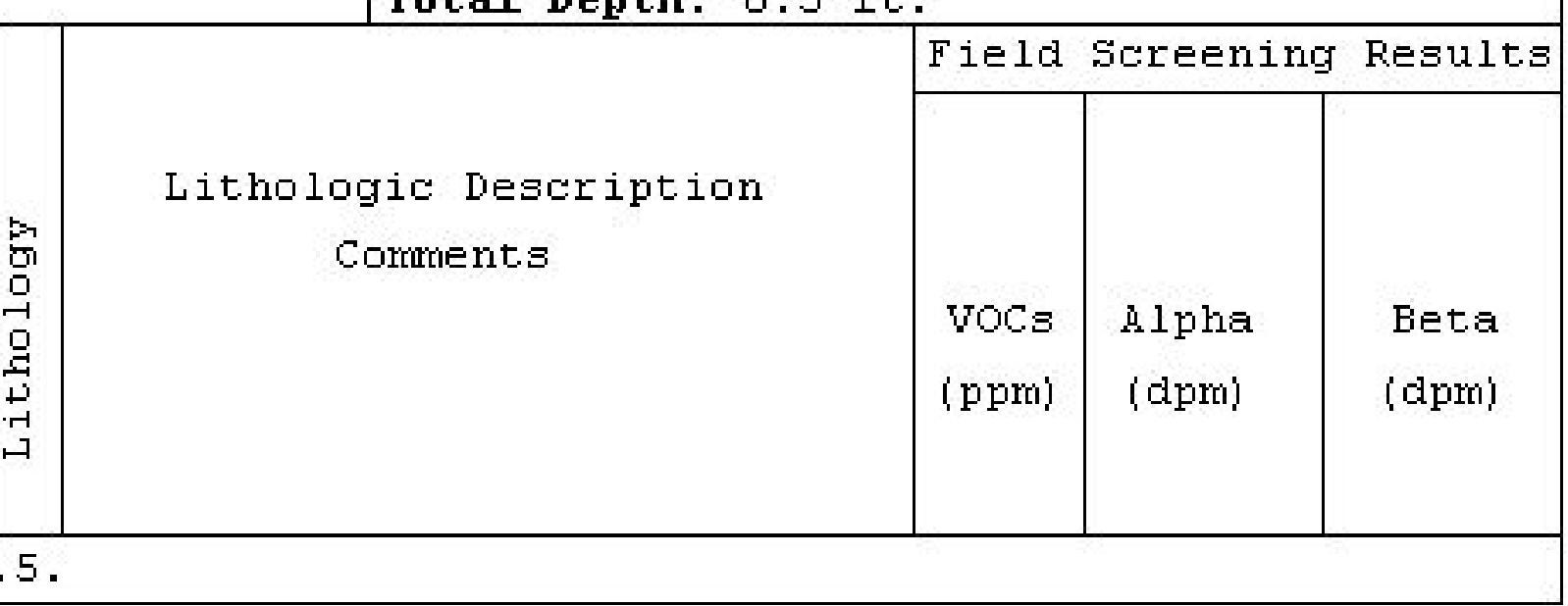

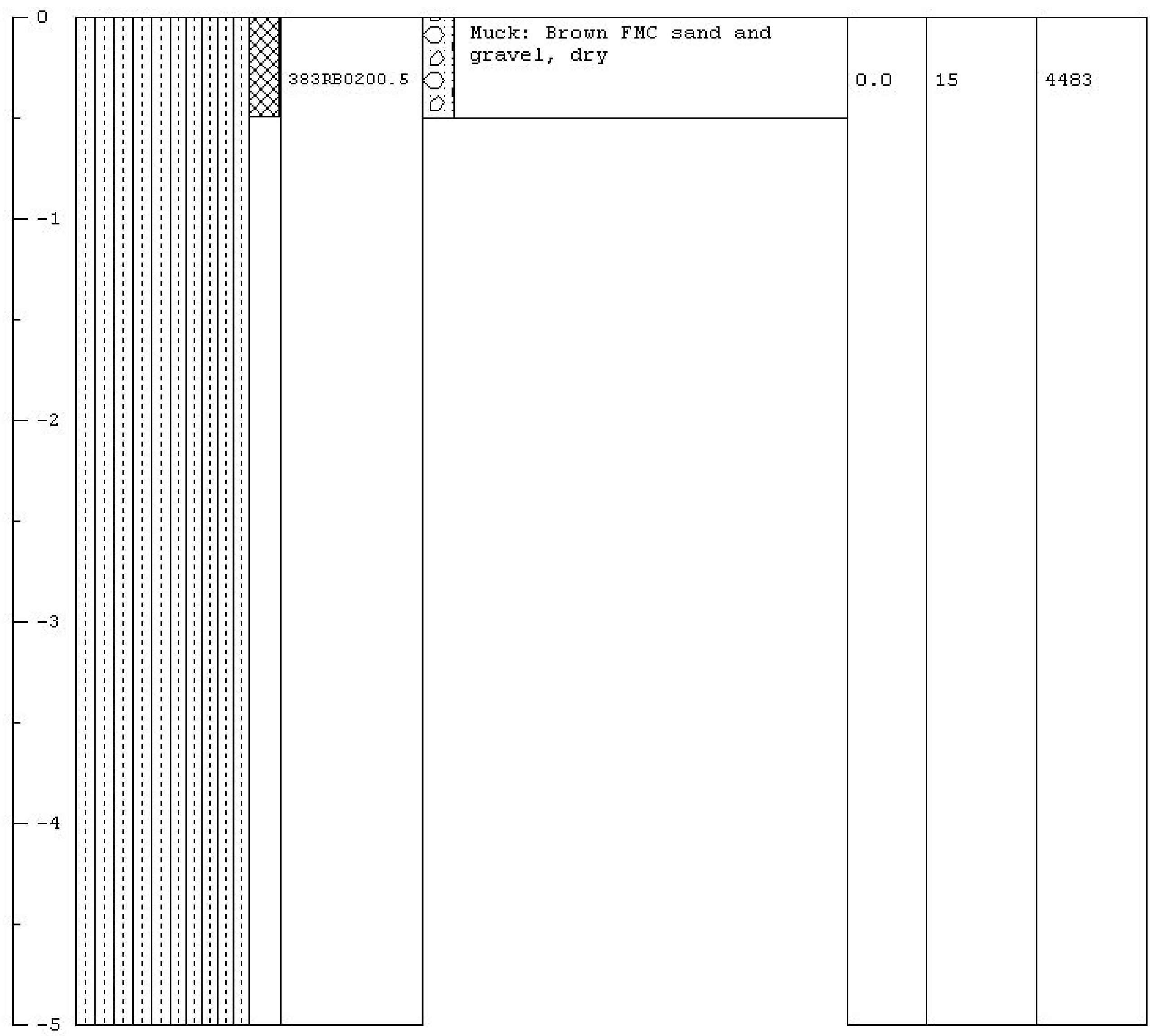


Project Name: CAU 383 E-Tunnel sites

Project Humber: DT04-320

Borehole Number: ERBO3

Logged By: $M$. McLane

Drilled By: M. McLane

Drilling Method: Hand Sampled
Date Started: $6 / 01 / 04$

Date completed: $6 / 01 / 04$

Elevation: 6121.3

Horthing: 887812.1

Easting: 637728.3

Total Depth: 0.5 ft.

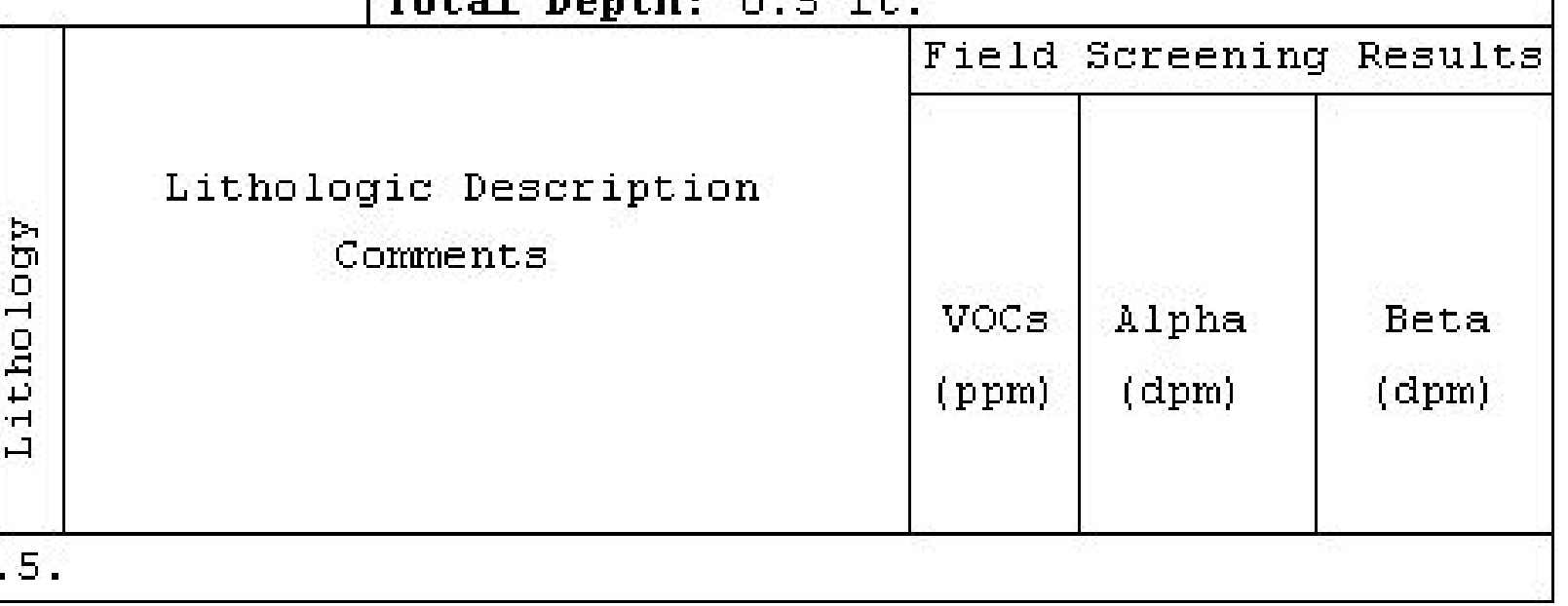

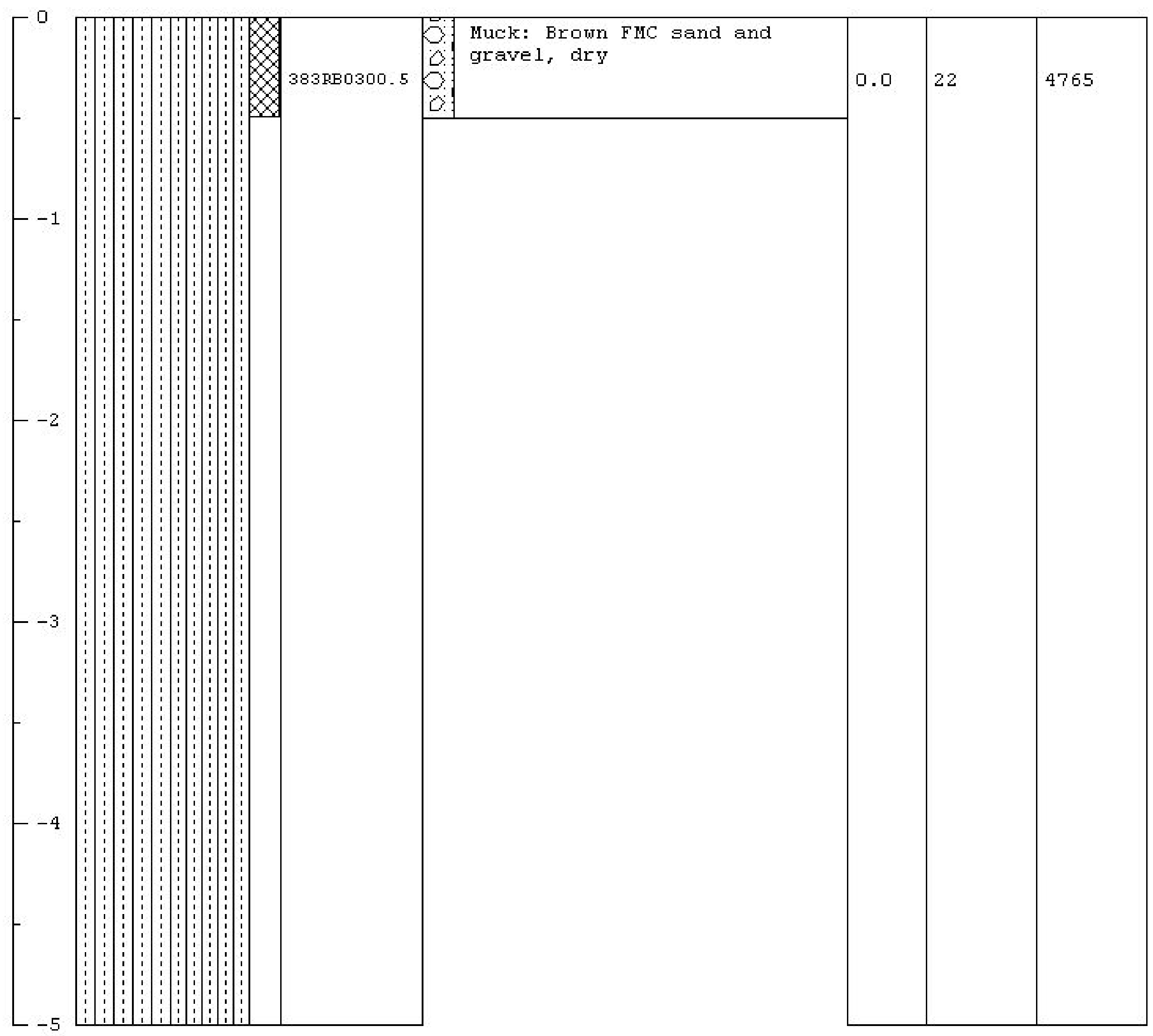


Project Name: CAU 383 E-Tunnel sites

Project Humber: DT04-320

Borehole Number: ERBO4

Logged By: $M$. McLane

Drilled By: M. McLane

Drilling Method: Hand Sampled
Date Started: $6 / 01 / 04$

Date completed: $6 / 01 / 04$

Elevation: 6118.6

Horthing: 887795.6

Easting: 637737.4

Total Depth: 0.5 ft.

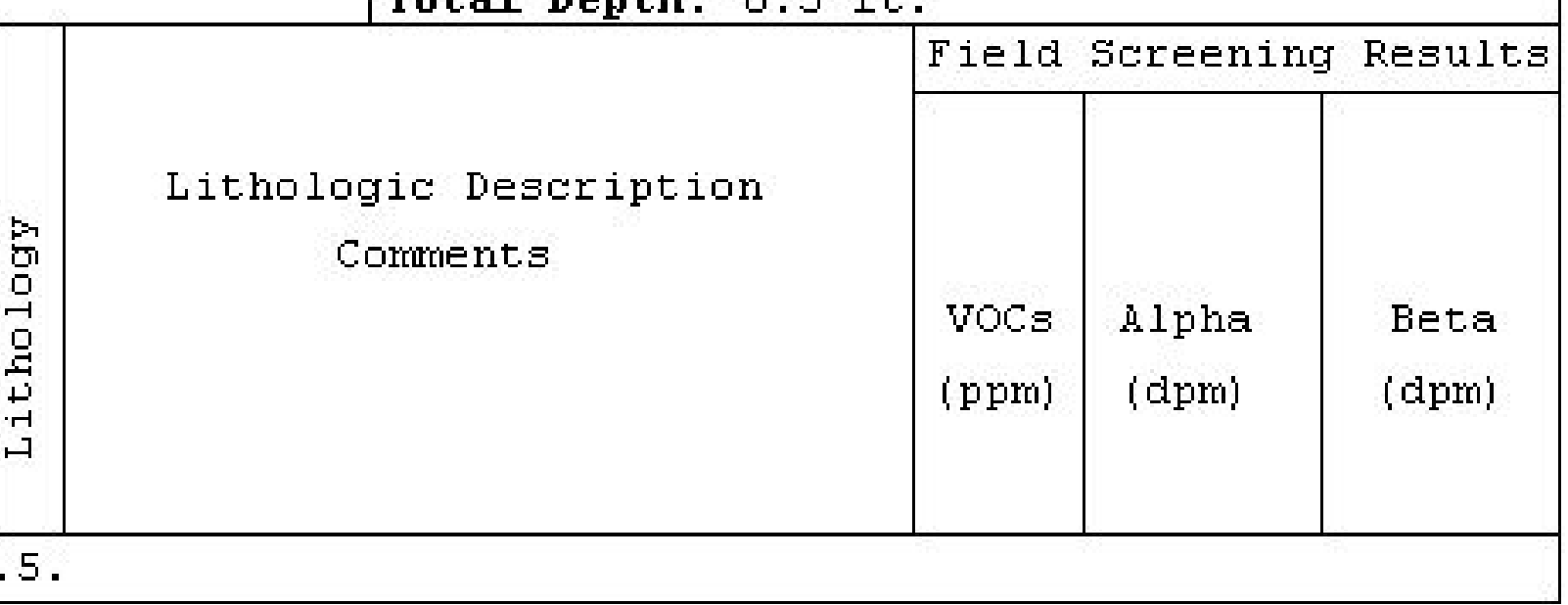

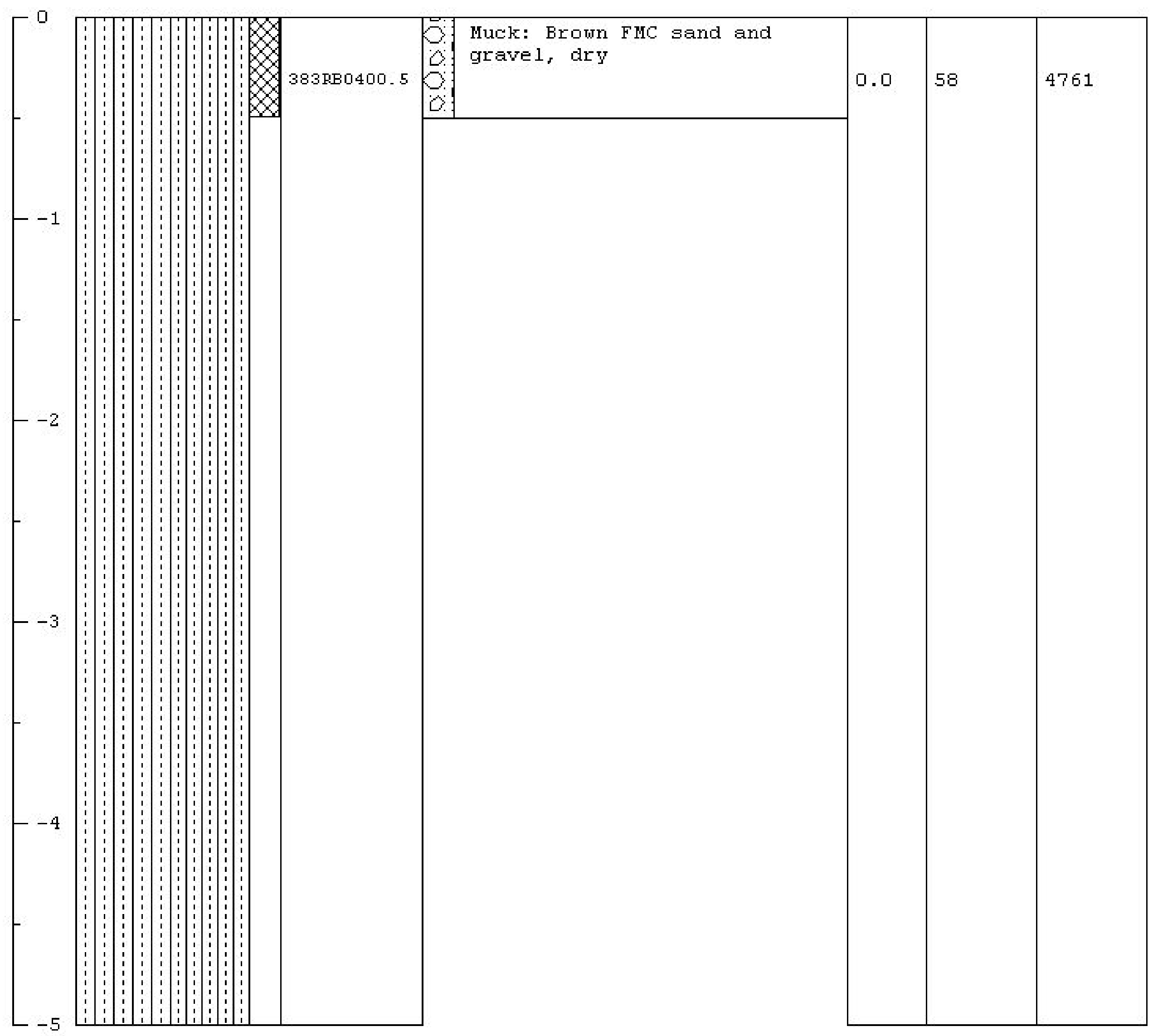




\section{Attachment B \\ Cross Sections}




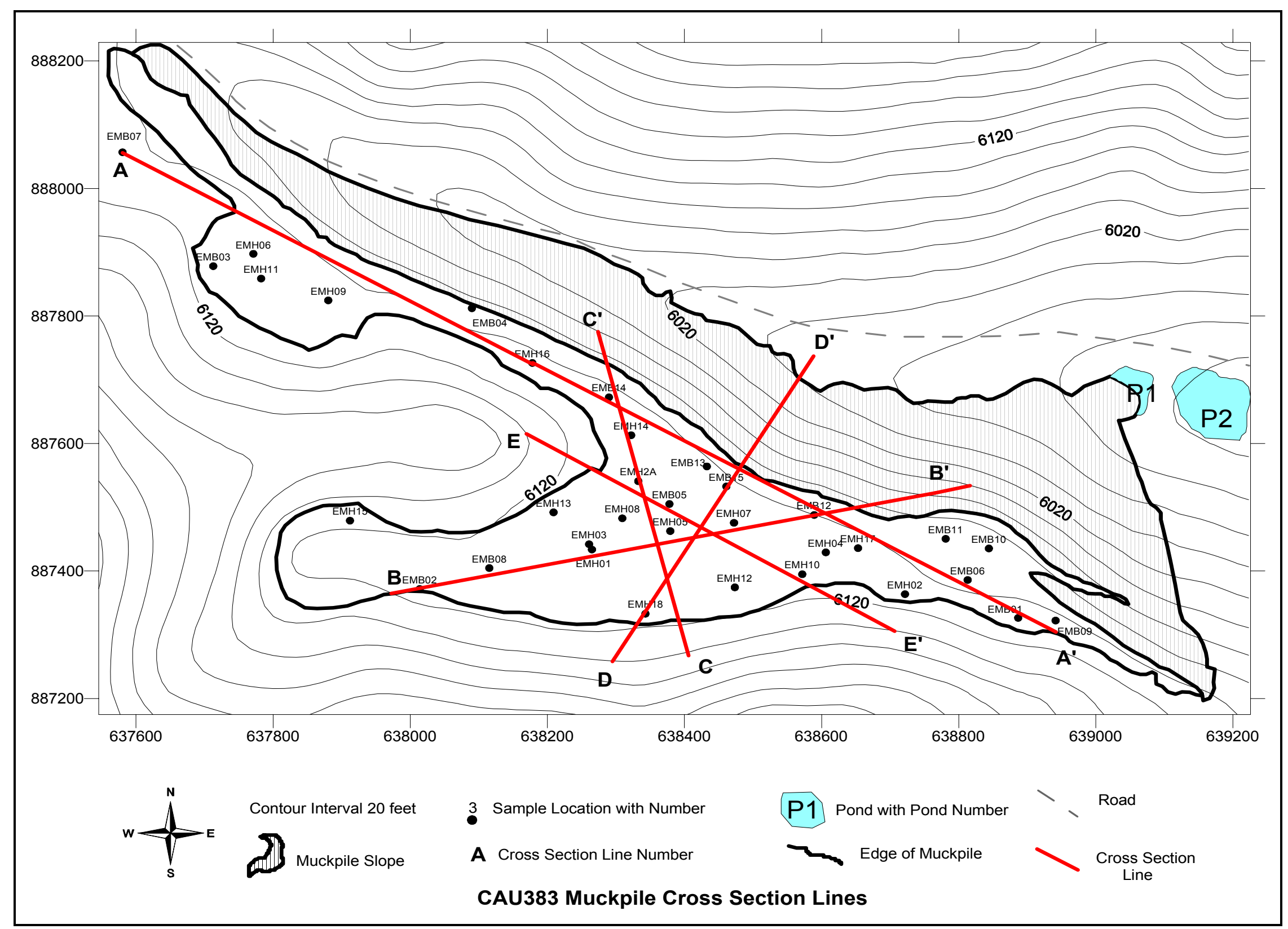




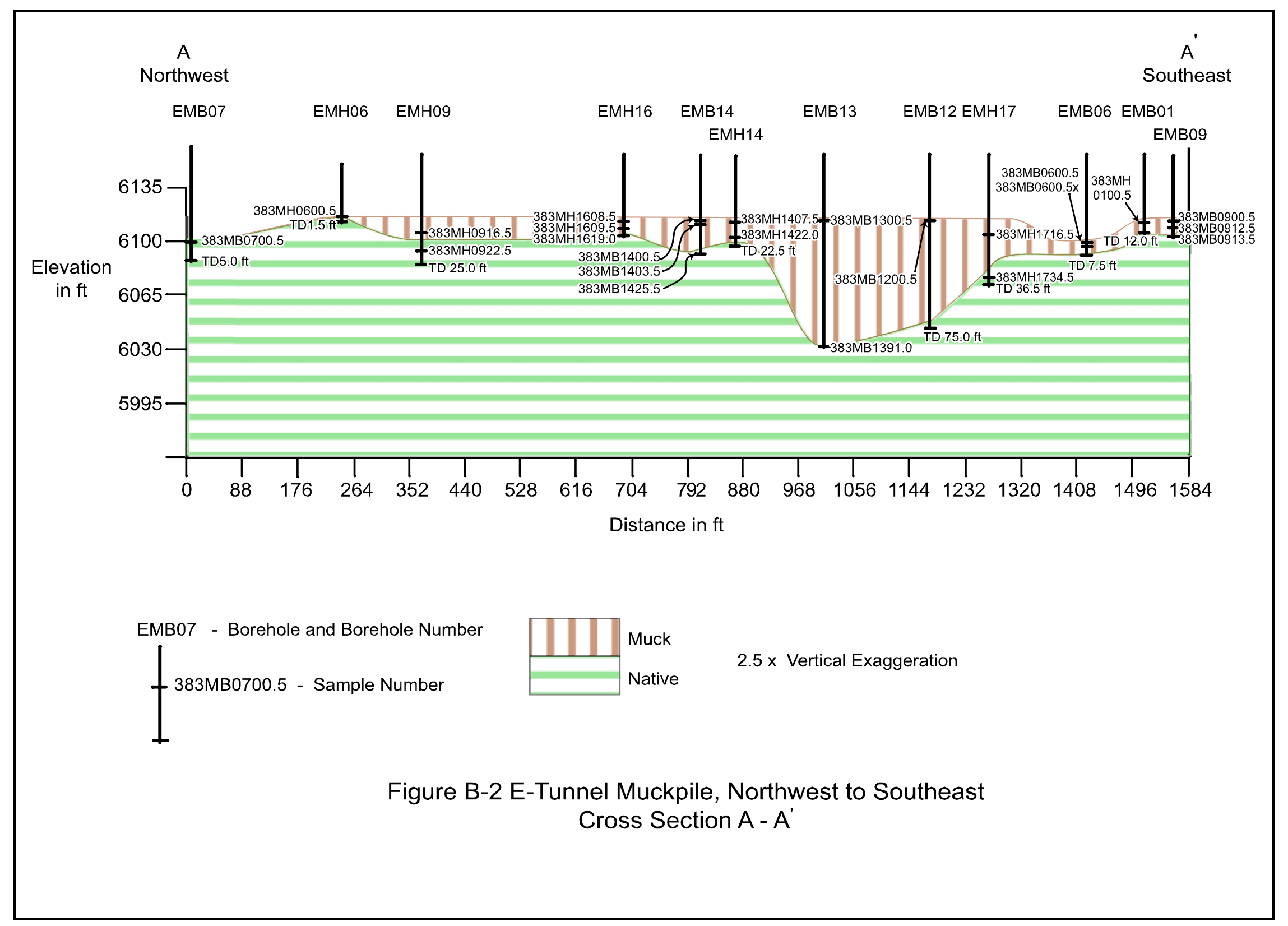




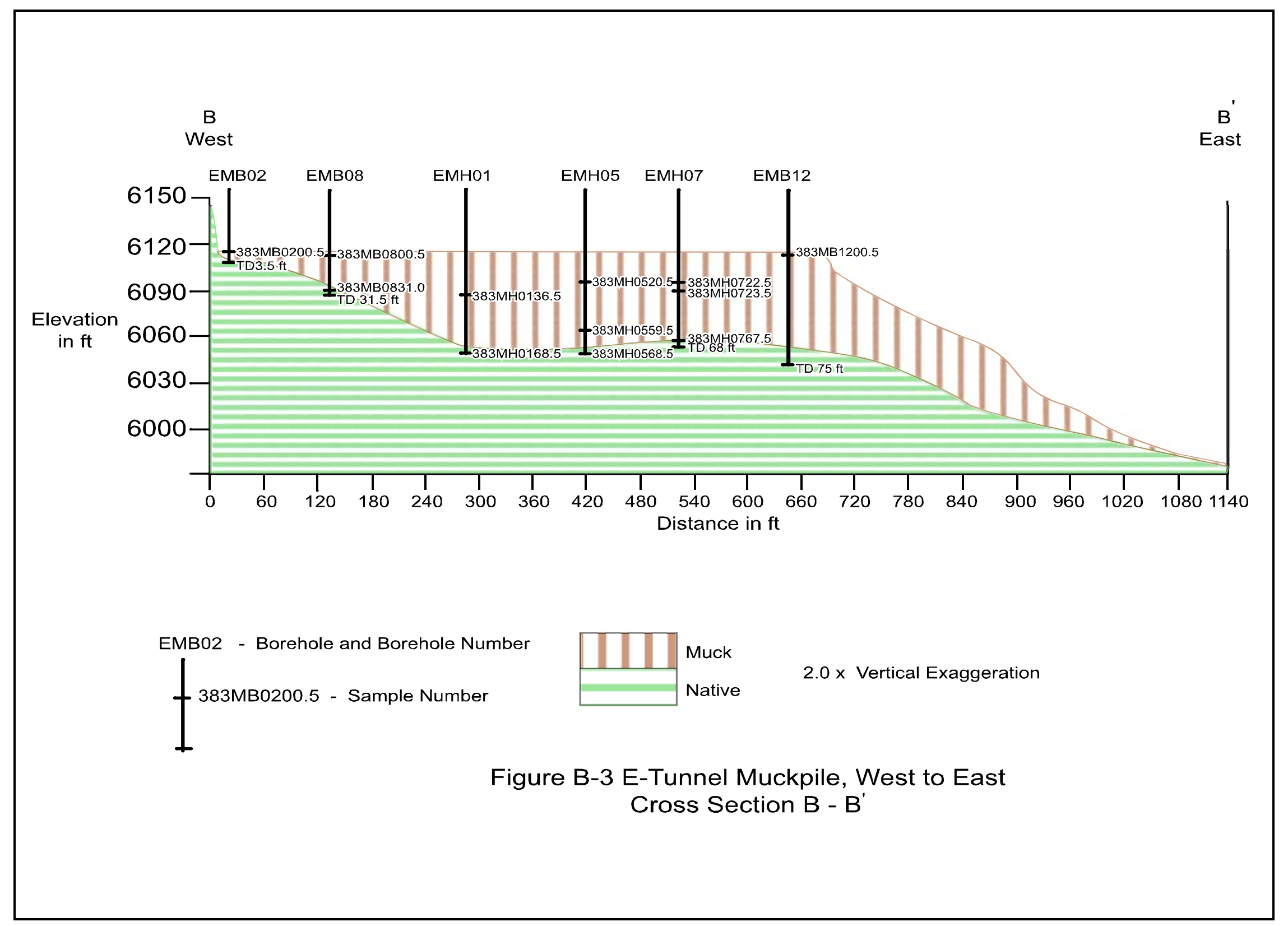




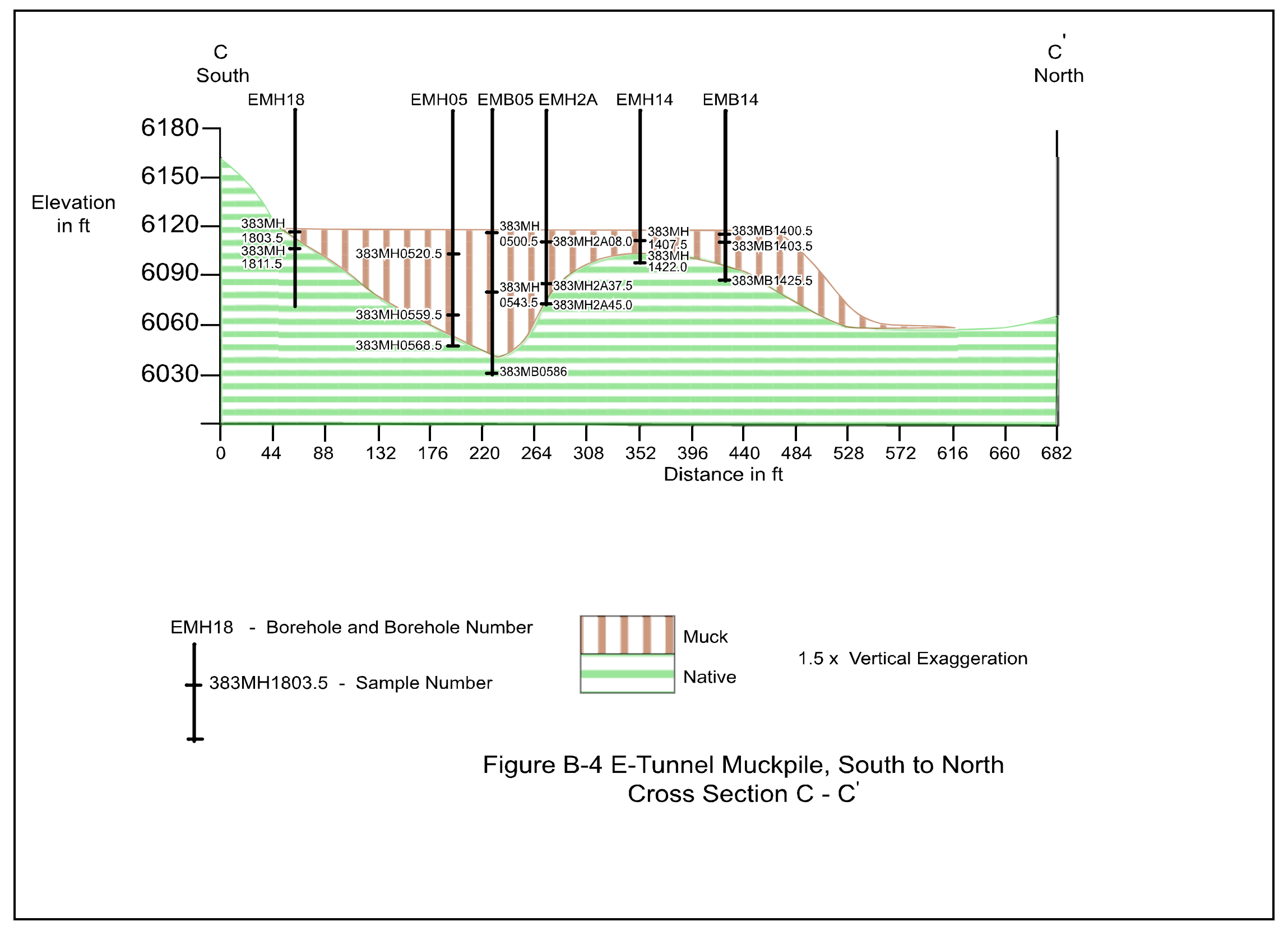




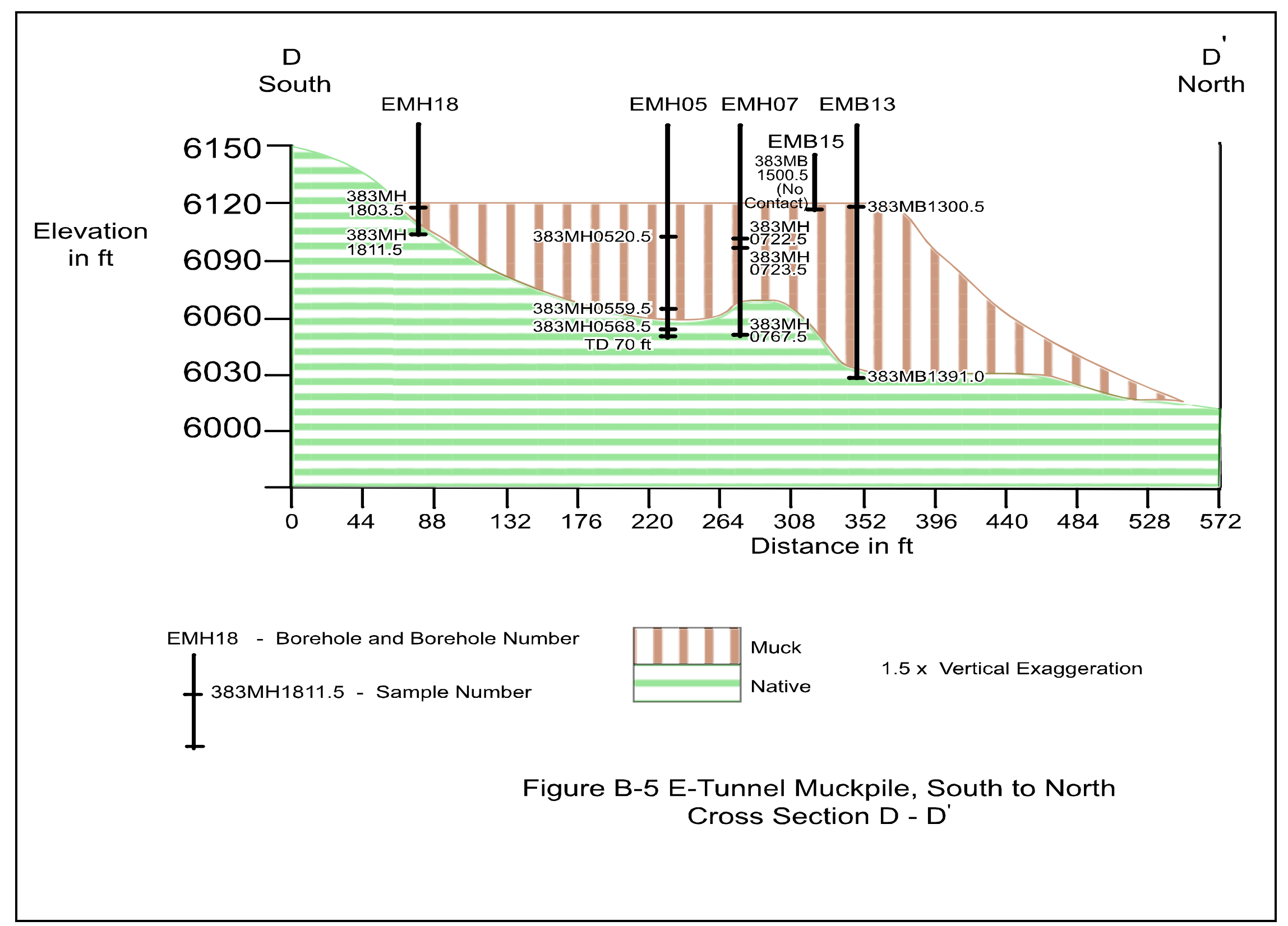




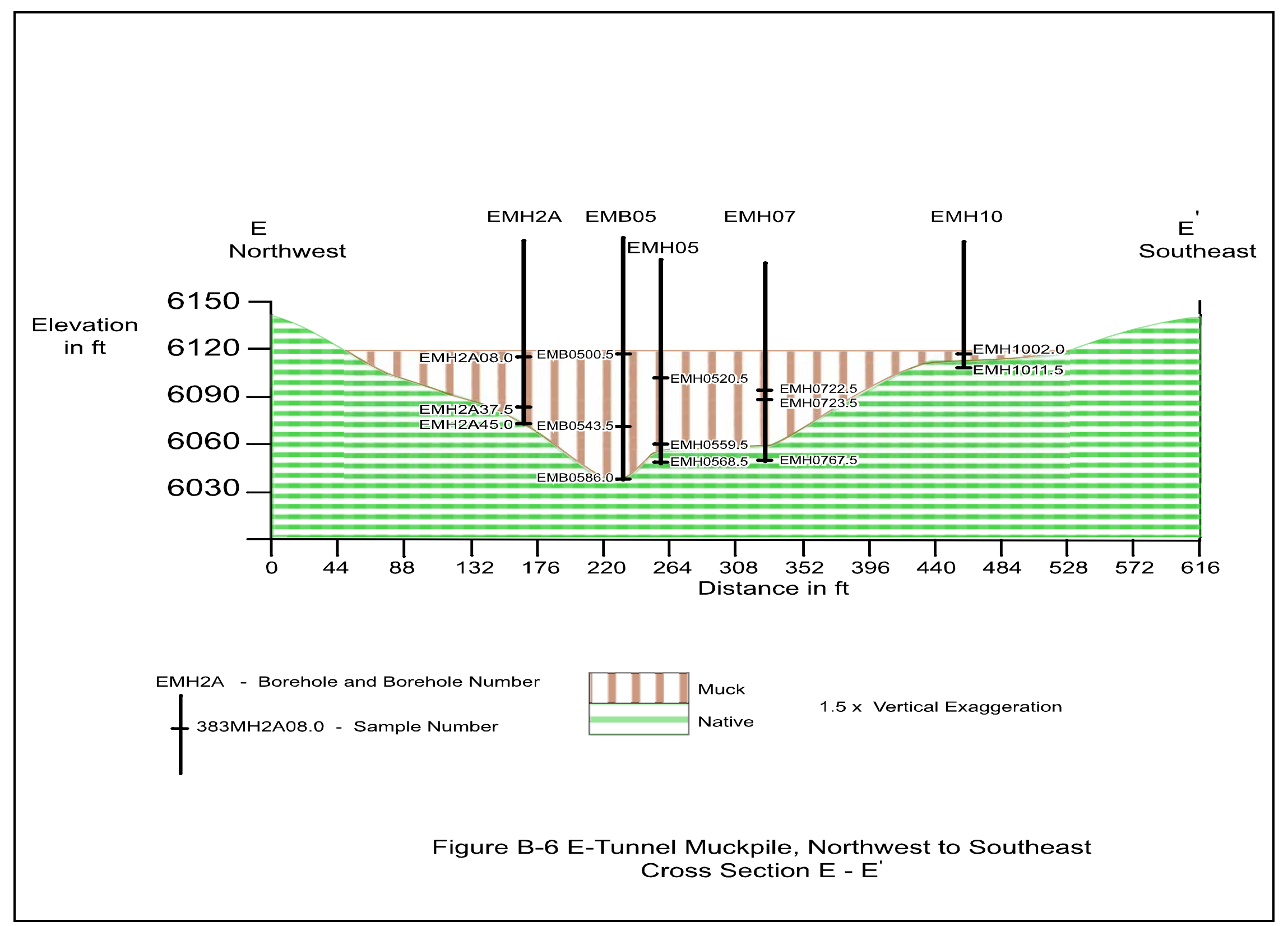




\section{Attachment C}

\section{Analytical Results Table}




\begin{tabular}{|c|c|c|c|c|c|c|c|c|c|c|}
\hline SAMPLE \# & $\begin{array}{l}\text { SAMPLE } \\
\text { DATE }\end{array}$ & MATRIX & $\begin{array}{l}\text { USER TEST } \\
\text { PANEL }\end{array}$ & $\begin{array}{l}\text { SAMPLE } \\
\text { PURPOSE }\end{array}$ & CAS \# & PARAMETER & RESULT & UNITS & Q & $\begin{array}{l}\text { DETECT } \\
\text { LIMIT }\end{array}$ \\
\hline 383CW01 & $5 / 23 / 2004$ & water & EPA6010 & SMQC & $7440-38-2$ & Arsenic & 0.0037 & MG/L & B & 0.01 \\
\hline 383CW01 & $5 / 23 / 2004$ & water & EPA6010 & SMQC & $7440-39-3$ & Barium & 0.012 & MG/L & B & 0.1 \\
\hline 383CW01 & $5 / 23 / 2004$ & water & EPA6010 & SMQC & $7440-41-7$ & Beryllium & 0.00006 & MG/L & B & 0.005 \\
\hline 383CW01 & $5 / 23 / 2004$ & water & EPA6010 & SMQC & $7440-47-3$ & Chromium & 0.0017 & MG/L & B & 0.01 \\
\hline 383CW01 & $5 / 23 / 2004$ & water & EPA7470 & SMQC & $7439-97-6$ & Mercury & 0.0000066 & $\mathrm{MG} / \mathrm{L}$ & B & 0.0002 \\
\hline 383CW01 & $5 / 23 / 2004$ & water & EPA6010 & SMQC & $7440-22-4$ & Silver & 0.0017 & MG/L & B & 0.01 \\
\hline 383CW02 & $5 / 23 / 2004$ & water & EPA8260 & ER & 67-64-1 & Acetone & 22 & UG/L & & 20 \\
\hline 383CW02 & $5 / 23 / 2004$ & water & EPA6010 & ER & $7440-38-2$ & Arsenic & 0.019 & MG/L & & 0.01 \\
\hline 383CW02 & $5 / 23 / 2004$ & water & EPA6010 & ER & $7440-39-3$ & Barium & 0.0047 & MG/L & B & 0.1 \\
\hline 383CW02 & $5 / 23 / 2004$ & water & EPA8270 & ER & 117-81-7 & Bis(2-Ethylhexyl)Phthalate & 15 & UG/L & & 9.5 \\
\hline 383CW02 & $5 / 23 / 2004$ & water & EPA7470 & ER & $7439-97-6$ & Mercury & 0.000007 & MG/L & B & 0.0002 \\
\hline 383CW02 & $5 / 23 / 2004$ & water & EPA6010 & ER & $7440-22-4$ & Silver & 0.0015 & MG/L & B & 0.01 \\
\hline 383CW03 & $5 / 24 / 2004$ & water & EPA8260 & FB & 67-64-1 & Acetone & 9.8 & UG/L & $\mathrm{J}$ & 20 \\
\hline 383CW03 & $5 / 24 / 2004$ & water & EPA6010 & FB & 7440-41-7 & Beryllium & 0.00009 & MG/L & B & 0.005 \\
\hline 383CW03 & $5 / 24 / 2004$ & water & EPA8270 & FB & 117-81-7 & Bis(2-Ethylhexyl)Phthalate & 5.9 & UG/L & $\mathrm{J}$ & 9.7 \\
\hline 383CW03 & $5 / 24 / 2004$ & water & EPA7470 & FB & $7439-97-6$ & Mercury & 0.0000097 & MG/L & B & 0.0002 \\
\hline 383CW04 & $6 / 1 / 2004$ & water & EPA8270 & FB & $117-81-7$ & Bis(2-Ethylhexyl)Phthalate & 16 & UG/L & & 11 \\
\hline 383CW04 & $6 / 1 / 2004$ & water & EPA6010 & FB & $7440-22-4$ & Silver & 0.00068 & MG/L & B & 0.01 \\
\hline 383CW05 & $6 / 1 / 2004$ & water & EPA8260 & ER & $67-64-1$ & Acetone & 8.4 & UG/L & $\mathrm{J}$ & 20 \\
\hline 383CW05 & $6 / 1 / 2004$ & water & EPA8270 & ER & 117-81-7 & Bis(2-Ethylhexyl)Phthalate & 17 & UG/L & & 9.5 \\
\hline 383CW05 & $6 / 1 / 2004$ & water & EPA6010 & ER & $7782-49-2$ & Selenium & 0.0024 & MG/L & B & 0.005 \\
\hline 383CW06 & $6 / 7 / 2004$ & water & EPA8270 & FB & 117-81-7 & Bis(2-Ethylhexyl)Phthalate & 8.8 & UG/L & $\mathrm{J}$ & 9.6 \\
\hline 383CW06 & $6 / 7 / 2004$ & water & EPA6010 & FB & $7440-22-4$ & Silver & 0.00094 & MG/L & B & 0.01 \\
\hline 383CW07 & $6 / 7 / 2004$ & water & EPA8260 & ER & $67-64-1$ & Acetone & 6.9 & UG/L & $\mathrm{J}$ & 20 \\
\hline 383CW07 & $6 / 7 / 2004$ & water & EPA6010 & ER & $7440-39-3$ & Barium & 0.0016 & MG/L & B & 0.1 \\
\hline 383CW07 & $6 / 7 / 2004$ & water & EPA8270 & ER & 117-81-7 & Bis(2-Ethylhexyl)Phthalate & 7.4 & UG/L & $\mathrm{J}$ & 9.6 \\
\hline 383CW07 & $6 / 7 / 2004$ & water & EPA8260 & ER & $74-87-3$ & Chloromethane & 0.58 & UG/L & $\mathrm{J}$ & 10 \\
\hline 383CW08 & $6 / 10 / 2004$ & water & EPA8260 & FB & $67-64-1$ & Acetone & 25 & UG/L & B & 20 \\
\hline 383CW08 & $6 / 10 / 2004$ & water & EPA8270 & FB & 117-81-7 & Bis(2-Ethylhexyl)Phthalate & 6.5 & UG/L & $\mathrm{J}$ & 9.6 \\
\hline 383CW08 & $6 / 10 / 2004$ & water & EPA7470 & FB & $7439-97-6$ & Mercury & 0.0000032 & MG/L & B & 0.0002 \\
\hline 383CW09 & $6 / 10 / 2004$ & water & EPA8260 & ER & $67-64-1$ & Acetone & 20 & UG/L & B & 20 \\
\hline 383CW09 & $6 / 10 / 2004$ & water & EPA8270 & ER & 117-81-7 & Bis(2-Ethylhexyl)Phthalate & 10 & UG/L & & 9.6 \\
\hline 383CW09 & $6 / 10 / 2004$ & water & EPA6010 & ER & $7440-43-9$ & Cadmium & 0.00018 & MG/L & B & 0.005 \\
\hline 383CW09 & $6 / 10 / 2004$ & water & EPA7470 & ER & $7439-97-6$ & Mercury & 0.0000035 & MG/L & B & 0.0002 \\
\hline 383CW09 & $6 / 10 / 2004$ & water & EPA6010 & ER & $7440-22-4$ & Silver & 0.00066 & MG/L & $B$ & 0.01 \\
\hline
\end{tabular}




\begin{tabular}{|c|c|c|c|c|c|c|c|c|c|c|}
\hline SAMPLE \# & $\begin{array}{l}\text { SAMPLE } \\
\text { DATE }\end{array}$ & MATRIX & $\begin{array}{l}\text { USER TEST } \\
\text { PANEL }\end{array}$ & $\begin{array}{l}\text { SAMPLE } \\
\text { PURPOSE }\end{array}$ & CAS \# & PARAMETER & RESULT & UNITS & Q & $\begin{array}{l}\text { DETECT } \\
\text { LIMIT }\end{array}$ \\
\hline 383CW10 & $6 / 12 / 2004$ & water & EPA8270 & FB & 117-81-7 & Bis(2-Ethylhexyl)Phthalate & 26 & UG/L & & 9.6 \\
\hline $383 C W 10$ & $6 / 12 / 2004$ & water & EPA8260 & FB & $75-09-2$ & Methylene Chloride & 0.79 & UG/L & $\mathrm{J}$ & 5 \\
\hline 383CW11 & $6 / 12 / 2004$ & water & EPA6010 & ER & $7440-39-3$ & Barium & 0.005 & MG/L & B & 0.1 \\
\hline 383CW11 & $6 / 12 / 2004$ & water & EPA8270 & ER & 117-81-7 & Bis(2-Ethylhexyl)Phthalate & 42 & UG/L & & 9.6 \\
\hline 383CW11 & $6 / 12 / 2004$ & water & EPA7470 & ER & $7439-97-6$ & Mercury & 0.0000042 & $\mathrm{MG} / \mathrm{L}$ & B & 0.0002 \\
\hline 383CW11 & $6 / 12 / 2004$ & water & EPA8260 & ER & $75-09-2$ & Methylene Chloride & 0.75 & UG/L & $\mathrm{J}$ & 5 \\
\hline 383CW12 & $6 / 16 / 2004$ & water & EPA8270 & FB & 117-81-7 & Bis(2-Ethylhexyl)Phthalate & 6 & UG/L & $\mathrm{J}$ & 9.5 \\
\hline 383CW12 & $6 / 16 / 2004$ & water & EPA7470 & FB & 7439-97-6 & Mercury & 0.00003 & MG/L & B & 0.0002 \\
\hline 383CW12 & $6 / 16 / 2004$ & water & EPA8260 & FB & $75-09-2$ & Methylene Chloride & 1.6 & UG/L & $\mathrm{J}$ & 5 \\
\hline $383 C W 13$ & $6 / 16 / 2004$ & water & EPA6010 & ER & $7440-39-3$ & Barium & 0.00049 & MG/L & B & 0.1 \\
\hline 383CW13 & $6 / 16 / 2004$ & water & EPA8270 & ER & 117-81-7 & Bis(2-Ethylhexyl)Phthalate & 24 & UG/L & & 9.6 \\
\hline 383CW13 & $6 / 16 / 2004$ & water & EPA8260 & ER & $74-87-3$ & Chloromethane & 0.71 & UG/L & $\mathrm{J}$ & 10 \\
\hline 383CW13 & $6 / 16 / 2004$ & water & EPA7470 & ER & 7439-97-6 & Mercury & 0.00003 & MG/L & B & 0.0002 \\
\hline 383CW13 & $6 / 16 / 2004$ & water & EPA8260 & ER & $75-09-2$ & Methylene Chloride & 1.4 & UG/L & $\mathrm{J}$ & 5 \\
\hline 383CW13 & $6 / 16 / 2004$ & water & EPA6010 & ER & $7440-22-4$ & Silver & 0.00087 & MG/L & B & 0.01 \\
\hline 383CW14 & $6 / 17 / 2004$ & water & EPA8270 & FB & 117-81-7 & Bis(2-Ethylhexyl)Phthalate & 10 & UG/L & & 9.5 \\
\hline 383CW14 & $6 / 17 / 2004$ & water & EPA7470 & FB & $7439-97-6$ & Mercury & 0.00003 & MG/L & B & 0.0002 \\
\hline 383CW14 & $6 / 17 / 2004$ & water & EPA8260 & FB & $75-09-2$ & Methylene Chloride & 1.5 & UG/L & $\mathrm{J}$ & 5 \\
\hline $383 C W 15$ & $6 / 17 / 2004$ & water & EPA6010 & ER & $7440-39-3$ & Barium & 0.013 & MG/L & B & 0.1 \\
\hline 383CW15 & $6 / 17 / 2004$ & water & EPA8270 & ER & 117-81-7 & Bis(2-Ethylhexyl)Phthalate & 18 & UG/L & & 9.6 \\
\hline 383CW15 & $6 / 17 / 2004$ & water & EPA7470 & ER & $7439-97-6$ & Mercury & 0.00003 & MG/L & $\mathrm{B}$ & 0.0002 \\
\hline 383CW15 & $6 / 17 / 2004$ & water & EPA8260 & ER & $75-09-2$ & Methylene Chloride & 1.3 & UG/L & $\mathrm{J}$ & 5 \\
\hline 383CW15 & $6 / 17 / 2004$ & water & EPA6010 & ER & $7440-22-4$ & Silver & 0.00069 & MG/L & B & 0.01 \\
\hline 383DH0100.5 & $6 / 7 / 2004$ & soil & HASL300 & REG & 14331-83-0 & Ac-228 & 2.21 & $\mathrm{PCl} / \mathrm{G}$ & G & 0.68 \\
\hline 383DH0100.5 & $6 / 7 / 2004$ & soil & EPA6010 & REG & $7440-38-2$ & Arsenic & 2.3 & MG/KG & & 1 \\
\hline 383DH0100.5 & $6 / 7 / 2004$ & soil & EPA6010 & REG & $7440-39-3$ & Barium & 750 & MG/KG & & 10 \\
\hline 383DH0100.5 & $6 / 7 / 2004$ & soil & EPA6010 & REG & $7440-41-7$ & Beryllium & 1.3 & MG/KG & & 0.52 \\
\hline 383DH0100.5 & $6 / 7 / 2004$ & soil & HASL300 & REG & 14733-03-0 & Bi-214 & 1.24 & $\mathrm{PCl} / \mathrm{G}$ & $\mathrm{G}, \mathrm{J}$ & 0.46 \\
\hline 383DH0100.5 & $6 / 7 / 2004$ & soil & EPA6010 & REG & $7440-43-9$ & Cadmium & 0.14 & MG/KG & $B$ & 0.52 \\
\hline 383DH0100.5 & $6 / 7 / 2004$ & soil & EPA6010 & REG & $7440-47-3$ & Chromium & 1.1 & MG/KG & & 1 \\
\hline 383DH0100.5 & $6 / 7 / 2004$ & soil & HASL300 & REG & 10045-97-3 & Cs-137 & 34.4 & $\mathrm{PCl} / \mathrm{G}$ & G & 0.2 \\
\hline 383DH0100.5 & $6 / 7 / 2004$ & soil & HASL300 & REG & 13966-00-2 & $\mathrm{K}-40$ & 34.5 & $\mathrm{PCl} / \mathrm{G}$ & G & 1.9 \\
\hline 383DH0100.5 & $6 / 7 / 2004$ & soil & EPA6010 & REG & 7439-92-1 & Lead & 20 & MG/KG & & 0.31 \\
\hline 383DH0100.5 & 6/7/2004 & soil & EPA8260 & REG & 136777-61-2 & M+P-Xylene & 2.1 & UG/KG & $\mathrm{J}$ & 5.2 \\
\hline 383DH0100.5 & $6 / 7 / 2004$ & soil & EPA7470 & REG & 7439-97-6 & Mercury & 0.01 & MG/KG & $B$ & 0.1 \\
\hline
\end{tabular}




\begin{tabular}{|c|c|c|c|c|c|c|c|c|c|c|}
\hline SAMPLE \# & $\begin{array}{l}\text { SAMPLE } \\
\text { DATE }\end{array}$ & MATRIX & $\begin{array}{l}\text { USER TEST } \\
\text { PANEL }\end{array}$ & $\begin{array}{l}\text { SAMPLE } \\
\text { PURPOSE }\end{array}$ & CAS \# & PARAMETER & RESULT & UNITS & Q & $\begin{array}{l}\text { DETECT } \\
\text { LIMIT }\end{array}$ \\
\hline 383DH0100.5 & $6 / 7 / 2004$ & soil & EPA8260 & REG & $75-09-2$ & Methylene Chloride & 0.83 & UG/KG & $\mathrm{J}$ & 5.2 \\
\hline 383DH0100.5 & $6 / 7 / 2004$ & soil & HASL300 & REG & 15092-94-1 & $\mathrm{Pb}-212$ & 2.51 & $\mathrm{PCl} / \mathrm{G}$ & $\mathrm{J}$ & 0.41 \\
\hline 383DH0100.5 & $6 / 7 / 2004$ & soil & HASL300 & REG & 15067-28-4 & $\mathrm{Pb}-214$ & 1.41 & $\mathrm{PCl} / \mathrm{G}$ & $\mathrm{G}, \mathrm{J}$ & 0.55 \\
\hline 383DH0100.5 & $6 / 7 / 2004$ & soil & UGTAISOPU & REG & 13981-16-3 & $\mathrm{Pu}-238$ & 0.73 & $\mathrm{PCl} / \mathrm{G}$ & & 0.02 \\
\hline 383DH0100.5 & $6 / 7 / 2004$ & soil & UGTAISOPU & REG & 15117-48-3 & Pu-239 & 3.03 & $\mathrm{PCl} / \mathrm{G}$ & $\mathrm{J}$ & 0.01 \\
\hline 383DH0100.5 & $6 / 7 / 2004$ & soil & SR7500 & REG & $10098-97-2$ & Sr-90 & 1.88 & $\mathrm{PCl} / \mathrm{G}$ & & 0.24 \\
\hline 383DH0100.5 & $6 / 7 / 2004$ & soil & HASL300 & REG & 14913-50-9 & TI-208 & 0.71 & $\mathrm{PCl} / \mathrm{G}$ & G & 0.22 \\
\hline 383DH0200.5 & $6 / 7 / 2004$ & soil & EPA8260 & REG & $78-93-3$ & 2-Butanone & 10 & UG/KG & $\mathrm{J}$ & 21 \\
\hline 383DH0200.5 & $6 / 7 / 2004$ & soil & HASL300 & REG & 14331-83-0 & Ac-228 & 2.26 & $\mathrm{PCl} / \mathrm{G}$ & $G$ & 0.71 \\
\hline 383DH0200.5 & $6 / 7 / 2004$ & soil & EPA8260 & REG & $67-64-1$ & Acetone & 39 & UG/KG & & 21 \\
\hline 383DH0200.5 & $6 / 7 / 2004$ & soil & HASL300 & REG & 14596-10-2 & Am-241 & 1.88 & $\mathrm{PCl} / \mathrm{G}$ & $\mathrm{J}$ & 0.48 \\
\hline 383DH0200.5 & $6 / 7 / 2004$ & soil & EPA6010 & REG & $7440-38-2$ & Arsenic & 2.6 & MG/KG & & 1 \\
\hline 383DH0200.5 & $6 / 7 / 2004$ & soil & EPA6010 & REG & $7440-39-3$ & Barium & 3100 & MG/KG & & 100 \\
\hline 383DH0200.5 & $6 / 7 / 2004$ & soil & EPA6010 & REG & $7440-41-7$ & Beryllium & 1.2 & MG/KG & & 0.51 \\
\hline 383DH0200.5 & $6 / 7 / 2004$ & soil & HASL300 & REG & 14733-03-0 & $\mathrm{Bi}-214$ & 1.27 & $\mathrm{PCl} / \mathrm{G}$ & $\mathrm{G}, \mathrm{J}$ & 0.47 \\
\hline 383DH0200.5 & $6 / 7 / 2004$ & soil & EPA6010 & REG & $7440-43-9$ & Cadmium & 0.16 & MG/KG & B & 0.51 \\
\hline 383DH0200.5 & $6 / 7 / 2004$ & soil & EPA6010 & REG & $7440-47-3$ & Chromium & 2.6 & MG/KG & & 1 \\
\hline 383DH0200.5 & $6 / 7 / 2004$ & soil & HASL300 & REG & $10045-97-3$ & Cs-137 & 45.8 & $\mathrm{PCl} / \mathrm{G}$ & G & 0.2 \\
\hline 383DH0200.5 & $6 / 7 / 2004$ & soil & EPA8260 & REG & $100-41-4$ & Ethylbenzene & 3.1 & UG/KG & $\mathrm{J}$ & 5.1 \\
\hline 383DH0200.5 & $6 / 7 / 2004$ & soil & HASL300 & REG & 13966-00-2 & $\mathrm{K}-40$ & 31.8 & $\mathrm{PCl} / \mathrm{G}$ & G & 2.2 \\
\hline 383DH0200.5 & $6 / 7 / 2004$ & soil & EPA6010 & REG & $7439-92-1$ & Lead & 15 & MG/KG & & 0.31 \\
\hline 383DH0200.5 & $6 / 7 / 2004$ & soil & EPA8260 & REG & 136777-61-2 & M+P-Xylene & 18 & UG/KG & & 5.1 \\
\hline 383DH0200.5 & $6 / 7 / 2004$ & soil & EPA7470 & REG & 7439-97-6 & Mercury & 0.015 & MG/KG & B & 0.1 \\
\hline 383DH0200.5 & $6 / 7 / 2004$ & soil & EPA8260 & REG & $75-09-2$ & Methylene Chloride & 4 & UG/KG & $\mathrm{J}$ & 5.1 \\
\hline 383DH0200.5 & $6 / 7 / 2004$ & soil & EPA8260 & REG & $95-47-6$ & O-Xylene & 6.7 & UG/KG & & 5.1 \\
\hline 383DH0200.5 & $6 / 7 / 2004$ & soil & HASL300 & REG & 15092-94-1 & $\mathrm{Pb}-212$ & 2.42 & $\mathrm{PCl} / \mathrm{G}$ & $\mathrm{J}$ & 0.44 \\
\hline 383DH0200.5 & $6 / 7 / 2004$ & soil & HASL300 & REG & 15067-28-4 & $\mathrm{Pb}-214$ & 1.4 & $\mathrm{PCl} / \mathrm{G}$ & $\mathrm{G}, \mathrm{J}$ & 0.58 \\
\hline 383DH0200.5 & $6 / 7 / 2004$ & soil & UGTAISOPU & REG & 13981-16-3 & Pu-238 & 0.156 & $\mathrm{PCl} / \mathrm{G}$ & & 0.029 \\
\hline 383DH0200.5 & $6 / 7 / 2004$ & soil & UGTAISOPU & REG & 15117-48-3 & Pu-239 & 1.46 & $\mathrm{PCl} / \mathrm{G}$ & $\mathrm{J}$ & 0.02 \\
\hline 383DH0200.5 & $6 / 7 / 2004$ & soil & SR7500 & REG & 10098-97-2 & Sr-90 & 1.23 & $\mathrm{PCl} / \mathrm{G}$ & Y1 & 0.22 \\
\hline 383DH0200.5 & $6 / 7 / 2004$ & soil & EPA8260 & REG & $100-42-5$ & Styrene & 1.2 & UG/KG & $\mathrm{J}$ & 5.1 \\
\hline 383DH0200.5 & $6 / 7 / 2004$ & soil & HASL300 & REG & 14913-50-9 & TI-208 & 0.7 & $\mathrm{PCl} / \mathrm{G}$ & G & 0.24 \\
\hline 383DH0200.5 & $6 / 7 / 2004$ & soil & EPA8260 & REG & $108-88-3$ & Toluene & 1.4 & UG/KG & $\mathrm{J}$ & 5.1 \\
\hline 383DH0300.5 & 6/7/2004 & soil & HASL300 & REG & 14331-83-0 & Ac-228 & 1.91 & $\mathrm{PCl} / \mathrm{G}$ & G & 0.53 \\
\hline 383DH0300.5 & $6 / 7 / 2004$ & soil & EPA8260 & REG & 67-64-1 & Acetone & 18 & UG/KG & $\mathrm{J}$ & 20 \\
\hline
\end{tabular}




\begin{tabular}{|c|c|c|c|c|c|c|c|c|c|c|}
\hline SAMPLE \# & $\begin{array}{l}\text { SAMPLE } \\
\text { DATE }\end{array}$ & MATRIX & $\begin{array}{l}\text { USER TEST } \\
\text { PANEL }\end{array}$ & $\begin{array}{l}\text { SAMPLE } \\
\text { PURPOSE }\end{array}$ & CAS \# & PARAMETER & RESULT & UNITS & Q & $\begin{array}{l}\text { DETECT } \\
\text { LIMIT }\end{array}$ \\
\hline 383DH0300.5 & $6 / 7 / 2004$ & soil & EPA6010 & REG & $7440-38-2$ & Arsenic & 2.7 & MG/KG & & 1 \\
\hline 383DH0300.5 & $6 / 7 / 2004$ & soil & EPA6010 & REG & $7440-39-3$ & Barium & 2800 & MG/KG & & 100 \\
\hline 383DH0300.5 & $6 / 7 / 2004$ & soil & EPA6010 & REG & $7440-41-7$ & Beryllium & 1.6 & MG/KG & & 0.51 \\
\hline 383DH0300.5 & $6 / 7 / 2004$ & soil & HASL300 & REG & 14733-03-0 & Bi-214 & 1.32 & $\mathrm{PCl} / \mathrm{G}$ & $\mathrm{G}, \mathrm{J}$ & 0.31 \\
\hline 383DH0300.5 & $6 / 7 / 2004$ & soil & EPA6010 & REG & $7440-43-9$ & Cadmium & 0.16 & MG/KG & B & 0.51 \\
\hline 383DH0300.5 & $6 / 7 / 2004$ & soil & EPA6010 & REG & $7440-47-3$ & Chromium & 3.9 & MG/KG & & 1 \\
\hline 383DH0300.5 & $6 / 7 / 2004$ & soil & HASL300 & REG & 10045-97-3 & Cs-137 & 3.72 & $\mathrm{PCl} / \mathrm{G}$ & G & 0.15 \\
\hline 383DH0300.5 & $6 / 7 / 2004$ & soil & EPA8260 & REG & $100-41-4$ & Ethylbenzene & 11 & UG/KG & & 5.1 \\
\hline 383DH0300.5 & $6 / 7 / 2004$ & soil & HASL300 & REG & 13966-00-2 & $\mathrm{K}-40$ & 27.6 & $\mathrm{PCl} / \mathrm{G}$ & G & 1.5 \\
\hline 383DH0300.5 & $6 / 7 / 2004$ & soil & EPA6010 & REG & $7439-92-1$ & Lead & 17 & MG/KG & & 0.31 \\
\hline 383DH0300.5 & $6 / 7 / 2004$ & soil & EPA8260 & REG & 136777-61-2 & M+P-Xylene & 57 & UG/KG & & 5.1 \\
\hline 383DH0300.5 & $6 / 7 / 2004$ & soil & EPA7470 & REG & $7439-97-6$ & Mercury & 0.0088 & MG/KG & B & 0.1 \\
\hline 383DH0300.5 & $6 / 7 / 2004$ & soil & EPA8260 & REG & $75-09-2$ & Methylene Chloride & 3.1 & UG/KG & $\mathrm{J}$ & 5.1 \\
\hline 383DH0300.5 & $6 / 7 / 2004$ & soil & EPA8260 & REG & $95-47-6$ & O-Xylene & 23 & UG/KG & & 5.1 \\
\hline 383DH0300.5 & $6 / 7 / 2004$ & soil & HASL300 & REG & 15092-94-1 & $\mathrm{Pb}-212$ & 2.08 & $\mathrm{PCl} / \mathrm{G}$ & $\mathrm{J}$ & 0.25 \\
\hline 383DH0300.5 & $6 / 7 / 2004$ & soil & HASL300 & REG & $15067-28-4$ & $\mathrm{~Pb}-214$ & 1.21 & $\mathrm{PCl} / \mathrm{G}$ & $\mathrm{G}, \mathrm{J}$ & 0.26 \\
\hline 383DH0300.5 & $6 / 7 / 2004$ & soil & UGTAISOPU & REG & 15117-48-3 & Pu-239 & 0.186 & $\mathrm{PCl} / \mathrm{G}$ & $\mathrm{J}$ & 0.025 \\
\hline 383DH0300.5 & $6 / 7 / 2004$ & soil & EPA8260 & REG & $100-42-5$ & Styrene & 3.6 & UG/KG & $\mathrm{J}$ & 5.1 \\
\hline 383DH0300.5 & $6 / 7 / 2004$ & soil & HASL300 & REG & 14913-50-9 & TI-208 & 0.58 & $\mathrm{PCl} / \mathrm{G}$ & G & 0.14 \\
\hline 383DH0300.5 & $6 / 7 / 2004$ & soil & EPA8260 & REG & $108-88-3$ & Toluene & 1.4 & UG/KG & $\mathrm{J}$ & 5.1 \\
\hline 383DH0400.5 & $6 / 7 / 2004$ & soil & HASL300 & REG & 14331-83-0 & Ac-228 & 1.8 & $\mathrm{PCl} / \mathrm{G}$ & G & 0.56 \\
\hline 383DH0400.5 & $6 / 7 / 2004$ & soil & EPA6010 & REG & 7440-38-2 & Arsenic & 3.1 & MG/KG & & 1 \\
\hline 383DH0400.5 & $6 / 7 / 2004$ & soil & EPA6010 & REG & $7440-39-3$ & Barium & 3300 & MG/KG & & 100 \\
\hline 383DH0400.5 & $6 / 7 / 2004$ & soil & EPA6010 & REG & $7440-41-7$ & Beryllium & 1.6 & MG/KG & & 0.52 \\
\hline 383DH0400.5 & $6 / 7 / 2004$ & soil & HASL300 & REG & 14733-03-0 & Bi-214 & 1.33 & $\mathrm{PCl} / \mathrm{G}$ & $\mathrm{G}, \mathrm{J}$ & 0.35 \\
\hline 383DH0400.5 & $6 / 7 / 2004$ & soil & EPA8270 & REG & $85-68-7$ & Butyl Benzyl Phthalate & 33 & UG/KG & $\mathrm{J}$ & 350 \\
\hline 383DH0400.5 & $6 / 7 / 2004$ & soil & EPA6010 & REG & $7440-43-9$ & Cadmium & 0.17 & MG/KG & B & 0.52 \\
\hline 383DH0400.5 & $6 / 7 / 2004$ & soil & EPA6010 & REG & $7440-47-3$ & Chromium & 4.3 & MG/KG & & 1 \\
\hline 383DH0400.5 & $6 / 7 / 2004$ & soil & HASL300 & REG & $10045-97-3$ & Cs-137 & 3.86 & $\mathrm{PCl} / \mathrm{G}$ & G & 0.18 \\
\hline 383DH0400.5 & $6 / 7 / 2004$ & soil & HASL300 & REG & 13966-00-2 & $\mathrm{K}-40$ & 30.8 & $\mathrm{PCl} / \mathrm{G}$ & G & 1.8 \\
\hline 383DH0400.5 & $6 / 7 / 2004$ & soil & EPA6010 & REG & $7439-92-1$ & Lead & 17 & MG/KG & & 0.31 \\
\hline 383DH0400.5 & $6 / 7 / 2004$ & soil & EPA7470 & REG & 7439-97-6 & Mercury & 0.012 & MG/KG & $B$ & 0.1 \\
\hline 383DH0400.5 & $6 / 7 / 2004$ & soil & EPA8260 & REG & $75-09-2$ & Methylene Chloride & 1.8 & UG/KG & $\mathrm{J}$ & 5.2 \\
\hline 383DH0400.5 & $6 / 7 / 2004$ & soil & HASL300 & REG & 15092-94-1 & $\mathrm{Pb}-212$ & 1.78 & $\mathrm{PCl} / \mathrm{G}$ & $\mathrm{J}$ & 0.29 \\
\hline 383DH0400.5 & $6 / 7 / 2004$ & soil & HASL300 & REG & 15067-28-4 & $\mathrm{Pb}-214$ & 1.4 & $\mathrm{PCl} / \mathrm{G}$ & $\mathrm{G}, \mathrm{J}$ & 0.3 \\
\hline
\end{tabular}




\begin{tabular}{|c|c|c|c|c|c|c|c|c|c|c|}
\hline SAMPLE \# & $\begin{array}{l}\text { SAMPLE } \\
\text { DATE }\end{array}$ & MATRIX & $\begin{array}{l}\text { USER TEST } \\
\text { PANEL }\end{array}$ & $\begin{array}{l}\text { SAMPLE } \\
\text { PURPOSE }\end{array}$ & CAS \# & PARAMETER & RESULT & UNITS & Q & $\begin{array}{l}\text { DETECT } \\
\text { LIMIT }\end{array}$ \\
\hline 383DH0400.5 & $6 / 7 / 2004$ & soil & UGTAISOPU & REG & 13981-16-3 & Pu-238 & 0.063 & $\mathrm{PCl} / \mathrm{G}$ & & 0.009 \\
\hline 383DH0400.5 & $6 / 7 / 2004$ & soil & UGTAISOPU & REG & 15117-48-3 & Pu-239 & 0.5 & $\mathrm{PCl} / \mathrm{G}$ & $\mathrm{J}$ & 0.02 \\
\hline 383DH0400.5 & $6 / 7 / 2004$ & soil & HASL300 & REG & $14913-50-9$ & TI-208 & 0.65 & $\mathrm{PCl} / \mathrm{G}$ & $\mathrm{G}$ & 0.17 \\
\hline 383DH0500.5 & $6 / 7 / 2004$ & soil & HASL300 & REG & 14331-83-0 & Ac-228 & 1.85 & $\mathrm{PCl} / \mathrm{G}$ & $\mathrm{G}$ & 0.56 \\
\hline 383DH0500.5 & $6 / 7 / 2004$ & soil & EPA8260 & REG & 67-64-1 & Acetone & 23 & UG/KG & & 20 \\
\hline 383DH0500.5 & $6 / 7 / 2004$ & soil & EPA6010 & REG & $7440-38-2$ & Arsenic & 3 & MG/KG & & 1 \\
\hline 383DH0500.5 & $6 / 7 / 2004$ & soil & EPA6010 & REG & $7440-39-3$ & Barium & 3200 & MG/KG & & 100 \\
\hline 383DH0500.5 & $6 / 7 / 2004$ & soil & EPA6010 & REG & $7440-41-7$ & Beryllium & 1.7 & MG/KG & & 0.51 \\
\hline 383DH0500.5 & $6 / 7 / 2004$ & soil & HASL300 & REG & 14733-03-0 & $\mathrm{Bi}-214$ & 1.41 & $\mathrm{PCl} / \mathrm{G}$ & G,J & 0.37 \\
\hline 383DH0500.5 & $6 / 7 / 2004$ & soil & EPA8270 & REG & $85-68-7$ & Butyl Benzyl Phthalate & 40 & UG/KG & $\mathrm{J}$ & 340 \\
\hline 383DH0500.5 & $6 / 7 / 2004$ & soil & EPA6010 & REG & $7440-43-9$ & Cadmium & 0.19 & MG/KG & B & 0.51 \\
\hline 383DH0500.5 & $6 / 7 / 2004$ & soil & EPA6010 & REG & $7440-47-3$ & Chromium & 3.6 & MG/KG & & 1 \\
\hline 383DH0500.5 & $6 / 7 / 2004$ & soil & HASL300 & REG & $10045-97-3$ & Cs-137 & 7.21 & $\mathrm{PCl} / \mathrm{G}$ & $\mathrm{G}$ & 0.15 \\
\hline 383DH0500.5 & $6 / 7 / 2004$ & soil & EPA8260 & REG & $100-41-4$ & Ethylbenzene & 8.2 & UG/KG & & 5.1 \\
\hline 383DH0500.5 & $6 / 7 / 2004$ & soil & HASL300 & REG & 13966-00-2 & $\mathrm{K}-40$ & 27.9 & $\mathrm{PCl} / \mathrm{G}$ & $G$ & 2.1 \\
\hline 383DH0500.5 & $6 / 7 / 2004$ & soil & EPA6010 & REG & 7439-92-1 & Lead & 25 & MG/KG & & 0.31 \\
\hline 383DH0500.5 & $6 / 7 / 2004$ & soil & EPA8260 & REG & 136777-61-2 & M+P-Xylene & 42 & UG/KG & & 5.1 \\
\hline 383DH0500.5 & $6 / 7 / 2004$ & soil & EPA7470 & REG & $7439-97-6$ & Mercury & 0.011 & $\mathrm{MG} / \mathrm{KG}$ & B & 0.1 \\
\hline 383DH0500.5 & $6 / 7 / 2004$ & soil & EPA8260 & REG & $75-09-2$ & Methylene Chloride & 2.2 & UG/KG & $\mathrm{J}$ & 5.1 \\
\hline 383DH0500.5 & $6 / 7 / 2004$ & soil & EPA8260 & REG & $95-47-6$ & O-Xylene & 15 & UG/KG & & 5.1 \\
\hline 383DH0500.5 & $6 / 7 / 2004$ & soil & HASL300 & REG & 15092-94-1 & $\mathrm{Pb}-212$ & 2.26 & $\mathrm{PCl} / \mathrm{G}$ & $\mathrm{J}$ & 0.26 \\
\hline 383DH0500.5 & $6 / 7 / 2004$ & soil & HASL300 & REG & 15067-28-4 & $\mathrm{Pb}-214$ & 1.25 & $\mathrm{PCl} / \mathrm{G}$ & $\mathrm{G}, \mathrm{J}$ & 0.31 \\
\hline 383DH0500.5 & $6 / 7 / 2004$ & soil & UGTAISOPU & REG & 13981-16-3 & Pu-238 & 0.064 & $\mathrm{PCl} / \mathrm{G}$ & & 0.018 \\
\hline 383DH0500.5 & $6 / 7 / 2004$ & soil & UGTAISOPU & REG & $15117-48-3$ & Pu-239 & 0.57 & $\mathrm{PCl} / \mathrm{G}$ & $\mathrm{J}$ & 0.01 \\
\hline 383DH0500.5 & $6 / 7 / 2004$ & soil & SR7500 & REG & 10098-97-2 & Sr-90 & 0.49 & $\mathrm{PCl} / \mathrm{G}$ & LT & 0.28 \\
\hline 383DH0500.5 & $6 / 7 / 2004$ & soil & EPA8260 & REG & $100-42-5$ & Styrene & 0.99 & UG/KG & $\mathrm{J}$ & 5.1 \\
\hline 383DH0500.5 & $6 / 7 / 2004$ & soil & HASL300 & REG & $15065-10-8$ & Th-234 & 2.4 & $\mathrm{PCl} / \mathrm{G}$ & $\mathrm{J}$ & 2.1 \\
\hline 383DH0500.5 & $6 / 7 / 2004$ & soil & HASL300 & REG & 14913-50-9 & TI-208 & 0.67 & $\mathrm{PCl} / \mathrm{G}$ & $G$ & 0.18 \\
\hline 383DH0500.5 & 6/7/2004 & soil & EPA8260 & REG & $108-88-3$ & Toluene & 1.6 & UG/KG & $\mathrm{J}$ & 5.1 \\
\hline 383DH0600.5 & $6 / 7 / 2004$ & soil & HASL300 & REG & 14331-83-0 & Ac-228 & 1.79 & $\mathrm{PCl} / \mathrm{G}$ & $\mathrm{G}$ & 0.7 \\
\hline 383DH0600.5 & 6/7/2004 & soil & EPA6010 & REG & $7440-38-2$ & Arsenic & 3.5 & MG/KG & & 1 \\
\hline 383DH0600.5 & $6 / 7 / 2004$ & soil & EPA6010 & REG & $7440-39-3$ & Barium & 3500 & MG/KG & & 100 \\
\hline 383DH0600.5 & $6 / 7 / 2004$ & soil & EPA6010 & REG & $7440-41-7$ & Beryllium & 1.4 & MG/KG & & 0.52 \\
\hline 383DH0600.5 & $6 / 7 / 2004$ & soil & HASL300 & REG & 14733-03-0 & Bi-214 & 0.94 & $\mathrm{PCl} / \mathrm{G}$ & $\mathrm{G}, \mathrm{J}$ & 0.34 \\
\hline 383DH0600.5 & $6 / 7 / 2004$ & soil & EPA8270 & REG & 117-81-7 & Bis(2-Ethylhexyl)Phthalate & 100 & UG/KG & $\mathrm{J}$ & 350 \\
\hline
\end{tabular}




\begin{tabular}{|c|c|c|c|c|c|c|c|c|c|c|}
\hline SAMPLE \# & $\begin{array}{l}\text { SAMPLE } \\
\text { DATE }\end{array}$ & MATRIX & $\begin{array}{l}\text { USER TEST } \\
\text { PANEL }\end{array}$ & $\begin{array}{l}\text { SAMPLE } \\
\text { PURPOSE }\end{array}$ & CAS \# & PARAMETER & RESULT & UNITS & Q & $\begin{array}{l}\text { DETECT } \\
\text { LIMIT }\end{array}$ \\
\hline 383DH0600.5 & $6 / 7 / 2004$ & soil & EPA6010 & REG & $7440-43-9$ & Cadmium & 0.23 & MG/KG & B & 0.52 \\
\hline 383DH0600.5 & $6 / 7 / 2004$ & soil & EPA6010 & REG & $7440-47-3$ & Chromium & 4.1 & MG/KG & & 1 \\
\hline 383DH0600.5 & $6 / 7 / 2004$ & soil & HASL300 & REG & $10045-97-3$ & Cs-137 & 18.4 & $\mathrm{PCl} / \mathrm{G}$ & G & 0.2 \\
\hline 383DH0600.5 & $6 / 7 / 2004$ & soil & EPA8260 & REG & $100-41-4$ & Ethylbenzene & 1.6 & UG/KG & $\mathrm{J}$ & 5.2 \\
\hline 383DH0600.5 & $6 / 7 / 2004$ & soil & HASL300 & REG & 13966-00-2 & $\mathrm{K}-40$ & 26.7 & $\mathrm{PCl} / \mathrm{G}$ & G & 2.1 \\
\hline 383DH0600.5 & $6 / 7 / 2004$ & soil & EPA6010 & REG & $7439-92-1$ & Lead & 22 & MG/KG & & 0.31 \\
\hline 383DH0600.5 & $6 / 7 / 2004$ & soil & EPA8260 & REG & 136777-61-2 & M+P-Xylene & 5.9 & UG/KG & & 5.2 \\
\hline 383DH0600.5 & $6 / 7 / 2004$ & soil & EPA7470 & REG & 7439-97-6 & Mercury & 0.019 & MG/KG & B & 0.1 \\
\hline 383DH0600.5 & $6 / 7 / 2004$ & soil & EPA8260 & REG & $95-47-6$ & O-Xylene & 1.6 & UG/KG & $\mathrm{J}$ & 5.2 \\
\hline 383DH0600.5 & $6 / 7 / 2004$ & soil & HASL300 & REG & 15092-94-1 & $\mathrm{Pb}-212$ & 2.54 & $\mathrm{PCl} / \mathrm{G}$ & $\mathrm{J}$ & 0.31 \\
\hline 383DH0600.5 & $6 / 7 / 2004$ & soil & HASL300 & REG & 15067-28-4 & $\mathrm{Pb}-214$ & 1.44 & $\mathrm{PCl} / \mathrm{G}$ & $\mathrm{G}, \mathrm{J}$ & 0.42 \\
\hline 383DH0600.5 & $6 / 7 / 2004$ & soil & UGTAISOPU & REG & 13981-16-3 & Pu-238 & 0.133 & $\mathrm{PCl} / \mathrm{G}$ & & 0.009 \\
\hline 383DH0600.5 & $6 / 7 / 2004$ & soil & UGTAISOPU & REG & 15117-48-3 & Pu-239 & 0.8 & $\mathrm{PCl} / \mathrm{G}$ & $\mathrm{J}$ & 0.02 \\
\hline 383DH0600.5 & $6 / 7 / 2004$ & soil & SR7500 & REG & 10098-97-2 & Sr-90 & 0.91 & $\mathrm{PCl} / \mathrm{G}$ & Y1 & 0.25 \\
\hline 383DH0600.5 & $6 / 7 / 2004$ & soil & HASL300 & REG & $14913-50-9$ & TI-208 & 0.67 & $\mathrm{PCl} / \mathrm{G}$ & $G$ & 0.19 \\
\hline 383DH0700.5 & $6 / 7 / 2004$ & soil & HASL300 & REG & 14331-83-0 & Ac-228 & 1.73 & $\mathrm{PCl} / \mathrm{G}$ & G & 0.47 \\
\hline 383DH0700.5 & $6 / 7 / 2004$ & soil & EPA6010 & REG & $7440-38-2$ & Arsenic & 2.7 & MG/KG & & 1 \\
\hline 383DH0700.5 & $6 / 7 / 2004$ & soil & EPA6010 & REG & $7440-39-3$ & Barium & 2500 & MG/KG & & 100 \\
\hline 383DH0700.5 & $6 / 7 / 2004$ & soil & EPA6010 & REG & $7440-41-7$ & Beryllium & 1.6 & MG/KG & & 0.51 \\
\hline 383DH0700.5 & $6 / 7 / 2004$ & soil & HASL300 & REG & 14733-03-0 & Bi-214 & 1.25 & $\mathrm{PCl} / \mathrm{G}$ & $\mathrm{G}, \mathrm{J}$ & 0.28 \\
\hline 383DH0700.5 & $6 / 7 / 2004$ & soil & EPA6010 & REG & 7440-43-9 & Cadmium & 0.27 & MG/KG & B & 0.51 \\
\hline 383DH0700.5 & $6 / 7 / 2004$ & soil & EPA6010 & REG & 7440-47-3 & Chromium & 2.8 & MG/KG & & 1 \\
\hline 383DH0700.5 & $6 / 7 / 2004$ & soil & HASL300 & REG & 10045-97-3 & Cs-137 & 2.72 & $\mathrm{PCl} / \mathrm{G}$ & G & 0.12 \\
\hline 383DH0700.5 & $6 / 7 / 2004$ & soil & HASL300 & REG & 13966-00-2 & $\mathrm{K}-40$ & 25.4 & $\mathrm{PCl} / \mathrm{G}$ & G & 1.5 \\
\hline 383DH0700.5 & $6 / 7 / 2004$ & soil & EPA6010 & REG & $7439-92-1$ & Lead & 22 & MG/KG & & 0.31 \\
\hline 383DH0700.5 & $6 / 7 / 2004$ & soil & EPA8260 & REG & $136777-61-2$ & M+P-Xylene & 2.5 & UG/KG & $\mathrm{J}$ & 5.1 \\
\hline 383DH0700.5 & $6 / 7 / 2004$ & soil & EPA7470 & REG & 7439-97-6 & Mercury & 0.014 & MG/KG & B & 0.1 \\
\hline 383DH0700.5 & $6 / 7 / 2004$ & soil & EPA8260 & REG & $75-09-2$ & Methylene Chloride & 1.6 & UG/KG & $\mathrm{J}$ & 5.1 \\
\hline 383DH0700.5 & $6 / 7 / 2004$ & soil & HASL300 & REG & 15092-94-1 & $\mathrm{Pb}-212$ & 1.82 & $\mathrm{PCl} / \mathrm{G}$ & $\mathrm{J}$ & 0.23 \\
\hline 383DH0700.5 & $6 / 7 / 2004$ & soil & HASL300 & REG & 15067-28-4 & $\mathrm{Pb}-214$ & 1.49 & $\mathrm{PCl} / \mathrm{G}$ & $\mathrm{G}, \mathrm{J}$ & 0.31 \\
\hline 383DH0700.5 & $6 / 7 / 2004$ & soil & UGTAISOPU & REG & 15117-48-3 & Pu-239 & 0.071 & $\mathrm{PCl} / \mathrm{G}$ & $\mathrm{J}$ & 0.016 \\
\hline 383DH0700.5 & $6 / 7 / 2004$ & soil & HASL300 & REG & 14913-50-9 & TI-208 & 0.65 & $\mathrm{PCl} / \mathrm{G}$ & G & 0.13 \\
\hline 383DH0700.5X & $6 / 7 / 2004$ & soil & HASL300 & FD & 14331-83-0 & Ac-228 & 1.92 & $\mathrm{PCl} / \mathrm{G}$ & G & 0.63 \\
\hline 383DH0700.5X & 6/7/2004 & soil & EPA8260 & FD & 67-64-1 & Acetone & 11 & UG/KG & $\mathrm{J}$ & 20 \\
\hline 383DH0700.5X & $6 / 7 / 2004$ & soil & EPA6010 & FD & $7440-38-2$ & Arsenic & 2.2 & MG/KG & & 1 \\
\hline
\end{tabular}




\begin{tabular}{|c|c|c|c|c|c|c|c|c|c|c|}
\hline SAMPLE \# & $\begin{array}{l}\text { SAMPLE } \\
\text { DATE }\end{array}$ & MATRIX & $\begin{array}{l}\text { USER TEST } \\
\text { PANEL }\end{array}$ & $\begin{array}{l}\text { SAMPLE } \\
\text { PURPOSE }\end{array}$ & CAS \# & PARAMETER & RESULT & UNITS & Q & $\begin{array}{l}\text { DETECT } \\
\text { LIMIT }\end{array}$ \\
\hline 383DH0700.5X & $6 / 7 / 2004$ & soil & EPA6010 & FD & $7440-39-3$ & Barium & 2500 & MG/KG & & 100 \\
\hline 383DH0700.5X & $6 / 7 / 2004$ & soil & EPA6010 & FD & $7440-41-7$ & Beryllium & 1.7 & MG/KG & & 0.51 \\
\hline 383DH0700.5X & $6 / 7 / 2004$ & soil & HASL300 & FD & 14733-03-0 & $\mathrm{Bi}-214$ & 1.36 & $\mathrm{PCl} / \mathrm{G}$ & $\mathrm{G}, \mathrm{J}$ & 0.23 \\
\hline 383DH0700.5X & $6 / 7 / 2004$ & soil & EPA6010 & FD & $7440-43-9$ & Cadmium & 0.23 & MG/KG & $B$ & 0.51 \\
\hline 383DH0700.5X & $6 / 7 / 2004$ & soil & EPA6010 & FD & $7440-47-3$ & Chromium & 2.9 & MG/KG & & 1 \\
\hline 383DH0700.5X & $6 / 7 / 2004$ & soil & HASL300 & FD & $10045-97-3$ & Cs-137 & 2.78 & $\mathrm{PCl} / \mathrm{G}$ & $G$ & 0.16 \\
\hline 383DH0700.5X & $6 / 7 / 2004$ & soil & HASL300 & FD & 13966-00-2 & $\mathrm{K}-40$ & 25.9 & $\mathrm{PCl} / \mathrm{G}$ & G & 1.6 \\
\hline 383DH0700.5X & $6 / 7 / 2004$ & soil & EPA6010 & FD & 7439-92-1 & Lead & 18 & MG/KG & & 0.31 \\
\hline 383DH0700.5X & $6 / 7 / 2004$ & soil & EPA7470 & FD & $7439-97-6$ & Mercury & 0.014 & MG/KG & B & 0.1 \\
\hline 383DH0700.5X & $6 / 7 / 2004$ & soil & EPA8260 & FD & $75-09-2$ & Methylene Chloride & 1.9 & UG/KG & $\mathrm{J}$ & 5.1 \\
\hline 383DH0700.5X & $6 / 7 / 2004$ & soil & HASL300 & FD & 15092-94-1 & $\mathrm{Pb}-212$ & 2.22 & $\mathrm{PCl} / \mathrm{G}$ & $\mathrm{J}$ & 0.21 \\
\hline 383DH0700.5X & $6 / 7 / 2004$ & soil & HASL300 & FD & $15067-28-4$ & $\mathrm{~Pb}-214$ & 1.2 & $\mathrm{PCl} / \mathrm{G}$ & $\mathrm{G}, \mathrm{J}$ & 0.26 \\
\hline 383DH0700.5X & $6 / 7 / 2004$ & soil & EPA6010 & FD & $7782-49-2$ & Selenium & 0.54 & MG/KG & & 0.51 \\
\hline 383DH0700.5X & $6 / 7 / 2004$ & soil & HASL300 & FD & 14913-50-9 & TI-208 & 0.57 & $\mathrm{PCl} / \mathrm{G}$ & $G$ & 0.14 \\
\hline 383DH0800.5 & $6 / 7 / 2004$ & soil & HASL300 & REG & $14331-83-0$ & Ac-228 & 1.72 & $\mathrm{PCl} / \mathrm{G}$ & $G$ & 0.48 \\
\hline 383DH0800.5 & $6 / 7 / 2004$ & soil & EPA6010 & REG & $7440-38-2$ & Arsenic & 4 & MG/KG & & 1 \\
\hline 383DH0800.5 & $6 / 7 / 2004$ & soil & EPA6010 & REG & $7440-39-3$ & Barium & 3900 & MG/KG & & 100 \\
\hline 383DH0800.5 & $6 / 7 / 2004$ & soil & EPA6010 & REG & $7440-41-7$ & Beryllium & 1.4 & MG/KG & & 0.51 \\
\hline 383DH0800.5 & $6 / 7 / 2004$ & soil & HASL300 & REG & 14733-03-0 & $\mathrm{Bi}-214$ & 1.33 & $\mathrm{PCl} / \mathrm{G}$ & $\mathrm{G}, \mathrm{J}$ & 0.39 \\
\hline 383DH0800.5 & $6 / 7 / 2004$ & soil & EPA8270 & REG & 117-81-7 & Bis(2-Ethylhexyl)Phthalate & 200 & UG/KG & $\mathrm{J}$ & 340 \\
\hline 383DH0800.5 & $6 / 7 / 2004$ & soil & EPA6010 & REG & $7440-43-9$ & Cadmium & 0.28 & MG/KG & B & 0.51 \\
\hline 383DH0800.5 & $6 / 7 / 2004$ & soil & EPA6010 & REG & $7440-47-3$ & Chromium & 3.9 & MG/KG & & 1 \\
\hline 383DH0800.5 & $6 / 7 / 2004$ & soil & HASL300 & REG & $10045-97-3$ & Cs-137 & 12.4 & $\mathrm{PCl} / \mathrm{G}$ & G & 0.2 \\
\hline 383DH0800.5 & $6 / 7 / 2004$ & soil & HASL300 & REG & 13966-00-2 & $\mathrm{K}-40$ & 30.9 & $\mathrm{PCl} / \mathrm{G}$ & $G$ & 1.6 \\
\hline 383DH0800.5 & $6 / 7 / 2004$ & soil & EPA6010 & REG & $7439-92-1$ & Lead & 22 & MG/KG & & 0.31 \\
\hline 383DH0800.5 & $6 / 7 / 2004$ & soil & EPA8260 & REG & $136777-61-2$ & M+P-Xylene & 3 & UG/KG & $J$ & 5.1 \\
\hline 383DH0800.5 & $6 / 7 / 2004$ & soil & EPA7470 & REG & 7439-97-6 & Mercury & 0.028 & MG/KG & B & 0.1 \\
\hline 383DH0800.5 & $6 / 7 / 2004$ & soil & EPA8260 & REG & $75-09-2$ & Methylene Chloride & 1.9 & UG/KG & $\mathrm{J}$ & 5.1 \\
\hline 383DH0800.5 & $6 / 7 / 2004$ & soil & HASL300 & REG & 15092-94-1 & $\mathrm{Pb}-212$ & 2.17 & $\mathrm{PCl} / \mathrm{G}$ & $\mathrm{J}$ & 0.31 \\
\hline 383DH0800.5 & $6 / 7 / 2004$ & soil & HASL300 & REG & 15067-28-4 & $\mathrm{Pb}-214$ & 1.16 & $\mathrm{PCl} / \mathrm{G}$ & $\mathrm{G}, \mathrm{J}$ & 0.4 \\
\hline 383DH0800.5 & $6 / 7 / 2004$ & soil & UGTAISOPU & REG & 13981-16-3 & Pu-238 & 0.059 & $\mathrm{PCl} / \mathrm{G}$ & & 0.009 \\
\hline 383DH0800.5 & $6 / 7 / 2004$ & soil & UGTAISOPU & REG & 15117-48-3 & Pu-239 & 1.87 & $\mathrm{PCl} / \mathrm{G}$ & $\mathrm{J}$ & 0.01 \\
\hline 383DH0800.5 & $6 / 7 / 2004$ & soil & SR7500 & REG & 10098-97-2 & Sr-90 & 0.55 & $\mathrm{PCl} / \mathrm{G}$ & Y1 & 0.25 \\
\hline 383DH0800.5 & $6 / 7 / 2004$ & soil & HASL300 & REG & 14913-50-9 & TI-208 & 0.6 & $\mathrm{PCl} / \mathrm{G}$ & G & 0.17 \\
\hline 383DH0800.5 & $6 / 7 / 2004$ & soil & EPA8260 & REG & 108-88-3 & Toluene & 1 & UG/KG & $\mathrm{J}$ & 5.1 \\
\hline
\end{tabular}




\begin{tabular}{|c|c|c|c|c|c|c|c|c|c|c|}
\hline SAMPLE \# & $\begin{array}{l}\text { SAMPLE } \\
\text { DATE }\end{array}$ & MATRIX & $\begin{array}{l}\text { USER TEST } \\
\text { PANEL }\end{array}$ & $\begin{array}{l}\text { SAMPLE } \\
\text { PURPOSE }\end{array}$ & CAS \# & PARAMETER & RESULT & UNITS & Q & $\begin{array}{l}\text { DETECT } \\
\text { LIMIT }\end{array}$ \\
\hline 383DH0900.5 & $6 / 7 / 2004$ & soil & HASL300 & REG & 14331-83-0 & Ac-228 & 1.7 & $\mathrm{PCl} / \mathrm{G}$ & G & 0.69 \\
\hline 383DH0900.5 & $6 / 7 / 2004$ & soil & EPA8260 & REG & $67-64-1$ & Acetone & 9.3 & UG/KG & J,B & 21 \\
\hline 383DH0900.5 & $6 / 7 / 2004$ & soil & EPA6010 & REG & $7440-38-2$ & Arsenic & 3.2 & MG/KG & & 1 \\
\hline 383DH0900.5 & $6 / 7 / 2004$ & soil & EPA6010 & REG & $7440-39-3$ & Barium & 3500 & MG/KG & & 100 \\
\hline 383DH0900.5 & $6 / 7 / 2004$ & soil & EPA6010 & REG & $7440-41-7$ & Beryllium & 1.2 & MG/KG & & 0.52 \\
\hline 383DH0900.5 & $6 / 7 / 2004$ & soil & HASL300 & REG & 14733-03-0 & $\mathrm{Bi}-214$ & 0.86 & $\mathrm{PCl} / \mathrm{G}$ & $\mathrm{G}, \mathrm{J}$ & 0.38 \\
\hline 383DH0900.5 & $6 / 7 / 2004$ & soil & EPA6010 & REG & $7440-43-9$ & Cadmium & 0.21 & MG/KG & B & 0.52 \\
\hline 383DH0900.5 & $6 / 7 / 2004$ & soil & EPA6010 & REG & $7440-47-3$ & Chromium & 2.9 & MG/KG & & 1 \\
\hline 383DH0900.5 & $6 / 7 / 2004$ & soil & HASL300 & REG & $10045-97-3$ & Cs-137 & 48.7 & $\mathrm{PCl} / \mathrm{G}$ & $G$ & 0.2 \\
\hline 383DH0900.5 & $6 / 7 / 2004$ & soil & HASL300 & REG & 13966-00-2 & $\mathrm{K}-40$ & 30.3 & $\mathrm{PCl} / \mathrm{G}$ & G & 1.5 \\
\hline 383DH0900.5 & $6 / 7 / 2004$ & soil & EPA6010 & REG & 7439-92-1 & Lead & 52 & MG/KG & & 0.31 \\
\hline 383DH0900.5 & $6 / 7 / 2004$ & soil & EPA7470 & REG & $7439-97-6$ & Mercury & 0.021 & MG/KG & B & 0.1 \\
\hline 383DH0900.5 & $6 / 7 / 2004$ & soil & HASL300 & REG & 15092-94-1 & $\mathrm{Pb}-212$ & 1.71 & $\mathrm{PCl} / \mathrm{G}$ & $\mathrm{J}$ & 0.5 \\
\hline 383DH0900.5 & $6 / 7 / 2004$ & soil & UGTAISOPU & REG & 13981-16-3 & Pu-238 & 0.201 & $\mathrm{PCl} / \mathrm{G}$ & & 0.011 \\
\hline 383DH0900.5 & $6 / 7 / 2004$ & soil & UGTAISOPU & REG & $15117-48-3$ & Pu-239 & 1.17 & $\mathrm{PCl} / \mathrm{G}$ & & 0.01 \\
\hline 383DH0900.5 & $6 / 7 / 2004$ & soil & SR7500 & REG & 10098-97-2 & Sr-90 & 0.92 & $\mathrm{PCl} / \mathrm{G}$ & $\mathrm{Y1}$ & 0.22 \\
\hline 383DH0900.5 & $6 / 7 / 2004$ & soil & HASL300 & REG & 14913-50-9 & TI-208 & 0.71 & $\mathrm{PCl} / \mathrm{G}$ & G & 0.24 \\
\hline 383DH1000.5 & $6 / 7 / 2004$ & soil & HASL300 & REG & 14331-83-0 & Ac-228 & 1.37 & $\mathrm{PCl} / \mathrm{G}$ & G & 0.51 \\
\hline 383DH1000.5 & $6 / 7 / 2004$ & soil & EPA8260 & REG & 67-64-1 & Acetone & 6.2 & UG/KG & J,B & 21 \\
\hline 383DH1000.5 & $6 / 7 / 2004$ & soil & EPA6010 & REG & $7440-38-2$ & Arsenic & 3 & MG/KG & & 1 \\
\hline 383DH1000.5 & $6 / 7 / 2004$ & soil & EPA6010 & REG & $7440-39-3$ & Barium & 3800 & MG/KG & & 100 \\
\hline 383DH1000.5 & $6 / 7 / 2004$ & soil & EPA6010 & REG & $7440-41-7$ & Beryllium & 1.2 & MG/KG & & 0.51 \\
\hline 383DH1000.5 & $6 / 7 / 2004$ & soil & HASL300 & REG & 14733-03-0 & Bi-214 & 0.85 & $\mathrm{PCl} / \mathrm{G}$ & $\mathrm{G}, \mathrm{J}$ & 0.33 \\
\hline 383DH1000.5 & $6 / 7 / 2004$ & soil & EPA6010 & REG & $7440-43-9$ & Cadmium & 0.15 & MG/KG & B & 0.51 \\
\hline 383DH1000.5 & $6 / 7 / 2004$ & soil & EPA6010 & REG & $7440-47-3$ & Chromium & 5.1 & MG/KG & & 1 \\
\hline 383DH1000.5 & $6 / 7 / 2004$ & soil & HASL300 & REG & $10045-97-3$ & Cs-137 & 23.7 & $\mathrm{PCl} / \mathrm{G}$ & G & 0.2 \\
\hline 383DH1000.5 & $6 / 7 / 2004$ & soil & HASL300 & REG & 13966-00-2 & $\mathrm{K}-40$ & 21.6 & $\mathrm{PCl} / \mathrm{G}$ & G & 1.2 \\
\hline 383DH1000.5 & $6 / 7 / 2004$ & soil & EPA6010 & REG & $7439-92-1$ & Lead & 22 & MG/KG & & 0.31 \\
\hline 383DH1000.5 & $6 / 7 / 2004$ & soil & EPA7470 & REG & $7439-97-6$ & Mercury & 0.027 & MG/KG & B & 0.1 \\
\hline 383DH1000.5 & $6 / 7 / 2004$ & soil & EPA8260 & REG & $75-09-2$ & Methylene Chloride & 3.4 & UG/KG & $\mathrm{J}$ & 5.1 \\
\hline 383DH1000.5 & $6 / 7 / 2004$ & soil & HASL300 & REG & 15092-94-1 & $\mathrm{Pb}-212$ & 1.57 & $\mathrm{PCl} / \mathrm{G}$ & $\mathrm{J}$ & 0.33 \\
\hline 383DH1000.5 & $6 / 7 / 2004$ & soil & HASL300 & REG & $15067-28-4$ & $\mathrm{~Pb}-214$ & 1.08 & $\mathrm{PCl} / \mathrm{G}$ & $\mathrm{G}, \mathrm{J}$ & 0.47 \\
\hline 383DH1000.5 & $6 / 7 / 2004$ & soil & UGTAISOPU & REG & 13981-16-3 & Pu-238 & 0.176 & $\mathrm{PCl} / \mathrm{G}$ & & 0.009 \\
\hline 383DH1000.5 & 6/7/2004 & soil & UGTAISOPU & REG & $15117-48-3$ & Pu-239 & 1.26 & $\mathrm{PCl} / \mathrm{G}$ & & 0.02 \\
\hline 383DH1000.5 & $6 / 7 / 2004$ & soil & SR7500 & REG & 10098-97-2 & Sr-90 & 0.96 & $\mathrm{PCl} / \mathrm{G}$ & & 0.23 \\
\hline
\end{tabular}




\begin{tabular}{|c|c|c|c|c|c|c|c|c|c|c|}
\hline SAMPLE \# & $\begin{array}{l}\text { SAMPLE } \\
\text { DATE }\end{array}$ & MATRIX & $\begin{array}{l}\text { USER TEST } \\
\text { PANEL }\end{array}$ & $\begin{array}{l}\text { SAMPLE } \\
\text { PURPOSE }\end{array}$ & CAS \# & PARAMETER & RESULT & UNITS & Q & $\begin{array}{l}\text { DETECT } \\
\text { LIMIT }\end{array}$ \\
\hline 383DH1000.5 & $6 / 7 / 2004$ & soil & HASL300 & REG & $14913-50-9$ & TI-208 & 0.47 & $\mathrm{PCl} / \mathrm{G}$ & G & 0.2 \\
\hline 383DH1100.5 & $6 / 7 / 2004$ & soil & HASL300 & REG & 14331-83-0 & Ac-228 & 1.95 & $\mathrm{PCl} / \mathrm{G}$ & G & 0.46 \\
\hline 383DH1100.5 & 6/7/2004 & soil & EPA6010 & REG & $7440-38-2$ & Arsenic & 2.2 & MG/KG & & 1 \\
\hline 383DH1100.5 & $6 / 7 / 2004$ & soil & EPA6010 & REG & 7440-39-3 & Barium & 2900 & MG/KG & & 100 \\
\hline 383DH1100.5 & $6 / 7 / 2004$ & soil & EPA6010 & REG & 7440-41-7 & Beryllium & 1.7 & MG/KG & & 0.51 \\
\hline 383DH1100.5 & $6 / 7 / 2004$ & soil & HASL300 & REG & 14733-03-0 & Bi-214 & 0.98 & $\mathrm{PCl} / \mathrm{G}$ & $\mathrm{G}, \mathrm{J}$ & 0.31 \\
\hline 383DH1100.5 & $6 / 7 / 2004$ & soil & EPA6010 & REG & $7440-43-9$ & Cadmium & 0.13 & MG/KG & $B$ & 0.51 \\
\hline 383DH1100.5 & $6 / 7 / 2004$ & soil & EPA6010 & REG & 7440-47-3 & Chromium & 4.3 & MG/KG & & 1 \\
\hline 383DH1100.5 & $6 / 7 / 2004$ & soil & HASL300 & REG & $10045-97-3$ & Cs-137 & 7.52 & $\mathrm{PCl} / \mathrm{G}$ & G & 0.16 \\
\hline 383DH1100.5 & $6 / 7 / 2004$ & soil & HASL300 & REG & 13966-00-2 & $\mathrm{K}-40$ & 26.3 & $\mathrm{PCl} / \mathrm{G}$ & G & 1.2 \\
\hline 383DH1100.5 & $6 / 7 / 2004$ & soil & EPA6010 & REG & $7439-92-1$ & Lead & 23 & MG/KG & & 0.31 \\
\hline 383DH1100.5 & $6 / 7 / 2004$ & soil & EPA8260 & REG & $136777-61-2$ & M+P-Xylene & 2.1 & UG/KG & $\mathrm{J}$ & 5.1 \\
\hline 383DH1100.5 & $6 / 7 / 2004$ & soil & EPA7470 & REG & 7439-97-6 & Mercury & 0.014 & MG/KG & B & 0.1 \\
\hline 383DH1100.5 & $6 / 7 / 2004$ & soil & EPA8260 & REG & $75-09-2$ & Methylene Chloride & 2.9 & UG/KG & $\mathrm{J}$ & 5.1 \\
\hline 383DH1100.5 & $6 / 7 / 2004$ & soil & HASL300 & REG & 15092-94-1 & $\mathrm{Pb}-212$ & 2.23 & $\mathrm{PCl} / \mathrm{G}$ & $\mathrm{J}$ & 0.26 \\
\hline 383DH1100.5 & $6 / 7 / 2004$ & soil & HASL300 & REG & $15067-28-4$ & $\mathrm{~Pb}-214$ & 1.33 & $\mathrm{PCl} / \mathrm{G}$ & $\mathrm{G}, \mathrm{J}$ & 0.32 \\
\hline 383DH1100.5 & $6 / 7 / 2004$ & soil & UGTAISOPU & REG & 13981-16-3 & Pu-238 & 0.048 & $\mathrm{PCl} / \mathrm{G}$ & LT & 0.01 \\
\hline 383DH1100.5 & $6 / 7 / 2004$ & soil & UGTAISOPU & REG & 15117-48-3 & Pu-239 & 0.7 & $\mathrm{PCl} / \mathrm{G}$ & & 0.02 \\
\hline 383DH1100.5 & $6 / 7 / 2004$ & soil & HASL300 & REG & 14913-50-9 & TI-208 & 0.66 & $\mathrm{PCl} / \mathrm{G}$ & G & 0.16 \\
\hline 383DH1200.5 & $6 / 7 / 2004$ & soil & HASL300 & REG & 14331-83-0 & Ac-228 & 1.75 & $\mathrm{PCl} / \mathrm{G}$ & G & 0.69 \\
\hline 383DH1200.5 & $6 / 7 / 2004$ & soil & EPA8260 & REG & 67-64-1 & Acetone & 6.3 & UG/KG & J,B & 21 \\
\hline 383DH1200.5 & $6 / 7 / 2004$ & soil & EPA6010 & REG & $7440-38-2$ & Arsenic & 3.2 & MG/KG & & 1 \\
\hline 383DH1200.5 & $6 / 7 / 2004$ & soil & EPA6010 & REG & $7440-39-3$ & Barium & 3600 & MG/KG & & 100 \\
\hline 383DH1200.5 & $6 / 7 / 2004$ & soil & EPA6010 & REG & $7440-41-7$ & Beryllium & 1.5 & MG/KG & & 0.52 \\
\hline 383DH1200.5 & $6 / 7 / 2004$ & soil & HASL300 & REG & 14733-03-0 & Bi-214 & 1.17 & $\mathrm{PCl} / \mathrm{G}$ & $\mathrm{G}, \mathrm{J}$ & 0.54 \\
\hline 383DH1200.5 & $6 / 7 / 2004$ & soil & EPA6010 & REG & $7440-43-9$ & Cadmium & 0.16 & MG/KG & $B$ & 0.52 \\
\hline 383DH1200.5 & $6 / 7 / 2004$ & soil & EPA6010 & REG & $7440-47-3$ & Chromium & 5 & MG/KG & & 1 \\
\hline 383DH1200.5 & $6 / 7 / 2004$ & soil & HASL300 & REG & $10045-97-3$ & Cs-137 & 33.8 & $\mathrm{PCl} / \mathrm{G}$ & G & 0.3 \\
\hline 383DH1200.5 & $6 / 7 / 2004$ & soil & EPA8015 & REG & 68334-30-5 & Diesel Range Organics & 2.7 & MG/KG & $\mathrm{J}$ & 5.2 \\
\hline 383DH1200.5 & $6 / 7 / 2004$ & soil & HASL300 & REG & 13966-00-2 & $\mathrm{K}-40$ & 24.1 & $\mathrm{PCl} / \mathrm{G}$ & G & 2.3 \\
\hline 383DH1200.5 & $6 / 7 / 2004$ & soil & EPA6010 & REG & $7439-92-1$ & Lead & 36 & MG/KG & & 0.31 \\
\hline 383DH1200.5 & $6 / 7 / 2004$ & soil & EPA7470 & REG & 7439-97-6 & Mercury & 0.049 & MG/KG & $B$ & 0.1 \\
\hline 383DH1200.5 & $6 / 7 / 2004$ & soil & EPA8260 & REG & $75-09-2$ & Methylene Chloride & 2.5 & UG/KG & $\mathrm{J}$ & 5.2 \\
\hline 383DH1200.5 & $6 / 7 / 2004$ & soil & HASL300 & REG & 15092-94-1 & $\mathrm{Pb}-212$ & 2.42 & $\mathrm{PCl} / \mathrm{G}$ & $\mathrm{J}$ & 0.45 \\
\hline 383DH1200.5 & $6 / 7 / 2004$ & soil & HASL300 & REG & 15067-28-4 & $\mathrm{Pb}-214$ & 1.31 & $\mathrm{PCl} / \mathrm{G}$ & $\mathrm{G}, \mathrm{J}$ & 0.69 \\
\hline
\end{tabular}




\begin{tabular}{|c|c|c|c|c|c|c|c|c|c|c|}
\hline SAMPLE \# & $\begin{array}{l}\text { SAMPLE } \\
\text { DATE }\end{array}$ & MATRIX & $\begin{array}{l}\text { USER TEST } \\
\text { PANEL }\end{array}$ & $\begin{array}{l}\text { SAMPLE } \\
\text { PURPOSE }\end{array}$ & CAS \# & PARAMETER & RESULT & UNITS & Q & $\begin{array}{l}\text { DETECT } \\
\text { LIMIT }\end{array}$ \\
\hline 383DH1200.5 & $6 / 7 / 2004$ & soil & UGTAISOPU & REG & 13981-16-3 & Pu-238 & 0.136 & $\mathrm{PCl} / \mathrm{G}$ & & 0.022 \\
\hline 383DH1200.5 & $6 / 7 / 2004$ & soil & UGTAISOPU & REG & 15117-48-3 & Pu-239 & 1.54 & $\mathrm{PCl} / \mathrm{G}$ & & 0.02 \\
\hline 383DH1200.5 & $6 / 7 / 2004$ & soil & SR7500 & REG & 10098-97-2 & Sr-90 & 0.97 & $\mathrm{PCl} / \mathrm{G}$ & Y1 & 0.25 \\
\hline 383DH1200.5 & $6 / 7 / 2004$ & soil & HASL300 & REG & 14913-50-9 & TI-208 & 0.72 & $\mathrm{PCl} / \mathrm{G}$ & G & 0.25 \\
\hline 383DH1300.5 & $6 / 7 / 2004$ & soil & HASL300 & REG & 14331-83-0 & Ac-228 & 1.29 & $\mathrm{PCl} / \mathrm{G}$ & G & 0.42 \\
\hline 383DH1300.5 & $6 / 7 / 2004$ & soil & EPA6010 & REG & $7440-38-2$ & Arsenic & 2.5 & MG/KG & & 1 \\
\hline 383DH1300.5 & $6 / 7 / 2004$ & soil & EPA6010 & REG & $7440-39-3$ & Barium & 2900 & MG/KG & & 100 \\
\hline 383DH1300.5 & $6 / 7 / 2004$ & soil & EPA6010 & REG & $7440-41-7$ & Beryllium & 1.6 & MG/KG & & 0.52 \\
\hline 383DH1300.5 & $6 / 7 / 2004$ & soil & HASL300 & REG & 14733-03-0 & Bi-214 & 1.1 & $\mathrm{PCl} / \mathrm{G}$ & $\mathrm{G}, \mathrm{J}$ & 0.28 \\
\hline 383DH1300.5 & $6 / 7 / 2004$ & soil & EPA6010 & REG & $7440-43-9$ & Cadmium & 0.11 & MG/KG & B & 0.52 \\
\hline 383DH1300.5 & $6 / 7 / 2004$ & soil & EPA6010 & REG & $7440-47-3$ & Chromium & 3.3 & MG/KG & & 1 \\
\hline 383DH1300.5 & $6 / 7 / 2004$ & soil & HASL300 & REG & $10045-97-3$ & Cs-137 & 4.87 & $\mathrm{PCl} / \mathrm{G}$ & G & 0.14 \\
\hline 383DH1300.5 & $6 / 7 / 2004$ & soil & HASL300 & REG & 13966-00-2 & $\mathrm{K}-40$ & 25.9 & $\mathrm{PCl} / \mathrm{G}$ & G & 1.1 \\
\hline 383DH1300.5 & $6 / 7 / 2004$ & soil & EPA6010 & REG & $7439-92-1$ & Lead & 22 & MG/KG & & 0.31 \\
\hline 383DH1300.5 & $6 / 7 / 2004$ & soil & EPA7470 & REG & 7439-97-6 & Mercury & 0.018 & MG/KG & $B$ & 0.1 \\
\hline 383DH1300.5 & $6 / 7 / 2004$ & soil & EPA8260 & REG & $75-09-2$ & Methylene Chloride & 0.84 & UG/KG & $\mathrm{J}$ & 5.2 \\
\hline 383DH1300.5 & $6 / 7 / 2004$ & soil & HASL300 & REG & 15092-94-1 & $\mathrm{Pb}-212$ & 2.06 & $\mathrm{PCl} / \mathrm{G}$ & $\mathrm{J}$ & 0.26 \\
\hline 383DH1300.5 & $6 / 7 / 2004$ & soil & HASL300 & REG & 15067-28-4 & $\mathrm{Pb}-214$ & 1.37 & $\mathrm{PCl} / \mathrm{G}$ & $\mathrm{G}, \mathrm{J}$ & 0.3 \\
\hline 383DH1300.5 & $6 / 7 / 2004$ & soil & UGTAISOPU & REG & 15117-48-3 & Pu-239 & 0.268 & $\mathrm{PCl} / \mathrm{G}$ & & 0.018 \\
\hline 383DH1300.5 & $6 / 7 / 2004$ & soil & HASL300 & REG & 14913-50-9 & TI-208 & 0.48 & $\mathrm{PCl} / \mathrm{G}$ & G & 0.13 \\
\hline 383DH1400.5 & $6 / 7 / 2004$ & soil & HASL300 & REG & 14331-83-0 & Ac-228 & 2.2 & $\mathrm{PCl} / \mathrm{G}$ & G & 0.74 \\
\hline 383DH1400.5 & $6 / 7 / 2004$ & soil & EPA8260 & REG & 67-64-1 & Acetone & 8.5 & UG/KG & J,B & 21 \\
\hline 383DH1400.5 & $6 / 7 / 2004$ & soil & EPA6010 & REG & $7440-38-2$ & Arsenic & 3.3 & MG/KG & & 1 \\
\hline 383DH1400.5 & $6 / 7 / 2004$ & soil & EPA6010 & REG & $7440-39-3$ & Barium & 3900 & MG/KG & & 100 \\
\hline 383DH1400.5 & $6 / 7 / 2004$ & soil & EPA6010 & REG & $7440-41-7$ & Beryllium & 2.1 & MG/KG & & 0.52 \\
\hline 383DH1400.5 & $6 / 7 / 2004$ & soil & HASL300 & REG & 14733-03-0 & Bi-214 & 1.48 & $\mathrm{PCl} / \mathrm{G}$ & $\mathrm{G}, \mathrm{J}$ & 0.62 \\
\hline 383DH1400.5 & $6 / 7 / 2004$ & soil & EPA6010 & REG & $7440-43-9$ & Cadmium & 0.12 & MG/KG & B & 0.52 \\
\hline 383DH1400.5 & $6 / 7 / 2004$ & soil & EPA6010 & REG & $7440-47-3$ & Chromium & 5.2 & MG/KG & & 1 \\
\hline 383DH1400.5 & $6 / 7 / 2004$ & soil & HASL300 & REG & 10045-97-3 & Cs-137 & 68.8 & $\mathrm{PCl} / \mathrm{G}$ & G & 0.3 \\
\hline 383DH1400.5 & $6 / 7 / 2004$ & soil & EPA8015 & REG & 68334-30-5 & Diesel Range Organics & 2.5 & MG/KG & $\mathrm{J}$ & 5.2 \\
\hline 383DH1400.5 & $6 / 7 / 2004$ & soil & EPA8260 & REG & $100-41-4$ & Ethylbenzene & 1.2 & UG/KG & $\mathrm{J}$ & 5.2 \\
\hline 383DH1400.5 & $6 / 7 / 2004$ & soil & HASL300 & REG & 13966-00-2 & $\mathrm{K}-40$ & 26.6 & $\mathrm{PCl} / \mathrm{G}$ & G & 2.5 \\
\hline 383DH1400.5 & $6 / 7 / 2004$ & soil & EPA6010 & REG & $7439-92-1$ & Lead & 33 & MG/KG & & 0.31 \\
\hline 383DH1400.5 & $6 / 7 / 2004$ & soil & EPA8260 & REG & 136777-61-2 & M+P-Xylene & 4.8 & UG/KG & $\mathrm{J}$ & 5.2 \\
\hline 383DH1400.5 & $6 / 7 / 2004$ & soil & EPA7470 & REG & 7439-97-6 & Mercury & 0.083 & MG/KG & $B$ & 0.1 \\
\hline
\end{tabular}




\begin{tabular}{|c|c|c|c|c|c|c|c|c|c|c|}
\hline SAMPLE \# & $\begin{array}{l}\text { SAMPLE } \\
\text { DATE }\end{array}$ & MATRIX & $\begin{array}{l}\text { USER TEST } \\
\text { PANEL }\end{array}$ & $\begin{array}{l}\text { SAMPLE } \\
\text { PURPOSE }\end{array}$ & CAS \# & PARAMETER & RESULT & UNITS & Q & $\begin{array}{l}\text { DETECT } \\
\text { LIMIT }\end{array}$ \\
\hline 383DH1400.5 & $6 / 7 / 2004$ & soil & EPA8260 & REG & $75-09-2$ & Methylene Chloride & 1.2 & UG/KG & $\mathrm{J}$ & 5.2 \\
\hline 383DH1400.5 & $6 / 7 / 2004$ & soil & EPA8260 & REG & $95-47-6$ & O-Xylene & 1.5 & UG/KG & $\mathrm{J}$ & 5.2 \\
\hline 383DH1400.5 & $6 / 7 / 2004$ & soil & HASL300 & REG & 15092-94-1 & $\mathrm{Pb}-212$ & 2.03 & $\mathrm{PCl} / \mathrm{G}$ & $\mathrm{J}$ & 0.54 \\
\hline 383DH1400.5 & $6 / 7 / 2004$ & soil & HASL300 & REG & $15067-28-4$ & $\mathrm{~Pb}-214$ & 1.68 & $\mathrm{PCl} / \mathrm{G}$ & $\mathrm{G}, \mathrm{J}$ & 0.9 \\
\hline 383DH1400.5 & $6 / 7 / 2004$ & soil & UGTAISOPU & REG & 13981-16-3 & Pu-238 & 0.68 & $\mathrm{PCl} / \mathrm{G}$ & & 0.02 \\
\hline 383DH1400.5 & $6 / 7 / 2004$ & soil & UGTAISOPU & REG & $15117-48-3$ & Pu-239 & 4.21 & $\mathrm{PCl} / \mathrm{G}$ & & 0.02 \\
\hline 383DH1400.5 & $6 / 7 / 2004$ & soil & SR7500 & REG & 10098-97-2 & Sr-90 & 1.14 & $\mathrm{PCl} / \mathrm{G}$ & Y1 & 0.25 \\
\hline 383DH1400.5 & $6 / 7 / 2004$ & soil & EPA8260 & REG & $100-42-5$ & Styrene & 1.3 & UG/KG & $\mathrm{J}$ & 5.2 \\
\hline 383DH1400.5 & $6 / 7 / 2004$ & soil & HASL300 & REG & $14913-50-9$ & TI-208 & 0.87 & $\mathrm{PCl} / \mathrm{G}$ & $G$ & 0.28 \\
\hline 383DH1500.5 & $6 / 7 / 2004$ & soil & HASL300 & REG & 14331-83-0 & Ac-228 & 1.71 & $\mathrm{PCl} / \mathrm{G}$ & G & 0.46 \\
\hline 383DH1500.5 & $6 / 7 / 2004$ & soil & EPA6010 & REG & $7440-38-2$ & Arsenic & 3.4 & MG/KG & & 1 \\
\hline 383DH1500.5 & $6 / 7 / 2004$ & soil & EPA6010 & REG & $7440-39-3$ & Barium & 4600 & MG/KG & & 100 \\
\hline 383DH1500.5 & $6 / 7 / 2004$ & soil & EPA6010 & REG & $7440-41-7$ & Beryllium & 1.3 & MG/KG & & 0.52 \\
\hline 383DH1500.5 & $6 / 7 / 2004$ & soil & HASL300 & REG & 14733-03-0 & $\mathrm{Bi}-214$ & 1.09 & $\mathrm{PCl} / \mathrm{G}$ & $\mathrm{G}, \mathrm{J}$ & 0.31 \\
\hline 383DH1500.5 & $6 / 7 / 2004$ & soil & EPA6010 & REG & 7440-43-9 & Cadmium & 0.19 & MG/KG & $B$ & 0.52 \\
\hline 383DH1500.5 & $6 / 7 / 2004$ & soil & EPA6010 & REG & $7440-47-3$ & Chromium & 6 & MG/KG & & 1 \\
\hline 383DH1500.5 & $6 / 7 / 2004$ & soil & HASL300 & REG & $10045-97-3$ & Cs-137 & 11.2 & $\mathrm{PCl} / \mathrm{G}$ & G & 0.2 \\
\hline 383DH1500.5 & $6 / 7 / 2004$ & soil & EPA8270 & REG & $206-44-0$ & Fluoranthene & 13 & UG/KG & $\mathrm{J}$ & 340 \\
\hline 383DH1500.5 & $6 / 7 / 2004$ & soil & HASL300 & REG & 13966-00-2 & $\mathrm{K}-40$ & 23.3 & $\mathrm{PCl} / \mathrm{G}$ & G & 1.3 \\
\hline 383DH1500.5 & $6 / 7 / 2004$ & soil & EPA6010 & REG & $7439-92-1$ & Lead & 31 & MG/KG & & 0.31 \\
\hline 383DH1500.5 & $6 / 7 / 2004$ & soil & EPA7470 & REG & $7439-97-6$ & Mercury & 0.025 & MG/KG & B & 0.1 \\
\hline 383DH1500.5 & $6 / 7 / 2004$ & soil & HASL300 & REG & 15092-94-1 & $\mathrm{Pb}-212$ & 1.69 & $\mathrm{PCl} / \mathrm{G}$ & $\mathrm{J}$ & 0.34 \\
\hline 383DH1500.5 & $6 / 7 / 2004$ & soil & HASL300 & REG & $15067-28-4$ & $\mathrm{~Pb}-214$ & 1.22 & $\mathrm{PCl} / \mathrm{G}$ & $\mathrm{G}, \mathrm{J}$ & 0.39 \\
\hline 383DH1500.5 & $6 / 7 / 2004$ & soil & UGTAISOPU & REG & 13981-16-3 & Pu-238 & 0.077 & $\mathrm{PCl} / \mathrm{G}$ & & 0.022 \\
\hline 383DH1500.5 & $6 / 7 / 2004$ & soil & UGTAISOPU & REG & 15117-48-3 & Pu-239 & 0.57 & $\mathrm{PCl} / \mathrm{G}$ & & 0.02 \\
\hline 383DH1500.5 & $6 / 7 / 2004$ & soil & SR7500 & REG & 10098-97-2 & Sr-90 & 0.53 & $\mathrm{PCl} / \mathrm{G}$ & $\mathrm{Y} 1$ & 0.23 \\
\hline 383DH1500.5 & $6 / 7 / 2004$ & soil & HASL300 & REG & 14913-50-9 & TI-208 & 0.59 & $\mathrm{PCl} / \mathrm{G}$ & G & 0.19 \\
\hline 383DH1600.5 & $6 / 7 / 2004$ & soil & HASL300 & REG & 14331-83-0 & Ac-228 & 1.56 & $\mathrm{PCl} / \mathrm{G}$ & G & 0.64 \\
\hline 383DH1600.5 & $6 / 7 / 2004$ & soil & HASL300 & REG & 14682-66-7 & Al-26 & 0.105 & $\mathrm{PCl} / \mathrm{G}$ & $\mathrm{G}, \mathrm{TI}$ & 0.032 \\
\hline 383DH1600.5 & $6 / 7 / 2004$ & soil & EPA6010 & REG & $7440-38-2$ & Arsenic & 3 & MG/KG & & 1 \\
\hline 383DH1600.5 & $6 / 7 / 2004$ & soil & EPA6010 & REG & 7440-39-3 & Barium & 4100 & MG/KG & & 100 \\
\hline 383DH1600.5 & $6 / 7 / 2004$ & soil & EPA6010 & REG & $7440-41-7$ & Beryllium & 1.4 & MG/KG & & 0.52 \\
\hline 383DH1600.5 & $6 / 7 / 2004$ & soil & HASL300 & REG & 14733-03-0 & Bi-214 & 1.07 & $\mathrm{PCl} / \mathrm{G}$ & $\mathrm{G}, \mathrm{J}$ & 0.41 \\
\hline 383DH1600.5 & 6/7/2004 & soil & EPA8270 & REG & 117-81-7 & Bis(2-Ethylhexyl)Phthalate & 130 & UG/KG & $\mathrm{J}$ & 350 \\
\hline 383DH1600.5 & $6 / 7 / 2004$ & soil & EPA6010 & REG & $7440-43-9$ & Cadmium & 0.17 & MG/KG & $B$ & 0.52 \\
\hline
\end{tabular}




\begin{tabular}{|c|c|c|c|c|c|c|c|c|c|c|}
\hline SAMPLE \# & $\begin{array}{l}\text { SAMPLE } \\
\text { DATE }\end{array}$ & MATRIX & $\begin{array}{l}\text { USER TEST } \\
\text { PANEL }\end{array}$ & $\begin{array}{l}\text { SAMPLE } \\
\text { PURPOSE }\end{array}$ & CAS \# & PARAMETER & RESULT & UNITS & Q & $\begin{array}{l}\text { DETECT } \\
\text { LIMIT }\end{array}$ \\
\hline 383DH1600.5 & $6 / 7 / 2004$ & soil & EPA6010 & REG & $7440-47-3$ & Chromium & 4.2 & MG/KG & & 1 \\
\hline 383DH1600.5 & $6 / 7 / 2004$ & soil & HASL300 & REG & $10045-97-3$ & Cs-137 & 20.5 & $\mathrm{PCl} / \mathrm{G}$ & G & 0.2 \\
\hline 383DH1600.5 & 6/7/2004 & soil & EPA8270 & REG & 84-74-2 & Di-N-Butyl Phthalate & 23 & UG/KG & $\mathrm{J}$ & 350 \\
\hline 383DH1600.5 & $6 / 7 / 2004$ & soil & EPA8260 & REG & $100-41-4$ & Ethylbenzene & 1.5 & UG/KG & $\mathrm{J}$ & 5.2 \\
\hline 383DH1600.5 & $6 / 7 / 2004$ & soil & HASL300 & REG & 13966-00-2 & $\mathrm{K}-40$ & 26.5 & $\mathrm{PCl} / \mathrm{G}$ & G & 1.8 \\
\hline 383DH1600.5 & $6 / 7 / 2004$ & soil & EPA6010 & REG & $7439-92-1$ & Lead & 24 & MG/KG & & 0.31 \\
\hline 383DH1600.5 & 6/7/2004 & soil & EPA8260 & REG & 136777-61-2 & M+P-Xylene & 5.8 & UG/KG & & 5.2 \\
\hline 383DH1600.5 & $6 / 7 / 2004$ & soil & EPA7470 & REG & $7439-97-6$ & Mercury & 0.038 & MG/KG & $B$ & 0.1 \\
\hline 383DH1600.5 & $6 / 7 / 2004$ & soil & EPA8260 & REG & $75-09-2$ & Methylene Chloride & 1 & UG/KG & $\mathrm{J}$ & 5.2 \\
\hline 383DH1600.5 & $6 / 7 / 2004$ & soil & EPA8260 & REG & $95-47-6$ & O-Xylene & 1.5 & UG/KG & $\mathrm{J}$ & 5.2 \\
\hline 383DH1600.5 & $6 / 7 / 2004$ & soil & HASL300 & REG & 15092-94-1 & $\mathrm{Pb}-212$ & 1.74 & $\mathrm{PCl} / \mathrm{G}$ & $\mathrm{J}$ & 0.4 \\
\hline 383DH1600.5 & $6 / 7 / 2004$ & soil & HASL300 & REG & 15067-28-4 & $\mathrm{Pb}-214$ & 1.32 & $\mathrm{PCl} / \mathrm{G}$ & $\mathrm{G}, \mathrm{J}$ & 0.51 \\
\hline 383DH1600.5 & $6 / 7 / 2004$ & soil & UGTAISOPU & REG & 13981-16-3 & Pu-238 & 0.13 & $\mathrm{PCl} / \mathrm{G}$ & & 0.022 \\
\hline 383DH1600.5 & $6 / 7 / 2004$ & soil & UGTAISOPU & REG & $15117-48-3$ & Pu-239 & 1.08 & $\mathrm{PCl} / \mathrm{G}$ & & 0.01 \\
\hline 383DH1600.5 & $6 / 7 / 2004$ & soil & SR7500 & REG & 10098-97-2 & Sr-90 & 0.54 & $\mathrm{PCl} / \mathrm{G}$ & Y1 & 0.25 \\
\hline 383DH1600.5 & $6 / 7 / 2004$ & soil & HASL300 & REG & 14913-50-9 & TI-208 & 0.55 & $\mathrm{PCl} / \mathrm{G}$ & G & 0.19 \\
\hline 383DH1700.5 & $6 / 7 / 2004$ & soil & HASL300 & REG & 14331-83-0 & Ac-228 & 1.8 & $\mathrm{PCl} / \mathrm{G}$ & G & 0.72 \\
\hline 383DH1700.5 & $6 / 7 / 2004$ & soil & EPA6010 & REG & $7440-38-2$ & Arsenic & 3.4 & MG/KG & & 1 \\
\hline 383DH1700.5 & $6 / 7 / 2004$ & soil & EPA6010 & REG & 7440-39-3 & Barium & 2800 & MG/KG & & 100 \\
\hline 383DH1700.5 & $6 / 7 / 2004$ & soil & EPA6010 & REG & $7440-41-7$ & Beryllium & 1.7 & MG/KG & & 0.51 \\
\hline 383DH1700.5 & $6 / 7 / 2004$ & soil & HASL300 & REG & 14733-03-0 & $\mathrm{Bi}-214$ & 1.12 & $\mathrm{PCl} / \mathrm{G}$ & $\mathrm{G}, \mathrm{J}$ & 0.59 \\
\hline 383DH1700.5 & $6 / 7 / 2004$ & soil & EPA6010 & REG & $7440-43-9$ & Cadmium & 0.37 & MG/KG & $B$ & 0.51 \\
\hline 383DH1700.5 & $6 / 7 / 2004$ & soil & EPA6010 & REG & $7440-47-3$ & Chromium & 5.8 & MG/KG & & 1 \\
\hline 383DH1700.5 & $6 / 7 / 2004$ & soil & HASL300 & REG & 10045-97-3 & Cs-137 & 68 & $\mathrm{PCl} / \mathrm{G}$ & G & 0.3 \\
\hline 383DH1700.5 & $6 / 7 / 2004$ & soil & HASL300 & REG & 13966-00-2 & $\mathrm{K}-40$ & 19.7 & $\mathrm{PCl} / \mathrm{G}$ & G & 2.7 \\
\hline 383DH1700.5 & $6 / 7 / 2004$ & soil & EPA6010 & REG & $7439-92-1$ & Lead & 49 & MG/KG & & 0.31 \\
\hline 383DH1700.5 & $6 / 7 / 2004$ & soil & EPA7470 & REG & 7439-97-6 & Mercury & 0.061 & MG/KG & B & 0.1 \\
\hline 383DH1700.5 & $6 / 7 / 2004$ & soil & EPA8260 & REG & $75-09-2$ & Methylene Chloride & 1.2 & UG/KG & $\mathrm{J}$ & 5.1 \\
\hline 383DH1700.5 & $6 / 7 / 2004$ & soil & HASL300 & REG & 15092-94-1 & $\mathrm{Pb}-212$ & 2.21 & $\mathrm{PCl} / \mathrm{G}$ & $\mathrm{J}$ & 0.58 \\
\hline 383DH1700.5 & $6 / 7 / 2004$ & soil & HASL300 & REG & $15067-28-4$ & $\mathrm{~Pb}-214$ & 1.32 & $\mathrm{PCl} / \mathrm{G}$ & $\mathrm{G}, \mathrm{J}$ & 0.84 \\
\hline 383DH1700.5 & $6 / 7 / 2004$ & soil & UGTAISOPU & REG & 13981-16-3 & Pu-238 & 0.41 & $\mathrm{PCl} / \mathrm{G}$ & & 0.03 \\
\hline 383DH1700.5 & $6 / 7 / 2004$ & soil & UGTAISOPU & REG & $15117-48-3$ & Pu-239 & 3.55 & $\mathrm{PCl} / \mathrm{G}$ & & 0.03 \\
\hline 383DH1700.5 & $6 / 7 / 2004$ & soil & EPA6010 & REG & $7782-49-2$ & Selenium & 0.87 & MG/KG & & 0.51 \\
\hline 383DH1700.5 & $6 / 7 / 2004$ & soil & SR7500 & REG & 10098-97-2 & Sr-90 & 1.09 & $\mathrm{PCl} / \mathrm{G}$ & Y1 & 0.24 \\
\hline 383DH1700.5 & $6 / 7 / 2004$ & soil & HASL300 & REG & 14913-50-9 & TI-208 & 0.7 & $\mathrm{PCl} / \mathrm{G}$ & G & 0.32 \\
\hline
\end{tabular}




\begin{tabular}{|c|c|c|c|c|c|c|c|c|c|c|}
\hline SAMPLE \# & $\begin{array}{l}\text { SAMPLE } \\
\text { DATE }\end{array}$ & MATRIX & $\begin{array}{l}\text { USER TEST } \\
\text { PANEL }\end{array}$ & $\begin{array}{l}\text { SAMPLE } \\
\text { PURPOSE }\end{array}$ & CAS \# & PARAMETER & RESULT & UNITS & Q & $\begin{array}{l}\text { DETECT } \\
\text { LIMIT }\end{array}$ \\
\hline 383DH1800.5 & $6 / 7 / 2004$ & soil & HASL300 & REG & 14331-83-0 & Ac-228 & 1.17 & $\mathrm{PCl} / \mathrm{G}$ & G & 0.64 \\
\hline 383DH1800.5 & $6 / 7 / 2004$ & soil & EPA6010 & REG & $7440-38-2$ & Arsenic & 3 & MG/KG & & 1 \\
\hline 383DH1800.5 & $6 / 7 / 2004$ & soil & EPA6010 & REG & $7440-39-3$ & Barium & 4200 & MG/KG & & 100 \\
\hline 383DH1800.5 & $6 / 7 / 2004$ & soil & EPA6010 & REG & $7440-41-7$ & Beryllium & 1.5 & MG/KG & & 0.52 \\
\hline 383DH1800.5 & $6 / 7 / 2004$ & soil & HASL300 & REG & 14733-03-0 & Bi-214 & 1.46 & $\mathrm{PCl} / \mathrm{G}$ & $\mathrm{G}, \mathrm{J}$ & 0.37 \\
\hline 383DH1800.5 & $6 / 7 / 2004$ & soil & EPA6010 & REG & $7440-43-9$ & Cadmium & 0.35 & MG/KG & $\mathrm{B}$ & 0.52 \\
\hline 383DH1800.5 & $6 / 7 / 2004$ & soil & EPA6010 & REG & $7440-47-3$ & Chromium & 5.5 & MG/KG & & 1 \\
\hline 383DH1800.5 & $6 / 7 / 2004$ & soil & HASL300 & REG & 10045-97-3 & Cs-137 & 23.1 & $\mathrm{PCl} / \mathrm{G}$ & G & 0.2 \\
\hline 383DH1800.5 & $6 / 7 / 2004$ & soil & HASL300 & REG & 13966-00-2 & $\mathrm{K}-40$ & 20.6 & $\mathrm{PCl} / \mathrm{G}$ & G & 1.3 \\
\hline 383DH1800.5 & $6 / 7 / 2004$ & soil & EPA6010 & REG & $7439-92-1$ & Lead & 21 & MG/KG & & 0.31 \\
\hline 383DH1800.5 & $6 / 7 / 2004$ & soil & EPA7470 & REG & $7439-97-6$ & Mercury & 0.039 & MG/KG & B & 0.1 \\
\hline 383DH1800.5 & $6 / 7 / 2004$ & soil & EPA8260 & REG & $75-09-2$ & Methylene Chloride & 1.1 & UG/KG & $\mathrm{J}$ & 5.2 \\
\hline 383DH1800.5 & $6 / 7 / 2004$ & soil & HASL300 & REG & 15092-94-1 & $\mathrm{Pb}-212$ & 1.69 & $\mathrm{PCl} / \mathrm{G}$ & $\mathrm{J}$ & 0.32 \\
\hline 383DH1800.5 & $6 / 7 / 2004$ & soil & HASL300 & REG & 15067-28-4 & $\mathrm{Pb}-214$ & 1.18 & $\mathrm{PCl} / \mathrm{G}$ & $\mathrm{G}, \mathrm{J}$ & 0.52 \\
\hline 383DH1800.5 & $6 / 7 / 2004$ & soil & UGTAISOPU & REG & 13981-16-3 & Pu-238 & 0.177 & $\mathrm{PCl} / \mathrm{G}$ & & 0.023 \\
\hline 383DH1800.5 & $6 / 7 / 2004$ & soil & UGTAISOPU & REG & 15117-48-3 & Pu-239 & 1.39 & $\mathrm{PCl} / \mathrm{G}$ & & 0.02 \\
\hline 383DH1800.5 & $6 / 7 / 2004$ & soil & SR7500 & REG & 10098-97-2 & Sr-90 & 0.69 & $\mathrm{PCl} / \mathrm{G}$ & Y1 & 0.25 \\
\hline 383DH1800.5 & $6 / 7 / 2004$ & soil & HASL300 & REG & 14913-50-9 & TI-208 & 0.52 & $\mathrm{PCl} / \mathrm{G}$ & G & 0.21 \\
\hline 383DH1900.5 & $6 / 7 / 2004$ & soil & HASL300 & REG & 14331-83-0 & Ac-228 & 1.82 & $\mathrm{PCl} / \mathrm{G}$ & G & 0.74 \\
\hline 383DH1900.5 & $6 / 7 / 2004$ & soil & EPA6010 & REG & $7440-38-2$ & Arsenic & 2.7 & MG/KG & & 1 \\
\hline 383DH1900.5 & $6 / 7 / 2004$ & soil & EPA6010 & REG & $7440-39-3$ & Barium & 4500 & MG/KG & & 100 \\
\hline 383DH1900.5 & $6 / 7 / 2004$ & soil & EPA6010 & REG & $7440-41-7$ & Beryllium & 1.7 & MG/KG & & 0.51 \\
\hline 383DH1900.5 & $6 / 7 / 2004$ & soil & HASL300 & REG & 14733-03-0 & Bi-214 & 1.27 & $\mathrm{PCl} / \mathrm{G}$ & $\mathrm{G}, \mathrm{J}$ & 0.47 \\
\hline 383DH1900.5 & $6 / 7 / 2004$ & soil & EPA8270 & REG & 117-81-7 & Bis(2-Ethylhexyl)Phthalate & 120 & UG/KG & $\mathrm{J}$ & 340 \\
\hline 383DH1900.5 & $6 / 7 / 2004$ & soil & EPA6010 & REG & 7440-43-9 & Cadmium & 0.33 & MG/KG & B & 0.51 \\
\hline 383DH1900.5 & $6 / 7 / 2004$ & soil & EPA6010 & REG & $7440-47-3$ & Chromium & 4.6 & MG/KG & & 1 \\
\hline 383DH1900.5 & $6 / 7 / 2004$ & soil & HASL300 & REG & $10045-97-3$ & Cs-137 & 42.4 & $\mathrm{PCl} / \mathrm{G}$ & G & 0.3 \\
\hline 383DH1900.5 & $6 / 7 / 2004$ & soil & HASL300 & REG & 13966-00-2 & $\mathrm{K}-40$ & 24.1 & $\mathrm{PCl} / \mathrm{G}$ & G & 1.7 \\
\hline 383DH1900.5 & $6 / 7 / 2004$ & soil & EPA6010 & REG & $7439-92-1$ & Lead & 25 & MG/KG & & 0.31 \\
\hline 383DH1900.5 & $6 / 7 / 2004$ & soil & EPA8260 & REG & 136777-61-2 & M+P-Xylene & 2.4 & UG/KG & $\mathrm{J}$ & 5.1 \\
\hline 383DH1900.5 & $6 / 7 / 2004$ & soil & EPA7470 & REG & 7439-97-6 & Mercury & 0.034 & MG/KG & $B$ & 0.1 \\
\hline 383DH1900.5 & $6 / 7 / 2004$ & soil & EPA8260 & REG & $75-09-2$ & Methylene Chloride & 1.2 & UG/KG & $\mathrm{J}$ & 5.1 \\
\hline 383DH1900.5 & $6 / 7 / 2004$ & soil & EPA8260 & REG & $95-47-6$ & O-Xylene & 1 & UG/KG & $\mathrm{J}$ & 5.1 \\
\hline 383DH1900.5 & $6 / 7 / 2004$ & soil & HASL300 & REG & 15092-94-1 & $\mathrm{Pb}-212$ & 1.78 & $\mathrm{PCl} / \mathrm{G}$ & $\mathrm{J}$ & 0.47 \\
\hline 383DH1900.5 & $6 / 7 / 2004$ & soil & HASL300 & REG & 15067-28-4 & $\mathrm{Pb}-214$ & 1.3 & $\mathrm{PCl} / \mathrm{G}$ & $\mathrm{G}, \mathrm{J}$ & 0.66 \\
\hline
\end{tabular}




\begin{tabular}{|c|c|c|c|c|c|c|c|c|c|c|}
\hline SAMPLE \# & $\begin{array}{l}\text { SAMPLE } \\
\text { DATE }\end{array}$ & MATRIX & $\begin{array}{l}\text { USER TEST } \\
\text { PANEL }\end{array}$ & $\begin{array}{l}\text { SAMPLE } \\
\text { PURPOSE }\end{array}$ & CAS \# & PARAMETER & RESULT & UNITS & Q & $\begin{array}{l}\text { DETECT } \\
\text { LIMIT }\end{array}$ \\
\hline 383DH1900.5 & $6 / 7 / 2004$ & soil & UGTAISOPU & REG & 13981-16-3 & Pu-238 & 0.32 & $\mathrm{PCl} / \mathrm{G}$ & & 0.019 \\
\hline 383DH1900.5 & $6 / 7 / 2004$ & soil & UGTAISOPU & REG & 15117-48-3 & Pu-239 & 2.28 & $\mathrm{PCl} / \mathrm{G}$ & & 0.01 \\
\hline 383DH1900.5 & 6/7/2004 & soil & SR7500 & REG & 10098-97-2 & Sr-90 & 1.14 & $\mathrm{PCl} / \mathrm{G}$ & Y1 & 0.26 \\
\hline 383DH1900.5 & $6 / 7 / 2004$ & soil & HASL300 & REG & 14913-50-9 & TI-208 & 0.65 & $\mathrm{PCl} / \mathrm{G}$ & G & 0.27 \\
\hline 383DH2000.5 & $6 / 7 / 2004$ & soil & HASL300 & REG & 14331-83-0 & Ac-228 & 1.47 & $\mathrm{PCl} / \mathrm{G}$ & G & 0.59 \\
\hline 383DH2000.5 & $6 / 7 / 2004$ & soil & EPA8260 & REG & 67-64-1 & Acetone & 6.6 & UG/KG & $\mathrm{J}$ & 21 \\
\hline 383DH2000.5 & $6 / 7 / 2004$ & soil & EPA6010 & REG & $7440-38-2$ & Arsenic & 3.3 & MG/KG & & 1 \\
\hline 383DH2000.5 & $6 / 7 / 2004$ & soil & EPA6010 & REG & 7440-39-3 & Barium & 5300 & MG/KG & & 100 \\
\hline 383DH2000.5 & $6 / 7 / 2004$ & soil & EPA6010 & REG & $7440-41-7$ & Beryllium & 1.3 & MG/KG & & 0.52 \\
\hline 383DH2000.5 & $6 / 7 / 2004$ & soil & HASL300 & REG & 14733-03-0 & $\mathrm{Bi}-214$ & 1.1 & $\mathrm{PCl} / \mathrm{G}$ & $\mathrm{G}, \mathrm{J}$ & 0.38 \\
\hline 383DH2000.5 & $6 / 7 / 2004$ & soil & EPA6010 & REG & $7440-43-9$ & Cadmium & 0.3 & MG/KG & B & 0.52 \\
\hline 383DH2000.5 & $6 / 7 / 2004$ & soil & EPA6010 & REG & 7440-47-3 & Chromium & 4.5 & MG/KG & & 1 \\
\hline 383DH2000.5 & $6 / 7 / 2004$ & soil & HASL300 & REG & 10045-97-3 & Cs-137 & 24.1 & $\mathrm{PCl} / \mathrm{G}$ & G & 0.2 \\
\hline 383DH2000.5 & $6 / 7 / 2004$ & soil & HASL300 & REG & 13966-00-2 & $\mathrm{K}-40$ & 22.8 & $\mathrm{PCl} / \mathrm{G}$ & G & 1.4 \\
\hline 383DH2000.5 & $6 / 7 / 2004$ & soil & EPA6010 & REG & $7439-92-1$ & Lead & 22 & MG/KG & & 0.31 \\
\hline 383DH2000.5 & $6 / 7 / 2004$ & soil & EPA7470 & REG & 7439-97-6 & Mercury & 0.031 & MG/KG & $B$ & 0.1 \\
\hline 383DH2000.5 & $6 / 7 / 2004$ & soil & HASL300 & REG & 15092-94-1 & $\mathrm{Pb}-212$ & 1.91 & $\mathrm{PCl} / \mathrm{G}$ & $\mathrm{J}$ & 0.33 \\
\hline 383DH2000.5 & $6 / 7 / 2004$ & soil & HASL300 & REG & 15067-28-4 & $\mathrm{Pb}-214$ & 1.16 & $\mathrm{PCl} / \mathrm{G}$ & $\mathrm{G}, \mathrm{J}$ & 0.43 \\
\hline 383DH2000.5 & $6 / 7 / 2004$ & soil & UGTAISOPU & REG & 13981-16-3 & Pu-238 & 0.165 & $\mathrm{PCl} / \mathrm{G}$ & & 0.02 \\
\hline 383DH2000.5 & $6 / 7 / 2004$ & soil & UGTAISOPU & REG & 15117-48-3 & Pu-239 & 1.44 & $\mathrm{PCl} / \mathrm{G}$ & & 0.01 \\
\hline 383DH2000.5 & $6 / 7 / 2004$ & soil & SR7500 & REG & 10098-97-2 & Sr-90 & 0.68 & $\mathrm{PCl} / \mathrm{G}$ & Y1 & 0.22 \\
\hline 383DH2000.5 & $6 / 7 / 2004$ & soil & HASL300 & REG & 14913-50-9 & TI-208 & 0.47 & $\mathrm{PCl} / \mathrm{G}$ & G & 0.24 \\
\hline 383DH2100.5 & $6 / 15 / 2004$ & soil & HASL300 & REG & 14331-83-0 & Ac-228 & 1.68 & $\mathrm{PCl} / \mathrm{G}$ & G & 0.65 \\
\hline 383DH2100.5 & $6 / 15 / 2004$ & soil & EPA8260 & REG & 67-64-1 & Acetone & 12 & UG/KG & $\mathrm{J}$ & 21 \\
\hline 383DH2100.5 & $6 / 15 / 2004$ & soil & EPA6010 & REG & $7440-38-2$ & Arsenic & 3.3 & MG/KG & & 1 \\
\hline 383DH2100.5 & $6 / 15 / 2004$ & soil & EPA6010 & REG & $7440-39-3$ & Barium & 5100 & MG/KG & & 520 \\
\hline 383DH2100.5 & $6 / 15 / 2004$ & soil & EPA6010 & REG & $7440-41-7$ & Beryllium & 1.6 & MG/KG & & 0.52 \\
\hline 383DH2100.5 & $6 / 15 / 2004$ & soil & HASL300 & REG & 14733-03-0 & Bi-214 & 1.37 & $\mathrm{PCl} / \mathrm{G}$ & $\mathrm{G}, \mathrm{J}$ & 0.6 \\
\hline 383DH2100.5 & $6 / 15 / 2004$ & soil & EPA8270 & REG & 117-81-7 & Bis(2-Ethylhexyl)Phthalate & 140 & UG/KG & $\mathrm{J}$ & 350 \\
\hline 383DH2100.5 & $6 / 15 / 2004$ & soil & EPA6010 & REG & $7440-43-9$ & Cadmium & 0.27 & MG/KG & B & 0.52 \\
\hline 383DH2100.5 & $6 / 15 / 2004$ & soil & EPA6010 & REG & $7440-47-3$ & Chromium & 4.7 & MG/KG & & 1 \\
\hline 383DH2100.5 & $6 / 15 / 2004$ & soil & HASL300 & REG & $10045-97-3$ & Cs-137 & 78.1 & $\mathrm{PCl} / \mathrm{G}$ & G & 0.3 \\
\hline 383DH2100.5 & $6 / 15 / 2004$ & soil & HASL300 & REG & 13966-00-2 & $\mathrm{K}-40$ & 22.9 & $\mathrm{PCl} / \mathrm{G}$ & G & 2.1 \\
\hline 383DH2100.5 & $6 / 15 / 2004$ & soil & EPA6010 & REG & 7439-92-1 & Lead & 30 & MG/KG & & 0.31 \\
\hline 383DH2100.5 & $6 / 15 / 2004$ & soil & EPA7470 & REG & 7439-97-6 & Mercury & 0.049 & MG/KG & B & 0.1 \\
\hline
\end{tabular}




\begin{tabular}{|c|c|c|c|c|c|c|c|c|c|c|}
\hline SAMPLE \# & $\begin{array}{l}\text { SAMPLE } \\
\text { DATE }\end{array}$ & MATRIX & $\begin{array}{l}\text { USER TEST } \\
\text { PANEL }\end{array}$ & $\begin{array}{l}\text { SAMPLE } \\
\text { PURPOSE }\end{array}$ & CAS \# & PARAMETER & RESULT & UNITS & Q & $\begin{array}{l}\text { DETECT } \\
\text { LIMIT }\end{array}$ \\
\hline 383DH2100.5 & $6 / 15 / 2004$ & soil & EPA8260 & REG & $75-09-2$ & Methylene Chloride & 1.2 & UG/KG & $\mathrm{J}, \mathrm{B}$ & 5.2 \\
\hline 383DH2100.5 & $6 / 15 / 2004$ & soil & HASL300 & REG & 15092-94-1 & $\mathrm{Pb}-212$ & 2.16 & $\mathrm{PCl} / \mathrm{G}$ & $\mathrm{J}$ & 0.56 \\
\hline 383DH2100.5 & $6 / 15 / 2004$ & soil & HASL300 & REG & $15067-28-4$ & $\mathrm{~Pb}-214$ & 1.26 & $\mathrm{PCl} / \mathrm{G}$ & $\mathrm{G}, \mathrm{J}$ & 0.66 \\
\hline 383DH2100.5 & $6 / 15 / 2004$ & soil & UGTAISOPU & REG & 13981-16-3 & Pu-238 & 0.341 & $\mathrm{PCl} / \mathrm{G}$ & & 0.021 \\
\hline 383DH2100.5 & $6 / 15 / 2004$ & soil & UGTAISOPU & REG & $15117-48-3$ & Pu-239 & 3.3 & $\mathrm{PCl} / \mathrm{G}$ & & 0.02 \\
\hline 383DH2100.5 & $6 / 15 / 2004$ & soil & EPA6010 & REG & $7782-49-2$ & Selenium & 1.1 & MG/KG & & 0.52 \\
\hline 383DH2100.5 & $6 / 15 / 2004$ & soil & SR7500 & REG & $10098-97-2$ & Sr-90 & 1.17 & $\mathrm{PCl} / \mathrm{G}$ & & 0.34 \\
\hline 383DH2100.5 & $6 / 15 / 2004$ & soil & HASL300 & REG & 14913-50-9 & TI-208 & 0.69 & $\mathrm{PCl} / \mathrm{G}$ & G & 0.28 \\
\hline 383DH2200.5 & $6 / 15 / 2004$ & soil & HASL300 & REG & 14331-83-0 & Ac-228 & 1.37 & $\mathrm{PCl} / \mathrm{G}$ & G & 0.53 \\
\hline 383DH2200.5 & $6 / 15 / 2004$ & soil & EPA6010 & REG & $7440-38-2$ & Arsenic & 3.3 & MG/KG & & 1 \\
\hline 383DH2200.5 & $6 / 15 / 2004$ & soil & EPA6010 & REG & $7440-39-3$ & Barium & 5300 & MG/KG & & 510 \\
\hline 383DH2200.5 & $6 / 15 / 2004$ & soil & EPA8270 & REG & $56-55-3$ & Benzo(A)Anthracene & 26 & UG/KG & $\mathrm{J}$ & 340 \\
\hline 383DH2200.5 & $6 / 15 / 2004$ & soil & EPA8270 & REG & $50-32-8$ & Benzo(A)Pyrene & 37 & UG/KG & $\mathrm{J}$ & 340 \\
\hline 383DH2200.5 & $6 / 15 / 2004$ & soil & EPA8270 & REG & $205-99-2$ & Benzo(B)Fluoranthene & 51 & UG/KG & $\mathrm{J}$ & 340 \\
\hline 383DH2200.5 & $6 / 15 / 2004$ & soil & EPA8270 & REG & $191-24-2$ & Benzo(G,H,I)Perylene & 46 & UG/KG & $\mathrm{J}$ & 340 \\
\hline 383DH2200.5 & $6 / 15 / 2004$ & soil & EPA8270 & REG & 207-08-9 & Benzo(K)Fluoranthene & 25 & UG/KG & $\mathrm{J}$ & 340 \\
\hline 383DH2200.5 & $6 / 15 / 2004$ & soil & EPA6010 & REG & $7440-41-7$ & Beryllium & 1.4 & MG/KG & & 0.51 \\
\hline 383DH2200.5 & $6 / 15 / 2004$ & soil & HASL300 & REG & 14733-03-0 & $\mathrm{Bi}-214$ & 1.3 & $\mathrm{PCl} / \mathrm{G}$ & $\mathrm{G}, \mathrm{J}$ & 0.42 \\
\hline 383DH2200.5 & $6 / 15 / 2004$ & soil & EPA8270 & REG & 117-81-7 & Bis(2-Ethylhexyl)Phthalate & 2400 & UG/KG & & 340 \\
\hline 383DH2200.5 & $6 / 15 / 2004$ & soil & EPA6010 & REG & $7440-43-9$ & Cadmium & 0.26 & MG/KG & $B$ & 0.51 \\
\hline 383DH2200.5 & $6 / 15 / 2004$ & soil & EPA6010 & REG & $7440-47-3$ & Chromium & 5 & MG/KG & & 1 \\
\hline 383DH2200.5 & $6 / 15 / 2004$ & soil & EPA8270 & REG & $218-01-9$ & Chrysene & 100 & UG/KG & $\mathrm{J}$ & 340 \\
\hline 383DH2200.5 & $6 / 15 / 2004$ & soil & HASL300 & REG & $10045-97-3$ & Cs-137 & 29.3 & $\mathrm{PCl} / \mathrm{G}$ & G & 0.2 \\
\hline 383DH2200.5 & $6 / 15 / 2004$ & soil & EPA8015 & REG & $68334-30-5$ & Diesel Range Organics & 8.5 & MG/KG & $\mathrm{Z}, \mathrm{H}$ & 5.1 \\
\hline 383DH2200.5 & $6 / 15 / 2004$ & soil & EPA8270 & REG & $131-11-3$ & Dimethyl Phthalate & 14 & UG/KG & $\mathrm{J}$ & 340 \\
\hline 383DH2200.5 & $6 / 15 / 2004$ & soil & EPA8270 & REG & $117-84-0$ & Di-N-Octyl Phthalate & 59 & UG/KG & $\mathrm{J}$ & 340 \\
\hline 383DH2200.5 & $6 / 15 / 2004$ & soil & EPA8270 & REG & $206-44-0$ & Fluoranthene & 23 & UG/KG & $\mathrm{J}$ & 340 \\
\hline 383DH2200.5 & $6 / 15 / 2004$ & soil & HASL300 & REG & $13966-00-2$ & $\mathrm{~K}-40$ & 25.6 & $\mathrm{PCl} / \mathrm{G}$ & G & 1.4 \\
\hline 383DH2200.5 & $6 / 15 / 2004$ & soil & EPA6010 & REG & $7439-92-1$ & Lead & 31 & MG/KG & & 0.31 \\
\hline 383DH2200.5 & $6 / 15 / 2004$ & soil & EPA7470 & REG & 7439-97-6 & Mercury & 0.038 & MG/KG & $B$ & 0.1 \\
\hline 383DH2200.5 & $6 / 15 / 2004$ & soil & EPA8260 & REG & $75-09-2$ & Methylene Chloride & 1.1 & UG/KG & $\mathrm{J}, \mathrm{B}$ & 5.1 \\
\hline 383DH2200.5 & $6 / 15 / 2004$ & soil & HASL300 & REG & 15092-94-1 & $\mathrm{Pb}-212$ & 2.09 & $\mathrm{PCl} / \mathrm{G}$ & $\mathrm{J}$ & 0.36 \\
\hline 383DH2200.5 & $6 / 15 / 2004$ & soil & HASL300 & REG & $15067-28-4$ & $\mathrm{~Pb}-214$ & 1.2 & $\mathrm{PCl} / \mathrm{G}$ & $\mathrm{G}, \mathrm{J}$ & 0.55 \\
\hline 383DH2200.5 & $6 / 15 / 2004$ & soil & UGTAISOPU & REG & 13981-16-3 & Pu-238 & 0.226 & $\mathrm{PCl} / \mathrm{G}$ & & 0.011 \\
\hline 383DH2200.5 & $6 / 15 / 2004$ & soil & UGTAISOPU & REG & 15117-48-3 & Pu-239 & 1.96 & $\mathrm{PCl} / \mathrm{G}$ & & 0.03 \\
\hline
\end{tabular}




\begin{tabular}{|c|c|c|c|c|c|c|c|c|c|c|}
\hline SAMPLE \# & $\begin{array}{l}\text { SAMPLE } \\
\text { DATE }\end{array}$ & MATRIX & $\begin{array}{l}\text { USER TEST } \\
\text { PANEL }\end{array}$ & $\begin{array}{l}\text { SAMPLE } \\
\text { PURPOSE }\end{array}$ & CAS \# & PARAMETER & RESULT & UNITS & Q & $\begin{array}{l}\text { DETECT } \\
\text { LIMIT }\end{array}$ \\
\hline 383DH2200.5 & $6 / 15 / 2004$ & soil & EPA8270 & REG & $129-00-0$ & Pyrene & 41 & UG/KG & $\mathrm{J}$ & 340 \\
\hline 383DH2200.5 & $6 / 15 / 2004$ & soil & EPA6010 & REG & $7782-49-2$ & Selenium & 0.4 & MG/KG & B & 0.51 \\
\hline 383DH2200.5 & $6 / 15 / 2004$ & soil & SR7500 & REG & 10098-97-2 & Sr-90 & 0.82 & $\mathrm{PCl} / \mathrm{G}$ & & 0.22 \\
\hline $383 \mathrm{DH} 2200.5$ & $6 / 15 / 2004$ & soil & HASL300 & REG & $14913-50-9$ & TI-208 & 0.62 & $\mathrm{PCl} / \mathrm{G}$ & G & 0.21 \\
\hline 383DH2200.5X & $6 / 15 / 2004$ & soil & HASL300 & FD & 14331-83-0 & Ac-228 & 1.52 & $\mathrm{PCl} / \mathrm{G}$ & G & 0.56 \\
\hline 383DH2200.5X & $6 / 15 / 2004$ & soil & EPA6010 & FD & $7440-38-2$ & Arsenic & 3.7 & MG/KG & & 1 \\
\hline 383DH2200.5X & $6 / 15 / 2004$ & soil & EPA6010 & FD & $7440-39-3$ & Barium & 5200 & MG/KG & & 510 \\
\hline 383DH2200.5X & $6 / 15 / 2004$ & soil & EPA8270 & FD & $56-55-3$ & Benzo(A)Anthracene & 24 & UG/KG & $\mathrm{J}$ & 340 \\
\hline 383DH2200.5X & $6 / 15 / 2004$ & soil & EPA8270 & FD & $50-32-8$ & Benzo(A)Pyrene & 40 & UG/KG & $\mathrm{J}$ & 340 \\
\hline 383DH2200.5X & $6 / 15 / 2004$ & soil & EPA8270 & FD & $205-99-2$ & Benzo(B)Fluoranthene & 39 & UG/KG & $\mathrm{J}$ & 340 \\
\hline 383DH2200.5X & $6 / 15 / 2004$ & soil & EPA8270 & FD & $191-24-2$ & Benzo(G,H,I)Perylene & 39 & UG/KG & $\mathrm{J}$ & 340 \\
\hline 383DH2200.5X & $6 / 15 / 2004$ & soil & EPA8270 & FD & $207-08-9$ & Benzo(K)Fluoranthene & 29 & UG/KG & $\mathrm{J}$ & 340 \\
\hline 383DH2200.5X & $6 / 15 / 2004$ & soil & EPA6010 & FD & $7440-41-7$ & Beryllium & 1.5 & MG/KG & & 0.51 \\
\hline 383DH2200.5X & $6 / 15 / 2004$ & soil & HASL300 & FD & 14733-03-0 & $\mathrm{Bi}-214$ & 1.23 & $\mathrm{PCl} / \mathrm{G}$ & $\mathrm{G}, \mathrm{J}$ & 0.39 \\
\hline 383DH2200.5X & $6 / 15 / 2004$ & soil & EPA8270 & FD & 117-81-7 & Bis(2-Ethylhexyl)Phthalate & 170 & UG/KG & $\mathrm{J}$ & 340 \\
\hline 383DH2200.5X & $6 / 15 / 2004$ & soil & EPA6010 & FD & $7440-43-9$ & Cadmium & 0.28 & MG/KG & B & 0.51 \\
\hline 383DH2200.5X & $6 / 15 / 2004$ & soil & EPA6010 & FD & $7440-47-3$ & Chromium & 5.2 & MG/KG & & 1 \\
\hline 383DH2200.5X & $6 / 15 / 2004$ & soil & EPA8270 & FD & $218-01-9$ & Chrysene & 95 & UG/KG & $\mathrm{J}$ & 340 \\
\hline 383DH2200.5X & $6 / 15 / 2004$ & soil & HASL300 & FD & $10045-97-3$ & Cs-137 & 28 & $\mathrm{PCl} / \mathrm{G}$ & G & 0.2 \\
\hline 383DH2200.5X & $6 / 15 / 2004$ & soil & EPA8015 & FD & $68334-30-5$ & Diesel Range Organics & 6.2 & MG/KG & $\mathrm{Z}, \mathrm{H}$ & 5.1 \\
\hline 383DH2200.5X & $6 / 15 / 2004$ & soil & EPA8270 & FD & $206-44-0$ & Fluoranthene & 23 & UG/KG & $\mathrm{J}$ & 340 \\
\hline 383DH2200.5X & $6 / 15 / 2004$ & soil & HASL300 & FD & 13966-00-2 & $\mathrm{K}-40$ & 21.8 & $\mathrm{PCl} / \mathrm{G}$ & G & 1.8 \\
\hline 383DH2200.5X & $6 / 15 / 2004$ & soil & EPA6010 & FD & $7439-92-1$ & Lead & 31 & MG/KG & & 0.31 \\
\hline 383DH2200.5X & $6 / 15 / 2004$ & soil & EPA7470 & FD & $7439-97-6$ & Mercury & 0.037 & MG/KG & $B$ & 0.1 \\
\hline 383DH2200.5X & $6 / 15 / 2004$ & soil & EPA8260 & FD & $75-09-2$ & Methylene Chloride & 0.87 & UG/KG & $\mathrm{J}, \mathrm{B}$ & 5.1 \\
\hline 383DH2200.5X & $6 / 15 / 2004$ & soil & HASL300 & FD & 15092-94-1 & $\mathrm{Pb}-212$ & 1.86 & $\mathrm{PCl} / \mathrm{G}$ & $\mathrm{J}$ & 0.42 \\
\hline 383DH2200.5X & $6 / 15 / 2004$ & soil & HASL300 & FD & 15067-28-4 & $\mathrm{Pb}-214$ & 1.05 & $\mathrm{PCl} / \mathrm{G}$ & G,J & 0.55 \\
\hline 383DH2200.5X & $6 / 15 / 2004$ & soil & UGTAISOPU & FD & 13981-16-3 & Pu-238 & 0.238 & $\mathrm{PCl} / \mathrm{G}$ & & 0.026 \\
\hline 383DH2200.5X & $6 / 15 / 2004$ & soil & UGTAISOPU & FD & $15117-48-3$ & Pu-239 & 1.87 & $\mathrm{PCl} / \mathrm{G}$ & & 0.03 \\
\hline 383DH2200.5X & $6 / 15 / 2004$ & soil & EPA6010 & FD & 7782-49-2 & Selenium & 0.43 & MG/KG & $B$ & 0.51 \\
\hline 383DH2200.5X & $6 / 15 / 2004$ & soil & SR7500 & FD & 10098-97-2 & Sr-90 & 0.76 & $\mathrm{PCl} / \mathrm{G}$ & & 0.22 \\
\hline 383DH2200.5X & $6 / 15 / 2004$ & soil & HASL300 & FD & 14913-50-9 & TI-208 & 0.64 & $\mathrm{PCl} / \mathrm{G}$ & G & 0.19 \\
\hline 383DH2300.5 & $6 / 15 / 2004$ & soil & HASL300 & REG & 14331-83-0 & Ac-228 & 1.55 & $\mathrm{PCl} / \mathrm{G}$ & G & 0.56 \\
\hline $383 \mathrm{DH} 2300.5$ & $6 / 15 / 2004$ & soil & EPA6010 & REG & $7440-38-2$ & Arsenic & 3.7 & MG/KG & & 1 \\
\hline 383DH2300.5 & $6 / 15 / 2004$ & soil & EPA6010 & REG & 7440-39-3 & Barium & 5400 & MG/KG & & 510 \\
\hline
\end{tabular}




\begin{tabular}{|c|c|c|c|c|c|c|c|c|c|c|}
\hline SAMPLE \# & $\begin{array}{l}\text { SAMPLE } \\
\text { DATE }\end{array}$ & MATRIX & $\begin{array}{l}\text { USER TEST } \\
\text { PANEL }\end{array}$ & $\begin{array}{l}\text { SAMPLE } \\
\text { PURPOSE }\end{array}$ & CAS \# & PARAMETER & RESULT & UNITS & Q & $\begin{array}{l}\text { DETECT } \\
\text { LIMIT }\end{array}$ \\
\hline 383DH2300.5 & $6 / 15 / 2004$ & soil & EPA8270 & REG & $56-55-3$ & Benzo(A)Anthracene & 38 & UG/KG & $\mathrm{J}$ & 340 \\
\hline 383DH2300.5 & $6 / 15 / 2004$ & soil & EPA8270 & REG & $50-32-8$ & Benzo(A)Pyrene & 38 & UG/KG & $\mathrm{J}$ & 340 \\
\hline 383DH2300.5 & $6 / 15 / 2004$ & soil & EPA8270 & REG & $205-99-2$ & Benzo(B)Fluoranthene & 61 & UG/KG & $\mathrm{J}$ & 340 \\
\hline 383DH2300.5 & $6 / 15 / 2004$ & soil & EPA8270 & REG & $207-08-9$ & Benzo(K)Fluoranthene & 44 & UG/KG & $\mathrm{J}$ & 340 \\
\hline 383DH2300.5 & $6 / 15 / 2004$ & soil & EPA6010 & REG & $7440-41-7$ & Beryllium & 1.3 & MG/KG & & 0.51 \\
\hline 383DH2300.5 & $6 / 15 / 2004$ & soil & HASL300 & REG & 14733-03-0 & Bi-214 & 0.92 & $\mathrm{PCl} / \mathrm{G}$ & G,J & 0.41 \\
\hline 383DH2300.5 & $6 / 15 / 2004$ & soil & EPA8270 & REG & $117-81-7$ & Bis(2-Ethylhexyl)Phthalate & 82 & UG/KG & $\mathrm{J}$ & 340 \\
\hline 383DH2300.5 & $6 / 15 / 2004$ & soil & EPA6010 & REG & $7440-43-9$ & Cadmium & 0.2 & MG/KG & B & 0.51 \\
\hline 383DH2300.5 & $6 / 15 / 2004$ & soil & EPA6010 & REG & $7440-47-3$ & Chromium & 5.6 & MG/KG & & 1 \\
\hline 383DH2300.5 & $6 / 15 / 2004$ & soil & EPA8270 & REG & 218-01-9 & Chrysene & 55 & UG/KG & $\mathrm{J}$ & 340 \\
\hline 383DH2300.5 & $6 / 15 / 2004$ & soil & HASL300 & REG & $10045-97-3$ & Cs-137 & 37.9 & $\mathrm{PCl} / \mathrm{G}$ & G & 0.2 \\
\hline 383DH2300.5 & $6 / 15 / 2004$ & soil & EPA8270 & REG & 206-44-0 & Fluoranthene & 33 & UG/KG & $\mathrm{J}$ & 340 \\
\hline 383DH2300.5 & $6 / 15 / 2004$ & soil & HASL300 & REG & $13966-00-2$ & $\mathrm{~K}-40$ & 21 & $\mathrm{PCl} / \mathrm{G}$ & G & 1.7 \\
\hline 383DH2300.5 & $6 / 15 / 2004$ & soil & EPA6010 & REG & $7439-92-1$ & Lead & 30 & MG/KG & & 0.31 \\
\hline 383DH2300.5 & $6 / 15 / 2004$ & soil & EPA7470 & REG & $7439-97-6$ & Mercury & 0.035 & MG/KG & B & 0.1 \\
\hline 383DH2300.5 & $6 / 15 / 2004$ & soil & EPA8260 & REG & $75-09-2$ & Methylene Chloride & 0.91 & UG/KG & $\mathrm{J}, \mathrm{B}$ & 5.1 \\
\hline 383DH2300.5 & $6 / 15 / 2004$ & soil & HASL300 & REG & 15092-94-1 & $\mathrm{Pb}-212$ & 1.7 & $\mathrm{PCl} / \mathrm{G}$ & $\mathrm{J}$ & 0.38 \\
\hline 383DH2300.5 & $6 / 15 / 2004$ & soil & HASL300 & REG & 15067-28-4 & $\mathrm{Pb}-214$ & 1.19 & $\mathrm{PCl} / \mathrm{G}$ & $\mathrm{G}, \mathrm{J}$ & 0.49 \\
\hline 383DH2300.5 & $6 / 15 / 2004$ & soil & UGTAISOPU & REG & 13981-16-3 & Pu-238 & 0.238 & $\mathrm{PCl} / \mathrm{G}$ & & 0.023 \\
\hline 383DH2300.5 & $6 / 15 / 2004$ & soil & UGTAISOPU & REG & $15117-48-3$ & Pu-239 & 2.7 & $\mathrm{PCl} / \mathrm{G}$ & & 0.02 \\
\hline 383DH2300.5 & $6 / 15 / 2004$ & soil & EPA8270 & REG & $129-00-0$ & Pyrene & 42 & UG/KG & $\mathrm{J}$ & 340 \\
\hline 383DH2300.5 & $6 / 15 / 2004$ & soil & EPA6010 & REG & $7782-49-2$ & Selenium & 0.7 & MG/KG & & 0.51 \\
\hline 383DH2300.5 & $6 / 15 / 2004$ & soil & SR7500 & REG & 10098-97-2 & Sr-90 & 1.65 & $\mathrm{PCl} / \mathrm{G}$ & & 0.28 \\
\hline 383DH2300.5 & $6 / 15 / 2004$ & soil & HASL300 & REG & 14913-50-9 & TI-208 & 0.51 & $\mathrm{PCl} / \mathrm{G}$ & $\mathrm{G}$ & 0.2 \\
\hline 383DH2400.5 & $6 / 15 / 2004$ & soil & HASL300 & REG & 14331-83-0 & Ac-228 & 1.29 & $\mathrm{PCl} / \mathrm{G}$ & G & 0.48 \\
\hline 383DH2400.5 & $6 / 15 / 2004$ & soil & EPA6010 & REG & $7440-38-2$ & Arsenic & 3.3 & MG/KG & & 1 \\
\hline 383DH2400.5 & $6 / 15 / 2004$ & soil & EPA6010 & REG & $7440-39-3$ & Barium & 5400 & MG/KG & & 520 \\
\hline 383DH2400.5 & $6 / 15 / 2004$ & soil & EPA6010 & REG & $7440-41-7$ & Beryllium & 1.2 & MG/KG & & 0.52 \\
\hline 383DH2400.5 & $6 / 15 / 2004$ & soil & HASL300 & REG & $14733-03-0$ & Bi-214 & 0.92 & $\mathrm{PCl} / \mathrm{G}$ & $\mathrm{G}, \mathrm{J}$ & 0.39 \\
\hline 383DH2400.5 & $6 / 15 / 2004$ & soil & EPA6010 & REG & $7440-43-9$ & Cadmium & 0.21 & MG/KG & $B$ & 0.52 \\
\hline 383DH2400.5 & $6 / 15 / 2004$ & soil & EPA6010 & REG & $7440-47-3$ & Chromium & 4.6 & MG/KG & & 1 \\
\hline 383DH2400.5 & $6 / 15 / 2004$ & soil & HASL300 & REG & $10045-97-3$ & Cs-137 & 32 & $\mathrm{PCl} / \mathrm{G}$ & G & 0.2 \\
\hline 383DH2400.5 & $6 / 15 / 2004$ & soil & HASL300 & REG & 13966-00-2 & $\mathrm{K}-40$ & 20.1 & $\mathrm{PCl} / \mathrm{G}$ & G & 1.5 \\
\hline 383DH2400.5 & $6 / 15 / 2004$ & soil & EPA6010 & REG & $7439-92-1$ & Lead & 22 & MG/KG & & 0.31 \\
\hline 383DH2400.5 & $6 / 15 / 2004$ & soil & EPA7470 & REG & $7439-97-6$ & Mercury & 0.034 & MG/KG & $B$ & 0.1 \\
\hline
\end{tabular}




\begin{tabular}{|c|c|c|c|c|c|c|c|c|c|c|}
\hline SAMPLE \# & $\begin{array}{l}\text { SAMPLE } \\
\text { DATE }\end{array}$ & MATRIX & $\begin{array}{l}\text { USER TEST } \\
\text { PANEL }\end{array}$ & $\begin{array}{l}\text { SAMPLE } \\
\text { PURPOSE }\end{array}$ & CAS \# & PARAMETER & RESULT & UNITS & Q & $\begin{array}{l}\text { DETECT } \\
\text { LIMIT }\end{array}$ \\
\hline 383DH2400.5 & $6 / 15 / 2004$ & soil & HASL300 & REG & 15092-94-1 & $\mathrm{Pb}-212$ & 1.76 & $\mathrm{PCl} / \mathrm{G}$ & $\mathrm{J}$ & 0.34 \\
\hline 383DH2400.5 & $6 / 15 / 2004$ & soil & HASL300 & REG & 15067-28-4 & $\mathrm{Pb}-214$ & 1.29 & $\mathrm{PCl} / \mathrm{G}$ & $\mathrm{G}, \mathrm{J}$ & 0.62 \\
\hline 383DH2400.5 & $6 / 15 / 2004$ & soil & UGTAISOPU & REG & 13981-16-3 & Pu-238 & 0.316 & $\mathrm{PCl} / \mathrm{G}$ & & 0.01 \\
\hline 383DH2400.5 & $6 / 15 / 2004$ & soil & UGTAISOPU & REG & 15117-48-3 & Pu-239 & 2.34 & $\mathrm{PCl} / \mathrm{G}$ & & 0.01 \\
\hline 383DH2400.5 & $6 / 15 / 2004$ & soil & SR7500 & REG & 10098-97-2 & Sr-90 & 1.73 & $\mathrm{PCl} / \mathrm{G}$ & & 0.26 \\
\hline 383DH2400.5 & $6 / 15 / 2004$ & soil & HASL300 & REG & 14913-50-9 & TI-208 & 0.3 & $\mathrm{PCl} / \mathrm{G}$ & G & 0.17 \\
\hline 383DH2500.5 & $6 / 15 / 2004$ & soil & HASL300 & REG & 14331-83-0 & Ac-228 & 1.62 & $\mathrm{PCl} / \mathrm{G}$ & G & 0.61 \\
\hline 383DH2500.5 & $6 / 15 / 2004$ & soil & EPA6010 & REG & 7440-38-2 & Arsenic & 3.3 & MG/KG & & 1 \\
\hline 383DH2500.5 & $6 / 15 / 2004$ & soil & EPA6010 & REG & 7440-39-3 & Barium & 5100 & MG/KG & & 520 \\
\hline 383DH2500.5 & $6 / 15 / 2004$ & soil & EPA6010 & REG & $7440-41-7$ & Beryllium & 1.5 & MG/KG & & 0.52 \\
\hline 383DH2500.5 & $6 / 15 / 2004$ & soil & HASL300 & REG & 14733-03-0 & Bi-214 & 1.19 & $\mathrm{PCl} / \mathrm{G}$ & $\mathrm{G}, \mathrm{J}$ & 0.48 \\
\hline 383DH2500.5 & $6 / 15 / 2004$ & soil & EPA6010 & REG & $7440-43-9$ & Cadmium & 0.17 & MG/KG & $B$ & 0.52 \\
\hline 383DH2500.5 & $6 / 15 / 2004$ & soil & EPA6010 & REG & $7440-47-3$ & Chromium & 4.8 & MG/KG & & 1 \\
\hline 383DH2500.5 & $6 / 15 / 2004$ & soil & HASL300 & REG & $10045-97-3$ & Cs-137 & 55.6 & $\mathrm{PCl} / \mathrm{G}$ & G & 0.3 \\
\hline 383DH2500.5 & $6 / 15 / 2004$ & soil & HASL300 & REG & 13966-00-2 & $\mathrm{K}-40$ & 26.3 & $\mathrm{PCl} / \mathrm{G}$ & G & 1.8 \\
\hline 383DH2500.5 & $6 / 15 / 2004$ & soil & EPA6010 & REG & $7439-92-1$ & Lead & 23 & MG/KG & & 0.31 \\
\hline 383DH2500.5 & $6 / 15 / 2004$ & soil & EPA7470 & REG & 7439-97-6 & Mercury & 0.052 & MG/KG & $B$ & 0.1 \\
\hline 383DH2500.5 & $6 / 15 / 2004$ & soil & EPA8260 & REG & $75-09-2$ & Methylene Chloride & 0.9 & UG/KG & J,B & 5.2 \\
\hline 383DH2500.5 & $6 / 15 / 2004$ & soil & HASL300 & REG & 15092-94-1 & $\mathrm{Pb}-212$ & 1.78 & $\mathrm{PCl} / \mathrm{G}$ & $\mathrm{J}$ & 0.44 \\
\hline 383DH2500.5 & $6 / 15 / 2004$ & soil & HASL300 & REG & 15067-28-4 & $\mathrm{Pb}-214$ & 1.28 & $\mathrm{PCl} / \mathrm{G}$ & $\mathrm{G}, \mathrm{J}$ & 0.73 \\
\hline 383DH2500.5 & $6 / 15 / 2004$ & soil & UGTAISOPU & REG & 13981-16-3 & Pu-238 & 0.265 & $\mathrm{PCl} / \mathrm{G}$ & & 0.021 \\
\hline 383DH2500.5 & $6 / 15 / 2004$ & soil & UGTAISOPU & REG & 15117-48-3 & Pu-239 & 3.1 & $\mathrm{PCl} / \mathrm{G}$ & & 0.02 \\
\hline 383DH2500.5 & $6 / 15 / 2004$ & soil & EPA6010 & REG & $7782-49-2$ & Selenium & 0.41 & MG/KG & $B$ & 0.52 \\
\hline 383DH2500.5 & $6 / 15 / 2004$ & soil & SR7500 & REG & 10098-97-2 & Sr-90 & 1.09 & $\mathrm{PCl} / \mathrm{G}$ & & 0.25 \\
\hline 383DH2500.5 & $6 / 15 / 2004$ & soil & HASL300 & REG & 14913-50-9 & TI-208 & 0.57 & $\mathrm{PCl} / \mathrm{G}$ & G & 0.3 \\
\hline 383DH2600.5 & $6 / 15 / 2004$ & soil & HASL300 & REG & 14331-83-0 & Ac-228 & 1.52 & $\mathrm{PCl} / \mathrm{G}$ & G & 0.61 \\
\hline 383DH2600.5 & $6 / 15 / 2004$ & soil & EPA6010 & REG & $7440-38-2$ & Arsenic & 3.4 & MG/KG & & 1 \\
\hline 383DH2600.5 & $6 / 15 / 2004$ & soil & EPA6010 & REG & $7440-39-3$ & Barium & 6100 & MG/KG & & 520 \\
\hline 383DH2600.5 & $6 / 15 / 2004$ & soil & EPA8270 & REG & $56-55-3$ & Benzo(A)Anthracene & 66 & UG/KG & $\mathrm{J}$ & 350 \\
\hline 383DH2600.5 & $6 / 15 / 2004$ & soil & EPA8270 & REG & $50-32-8$ & Benzo(A)Pyrene & 64 & UG/KG & $\mathrm{J}$ & 350 \\
\hline 383DH2600.5 & $6 / 15 / 2004$ & soil & EPA8270 & REG & $205-99-2$ & Benzo(B)Fluoranthene & 62 & UG/KG & $\mathrm{J}$ & 350 \\
\hline 383DH2600.5 & $6 / 15 / 2004$ & soil & EPA8270 & REG & 207-08-9 & Benzo(K)Fluoranthene & 57 & UG/KG & $\mathrm{J}$ & 350 \\
\hline 383DH2600.5 & $6 / 15 / 2004$ & soil & EPA6010 & REG & $7440-41-7$ & Beryllium & 1.5 & MG/KG & & 0.52 \\
\hline 383DH2600.5 & $6 / 15 / 2004$ & soil & HASL300 & REG & 14733-03-0 & Bi-214 & 1.13 & $\mathrm{PCl} / \mathrm{G}$ & $\mathrm{G}, \mathrm{J}$ & 0.47 \\
\hline 383DH2600.5 & $6 / 15 / 2004$ & soil & EPA6010 & REG & $7440-43-9$ & Cadmium & 0.25 & MG/KG & $B$ & 0.52 \\
\hline
\end{tabular}




\begin{tabular}{|c|c|c|c|c|c|c|c|c|c|c|}
\hline SAMPLE \# & $\begin{array}{l}\text { SAMPLE } \\
\text { DATE }\end{array}$ & MATRIX & $\begin{array}{l}\text { USER TEST } \\
\text { PANEL }\end{array}$ & $\begin{array}{l}\text { SAMPLE } \\
\text { PURPOSE }\end{array}$ & CAS \# & PARAMETER & RESULT & UNITS & Q & $\begin{array}{l}\text { DETECT } \\
\text { LIMIT }\end{array}$ \\
\hline 383DH2600.5 & $6 / 15 / 2004$ & soil & EPA6010 & REG & $7440-47-3$ & Chromium & 5 & MG/KG & & 1 \\
\hline 383DH2600.5 & $6 / 15 / 2004$ & soil & EPA8270 & REG & $218-01-9$ & Chrysene & 82 & UG/KG & $\mathrm{J}$ & 350 \\
\hline 383DH2600.5 & $6 / 15 / 2004$ & soil & HASL300 & REG & 10045-97-3 & Cs-137 & 28.4 & $\mathrm{PCl} / \mathrm{G}$ & G & 0.2 \\
\hline 383DH2600.5 & $6 / 15 / 2004$ & soil & EPA8015 & REG & $68334-30-5$ & Diesel Range Organics & 2.5 & MG/KG & $\mathrm{J}$ & 5.2 \\
\hline 383DH2600.5 & $6 / 15 / 2004$ & soil & EPA8270 & REG & $206-44-0$ & Fluoranthene & 120 & UG/KG & $\mathrm{J}$ & 350 \\
\hline 383DH2600.5 & $6 / 15 / 2004$ & soil & HASL300 & REG & 13966-00-2 & $\mathrm{K}-40$ & 24.8 & $\mathrm{PCl} / \mathrm{G}$ & G & 1.5 \\
\hline 383DH2600.5 & $6 / 15 / 2004$ & soil & EPA6010 & REG & $7439-92-1$ & Lead & 28 & MG/KG & & 0.31 \\
\hline 383DH2600.5 & $6 / 15 / 2004$ & soil & EPA7470 & REG & 7439-97-6 & Mercury & 0.038 & MG/KG & $B$ & 0.1 \\
\hline 383DH2600.5 & $6 / 15 / 2004$ & soil & EPA8260 & REG & $75-09-2$ & Methylene Chloride & 0.99 & UG/KG & J,B & 5.2 \\
\hline 383DH2600.5 & $6 / 15 / 2004$ & soil & HASL300 & REG & 15092-94-1 & $\mathrm{Pb}-212$ & 2.01 & $\mathrm{PCl} / \mathrm{G}$ & $\mathrm{J}$ & 0.34 \\
\hline 383DH2600.5 & $6 / 15 / 2004$ & soil & HASL300 & REG & 15067-28-4 & $\mathrm{Pb}-214$ & 1.4 & $\mathrm{PCl} / \mathrm{G}$ & $\mathrm{G}, \mathrm{J}$ & 0.49 \\
\hline 383DH2600.5 & $6 / 15 / 2004$ & soil & EPA8270 & REG & $85-01-8$ & Phenanthrene & 49 & UG/KG & $\mathrm{J}$ & 350 \\
\hline 383DH2600.5 & $6 / 15 / 2004$ & soil & UGTAISOPU & REG & 13981-16-3 & Pu-238 & 0.188 & $\mathrm{PCl} / \mathrm{G}$ & & 0.021 \\
\hline 383DH2600.5 & $6 / 15 / 2004$ & soil & UGTAISOPU & REG & 15117-48-3 & Pu-239 & 1.82 & $\mathrm{PCl} / \mathrm{G}$ & & 0.01 \\
\hline 383DH2600.5 & $6 / 15 / 2004$ & soil & EPA8270 & REG & $129-00-0$ & Pyrene & 190 & UG/KG & $\mathrm{J}$ & 350 \\
\hline 383DH2600.5 & $6 / 15 / 2004$ & soil & SR7500 & REG & 10098-97-2 & Sr-90 & 0.93 & $\mathrm{PCl} / \mathrm{G}$ & & 0.25 \\
\hline 383DH2600.5 & $6 / 15 / 2004$ & soil & HASL300 & REG & 14913-50-9 & TI-208 & 0.46 & $\mathrm{PCl} / \mathrm{G}$ & G & 0.19 \\
\hline 383DH2700.5 & $6 / 16 / 2004$ & soil & HASL300 & REG & 14331-83-0 & Ac-228 & 1.66 & $\mathrm{PCl} / \mathrm{G}$ & G & 0.67 \\
\hline 383DH2700.5 & $6 / 16 / 2004$ & soil & EPA6010 & REG & $7440-38-2$ & Arsenic & 3.4 & MG/KG & & 1 \\
\hline 383DH2700.5 & $6 / 16 / 2004$ & soil & EPA6010 & REG & $7440-39-3$ & Barium & 5600 & MG/KG & & 520 \\
\hline 383DH2700.5 & $6 / 16 / 2004$ & soil & EPA6010 & REG & $7440-41-7$ & Beryllium & 1.4 & MG/KG & & 0.52 \\
\hline 383DH2700.5 & $6 / 16 / 2004$ & soil & HASL300 & REG & 14733-03-0 & $\mathrm{Bi}-214$ & 1.43 & $\mathrm{PCl} / \mathrm{G}$ & $\mathrm{G}, \mathrm{J}$ & 0.36 \\
\hline 383DH2700.5 & $6 / 16 / 2004$ & soil & EPA8270 & REG & $117-81-7$ & Bis(2-Ethylhexyl)Phthalate & 100 & UG/KG & $\mathrm{J}$ & 350 \\
\hline 383DH2700.5 & $6 / 16 / 2004$ & soil & EPA6010 & REG & $7440-43-9$ & Cadmium & 0.2 & MG/KG & B & 0.52 \\
\hline 383DH2700.5 & $6 / 16 / 2004$ & soil & EPA6010 & REG & $7440-47-3$ & Chromium & 5.3 & MG/KG & & 1 \\
\hline 383DH2700.5 & $6 / 16 / 2004$ & soil & HASL300 & REG & 10045-97-3 & Cs-137 & 41.7 & $\mathrm{PCl} / \mathrm{G}$ & G & 0.2 \\
\hline 383DH2700.5 & $6 / 16 / 2004$ & soil & EPA8015 & REG & 68334-30-5 & Diesel Range Organics & 1.8 & MG/KG & $\mathrm{J}$ & 5.2 \\
\hline 383DH2700.5 & $6 / 16 / 2004$ & soil & EPA8260 & REG & $87-68-3$ & Hexachlorobutadiene & 1.6 & UG/KG & $\mathrm{J}$ & 5.2 \\
\hline 383DH2700.5 & $6 / 16 / 2004$ & soil & HASL300 & REG & 13966-00-2 & $\mathrm{K}-40$ & 20.9 & $\mathrm{PCl} / \mathrm{G}$ & G & 1.7 \\
\hline 383DH2700.5 & $6 / 16 / 2004$ & soil & EPA6010 & REG & $7439-92-1$ & Lead & 25 & MG/KG & & 0.31 \\
\hline 383DH2700.5 & $6 / 16 / 2004$ & soil & EPA7470 & REG & 7439-97-6 & Mercury & 0.043 & MG/KG & B & 0.1 \\
\hline 383DH2700.5 & $6 / 16 / 2004$ & soil & EPA8260 & REG & $75-09-2$ & Methylene Chloride & 1.1 & UG/KG & J,B & 5.2 \\
\hline 383DH2700.5 & $6 / 16 / 2004$ & soil & HASL300 & REG & 15092-94-1 & $\mathrm{Pb}-212$ & 1.58 & $\mathrm{PCl} / \mathrm{G}$ & $\mathrm{J}$ & 0.38 \\
\hline 383DH2700.5 & $6 / 16 / 2004$ & soil & HASL300 & REG & 15067-28-4 & $\mathrm{Pb}-214$ & 1.15 & $\mathrm{PCl} / \mathrm{G}$ & $\mathrm{G}, \mathrm{J}$ & 0.48 \\
\hline 383DH2700.5 & $6 / 16 / 2004$ & soil & UGTAISOPU & REG & 13981-16-3 & Pu-238 & 0.271 & $\mathrm{PCl} / \mathrm{G}$ & & 0.019 \\
\hline
\end{tabular}




\begin{tabular}{|c|c|c|c|c|c|c|c|c|c|c|}
\hline SAMPLE \# & $\begin{array}{l}\text { SAMPLE } \\
\text { DATE }\end{array}$ & MATRIX & $\begin{array}{l}\text { USER TEST } \\
\text { PANEL }\end{array}$ & $\begin{array}{l}\text { SAMPLE } \\
\text { PURPOSE }\end{array}$ & CAS \# & PARAMETER & RESULT & UNITS & Q & $\begin{array}{l}\text { DETECT } \\
\text { LIMIT }\end{array}$ \\
\hline 383DH2700.5 & $6 / 16 / 2004$ & soil & UGTAISOPU & REG & $15117-48-3$ & Pu-239 & 3.31 & $\mathrm{PCl} / \mathrm{G}$ & & 0.02 \\
\hline 383DH2700.5 & $6 / 16 / 2004$ & soil & EPA6010 & REG & $7782-49-2$ & Selenium & 0.43 & MG/KG & $B$ & 0.52 \\
\hline 383DH2700.5 & $6 / 16 / 2004$ & soil & SR7500 & REG & 10098-97-2 & Sr-90 & 1.55 & $\mathrm{PCl} / \mathrm{G}$ & & 0.25 \\
\hline 383DH2700.5 & $6 / 16 / 2004$ & soil & HASL300 & REG & 14913-50-9 & TI-208 & 0.51 & $\mathrm{PCl} / \mathrm{G}$ & G & 0.2 \\
\hline 383DH2800.5 & $6 / 16 / 2004$ & soil & HASL300 & REG & 14331-83-0 & Ac-228 & 1.72 & $\mathrm{PCl} / \mathrm{G}$ & G & 0.62 \\
\hline 383DH2800.5 & $6 / 16 / 2004$ & soil & EPA6010 & REG & $7440-38-2$ & Arsenic & 2.8 & MG/KG & & 1 \\
\hline 383DH2800.5 & $6 / 16 / 2004$ & soil & EPA6010 & REG & $7440-39-3$ & Barium & 5300 & MG/KG & & 520 \\
\hline 383DH2800.5 & $6 / 16 / 2004$ & soil & EPA6010 & REG & 7440-41-7 & Beryllium & 1.7 & MG/KG & & 0.52 \\
\hline 383DH2800.5 & $6 / 16 / 2004$ & soil & HASL300 & REG & 14733-03-0 & $\mathrm{Bi}-214$ & 1.26 & $\mathrm{PCl} / \mathrm{G}$ & $\mathrm{G}, \mathrm{J}$ & 0.36 \\
\hline 383DH2800.5 & $6 / 16 / 2004$ & soil & EPA6010 & REG & $7440-43-9$ & Cadmium & 0.16 & MG/KG & $B$ & 0.52 \\
\hline 383DH2800.5 & $6 / 16 / 2004$ & soil & EPA6010 & REG & $7440-47-3$ & Chromium & 4 & MG/KG & & 1 \\
\hline 383DH2800.5 & $6 / 16 / 2004$ & soil & HASL300 & REG & 10045-97-3 & Cs-137 & 14 & $\mathrm{PCl} / \mathrm{G}$ & G & 0.2 \\
\hline 383DH2800.5 & $6 / 16 / 2004$ & soil & HASL300 & REG & 13966-00-2 & $\mathrm{K}-40$ & 27.7 & $\mathrm{PCl} / \mathrm{G}$ & G & 1.8 \\
\hline 383DH2800.5 & $6 / 16 / 2004$ & soil & EPA6010 & REG & $7439-92-1$ & Lead & 27 & MG/KG & & 0.31 \\
\hline 383DH2800.5 & $6 / 16 / 2004$ & soil & EPA7470 & REG & 7439-97-6 & Mercury & 0.023 & MG/KG & $B$ & 0.1 \\
\hline 383DH2800.5 & $6 / 16 / 2004$ & soil & EPA8260 & REG & $75-09-2$ & Methylene Chloride & 1.4 & UG/KG & $\mathrm{J}, \mathrm{B}$ & 5.2 \\
\hline 383DH2800.5 & $6 / 16 / 2004$ & soil & HASL300 & REG & 15092-94-1 & $\mathrm{Pb}-212$ & 2.19 & $\mathrm{PCl} / \mathrm{G}$ & $\mathrm{J}$ & 0.28 \\
\hline 383DH2800.5 & $6 / 16 / 2004$ & soil & HASL300 & REG & 15067-28-4 & $\mathrm{Pb}-214$ & 1.4 & $\mathrm{PCl} / \mathrm{G}$ & $\mathrm{G}, \mathrm{J}$ & 0.47 \\
\hline 383DH2800.5 & $6 / 16 / 2004$ & soil & UGTAISOPU & REG & 13981-16-3 & Pu-238 & 0.068 & $\mathrm{PCl} / \mathrm{G}$ & & 0.021 \\
\hline 383DH2800.5 & $6 / 16 / 2004$ & soil & UGTAISOPU & REG & $15117-48-3$ & Pu-239 & 0.93 & $\mathrm{PCl} / \mathrm{G}$ & & 0.01 \\
\hline 383DH2800.5 & $6 / 16 / 2004$ & soil & SR7500 & REG & 10098-97-2 & Sr-90 & 0.45 & $\mathrm{PCl} / \mathrm{G}$ & $\mathrm{Y} 1, \mathrm{LT}$ & 0.22 \\
\hline 383DH2800.5 & $6 / 16 / 2004$ & soil & HASL300 & REG & 14913-50-9 & TI-208 & 0.67 & $\mathrm{PCl} / \mathrm{G}$ & G & 0.18 \\
\hline 383DH2900.5 & $6 / 16 / 2004$ & soil & HASL300 & REG & $14331-83-0$ & Ac-228 & 1.59 & $\mathrm{PCl} / \mathrm{G}$ & G & 0.5 \\
\hline 383DH2900.5 & $6 / 16 / 2004$ & soil & EPA8260 & REG & $67-64-1$ & Acetone & 7.1 & UG/KG & $\mathrm{J}$ & 21 \\
\hline 383DH2900.5 & $6 / 16 / 2004$ & soil & EPA6010 & REG & $7440-38-2$ & Arsenic & 3.5 & MG/KG & & 1 \\
\hline 383DH2900.5 & $6 / 16 / 2004$ & soil & EPA6010 & REG & 7440-39-3 & Barium & 5900 & MG/KG & & 520 \\
\hline 383DH2900.5 & $6 / 16 / 2004$ & soil & EPA6010 & REG & $7440-41-7$ & Beryllium & 1.3 & MG/KG & & 0.52 \\
\hline 383DH2900.5 & $6 / 16 / 2004$ & soil & HASL300 & REG & 14733-03-0 & Bi-214 & 1.12 & $\mathrm{PCl} / \mathrm{G}$ & $\mathrm{G}, \mathrm{J}$ & 0.47 \\
\hline 383DH2900.5 & $6 / 16 / 2004$ & soil & EPA6010 & REG & $7440-43-9$ & Cadmium & 0.2 & MG/KG & $B$ & 0.52 \\
\hline 383DH2900.5 & $6 / 16 / 2004$ & soil & EPA6010 & REG & $7440-47-3$ & Chromium & 5.6 & MG/KG & & 1 \\
\hline 383DH2900.5 & $6 / 16 / 2004$ & soil & HASL300 & REG & $10045-97-3$ & Cs-137 & 33.1 & $\mathrm{PCl} / \mathrm{G}$ & G & 0.2 \\
\hline 383DH2900.5 & $6 / 16 / 2004$ & soil & HASL300 & REG & $13966-00-2$ & $\mathrm{~K}-40$ & 22.1 & $\mathrm{PCl} / \mathrm{G}$ & G & 2.1 \\
\hline 383DH2900.5 & $6 / 16 / 2004$ & soil & EPA6010 & REG & $7439-92-1$ & Lead & 29 & MG/KG & & 0.31 \\
\hline 383DH2900.5 & $6 / 16 / 2004$ & soil & EPA7470 & REG & 7439-97-6 & Mercury & 0.042 & MG/KG & $B$ & 0.1 \\
\hline 383DH2900.5 & $6 / 16 / 2004$ & soil & EPA8260 & REG & $75-09-2$ & Methylene Chloride & 1.4 & UG/KG & $\mathrm{J}, \mathrm{B}$ & 5.2 \\
\hline
\end{tabular}




\begin{tabular}{|c|c|c|c|c|c|c|c|c|c|c|}
\hline SAMPLE \# & $\begin{array}{l}\text { SAMPLE } \\
\text { DATE }\end{array}$ & MATRIX & $\begin{array}{l}\text { USER TEST } \\
\text { PANEL }\end{array}$ & $\begin{array}{l}\text { SAMPLE } \\
\text { PURPOSE }\end{array}$ & CAS \# & PARAMETER & RESULT & UNITS & Q & $\begin{array}{l}\text { DETECT } \\
\text { LIMIT }\end{array}$ \\
\hline 383DH2900.5 & $6 / 16 / 2004$ & soil & HASL300 & REG & 15092-94-1 & $\mathrm{Pb}-212$ & 1.75 & $\mathrm{PCl} / \mathrm{G}$ & $\mathrm{J}$ & 0.37 \\
\hline 383DH2900.5 & $6 / 16 / 2004$ & soil & HASL300 & REG & 15067-28-4 & $\mathrm{Pb}-214$ & 1.06 & $\mathrm{PCl} / \mathrm{G}$ & G,J & 0.44 \\
\hline 383DH2900.5 & $6 / 16 / 2004$ & soil & UGTAISOPU & REG & 13981-16-3 & Pu-238 & 0.237 & $\mathrm{PCl} / \mathrm{G}$ & & 0.017 \\
\hline 383DH2900.5 & $6 / 16 / 2004$ & soil & UGTAISOPU & REG & 15117-48-3 & Pu-239 & 2.14 & $\mathrm{PCl} / \mathrm{G}$ & & 0.02 \\
\hline 383DH2900.5 & $6 / 16 / 2004$ & soil & SR7500 & REG & 10098-97-2 & Sr-90 & 0.76 & $\mathrm{PCl} / \mathrm{G}$ & & 0.25 \\
\hline 383DH2900.5 & $6 / 16 / 2004$ & soil & HASL300 & REG & 14913-50-9 & TI-208 & 0.47 & $\mathrm{PCl} / \mathrm{G}$ & $\mathrm{G}$ & 0.2 \\
\hline 383DH3000.5 & $6 / 16 / 2004$ & soil & HASL300 & REG & 14331-83-0 & Ac-228 & 1.41 & $\mathrm{PCl} / \mathrm{G}$ & $\mathrm{G}$ & 0.56 \\
\hline 383DH3000.5 & $6 / 16 / 2004$ & soil & EPA6010 & REG & $7440-38-2$ & Arsenic & 2.4 & MG/KG & & 1 \\
\hline 383DH3000.5 & $6 / 16 / 2004$ & soil & EPA6010 & REG & $7440-39-3$ & Barium & 5100 & MG/KG & & 510 \\
\hline 383DH3000.5 & $6 / 16 / 2004$ & soil & EPA6010 & REG & $7440-41-7$ & Beryllium & 1.2 & MG/KG & & 0.51 \\
\hline 383DH3000.5 & $6 / 16 / 2004$ & soil & HASL300 & REG & 14733-03-0 & Bi-214 & 0.89 & $\mathrm{PCl} / \mathrm{G}$ & $\mathrm{G}, \mathrm{J}$ & 0.27 \\
\hline 383DH3000.5 & $6 / 16 / 2004$ & soil & EPA6010 & REG & $7440-43-9$ & Cadmium & 0.21 & MG/KG & B & 0.51 \\
\hline 383DH3000.5 & $6 / 16 / 2004$ & soil & EPA6010 & REG & $7440-47-3$ & Chromium & 5.8 & MG/KG & & 1 \\
\hline 383DH3000.5 & $6 / 16 / 2004$ & soil & HASL300 & REG & $10045-97-3$ & Cs-137 & 7.67 & $\mathrm{PCl} / \mathrm{G}$ & G & 0.13 \\
\hline 383DH3000.5 & $6 / 16 / 2004$ & soil & HASL300 & REG & 13966-00-2 & $\mathrm{K}-40$ & 25.6 & $\mathrm{PCl} / \mathrm{G}$ & $\mathrm{G}$ & 1.7 \\
\hline 383DH3000.5 & $6 / 16 / 2004$ & soil & EPA6010 & REG & $7439-92-1$ & Lead & 20 & MG/KG & & 0.31 \\
\hline 383DH3000.5 & $6 / 16 / 2004$ & soil & EPA7470 & REG & $7439-97-6$ & Mercury & 0.015 & MG/KG & B & 0.1 \\
\hline 383DH3000.5 & $6 / 16 / 2004$ & soil & EPA8260 & REG & $75-09-2$ & Methylene Chloride & 1.4 & UG/KG & $J, B$ & 5.1 \\
\hline 383DH3000.5 & $6 / 16 / 2004$ & soil & HASL300 & REG & 15092-94-1 & $\mathrm{Pb}-212$ & 1.81 & $\mathrm{PCl} / \mathrm{G}$ & $\mathrm{J}$ & 0.25 \\
\hline 383DH3000.5 & $6 / 16 / 2004$ & soil & HASL300 & REG & $15067-28-4$ & $\mathrm{~Pb}-214$ & 1.22 & $\mathrm{PCl} / \mathrm{G}$ & $\mathrm{G}, \mathrm{J}$ & 0.32 \\
\hline 383DH3000.5 & $6 / 16 / 2004$ & soil & UGTAISOPU & REG & 13981-16-3 & Pu-238 & 0.042 & $\mathrm{PCl} / \mathrm{G}$ & LT & 0.009 \\
\hline 383DH3000.5 & $6 / 16 / 2004$ & soil & UGTAISOPU & REG & 15117-48-3 & Pu-239 & 0.286 & $\mathrm{PCl} / \mathrm{G}$ & & 0.022 \\
\hline 383DH3000.5 & $6 / 16 / 2004$ & soil & HASL300 & REG & $14913-50-9$ & TI-208 & 0.55 & $\mathrm{PCl} / \mathrm{G}$ & G & 0.15 \\
\hline 383DH3100.5 & $6 / 16 / 2004$ & soil & HASL300 & REG & 14331-83-0 & Ac-228 & 0.91 & $\mathrm{PCl} / \mathrm{G}$ & $\mathrm{G}$ & 0.39 \\
\hline 383DH3100.5 & $6 / 16 / 2004$ & soil & EPA6010 & REG & $7440-38-2$ & Arsenic & 5.3 & MG/KG & & 1 \\
\hline 383DH3100.5 & $6 / 16 / 2004$ & soil & EPA6010 & REG & $7440-39-3$ & Barium & 6500 & MG/KG & & 510 \\
\hline 383DH3100.5 & $6 / 16 / 2004$ & soil & EPA6010 & REG & $7440-41-7$ & Beryllium & 0.77 & MG/KG & & 0.51 \\
\hline 383DH3100.5 & $6 / 16 / 2004$ & soil & HASL300 & REG & 14733-03-0 & Bi-214 & 0.84 & $\mathrm{PCl} / \mathrm{G}$ & G,J & 0.28 \\
\hline 383DH3100.5 & $6 / 16 / 2004$ & soil & EPA6010 & REG & $7440-43-9$ & Cadmium & 0.22 & MG/KG & B & 0.51 \\
\hline 383DH3100.5 & $6 / 16 / 2004$ & soil & EPA6010 & REG & $7440-47-3$ & Chromium & 8.4 & MG/KG & & 1 \\
\hline 383DH3100.5 & $6 / 16 / 2004$ & soil & HASL300 & REG & 10045-97-3 & Cs-137 & 8.4 & $\mathrm{PCl} / \mathrm{G}$ & G & 0.1 \\
\hline 383DH3100.5 & $6 / 16 / 2004$ & soil & HASL300 & REG & $13966-00-2$ & $\mathrm{~K}-40$ & 17.2 & $\mathrm{PCl} / \mathrm{G}$ & G & 1.3 \\
\hline 383DH3100.5 & $6 / 16 / 2004$ & soil & EPA6010 & REG & $7439-92-1$ & Lead & 18 & MG/KG & & 0.61 \\
\hline 383DH3100.5 & $6 / 16 / 2004$ & soil & EPA7470 & REG & 7439-97-6 & Mercury & 0.021 & MG/KG & B & 0.1 \\
\hline 383DH3100.5 & $6 / 16 / 2004$ & soil & EPA8260 & REG & $75-09-2$ & Methylene Chloride & 1.2 & UG/KG & $\mathrm{J}, \mathrm{B}$ & 5.1 \\
\hline
\end{tabular}




\begin{tabular}{|c|c|c|c|c|c|c|c|c|c|c|}
\hline SAMPLE \# & $\begin{array}{l}\text { SAMPLE } \\
\text { DATE }\end{array}$ & MATRIX & $\begin{array}{l}\text { USER TEST } \\
\text { PANEL }\end{array}$ & $\begin{array}{l}\text { SAMPLE } \\
\text { PURPOSE }\end{array}$ & CAS \# & PARAMETER & RESULT & UNITS & Q & $\begin{array}{l}\text { DETECT } \\
\text { LIMIT }\end{array}$ \\
\hline 383DH3100.5 & $6 / 16 / 2004$ & soil & HASL300 & REG & 15092-94-1 & $\mathrm{Pb}-212$ & 0.74 & $\mathrm{PCl} / \mathrm{G}$ & $\mathrm{J}$ & 0.26 \\
\hline 383DH3100.5 & $6 / 16 / 2004$ & soil & HASL300 & REG & 15067-28-4 & $\mathrm{Pb}-214$ & 0.87 & $\mathrm{PCl} / \mathrm{G}$ & $\mathrm{G}, \mathrm{J}$ & 0.36 \\
\hline 383DH3100.5 & $6 / 16 / 2004$ & soil & UGTAISOPU & REG & 13981-16-3 & Pu-238 & 0.08 & $\mathrm{PCl} / \mathrm{G}$ & & 0.018 \\
\hline 383DH3100.5 & $6 / 16 / 2004$ & soil & UGTAISOPU & REG & 15117-48-3 & Pu-239 & 0.51 & $\mathrm{PCl} / \mathrm{G}$ & & 0.01 \\
\hline 383DH3100.5 & $6 / 16 / 2004$ & soil & EPA6010 & REG & 7782-49-2 & Selenium & 0.37 & MG/KG & $B$ & 0.51 \\
\hline 383DH3200.5 & $6 / 16 / 2004$ & soil & HASL300 & REG & 14331-83-0 & Ac-228 & 0.7 & $\mathrm{PCl} / \mathrm{G}$ & G & 0.38 \\
\hline 383DH3200.5 & $6 / 16 / 2004$ & soil & EPA6010 & REG & $7440-38-2$ & Arsenic & 4.6 & MG/KG & & 1 \\
\hline 383DH3200.5 & $6 / 16 / 2004$ & soil & EPA6010 & REG & 7440-39-3 & Barium & 6600 & MG/KG & & 510 \\
\hline 383DH3200.5 & $6 / 16 / 2004$ & soil & EPA6010 & REG & $7440-41-7$ & Beryllium & 0.83 & MG/KG & & 0.51 \\
\hline 383DH3200.5 & $6 / 16 / 2004$ & soil & HASL300 & REG & 14733-03-0 & $\mathrm{Bi}-214$ & 1 & $\mathrm{PCl} / \mathrm{G}$ & $\mathrm{G}, \mathrm{J}$ & 0.3 \\
\hline 383DH3200.5 & $6 / 16 / 2004$ & soil & EPA6010 & REG & $7440-43-9$ & Cadmium & 0.23 & MG/KG & B & 0.51 \\
\hline 383DH3200.5 & $6 / 16 / 2004$ & soil & EPA6010 & REG & 7440-47-3 & Chromium & 9.2 & MG/KG & & 1 \\
\hline 383DH3200.5 & $6 / 16 / 2004$ & soil & HASL300 & REG & 10045-97-3 & Cs-137 & 12.7 & $\mathrm{PCl} / \mathrm{G}$ & G & 0.1 \\
\hline 383DH3200.5 & $6 / 16 / 2004$ & soil & HASL300 & REG & 13966-00-2 & $\mathrm{K}-40$ & 17 & $\mathrm{PCl} / \mathrm{G}$ & G & 1.4 \\
\hline 383DH3200.5 & $6 / 16 / 2004$ & soil & EPA6010 & REG & $7439-92-1$ & Lead & 21 & MG/KG & & 0.61 \\
\hline 383DH3200.5 & $6 / 16 / 2004$ & soil & EPA7470 & REG & 7439-97-6 & Mercury & 0.021 & MG/KG & $B$ & 0.1 \\
\hline 383DH3200.5 & $6 / 16 / 2004$ & soil & EPA8260 & REG & $75-09-2$ & Methylene Chloride & 0.82 & UG/KG & J,B & 5.1 \\
\hline 383DH3200.5 & $6 / 16 / 2004$ & soil & HASL300 & REG & 15092-94-1 & $\mathrm{Pb}-212$ & 0.85 & $\mathrm{PCl} / \mathrm{G}$ & $\mathrm{J}$ & 0.27 \\
\hline 383DH3200.5 & $6 / 16 / 2004$ & soil & HASL300 & REG & 15067-28-4 & $\mathrm{Pb}-214$ & 0.8 & $\mathrm{PCl} / \mathrm{G}$ & $\mathrm{G}, \mathrm{J}$ & 0.46 \\
\hline 383DH3200.5 & $6 / 16 / 2004$ & soil & UGTAISOPU & REG & 13981-16-3 & Pu-238 & 0.058 & $\mathrm{PCl} / \mathrm{G}$ & & 0.021 \\
\hline 383DH3200.5 & $6 / 16 / 2004$ & soil & UGTAISOPU & REG & 15117-48-3 & Pu-239 & 0.66 & $\mathrm{PCl} / \mathrm{G}$ & & 0.02 \\
\hline 383DH3200.5 & $6 / 16 / 2004$ & soil & EPA6010 & REG & $7782-49-2$ & Selenium & 0.29 & MG/KG & $B$ & 0.51 \\
\hline 383DH3200.5 & $6 / 16 / 2004$ & soil & HASL300 & REG & 14913-50-9 & TI-208 & 0.41 & $\mathrm{PCl} / \mathrm{G}$ & G & 0.18 \\
\hline 383DH3300.5 & $6 / 16 / 2004$ & soil & HASL300 & REG & 14331-83-0 & Ac-228 & 1.9 & $\mathrm{PCl} / \mathrm{G}$ & G & 0.66 \\
\hline 383DH3300.5 & $6 / 16 / 2004$ & soil & EPA6010 & REG & $7440-38-2$ & Arsenic & 3.9 & MG/KG & & 1 \\
\hline 383DH3300.5 & $6 / 16 / 2004$ & soil & EPA6010 & REG & $7440-39-3$ & Barium & 5900 & MG/KG & & 510 \\
\hline 383DH3300.5 & $6 / 16 / 2004$ & soil & EPA6010 & REG & $7440-41-7$ & Beryllium & 1.4 & MG/KG & & 0.51 \\
\hline 383DH3300.5 & $6 / 16 / 2004$ & soil & HASL300 & REG & 14733-03-0 & Bi-214 & 1.06 & $\mathrm{PCl} / \mathrm{G}$ & $\mathrm{G}, \mathrm{J}$ & 0.36 \\
\hline 383DH3300.5 & $6 / 16 / 2004$ & soil & EPA6010 & REG & $7440-43-9$ & Cadmium & 0.26 & MG/KG & $B$ & 0.51 \\
\hline 383DH3300.5 & $6 / 16 / 2004$ & soil & EPA6010 & REG & $7440-47-3$ & Chromium & 5.5 & MG/KG & & 1 \\
\hline 383DH3300.5 & $6 / 16 / 2004$ & soil & HASL300 & REG & 10045-97-3 & Cs-137 & 7.5 & $\mathrm{PCl} / \mathrm{G}$ & G & 0.2 \\
\hline 383DH3300.5 & $6 / 16 / 2004$ & soil & EPA8015 & REG & 68334-30-5 & Diesel Range Organics & 2.2 & MG/KG & $\mathrm{J}$ & 5.1 \\
\hline 383DH3300.5 & $6 / 16 / 2004$ & soil & HASL300 & REG & 13966-00-2 & $\mathrm{K}-40$ & 25.3 & $\mathrm{PCl} / \mathrm{G}$ & G & 2 \\
\hline 383DH3300.5 & $6 / 16 / 2004$ & soil & EPA6010 & REG & $7439-92-1$ & Lead & 30 & MG/KG & & 0.31 \\
\hline 383DH3300.5 & $6 / 16 / 2004$ & soil & EPA7470 & REG & 7439-97-6 & Mercury & 0.019 & MG/KG & B & 0.1 \\
\hline
\end{tabular}




\begin{tabular}{|c|c|c|c|c|c|c|c|c|c|c|}
\hline SAMPLE \# & $\begin{array}{l}\text { SAMPLE } \\
\text { DATE }\end{array}$ & MATRIX & $\begin{array}{l}\text { USER TEST } \\
\text { PANEL }\end{array}$ & $\begin{array}{l}\text { SAMPLE } \\
\text { PURPOSE }\end{array}$ & CAS \# & PARAMETER & RESULT & UNITS & Q & $\begin{array}{l}\text { DETECT } \\
\text { LIMIT }\end{array}$ \\
\hline 383DH3300.5 & $6 / 16 / 2004$ & soil & EPA8260 & REG & $75-09-2$ & Methylene Chloride & 1.3 & UG/KG & $J, B$ & 5.1 \\
\hline 383DH3300.5 & $6 / 16 / 2004$ & soil & HASL300 & REG & 15092-94-1 & $\mathrm{Pb}-212$ & 1.88 & $\mathrm{PCl} / \mathrm{G}$ & $\mathrm{J}$ & 0.32 \\
\hline 383DH3300.5 & $6 / 16 / 2004$ & soil & HASL300 & REG & $15067-28-4$ & $\mathrm{~Pb}-214$ & 1.26 & $\mathrm{PCl} / \mathrm{G}$ & $\mathrm{G}, \mathrm{J}$ & 0.38 \\
\hline 383DH3300.5 & $6 / 16 / 2004$ & soil & UGTAISOPU & REG & 13981-16-3 & Pu-238 & 0.04 & $\mathrm{PCl} / \mathrm{G}$ & LT & 0.01 \\
\hline 383DH3300.5 & $6 / 16 / 2004$ & soil & UGTAISOPU & REG & $15117-48-3$ & Pu-239 & 0.261 & $\mathrm{PCl} / \mathrm{G}$ & & 0.01 \\
\hline 383DH3300.5 & $6 / 16 / 2004$ & soil & EPA6010 & REG & $7782-49-2$ & Selenium & 0.44 & MG/KG & B & 0.51 \\
\hline 383DH3300.5 & $6 / 16 / 2004$ & soil & HASL300 & REG & $14913-50-9$ & TI-208 & 0.47 & $\mathrm{PCl} / \mathrm{G}$ & $\mathrm{G}$ & 0.18 \\
\hline 383DH3400.5 & $6 / 10 / 2004$ & soil & HASL300 & REG & 14331-83-0 & Ac-228 & 1.6 & $\mathrm{PCl} / \mathrm{G}$ & $\mathrm{G}$ & 0.5 \\
\hline 383DH3400.5 & $6 / 10 / 2004$ & soil & EPA8260 & REG & 67-64-1 & Acetone & 6.5 & UG/KG & $\mathrm{J}$ & 21 \\
\hline 383DH3400.5 & $6 / 10 / 2004$ & soil & EPA6010 & REG & $7440-38-2$ & Arsenic & 2.6 & MG/KG & & 1.1 \\
\hline 383DH3400.5 & $6 / 10 / 2004$ & soil & EPA6010 & REG & 7440-39-3 & Barium & 3900 & MG/KG & & 110 \\
\hline 383DH3400.5 & $6 / 10 / 2004$ & soil & EPA6010 & REG & $7440-41-7$ & Beryllium & 1 & MG/KG & & 0.53 \\
\hline 383DH3400.5 & $6 / 10 / 2004$ & soil & HASL300 & REG & 14733-03-0 & $\mathrm{Bi}-214$ & 1.01 & $\mathrm{PCl} / \mathrm{G}$ & G,J & 0.28 \\
\hline 383DH3400.5 & $6 / 10 / 2004$ & soil & EPA6010 & REG & $7440-43-9$ & Cadmium & 0.22 & MG/KG & B & 0.53 \\
\hline 383DH3400.5 & $6 / 10 / 2004$ & soil & EPA6010 & REG & $7440-47-3$ & Chromium & 3.9 & MG/KG & & 1.1 \\
\hline 383DH3400.5 & $6 / 10 / 2004$ & soil & HASL300 & REG & $10045-97-3$ & Cs-137 & 11.7 & $\mathrm{PCl} / \mathrm{G}$ & $\mathrm{G}$ & 0.1 \\
\hline 383DH3400.5 & $6 / 10 / 2004$ & soil & EPA8015 & REG & $68334-30-5$ & Diesel Range Organics & 51 & MG/KG & $\mathrm{Z}, \mathrm{H}$ & 5.3 \\
\hline 383DH3400.5 & $6 / 10 / 2004$ & soil & HASL300 & REG & 13966-00-2 & $\mathrm{K}-40$ & 22.8 & $\mathrm{PCl} / \mathrm{G}$ & $\mathrm{G}$ & 1.4 \\
\hline 383DH3400.5 & $6 / 10 / 2004$ & soil & EPA6010 & REG & 7439-92-1 & Lead & 14 & MG/KG & & 0.32 \\
\hline 383DH3400.5 & $6 / 10 / 2004$ & soil & EPA7470 & REG & 7439-97-6 & Mercury & 0.025 & MG/KG & B & 0.11 \\
\hline 383DH3400.5 & $6 / 10 / 2004$ & soil & HASL300 & REG & 15092-94-1 & $\mathrm{Pb}-212$ & 1.7 & $\mathrm{PCl} / \mathrm{G}$ & $\mathrm{J}$ & 0.3 \\
\hline 383DH3400.5 & $6 / 10 / 2004$ & soil & HASL300 & REG & $15067-28-4$ & $\mathrm{~Pb}-214$ & 1.26 & $\mathrm{PCl} / \mathrm{G}$ & $\mathrm{G}, \mathrm{J}$ & 0.34 \\
\hline 383DH3400.5 & $6 / 10 / 2004$ & soil & UGTAISOPU & REG & 13981-16-3 & Pu-238 & 0.143 & $\mathrm{PCl} / \mathrm{G}$ & & 0.019 \\
\hline 383DH3400.5 & $6 / 10 / 2004$ & soil & UGTAISOPU & REG & 15117-48-3 & Pu-239 & 1.01 & $\mathrm{PCl} / \mathrm{G}$ & $\mathrm{J}$ & 0.02 \\
\hline 383DH3400.5 & $6 / 10 / 2004$ & soil & SR7500 & REG & $10098-97-2$ & Sr-90 & 0.69 & $\mathrm{PCl} / \mathrm{G}$ & & 0.23 \\
\hline 383DH3400.5 & $6 / 10 / 2004$ & soil & HASL300 & REG & 14913-50-9 & TI-208 & 0.47 & $\mathrm{PCl} / \mathrm{G}$ & $\mathrm{G}$ & 0.16 \\
\hline 383DH3500.5 & $6 / 10 / 2004$ & soil & HASL300 & REG & 14331-83-0 & Ac-228 & 1.42 & $\mathrm{PCl} / \mathrm{G}$ & $G$ & 0.52 \\
\hline 383DH3500.5 & $6 / 10 / 2004$ & soil & EPA8260 & REG & 67-64-1 & Acetone & 6.6 & UG/KG & $\mathrm{J}$ & 23 \\
\hline 383DH3500.5 & $6 / 10 / 2004$ & soil & EPA6010 & REG & 7440-38-2 & Arsenic & 4.5 & MG/KG & & 1.2 \\
\hline 383DH3500.5 & $6 / 10 / 2004$ & soil & EPA6010 & REG & $7440-39-3$ & Barium & 4600 & MG/KG & & 120 \\
\hline 383DH3500.5 & $6 / 10 / 2004$ & soil & EPA6010 & REG & $7440-41-7$ & Beryllium & 0.9 & MG/KG & & 0.58 \\
\hline 383DH3500.5 & $6 / 10 / 2004$ & soil & HASL300 & REG & 14733-03-0 & Bi-214 & 0.76 & $\mathrm{PCl} / \mathrm{G}$ & $\mathrm{G}, \mathrm{J}$ & 0.28 \\
\hline 383DH3500.5 & $6 / 10 / 2004$ & soil & EPA6010 & REG & $7440-43-9$ & Cadmium & 0.28 & MG/KG & B & 0.58 \\
\hline 383DH3500.5 & $6 / 10 / 2004$ & soil & EPA6010 & REG & $7440-47-3$ & Chromium & 5.8 & MG/KG & & 1.2 \\
\hline 383DH3500.5 & $6 / 10 / 2004$ & soil & HASL300 & REG & 10045-97-3 & Cs-137 & 5.97 & $\mathrm{PCl} / \mathrm{G}$ & $\mathrm{G}$ & 0.14 \\
\hline
\end{tabular}




\begin{tabular}{|c|c|c|c|c|c|c|c|c|c|c|}
\hline SAMPLE \# & $\begin{array}{l}\text { SAMPLE } \\
\text { DATE }\end{array}$ & MATRIX & $\begin{array}{l}\text { USER TEST } \\
\text { PANEL }\end{array}$ & $\begin{array}{l}\text { SAMPLE } \\
\text { PURPOSE }\end{array}$ & CAS \# & PARAMETER & RESULT & UNITS & Q & $\begin{array}{l}\text { DETECT } \\
\text { LIMIT }\end{array}$ \\
\hline 383DH3500.5 & $6 / 10 / 2004$ & soil & EPA8015 & REG & $68334-30-5$ & Diesel Range Organics & 68 & MG/KG & $\mathrm{Z}, \mathrm{H}$ & 5.8 \\
\hline 383DH3500.5 & $6 / 10 / 2004$ & soil & HASL300 & REG & $13966-00-2$ & $\mathrm{~K}-40$ & 17 & $\mathrm{PCl} / \mathrm{G}$ & G & 1.6 \\
\hline 383DH3500.5 & $6 / 10 / 2004$ & soil & EPA6010 & REG & $7439-92-1$ & Lead & 19 & MG/KG & & 3.5 \\
\hline 383DH3500.5 & $6 / 10 / 2004$ & soil & EPA7470 & REG & 7439-97-6 & Mercury & 0.026 & MG/KG & B & 0.12 \\
\hline 383DH3500.5 & $6 / 10 / 2004$ & soil & HASL300 & REG & 15092-94-1 & $\mathrm{Pb}-212$ & 1.44 & $\mathrm{PCl} / \mathrm{G}$ & $\mathrm{J}$ & 0.26 \\
\hline 383DH3500.5 & $6 / 10 / 2004$ & soil & HASL300 & REG & $15067-28-4$ & $\mathrm{~Pb}-214$ & 1.14 & $\mathrm{PCl} / \mathrm{G}$ & $\mathrm{G}, \mathrm{J}$ & 0.31 \\
\hline 383DH3500.5 & $6 / 10 / 2004$ & soil & UGTAISOPU & REG & 13981-16-3 & Pu-238 & 0.147 & $\mathrm{PCl} / \mathrm{G}$ & & 0.02 \\
\hline 383DH3500.5 & $6 / 10 / 2004$ & soil & UGTAISOPU & REG & $15117-48-3$ & Pu-239 & 0.51 & $\mathrm{PCl} / \mathrm{G}$ & $\mathrm{J}$ & 0.01 \\
\hline 383DH3500.5 & $6 / 10 / 2004$ & soil & HASL300 & REG & $14913-50-9$ & TI-208 & 0.5 & $\mathrm{PCl} / \mathrm{G}$ & $G$ & 0.13 \\
\hline 383GB0100.5 & $2 / 4 / 2004$ & soil & HASL300 & REG & 14331-83-0 & Ac-228 & 2.14 & $\mathrm{PCl} / \mathrm{G}$ & G & 1.04 \\
\hline 383GB0100.5 & $2 / 4 / 2004$ & soil & EPA6010 & REG & $7440-38-2$ & Arsenic & 4.4 & MG/KG & & 1.2 \\
\hline 383GB0100.5 & $2 / 4 / 2004$ & soil & EPA6010 & REG & $7440-41-7$ & Beryllium & 1.3 & MG/KG & & 0.58 \\
\hline 383GB0100.5 & $2 / 4 / 2004$ & soil & HASL300 & REG & 14733-03-0 & $\mathrm{Bi}-214$ & 1.41 & $\mathrm{PCl} / \mathrm{G}$ & G & 0.73 \\
\hline 383GB0100.5 & $2 / 4 / 2004$ & soil & HASL300 & REG & 10045-97-3 & Cs-137 & 0.78 & $\mathrm{PCl} / \mathrm{G}$ & G & 0.31 \\
\hline 383GB0100.5 & $2 / 4 / 2004$ & soil & HASL300 & REG & 13966-00-2 & $\mathrm{K}-40$ & 18.9 & $\mathrm{PCl} / \mathrm{G}$ & G & 4.4 \\
\hline 383GB0100.5 & $2 / 4 / 2004$ & soil & EPA6010 & REG & $7439-92-1$ & Lead & 12 & MG/KG & & 0.35 \\
\hline 383GB0100.5 & $2 / 4 / 2004$ & soil & HASL300 & REG & 15092-94-1 & $\mathrm{Pb}-212$ & 1.66 & $\mathrm{PCl} / \mathrm{G}$ & $\mathrm{J}$ & 0.52 \\
\hline 383GB0100.5 & $2 / 4 / 2004$ & soil & UGTAISOPU & REG & 15117-48-3 & Pu-239 & 0.099 & $\mathrm{PCl} / \mathrm{G}$ & $\mathrm{J}$ & 0.009 \\
\hline 383GB0100.5 & $2 / 4 / 2004$ & soil & HASL300 & REG & 14913-50-9 & TI-208 & 0.64 & $\mathrm{PCl} / \mathrm{G}$ & G & 0.27 \\
\hline 383GB0200.5 & $2 / 4 / 2004$ & soil & HASL300 & REG & 14331-83-0 & Ac-228 & 1.85 & $\mathrm{PCl} / \mathrm{G}$ & $\mathrm{TI}, \mathrm{G}$ & 0.85 \\
\hline 383GB0200.5 & $2 / 4 / 2004$ & soil & EPA6010 & REG & $7440-38-2$ & Arsenic & 4.3 & MG/KG & & 1.1 \\
\hline 383GB0200.5 & $2 / 4 / 2004$ & soil & EPA6010 & REG & $7440-41-7$ & Beryllium & 0.72 & MG/KG & & 0.55 \\
\hline 383GB0200.5 & $2 / 4 / 2004$ & soil & HASL300 & REG & $10045-97-3$ & Cs-137 & 4.39 & $\mathrm{PCl} / \mathrm{G}$ & G & 0.23 \\
\hline 383GB0200.5 & $2 / 4 / 2004$ & soil & HASL300 & REG & 13966-00-2 & $\mathrm{K}-40$ & 17.8 & $\mathrm{PCl} / \mathrm{G}$ & G & 2.8 \\
\hline 383GB0200.5 & $2 / 4 / 2004$ & soil & EPA6010 & REG & $7439-92-1$ & Lead & 75 & MG/KG & & 0.33 \\
\hline 383GB0200.5 & $2 / 4 / 2004$ & soil & HASL300 & REG & 15092-94-1 & $\mathrm{Pb}-212$ & 1.3 & $\mathrm{PCl} / \mathrm{G}$ & $\mathrm{J}$ & 0.42 \\
\hline 383GB0200.5 & $2 / 4 / 2004$ & soil & UGTAISOPU & REG & 13981-16-3 & Pu-238 & 0.032 & $\mathrm{PCl} / \mathrm{G}$ & LT & 0.01 \\
\hline 383GB0200.5 & $2 / 4 / 2004$ & soil & UGTAISOPU & REG & 15117-48-3 & Pu-239 & 0.318 & $\mathrm{PCl} / \mathrm{G}$ & $\mathrm{J}$ & 0.018 \\
\hline 383GB0200.5 & $2 / 4 / 2004$ & soil & HASL300 & REG & 14913-50-9 & TI-208 & 0.43 & $\mathrm{PCl} / \mathrm{G}$ & G & 0.22 \\
\hline 383GB0300.5 & $2 / 4 / 2004$ & soil & EPA6010 & REG & $7440-38-2$ & Arsenic & 3 & MG/KG & & 1.1 \\
\hline 383GB0300.5 & $2 / 4 / 2004$ & soil & EPA6010 & REG & $7440-41-7$ & Beryllium & 0.79 & MG/KG & & 0.57 \\
\hline 383GB0300.5 & $2 / 4 / 2004$ & soil & HASL300 & REG & 13966-00-2 & $\mathrm{K}-40$ & 24 & $\mathrm{PCl} / \mathrm{G}$ & G & 3.1 \\
\hline 383GB0300.5 & $2 / 4 / 2004$ & soil & EPA6010 & REG & 7439-92-1 & Lead & 26 & MG/KG & & 0.34 \\
\hline 383GB0300.5 & $2 / 4 / 2004$ & soil & HASL300 & REG & 15092-94-1 & $\mathrm{Pb}-212$ & 1.87 & $\mathrm{PCl} / \mathrm{G}$ & $\mathrm{J}$ & 0.38 \\
\hline 383GB0300.5 & $2 / 4 / 2004$ & soil & HASL300 & REG & 15067-28-4 & $\mathrm{Pb}-214$ & 0.81 & $\mathrm{PCl} / \mathrm{G}$ & $\mathrm{J}$ & 0.43 \\
\hline
\end{tabular}




\begin{tabular}{|c|c|c|c|c|c|c|c|c|c|c|}
\hline SAMPLE \# & $\begin{array}{l}\text { SAMPLE } \\
\text { DATE }\end{array}$ & MATRIX & $\begin{array}{l}\text { USER TEST } \\
\text { PANEL }\end{array}$ & $\begin{array}{l}\text { SAMPLE } \\
\text { PURPOSE }\end{array}$ & CAS \# & PARAMETER & RESULT & UNITS & Q & $\begin{array}{l}\text { DETECT } \\
\text { LIMIT }\end{array}$ \\
\hline 383GB0300.5 & $2 / 4 / 2004$ & soil & UGTAISOPU & REG & $15117-48-3$ & Pu-239 & 0.241 & $\mathrm{PCl} / \mathrm{G}$ & $\mathrm{J}$ & 0.018 \\
\hline 383GB0400.5 & $2 / 4 / 2004$ & soil & HASL300 & REG & 14331-83-0 & Ac-228 & 2.09 & $\mathrm{PCl} / \mathrm{G}$ & G & 1.05 \\
\hline 383GB0400.5 & $2 / 4 / 2004$ & soil & EPA6010 & REG & $7440-38-2$ & Arsenic & 3.8 & MG/KG & & 1.1 \\
\hline 383GB0400.5 & $2 / 4 / 2004$ & soil & EPA6010 & REG & $7440-41-7$ & Beryllium & 0.82 & MG/KG & & 0.57 \\
\hline 383GB0400.5 & $2 / 4 / 2004$ & soil & HASL300 & REG & $10045-97-3$ & Cs-137 & 1.86 & $\mathrm{PCl} / \mathrm{G}$ & G & 0.27 \\
\hline 383GB0400.5 & $2 / 4 / 2004$ & soil & HASL300 & REG & 13966-00-2 & $\mathrm{K}-40$ & 26 & $\mathrm{PCl} / \mathrm{G}$ & G & 3.2 \\
\hline 383GB0400.5 & $2 / 4 / 2004$ & soil & EPA6010 & REG & $7439-92-1$ & Lead & 60 & MG/KG & & 0.34 \\
\hline 383GB0400.5 & $2 / 4 / 2004$ & soil & HASL300 & REG & $15092-94-1$ & $\mathrm{~Pb}-212$ & 1.78 & $\mathrm{PCl} / \mathrm{G}$ & $\mathrm{J}$ & 0.3 \\
\hline 383GB0400.5 & $2 / 4 / 2004$ & soil & UGTAISOPU & REG & $15117-48-3$ & Pu-239 & 0.093 & $\mathrm{PCl} / \mathrm{G}$ & $\mathrm{J}$ & 0.022 \\
\hline 383GB0500.5 & $2 / 4 / 2004$ & soil & HASL300 & REG & 14331-83-0 & Ac-228 & 2.27 & $\mathrm{PCl} / \mathrm{G}$ & G & 0.55 \\
\hline 383GB0500.5 & $2 / 4 / 2004$ & soil & EPA6010 & REG & $7440-38-2$ & Arsenic & 5.3 & MG/KG & & 1.1 \\
\hline 383GB0500.5 & $2 / 4 / 2004$ & soil & EPA6010 & REG & $7440-39-3$ & Barium & 130 & MG/KG & & 11 \\
\hline 383GB0500.5 & $2 / 4 / 2004$ & soil & EPA6010 & REG & $7440-41-7$ & Beryllium & 1 & MG/KG & & 0.57 \\
\hline 383GB0500.5 & $2 / 4 / 2004$ & soil & HASL300 & REG & 14733-03-0 & Bi-214 & 1.24 & $\mathrm{PCl} / \mathrm{G}$ & G & 0.33 \\
\hline 383GB0500.5 & $2 / 4 / 2004$ & soil & EPA6010 & REG & $7440-47-3$ & Chromium & 8.2 & MG/KG & & 1.1 \\
\hline 383GB0500.5 & $2 / 4 / 2004$ & soil & HASL300 & REG & $10045-97-3$ & Cs-137 & 1.27 & $\mathrm{PCl} / \mathrm{G}$ & G & 0.18 \\
\hline 383GB0500.5 & $2 / 4 / 2004$ & soil & HASL300 & REG & 13966-00-2 & $\mathrm{K}-40$ & 30.7 & $\mathrm{PCl} / \mathrm{G}$ & G & 1.6 \\
\hline 383GB0500.5 & $2 / 4 / 2004$ & soil & EPA6010 & REG & $7439-92-1$ & Lead & 15 & MG/KG & & 0.34 \\
\hline 383GB0500.5 & $2 / 4 / 2004$ & soil & EPA7470 & REG & $7439-97-6$ & Mercury & 0.065 & MG/KG & B & 0.11 \\
\hline 383GB0500.5 & $2 / 4 / 2004$ & soil & HASL300 & REG & 15092-94-1 & $\mathrm{Pb}-212$ & 2.47 & $\mathrm{PCl} / \mathrm{G}$ & $\mathrm{J}$ & 0.24 \\
\hline 383GB0500.5 & $2 / 4 / 2004$ & soil & HASL300 & REG & $15067-28-4$ & $\mathrm{~Pb}-214$ & 1.07 & $\mathrm{PCl} / \mathrm{G}$ & $\mathrm{J}$ & 0.32 \\
\hline 383GB0500.5 & $2 / 4 / 2004$ & soil & UGTAISOPU & REG & 13981-16-3 & Pu-238 & 0.38 & $\mathrm{PCl} / \mathrm{G}$ & & 0.03 \\
\hline 383GB0500.5 & $2 / 4 / 2004$ & soil & UGTAISOPU & REG & $15117-48-3$ & Pu-239 & 1.41 & $\mathrm{PCl} / \mathrm{G}$ & & 0.02 \\
\hline 383GB0500.5 & $2 / 4 / 2004$ & soil & EPA6010 & REG & 7782-49-2 & Selenium & 0.52 & MG/KG & B & 0.57 \\
\hline 383GB0500.5 & $2 / 4 / 2004$ & soil & HASL300 & REG & 14913-50-9 & TI-208 & 0.83 & $\mathrm{PCl} / \mathrm{G}$ & G & 0.17 \\
\hline 383GB0600.5 & $2 / 4 / 2004$ & soil & HASL300 & REG & 14331-83-0 & Ac-228 & 1.44 & $\mathrm{PCl} / \mathrm{G}$ & G & 0.53 \\
\hline 383GB0600.5 & $2 / 4 / 2004$ & soil & EPA6010 & REG & $7440-38-2$ & Arsenic & 4 & MG/KG & & 1.1 \\
\hline 383GB0600.5 & $2 / 4 / 2004$ & soil & EPA6010 & REG & 7440-39-3 & Barium & 290 & MG/KG & & 11 \\
\hline 383GB0600.5 & $2 / 4 / 2004$ & soil & EPA6010 & REG & $7440-41-7$ & Beryllium & 0.88 & MG/KG & & 0.57 \\
\hline 383GB0600.5 & $2 / 4 / 2004$ & soil & HASL300 & REG & 14733-03-0 & Bi-214 & 0.79 & $\mathrm{PCl} / \mathrm{G}$ & G & 0.29 \\
\hline 383GB0600.5 & $2 / 4 / 2004$ & soil & EPA6010 & REG & $7440-43-9$ & Cadmium & 0.11 & MG/KG & $\mathrm{J}$ & 0.57 \\
\hline 383GB0600.5 & $2 / 4 / 2004$ & soil & EPA6010 & REG & $7440-47-3$ & Chromium & 8.4 & MG/KG & & 1.1 \\
\hline 383GB0600.5 & $2 / 4 / 2004$ & soil & HASL300 & REG & $10045-97-3$ & Cs-137 & 2.7 & $\mathrm{PCl} / \mathrm{G}$ & G & 0.14 \\
\hline 383GB0600.5 & 2/4/2004 & soil & HASL300 & REG & 13966-00-2 & $\mathrm{K}-40$ & 16.3 & $\mathrm{PCl} / \mathrm{G}$ & G & 1.3 \\
\hline 383GB0600.5 & 2/4/2004 & soil & EPA6010 & REG & $7439-92-1$ & Lead & 16 & MG/KG & & 0.68 \\
\hline
\end{tabular}




\begin{tabular}{|c|c|c|c|c|c|c|c|c|c|c|}
\hline SAMPLE \# & $\begin{array}{l}\text { SAMPLE } \\
\text { DATE }\end{array}$ & MATRIX & $\begin{array}{l}\text { USER TEST } \\
\text { PANEL }\end{array}$ & $\begin{array}{l}\text { SAMPLE } \\
\text { PURPOSE }\end{array}$ & CAS \# & PARAMETER & RESULT & UNITS & Q & $\begin{array}{l}\text { DETECT } \\
\text { LIMIT }\end{array}$ \\
\hline 383GB0600.5 & $2 / 4 / 2004$ & soil & EPA7470 & REG & 7439-97-6 & Mercury & 0.032 & MG/KG & B & 0.11 \\
\hline 383GB0600.5 & $2 / 4 / 2004$ & soil & HASL300 & REG & 15092-94-1 & $\mathrm{Pb}-212$ & 1.48 & $\mathrm{PCl} / \mathrm{G}$ & $\mathrm{J}$ & 0.2 \\
\hline 383GB0600.5 & $2 / 4 / 2004$ & soil & HASL300 & REG & $15067-28-4$ & $\mathrm{~Pb}-214$ & 0.89 & $\mathrm{PCl} / \mathrm{G}$ & $\mathrm{J}$ & 0.28 \\
\hline 383GB0600.5 & $2 / 4 / 2004$ & soil & UGTAISOPU & REG & 13981-16-3 & Pu-238 & 0.71 & $\mathrm{PCl} / \mathrm{G}$ & & 0.02 \\
\hline 383GB0600.5 & $2 / 4 / 2004$ & soil & UGTAISOPU & REG & $15117-48-3$ & Pu-239 & 5.08 & $\mathrm{PCl} / \mathrm{G}$ & & 0.02 \\
\hline 383GB0600.5 & $2 / 4 / 2004$ & soil & EPA6010 & REG & $7782-49-2$ & Selenium & 0.71 & MG/KG & & 0.57 \\
\hline 383GB0600.5 & $2 / 4 / 2004$ & soil & HASL300 & REG & 14913-50-9 & TI-208 & 0.38 & $\mathrm{PCl} / \mathrm{G}$ & G & 0.13 \\
\hline 383GB0700.5 & $2 / 4 / 2004$ & soil & EPA6010 & REG & $7440-38-2$ & Arsenic & 2.6 & MG/KG & & 1.1 \\
\hline 383GB0700.5 & $2 / 4 / 2004$ & soil & EPA6010 & REG & $7440-39-3$ & Barium & 170 & MG/KG & & 11 \\
\hline 383GB0700.5 & $2 / 4 / 2004$ & soil & HASL300 & REG & 14733-03-0 & Bi-214 & 0.45 & $\mathrm{PCl} / \mathrm{G}$ & G & 0.22 \\
\hline 383GB0700.5 & $2 / 4 / 2004$ & soil & EPA6010 & REG & $7440-43-9$ & Cadmium & 0.32 & MG/KG & B & 0.54 \\
\hline 383GB0700.5 & $2 / 4 / 2004$ & soil & EPA6010 & REG & $7440-47-3$ & Chromium & 5 & MG/KG & & 1.1 \\
\hline 383GB0700.5 & $2 / 4 / 2004$ & soil & HASL300 & REG & $10045-97-3$ & Cs-137 & 0.46 & $\mathrm{PCl} / \mathrm{G}$ & LT,G & 0.1 \\
\hline 383GB0700.5 & $2 / 4 / 2004$ & soil & HASL300 & REG & 13966-00-2 & $\mathrm{K}-40$ & 8.8 & $\mathrm{PCl} / \mathrm{G}$ & G & 0.8 \\
\hline 383GB0700.5 & $2 / 4 / 2004$ & soil & EPA6010 & REG & $7439-92-1$ & Lead & 21 & MG/KG & & 1.6 \\
\hline 383GB0700.5 & $2 / 4 / 2004$ & soil & EPA7470 & REG & 7439-97-6 & Mercury & 0.032 & MG/KG & B & 0.11 \\
\hline 383GB0700.5 & $2 / 4 / 2004$ & soil & HASL300 & REG & 15092-94-1 & $\mathrm{Pb}-212$ & 0.72 & $\mathrm{PCl} / \mathrm{G}$ & $\mathrm{J}$ & 0.16 \\
\hline 383GB0700.5 & $2 / 4 / 2004$ & soil & HASL300 & REG & $15067-28-4$ & $\mathrm{~Pb}-214$ & 0.6 & $\mathrm{PCl} / \mathrm{G}$ & $\mathrm{J}$ & 0.21 \\
\hline 383GB0700.5 & $2 / 4 / 2004$ & soil & UGTAISOPU & REG & 13981-16-3 & Pu-238 & 0.088 & $\mathrm{PCl} / \mathrm{G}$ & & 0.018 \\
\hline 383GB0700.5 & $2 / 4 / 2004$ & soil & UGTAISOPU & REG & $15117-48-3$ & Pu-239 & 0.242 & $\mathrm{PCl} / \mathrm{G}$ & & 0.009 \\
\hline 383GB0700.5 & $2 / 4 / 2004$ & soil & HASL300 & REG & 14913-50-9 & TI-208 & 0.247 & $\mathrm{PCl} / \mathrm{G}$ & G & 0.113 \\
\hline 383GB0800.5 & $2 / 4 / 2004$ & soil & HASL300 & FD & 14331-83-0 & Ac-228 & 1.36 & $\mathrm{PCl} / \mathrm{G}$ & G & 0.52 \\
\hline 383GB0800.5 & $2 / 4 / 2004$ & soil & HASL300 & FD & 14596-10-2 & Am-241 & 0.62 & $\mathrm{PCl} / \mathrm{G}$ & $\mathrm{J}$ & 0.28 \\
\hline 383GB0800.5 & $2 / 4 / 2004$ & soil & EPA6010 & FD & $7440-38-2$ & Arsenic & 4.5 & MG/KG & & 1.2 \\
\hline 383GB0800.5 & $2 / 4 / 2004$ & soil & EPA6010 & FD & $7440-39-3$ & Barium & 260 & MG/KG & & 12 \\
\hline 383GB0800.5 & $2 / 4 / 2004$ & soil & EPA6010 & FD & $7440-41-7$ & Beryllium & 0.87 & MG/KG & & 0.58 \\
\hline 383GB0800.5 & $2 / 4 / 2004$ & soil & HASL300 & FD & 14733-03-0 & Bi-214 & 0.73 & $\mathrm{PCl} / \mathrm{G}$ & G & 0.3 \\
\hline 383GB0800.5 & $2 / 4 / 2004$ & soil & EPA6010 & FD & 7440-43-9 & Cadmium & 0.081 & MG/KG & $\mathrm{J}$ & 0.58 \\
\hline 383GB0800.5 & $2 / 4 / 2004$ & soil & EPA6010 & FD & $7440-47-3$ & Chromium & 8.4 & MG/KG & & 1.2 \\
\hline 383GB0800.5 & $2 / 4 / 2004$ & soil & HASL300 & FD & $10045-97-3$ & Cs-137 & 2.25 & $\mathrm{PCl} / \mathrm{G}$ & G & 0.2 \\
\hline 383GB0800.5 & $2 / 4 / 2004$ & soil & HASL300 & FD & 13966-00-2 & $\mathrm{K}-40$ & 12.9 & $\mathrm{PCl} / \mathrm{G}$ & G & 2 \\
\hline 383GB0800.5 & $2 / 4 / 2004$ & soil & EPA6010 & FD & $7439-92-1$ & Lead & 17 & MG/KG & & 1.7 \\
\hline 383GB0800.5 & $2 / 4 / 2004$ & soil & EPA7470 & FD & 7439-97-6 & Mercury & 0.035 & MG/KG & B & 0.12 \\
\hline 383GB0800.5 & 2/4/2004 & soil & HASL300 & FD & 15092-94-1 & $\mathrm{Pb}-212$ & 1.26 & $\mathrm{PCl} / \mathrm{G}$ & $\mathrm{J}$ & 0.26 \\
\hline 383GB0800.5 & 2/4/2004 & soil & HASL300 & FD & 15067-28-4 & $\mathrm{Pb}-214$ & 0.97 & $\mathrm{PCl} / \mathrm{G}$ & $\mathrm{J}$ & 0.36 \\
\hline
\end{tabular}




\begin{tabular}{|c|c|c|c|c|c|c|c|c|c|c|}
\hline SAMPLE \# & $\begin{array}{l}\text { SAMPLE } \\
\text { DATE }\end{array}$ & MATRIX & $\begin{array}{l}\text { USER TEST } \\
\text { PANEL }\end{array}$ & $\begin{array}{l}\text { SAMPLE } \\
\text { PURPOSE }\end{array}$ & CAS \# & PARAMETER & RESULT & UNITS & Q & $\begin{array}{l}\text { DETECT } \\
\text { LIMIT }\end{array}$ \\
\hline 383GB0800.5 & $2 / 4 / 2004$ & soil & UGTAISOPU & FD & 13981-16-3 & Pu-238 & 0.71 & $\mathrm{PCl} / \mathrm{G}$ & & 0.01 \\
\hline 383GB0800.5 & $2 / 4 / 2004$ & soil & UGTAISOPU & FD & 15117-48-3 & Pu-239 & 2.43 & $\mathrm{PCl} / \mathrm{G}$ & & 0.02 \\
\hline 383GB0800.5 & $2 / 4 / 2004$ & soil & HASL300 & FD & 14913-50-9 & TI-208 & 0.51 & $\mathrm{PCl} / \mathrm{G}$ & G & 0.16 \\
\hline 383GB0901 & $3 / 10 / 2004$ & soil & HASL300 & REG & 14331-83-0 & Ac-228 & 2.27 & $\mathrm{PCl} / \mathrm{G}$ & G & 0.69 \\
\hline 383GB0901 & $3 / 10 / 2004$ & soil & HASL300 & REG & 14596-10-2 & Am-241 & 2.83 & $\mathrm{PCl} / \mathrm{G}$ & $\mathrm{J}$ & 0.65 \\
\hline 383GB0901 & $3 / 10 / 2004$ & soil & HASL300 & REG & $10045-97-3$ & Cs-137 & 180 & $\mathrm{PCl} / \mathrm{G}$ & G & 0 \\
\hline 383GB0901 & $3 / 10 / 2004$ & soil & HASL300 & REG & $13966-00-2$ & $\mathrm{~K}-40$ & 29.2 & $\mathrm{PCl} / \mathrm{G}$ & G & 2 \\
\hline 383GB0901 & $3 / 10 / 2004$ & soil & HASL300 & REG & 15092-94-1 & $\mathrm{Pb}-212$ & 2.12 & $\mathrm{PCl} / \mathrm{G}$ & $\mathrm{J}$ & 0.74 \\
\hline 383GB0901 & $3 / 10 / 2004$ & soil & UGTAISOPU & REG & 13981-16-3 & Pu-238 & 11.3 & $\mathrm{PCl} / \mathrm{G}$ & & 0 \\
\hline 383GB0901 & $3 / 10 / 2004$ & soil & UGTAISOPU & REG & $15117-48-3$ & Pu-239 & 61 & $\mathrm{PCl} / \mathrm{G}$ & M3 & 0 \\
\hline 383GB0901 & $3 / 10 / 2004$ & soil & SR7500 & REG & 10098-97-2 & Sr-90 & 18.8 & $\mathrm{PCl} / \mathrm{G}$ & & 0.3 \\
\hline 383MB0100.5 & $6 / 12 / 2004$ & soil & EPA6010 & REG & 7440-38-2 & Arsenic & 0.7 & MG/KG & $B$ & 1 \\
\hline 383MB0100.5 & $6 / 12 / 2004$ & soil & EPA6010 & REG & 7440-39-3 & Barium & 470 & MG/KG & & 10 \\
\hline 383MB0100.5 & $6 / 12 / 2004$ & soil & EPA6010 & REG & 7440-41-7 & Beryllium & 0.49 & MG/KG & B & 0.51 \\
\hline 383MB0100.5 & $6 / 12 / 2004$ & soil & EPA6010 & REG & $7440-43-9$ & Cadmium & 0.099 & MG/KG & $B$ & 0.51 \\
\hline 383MB0100.5 & $6 / 12 / 2004$ & soil & EPA6010 & REG & $7440-47-3$ & Chromium & 2 & MG/KG & & 1 \\
\hline 383MB0100.5 & $6 / 12 / 2004$ & soil & HASL300 & REG & $10045-97-3$ & Cs-137 & 0.94 & $\mathrm{PCl} / \mathrm{G}$ & & 0.05 \\
\hline 383MB0100.5 & $6 / 12 / 2004$ & soil & HASL300 & REG & 13966-00-2 & $\mathrm{K}-40$ & 3.7 & $\mathrm{PCl} / \mathrm{G}$ & & 0.9 \\
\hline 383MB0100.5 & $6 / 12 / 2004$ & soil & EPA6010 & REG & $7439-92-1$ & Lead & 3.4 & MG/KG & & 1.5 \\
\hline 383MB0100.5 & $6 / 12 / 2004$ & soil & EPA7470 & REG & 7439-97-6 & Mercury & 0.073 & MG/KG & $B$ & 0.1 \\
\hline 383MB0100.5 & $6 / 12 / 2004$ & soil & EPA8260 & REG & $75-09-2$ & Methylene Chloride & 0.95 & UG/KG & J,B & 5.1 \\
\hline 383MB0100.5 & $6 / 12 / 2004$ & soil & HASL300 & REG & 15092-94-1 & $\mathrm{Pb}-212$ & 0.35 & $\mathrm{PCl} / \mathrm{G}$ & $\mathrm{J}$ & 0.12 \\
\hline 383MB0100.5 & $6 / 12 / 2004$ & soil & HASL300 & REG & $15067-28-4$ & $\mathrm{~Pb}-214$ & 0.3 & $\mathrm{PCl} / \mathrm{G}$ & $\mathrm{J}$ & 0.16 \\
\hline 383MB0100.5 & $6 / 12 / 2004$ & soil & EPA8260 & REG & $99-87-6$ & P-Isopropyltoluene & 1.3 & UG/KG & $\mathrm{J}$ & 5.1 \\
\hline 383MB0200.5 & $5 / 24 / 2004$ & soil & HASL300 & REG & 14331-83-0 & Ac-228 & 0.85 & $\mathrm{PCl} / \mathrm{G}$ & G & 0.34 \\
\hline 383MB0200.5 & $5 / 24 / 2004$ & soil & EPA8260 & REG & $67-64-1$ & Acetone & 7.9 & UG/KG & $\mathrm{J}$ & 22 \\
\hline 383MB0200.5 & $5 / 24 / 2004$ & soil & EPA6010 & REG & $7440-38-2$ & Arsenic & 1.9 & MG/KG & & 1.1 \\
\hline 383MB0200.5 & $5 / 24 / 2004$ & soil & EPA6010 & REG & $7440-39-3$ & Barium & 910 & MG/KG & & 11 \\
\hline 383MB0200.5 & $5 / 24 / 2004$ & soil & EPA8270 & REG & $56-55-3$ & Benzo(A)Anthracene & 38 & UG/KG & $\mathrm{J}$ & 720 \\
\hline 383MB0200.5 & $5 / 24 / 2004$ & soil & EPA6010 & REG & $7440-41-7$ & Beryllium & 0.47 & MG/KG & $B$ & 0.54 \\
\hline 383MB0200.5 & $5 / 24 / 2004$ & soil & HASL300 & REG & 14733-03-0 & Bi-214 & 0.64 & $\mathrm{PCl} / \mathrm{G}$ & $\mathrm{G}, \mathrm{J}$ & 0.24 \\
\hline 383MB0200.5 & $5 / 24 / 2004$ & soil & EPA8270 & REG & $117-81-7$ & Bis(2-Ethylhexyl)Phthalate & 170 & UG/KG & $\mathrm{J}$ & 720 \\
\hline 383MB0200.5 & $5 / 24 / 2004$ & soil & EPA6010 & REG & $7440-43-9$ & Cadmium & 0.74 & MG/KG & & 0.54 \\
\hline 383MB0200.5 & $5 / 24 / 2004$ & soil & EPA6010 & REG & $7440-47-3$ & Chromium & 33 & MG/KG & & 1.1 \\
\hline 383MB0200.5 & $5 / 24 / 2004$ & soil & HASL300 & REG & $10045-97-3$ & Cs-137 & 18.4 & $\mathrm{PCl} / \mathrm{G}$ & G & 0.1 \\
\hline
\end{tabular}




\begin{tabular}{|c|c|c|c|c|c|c|c|c|c|c|}
\hline SAMPLE \# & $\begin{array}{l}\text { SAMPLE } \\
\text { DATE }\end{array}$ & MATRIX & $\begin{array}{l}\text { USER TEST } \\
\text { PANEL }\end{array}$ & $\begin{array}{l}\text { SAMPLE } \\
\text { PURPOSE }\end{array}$ & CAS \# & PARAMETER & RESULT & UNITS & Q & $\begin{array}{l}\text { DETECT } \\
\text { LIMIT }\end{array}$ \\
\hline 383MB0200.5 & $5 / 24 / 2004$ & soil & EPA8015 & REG & $68334-30-5$ & Diesel Range Organics & 45 & MG/KG & $\mathrm{M}, \mathrm{H}$ & 5.4 \\
\hline 383MB0200.5 & $5 / 24 / 2004$ & soil & HASL300 & REG & $13966-00-2$ & $\mathrm{~K}-40$ & 9.8 & $\mathrm{PCl} / \mathrm{G}$ & $\mathrm{G}$ & 1.3 \\
\hline 383MB0200.5 & $5 / 24 / 2004$ & soil & EPA6010 & REG & $7439-92-1$ & Lead & 39 & MG/KG & & 1.6 \\
\hline 383MB0200.5 & $5 / 24 / 2004$ & soil & EPA7470 & REG & $7439-97-6$ & Mercury & 0.052 & MG/KG & $B$ & 0.11 \\
\hline 383MB0200.5 & $5 / 24 / 2004$ & soil & EPA8260 & REG & $75-09-2$ & Methylene Chloride & 0.88 & UG/KG & $\mathrm{J}, \mathrm{B}$ & 5.4 \\
\hline 383MB0200.5 & $5 / 24 / 2004$ & soil & HASL300 & REG & 15092-94-1 & $\mathrm{Pb}-212$ & 0.68 & $\mathrm{PCl} / \mathrm{G}$ & $\mathrm{J}$ & 0.27 \\
\hline 383MB0200.5 & $5 / 24 / 2004$ & soil & HASL300 & REG & $15067-28-4$ & $\mathrm{~Pb}-214$ & 0.71 & $\mathrm{PCl} / \mathrm{G}$ & $\mathrm{G}, \mathrm{J}$ & 0.36 \\
\hline 383MB0200.5 & $5 / 24 / 2004$ & soil & UGTAISOPU & REG & 13981-16-3 & Pu-238 & 0.128 & $\mathrm{PCl} / \mathrm{G}$ & & 0.018 \\
\hline 383MB0200.5 & $5 / 24 / 2004$ & soil & UGTAISOPU & REG & 15117-48-3 & Pu-239 & 1.33 & $\mathrm{PCl} / \mathrm{G}$ & & 0.02 \\
\hline 383MB0300.5 & $6 / 11 / 2004$ & soil & HASL300 & REG & 14331-83-0 & Ac-228 & 0.5 & $\mathrm{PCl} / \mathrm{G}$ & & 0.39 \\
\hline 383MB0300.5 & $6 / 11 / 2004$ & soil & EPA6010 & REG & $7440-38-2$ & Arsenic & 3.5 & MG/KG & & 1 \\
\hline 383MB0300.5 & $6 / 11 / 2004$ & soil & EPA6010 & REG & $7440-39-3$ & Barium & 110 & MG/KG & & 10 \\
\hline 383MB0300.5 & $6 / 11 / 2004$ & soil & EPA8270 & REG & $56-55-3$ & Benzo(A)Anthracene & 83 & UG/KG & $\mathrm{J}$ & 340 \\
\hline 383MB0300.5 & $6 / 11 / 2004$ & soil & EPA8270 & REG & $50-32-8$ & Benzo(A)Pyrene & 78 & UG/KG & $\mathrm{J}$ & 340 \\
\hline 383MB0300.5 & $6 / 11 / 2004$ & soil & EPA8270 & REG & $205-99-2$ & Benzo(B)Fluoranthene & 140 & UG/KG & $\mathrm{J}$ & 340 \\
\hline 383MB0300.5 & $6 / 11 / 2004$ & soil & EPA8270 & REG & $191-24-2$ & Benzo(G,H,I)Perylene & 68 & UG/KG & $\mathrm{J}$ & 340 \\
\hline 383MB0300.5 & $6 / 11 / 2004$ & soil & EPA8270 & REG & $207-08-9$ & Benzo(K)Fluoranthene & 55 & UG/KG & $\mathrm{J}$ & 340 \\
\hline 383MB0300.5 & $6 / 11 / 2004$ & soil & EPA6010 & REG & $7440-41-7$ & Beryllium & 0.44 & MG/KG & B & 0.52 \\
\hline 383MB0300.5 & $6 / 11 / 2004$ & soil & HASL300 & REG & $14913-49-6$ & $\mathrm{Bi}-212$ & 1.77 & $\mathrm{PCl} / \mathrm{G}$ & $\mathrm{TI}$ & 1.03 \\
\hline 383MB0300.5 & $6 / 11 / 2004$ & soil & HASL300 & REG & $14733-03-0$ & $\mathrm{Bi}-214$ & 0.39 & $\mathrm{PCl} / \mathrm{G}$ & $\mathrm{J}$ & 0.21 \\
\hline 383MB0300.5 & $6 / 11 / 2004$ & soil & EPA6010 & REG & $7440-43-9$ & Cadmium & 0.5 & MG/KG & $B$ & 0.52 \\
\hline 383MB0300.5 & $6 / 11 / 2004$ & soil & EPA6010 & REG & $7440-47-3$ & Chromium & 4.1 & MG/KG & & 1 \\
\hline 383MB0300.5 & $6 / 11 / 2004$ & soil & EPA8270 & REG & $218-01-9$ & Chrysene & 120 & UG/KG & $\mathrm{J}$ & 340 \\
\hline 383MB0300.5 & $6 / 11 / 2004$ & soil & HASL300 & REG & $10045-97-3$ & Cs-137 & 24.2 & $\mathrm{PCl} / \mathrm{G}$ & & 0.1 \\
\hline 383MB0300.5 & $6 / 11 / 2004$ & soil & EPA8015 & REG & $68334-30-5$ & Diesel Range Organics & 3.2 & MG/KG & $\mathrm{J}$ & 5.2 \\
\hline 383MB0300.5 & $6 / 11 / 2004$ & soil & EPA8270 & REG & $206-44-0$ & Fluoranthene & 110 & UG/KG & $\mathrm{J}$ & 340 \\
\hline 383MB0300.5 & $6 / 11 / 2004$ & soil & EPA8270 & REG & 193-39-5 & Indeno(1,2,3-Cd)Pyrene & 45 & UG/KG & $\mathrm{J}$ & 340 \\
\hline 383MB0300.5 & $6 / 11 / 2004$ & soil & HASL300 & REG & $13966-00-2$ & $\mathrm{~K}-40$ & 8.4 & $\mathrm{PCl} / \mathrm{G}$ & & 0.7 \\
\hline 383MB0300.5 & $6 / 11 / 2004$ & soil & EPA6010 & REG & $7439-92-1$ & Lead & 18 & MG/KG & & 1.6 \\
\hline 383MB0300.5 & $6 / 11 / 2004$ & soil & EPA7470 & REG & 7439-97-6 & Mercury & 0.11 & MG/KG & & 0.1 \\
\hline 383MB0300.5 & $6 / 11 / 2004$ & soil & HASL300 & REG & 15092-94-1 & $\mathrm{Pb}-212$ & 0.79 & $\mathrm{PCl} / \mathrm{G}$ & & 0.24 \\
\hline 383MB0300.5 & $6 / 11 / 2004$ & soil & HASL300 & REG & $15067-28-4$ & $\mathrm{~Pb}-214$ & 0.53 & $\mathrm{PCl} / \mathrm{G}$ & $\mathrm{J}$ & 0.33 \\
\hline 383MB0300.5 & $6 / 11 / 2004$ & soil & EPA8270 & REG & 85-01-8 & Phenanthrene & 30 & UG/KG & $\mathrm{J}$ & 340 \\
\hline 383MB0300.5 & $6 / 11 / 2004$ & soil & UGTAISOPU & REG & 13981-16-3 & Pu-238 & 0.158 & $\mathrm{PCl} / \mathrm{G}$ & & 0.011 \\
\hline 383MB0300.5 & $6 / 11 / 2004$ & soil & UGTAISOPU & REG & 15117-48-3 & Pu-239 & 1.35 & $\mathrm{PCl} / \mathrm{G}$ & & 0.03 \\
\hline
\end{tabular}




\begin{tabular}{|c|c|c|c|c|c|c|c|c|c|c|}
\hline SAMPLE \# & $\begin{array}{l}\text { SAMPLE } \\
\text { DATE }\end{array}$ & MATRIX & $\begin{array}{l}\text { USER TEST } \\
\text { PANEL }\end{array}$ & $\begin{array}{l}\text { SAMPLE } \\
\text { PURPOSE }\end{array}$ & CAS \# & PARAMETER & RESULT & UNITS & Q & $\begin{array}{l}\text { DETECT } \\
\text { LIMIT }\end{array}$ \\
\hline 383MB0300.5 & $6 / 11 / 2004$ & soil & EPA8270 & REG & $129-00-0$ & Pyrene & 100 & UG/KG & $\mathrm{J}$ & 340 \\
\hline 383MB0300.5 & $6 / 11 / 2004$ & soil & HASL300 & REG & 14913-50-9 & TI-208 & 0.131 & $\mathrm{PCl} / \mathrm{G}$ & & 0.117 \\
\hline 383MB0305.5 & $6 / 11 / 2004$ & soil & EPA6010 & REG & $7440-38-2$ & Arsenic & 2.8 & MG/KG & & 1 \\
\hline 383MB0305.5 & $6 / 11 / 2004$ & soil & EPA6010 & REG & $7440-39-3$ & Barium & 45 & MG/KG & & 10 \\
\hline 383MB0305.5 & $6 / 11 / 2004$ & soil & EPA8270 & REG & $56-55-3$ & Benzo(A)Anthracene & 22 & UG/KG & $\mathrm{J}$ & 340 \\
\hline 383MB0305.5 & $6 / 11 / 2004$ & soil & EPA8270 & REG & $50-32-8$ & Benzo(A)Pyrene & 16 & UG/KG & $\mathrm{J}$ & 340 \\
\hline 383MB0305.5 & $6 / 11 / 2004$ & soil & EPA8270 & REG & $205-99-2$ & Benzo(B)Fluoranthene & 28 & UG/KG & $\mathrm{J}$ & 340 \\
\hline 383MB0305.5 & $6 / 11 / 2004$ & soil & EPA6010 & REG & $7440-41-7$ & Beryllium & 0.3 & MG/KG & B & 0.51 \\
\hline 383MB0305.5 & $6 / 11 / 2004$ & soil & HASL300 & REG & 14733-03-0 & Bi-214 & 0.26 & $\mathrm{PCl} / \mathrm{G}$ & $\mathrm{J}$ & 0.17 \\
\hline 383MB0305.5 & $6 / 11 / 2004$ & soil & EPA6010 & REG & $7440-43-9$ & Cadmium & 0.15 & MG/KG & B & 0.51 \\
\hline 383MB0305.5 & $6 / 11 / 2004$ & soil & EPA6010 & REG & $7440-47-3$ & Chromium & 2.8 & MG/KG & & 1 \\
\hline 383MB0305.5 & $6 / 11 / 2004$ & soil & EPA8270 & REG & $218-01-9$ & Chrysene & 23 & UG/KG & $\mathrm{J}$ & 340 \\
\hline 383MB0305.5 & $6 / 11 / 2004$ & soil & HASL300 & REG & $10045-97-3$ & Cs-137 & 4.65 & $\mathrm{PCl} / \mathrm{G}$ & & 0.09 \\
\hline 383MB0305.5 & $6 / 11 / 2004$ & soil & EPA8270 & REG & $206-44-0$ & Fluoranthene & 23 & UG/KG & $\mathrm{J}$ & 340 \\
\hline 383MB0305.5 & $6 / 11 / 2004$ & soil & HASL300 & REG & 13966-00-2 & $\mathrm{K}-40$ & 2.5 & $\mathrm{PCl} / \mathrm{G}$ & & 1.08 \\
\hline 383MB0305.5 & $6 / 11 / 2004$ & soil & EPA6010 & REG & $7439-92-1$ & Lead & 6.1 & MG/KG & & 1.5 \\
\hline 383MB0305.5 & $6 / 11 / 2004$ & soil & EPA7470 & REG & 7439-97-6 & Mercury & 0.2 & MG/KG & & 0.1 \\
\hline 383MB0305.5 & $6 / 11 / 2004$ & soil & HASL300 & REG & 15092-94-1 & $\mathrm{Pb}-212$ & 0.3 & $\mathrm{PCl} / \mathrm{G}$ & & 0.14 \\
\hline 383MB0305.5 & $6 / 11 / 2004$ & soil & HASL300 & REG & $15067-28-4$ & $\mathrm{~Pb}-214$ & 0.33 & $\mathrm{PCl} / \mathrm{G}$ & $\mathrm{J}$ & 0.22 \\
\hline 383MB0305.5 & $6 / 11 / 2004$ & soil & UGTAISOPU & REG & 13981-16-3 & Pu-238 & 0.102 & $\mathrm{PCl} / \mathrm{G}$ & & 0.011 \\
\hline 383MB0305.5 & $6 / 11 / 2004$ & soil & UGTAISOPU & REG & 15117-48-3 & Pu-239 & 0.79 & $\mathrm{PCl} / \mathrm{G}$ & & 0.02 \\
\hline 383MB0305.5 & $6 / 11 / 2004$ & soil & HASL300 & REG & 14913-50-9 & TI-208 & 0.103 & $\mathrm{PCl} / \mathrm{G}$ & & 0.098 \\
\hline 383MB0400.5 & $6 / 1 / 2004$ & soil & HASL300 & REG & $14331-83-0$ & Ac-228 & 2.89 & $\mathrm{PCl} / \mathrm{G}$ & $\mathrm{G}, \mathrm{Tl}$ & 0.53 \\
\hline 383MB0400.5 & $6 / 1 / 2004$ & soil & HASL300 & REG & 14596-10-2 & Am-241 & 6.2 & $\mathrm{PCl} / \mathrm{G}$ & $\mathrm{J}$ & 3.1 \\
\hline 383MB0400.5 & $6 / 1 / 2004$ & soil & EPA6010 & REG & $7440-38-2$ & Arsenic & 2.9 & MG/KG & & 1.1 \\
\hline 383MB0400.5 & $6 / 1 / 2004$ & soil & EPA6010 & REG & $7440-39-3$ & Barium & 440 & MG/KG & & 11 \\
\hline 383MB0400.5 & $6 / 1 / 2004$ & soil & EPA8270 & REG & $56-55-3$ & Benzo(A)Anthracene & 90 & UG/KG & $\mathrm{J}$ & 360 \\
\hline 383MB0400.5 & $6 / 1 / 2004$ & soil & EPA8270 & REG & $50-32-8$ & Benzo(A)Pyrene & 73 & UG/KG & $\mathrm{J}$ & 360 \\
\hline 383MB0400.5 & $6 / 1 / 2004$ & soil & EPA8270 & REG & $205-99-2$ & Benzo(B)Fluoranthene & 130 & UG/KG & $\mathrm{J}$ & 360 \\
\hline 383MB0400.5 & $6 / 1 / 2004$ & soil & EPA8270 & REG & $207-08-9$ & Benzo(K)Fluoranthene & 60 & UG/KG & $\mathrm{J}$ & 360 \\
\hline 383MB0400.5 & $6 / 1 / 2004$ & soil & EPA6010 & REG & $7440-41-7$ & Beryllium & 0.92 & MG/KG & & 0.53 \\
\hline 383MB0400.5 & $6 / 1 / 2004$ & soil & EPA6010 & REG & $7440-43-9$ & Cadmium & 15 & MG/KG & & 0.53 \\
\hline 383MB0400.5 & 6/1/2004 & soil & EPA6010 & REG & $7440-47-3$ & Chromium & 2.2 & MG/KG & & 1.1 \\
\hline 383MB0400.5 & 6/1/2004 & soil & EPA8270 & REG & $218-01-9$ & Chrysene & 95 & UG/KG & $\mathrm{J}$ & 360 \\
\hline 383MB0400.5 & $6 / 1 / 2004$ & soil & HASL300 & REG & 10045-97-3 & Cs-137 & 393 & $\mathrm{PCl} / \mathrm{G}$ & $\mathrm{M} 3, \mathrm{G}$ & 1 \\
\hline
\end{tabular}




\begin{tabular}{|c|c|c|c|c|c|c|c|c|c|c|}
\hline SAMPLE \# & $\begin{array}{l}\text { SAMPLE } \\
\text { DATE }\end{array}$ & MATRIX & $\begin{array}{l}\text { USER TEST } \\
\text { PANEL }\end{array}$ & $\begin{array}{l}\text { SAMPLE } \\
\text { PURPOSE }\end{array}$ & CAS \# & PARAMETER & RESULT & UNITS & Q & $\begin{array}{l}\text { DETECT } \\
\text { LIMIT }\end{array}$ \\
\hline 383MB0400.5 & $6 / 1 / 2004$ & soil & EPA8270 & REG & $206-44-0$ & Fluoranthene & 98 & UG/KG & $\mathrm{J}$ & 360 \\
\hline 383MB0400.5 & $6 / 1 / 2004$ & soil & HASL300 & REG & 13966-00-2 & $\mathrm{K}-40$ & 34.2 & $\mathrm{PCl} / \mathrm{G}$ & G & 1.6 \\
\hline 383MB0400.5 & 6/1/2004 & soil & EPA6010 & REG & $7439-92-1$ & Lead & 28 & MG/KG & & 0.32 \\
\hline 383MB0400.5 & $6 / 1 / 2004$ & soil & HASL300 & REG & 15092-94-1 & $\mathrm{Pb}-212$ & 2.22 & $\mathrm{PCl} / \mathrm{G}$ & $\mathrm{J}$ & 1.07 \\
\hline 383MB0400.5 & $6 / 1 / 2004$ & soil & EPA8270 & REG & $85-01-8$ & Phenanthrene & 37 & UG/KG & $\mathrm{J}$ & 360 \\
\hline 383MB0400.5 & $6 / 1 / 2004$ & soil & UGTAISOPU & REG & 13981-16-3 & Pu-238 & 1.86 & $\mathrm{PCl} / \mathrm{G}$ & & 0.02 \\
\hline 383MB0400.5 & 6/1/2004 & soil & UGTAISOPU & REG & 15117-48-3 & Pu-239 & 17.8 & $\mathrm{PCl} / \mathrm{G}$ & $\mathrm{J}$ & 0 \\
\hline 383MB0400.5 & $6 / 1 / 2004$ & soil & EPA8270 & REG & $129-00-0$ & Pyrene & 91 & UG/KG & $\mathrm{J}$ & 360 \\
\hline 383MB0400.5 & $6 / 1 / 2004$ & soil & EPA6010 & REG & $7782-49-2$ & Selenium & 0.38 & MG/KG & $B$ & 0.53 \\
\hline 383MB0400.5 & $6 / 1 / 2004$ & soil & SR7500 & REG & 10098-97-2 & Sr-90 & 2.5 & $\mathrm{PCl} / \mathrm{G}$ & Y1 & 0.13 \\
\hline 383MB0500.5 & $6 / 3 / 2004$ & soil & HASL300 & REG & 14331-83-0 & Ac-228 & 2 & $\mathrm{PCl} / \mathrm{G}$ & G & 0.75 \\
\hline 383MB0500.5 & $6 / 3 / 2004$ & soil & EPA6010 & REG & 7440-38-2 & Arsenic & 3.2 & MG/KG & & 1.1 \\
\hline 383MB0500.5 & $6 / 3 / 2004$ & soil & EPA6010 & REG & 7440-39-3 & Barium & 2500 & MG/KG & & 110 \\
\hline 383MB0500.5 & $6 / 3 / 2004$ & soil & EPA6010 & REG & $7440-41-7$ & Beryllium & 1 & MG/KG & & 0.56 \\
\hline 383MB0500.5 & $6 / 3 / 2004$ & soil & HASL300 & REG & 14733-03-0 & $\mathrm{Bi}-214$ & 1.1 & $\mathrm{PCl} / \mathrm{G}$ & $\mathrm{G}, \mathrm{J}$ & 0.45 \\
\hline 383MB0500.5 & $6 / 3 / 2004$ & soil & EPA6010 & REG & $7440-43-9$ & Cadmium & 0.2 & MG/KG & $B$ & 0.56 \\
\hline 383MB0500.5 & $6 / 3 / 2004$ & soil & EPA6010 & REG & $7440-47-3$ & Chromium & 3 & MG/KG & & 1.1 \\
\hline 383MB0500.5 & $6 / 3 / 2004$ & soil & HASL300 & REG & 10045-97-3 & Cs-137 & 29.3 & $\mathrm{PCl} / \mathrm{G}$ & G & 0.2 \\
\hline 383MB0500.5 & $6 / 3 / 2004$ & soil & EPA8015 & REG & 68334-30-5 & Diesel Range Organics & 160 & MG/KG & $\mathrm{Z}, \mathrm{H}$ & 5.6 \\
\hline 383MB0500.5 & $6 / 3 / 2004$ & soil & HASL300 & REG & 13966-00-2 & $\mathrm{K}-40$ & 24.6 & $\mathrm{PCl} / \mathrm{G}$ & G & 1.9 \\
\hline 383MB0500.5 & $6 / 3 / 2004$ & soil & EPA6010 & REG & $7439-92-1$ & Lead & 29 & MG/KG & & 0.34 \\
\hline 383MB0500.5 & $6 / 3 / 2004$ & soil & EPA7470 & REG & 7439-97-6 & Mercury & 0.016 & MG/KG & $B$ & 0.11 \\
\hline 383MB0500.5 & $6 / 3 / 2004$ & soil & EPA8260 & REG & $75-09-2$ & Methylene Chloride & 1.1 & UG/KG & $\mathrm{J}$ & 5.6 \\
\hline 383MB0500.5 & $6 / 3 / 2004$ & soil & EPA8260 & REG & $95-47-6$ & O-Xylene & 1.2 & UG/KG & $\mathrm{J}$ & 5.6 \\
\hline 383MB0500.5 & $6 / 3 / 2004$ & soil & HASL300 & REG & 15092-94-1 & $\mathrm{Pb}-212$ & 2.03 & $\mathrm{PCl} / \mathrm{G}$ & $\mathrm{J}$ & 0.33 \\
\hline 383MB0500.5 & $6 / 3 / 2004$ & soil & HASL300 & REG & 15067-28-4 & $\mathrm{Pb}-214$ & 1.37 & $\mathrm{PCl} / \mathrm{G}$ & $\mathrm{G}, \mathrm{J}$ & 0.52 \\
\hline 383MB0500.5 & $6 / 3 / 2004$ & soil & UGTAISOPU & REG & 13981-16-3 & Pu-238 & 0.191 & $\mathrm{PCl} / \mathrm{G}$ & & 0.011 \\
\hline 383MB0500.5 & $6 / 3 / 2004$ & soil & UGTAISOPU & REG & 15117-48-3 & Pu-239 & 0.73 & $\mathrm{PCl} / \mathrm{G}$ & $\mathrm{J}$ & 0.02 \\
\hline 383MB0500.5 & $6 / 3 / 2004$ & soil & SR7500 & REG & 10098-97-2 & Sr-90 & 0.51 & $\mathrm{PCl} / \mathrm{G}$ & Y1 & 0.25 \\
\hline 383MB0500.5 & $6 / 3 / 2004$ & soil & HASL300 & REG & 14913-50-9 & TI-208 & 0.57 & $\mathrm{PCl} / \mathrm{G}$ & G & 0.2 \\
\hline 383MB0543.5 & $6 / 3 / 2004$ & soil & HASL300 & REG & 14331-83-0 & Ac-228 & 2.81 & $\mathrm{PCl} / \mathrm{G}$ & G & 0.78 \\
\hline 383MB0543.5 & $6 / 3 / 2004$ & soil & HASL300 & REG & 14596-10-2 & Am-241 & 28.8 & $\mathrm{PCl} / \mathrm{G}$ & $\mathrm{J}$ & 1.6 \\
\hline 383MB0543.5 & $6 / 3 / 2004$ & soil & EPA6010 & REG & $7440-38-2$ & Arsenic & 2.2 & MG/KG & & 1.2 \\
\hline 383MB0543.5 & $6 / 3 / 2004$ & soil & EPA6010 & REG & $7440-39-3$ & Barium & 85 & MG/KG & & 12 \\
\hline 383MB0543.5 & $6 / 3 / 2004$ & soil & EPA6010 & REG & $7440-41-7$ & Beryllium & 1 & MG/KG & & 0.59 \\
\hline
\end{tabular}




\begin{tabular}{|c|c|c|c|c|c|c|c|c|c|c|}
\hline SAMPLE \# & $\begin{array}{l}\text { SAMPLE } \\
\text { DATE }\end{array}$ & MATRIX & $\begin{array}{l}\text { USER TEST } \\
\text { PANEL }\end{array}$ & $\begin{array}{l}\text { SAMPLE } \\
\text { PURPOSE }\end{array}$ & CAS \# & PARAMETER & RESULT & UNITS & Q & $\begin{array}{l}\text { DETECT } \\
\text { LIMIT }\end{array}$ \\
\hline 383MB0543.5 & $6 / 3 / 2004$ & soil & EPA6010 & REG & $7440-43-9$ & Cadmium & 0.078 & MG/KG & B & 0.59 \\
\hline 383MB0543.5 & $6 / 3 / 2004$ & soil & EPA6010 & REG & $7440-47-3$ & Chromium & 1.1 & MG/KG & B & 1.2 \\
\hline 383MB0543.5 & $6 / 3 / 2004$ & soil & HASL300 & REG & $10045-97-3$ & Cs-137 & 1060 & $\mathrm{PCl} / \mathrm{G}$ & M3,G & 0 \\
\hline 383MB0543.5 & $6 / 3 / 2004$ & soil & HASL300 & REG & 14683-23-9 & Eu-152 & 2.64 & $\mathrm{PCl} / \mathrm{G}$ & G & 0.81 \\
\hline 383MB0543.5 & $6 / 3 / 2004$ & soil & HASL300 & REG & 13966-00-2 & $\mathrm{K}-40$ & 39.8 & $\mathrm{PCl} / \mathrm{G}$ & G & 1.7 \\
\hline 383MB0543.5 & $6 / 3 / 2004$ & soil & EPA6010 & REG & 7439-92-1 & Lead & 22 & MG/KG & & 0.36 \\
\hline 383MB0543.5 & $6 / 3 / 2004$ & soil & EPA7470 & REG & 7439-97-6 & Mercury & 0.0098 & MG/KG & B & 0.12 \\
\hline 383MB0543.5 & $6 / 3 / 2004$ & soil & EPA8260 & REG & $75-09-2$ & Methylene Chloride & 0.96 & UG/KG & $\mathrm{J}$ & 5.9 \\
\hline 383MB0543.5 & $6 / 3 / 2004$ & soil & UGTAISOPU & REG & 13981-16-3 & Pu-238 & 15.6 & $\mathrm{PCl} / \mathrm{G}$ & $\mathrm{J}$ & 0 \\
\hline 383MB0543.5 & $6 / 3 / 2004$ & soil & UGTAISOPU & REG & $15117-48-3$ & Pu-239 & 84 & $\mathrm{PCl} / \mathrm{G}$ & $\mathrm{J}$ & 0 \\
\hline 383MB0543.5 & $6 / 3 / 2004$ & soil & SR7500 & REG & 10098-97-2 & Sr-90 & 34.1 & $\mathrm{PCl} / \mathrm{G}$ & & 0.2 \\
\hline 383MB0586.0 & $6 / 3 / 2004$ & soil & EPA8260 & REG & $67-64-1$ & Acetone & 8.8 & UG/KG & $\mathrm{J}$ & 20 \\
\hline 383MB0586.0 & $6 / 3 / 2004$ & soil & EPA6010 & REG & $7440-38-2$ & Arsenic & 0.31 & MG/KG & $B$ & 1 \\
\hline 383MB0586.0 & $6 / 3 / 2004$ & soil & EPA6010 & REG & $7440-39-3$ & Barium & 4.8 & MG/KG & B & 10 \\
\hline 383MB0586.0 & $6 / 3 / 2004$ & soil & EPA6010 & REG & $7440-41-7$ & Beryllium & 0.2 & MG/KG & $B$ & 0.5 \\
\hline 383MB0586.0 & $6 / 3 / 2004$ & soil & EPA6010 & REG & $7440-43-9$ & Cadmium & 0.042 & MG/KG & B & 0.5 \\
\hline 383MB0586.0 & $6 / 3 / 2004$ & soil & EPA6010 & REG & 7440-47-3 & Chromium & 1.4 & MG/KG & & 1 \\
\hline 383MB0586.0 & $6 / 3 / 2004$ & soil & EPA7470 & REG & 7439-97-6 & Mercury & 0.008 & MG/KG & $B$ & 0.1 \\
\hline 383MB0586.0 & $6 / 3 / 2004$ & soil & EPA8260 & REG & $75-09-2$ & Methylene Chloride & 1.3 & UG/KG & $\mathrm{J}$ & 5 \\
\hline 383MB0586.0 & $6 / 3 / 2004$ & soil & HASL300 & REG & $15067-28-4$ & $\mathrm{~Pb}-214$ & 0.313 & $\mathrm{PCl} / \mathrm{G}$ & $\mathrm{J}$ & 0.122 \\
\hline 383MB0586.0 & $6 / 3 / 2004$ & soil & EPA6010 & REG & $7440-22-4$ & Silver & 0.19 & MG/KG & $B$ & 1 \\
\hline 383MB0600.5 & $6 / 12 / 2004$ & soil & HASL300 & REG & 14331-83-0 & Ac-228 & 1.01 & $\mathrm{PCl} / \mathrm{G}$ & G & 0.51 \\
\hline 383MB0600.5 & $6 / 12 / 2004$ & soil & EPA8260 & REG & $67-64-1$ & Acetone & 8.1 & UG/KG & $\mathrm{J}$ & 24 \\
\hline 383MB0600.5 & $6 / 12 / 2004$ & soil & EPA6010 & REG & $7440-38-2$ & Arsenic & 4.4 & MG/KG & & 1.2 \\
\hline 383MB0600.5 & $6 / 12 / 2004$ & soil & EPA6010 & REG & $7440-39-3$ & Barium & 7000 & MG/KG & & 600 \\
\hline 383MB0600.5 & $6 / 12 / 2004$ & soil & EPA6010 & REG & $7440-41-7$ & Beryllium & 0.94 & MG/KG & & 0.6 \\
\hline 383MB0600.5 & $6 / 12 / 2004$ & soil & HASL300 & REG & 14733-03-0 & $\mathrm{Bi}-214$ & 1.09 & $\mathrm{PCl} / \mathrm{G}$ & $\mathrm{G}, \mathrm{J}$ & 0.29 \\
\hline 383MB0600.5 & $6 / 12 / 2004$ & soil & EPA6010 & REG & $7440-43-9$ & Cadmium & 0.13 & MG/KG & $B$ & 0.6 \\
\hline 383MB0600.5 & $6 / 12 / 2004$ & soil & EPA6010 & REG & $7440-47-3$ & Chromium & 6.8 & MG/KG & & 1.2 \\
\hline 383MB0600.5 & $6 / 12 / 2004$ & soil & HASL300 & REG & 10045-97-3 & Cs-137 & 0.6 & $\mathrm{PCl} / \mathrm{G}$ & G & 0.13 \\
\hline 383MB0600.5 & $6 / 12 / 2004$ & soil & HASL300 & REG & 13966-00-2 & $\mathrm{K}-40$ & 27.7 & $\mathrm{PCl} / \mathrm{G}$ & G & 1.6 \\
\hline 383MB0600.5 & $6 / 12 / 2004$ & soil & EPA6010 & REG & 7439-92-1 & Lead & 22 & MG/KG & & 0.36 \\
\hline 383MB0600.5 & $6 / 12 / 2004$ & soil & EPA7470 & REG & 7439-97-6 & Mercury & 0.0086 & MG/KG & $B$ & 0.12 \\
\hline 383MB0600.5 & $6 / 12 / 2004$ & soil & EPA8260 & REG & $75-09-2$ & Methylene Chloride & 0.99 & UG/KG & $\mathrm{J}, \mathrm{B}$ & 6 \\
\hline 383MB0600.5 & $6 / 12 / 2004$ & soil & HASL300 & REG & 15092-94-1 & $\mathrm{Pb}-212$ & 1.6 & $\mathrm{PCl} / \mathrm{G}$ & $\mathrm{J}$ & 0.29 \\
\hline
\end{tabular}




\begin{tabular}{|c|c|c|c|c|c|c|c|c|c|c|}
\hline SAMPLE \# & $\begin{array}{l}\text { SAMPLE } \\
\text { DATE }\end{array}$ & MATRIX & $\begin{array}{l}\text { USER TEST } \\
\text { PANEL }\end{array}$ & $\begin{array}{l}\text { SAMPLE } \\
\text { PURPOSE }\end{array}$ & CAS \# & PARAMETER & RESULT & UNITS & Q & $\begin{array}{l}\text { DETECT } \\
\text { LIMIT }\end{array}$ \\
\hline 383MB0600.5 & $6 / 12 / 2004$ & soil & HASL300 & REG & 15067-28-4 & $\mathrm{Pb}-214$ & 1.14 & $\mathrm{PCl} / \mathrm{G}$ & $\mathrm{G}, \mathrm{J}$ & 0.27 \\
\hline 383MB0600.5 & $6 / 12 / 2004$ & soil & UGTAISOPU & REG & 15117-48-3 & Pu-239 & 0.08 & $\mathrm{PCl} / \mathrm{G}$ & & 0.009 \\
\hline 383MB0600.5 & $6 / 12 / 2004$ & soil & HASL300 & REG & 14913-50-9 & TI-208 & 0.55 & $\mathrm{PCl} / \mathrm{G}$ & $\mathrm{G}, \mathrm{TI}$ & 0.17 \\
\hline 383MB0600.5X & $6 / 12 / 2004$ & soil & HASL300 & REG & 14331-83-0 & Ac-228 & 1.68 & $\mathrm{PCl} / \mathrm{G}$ & G & 0.73 \\
\hline 383MB0600.5X & $6 / 12 / 2004$ & soil & EPA6010 & REG & $7440-38-2$ & Arsenic & 3.4 & MG/KG & & 1.2 \\
\hline 383MB0600.5X & $6 / 12 / 2004$ & soil & EPA6010 & REG & $7440-39-3$ & Barium & 6600 & MG/KG & & 590 \\
\hline 383MB0600.5X & $6 / 12 / 2004$ & soil & EPA6010 & REG & $7440-41-7$ & Beryllium & 0.91 & MG/KG & & 0.59 \\
\hline 383MB0600.5X & $6 / 12 / 2004$ & soil & HASL300 & REG & 14733-03-0 & $\mathrm{Bi}-214$ & 1.15 & $\mathrm{PCl} / \mathrm{G}$ & $\mathrm{G}, \mathrm{J}$ & 0.4 \\
\hline 383MB0600.5X & $6 / 12 / 2004$ & soil & EPA8270 & REG & 117-81-7 & Bis(2-Ethylhexyl)Phthalate & 630 & UG/KG & & 400 \\
\hline 383MB0600.5X & $6 / 12 / 2004$ & soil & EPA6010 & REG & $7440-43-9$ & Cadmium & 0.12 & MG/KG & B & 0.59 \\
\hline 383MB0600.5X & $6 / 12 / 2004$ & soil & EPA6010 & REG & $7440-47-3$ & Chromium & 5.8 & MG/KG & & 1.2 \\
\hline 383MB0600.5X & $6 / 12 / 2004$ & soil & HASL300 & REG & $10045-97-3$ & Cs-137 & 0.67 & $\mathrm{PCl} / \mathrm{G}$ & G & 0.16 \\
\hline 383MB0600.5X & $6 / 12 / 2004$ & soil & HASL300 & REG & 13966-00-2 & $\mathrm{K}-40$ & 30 & $\mathrm{PCl} / \mathrm{G}$ & G & 1.7 \\
\hline 383MB0600.5X & $6 / 12 / 2004$ & soil & EPA6010 & REG & $7439-92-1$ & Lead & 22 & MG/KG & & 0.36 \\
\hline 383MB0600.5X & $6 / 12 / 2004$ & soil & EPA7470 & REG & 7439-97-6 & Mercury & 0.011 & MG/KG & $B$ & 0.12 \\
\hline 383MB0600.5X & $6 / 12 / 2004$ & soil & EPA8260 & REG & $75-09-2$ & Methylene Chloride & 1.1 & UG/KG & $\mathrm{J}, \mathrm{B}$ & 5.9 \\
\hline 383MB0600.5X & $6 / 12 / 2004$ & soil & HASL300 & REG & 15092-94-1 & $\mathrm{Pb}-212$ & 1.85 & $\mathrm{PCl} / \mathrm{G}$ & $\mathrm{J}$ & 0.24 \\
\hline 383MB0600.5X & $6 / 12 / 2004$ & soil & HASL300 & REG & 15067-28-4 & $\mathrm{Pb}-214$ & 1.14 & $\mathrm{PCl} / \mathrm{G}$ & $\mathrm{G}, \mathrm{J}$ & 0.32 \\
\hline 383MB0600.5X & $6 / 12 / 2004$ & soil & UGTAISOPU & REG & 15117-48-3 & Pu-239 & 0.068 & $\mathrm{PCl} / \mathrm{G}$ & & 0.023 \\
\hline 383MB0600.5X & $6 / 12 / 2004$ & soil & SR7500 & REG & 10098-97-2 & Sr-90 & 0.53 & $\mathrm{PCl} / \mathrm{G}$ & & 0.23 \\
\hline 383MB0600.5X & $6 / 12 / 2004$ & soil & HASL300 & REG & 14913-50-9 & TI-208 & 0.57 & $\mathrm{PCl} / \mathrm{G}$ & G & 0.17 \\
\hline 383MB0700.5 & $5 / 19 / 2004$ & soil & HASL300 & REG & 14331-83-0 & Ac-228 & 0.72 & $\mathrm{PCl} / \mathrm{G}$ & & 0.39 \\
\hline 383MB0700.5 & $5 / 19 / 2004$ & soil & EPA6010 & REG & $7440-38-2$ & Arsenic & 2.4 & MG/KG & & 1 \\
\hline 383MB0700.5 & $5 / 19 / 2004$ & soil & EPA6010 & REG & 7440-39-3 & Barium & 73 & MG/KG & & 10 \\
\hline 383MB0700.5 & $5 / 19 / 2004$ & soil & EPA6010 & REG & $7440-41-7$ & Beryllium & 0.5 & MG/KG & $B$ & 0.52 \\
\hline 383MB0700.5 & $5 / 19 / 2004$ & soil & HASL300 & REG & 14733-03-0 & Bi-214 & 0.63 & $\mathrm{PCl} / \mathrm{G}$ & $\mathrm{J}$ & 0.23 \\
\hline 383MB0700.5 & $5 / 19 / 2004$ & soil & EPA6010 & REG & $7440-43-9$ & Cadmium & 0.065 & MG/KG & $B$ & 0.52 \\
\hline 383MB0700.5 & $5 / 19 / 2004$ & soil & EPA6010 & REG & $7440-47-3$ & Chromium & 3.5 & MG/KG & & 1 \\
\hline 383MB0700.5 & $5 / 19 / 2004$ & soil & HASL300 & REG & $10045-97-3$ & Cs-137 & 0.58 & $\mathrm{PCl} / \mathrm{G}$ & & 0.09 \\
\hline 383MB0700.5 & $5 / 19 / 2004$ & soil & EPA8270 & REG & 84-74-2 & Di-N-Butyl Phthalate & 26 & UG/KG & $\mathrm{J}$ & 350 \\
\hline 383MB0700.5 & $5 / 19 / 2004$ & soil & HASL300 & REG & 13966-00-2 & $\mathrm{K}-40$ & 6.6 & $\mathrm{PCl} / \mathrm{G}$ & & 1.2 \\
\hline 383MB0700.5 & $5 / 19 / 2004$ & soil & EPA6010 & REG & 7439-92-1 & Lead & 17 & MG/KG & & 1.6 \\
\hline 383MB0700.5 & $5 / 19 / 2004$ & soil & EPA7470 & REG & 7439-97-6 & Mercury & 0.11 & MG/KG & & 0.1 \\
\hline 383MB0700.5 & $5 / 19 / 2004$ & soil & HASL300 & REG & 15092-94-1 & $\mathrm{Pb}-212$ & 0.68 & $\mathrm{PCl} / \mathrm{G}$ & $\mathrm{J}$ & 0.14 \\
\hline 383MB0700.5 & $5 / 19 / 2004$ & soil & HASL300 & REG & 15067-28-4 & $\mathrm{Pb}-214$ & 0.74 & $\mathrm{PCl} / \mathrm{G}$ & $\mathrm{J}$ & 0.15 \\
\hline
\end{tabular}




\begin{tabular}{|c|c|c|c|c|c|c|c|c|c|c|}
\hline SAMPLE \# & $\begin{array}{l}\text { SAMPLE } \\
\text { DATE }\end{array}$ & MATRIX & $\begin{array}{l}\text { USER TEST } \\
\text { PANEL }\end{array}$ & $\begin{array}{l}\text { SAMPLE } \\
\text { PURPOSE }\end{array}$ & CAS \# & PARAMETER & RESULT & UNITS & Q & $\begin{array}{l}\text { DETECT } \\
\text { LIMIT }\end{array}$ \\
\hline 383MB0700.5 & $5 / 19 / 2004$ & soil & UGTAISOPU & REG & 13981-16-3 & Pu-238 & 0.049 & $\mathrm{PCl} / \mathrm{G}$ & $\mathrm{LT}$ & 0.01 \\
\hline 383MB0700.5 & $5 / 19 / 2004$ & soil & UGTAISOPU & REG & 15117-48-3 & Pu-239 & 0.217 & $\mathrm{PCl} / \mathrm{G}$ & & 0.01 \\
\hline 383MB0700.5 & $5 / 19 / 2004$ & soil & HASL300 & REG & 14913-50-9 & TI-208 & 0.166 & $\mathrm{PCl} / \mathrm{G}$ & & 0.085 \\
\hline 383MB0800.5 & $5 / 24 / 2004$ & soil & EPA8260 & REG & $95-63-6$ & 1,2,4-Trimethylbenzene & 7.2 & UG/KG & & 5.9 \\
\hline 383MB0800.5 & $5 / 24 / 2004$ & soil & EPA8260 & REG & $108-67-8$ & 1,3,5-Trimethylbenzene & 7.2 & UG/KG & & 5.9 \\
\hline 383MB0800.5 & $5 / 24 / 2004$ & soil & HASL300 & REG & 14331-83-0 & Ac-228 & 1.69 & $\mathrm{PCl} / \mathrm{G}$ & G & 0.71 \\
\hline 383MB0800.5 & $5 / 24 / 2004$ & soil & EPA6010 & REG & $7440-38-2$ & Arsenic & 4.1 & MG/KG & & 1.2 \\
\hline 383MB0800.5 & $5 / 24 / 2004$ & soil & EPA6010 & REG & $7440-39-3$ & Barium & 5000 & MG/KG & & 120 \\
\hline 383MB0800.5 & $5 / 24 / 2004$ & soil & EPA6010 & REG & $7440-41-7$ & Beryllium & 0.77 & MG/KG & & 0.59 \\
\hline 383MB0800.5 & $5 / 24 / 2004$ & soil & HASL300 & REG & 14733-03-0 & Bi-214 & 0.89 & $\mathrm{PCl} / \mathrm{G}$ & $\mathrm{G}, \mathrm{J}$ & 0.31 \\
\hline 383MB0800.5 & $5 / 24 / 2004$ & soil & EPA6010 & REG & $7440-43-9$ & Cadmium & 2.1 & MG/KG & & 0.59 \\
\hline 383MB0800.5 & $5 / 24 / 2004$ & soil & EPA6010 & REG & 7440-47-3 & Chromium & 8.6 & MG/KG & & 1.2 \\
\hline 383MB0800.5 & $5 / 24 / 2004$ & soil & HASL300 & REG & 10045-97-3 & Cs-137 & 3.16 & $\mathrm{PCl} / \mathrm{G}$ & G & 0.16 \\
\hline 383MB0800.5 & $5 / 24 / 2004$ & soil & EPA8015 & REG & 68334-30-5 & Diesel Range Organics & 300 & MG/KG & $L, H, M$ & 5.9 \\
\hline 383MB0800.5 & $5 / 24 / 2004$ & soil & EPA8260 & REG & $100-41-4$ & Ethylbenzene & 120 & UG/KG & & 5.9 \\
\hline 383MB0800.5 & $5 / 24 / 2004$ & soil & HASL300 & REG & 13966-00-2 & $\mathrm{K}-40$ & 22.2 & $\mathrm{PCl} / \mathrm{G}$ & G & 2 \\
\hline 383MB0800.5 & $5 / 24 / 2004$ & soil & EPA6010 & REG & 7439-92-1 & Lead & 53 & MG/KG & & 0.35 \\
\hline 383MB0800.5 & $5 / 24 / 2004$ & soil & EPA8260 & REG & $136777-61-2$ & M+P-Xylene & 1500 & UG/KG & $E$ & 5.9 \\
\hline 383MB0800.5 & $5 / 24 / 2004$ & soil & EPA7470 & REG & 7439-97-6 & Mercury & 0.055 & MG/KG & B & 0.12 \\
\hline 383MB0800.5 & $5 / 24 / 2004$ & soil & EPA8260 & REG & $75-09-2$ & Methylene Chloride & 1.9 & UG/KG & $\mathrm{J}, \mathrm{B}$ & 5.9 \\
\hline 383MB0800.5 & $5 / 24 / 2004$ & soil & EPA8260 & REG & $103-65-1$ & N-Propylbenzene & 5.9 & UG/KG & & 5.9 \\
\hline 383MB0800.5 & $5 / 24 / 2004$ & soil & EPA8260 & REG & $95-47-6$ & O-Xylene & 1000 & UG/KG & $E$ & 5.9 \\
\hline 383MB0800.5 & $5 / 24 / 2004$ & soil & HASL300 & REG & 15092-94-1 & $\mathrm{Pb}-212$ & 1.9 & $\mathrm{PCl} / \mathrm{G}$ & $\mathrm{J}$ & 0.31 \\
\hline 383MB0800.5 & $5 / 24 / 2004$ & soil & HASL300 & REG & 15067-28-4 & $\mathrm{Pb}-214$ & 0.95 & $\mathrm{PCl} / \mathrm{G}$ & $\mathrm{G}, \mathrm{J}$ & 0.32 \\
\hline 383MB0800.5 & $5 / 24 / 2004$ & soil & UGTAISOPU & REG & 13981-16-3 & Pu-238 & 0.156 & $\mathrm{PCl} / \mathrm{G}$ & & 0.009 \\
\hline 383MB0800.5 & $5 / 24 / 2004$ & soil & UGTAISOPU & REG & 15117-48-3 & Pu-239 & 0.66 & $\mathrm{PCl} / \mathrm{G}$ & & 0.02 \\
\hline 383MB0800.5 & $5 / 24 / 2004$ & soil & HASL300 & REG & 14913-50-9 & TI-208 & 0.47 & $\mathrm{PCl} / \mathrm{G}$ & G & 0.21 \\
\hline 383MB0800.5RR1 & $5 / 24 / 2004$ & soil & EPA8260 & $\mathrm{RE}$ & $100-41-4$ & Ethylbenzene & 80 & UG/KG & & 59 \\
\hline 383MB0800.5RR1 & $5 / 24 / 2004$ & soil & EPA8260 & $\mathrm{RE}$ & 136777-61-2 & M+P-Xylene & 1200 & UG/KG & & 59 \\
\hline 383MB0800.5RR1 & $5 / 24 / 2004$ & soil & EPA8260 & RE & $75-09-2$ & Methylene Chloride & 9.5 & UG/KG & $\mathrm{J}, \mathrm{B}$ & 59 \\
\hline 383MB0800.5RR1 & $5 / 24 / 2004$ & soil & EPA8260 & RE & $95-47-6$ & O-Xylene & 780 & UG/KG & & 59 \\
\hline 383MB0831.0 & $5 / 24 / 2004$ & soil & EPA8260 & REG & 67-64-1 & Acetone & 8.9 & UG/KG & $\mathrm{J}$ & 20 \\
\hline 383MB0831.0 & $5 / 24 / 2004$ & soil & EPA6010 & REG & $7440-38-2$ & Arsenic & 0.73 & MG/KG & $B$ & 1 \\
\hline 383MB0831.0 & $5 / 24 / 2004$ & soil & EPA6010 & REG & 7440-39-3 & Barium & 7.3 & MG/KG & $B$ & 10 \\
\hline 383MB0831.0 & $5 / 24 / 2004$ & soil & EPA6010 & REG & $7440-41-7$ & Beryllium & 0.25 & MG/KG & $B$ & 0.51 \\
\hline
\end{tabular}




\begin{tabular}{|c|c|c|c|c|c|c|c|c|c|c|}
\hline SAMPLE \# & $\begin{array}{l}\text { SAMPLE } \\
\text { DATE }\end{array}$ & MATRIX & $\begin{array}{l}\text { USER TEST } \\
\text { PANEL }\end{array}$ & $\begin{array}{l}\text { SAMPLE } \\
\text { PURPOSE }\end{array}$ & CAS \# & PARAMETER & RESULT & UNITS & $Q$ & $\begin{array}{l}\text { DETECT } \\
\text { LIMIT }\end{array}$ \\
\hline 383MB0831.0 & $5 / 24 / 2004$ & soil & EPA6010 & REG & $7440-43-9$ & Cadmium & 0.066 & MG/KG & B & 0.51 \\
\hline 383MB0831.0 & $5 / 24 / 2004$ & soil & EPA6010 & REG & $7440-47-3$ & Chromium & 0.94 & MG/KG & B & 1 \\
\hline 383MB0831.0 & $5 / 24 / 2004$ & soil & EPA7470 & REG & $7439-97-6$ & Mercury & 0.094 & MG/KG & B & 0.1 \\
\hline 383MB0831.0 & $5 / 24 / 2004$ & soil & EPA8260 & REG & $75-09-2$ & Methylene Chloride & 0.82 & UG/KG & $\mathrm{J}, \mathrm{B}$ & 5.1 \\
\hline 383MB0831.0 & $5 / 24 / 2004$ & soil & HASL300 & REG & $15067-28-4$ & $\mathrm{~Pb}-214$ & 0.238 & $\mathrm{PCl} / \mathrm{G}$ & $\mathrm{J}$ & 0.118 \\
\hline 383MB0900.5 & $6 / 12 / 2004$ & soil & HASL300 & REG & 14331-83-0 & Ac-228 & 2.45 & $\mathrm{PCl} / \mathrm{G}$ & $\mathrm{G}$ & 0.65 \\
\hline 383MB0900.5 & $6 / 12 / 2004$ & soil & EPA6010 & REG & $7440-38-2$ & Arsenic & 1.9 & MG/KG & & 1.1 \\
\hline 383MB0900.5 & $6 / 12 / 2004$ & soil & EPA6010 & REG & $7440-39-3$ & Barium & 120 & MG/KG & & 11 \\
\hline 383MB0900.5 & $6 / 12 / 2004$ & soil & EPA6010 & REG & $7440-41-7$ & Beryllium & 2.4 & MG/KG & & 0.57 \\
\hline 383MB0900.5 & $6 / 12 / 2004$ & soil & HASL300 & REG & 14733-03-0 & $\mathrm{Bi}-214$ & 2.08 & $\mathrm{PCl} / \mathrm{G}$ & $\mathrm{G}, \mathrm{J}$ & 0.38 \\
\hline 383MB0900.5 & $6 / 12 / 2004$ & soil & EPA6010 & REG & $7440-43-9$ & Cadmium & 0.1 & MG/KG & $B$ & 0.57 \\
\hline 383MB0900.5 & $6 / 12 / 2004$ & soil & EPA6010 & REG & $7440-47-3$ & Chromium & 0.89 & MG/KG & B & 1.1 \\
\hline 383MB0900.5 & $6 / 12 / 2004$ & soil & HASL300 & REG & $10045-97-3$ & Cs-137 & 0.27 & $\mathrm{PCl} / \mathrm{G}$ & LT,G & 0.15 \\
\hline 383MB0900.5 & $6 / 12 / 2004$ & soil & HASL300 & REG & $13966-00-2$ & $\mathrm{~K}-40$ & 30.9 & $\mathrm{PCl} / \mathrm{G}$ & G & 2.1 \\
\hline 383MB0900.5 & $6 / 12 / 2004$ & soil & EPA6010 & REG & 7439-92-1 & Lead & 19 & MG/KG & & 0.34 \\
\hline 383MB0900.5 & $6 / 12 / 2004$ & soil & EPA7470 & REG & $7439-97-6$ & Mercury & 0.0025 & MG/KG & B & 0.11 \\
\hline 383MB0900.5 & $6 / 12 / 2004$ & soil & EPA8260 & REG & $75-09-2$ & Methylene Chloride & 1.1 & UG/KG & J,B & 5.7 \\
\hline 383MB0900.5 & $6 / 12 / 2004$ & soil & HASL300 & REG & 15092-94-1 & $\mathrm{Pb}-212$ & 3.37 & $\mathrm{PCl} / \mathrm{G}$ & $\mathrm{J}$ & 0.27 \\
\hline 383MB0900.5 & $6 / 12 / 2004$ & soil & HASL300 & REG & 15067-28-4 & $\mathrm{Pb}-214$ & 2.08 & $\mathrm{PCl} / \mathrm{G}$ & $\mathrm{G}, \mathrm{J}$ & 0.33 \\
\hline 383MB0900.5 & $6 / 12 / 2004$ & soil & EPA6010 & REG & $7440-22-4$ & Silver & 0.26 & MG/KG & $B$ & 1.1 \\
\hline 383MB0900.5 & $6 / 12 / 2004$ & soil & HASL300 & REG & 14913-50-9 & TI-208 & 1.02 & $\mathrm{PCl} / \mathrm{G}$ & $\mathrm{G}$ & 0.19 \\
\hline 383MB0912.5 & $6 / 12 / 2004$ & soil & HASL300 & REG & 14331-83-0 & Ac-228 & 1.81 & $\mathrm{PCl} / \mathrm{G}$ & G & 0.56 \\
\hline 383MB0912.5 & $6 / 12 / 2004$ & soil & HASL300 & REG & $14596-10-2$ & Am-241 & 1.39 & $\mathrm{PCl} / \mathrm{G}$ & $\mathrm{J}$ & 0.67 \\
\hline 383MB0912.5 & $6 / 12 / 2004$ & soil & EPA6010 & REG & $7440-38-2$ & Arsenic & 3.1 & MG/KG & & 1.1 \\
\hline 383MB0912.5 & $6 / 12 / 2004$ & soil & EPA6010 & REG & $7440-39-3$ & Barium & 2100 & MG/KG & & 550 \\
\hline 383MB0912.5 & $6 / 12 / 2004$ & soil & EPA6010 & REG & $7440-41-7$ & Beryllium & 1.4 & MG/KG & & 0.55 \\
\hline 383MB0912.5 & $6 / 12 / 2004$ & soil & HASL300 & REG & 14733-03-0 & Bi-214 & 1.17 & $\mathrm{PCl} / \mathrm{G}$ & $\mathrm{G}, \mathrm{J}$ & 0.51 \\
\hline 383MB0912.5 & $6 / 12 / 2004$ & soil & EPA6010 & REG & $7440-43-9$ & Cadmium & 0.26 & MG/KG & $B$ & 0.55 \\
\hline 383MB0912.5 & $6 / 12 / 2004$ & soil & EPA6010 & REG & $7440-47-3$ & Chromium & 1.7 & MG/KG & & 1.1 \\
\hline 383MB0912.5 & $6 / 12 / 2004$ & soil & HASL300 & REG & $10045-97-3$ & Cs-137 & 135 & $\mathrm{PCl} / \mathrm{G}$ & $G$ & 0 \\
\hline 383MB0912.5 & $6 / 12 / 2004$ & soil & EPA8015 & REG & $68334-30-5$ & Diesel Range Organics & 14 & MG/KG & $\mathrm{M}$ & 5.5 \\
\hline 383MB0912.5 & $6 / 12 / 2004$ & soil & HASL300 & REG & $13966-00-2$ & $\mathrm{~K}-40$ & 32 & $\mathrm{PCl} / \mathrm{G}$ & G & 1.5 \\
\hline 383MB0912.5 & $6 / 12 / 2004$ & soil & EPA6010 & REG & $7439-92-1$ & Lead & 120 & MG/KG & & 17 \\
\hline 383MB0912.5 & $6 / 12 / 2004$ & soil & EPA7470 & REG & 7439-97-6 & Mercury & 0.017 & MG/KG & B & 0.11 \\
\hline 383MB0912.5 & $6 / 12 / 2004$ & soil & EPA8260 & REG & $75-09-2$ & Methylene Chloride & 1.2 & UG/KG & $\mathrm{J}, \mathrm{B}$ & 5.5 \\
\hline
\end{tabular}




\begin{tabular}{|c|c|c|c|c|c|c|c|c|c|c|}
\hline SAMPLE \# & $\begin{array}{l}\text { SAMPLE } \\
\text { DATE }\end{array}$ & MATRIX & $\begin{array}{l}\text { USER TEST } \\
\text { PANEL }\end{array}$ & $\begin{array}{l}\text { SAMPLE } \\
\text { PURPOSE }\end{array}$ & CAS \# & PARAMETER & RESULT & UNITS & Q & $\begin{array}{l}\text { DETECT } \\
\text { LIMIT }\end{array}$ \\
\hline 383MB0912.5 & $6 / 12 / 2004$ & soil & HASL300 & REG & 15092-94-1 & $\mathrm{Pb}-212$ & 2.02 & $\mathrm{PCl} / \mathrm{G}$ & $\mathrm{J}$ & 0.65 \\
\hline 383MB0912.5 & $6 / 12 / 2004$ & soil & UGTAISOPU & REG & 13981-16-3 & Pu-238 & 3.32 & $\mathrm{PCl} / \mathrm{G}$ & & 0.01 \\
\hline 383MB0912.5 & $6 / 12 / 2004$ & soil & UGTAISOPU & REG & $15117-48-3$ & Pu-239 & 4.11 & $\mathrm{PCl} / \mathrm{G}$ & & 0.02 \\
\hline 383MB0912.5 & $6 / 12 / 2004$ & soil & EPA6010 & REG & $7782-49-2$ & Selenium & 0.7 & MG/KG & & 0.55 \\
\hline 383MB0912.5 & $6 / 12 / 2004$ & soil & SR7500 & REG & 10098-97-2 & Sr-90 & 5.5 & $\mathrm{PCl} / \mathrm{G}$ & $\mathrm{J}$ & 0.2 \\
\hline 383MB0912.5 & $6 / 12 / 2004$ & soil & HASL300 & REG & 14913-50-9 & TI-208 & 0.68 & $\mathrm{PCl} / \mathrm{G}$ & G & 0.39 \\
\hline 383MB0913.5 & $6 / 12 / 2004$ & soil & EPA8260 & REG & $67-64-1$ & Acetone & 5.7 & UG/KG & $\mathrm{J}$ & 20 \\
\hline 383MB0913.5 & $6 / 12 / 2004$ & soil & EPA6010 & REG & 7440-39-3 & Barium & 14 & MG/KG & & 10 \\
\hline 383MB0913.5 & $6 / 12 / 2004$ & soil & EPA6010 & REG & $7440-41-7$ & Beryllium & 0.2 & MG/KG & $B$ & 0.5 \\
\hline 383MB0913.5 & $6 / 12 / 2004$ & soil & EPA6010 & REG & $7440-43-9$ & Cadmium & 0.039 & MG/KG & $B$ & 0.5 \\
\hline 383MB0913.5 & $6 / 12 / 2004$ & soil & EPA6010 & REG & $7440-47-3$ & Chromium & 1.6 & MG/KG & & 1 \\
\hline 383MB0913.5 & $6 / 12 / 2004$ & soil & EPA7470 & REG & 7439-97-6 & Mercury & 0.026 & MG/KG & $B$ & 0.1 \\
\hline 383MB0913.5 & $6 / 12 / 2004$ & soil & EPA8260 & REG & $75-09-2$ & Methylene Chloride & 1 & UG/KG & $\mathrm{J}, \mathrm{B}$ & 5 \\
\hline 383MB0913.5 & $6 / 12 / 2004$ & soil & EPA6010 & REG & $7440-22-4$ & Silver & 0.13 & MG/KG & $B$ & 1 \\
\hline 383MB1000.5 & $6 / 12 / 2004$ & soil & EPA8270 & REG & $91-57-6$ & 2-Methylnaphthalene & 68 & UG/KG & $\mathrm{J}$ & 380 \\
\hline 383MB1000.5 & $6 / 12 / 2004$ & soil & HASL300 & REG & 14331-83-0 & Ac-228 & 1.35 & $\mathrm{PCl} / \mathrm{G}$ & G & 0.45 \\
\hline 383MB1000.5 & $6 / 12 / 2004$ & soil & EPA8270 & REG & $83-32-9$ & Acenaphthene & 22 & UG/KG & $\mathrm{J}$ & 380 \\
\hline 383MB1000.5 & $6 / 12 / 2004$ & soil & EPA8270 & REG & $208-96-8$ & Acenaphthylene & 14 & UG/KG & $\mathrm{J}$ & 380 \\
\hline 383MB1000.5 & $6 / 12 / 2004$ & soil & EPA8270 & REG & $120-12-7$ & Anthracene & 170 & UG/KG & $\mathrm{J}$ & 380 \\
\hline 383MB1000.5 & $6 / 12 / 2004$ & soil & EPA6010 & REG & $7440-38-2$ & Arsenic & 4.7 & MG/KG & & 1.1 \\
\hline 383MB1000.5 & $6 / 12 / 2004$ & soil & EPA6010 & REG & 7440-39-3 & Barium & 4900 & MG/KG & & 560 \\
\hline 383MB1000.5 & $6 / 12 / 2004$ & soil & EPA8270 & REG & $56-55-3$ & Benzo(A)Anthracene & 1200 & UG/KG & & 380 \\
\hline 383MB1000.5 & $6 / 12 / 2004$ & soil & EPA8270 & REG & $50-32-8$ & Benzo(A)Pyrene & 1300 & UG/KG & & 380 \\
\hline 383MB1000.5 & $6 / 12 / 2004$ & soil & EPA8270 & REG & $205-99-2$ & Benzo(B)Fluoranthene & 1700 & UG/KG & & 380 \\
\hline 383MB1000.5 & $6 / 12 / 2004$ & soil & EPA8270 & REG & $191-24-2$ & Benzo(G,H,I)Perylene & 760 & UG/KG & & 380 \\
\hline 383MB1000.5 & $6 / 12 / 2004$ & soil & EPA8270 & REG & 207-08-9 & Benzo(K)Fluoranthene & 650 & UG/KG & & 380 \\
\hline 383MB1000.5 & $6 / 12 / 2004$ & soil & EPA6010 & REG & $7440-41-7$ & Beryllium & 0.94 & MG/KG & & 0.56 \\
\hline 383MB1000.5 & $6 / 12 / 2004$ & soil & HASL300 & REG & 14733-03-0 & Bi-214 & 1.02 & $\mathrm{PCl} / \mathrm{G}$ & $\mathrm{G}, \mathrm{J}$ & 0.24 \\
\hline 383MB1000.5 & $6 / 12 / 2004$ & soil & EPA8270 & REG & 117-81-7 & Bis(2-Ethylhexyl)Phthalate & 130 & UG/KG & $\mathrm{J}$ & 380 \\
\hline 383MB1000.5 & $6 / 12 / 2004$ & soil & EPA6010 & REG & $7440-43-9$ & Cadmium & 0.34 & MG/KG & $B$ & 0.56 \\
\hline 383MB1000.5 & $6 / 12 / 2004$ & soil & EPA8270 & REG & $86-74-8$ & Carbazole & 57 & UG/KG & $\mathrm{J}$ & 380 \\
\hline 383MB1000.5 & $6 / 12 / 2004$ & soil & EPA6010 & REG & $7440-47-3$ & Chromium & 11 & MG/KG & & 1.1 \\
\hline 383MB1000.5 & $6 / 12 / 2004$ & soil & EPA8270 & REG & 218-01-9 & Chrysene & 1000 & UG/KG & & 380 \\
\hline 383MB1000.5 & $6 / 12 / 2004$ & soil & HASL300 & REG & $10045-97-3$ & Cs-137 & 0.33 & $\mathrm{PCl} / \mathrm{G}$ & LT,G & 0.16 \\
\hline 383MB1000.5 & $6 / 12 / 2004$ & soil & EPA8270 & REG & $132-64-9$ & Dibenzofuran & 65 & UG/KG & $\mathrm{J}$ & 380 \\
\hline
\end{tabular}




\begin{tabular}{|c|c|c|c|c|c|c|c|c|c|c|}
\hline SAMPLE \# & $\begin{array}{l}\text { SAMPLE } \\
\text { DATE }\end{array}$ & MATRIX & $\begin{array}{l}\text { USER TEST } \\
\text { PANEL }\end{array}$ & $\begin{array}{l}\text { SAMPLE } \\
\text { PURPOSE }\end{array}$ & CAS \# & PARAMETER & RESULT & UNITS & Q & $\begin{array}{l}\text { DETECT } \\
\text { LIMIT }\end{array}$ \\
\hline 383MB1000.5 & $6 / 12 / 2004$ & soil & EPA8015 & REG & $68334-30-5$ & Diesel Range Organics & 9.9 & MG/KG & Z,H & 5.6 \\
\hline 383MB1000.5 & $6 / 12 / 2004$ & soil & EPA8270 & REG & $206-44-0$ & Fluoranthene & 1500 & UG/KG & & 380 \\
\hline 383MB1000.5 & $6 / 12 / 2004$ & soil & EPA8270 & REG & $86-73-7$ & Fluorene & 16 & UG/KG & $\mathrm{J}$ & 380 \\
\hline 383MB1000.5 & $6 / 12 / 2004$ & soil & EPA8270 & REG & $193-39-5$ & Indeno(1,2,3-Cd)Pyrene & 630 & UG/KG & & 380 \\
\hline 383MB1000.5 & $6 / 12 / 2004$ & soil & HASL300 & REG & 13966-00-2 & $\mathrm{K}-40$ & 22.3 & $\mathrm{PCl} / \mathrm{G}$ & G & 1 \\
\hline 383MB1000.5 & $6 / 12 / 2004$ & soil & EPA6010 & REG & $7439-92-1$ & Lead & 51 & MG/KG & & 0.68 \\
\hline 383MB1000.5 & $6 / 12 / 2004$ & soil & EPA7470 & REG & $7439-97-6$ & Mercury & 0.047 & MG/KG & $B$ & 0.11 \\
\hline 383MB1000.5 & $6 / 12 / 2004$ & soil & EPA8260 & REG & $75-09-2$ & Methylene Chloride & 1.3 & UG/KG & J,B & 5.6 \\
\hline 383MB1000.5 & $6 / 12 / 2004$ & soil & EPA8270 & REG & $91-20-3$ & Naphthalene & 19 & UG/KG & $\mathrm{J}$ & 380 \\
\hline 383MB1000.5 & $6 / 12 / 2004$ & soil & HASL300 & REG & 15092-94-1 & $\mathrm{Pb}-212$ & 1.5 & $\mathrm{PCl} / \mathrm{G}$ & $\mathrm{J}$ & 0.18 \\
\hline 383MB1000.5 & $6 / 12 / 2004$ & soil & HASL300 & REG & 15067-28-4 & $\mathrm{Pb}-214$ & 1.13 & $\mathrm{PCl} / \mathrm{G}$ & $\mathrm{G}, \mathrm{J}$ & 0.27 \\
\hline 383MB1000.5 & $6 / 12 / 2004$ & soil & EPA8270 & REG & $85-01-8$ & Phenanthrene & 350 & UG/KG & $\mathrm{J}$ & 380 \\
\hline 383MB1000.5 & $6 / 12 / 2004$ & soil & UGTAISOPU & REG & 15117-48-3 & Pu-239 & 0.117 & $\mathrm{PCl} / \mathrm{G}$ & & 0.022 \\
\hline 383MB1000.5 & $6 / 12 / 2004$ & soil & EPA8270 & REG & $129-00-0$ & Pyrene & 1600 & UG/KG & & 380 \\
\hline 383MB1000.5 & $6 / 12 / 2004$ & soil & EPA6010 & REG & $7782-49-2$ & Selenium & 0.24 & MG/KG & B & 0.56 \\
\hline 383MB1000.5 & $6 / 12 / 2004$ & soil & EPA6010 & REG & $7440-22-4$ & Silver & 0.34 & MG/KG & $B$ & 1.1 \\
\hline 383MB1000.5 & $6 / 12 / 2004$ & soil & HASL300 & REG & 14913-50-9 & TI-208 & 0.46 & $\mathrm{PCl} / \mathrm{G}$ & G & 0.12 \\
\hline 383MB1100.5 & $6 / 12 / 2004$ & soil & HASL300 & REG & 14331-83-0 & Ac-228 & 1.65 & $\mathrm{PCl} / \mathrm{G}$ & G & 0.56 \\
\hline 383MB1100.5 & $6 / 12 / 2004$ & soil & EPA6010 & REG & $7440-38-2$ & Arsenic & 4.3 & MG/KG & & 1.1 \\
\hline 383MB1100.5 & $6 / 12 / 2004$ & soil & EPA6010 & REG & $7440-39-3$ & Barium & 5300 & MG/KG & & 550 \\
\hline 383MB1100.5 & $6 / 12 / 2004$ & soil & EPA6010 & REG & $7440-41-7$ & Beryllium & 1.4 & MG/KG & & 0.55 \\
\hline 383MB1100.5 & $6 / 12 / 2004$ & soil & HASL300 & REG & 14733-03-0 & $\mathrm{Bi}-214$ & 1.3 & $\mathrm{PCl} / \mathrm{G}$ & $\mathrm{G}, \mathrm{J}$ & 0.27 \\
\hline 383MB1100.5 & $6 / 12 / 2004$ & soil & EPA6010 & REG & $7440-43-9$ & Cadmium & 0.23 & MG/KG & $B$ & 0.55 \\
\hline 383MB1100.5 & $6 / 12 / 2004$ & soil & EPA6010 & REG & $7440-47-3$ & Chromium & 5.6 & MG/KG & & 1.1 \\
\hline 383MB1100.5 & $6 / 12 / 2004$ & soil & HASL300 & REG & 10045-97-3 & Cs-137 & 0.87 & $\mathrm{PCl} / \mathrm{G}$ & G & 0.15 \\
\hline 383MB1100.5 & $6 / 12 / 2004$ & soil & EPA8015 & REG & 68334-30-5 & Diesel Range Organics & 13 & MG/KG & $\mathrm{M}$ & 5.5 \\
\hline 383MB1100.5 & $6 / 12 / 2004$ & soil & HASL300 & REG & 13966-00-2 & $\mathrm{K}-40$ & 25.6 & $\mathrm{PCl} / \mathrm{G}$ & G & 1.6 \\
\hline 383MB1100.5 & $6 / 12 / 2004$ & soil & EPA6010 & REG & 7439-92-1 & Lead & 20 & MG/KG & & 0.33 \\
\hline 383MB1100.5 & $6 / 12 / 2004$ & soil & EPA7470 & REG & $7439-97-6$ & Mercury & 0.016 & MG/KG & $B$ & 0.11 \\
\hline 383MB1100.5 & $6 / 12 / 2004$ & soil & EPA8260 & REG & $75-09-2$ & Methylene Chloride & 1.1 & UG/KG & J,B & 5.5 \\
\hline 383MB1100.5 & $6 / 12 / 2004$ & soil & HASL300 & REG & 15092-94-1 & $\mathrm{Pb}-212$ & 1.89 & $\mathrm{PCl} / \mathrm{G}$ & $\mathrm{J}$ & 0.24 \\
\hline 383MB1100.5 & $6 / 12 / 2004$ & soil & HASL300 & REG & 15067-28-4 & $\mathrm{Pb}-214$ & 1.46 & $\mathrm{PCl} / \mathrm{G}$ & $\mathrm{G}, \mathrm{J}$ & 0.27 \\
\hline 383MB1100.5 & $6 / 12 / 2004$ & soil & EPA6010 & REG & $7782-49-2$ & Selenium & 0.74 & MG/KG & & 0.55 \\
\hline 383MB1100.5 & $6 / 12 / 2004$ & soil & HASL300 & REG & 14913-50-9 & TI-208 & 0.66 & $\mathrm{PCl} / \mathrm{G}$ & G & 0.14 \\
\hline 383MB1114.5 & $6 / 12 / 2004$ & soil & HASL300 & REG & 14331-83-0 & Ac-228 & 1.54 & $\mathrm{PCl} / \mathrm{G}$ & G & 0.52 \\
\hline
\end{tabular}




\begin{tabular}{|c|c|c|c|c|c|c|c|c|c|c|}
\hline SAMPLE \# & $\begin{array}{l}\text { SAMPLE } \\
\text { DATE }\end{array}$ & MATRIX & $\begin{array}{l}\text { USER TEST } \\
\text { PANEL }\end{array}$ & $\begin{array}{l}\text { SAMPLE } \\
\text { PURPOSE }\end{array}$ & CAS \# & PARAMETER & RESULT & UNITS & Q & $\begin{array}{l}\text { DETECT } \\
\text { LIMIT }\end{array}$ \\
\hline 383MB1114.5 & $6 / 12 / 2004$ & soil & EPA6010 & REG & $7440-38-2$ & Arsenic & 2.5 & MG/KG & & 1.1 \\
\hline 383MB1114.5 & $6 / 12 / 2004$ & soil & EPA6010 & REG & 7440-39-3 & Barium & 470 & MG/KG & & 11 \\
\hline 383MB1114.5 & $6 / 12 / 2004$ & soil & EPA6010 & REG & $7440-41-7$ & Beryllium & 1.1 & MG/KG & & 0.56 \\
\hline 383MB1114.5 & $6 / 12 / 2004$ & soil & HASL300 & REG & 14733-03-0 & Bi-214 & 1.27 & $\mathrm{PCl} / \mathrm{G}$ & $\mathrm{G}, \mathrm{J}$ & 0.41 \\
\hline 383MB1114.5 & $6 / 12 / 2004$ & soil & EPA6010 & REG & $7440-43-9$ & Cadmium & 0.13 & MG/KG & $B$ & 0.56 \\
\hline 383MB1114.5 & $6 / 12 / 2004$ & soil & EPA6010 & REG & $7440-47-3$ & Chromium & 1.4 & MG/KG & & 1.1 \\
\hline 383MB1114.5 & $6 / 12 / 2004$ & soil & HASL300 & REG & $10045-97-3$ & Cs-137 & 37.4 & $\mathrm{PCl} / \mathrm{G}$ & G & 0.2 \\
\hline 383MB1114.5 & $6 / 12 / 2004$ & soil & EPA8015 & REG & 68334-30-5 & Diesel Range Organics & 8.8 & MG/KG & $\mathrm{M}$ & 5.6 \\
\hline 383MB1114.5 & $6 / 12 / 2004$ & soil & HASL300 & REG & 13966-00-2 & $\mathrm{K}-40$ & 37.7 & $\mathrm{PCl} / \mathrm{G}$ & G & 1.5 \\
\hline 383MB1114.5 & $6 / 12 / 2004$ & soil & EPA6010 & REG & $7439-92-1$ & Lead & 15 & MG/KG & & 0.34 \\
\hline 383MB1114.5 & $6 / 12 / 2004$ & soil & EPA7470 & REG & $7439-97-6$ & Mercury & 0.006 & MG/KG & B & 0.11 \\
\hline 383MB1114.5 & $6 / 12 / 2004$ & soil & EPA8260 & REG & $75-09-2$ & Methylene Chloride & 1.2 & UG/KG & $\mathrm{J}, \mathrm{B}$ & 5.6 \\
\hline 383MB1114.5 & $6 / 12 / 2004$ & soil & HASL300 & REG & 15092-94-1 & $\mathrm{Pb}-212$ & 1.86 & $\mathrm{PCl} / \mathrm{G}$ & $\mathrm{J}$ & 0.39 \\
\hline 383MB1114.5 & $6 / 12 / 2004$ & soil & HASL300 & REG & $15067-28-4$ & $\mathrm{~Pb}-214$ & 1.24 & $\mathrm{PCl} / \mathrm{G}$ & $\mathrm{G}, \mathrm{J}$ & 0.57 \\
\hline 383MB1114.5 & $6 / 12 / 2004$ & soil & UGTAISOPU & REG & 15117-48-3 & Pu-239 & 0.206 & $\mathrm{PCl} / \mathrm{G}$ & & 0.021 \\
\hline 383MB1114.5 & $6 / 12 / 2004$ & soil & EPA6010 & REG & $7782-49-2$ & Selenium & 0.53 & MG/KG & $B$ & 0.56 \\
\hline 383MB1114.5 & $6 / 12 / 2004$ & soil & SR7500 & REG & 10098-97-2 & Sr-90 & 1.08 & $\mathrm{PCl} / \mathrm{G}$ & Y1 & 0.25 \\
\hline 383MB1114.5 & $6 / 12 / 2004$ & soil & HASL300 & REG & 14913-50-9 & TI-208 & 0.7 & $\mathrm{PCl} / \mathrm{G}$ & G & 0.21 \\
\hline 383MB1119.5 & $6 / 12 / 2004$ & soil & EPA6010 & REG & $7440-38-2$ & Arsenic & 1.4 & MG/KG & & 1 \\
\hline 383MB1119.5 & $6 / 12 / 2004$ & soil & EPA6010 & REG & $7440-39-3$ & Barium & 36 & MG/KG & & 10 \\
\hline 383MB1119.5 & $6 / 12 / 2004$ & soil & EPA6010 & REG & $7440-41-7$ & Beryllium & 0.23 & MG/KG & $B$ & 0.51 \\
\hline 383MB1119.5 & $6 / 12 / 2004$ & soil & EPA6010 & REG & $7440-43-9$ & Cadmium & 0.17 & MG/KG & $B$ & 0.51 \\
\hline 383MB1119.5 & $6 / 12 / 2004$ & soil & EPA6010 & REG & $7440-47-3$ & Chromium & 2.1 & MG/KG & & 1 \\
\hline 383MB1119.5 & $6 / 12 / 2004$ & soil & HASL300 & REG & 10045-97-3 & Cs-137 & 0.121 & $\mathrm{PCl} / \mathrm{G}$ & LT & 0.062 \\
\hline 383MB1119.5 & $6 / 12 / 2004$ & soil & EPA6010 & REG & $7439-92-1$ & Lead & 1.1 & MG/KG & $B$ & 1.5 \\
\hline 383MB1119.5 & $6 / 12 / 2004$ & soil & EPA7470 & REG & $7439-97-6$ & Mercury & 0.037 & MG/KG & $B$ & 0.1 \\
\hline 383MB1119.5 & $6 / 12 / 2004$ & soil & EPA8260 & REG & $75-09-2$ & Methylene Chloride & 0.97 & UG/KG & $\mathrm{J}, \mathrm{B}$ & 5.1 \\
\hline 383MB1119.5 & $6 / 12 / 2004$ & soil & HASL300 & REG & 15067-28-4 & $\mathrm{Pb}-214$ & 0.38 & $\mathrm{PCl} / \mathrm{G}$ & $\mathrm{J}$ & 0.15 \\
\hline 383MB1119.5 & $6 / 12 / 2004$ & soil & EPA6010 & REG & $7440-22-4$ & Silver & 0.17 & MG/KG & $B$ & 1 \\
\hline 383MB1200.5 & $6 / 13 / 2004$ & soil & HASL300 & REG & 14331-83-0 & Ac-228 & 1.98 & $\mathrm{PCl} / \mathrm{G}$ & G & 0.8 \\
\hline 383MB1200.5 & $6 / 13 / 2004$ & soil & HASL300 & REG & 14682-66-7 & $\mathrm{Al}-26$ & 0.108 & $\mathrm{PCl} / \mathrm{G}$ & $\mathrm{G}, \mathrm{TI}$ & 0.103 \\
\hline 383MB1200.5 & $6 / 13 / 2004$ & soil & EPA6010 & REG & $7440-38-2$ & Arsenic & 2.3 & MG/KG & & 1.1 \\
\hline 383MB1200.5 & $6 / 13 / 2004$ & soil & EPA6010 & REG & $7440-39-3$ & Barium & 640 & MG/KG & & 11 \\
\hline 383MB1200.5 & $6 / 13 / 2004$ & soil & EPA6010 & REG & $7440-41-7$ & Beryllium & 2.4 & MG/KG & & 0.56 \\
\hline 383MB1200.5 & $6 / 13 / 2004$ & soil & HASL300 & REG & 14913-49-6 & $\mathrm{Bi}-212$ & 2.5 & $\mathrm{PCl} / \mathrm{G}$ & G & 2.1 \\
\hline
\end{tabular}




\begin{tabular}{|c|c|c|c|c|c|c|c|c|c|c|}
\hline SAMPLE \# & $\begin{array}{l}\text { SAMPLE } \\
\text { DATE }\end{array}$ & MATRIX & $\begin{array}{l}\text { USER TEST } \\
\text { PANEL }\end{array}$ & $\begin{array}{l}\text { SAMPLE } \\
\text { PURPOSE }\end{array}$ & CAS \# & PARAMETER & RESULT & UNITS & Q & $\begin{array}{l}\text { DETECT } \\
\text { LIMIT }\end{array}$ \\
\hline 383MB1200.5 & $6 / 13 / 2004$ & soil & HASL300 & REG & 14733-03-0 & Bi-214 & 1.84 & $\mathrm{PCl} / \mathrm{G}$ & $\mathrm{G}, \mathrm{J}$ & 0.35 \\
\hline 383MB1200.5 & $6 / 13 / 2004$ & soil & EPA6010 & REG & $7440-43-9$ & Cadmium & 0.17 & MG/KG & $B$ & 0.56 \\
\hline 383MB1200.5 & $6 / 13 / 2004$ & soil & EPA6010 & REG & $7440-47-3$ & Chromium & 2.1 & MG/KG & & 1.1 \\
\hline 383MB1200.5 & $6 / 13 / 2004$ & soil & HASL300 & REG & 10045-97-3 & Cs-137 & 0.23 & $\mathrm{PCl} / \mathrm{G}$ & LT,G & 0.14 \\
\hline 383MB1200.5 & $6 / 13 / 2004$ & soil & HASL300 & REG & 13966-00-2 & $\mathrm{K}-40$ & 30.4 & $\mathrm{PCl} / \mathrm{G}$ & G & 2 \\
\hline 383MB1200.5 & $6 / 13 / 2004$ & soil & EPA6010 & REG & $7439-92-1$ & Lead & 20 & MG/KG & & 0.34 \\
\hline 383MB1200.5 & $6 / 13 / 2004$ & soil & EPA7470 & REG & 7439-97-6 & Mercury & 0.0079 & MG/KG & $B$ & 0.11 \\
\hline 383MB1200.5 & $6 / 13 / 2004$ & soil & HASL300 & REG & 15092-94-1 & $\mathrm{Pb}-212$ & 2.99 & $\mathrm{PCl} / \mathrm{G}$ & G & 0.26 \\
\hline 383MB1200.5 & $6 / 13 / 2004$ & soil & HASL300 & REG & 15067-28-4 & $\mathrm{Pb}-214$ & 2.04 & $\mathrm{PCl} / \mathrm{G}$ & $\mathrm{G}, \mathrm{J}$ & 0.31 \\
\hline 383MB1200.5 & $6 / 13 / 2004$ & soil & UGTAISOPU & REG & 13981-16-3 & Pu-238 & 0.017 & $\mathrm{PCl} / \mathrm{G}$ & LT & 0.009 \\
\hline 383MB1200.5 & $6 / 13 / 2004$ & soil & EPA6010 & REG & $7782-49-2$ & Selenium & 0.36 & MG/KG & B & 0.56 \\
\hline 383MB1200.5 & $6 / 13 / 2004$ & soil & HASL300 & REG & 14913-50-9 & TI-208 & 0.85 & $\mathrm{PCl} / \mathrm{G}$ & G & 0.16 \\
\hline 383MB1300.5 & $6 / 13 / 2004$ & soil & HASL300 & REG & 14331-83-0 & Ac-228 & 2.05 & $\mathrm{PCl} / \mathrm{G}$ & G & 0.58 \\
\hline 383MB1300.5 & $6 / 13 / 2004$ & soil & EPA6010 & REG & 7440-38-2 & Arsenic & 3.5 & MG/KG & & 1.1 \\
\hline 383MB1300.5 & $6 / 13 / 2004$ & soil & EPA6010 & REG & 7440-39-3 & Barium & 660 & MG/KG & & 11 \\
\hline 383MB1300.5 & $6 / 13 / 2004$ & soil & EPA6010 & REG & $7440-41-7$ & Beryllium & 1.1 & MG/KG & & 0.56 \\
\hline 383MB1300.5 & $6 / 13 / 2004$ & soil & HASL300 & REG & 14913-49-6 & $\mathrm{Bi}-212$ & 2.3 & $\mathrm{PCl} / \mathrm{G}$ & G & 2.1 \\
\hline 383MB1300.5 & $6 / 13 / 2004$ & soil & HASL300 & REG & 14733-03-0 & Bi-214 & 1.07 & $\mathrm{PCl} / \mathrm{G}$ & $\mathrm{G}, \mathrm{J}$ & 0.27 \\
\hline 383MB1300.5 & $6 / 13 / 2004$ & soil & EPA6010 & REG & $7440-43-9$ & Cadmium & 0.03 & MG/KG & $B$ & 0.56 \\
\hline 383MB1300.5 & $6 / 13 / 2004$ & soil & EPA6010 & REG & $7440-47-3$ & Chromium & 2 & MG/KG & & 1.1 \\
\hline 383MB1300.5 & $6 / 13 / 2004$ & soil & HASL300 & REG & 13966-00-2 & $\mathrm{K}-40$ & 25.7 & $\mathrm{PCl} / \mathrm{G}$ & G & 1.5 \\
\hline 383MB1300.5 & $6 / 13 / 2004$ & soil & EPA6010 & REG & $7439-92-1$ & Lead & 13 & MG/KG & & 0.33 \\
\hline 383MB1300.5 & $6 / 13 / 2004$ & soil & EPA7470 & REG & 7439-97-6 & Mercury & 0.013 & MG/KG & $B$ & 0.11 \\
\hline 383MB1300.5 & $6 / 13 / 2004$ & soil & HASL300 & REG & 15092-94-1 & $\mathrm{Pb}-212$ & 2.1 & $\mathrm{PCl} / \mathrm{G}$ & G & 0.26 \\
\hline 383MB1300.5 & $6 / 13 / 2004$ & soil & HASL300 & REG & 15067-28-4 & $\mathrm{Pb}-214$ & 0.97 & $\mathrm{PCl} / \mathrm{G}$ & $\mathrm{G}, \mathrm{J}$ & 0.28 \\
\hline 383MB1300.5 & $6 / 13 / 2004$ & soil & EPA6010 & REG & $7782-49-2$ & Selenium & 0.27 & MG/KG & $B$ & 0.56 \\
\hline 383MB1300.5 & $6 / 13 / 2004$ & soil & HASL300 & REG & 15065-10-8 & Th-234 & 2.7 & $\mathrm{PCl} / \mathrm{G}$ & $\mathrm{G}, \mathrm{TI}$ & 2 \\
\hline 383MB1300.5 & $6 / 13 / 2004$ & soil & HASL300 & REG & 14913-50-9 & TI-208 & 0.79 & $\mathrm{PCl} / \mathrm{G}$ & G & 0.15 \\
\hline 383MB1391.0 & $6 / 14 / 2004$ & soil & HASL300 & REG & 14331-83-0 & Ac-228 & 0.71 & $\mathrm{PCl} / \mathrm{G}$ & G & 0.4 \\
\hline 383MB1391.0 & $6 / 14 / 2004$ & soil & EPA6010 & REG & $7440-38-2$ & Arsenic & 2.3 & MG/KG & & 1.1 \\
\hline 383MB1391.0 & $6 / 14 / 2004$ & soil & EPA6010 & REG & 7440-39-3 & Barium & 67 & MG/KG & & 11 \\
\hline 383MB1391.0 & $6 / 14 / 2004$ & soil & EPA6010 & REG & $7440-41-7$ & Beryllium & 0.65 & MG/KG & & 0.54 \\
\hline 383MB1391.0 & $6 / 14 / 2004$ & soil & HASL300 & REG & 14733-03-0 & Bi-214 & 0.65 & $\mathrm{PCl} / \mathrm{G}$ & $\mathrm{G}, \mathrm{J}$ & 0.2 \\
\hline 383MB1391.0 & $6 / 14 / 2004$ & soil & EPA6010 & REG & $7440-43-9$ & Cadmium & 0.092 & MG/KG & B & 0.54 \\
\hline 383MB1391.0 & $6 / 14 / 2004$ & soil & EPA6010 & REG & $7440-47-3$ & Chromium & 5.8 & MG/KG & & 1.1 \\
\hline
\end{tabular}




\begin{tabular}{|c|c|c|c|c|c|c|c|c|c|c|}
\hline SAMPLE \# & $\begin{array}{l}\text { SAMPLE } \\
\text { DATE }\end{array}$ & MATRIX & $\begin{array}{l}\text { USER TEST } \\
\text { PANEL }\end{array}$ & $\begin{array}{l}\text { SAMPLE } \\
\text { PURPOSE }\end{array}$ & CAS \# & PARAMETER & RESULT & UNITS & Q & $\begin{array}{l}\text { DETECT } \\
\text { LIMIT }\end{array}$ \\
\hline 383MB1391.0 & $6 / 14 / 2004$ & soil & HASL300 & REG & $13966-00-2$ & $\mathrm{~K}-40$ & 13 & $\mathrm{PCl} / \mathrm{G}$ & G & 1.1 \\
\hline 383MB1391.0 & $6 / 14 / 2004$ & soil & EPA6010 & REG & $7439-92-1$ & Lead & 8.6 & MG/KG & & 1.6 \\
\hline 383MB1391.0 & $6 / 14 / 2004$ & soil & EPA7470 & REG & 7439-97-6 & Mercury & 0.014 & MG/KG & $B$ & 0.11 \\
\hline 383MB1391.0 & $6 / 14 / 2004$ & soil & EPA8260 & REG & $75-09-2$ & Methylene Chloride & 0.92 & UG/KG & $\mathrm{J}, \mathrm{B}$ & 5.4 \\
\hline 383MB1391.0 & $6 / 14 / 2004$ & soil & HASL300 & REG & 15092-94-1 & $\mathrm{Pb}-212$ & 0.8 & $\mathrm{PCl} / \mathrm{G}$ & G & 0.17 \\
\hline 383MB1391.0 & $6 / 14 / 2004$ & soil & HASL300 & REG & $15067-28-4$ & $\mathrm{~Pb}-214$ & 0.76 & $\mathrm{PCl} / \mathrm{G}$ & $\mathrm{G}, \mathrm{J}$ & 0.18 \\
\hline 383MB1391.0 & $6 / 14 / 2004$ & soil & UGTAISOPU & REG & 13981-16-3 & Pu-238 & 0.02 & $\mathrm{PCl} / \mathrm{G}$ & LT & 0.009 \\
\hline 383MB1391.0 & $6 / 14 / 2004$ & soil & UGTAISOPU & REG & 15117-48-3 & Pu-239 & 0.03 & $\mathrm{PCl} / \mathrm{G}$ & LT & 0.009 \\
\hline 383MB1391.0 & $6 / 14 / 2004$ & soil & EPA6010 & REG & $7440-22-4$ & Silver & 1.6 & MG/KG & & 1.1 \\
\hline 383MB1391.0 & $6 / 14 / 2004$ & soil & HASL300 & REG & $14913-50-9$ & TI-208 & 0.183 & $\mathrm{PCl} / \mathrm{G}$ & G & 0.089 \\
\hline 383MB1400.5 & $6 / 14 / 2004$ & soil & HASL300 & REG & 14331-83-0 & Ac-228 & 0.74 & $\mathrm{PCl} / \mathrm{G}$ & & 0.4 \\
\hline 383MB1400.5 & $6 / 14 / 2004$ & soil & EPA6010 & REG & $7440-38-2$ & Arsenic & 3.4 & MG/KG & & 1 \\
\hline 383MB1400.5 & $6 / 14 / 2004$ & soil & EPA6010 & REG & 7440-39-3 & Barium & 300 & MG/KG & & 10 \\
\hline 383MB1400.5 & $6 / 14 / 2004$ & soil & EPA8270 & REG & $56-55-3$ & Benzo(A)Anthracene & 18 & UG/KG & $\mathrm{J}$ & 340 \\
\hline 383MB1400.5 & $6 / 14 / 2004$ & soil & EPA8270 & REG & $50-32-8$ & Benzo(A)Pyrene & 15 & UG/KG & $\mathrm{J}$ & 340 \\
\hline 383MB1400.5 & $6 / 14 / 2004$ & soil & EPA8270 & REG & $205-99-2$ & Benzo(B)Fluoranthene & 27 & UG/KG & $\mathrm{J}$ & 340 \\
\hline 383MB1400.5 & $6 / 14 / 2004$ & soil & EPA6010 & REG & $7440-41-7$ & Beryllium & 0.53 & MG/KG & & 0.52 \\
\hline 383MB1400.5 & $6 / 14 / 2004$ & soil & HASL300 & REG & 14733-03-0 & $\mathrm{Bi}-214$ & 0.46 & $\mathrm{PCl} / \mathrm{G}$ & $\mathrm{J}$ & 0.29 \\
\hline 383MB1400.5 & $6 / 14 / 2004$ & soil & EPA8270 & REG & 117-81-7 & Bis(2-Ethylhexyl)Phthalate & 72 & UG/KG & $\mathrm{J}$ & 340 \\
\hline 383MB1400.5 & $6 / 14 / 2004$ & soil & EPA6010 & REG & $7440-43-9$ & Cadmium & 0.3 & MG/KG & B & 0.52 \\
\hline 383MB1400.5 & $6 / 14 / 2004$ & soil & EPA6010 & REG & $7440-47-3$ & Chromium & 3.4 & MG/KG & & 1 \\
\hline 383MB1400.5 & $6 / 14 / 2004$ & soil & EPA8270 & REG & $218-01-9$ & Chrysene & 21 & UG/KG & $\mathrm{J}$ & 340 \\
\hline 383MB1400.5 & $6 / 14 / 2004$ & soil & HASL300 & REG & $10045-97-3$ & Cs-137 & 8.5 & $\mathrm{PCl} / \mathrm{G}$ & & 0.1 \\
\hline 383MB1400.5 & $6 / 14 / 2004$ & soil & EPA8015 & REG & $68334-30-5$ & Diesel Range Organics & 45 & MG/KG & $\mathrm{Z}, \mathrm{H}$ & 5.1 \\
\hline 383MB1400.5 & $6 / 14 / 2004$ & soil & EPA8270 & REG & $206-44-0$ & Fluoranthene & 21 & UG/KG & $\mathrm{J}$ & 340 \\
\hline 383MB1400.5 & $6 / 14 / 2004$ & soil & HASL300 & REG & $13966-00-2$ & $\mathrm{~K}-40$ & 9.5 & $\mathrm{PCl} / \mathrm{G}$ & & 1.6 \\
\hline 383MB1400.5 & $6 / 14 / 2004$ & soil & EPA6010 & REG & 7439-92-1 & Lead & 20 & MG/KG & & 1.5 \\
\hline 383MB1400.5 & $6 / 14 / 2004$ & soil & EPA7470 & REG & 7439-97-6 & Mercury & 0.21 & MG/KG & & 0.1 \\
\hline 383MB1400.5 & $6 / 14 / 2004$ & soil & EPA8260 & REG & $75-09-2$ & Methylene Chloride & 0.83 & UG/KG & $\mathrm{J}, \mathrm{B}$ & 5.2 \\
\hline 383MB1400.5 & $6 / 14 / 2004$ & soil & HASL300 & REG & 15092-94-1 & $\mathrm{Pb}-212$ & 0.62 & $\mathrm{PCl} / \mathrm{G}$ & & 0.23 \\
\hline 383MB1400.5 & $6 / 14 / 2004$ & soil & HASL300 & REG & $15067-28-4$ & $\mathrm{~Pb}-214$ & 0.51 & $\mathrm{PCl} / \mathrm{G}$ & $\mathrm{J}$ & 0.27 \\
\hline 383MB1400.5 & $6 / 14 / 2004$ & soil & EPA8260 & REG & $99-87-6$ & P-Isopropyltoluene & 13 & UG/KG & & 5.2 \\
\hline 383MB1400.5 & $6 / 14 / 2004$ & soil & UGTAISOPU & REG & 13981-16-3 & Pu-238 & 0.88 & $\mathrm{PCl} / \mathrm{G}$ & & 0.02 \\
\hline 383MB1400.5 & $6 / 14 / 2004$ & soil & UGTAISOPU & REG & 15117-48-3 & Pu-239 & 2.84 & $\mathrm{PCl} / \mathrm{G}$ & & 0.02 \\
\hline 383MB1400.5 & $6 / 14 / 2004$ & soil & SR7500 & REG & 10098-97-2 & Sr-90 & 0.36 & $\mathrm{PCl} / \mathrm{G}$ & $\mathrm{Y} 1, \mathrm{LT}$ & 0.22 \\
\hline
\end{tabular}




\begin{tabular}{|c|c|c|c|c|c|c|c|c|c|c|}
\hline SAMPLE \# & $\begin{array}{l}\text { SAMPLE } \\
\text { DATE }\end{array}$ & MATRIX & $\begin{array}{l}\text { USER TEST } \\
\text { PANEL }\end{array}$ & $\begin{array}{l}\text { SAMPLE } \\
\text { PURPOSE }\end{array}$ & CAS \# & PARAMETER & RESULT & UNITS & Q & $\begin{array}{l}\text { DETECT } \\
\text { LIMIT }\end{array}$ \\
\hline 383MB1400.5 & $6 / 14 / 2004$ & soil & HASL300 & REG & 14913-50-9 & TI-208 & 0.222 & $\mathrm{PCl} / \mathrm{G}$ & & 0.11 \\
\hline 383MB1403.5 & $6 / 14 / 2004$ & soil & HASL300 & REG & 14331-83-0 & Ac-228 & 1.9 & $\mathrm{PCl} / \mathrm{G}$ & G & 0.52 \\
\hline 383MB1403.5 & $6 / 14 / 2004$ & soil & HASL300 & REG & 14596-10-2 & Am-241 & 1.57 & $\mathrm{PCl} / \mathrm{G}$ & G & 0.7 \\
\hline 383MB1403.5 & $6 / 14 / 2004$ & soil & EPA6010 & REG & $7440-38-2$ & Arsenic & 2.1 & MG/KG & & 1.1 \\
\hline 383MB1403.5 & $6 / 14 / 2004$ & soil & EPA6010 & REG & 7440-39-3 & Barium & 87 & MG/KG & & 11 \\
\hline 383MB1403.5 & $6 / 14 / 2004$ & soil & EPA6010 & REG & $7440-41-7$ & Beryllium & 1.3 & MG/KG & & 0.57 \\
\hline 383MB1403.5 & $6 / 14 / 2004$ & soil & HASL300 & REG & $14733-03-0$ & Bi-214 & 1.24 & $\mathrm{PCl} / \mathrm{G}$ & $\mathrm{G}, \mathrm{J}$ & 0.79 \\
\hline 383MB1403.5 & $6 / 14 / 2004$ & soil & EPA8270 & REG & 117-81-7 & Bis(2-Ethylhexyl)Phthalate & 93 & UG/KG & $\mathrm{J}$ & 380 \\
\hline 383MB1403.5 & $6 / 14 / 2004$ & soil & EPA6010 & REG & $7440-43-9$ & Cadmium & 0.069 & MG/KG & B & 0.57 \\
\hline 383MB1403.5 & $6 / 14 / 2004$ & soil & EPA6010 & REG & $7440-47-3$ & Chromium & 0.71 & MG/KG & $B$ & 1.1 \\
\hline 383MB1403.5 & $6 / 14 / 2004$ & soil & HASL300 & REG & $10045-97-3$ & Cs-137 & 240 & $\mathrm{PCl} / \mathrm{G}$ & G & 0 \\
\hline 383MB1403.5 & $6 / 14 / 2004$ & soil & HASL300 & REG & $13966-00-2$ & $\mathrm{~K}-40$ & 30.7 & $\mathrm{PCl} / \mathrm{G}$ & G & 2.1 \\
\hline 383MB1403.5 & $6 / 14 / 2004$ & soil & EPA6010 & REG & $7439-92-1$ & Lead & 27 & MG/KG & & 0.34 \\
\hline 383MB1403.5 & $6 / 14 / 2004$ & soil & EPA7470 & REG & $7439-97-6$ & Mercury & 0.017 & MG/KG & $B$ & 0.11 \\
\hline 383MB1403.5 & $6 / 14 / 2004$ & soil & EPA8260 & REG & $75-09-2$ & Methylene Chloride & 0.97 & UG/KG & J,B & 5.7 \\
\hline 383MB1403.5 & $6 / 14 / 2004$ & soil & HASL300 & REG & 15092-94-1 & $\mathrm{Pb}-212$ & 2.15 & $\mathrm{PCl} / \mathrm{G}$ & G & 0.96 \\
\hline 383MB1403.5 & $6 / 14 / 2004$ & soil & HASL300 & REG & $15067-28-4$ & $\mathrm{~Pb}-214$ & 1.49 & $\mathrm{PCl} / \mathrm{G}$ & $\mathrm{G}, \mathrm{J}$ & 1.26 \\
\hline 383MB1403.5 & $6 / 14 / 2004$ & soil & UGTAISOPU & REG & 13981-16-3 & Pu-238 & 1.11 & $\mathrm{PCl} / \mathrm{G}$ & & 0.02 \\
\hline 383MB1403.5 & $6 / 14 / 2004$ & soil & UGTAISOPU & REG & $15117-48-3$ & Pu-239 & 5.34 & $\mathrm{PCl} / \mathrm{G}$ & & 0.02 \\
\hline 383MB1403.5 & $6 / 14 / 2004$ & soil & EPA6010 & REG & $7782-49-2$ & Selenium & 0.3 & MG/KG & $B$ & 0.57 \\
\hline 383MB1403.5 & $6 / 14 / 2004$ & soil & SR7500 & REG & 10098-97-2 & Sr-90 & 6.9 & $\mathrm{PCl} / \mathrm{G}$ & Y1 & 0.2 \\
\hline 383MB1403.5 & $6 / 14 / 2004$ & soil & HASL300 & REG & 14913-50-9 & TI-208 & 0.76 & $\mathrm{PCl} / \mathrm{G}$ & G & 0.45 \\
\hline 383MB1425.5 & $6 / 14 / 2004$ & soil & HASL300 & REG & 14331-83-0 & Ac-228 & 1.25 & $\mathrm{PCl} / \mathrm{G}$ & G & 0.53 \\
\hline 383MB1425.5 & $6 / 14 / 2004$ & soil & EPA6010 & REG & 7440-38-2 & Arsenic & 1.9 & MG/KG & & 1.1 \\
\hline 383MB1425.5 & $6 / 14 / 2004$ & soil & EPA6010 & REG & 7440-39-3 & Barium & 30 & MG/KG & & 11 \\
\hline 383MB1425.5 & $6 / 14 / 2004$ & soil & EPA6010 & REG & $7440-41-7$ & Beryllium & 0.77 & MG/KG & & 0.54 \\
\hline 383MB1425.5 & $6 / 14 / 2004$ & soil & HASL300 & REG & 14733-03-0 & Bi-214 & 0.7 & $\mathrm{PCl} / \mathrm{G}$ & $\mathrm{G}, \mathrm{J}$ & 0.32 \\
\hline 383MB1425.5 & $6 / 14 / 2004$ & soil & EPA8270 & REG & $117-81-7$ & Bis(2-Ethylhexyl)Phthalate & 220 & UG/KG & $\mathrm{J}$ & 360 \\
\hline 383MB1425.5 & $6 / 14 / 2004$ & soil & EPA6010 & REG & $7440-43-9$ & Cadmium & 0.045 & MG/KG & B & 0.54 \\
\hline 383MB1425.5 & $6 / 14 / 2004$ & soil & EPA6010 & REG & $7440-47-3$ & Chromium & 1.3 & MG/KG & & 1.1 \\
\hline 383MB1425.5 & $6 / 14 / 2004$ & soil & HASL300 & REG & $10045-97-3$ & Cs-137 & 3.18 & $\mathrm{PCl} / \mathrm{G}$ & G & 0.14 \\
\hline 383MB1425.5 & $6 / 14 / 2004$ & soil & HASL300 & REG & $13966-00-2$ & $\mathrm{~K}-40$ & 19.6 & $\mathrm{PCl} / \mathrm{G}$ & G & 1.7 \\
\hline 383MB1425.5 & $6 / 14 / 2004$ & soil & EPA6010 & REG & 7439-92-1 & Lead & 95 & MG/KG & & 1.6 \\
\hline 383MB1425.5 & $6 / 14 / 2004$ & soil & EPA7470 & REG & 7439-97-6 & Mercury & 0.013 & MG/KG & B & 0.11 \\
\hline 383MB1425.5 & $6 / 14 / 2004$ & soil & EPA8260 & REG & $75-09-2$ & Methylene Chloride & 0.86 & UG/KG & $\mathrm{J}, \mathrm{B}$ & 5.4 \\
\hline
\end{tabular}




\begin{tabular}{|c|c|c|c|c|c|c|c|c|c|c|}
\hline SAMPLE \# & $\begin{array}{l}\text { SAMPLE } \\
\text { DATE }\end{array}$ & MATRIX & $\begin{array}{l}\text { USER TEST } \\
\text { PANEL }\end{array}$ & $\begin{array}{l}\text { SAMPLE } \\
\text { PURPOSE }\end{array}$ & CAS \# & PARAMETER & RESULT & UNITS & Q & $\begin{array}{l}\text { DETECT } \\
\text { LIMIT }\end{array}$ \\
\hline 383MB1425.5 & $6 / 14 / 2004$ & soil & HASL300 & REG & 15092-94-1 & $\mathrm{Pb}-212$ & 1.44 & $\mathrm{PCl} / \mathrm{G}$ & G & 0.26 \\
\hline 383MB1425.5 & $6 / 14 / 2004$ & soil & HASL300 & REG & 15067-28-4 & $\mathrm{Pb}-214$ & 0.91 & $\mathrm{PCl} / \mathrm{G}$ & $\mathrm{G}, \mathrm{J}$ & 0.27 \\
\hline 383MB1425.5 & $6 / 14 / 2004$ & soil & UGTAISOPU & REG & 13981-16-3 & Pu-238 & 0.051 & $\mathrm{PCl} / \mathrm{G}$ & & 0.021 \\
\hline 383MB1425.5 & $6 / 14 / 2004$ & soil & UGTAISOPU & REG & $15117-48-3$ & Pu-239 & 0.203 & $\mathrm{PCl} / \mathrm{G}$ & & 0.009 \\
\hline 383MB1425.5 & $6 / 14 / 2004$ & soil & SR7500 & REG & 10098-97-2 & Sr-90 & 0.33 & $\mathrm{PCl} / \mathrm{G}$ & $\mathrm{Y} 1, \mathrm{LT}$ & 0.25 \\
\hline 383MB1425.5 & $6 / 14 / 2004$ & soil & HASL300 & REG & 14913-50-9 & TI-208 & 0.48 & $\mathrm{PCl} / \mathrm{G}$ & G & 0.13 \\
\hline 383MB1500.5 & $6 / 17 / 2004$ & soil & HASL300 & REG & 14331-83-0 & Ac-228 & 1.63 & $\mathrm{PCl} / \mathrm{G}$ & G & 0.51 \\
\hline 383MB1500.5 & $6 / 17 / 2004$ & soil & EPA6010 & REG & $7440-38-2$ & Arsenic & 3.2 & MG/KG & & 1.1 \\
\hline 383MB1500.5 & $6 / 17 / 2004$ & soil & EPA6010 & REG & $7440-39-3$ & Barium & 3700 & MG/KG & & 540 \\
\hline 383MB1500.5 & $6 / 17 / 2004$ & soil & EPA6010 & REG & $7440-41-7$ & Beryllium & 0.73 & MG/KG & & 0.54 \\
\hline 383MB1500.5 & $6 / 17 / 2004$ & soil & HASL300 & REG & $14733-03-0$ & Bi-214 & 0.7 & $\mathrm{PCl} / \mathrm{G}$ & $\mathrm{G}, \mathrm{J}$ & 0.32 \\
\hline 383MB1500.5 & $6 / 17 / 2004$ & soil & EPA6010 & REG & $7440-43-9$ & Cadmium & 0.17 & MG/KG & B & 0.54 \\
\hline 383MB1500.5 & $6 / 17 / 2004$ & soil & EPA6010 & REG & $7440-47-3$ & Chromium & 3.7 & MG/KG & & 1.1 \\
\hline 383MB1500.5 & $6 / 17 / 2004$ & soil & HASL300 & REG & $10045-97-3$ & Cs-137 & 20.7 & $\mathrm{PCl} / \mathrm{G}$ & G & 0.2 \\
\hline 383MB1500.5 & $6 / 17 / 2004$ & soil & EPA8015 & REG & $68334-30-5$ & Diesel Range Organics & 19 & MG/KG & $\mathrm{Z}, \mathrm{H}$ & 5.4 \\
\hline 383MB1500.5 & $6 / 17 / 2004$ & soil & HASL300 & REG & 13966-00-2 & $\mathrm{K}-40$ & 23.8 & $\mathrm{PCl} / \mathrm{G}$ & G & 1.3 \\
\hline 383MB1500.5 & $6 / 17 / 2004$ & soil & EPA6010 & REG & $7439-92-1$ & Lead & 30 & MG/KG & & 0.33 \\
\hline 383MB1500.5 & $6 / 17 / 2004$ & soil & EPA7470 & REG & $7439-97-6$ & Mercury & 0.024 & MG/KG & $B$ & 0.11 \\
\hline 383MB1500.5 & $6 / 17 / 2004$ & soil & EPA8260 & REG & $75-09-2$ & Methylene Chloride & 0.99 & UG/KG & J,B & 5.4 \\
\hline 383MB1500.5 & $6 / 17 / 2004$ & soil & HASL300 & REG & 15092-94-1 & $\mathrm{Pb}-212$ & 1.47 & $\mathrm{PCl} / \mathrm{G}$ & $\mathrm{G}$ & 0.28 \\
\hline 383MB1500.5 & $6 / 17 / 2004$ & soil & HASL300 & REG & 15067-28-4 & $\mathrm{Pb}-214$ & 0.73 & $\mathrm{PCl} / \mathrm{G}$ & $\mathrm{G}, \mathrm{J}$ & 0.38 \\
\hline 383MB1500.5 & $6 / 17 / 2004$ & soil & UGTAISOPU & REG & 13981-16-3 & Pu-238 & 0.11 & $\mathrm{PCl} / \mathrm{G}$ & & 0.021 \\
\hline 383MB1500.5 & $6 / 17 / 2004$ & soil & UGTAISOPU & REG & $15117-48-3$ & Pu-239 & 1.15 & $\mathrm{PCl} / \mathrm{G}$ & & 0.01 \\
\hline 383MB1500.5 & $6 / 17 / 2004$ & soil & SR7500 & REG & 10098-97-2 & Sr-90 & 0.67 & $\mathrm{PCl} / \mathrm{G}$ & Y1 & 0.24 \\
\hline 383MB1500.5 & $6 / 17 / 2004$ & soil & HASL300 & REG & 14913-50-9 & TI-208 & 0.33 & $\mathrm{PCl} / \mathrm{G}$ & G & 0.19 \\
\hline 383МH0136.5 & $5 / 25 / 2004$ & soil & HASL300 & REG & 14331-83-0 & Ac-228 & 1.67 & $\mathrm{PCl} / \mathrm{G}$ & G & 0.69 \\
\hline 383МH0136.5 & $5 / 25 / 2004$ & soil & EPA8260 & REG & 67-64-1 & Acetone & 10 & UG/KG & $\mathrm{J}$ & 24 \\
\hline 383МH0136.5 & $5 / 25 / 2004$ & soil & EPA6010 & REG & $7440-38-2$ & Arsenic & 3.3 & MG/KG & & 1.2 \\
\hline 383МH0136.5 & $5 / 25 / 2004$ & soil & EPA6010 & REG & 7440-39-3 & Barium & 150 & MG/KG & & 12 \\
\hline 383МH0136.5 & $5 / 25 / 2004$ & soil & EPA6010 & REG & $7440-41-7$ & Beryllium & 1.2 & MG/KG & & 0.59 \\
\hline 383МH0136.5 & $5 / 25 / 2004$ & soil & HASL300 & REG & 14733-03-0 & $\mathrm{Bi}-214$ & 0.88 & $\mathrm{PCl} / \mathrm{G}$ & $\mathrm{G}, \mathrm{J}$ & 0.39 \\
\hline 383МH0136.5 & $5 / 25 / 2004$ & soil & EPA6010 & REG & $7440-47-3$ & Chromium & 2.3 & MG/KG & & 1.2 \\
\hline 383МH0136.5 & $5 / 25 / 2004$ & soil & HASL300 & REG & 10045-97-3 & Cs-137 & 4.11 & $\mathrm{PCl} / \mathrm{G}$ & G & 0.2 \\
\hline 383MH0136.5 & $5 / 25 / 2004$ & soil & HASL300 & REG & 13966-00-2 & $\mathrm{K}-40$ & 26.9 & $\mathrm{PCl} / \mathrm{G}$ & G & 2.1 \\
\hline 383MH0136.5 & $5 / 25 / 2004$ & soil & EPA6010 & REG & $7439-92-1$ & Lead & 14 & MG/KG & & 0.35 \\
\hline
\end{tabular}




\begin{tabular}{|c|c|c|c|c|c|c|c|c|c|c|}
\hline SAMPLE \# & $\begin{array}{l}\text { SAMPLE } \\
\text { DATE }\end{array}$ & MATRIX & $\begin{array}{l}\text { USER TEST } \\
\text { PANEL }\end{array}$ & $\begin{array}{l}\text { SAMPLE } \\
\text { PURPOSE }\end{array}$ & CAS \# & PARAMETER & RESULT & UNITS & Q & $\begin{array}{l}\text { DETECT } \\
\text { LIMIT }\end{array}$ \\
\hline 383MH0136.5 & $5 / 25 / 2004$ & soil & EPA7470 & REG & $7439-97-6$ & Mercury & 0.079 & MG/KG & $B$ & 0.12 \\
\hline 383MH0136.5 & $5 / 25 / 2004$ & soil & HASL300 & REG & 15092-94-1 & $\mathrm{Pb}-212$ & 2.55 & $\mathrm{PCl} / \mathrm{G}$ & $\mathrm{J}$ & 0.38 \\
\hline 383MH0136.5 & $5 / 25 / 2004$ & soil & HASL300 & REG & 15067-28-4 & $\mathrm{Pb}-214$ & 1.13 & $\mathrm{PCl} / \mathrm{G}$ & $\mathrm{G}, \mathrm{J}$ & 0.4 \\
\hline 383МH0136.5 & $5 / 25 / 2004$ & soil & UGTAISOPU & REG & $15117-48-3$ & Pu-239 & 0.141 & $\mathrm{PCl} / \mathrm{G}$ & & 0.01 \\
\hline 383MH0136.5 & $5 / 25 / 2004$ & soil & HASL300 & REG & 14913-50-9 & TI-208 & 0.65 & $\mathrm{PCl} / \mathrm{G}$ & G & 0.18 \\
\hline 383MH0168.5 & $5 / 25 / 2004$ & soil & EPA6010 & REG & $7440-38-2$ & Arsenic & 0.44 & MG/KG & $B$ & 1 \\
\hline 383МH0168.5 & $5 / 25 / 2004$ & soil & EPA6010 & REG & $7440-39-3$ & Barium & 5.3 & MG/KG & $B$ & 10 \\
\hline 383MH0168.5 & $5 / 25 / 2004$ & soil & EPA6010 & REG & 7440-41-7 & Beryllium & 0.23 & MG/KG & $B$ & 0.52 \\
\hline 383МH0168.5 & $5 / 25 / 2004$ & soil & EPA6010 & REG & $7440-43-9$ & Cadmium & 0.036 & MG/KG & $B$ & 0.52 \\
\hline 383МH0168.5 & $5 / 25 / 2004$ & soil & EPA6010 & REG & $7440-47-3$ & Chromium & 1.3 & MG/KG & & 1 \\
\hline 383MH0168.5 & $5 / 25 / 2004$ & soil & EPA7470 & REG & 7439-97-6 & Mercury & 0.052 & MG/KG & B & 0.1 \\
\hline 383MH0168.5 & $5 / 25 / 2004$ & soil & EPA6010 & REG & $7440-22-4$ & Silver & 0.57 & MG/KG & $B$ & 1 \\
\hline 383МH0313.0 & $5 / 25 / 2004$ & soil & HASL300 & REG & 14331-83-0 & Ac-228 & 1.9 & $\mathrm{PCl} / \mathrm{G}$ & G & 0.73 \\
\hline 383МH0313.0 & $5 / 25 / 2004$ & soil & EPA8260 & REG & $67-64-1$ & Acetone & 10 & UG/KG & $\mathrm{J}$ & 24 \\
\hline 383MH0313.0 & $5 / 25 / 2004$ & soil & EPA6010 & REG & $7440-38-2$ & Arsenic & 1.7 & MG/KG & & 1.2 \\
\hline 383МH0313.0 & $5 / 25 / 2004$ & soil & EPA6010 & REG & $7440-39-3$ & Barium & 250 & MG/KG & & 12 \\
\hline 383МH0313.0 & $5 / 25 / 2004$ & soil & EPA6010 & REG & $7440-41-7$ & Beryllium & 1.5 & MG/KG & & 0.61 \\
\hline 383MH0313.0 & $5 / 25 / 2004$ & soil & HASL300 & REG & 14733-03-0 & Bi-214 & 1.27 & $\mathrm{PCl} / \mathrm{G}$ & $\mathrm{G}, \mathrm{J}$ & 0.38 \\
\hline 383МH0313.0 & $5 / 25 / 2004$ & soil & EPA6010 & REG & $7440-43-9$ & Cadmium & 0.045 & MG/KG & $B$ & 0.61 \\
\hline 383МH0313.0 & $5 / 25 / 2004$ & soil & EPA6010 & REG & $7440-47-3$ & Chromium & 0.57 & MG/KG & $B$ & 1.2 \\
\hline 383MH0313.0 & $5 / 25 / 2004$ & soil & HASL300 & REG & 10045-97-3 & Cs-137 & 0.43 & $\mathrm{PCl} / \mathrm{G}$ & LT,G & 0.17 \\
\hline 383МH0313.0 & $5 / 25 / 2004$ & soil & HASL300 & REG & 13966-00-2 & $\mathrm{K}-40$ & 35.9 & $\mathrm{PCl} / \mathrm{G}$ & G & 1.7 \\
\hline 383МH0313.0 & $5 / 25 / 2004$ & soil & EPA6010 & REG & $7439-92-1$ & Lead & 35 & MG/KG & & 0.37 \\
\hline 383МH0313.0 & $5 / 25 / 2004$ & soil & EPA7470 & REG & 7439-97-6 & Mercury & 0.0037 & MG/KG & $B$ & 0.12 \\
\hline 383МH0313.0 & $5 / 25 / 2004$ & soil & HASL300 & REG & 15092-94-1 & $\mathrm{Pb}-212$ & 2.4 & $\mathrm{PCl} / \mathrm{G}$ & $\mathrm{J}$ & 0.26 \\
\hline 383МH0313.0 & $5 / 25 / 2004$ & soil & HASL300 & REG & 15067-28-4 & $\mathrm{Pb}-214$ & 1.32 & $\mathrm{PCl} / \mathrm{G}$ & $\mathrm{G}, \mathrm{J}$ & 0.29 \\
\hline 383MH0313.0 & $5 / 25 / 2004$ & soil & EPA6010 & REG & 7782-49-2 & Selenium & 0.31 & MG/KG & B & 0.61 \\
\hline 383МH0313.0 & $5 / 25 / 2004$ & soil & HASL300 & REG & 14913-50-9 & TI-208 & 0.81 & $\mathrm{PCl} / \mathrm{G}$ & G & 0.19 \\
\hline 383MH0353.5 & $5 / 25 / 2004$ & soil & HASL300 & REG & 14331-83-0 & Ac-228 & 2.4 & $\mathrm{PCl} / \mathrm{G}$ & G & 0.47 \\
\hline 383МH0353.5 & $5 / 25 / 2004$ & soil & HASL300 & REG & 14596-10-2 & Am-241 & 3.2 & $\mathrm{PCl} / \mathrm{G}$ & $\mathrm{J}$ & 1.8 \\
\hline 383MH0353.5 & $5 / 25 / 2004$ & soil & EPA6010 & REG & $7440-38-2$ & Arsenic & 6 & MG/KG & & 1.1 \\
\hline 383МH0353.5 & $5 / 25 / 2004$ & soil & EPA6010 & REG & $7440-39-3$ & Barium & 350 & MG/KG & & 11 \\
\hline 383МH0353.5 & $5 / 25 / 2004$ & soil & EPA6010 & REG & $7440-41-7$ & Beryllium & 1.2 & MG/KG & & 0.57 \\
\hline 383MH0353.5 & $5 / 25 / 2004$ & soil & HASL300 & REG & 14733-03-0 & Bi-214 & 0.89 & $\mathrm{PCl} / \mathrm{G}$ & $\mathrm{G}, \mathrm{J}$ & 0.48 \\
\hline 383МH0353.5 & $5 / 25 / 2004$ & soil & EPA6010 & REG & $7440-43-9$ & Cadmium & 0.079 & MG/KG & B & 0.57 \\
\hline
\end{tabular}




\begin{tabular}{|c|c|c|c|c|c|c|c|c|c|c|}
\hline SAMPLE \# & $\begin{array}{l}\text { SAMPLE } \\
\text { DATE }\end{array}$ & MATRIX & $\begin{array}{l}\text { USER TEST } \\
\text { PANEL }\end{array}$ & $\begin{array}{l}\text { SAMPLE } \\
\text { PURPOSE }\end{array}$ & CAS \# & PARAMETER & RESULT & UNITS & Q & $\begin{array}{l}\text { DETECT } \\
\text { LIMIT }\end{array}$ \\
\hline 383МH0353.5 & $5 / 25 / 2004$ & soil & EPA6010 & REG & $7440-47-3$ & Chromium & 2.4 & MG/KG & & 1.1 \\
\hline 383MH0353.5 & $5 / 25 / 2004$ & soil & HASL300 & REG & $10045-97-3$ & Cs-137 & 144 & $\mathrm{PCl} / \mathrm{G}$ & G & 0 \\
\hline 383MH0353.5 & $5 / 25 / 2004$ & soil & EPA8015 & REG & 68334-30-5 & Diesel Range Organics & 3.5 & MG/KG & $\mathrm{J}$ & 5.7 \\
\hline 383MH0353.5 & $5 / 25 / 2004$ & soil & HASL300 & REG & 13966-00-2 & $\mathrm{K}-40$ & 28.7 & $\mathrm{PCl} / \mathrm{G}$ & G & 1.3 \\
\hline 383МH0353.5 & $5 / 25 / 2004$ & soil & EPA6010 & REG & $7439-92-1$ & Lead & 40 & MG/KG & & 1.7 \\
\hline 383MH0353.5 & $5 / 25 / 2004$ & soil & EPA7470 & REG & 7439-97-6 & Mercury & 0.51 & MG/KG & & 0.11 \\
\hline 383МH0353.5 & $5 / 25 / 2004$ & soil & HASL300 & REG & 15092-94-1 & $\mathrm{Pb}-212$ & 2.23 & $\mathrm{PCl} / \mathrm{G}$ & $\mathrm{J}$ & 0.6 \\
\hline 383MH0353.5 & $5 / 25 / 2004$ & soil & UGTAISOPU & REG & 13981-16-3 & Pu-238 & 0.77 & $\mathrm{PCl} / \mathrm{G}$ & & 0.01 \\
\hline 383MH0353.5 & $5 / 25 / 2004$ & soil & UGTAISOPU & REG & $15117-48-3$ & Pu-239 & 7.3 & $\mathrm{PCl} / \mathrm{G}$ & & 0 \\
\hline 383МH0353.5 & $5 / 25 / 2004$ & soil & EPA6010 & REG & 7782-49-2 & Selenium & 0.67 & MG/KG & & 0.57 \\
\hline 383МH0353.5 & $5 / 25 / 2004$ & soil & SR7500 & REG & 10098-97-2 & Sr-90 & 0.94 & $\mathrm{PCl} / \mathrm{G}$ & & 0.22 \\
\hline 383MH0353.5 & $5 / 25 / 2004$ & soil & HASL300 & REG & 14913-50-9 & TI-208 & 0.78 & $\mathrm{PCl} / \mathrm{G}$ & G & 0.3 \\
\hline 383MH0361.0 & $5 / 25 / 2004$ & soil & EPA6010 & REG & $7440-38-2$ & Arsenic & 0.48 & MG/KG & B & 1 \\
\hline 383МH0361.0 & $5 / 25 / 2004$ & soil & EPA6010 & REG & 7440-39-3 & Barium & 2.6 & MG/KG & $B$ & 10 \\
\hline 383МH0361.0 & $5 / 25 / 2004$ & soil & EPA6010 & REG & $7440-41-7$ & Beryllium & 0.2 & MG/KG & $B$ & 0.5 \\
\hline 383МH0361.0 & $5 / 25 / 2004$ & soil & EPA6010 & REG & $7440-47-3$ & Chromium & 1.2 & MG/KG & & 1 \\
\hline 383МH0361.0 & $5 / 25 / 2004$ & soil & EPA7470 & REG & $7439-97-6$ & Mercury & 0.016 & MG/KG & B & 0.1 \\
\hline 383МH0361.0 & $5 / 25 / 2004$ & soil & EPA6010 & REG & $7440-22-4$ & Silver & 0.23 & MG/KG & $B$ & 1 \\
\hline 383MH0432.0 & $5 / 22 / 2004$ & soil & HASL300 & REG & 14331-83-0 & Ac-228 & 2.18 & $\mathrm{PCl} / \mathrm{G}$ & G & 0.65 \\
\hline 383МH0432.0 & $5 / 22 / 2004$ & soil & EPA6010 & REG & $7440-38-2$ & Arsenic & 2.5 & MG/KG & & 1.2 \\
\hline 383МH0432.0 & $5 / 22 / 2004$ & soil & EPA6010 & REG & 7440-39-3 & Barium & 160 & MG/KG & & 12 \\
\hline 383МH0432.0 & $5 / 22 / 2004$ & soil & EPA6010 & REG & $7440-41-7$ & Beryllium & 1.2 & MG/KG & & 0.59 \\
\hline 383МH0432.0 & $5 / 22 / 2004$ & soil & HASL300 & REG & 14733-03-0 & $\mathrm{Bi}-214$ & 1.49 & $\mathrm{PCl} / \mathrm{G}$ & $\mathrm{G}, \mathrm{J}$ & 0.29 \\
\hline 383МH0432.0 & $5 / 22 / 2004$ & soil & EPA6010 & REG & 7440-47-3 & Chromium & 2.2 & MG/KG & & 1.2 \\
\hline 383МH0432.0 & $5 / 22 / 2004$ & soil & HASL300 & REG & $10045-97-3$ & Cs-137 & 2.96 & $\mathrm{PCl} / \mathrm{G}$ & G & 0.18 \\
\hline 383МH0432.0 & $5 / 22 / 2004$ & soil & HASL300 & REG & 13966-00-2 & $\mathrm{K}-40$ & 36 & $\mathrm{PCl} / \mathrm{G}$ & G & 1.8 \\
\hline 383МH0432.0 & $5 / 22 / 2004$ & soil & EPA6010 & REG & $7439-92-1$ & Lead & 15 & MG/KG & & 0.36 \\
\hline 383МH0432.0 & $5 / 22 / 2004$ & soil & EPA7470 & REG & 7439-97-6 & Mercury & 0.0012 & MG/KG & $B$ & 0.12 \\
\hline $383 \mathrm{MH} 0432.0$ & $5 / 22 / 2004$ & soil & HASL300 & REG & 15092-94-1 & $\mathrm{Pb}-212$ & 2.68 & $\mathrm{PCl} / \mathrm{G}$ & $\mathrm{J}$ & 0.27 \\
\hline 383МH0432.0 & $5 / 22 / 2004$ & soil & HASL300 & REG & 15067-28-4 & $\mathrm{Pb}-214$ & 1.69 & $\mathrm{PCl} / \mathrm{G}$ & $\mathrm{G}, \mathrm{J}$ & 0.32 \\
\hline 383МH0432.0 & $5 / 22 / 2004$ & soil & HASL300 & REG & 14913-50-9 & TI-208 & 0.74 & $\mathrm{PCl} / \mathrm{G}$ & $G$ & 0.18 \\
\hline 383МH0441.5 & $5 / 22 / 2004$ & soil & EPA6010 & REG & $7440-38-2$ & Arsenic & 0.76 & MG/KG & B & 1 \\
\hline 383МH0441.5 & $5 / 22 / 2004$ & soil & EPA6010 & REG & 7440-39-3 & Barium & 5 & MG/KG & $B$ & 10 \\
\hline 383MH0441.5 & $5 / 22 / 2004$ & soil & EPA6010 & REG & $7440-41-7$ & Beryllium & 0.18 & MG/KG & $B$ & 0.5 \\
\hline 383MH0441.5 & $5 / 22 / 2004$ & soil & EPA6010 & REG & $7440-43-9$ & Cadmium & 0.46 & MG/KG & $B$ & 0.5 \\
\hline
\end{tabular}




\begin{tabular}{|c|c|c|c|c|c|c|c|c|c|c|}
\hline SAMPLE \# & $\begin{array}{l}\text { SAMPLE } \\
\text { DATE }\end{array}$ & MATRIX & $\begin{array}{l}\text { USER TEST } \\
\text { PANEL }\end{array}$ & $\begin{array}{l}\text { SAMPLE } \\
\text { PURPOSE }\end{array}$ & CAS \# & PARAMETER & RESULT & UNITS & Q & $\begin{array}{l}\text { DETECT } \\
\text { LIMIT }\end{array}$ \\
\hline 383МH0441.5 & $5 / 22 / 2004$ & soil & EPA6010 & REG & $7440-47-3$ & Chromium & 1.6 & MG/KG & & 1 \\
\hline 383MH0441.5 & $5 / 22 / 2004$ & soil & EPA6010 & REG & $7439-92-1$ & Lead & 56 & MG/KG & & 1.5 \\
\hline 383MH0441.5 & $5 / 22 / 2004$ & soil & EPA7470 & REG & $7439-97-6$ & Mercury & 0.22 & MG/KG & & 0.1 \\
\hline 383MH0441.5 & $5 / 22 / 2004$ & soil & EPA8260 & REG & $75-09-2$ & Methylene Chloride & 0.78 & UG/KG & J,B & 5 \\
\hline 383МH0441.5 & $5 / 22 / 2004$ & soil & EPA6010 & REG & $7440-22-4$ & Silver & 1.3 & MG/KG & & 1 \\
\hline 383MH0520.5 & $5 / 27 / 2004$ & soil & HASL300 & REG & 14331-83-0 & Ac-228 & 2.01 & $\mathrm{PCl} / \mathrm{G}$ & G & 0.57 \\
\hline 383МH0520.5 & $5 / 27 / 2004$ & soil & EPA8260 & REG & $67-64-1$ & Acetone & 7.2 & UG/KG & $\mathrm{J}$ & 23 \\
\hline 383MH0520.5 & $5 / 27 / 2004$ & soil & EPA6010 & REG & $7440-38-2$ & Arsenic & 2.2 & MG/KG & & 1.2 \\
\hline 383MH0520.5 & $5 / 27 / 2004$ & soil & EPA6010 & REG & $7440-39-3$ & Barium & 63 & MG/KG & & 12 \\
\hline 383МH0520.5 & $5 / 27 / 2004$ & soil & EPA6010 & REG & $7440-41-7$ & Beryllium & 1.2 & MG/KG & & 0.59 \\
\hline 383MH0520.5 & $5 / 27 / 2004$ & soil & HASL300 & REG & 14733-03-0 & Bi-214 & 1.38 & $\mathrm{PCl} / \mathrm{G}$ & $\mathrm{G}, \mathrm{J}$ & 0.33 \\
\hline 383MH0520.5 & $5 / 27 / 2004$ & soil & EPA6010 & REG & $7440-47-3$ & Chromium & 0.73 & MG/KG & B & 1.2 \\
\hline 383МH0520.5 & $5 / 27 / 2004$ & soil & HASL300 & REG & $10045-97-3$ & Cs-137 & 3.95 & $\mathrm{PCl} / \mathrm{G}$ & G & 0.18 \\
\hline 383МH0520.5 & $5 / 27 / 2004$ & soil & HASL300 & REG & 13966-00-2 & $\mathrm{K}-40$ & 41.6 & $\mathrm{PCl} / \mathrm{G}$ & G & 1.6 \\
\hline 383МH0520.5 & $5 / 27 / 2004$ & soil & EPA6010 & REG & $7439-92-1$ & Lead & 10 & MG/KG & & 0.35 \\
\hline 383МH0520.5 & $5 / 27 / 2004$ & soil & EPA7470 & REG & $7439-97-6$ & Mercury & 0.0014 & MG/KG & B & 0.12 \\
\hline 383МH0520.5 & $5 / 27 / 2004$ & soil & EPA8260 & REG & $75-09-2$ & Methylene Chloride & 1.7 & UG/KG & $\mathrm{J}, \mathrm{B}$ & 5.9 \\
\hline 383MH0520.5 & $5 / 27 / 2004$ & soil & HASL300 & REG & 15092-94-1 & $\mathrm{Pb}-212$ & 2.48 & $\mathrm{PCl} / \mathrm{G}$ & $\mathrm{J}$ & 0.25 \\
\hline 383MH0520.5 & $5 / 27 / 2004$ & soil & HASL300 & REG & $15067-28-4$ & $\mathrm{~Pb}-214$ & 1.65 & $\mathrm{PCl} / \mathrm{G}$ & $\mathrm{G}, \mathrm{J}$ & 0.34 \\
\hline 383МH0520.5 & $5 / 27 / 2004$ & soil & UGTAISOPU & REG & $15117-48-3$ & Pu-239 & 0.189 & $\mathrm{PCl} / \mathrm{G}$ & & 0.017 \\
\hline 383МH0520.5 & $5 / 27 / 2004$ & soil & HASL300 & REG & 14913-50-9 & TI-208 & 0.73 & $\mathrm{PCl} / \mathrm{G}$ & G & 0.14 \\
\hline 383МH0559.5 & $5 / 27 / 2004$ & soil & HASL300 & REG & 14331-83-0 & Ac-228 & 2.27 & $\mathrm{PCl} / \mathrm{G}$ & G, TI & 1.01 \\
\hline 383МH0559.5 & $5 / 27 / 2004$ & soil & HASL300 & REG & 14596-10-2 & Am-241 & 12.8 & $\mathrm{PCl} / \mathrm{G}$ & $\mathrm{J}$ & 4.6 \\
\hline 383МH0559.5 & $5 / 27 / 2004$ & soil & EPA6010 & REG & $7440-38-2$ & Arsenic & 3.6 & MG/KG & & 1.3 \\
\hline 383MH0559.5 & $5 / 27 / 2004$ & soil & EPA6010 & REG & 7440-39-3 & Barium & 56 & MG/KG & & 13 \\
\hline 383МH0559.5 & $5 / 27 / 2004$ & soil & EPA8270 & REG & $56-55-3$ & Benzo(A)Anthracene & 140 & UG/KG & $\mathrm{J}$ & 420 \\
\hline 383МH0559.5 & $5 / 27 / 2004$ & soil & EPA8270 & REG & $50-32-8$ & Benzo(A)Pyrene & 120 & UG/KG & $\mathrm{J}$ & 420 \\
\hline 383МH0559.5 & $5 / 27 / 2004$ & soil & EPA8270 & REG & $205-99-2$ & Benzo(B)Fluoranthene & 180 & UG/KG & $\mathrm{J}$ & 420 \\
\hline 383MH0559.5 & $5 / 27 / 2004$ & soil & EPA8270 & REG & 191-24-2 & Benzo(G,H,I)Perylene & 85 & UG/KG & $\mathrm{J}$ & 420 \\
\hline 383МH0559.5 & $5 / 27 / 2004$ & soil & EPA8270 & REG & $207-08-9$ & Benzo(K)Fluoranthene & 80 & UG/KG & $\mathrm{J}$ & 420 \\
\hline 383МH0559.5 & $5 / 27 / 2004$ & soil & EPA6010 & REG & $7440-41-7$ & Beryllium & 1.2 & MG/KG & & 0.64 \\
\hline 383МH0559.5 & $5 / 27 / 2004$ & soil & EPA6010 & REG & $7440-47-3$ & Chromium & 2.5 & MG/KG & & 1.3 \\
\hline 383МH0559.5 & $5 / 27 / 2004$ & soil & EPA8270 & REG & $218-01-9$ & Chrysene & 170 & UG/KG & $\mathrm{J}$ & 420 \\
\hline 383МH0559.5 & $5 / 27 / 2004$ & soil & HASL300 & REG & $10045-97-3$ & Cs-137 & 471 & $\mathrm{PCl} / \mathrm{G}$ & M3,G & 1 \\
\hline 383MH0559.5 & $5 / 27 / 2004$ & soil & EPA8270 & REG & 206-44-0 & Fluoranthene & 150 & UG/KG & $\mathrm{J}$ & 420 \\
\hline
\end{tabular}




\begin{tabular}{|c|c|c|c|c|c|c|c|c|c|c|}
\hline SAMPLE \# & $\begin{array}{l}\text { SAMPLE } \\
\text { DATE }\end{array}$ & MATRIX & $\begin{array}{l}\text { USER TEST } \\
\text { PANEL }\end{array}$ & $\begin{array}{l}\text { SAMPLE } \\
\text { PURPOSE }\end{array}$ & CAS \# & PARAMETER & RESULT & UNITS & Q & $\begin{array}{l}\text { DETECT } \\
\text { LIMIT }\end{array}$ \\
\hline 383MH0559.5 & $5 / 27 / 2004$ & soil & EPA8270 & REG & $193-39-5$ & Indeno(1,2,3-Cd)Pyrene & 60 & UG/KG & $\mathrm{J}$ & 420 \\
\hline 383MH0559.5 & $5 / 27 / 2004$ & soil & HASL300 & REG & 13966-00-2 & $\mathrm{K}-40$ & 31.2 & $\mathrm{PCl} / \mathrm{G}$ & G & 2.6 \\
\hline 383MH0559.5 & $5 / 27 / 2004$ & soil & EPA6010 & REG & 7439-92-1 & Lead & 20 & MG/KG & & 0.38 \\
\hline 383MH0559.5 & $5 / 27 / 2004$ & soil & EPA7470 & REG & $7439-97-6$ & Mercury & 0.013 & MG/KG & $\mathrm{B}$ & 0.13 \\
\hline 383MH0559.5 & $5 / 27 / 2004$ & soil & EPA8260 & REG & $75-09-2$ & Methylene Chloride & 2 & UG/KG & J,B & 6.4 \\
\hline 383MH0559.5 & $5 / 27 / 2004$ & soil & EPA8270 & REG & $85-01-8$ & Phenanthrene & 52 & UG/KG & $\mathrm{J}$ & 420 \\
\hline 383MH0559.5 & $5 / 27 / 2004$ & soil & UGTAISOPU & REG & 13981-16-3 & Pu-238 & 5.9 & $\mathrm{PCl} / \mathrm{G}$ & & 0 \\
\hline 383MH0559.5 & $5 / 27 / 2004$ & soil & UGTAISOPU & REG & $15117-48-3$ & Pu-239 & 25.3 & $\mathrm{PCl} / \mathrm{G}$ & & 0 \\
\hline 383MH0559.5 & $5 / 27 / 2004$ & soil & EPA8270 & REG & $129-00-0$ & Pyrene & 310 & UG/KG & $\mathrm{J}$ & 420 \\
\hline 383MH0559.5 & $5 / 27 / 2004$ & soil & EPA6010 & REG & 7782-49-2 & Selenium & 0.99 & MG/KG & & 0.64 \\
\hline 383МH0559.5 & $5 / 27 / 2004$ & soil & EPA6010 & REG & $7440-22-4$ & Silver & 0.47 & MG/KG & B & 1.3 \\
\hline 383МH0559.5 & $5 / 27 / 2004$ & soil & SR7500 & REG & 10098-97-2 & Sr-90 & 54 & $\mathrm{PCl} / \mathrm{G}$ & & 0 \\
\hline 383МH0568.5 & $5 / 27 / 2004$ & soil & HASL300 & REG & 14331-83-0 & Ac-228 & 0.42 & $\mathrm{PCl} / \mathrm{G}$ & & 0.24 \\
\hline 383MH0568.5 & $5 / 27 / 2004$ & soil & EPA6010 & REG & $7440-38-2$ & Arsenic & 2.4 & MG/KG & & 1 \\
\hline 383MH0568.5 & $5 / 27 / 2004$ & soil & EPA6010 & REG & 7440-39-3 & Barium & 29 & MG/KG & & 10 \\
\hline 383MH0568.5 & $5 / 27 / 2004$ & soil & EPA6010 & REG & $7440-41-7$ & Beryllium & 0.42 & MG/KG & B & 0.52 \\
\hline 383MH0568.5 & $5 / 27 / 2004$ & soil & HASL300 & REG & 14733-03-0 & Bi-214 & 0.45 & $\mathrm{PCl} / \mathrm{G}$ & $\mathrm{J}$ & 0.17 \\
\hline 383МH0568.5 & $5 / 27 / 2004$ & soil & EPA6010 & REG & $7440-43-9$ & Cadmium & 0.066 & MG/KG & $B$ & 0.52 \\
\hline 383MH0568.5 & $5 / 27 / 2004$ & soil & EPA6010 & REG & 7440-47-3 & Chromium & 4.2 & MG/KG & & 1 \\
\hline 383MH0568.5 & $5 / 27 / 2004$ & soil & HASL300 & REG & $10045-97-3$ & Cs-137 & 0.348 & $\mathrm{PCl} / \mathrm{G}$ & LT & 0.072 \\
\hline 383MH0568.5 & $5 / 27 / 2004$ & soil & HASL300 & REG & 13966-00-2 & $\mathrm{K}-40$ & 3.62 & $\mathrm{PCl} / \mathrm{G}$ & & 0.8 \\
\hline 383MH0568.5 & $5 / 27 / 2004$ & soil & EPA6010 & REG & 7439-92-1 & Lead & 13 & MG/KG & & 1.5 \\
\hline 383MH0568.5 & $5 / 27 / 2004$ & soil & EPA7470 & REG & $7439-97-6$ & Mercury & 0.22 & MG/KG & & 0.1 \\
\hline 383MH0568.5 & $5 / 27 / 2004$ & soil & EPA8260 & REG & $75-09-2$ & Methylene Chloride & 1.4 & UG/KG & J,B & 5.2 \\
\hline 383МH0568.5 & $5 / 27 / 2004$ & soil & HASL300 & REG & 15092-94-1 & $\mathrm{Pb}-212$ & 0.29 & $\mathrm{PCl} / \mathrm{G}$ & $\mathrm{J}$ & 0.14 \\
\hline 383MH0568.5 & $5 / 27 / 2004$ & soil & HASL300 & REG & $15067-28-4$ & $\mathrm{~Pb}-214$ & 0.5 & $\mathrm{PCl} / \mathrm{G}$ & $\mathrm{J}$ & 0.14 \\
\hline 383MH0568.5 & $5 / 27 / 2004$ & soil & EPA6010 & REG & $7440-22-4$ & Silver & 0.5 & MG/KG & $B$ & 1 \\
\hline 383MH0600.5 & $6 / 11 / 2004$ & soil & HASL300 & REG & 14331-83-0 & Ac-228 & 0.43 & $\mathrm{PCl} / \mathrm{G}$ & $\mathrm{TI}$ & 0.31 \\
\hline 383MH0600.5 & $6 / 11 / 2004$ & soil & EPA6010 & REG & $7440-38-2$ & Arsenic & 1.8 & MG/KG & & 1 \\
\hline 383MH0600.5 & $6 / 11 / 2004$ & soil & EPA6010 & REG & 7440-39-3 & Barium & 370 & MG/KG & & 10 \\
\hline 383MH0600.5 & $6 / 11 / 2004$ & soil & EPA6010 & REG & $7440-41-7$ & Beryllium & 0.33 & MG/KG & B & 0.51 \\
\hline 383MH0600.5 & $6 / 11 / 2004$ & soil & HASL300 & REG & 14733-03-0 & Bi-214 & 0.49 & $\mathrm{PCl} / \mathrm{G}$ & $\mathrm{J}$ & 0.18 \\
\hline 383MH0600.5 & $6 / 11 / 2004$ & soil & EPA6010 & REG & 7440-43-9 & Cadmium & 0.44 & MG/KG & $B$ & 0.51 \\
\hline 383МH0600.5 & $6 / 11 / 2004$ & soil & EPA6010 & REG & 7440-47-3 & Chromium & 2.6 & MG/KG & & 1 \\
\hline 383MH0600.5 & $6 / 11 / 2004$ & soil & HASL300 & REG & 10045-97-3 & Cs-137 & 1.65 & $\mathrm{PCl} / \mathrm{G}$ & & 0.08 \\
\hline
\end{tabular}




\begin{tabular}{|c|c|c|c|c|c|c|c|c|c|c|}
\hline SAMPLE \# & $\begin{array}{l}\text { SAMPLE } \\
\text { DATE }\end{array}$ & MATRIX & $\begin{array}{l}\text { USER TEST } \\
\text { PANEL }\end{array}$ & $\begin{array}{l}\text { SAMPLE } \\
\text { PURPOSE }\end{array}$ & CAS \# & PARAMETER & RESULT & UNITS & Q & $\begin{array}{l}\text { DETECT } \\
\text { LIMIT }\end{array}$ \\
\hline 383MH0600.5 & $6 / 11 / 2004$ & soil & EPA8015 & REG & $68334-30-5$ & Diesel Range Organics & 700 & MG/KG & $M$ & 5.1 \\
\hline 383MH0600.5 & $6 / 11 / 2004$ & soil & HASL300 & REG & 13966-00-2 & $\mathrm{K}-40$ & 2.68 & $\mathrm{PCl} / \mathrm{G}$ & & 0.91 \\
\hline 383MH0600.5 & $6 / 11 / 2004$ & soil & EPA6010 & REG & $7439-92-1$ & Lead & 5.9 & MG/KG & & 1.5 \\
\hline 383MH0600.5 & $6 / 11 / 2004$ & soil & EPA7470 & REG & $7439-97-6$ & Mercury & 0.067 & MG/KG & B & 0.1 \\
\hline 383MH0600.5 & $6 / 11 / 2004$ & soil & HASL300 & REG & 15092-94-1 & $\mathrm{Pb}-212$ & 0.29 & $\mathrm{PCl} / \mathrm{G}$ & & 0.14 \\
\hline 383MH0600.5 & $6 / 11 / 2004$ & soil & HASL300 & REG & $15067-28-4$ & $\mathrm{~Pb}-214$ & 0.58 & $\mathrm{PCl} / \mathrm{G}$ & $\mathrm{J}$ & 0.14 \\
\hline 383MH0600.5 & $6 / 11 / 2004$ & soil & UGTAISOPU & REG & $15117-48-3$ & Pu-239 & 0.04 & $\mathrm{PCl} / \mathrm{G}$ & LT & 0.02 \\
\hline 383MH0600.5 & $6 / 11 / 2004$ & soil & EPA6010 & REG & $7440-22-4$ & Silver & 0.17 & MG/KG & $\mathrm{B}$ & 1 \\
\hline 383MH0600.5 & $6 / 11 / 2004$ & soil & SR7500 & REG & 10098-97-2 & Sr-90 & 0.048 & $\mathrm{PCl} / \mathrm{G}$ & $\mathrm{Y1}, \mathrm{U}$ & 0.182 \\
\hline 383MH0600.5 & $6 / 11 / 2004$ & soil & HASL300 & REG & 14913-50-9 & TI-208 & 0.093 & $\mathrm{PCl} / \mathrm{G}$ & & 0.075 \\
\hline 383МH0722.5 & $5 / 21 / 2004$ & soil & HASL300 & REG & 14331-83-0 & Ac-228 & 2.1 & $\mathrm{PCl} / \mathrm{G}$ & G & 0.61 \\
\hline 383MH0722.5 & $5 / 21 / 2004$ & soil & EPA8260 & REG & $67-64-1$ & Acetone & 8.3 & UG/KG & $\mathrm{J}$ & 23 \\
\hline 383MH0722.5 & $5 / 21 / 2004$ & soil & EPA6010 & REG & $7440-38-2$ & Arsenic & 2.5 & MG/KG & & 1.2 \\
\hline 383MH0722.5 & $5 / 21 / 2004$ & soil & EPA6010 & REG & 7440-39-3 & Barium & 200 & MG/KG & & 12 \\
\hline 383MH0722.5 & $5 / 21 / 2004$ & soil & EPA6010 & REG & $7440-41-7$ & Beryllium & 1.3 & MG/KG & & 0.59 \\
\hline 383MH0722.5 & $5 / 21 / 2004$ & soil & HASL300 & REG & 14733-03-0 & Bi-214 & 1.38 & $\mathrm{PCl} / \mathrm{G}$ & $\mathrm{G}, \mathrm{J}$ & 0.41 \\
\hline 383MH0722.5 & $5 / 21 / 2004$ & soil & EPA6010 & REG & $7440-47-3$ & Chromium & 2 & MG/KG & & 1.2 \\
\hline 383MH0722.5 & $5 / 21 / 2004$ & soil & HASL300 & REG & $10045-97-3$ & Cs-137 & 26.9 & $\mathrm{PCl} / \mathrm{G}$ & G & 0.2 \\
\hline 383MH0722.5 & $5 / 21 / 2004$ & soil & HASL300 & REG & 13966-00-2 & $\mathrm{K}-40$ & 33.2 & $\mathrm{PCl} / \mathrm{G}$ & G & 2.2 \\
\hline 383MH0722.5 & $5 / 21 / 2004$ & soil & EPA6010 & REG & $7439-92-1$ & Lead & 12 & MG/KG & & 0.35 \\
\hline 383MH0722.5 & $5 / 21 / 2004$ & soil & EPA7470 & REG & 7439-97-6 & Mercury & 0.055 & MG/KG & $B$ & 0.12 \\
\hline 383MH0722.5 & $5 / 21 / 2004$ & soil & HASL300 & REG & 15092-94-1 & $\mathrm{Pb}-212$ & 2.48 & $\mathrm{PCl} / \mathrm{G}$ & $\mathrm{J}$ & 0.37 \\
\hline 383MH0722.5 & $5 / 21 / 2004$ & soil & HASL300 & REG & 15067-28-4 & $\mathrm{Pb}-214$ & 1.28 & $\mathrm{PCl} / \mathrm{G}$ & $\mathrm{G}, \mathrm{J}$ & 0.46 \\
\hline 383MH0722.5 & $5 / 21 / 2004$ & soil & UGTAISOPU & REG & 15117-48-3 & Pu-239 & 0.173 & $\mathrm{PCl} / \mathrm{G}$ & & 0.01 \\
\hline 383MH0722.5 & $5 / 21 / 2004$ & soil & HASL300 & REG & 14913-50-9 & TI-208 & 0.79 & $\mathrm{PCl} / \mathrm{G}$ & G & 0.24 \\
\hline 383MH0723.5 & $5 / 21 / 2004$ & soil & HASL300 & REG & 14331-83-0 & Ac-228 & 2.01 & $\mathrm{PCl} / \mathrm{G}$ & G & 0.84 \\
\hline 383MH0723.5 & $5 / 21 / 2004$ & soil & EPA8260 & REG & 67-64-1 & Acetone & 10 & UG/KG & $\mathrm{J}$ & 23 \\
\hline 383MH0723.5 & $5 / 21 / 2004$ & soil & EPA6010 & REG & $7440-38-2$ & Arsenic & 2.5 & MG/KG & & 1.2 \\
\hline 383MH0723.5 & $5 / 21 / 2004$ & soil & EPA6010 & REG & 7440-39-3 & Barium & 200 & MG/KG & & 12 \\
\hline 383MH0723.5 & $5 / 21 / 2004$ & soil & EPA6010 & REG & $7440-41-7$ & Beryllium & 1.3 & MG/KG & & 0.59 \\
\hline 383MH0723.5 & $5 / 21 / 2004$ & soil & HASL300 & REG & 14733-03-0 & $\mathrm{Bi}-214$ & 1.32 & $\mathrm{PCl} / \mathrm{G}$ & $\mathrm{G}, \mathrm{J}$ & 0.69 \\
\hline 383MH0723.5 & $5 / 21 / 2004$ & soil & EPA6010 & REG & $7440-47-3$ & Chromium & 1.7 & MG/KG & & 1.2 \\
\hline 383MH0723.5 & $5 / 21 / 2004$ & soil & HASL300 & REG & 10045-97-3 & Cs-137 & 83.7 & $\mathrm{PCl} / \mathrm{G}$ & G & 0.3 \\
\hline 383МH0723.5 & $5 / 21 / 2004$ & soil & HASL300 & REG & 13966-00-2 & $\mathrm{K}-40$ & 40.7 & $\mathrm{PCl} / \mathrm{G}$ & G & 1.8 \\
\hline 383MH0723.5 & $5 / 21 / 2004$ & soil & EPA6010 & REG & $7439-92-1$ & Lead & 13 & MG/KG & & 0.35 \\
\hline
\end{tabular}




\begin{tabular}{|c|c|c|c|c|c|c|c|c|c|c|}
\hline SAMPLE \# & $\begin{array}{l}\text { SAMPLE } \\
\text { DATE }\end{array}$ & MATRIX & $\begin{array}{l}\text { USER TEST } \\
\text { PANEL }\end{array}$ & $\begin{array}{l}\text { SAMPLE } \\
\text { PURPOSE }\end{array}$ & CAS \# & PARAMETER & RESULT & UNITS & Q & $\begin{array}{l}\text { DETECT } \\
\text { LIMIT }\end{array}$ \\
\hline 383МH0723.5 & $5 / 21 / 2004$ & soil & EPA7470 & REG & $7439-97-6$ & Mercury & 0.0021 & MG/KG & B & 0.12 \\
\hline 383MH0723.5 & $5 / 21 / 2004$ & soil & EPA8260 & REG & $75-09-2$ & Methylene Chloride & 0.98 & UG/KG & J,B & 5.9 \\
\hline 383MH0723.5 & $5 / 21 / 2004$ & soil & HASL300 & REG & 15092-94-1 & $\mathrm{Pb}-212$ & 2.48 & $\mathrm{PCl} / \mathrm{G}$ & $\mathrm{J}$ & 0.55 \\
\hline 383MH0723.5 & $5 / 21 / 2004$ & soil & UGTAISOPU & REG & 13981-16-3 & Pu-238 & 0.059 & $\mathrm{PCl} / \mathrm{G}$ & & 0.021 \\
\hline 383МH0723.5 & $5 / 21 / 2004$ & soil & UGTAISOPU & REG & $15117-48-3$ & Pu-239 & 0.262 & $\mathrm{PCl} / \mathrm{G}$ & & 0.009 \\
\hline 383MH0723.5 & $5 / 21 / 2004$ & soil & SR7500 & REG & 10098-97-2 & Sr-90 & 0.85 & $\mathrm{PCl} / \mathrm{G}$ & & 0.19 \\
\hline 383МH0723.5 & $5 / 21 / 2004$ & soil & HASL300 & REG & 14913-50-9 & TI-208 & 0.87 & $\mathrm{PCl} / \mathrm{G}$ & G & 0.35 \\
\hline 383MH0767.5 & $5 / 21 / 2004$ & soil & EPA6010 & REG & $7440-38-2$ & Arsenic & 1.1 & MG/KG & & 1 \\
\hline 383MH0767.5 & $5 / 21 / 2004$ & soil & EPA6010 & REG & $7440-39-3$ & Barium & 24 & MG/KG & & 10 \\
\hline 383МH0767.5 & $5 / 21 / 2004$ & soil & EPA6010 & REG & $7440-41-7$ & Beryllium & 0.28 & MG/KG & B & 0.51 \\
\hline 383МH0767.5 & $5 / 21 / 2004$ & soil & HASL300 & REG & 14733-03-0 & Bi-214 & 0.27 & $\mathrm{PCl} / \mathrm{G}$ & $\mathrm{J}$ & 0.14 \\
\hline 383MH0767.5 & $5 / 21 / 2004$ & soil & EPA6010 & REG & $7440-43-9$ & Cadmium & 0.046 & MG/KG & B & 0.51 \\
\hline 383МH0767.5 & $5 / 21 / 2004$ & soil & EPA6010 & REG & $7440-47-3$ & Chromium & 2.5 & MG/KG & & 1 \\
\hline 383МH0767.5 & $5 / 21 / 2004$ & soil & HASL300 & REG & 13966-00-2 & $\mathrm{K}-40$ & 2.21 & $\mathrm{PCl} / \mathrm{G}$ & & 0.83 \\
\hline 383МH0767.5 & $5 / 21 / 2004$ & soil & EPA6010 & REG & $7439-92-1$ & Lead & 8.9 & MG/KG & & 1.5 \\
\hline 383МH0767.5 & $5 / 21 / 2004$ & soil & EPA7470 & REG & 7439-97-6 & Mercury & 0.056 & MG/KG & B & 0.1 \\
\hline 383МH0767.5 & $5 / 21 / 2004$ & soil & HASL300 & REG & 15092-94-1 & $\mathrm{Pb}-212$ & 0.212 & $\mathrm{PCl} / \mathrm{G}$ & $\mathrm{J}$ & 0.108 \\
\hline 383МH0767.5 & $5 / 21 / 2004$ & soil & HASL300 & REG & 15067-28-4 & $\mathrm{Pb}-214$ & 0.37 & $\mathrm{PCl} / \mathrm{G}$ & $\mathrm{J}$ & 0.14 \\
\hline 383MH0767.5 & $5 / 21 / 2004$ & soil & EPA6010 & REG & $7440-22-4$ & Silver & 0.35 & MG/KG & B & 1 \\
\hline 383МH0832.5 & $5 / 26 / 2004$ & soil & EPA6010 & REG & $7440-38-2$ & Arsenic & 2.7 & MG/KG & & 1 \\
\hline 383МH0832.5 & $5 / 26 / 2004$ & soil & EPA6010 & REG & 7440-39-3 & Barium & 22 & MG/KG & & 10 \\
\hline 383MH0832.5 & $5 / 26 / 2004$ & soil & EPA6010 & REG & $7440-41-7$ & Beryllium & 0.42 & MG/KG & B & 0.52 \\
\hline 383МH0832.5 & $5 / 26 / 2004$ & soil & HASL300 & REG & 14733-03-0 & $\mathrm{Bi}-214$ & 0.56 & $\mathrm{PCl} / \mathrm{G}$ & $\mathrm{G}, \mathrm{J}$ & 0.26 \\
\hline 383МH0832.5 & $5 / 26 / 2004$ & soil & EPA6010 & REG & 7440-47-3 & Chromium & 2.1 & MG/KG & & 1 \\
\hline 383MH0832.5 & $5 / 26 / 2004$ & soil & HASL300 & REG & 10045-97-3 & Cs-137 & 0.84 & $\mathrm{PCl} / \mathrm{G}$ & G & 0.12 \\
\hline 383МH0832.5 & $5 / 26 / 2004$ & soil & HASL300 & REG & 13966-00-2 & $\mathrm{K}-40$ & 7.1 & $\mathrm{PCl} / \mathrm{G}$ & G & 1.5 \\
\hline 383MH0832.5 & $5 / 26 / 2004$ & soil & EPA6010 & REG & $7439-92-1$ & Lead & 6.3 & MG/KG & & 1.6 \\
\hline 383МH0832.5 & $5 / 26 / 2004$ & soil & EPA7470 & REG & $7439-97-6$ & Mercury & 0.055 & MG/KG & B & 0.1 \\
\hline 383MH0832.5 & $5 / 26 / 2004$ & soil & EPA8260 & REG & $75-09-2$ & Methylene Chloride & 1.4 & UG/KG & J,B & 5.2 \\
\hline 383МH0832.5 & $5 / 26 / 2004$ & soil & HASL300 & REG & 15092-94-1 & $\mathrm{Pb}-212$ & 0.56 & $\mathrm{PCl} / \mathrm{G}$ & $\mathrm{J}$ & 0.17 \\
\hline 383MH0832.5 & $5 / 26 / 2004$ & soil & HASL300 & REG & $15067-28-4$ & $\mathrm{~Pb}-214$ & 0.64 & $\mathrm{PCl} / \mathrm{G}$ & $\mathrm{G}, \mathrm{J}$ & 0.21 \\
\hline 383МH0858.0 & $5 / 26 / 2004$ & soil & EPA8260 & REG & 67-64-1 & Acetone & 7.3 & UG/KG & $\mathrm{J}$ & 20 \\
\hline 383МH0858.0 & $5 / 26 / 2004$ & soil & EPA6010 & REG & $7440-38-2$ & Arsenic & 0.68 & MG/KG & $B$ & 1 \\
\hline 383МH0858.0 & $5 / 26 / 2004$ & soil & EPA6010 & REG & $7440-39-3$ & Barium & 3 & MG/KG & $B$ & 10 \\
\hline 383МH0858.0 & $5 / 26 / 2004$ & soil & EPA6010 & REG & $7440-41-7$ & Beryllium & 0.19 & MG/KG & $B$ & 0.5 \\
\hline
\end{tabular}




\begin{tabular}{|c|c|c|c|c|c|c|c|c|c|c|}
\hline SAMPLE \# & $\begin{array}{l}\text { SAMPLE } \\
\text { DATE }\end{array}$ & MATRIX & $\begin{array}{l}\text { USER TEST } \\
\text { PANEL }\end{array}$ & $\begin{array}{l}\text { SAMPLE } \\
\text { PURPOSE }\end{array}$ & CAS \# & PARAMETER & RESULT & UNITS & Q & $\begin{array}{l}\text { DETECT } \\
\text { LIMIT }\end{array}$ \\
\hline 383МH0858.0 & $5 / 26 / 2004$ & soil & EPA6010 & REG & $7440-43-9$ & Cadmium & 0.026 & MG/KG & $B$ & 0.5 \\
\hline 383МH0858.0 & $5 / 26 / 2004$ & soil & EPA6010 & REG & $7440-47-3$ & Chromium & 1.2 & MG/KG & & 1 \\
\hline 383МH0858.0 & $5 / 26 / 2004$ & soil & EPA7470 & REG & 7439-97-6 & Mercury & 0.017 & MG/KG & $B$ & 0.1 \\
\hline 383МH0916.5 & $6 / 11 / 2004$ & soil & HASL300 & REG & 14331-83-0 & Ac-228 & 1.5 & $\mathrm{PCl} / \mathrm{G}$ & G & 0.49 \\
\hline 383MH0916.5 & $6 / 11 / 2004$ & soil & EPA6010 & REG & $7440-38-2$ & Arsenic & 2.4 & MG/KG & & 1.1 \\
\hline 383MH0916.5 & $6 / 11 / 2004$ & soil & EPA6010 & REG & 7440-39-3 & Barium & 81 & MG/KG & & 11 \\
\hline 383МH0916.5 & $6 / 11 / 2004$ & soil & EPA6010 & REG & $7440-41-7$ & Beryllium & 0.67 & MG/KG & & 0.55 \\
\hline 383MH0916.5 & $6 / 11 / 2004$ & soil & HASL300 & REG & 14733-03-0 & $\mathrm{Bi}-214$ & 0.71 & $\mathrm{PCl} / \mathrm{G}$ & $\mathrm{G}, \mathrm{J}$ & 0.27 \\
\hline 383МH0916.5 & $6 / 11 / 2004$ & soil & EPA6010 & REG & $7440-47-3$ & Chromium & 4.3 & MG/KG & & 1.1 \\
\hline 383МH0916.5 & $6 / 11 / 2004$ & soil & HASL300 & REG & 10045-97-3 & Cs-137 & 0.15 & $\mathrm{PCl} / \mathrm{G}$ & LT,G & 0.143 \\
\hline 383МH0916.5 & $6 / 11 / 2004$ & soil & HASL300 & REG & 13966-00-2 & $\mathrm{K}-40$ & 27 & $\mathrm{PCl} / \mathrm{G}$ & G & 1.2 \\
\hline 383MH0916.5 & $6 / 11 / 2004$ & soil & EPA6010 & REG & $7439-92-1$ & Lead & 9.6 & MG/KG & & 0.33 \\
\hline 383МH0916.5 & $6 / 11 / 2004$ & soil & EPA7470 & REG & 7439-97-6 & Mercury & 0.088 & MG/KG & $B$ & 0.11 \\
\hline 383МH0916.5 & $6 / 11 / 2004$ & soil & HASL300 & REG & 15092-94-1 & $\mathrm{Pb}-212$ & 1.83 & $\mathrm{PCl} / \mathrm{G}$ & G & 0.18 \\
\hline 383MH0916.5 & $6 / 11 / 2004$ & soil & HASL300 & REG & 15067-28-4 & $\mathrm{Pb}-214$ & 0.69 & $\mathrm{PCl} / \mathrm{G}$ & $\mathrm{G}, \mathrm{J}$ & 0.23 \\
\hline 383МH0916.5 & $6 / 11 / 2004$ & soil & UGTAISOPU & REG & 15117-48-3 & Pu-239 & 0.016 & $\mathrm{PCl} / \mathrm{G}$ & LT & 0.009 \\
\hline 383МH0916.5 & $6 / 11 / 2004$ & soil & HASL300 & REG & 14913-50-9 & TI-208 & 0.64 & $\mathrm{PCl} / \mathrm{G}$ & G & 0.11 \\
\hline 383MH0922.5 & $6 / 11 / 2004$ & soil & HASL300 & REG & 14331-83-0 & Ac-228 & 2.14 & $\mathrm{PCl} / \mathrm{G}$ & G & 0.54 \\
\hline 383МH0922.5 & $6 / 11 / 2004$ & soil & EPA6010 & REG & $7440-38-2$ & Arsenic & 3.8 & MG/KG & & 1.1 \\
\hline 383МH0922.5 & $6 / 11 / 2004$ & soil & EPA6010 & REG & $7440-39-3$ & Barium & 160 & MG/KG & & 11 \\
\hline 383MH0922.5 & $6 / 11 / 2004$ & soil & EPA6010 & REG & $7440-41-7$ & Beryllium & 1 & MG/KG & & 0.56 \\
\hline 383MH0922.5 & $6 / 11 / 2004$ & soil & HASL300 & REG & 14733-03-0 & $\mathrm{Bi}-214$ & 0.92 & $\mathrm{PCl} / \mathrm{G}$ & $\mathrm{G}, \mathrm{J}$ & 0.26 \\
\hline 383МH0922.5 & $6 / 11 / 2004$ & soil & EPA6010 & REG & $7440-47-3$ & Chromium & 4.3 & MG/KG & & 1.1 \\
\hline 383MH0922.5 & $6 / 11 / 2004$ & soil & HASL300 & REG & 14391-16-3 & Eu-155 & 0.32 & $\mathrm{PCl} / \mathrm{G}$ & $\mathrm{G}, \mathrm{TI}$ & 0.3 \\
\hline 383МH0922.5 & $6 / 11 / 2004$ & soil & HASL300 & REG & 13966-00-2 & $\mathrm{K}-40$ & 31.7 & $\mathrm{PCl} / \mathrm{G}$ & G & 1.4 \\
\hline 383МH0922.5 & $6 / 11 / 2004$ & soil & EPA6010 & REG & $7439-92-1$ & Lead & 13 & MG/KG & & 0.33 \\
\hline 383МH0922.5 & $6 / 11 / 2004$ & soil & EPA7470 & REG & $7439-97-6$ & Mercury & 0.07 & MG/KG & B & 0.11 \\
\hline 383МH0922.5 & $6 / 11 / 2004$ & soil & HASL300 & REG & 15092-94-1 & $\mathrm{Pb}-212$ & 2.27 & $\mathrm{PCl} / \mathrm{G}$ & G & 0.23 \\
\hline 383MH0922.5 & $6 / 11 / 2004$ & soil & HASL300 & REG & 15067-28-4 & $\mathrm{Pb}-214$ & 0.72 & $\mathrm{PCl} / \mathrm{G}$ & $\mathrm{G}, \mathrm{J}$ & 0.25 \\
\hline 383МH0922.5 & $6 / 11 / 2004$ & soil & EPA6010 & REG & 7782-49-2 & Selenium & 0.26 & MG/KG & $B$ & 0.56 \\
\hline 383MH0922.5 & $6 / 11 / 2004$ & soil & EPA6010 & REG & $7440-22-4$ & Silver & 0.15 & MG/KG & $B$ & 1.1 \\
\hline 383МH0922.5 & $6 / 11 / 2004$ & soil & HASL300 & REG & 15065-10-8 & Th-234 & 1.8 & $\mathrm{PCl} / \mathrm{G}$ & G & 1.67 \\
\hline 383МH0922.5 & $6 / 11 / 2004$ & soil & HASL300 & REG & 14913-50-9 & TI-208 & 0.76 & $\mathrm{PCl} / \mathrm{G}$ & G & 0.15 \\
\hline 383MH1002.0 & $5 / 22 / 2004$ & soil & HASL300 & REG & 14331-83-0 & Ac-228 & 0.7 & $\mathrm{PCl} / \mathrm{G}$ & G & 0.31 \\
\hline 383MH1002.0 & $5 / 22 / 2004$ & soil & EPA6010 & REG & $7440-38-2$ & Arsenic & 3 & MG/KG & & 1.1 \\
\hline
\end{tabular}




\begin{tabular}{|c|c|c|c|c|c|c|c|c|c|c|}
\hline SAMPLE \# & $\begin{array}{l}\text { SAMPLE } \\
\text { DATE }\end{array}$ & MATRIX & $\begin{array}{l}\text { USER TEST } \\
\text { PANEL }\end{array}$ & $\begin{array}{l}\text { SAMPLE } \\
\text { PURPOSE }\end{array}$ & CAS \# & PARAMETER & RESULT & UNITS & Q & $\begin{array}{l}\text { DETECT } \\
\text { LIMIT }\end{array}$ \\
\hline 383МH1002.0 & $5 / 22 / 2004$ & soil & EPA6010 & REG & $7440-39-3$ & Barium & 190 & MG/KG & & 11 \\
\hline 383MH1002.0 & $5 / 22 / 2004$ & soil & EPA6010 & REG & $7440-41-7$ & Beryllium & 0.78 & MG/KG & & 0.53 \\
\hline 383MH1002.0 & $5 / 22 / 2004$ & soil & HASL300 & REG & 14733-03-0 & $\mathrm{Bi}-214$ & 0.48 & $\mathrm{PCl} / \mathrm{G}$ & $\mathrm{G}, \mathrm{J}$ & 0.18 \\
\hline 383MH1002.0 & $5 / 22 / 2004$ & soil & EPA6010 & REG & $7440-47-3$ & Chromium & 2.8 & MG/KG & & 1.1 \\
\hline 383МH1002.0 & $5 / 22 / 2004$ & soil & HASL300 & REG & $10045-97-3$ & Cs-137 & 1.04 & $\mathrm{PCl} / \mathrm{G}$ & G & 0.09 \\
\hline 383MH1002.0 & $5 / 22 / 2004$ & soil & HASL300 & REG & 13966-00-2 & $\mathrm{K}-40$ & 13.8 & $\mathrm{PCl} / \mathrm{G}$ & G & 1 \\
\hline 383МH1002.0 & $5 / 22 / 2004$ & soil & EPA6010 & REG & $7439-92-1$ & Lead & 17 & MG/KG & & 1.6 \\
\hline 383MH1002.0 & $5 / 22 / 2004$ & soil & EPA7470 & REG & $7439-97-6$ & Mercury & 0.056 & MG/KG & B & 0.11 \\
\hline 383MH1002.0 & $5 / 22 / 2004$ & soil & HASL300 & REG & 15092-94-1 & $\mathrm{Pb}-212$ & 0.83 & $\mathrm{PCl} / \mathrm{G}$ & $\mathrm{J}$ & 0.15 \\
\hline 383МH1002.0 & $5 / 22 / 2004$ & soil & HASL300 & REG & $15067-28-4$ & $\mathrm{~Pb}-214$ & 0.61 & $\mathrm{PCl} / \mathrm{G}$ & $\mathrm{G}, \mathrm{J}$ & 0.17 \\
\hline 383MH1002.0 & $5 / 22 / 2004$ & soil & HASL300 & REG & 14913-50-9 & TI-208 & 0.293 & $\mathrm{PCl} / \mathrm{G}$ & G & 0.078 \\
\hline 383MH1011.5 & $5 / 22 / 2004$ & soil & EPA6010 & REG & $7440-38-2$ & Arsenic & 1.9 & MG/KG & & 1.1 \\
\hline 383MH1011.5 & $5 / 22 / 2004$ & soil & EPA6010 & REG & $7440-39-3$ & Barium & 56 & MG/KG & & 11 \\
\hline 383МH1011.5 & $5 / 22 / 2004$ & soil & EPA6010 & REG & $7440-41-7$ & Beryllium & 0.47 & MG/KG & B & 0.54 \\
\hline 383MH1011.5 & $5 / 22 / 2004$ & soil & HASL300 & REG & 14733-03-0 & $\mathrm{Bi}-214$ & 0.67 & $\mathrm{PCl} / \mathrm{G}$ & $\mathrm{G}, \mathrm{J}$ & 0.27 \\
\hline 383MH1011.5 & $5 / 22 / 2004$ & soil & EPA6010 & REG & $7440-47-3$ & Chromium & 3.8 & MG/KG & & 1.1 \\
\hline 383MH1011.5 & $5 / 22 / 2004$ & soil & HASL300 & REG & $10045-97-3$ & Cs-137 & 0.42 & $\mathrm{PCl} / \mathrm{G}$ & LT,G & 0.1 \\
\hline 383MH1011.5 & $5 / 22 / 2004$ & soil & HASL300 & REG & 13966-00-2 & $\mathrm{K}-40$ & 5.3 & $\mathrm{PCl} / \mathrm{G}$ & $\mathrm{G}$ & 1.5 \\
\hline 383MH1011.5 & $5 / 22 / 2004$ & soil & EPA6010 & REG & 7439-92-1 & Lead & 5.1 & MG/KG & & 1.6 \\
\hline 383MH1011.5 & $5 / 22 / 2004$ & soil & EPA7470 & REG & 7439-97-6 & Mercury & 0.073 & MG/KG & B & 0.11 \\
\hline 383MH1011.5 & $5 / 22 / 2004$ & soil & HASL300 & REG & 15092-94-1 & $\mathrm{Pb}-212$ & 0.49 & $\mathrm{PCl} / \mathrm{G}$ & $\mathrm{J}$ & 0.21 \\
\hline 383MH1011.5 & $5 / 22 / 2004$ & soil & HASL300 & REG & $15067-28-4$ & $\mathrm{~Pb}-214$ & 0.56 & $\mathrm{PCl} / \mathrm{G}$ & $\mathrm{G}, \mathrm{J}$ & 0.25 \\
\hline 383МH1011.5 & $5 / 22 / 2004$ & soil & EPA6010 & REG & $7440-22-4$ & Silver & 0.076 & MG/KG & B & 1.1 \\
\hline 383MH1100.5 & $6 / 11 / 2004$ & soil & HASL300 & REG & 14331-83-0 & Ac-228 & 1.47 & $\mathrm{PCl} / \mathrm{G}$ & G & 0.49 \\
\hline 383MH1100.5 & $6 / 11 / 2004$ & soil & HASL300 & REG & 14596-10-2 & Am-241 & 0.95 & $\mathrm{PCl} / \mathrm{G}$ & G & 0.33 \\
\hline 383MH1100.5 & $6 / 11 / 2004$ & soil & EPA6010 & REG & $7440-38-2$ & Arsenic & 5.7 & MG/KG & & 1.1 \\
\hline 383MH1100.5 & $6 / 11 / 2004$ & soil & EPA6010 & REG & $7440-39-3$ & Barium & 210 & MG/KG & & 11 \\
\hline 383MH1100.5 & $6 / 11 / 2004$ & soil & EPA8270 & REG & $56-55-3$ & Benzo(A)Anthracene & 130 & UG/KG & $\mathrm{J}$ & 350 \\
\hline 383MH1100.5 & $6 / 11 / 2004$ & soil & EPA8270 & REG & $50-32-8$ & Benzo(A)Pyrene & 110 & UG/KG & $\mathrm{J}$ & 350 \\
\hline 383МH1100.5 & $6 / 11 / 2004$ & soil & EPA8270 & REG & $205-99-2$ & Benzo(B)Fluoranthene & 210 & UG/KG & $\mathrm{J}$ & 350 \\
\hline 383MH1100.5 & $6 / 11 / 2004$ & soil & EPA8270 & REG & $191-24-2$ & Benzo(G,H,I)Perylene & 83 & UG/KG & $\mathrm{J}$ & 350 \\
\hline 383МH1100.5 & $6 / 11 / 2004$ & soil & EPA8270 & REG & $207-08-9$ & Benzo(K)Fluoranthene & 94 & UG/KG & $\mathrm{J}$ & 350 \\
\hline 383MH1100.5 & $6 / 11 / 2004$ & soil & EPA6010 & REG & $7440-41-7$ & Beryllium & 0.71 & MG/KG & & 0.53 \\
\hline 383MH1100.5 & $6 / 11 / 2004$ & soil & HASL300 & REG & 14913-49-6 & $\mathrm{Bi}-212$ & 2.1 & $\mathrm{PCl} / \mathrm{G}$ & $\mathrm{G}, \mathrm{TI}$ & 1.8 \\
\hline 383MH1100.5 & $6 / 11 / 2004$ & soil & HASL300 & REG & 14733-03-0 & Bi-214 & 0.86 & $\mathrm{PCl} / \mathrm{G}$ & $\mathrm{G}, \mathrm{J}$ & 0.41 \\
\hline
\end{tabular}




\begin{tabular}{|c|c|c|c|c|c|c|c|c|c|c|}
\hline SAMPLE \# & $\begin{array}{l}\text { SAMPLE } \\
\text { DATE }\end{array}$ & MATRIX & $\begin{array}{l}\text { USER TEST } \\
\text { PANEL }\end{array}$ & $\begin{array}{l}\text { SAMPLE } \\
\text { PURPOSE }\end{array}$ & CAS \# & PARAMETER & RESULT & UNITS & Q & $\begin{array}{l}\text { DETECT } \\
\text { LIMIT }\end{array}$ \\
\hline 383MH1100.5 & $6 / 11 / 2004$ & soil & EPA8270 & REG & 117-81-7 & Bis(2-Ethylhexyl)Phthalate & 110 & UG/KG & $\mathrm{J}$ & 350 \\
\hline 383MH1100.5 & $6 / 11 / 2004$ & soil & EPA8270 & REG & $85-68-7$ & Butyl Benzyl Phthalate & 35 & UG/KG & $\mathrm{J}$ & 350 \\
\hline 383MH1100.5 & $6 / 11 / 2004$ & soil & EPA6010 & REG & $7440-43-9$ & Cadmium & 0.35 & MG/KG & $B$ & 0.53 \\
\hline 383MH1100.5 & $6 / 11 / 2004$ & soil & EPA6010 & REG & $7440-47-3$ & Chromium & 6.9 & MG/KG & & 1.1 \\
\hline 383MH1100.5 & $6 / 11 / 2004$ & soil & EPA8270 & REG & $218-01-9$ & Chrysene & 170 & UG/KG & $\mathrm{J}$ & 350 \\
\hline 383MH1100.5 & $6 / 11 / 2004$ & soil & HASL300 & REG & $10045-97-3$ & Cs-137 & 47.3 & $\mathrm{PCl} / \mathrm{G}$ & G & 0.2 \\
\hline 383MH1100.5 & $6 / 11 / 2004$ & soil & EPA8015 & REG & $68334-30-5$ & Diesel Range Organics & 81 & MG/KG & Z,H & 5.3 \\
\hline 383MH1100.5 & $6 / 11 / 2004$ & soil & EPA8270 & REG & 84-74-2 & Di-N-Butyl Phthalate & 26 & UG/KG & $\mathrm{J}$ & 350 \\
\hline 383MH1100.5 & $6 / 11 / 2004$ & soil & EPA8270 & REG & $206-44-0$ & Fluoranthene & 170 & UG/KG & $\mathrm{J}$ & 350 \\
\hline 383MH1100.5 & $6 / 11 / 2004$ & soil & EPA8270 & REG & $193-39-5$ & Indeno(1,2,3-Cd)Pyrene & 54 & UG/KG & $\mathrm{J}$ & 350 \\
\hline 383MH1100.5 & $6 / 11 / 2004$ & soil & HASL300 & REG & $13966-00-2$ & $\mathrm{~K}-40$ & 19.6 & $\mathrm{PCl} / \mathrm{G}$ & G & 1.4 \\
\hline 383MH1100.5 & $6 / 11 / 2004$ & soil & EPA6010 & REG & $7439-92-1$ & Lead & 38 & MG/KG & & 1.6 \\
\hline 383MH1100.5 & $6 / 11 / 2004$ & soil & EPA7470 & REG & 7439-97-6 & Mercury & 0.033 & MG/KG & $B$ & 0.11 \\
\hline 383MH1100.5 & $6 / 11 / 2004$ & soil & HASL300 & REG & 15092-94-1 & $\mathrm{Pb}-212$ & 1.67 & $\mathrm{PCl} / \mathrm{G}$ & G & 0.38 \\
\hline 383MH1100.5 & $6 / 11 / 2004$ & soil & HASL300 & REG & 15067-28-4 & $\mathrm{Pb}-214$ & 0.8 & $\mathrm{PCl} / \mathrm{G}$ & $\mathrm{G}, \mathrm{J}$ & 0.48 \\
\hline 383MH1100.5 & $6 / 11 / 2004$ & soil & EPA8270 & REG & $85-01-8$ & Phenanthrene & 56 & UG/KG & $\mathrm{J}$ & 350 \\
\hline 383MH1100.5 & $6 / 11 / 2004$ & soil & UGTAISOPU & REG & 13981-16-3 & Pu-238 & 0.46 & $\mathrm{PCl} / \mathrm{G}$ & & 0.01 \\
\hline 383MH1100.5 & $6 / 11 / 2004$ & soil & UGTAISOPU & REG & 15117-48-3 & Pu-239 & 3.04 & $\mathrm{PCl} / \mathrm{G}$ & & 0.03 \\
\hline 383MH1100.5 & $6 / 11 / 2004$ & soil & EPA8270 & REG & $129-00-0$ & Pyrene & 190 & UG/KG & $\mathrm{J}$ & 350 \\
\hline 383MH1100.5 & $6 / 11 / 2004$ & soil & SR7500 & REG & 10098-97-2 & Sr-90 & 0.62 & $\mathrm{PCl} / \mathrm{G}$ & Y1 & 0.19 \\
\hline 383MH1100.5 & $6 / 11 / 2004$ & soil & HASL300 & REG & 14913-50-9 & TI-208 & 0.36 & $\mathrm{PCl} / \mathrm{G}$ & G & 0.18 \\
\hline 383MH1104.0 & $6 / 11 / 2004$ & soil & EPA6010 & REG & $7440-38-2$ & Arsenic & 0.69 & MG/KG & B & 1 \\
\hline 383MH1104.0 & $6 / 11 / 2004$ & soil & EPA6010 & REG & $7440-39-3$ & Barium & 15 & MG/KG & & 10 \\
\hline 383MH1104.0 & $6 / 11 / 2004$ & soil & EPA6010 & REG & $7440-41-7$ & Beryllium & 0.42 & MG/KG & B & 0.51 \\
\hline 383MH1104.0 & $6 / 11 / 2004$ & soil & HASL300 & REG & $14733-03-0$ & Bi-214 & 0.33 & $\mathrm{PCl} / \mathrm{G}$ & $\mathrm{J}$ & 0.22 \\
\hline 383MH1104.0 & $6 / 11 / 2004$ & soil & EPA8270 & REG & 117-81-7 & Bis(2-Ethylhexyl)Phthalate & 84 & UG/KG & $\mathrm{J}$ & 340 \\
\hline 383МH1104.0 & $6 / 11 / 2004$ & soil & EPA6010 & REG & $7440-43-9$ & Cadmium & 0.037 & MG/KG & B & 0.51 \\
\hline 383МH1104.0 & $6 / 11 / 2004$ & soil & EPA6010 & REG & $7440-47-3$ & Chromium & 2.1 & MG/KG & & 1 \\
\hline 383MH1104.0 & $6 / 11 / 2004$ & soil & HASL300 & REG & $10045-97-3$ & Cs-137 & 1.83 & $\mathrm{PCl} / \mathrm{G}$ & & 0.1 \\
\hline 383МH1104.0 & $6 / 11 / 2004$ & soil & EPA6010 & REG & $7439-92-1$ & Lead & 4 & MG/KG & & 1.5 \\
\hline 383MH1104.0 & $6 / 11 / 2004$ & soil & EPA7470 & REG & 7439-97-6 & Mercury & 0.06 & MG/KG & B & 0.1 \\
\hline 383MH1104.0 & $6 / 11 / 2004$ & soil & HASL300 & REG & 15092-94-1 & $\mathrm{Pb}-212$ & 0.212 & $\mathrm{PCl} / \mathrm{G}$ & & 0.134 \\
\hline 383МH1104.0 & $6 / 11 / 2004$ & soil & HASL300 & REG & $15067-28-4$ & $\mathrm{~Pb}-214$ & 0.41 & $\mathrm{PCl} / \mathrm{G}$ & $\mathrm{J}$ & 0.18 \\
\hline 383MH1104.0 & $6 / 11 / 2004$ & soil & UGTAISOPU & REG & $15117-48-3$ & Pu-239 & 0.06 & $\mathrm{PCl} / \mathrm{G}$ & & 0.009 \\
\hline 383MH1104.0 & $6 / 11 / 2004$ & soil & EPA6010 & REG & $7440-22-4$ & Silver & 0.071 & MG/KG & B & 1 \\
\hline
\end{tabular}




\begin{tabular}{|c|c|c|c|c|c|c|c|c|c|c|}
\hline SAMPLE \# & $\begin{array}{l}\text { SAMPLE } \\
\text { DATE }\end{array}$ & MATRIX & $\begin{array}{l}\text { USER TEST } \\
\text { PANEL }\end{array}$ & $\begin{array}{l}\text { SAMPLE } \\
\text { PURPOSE }\end{array}$ & CAS \# & PARAMETER & RESULT & UNITS & $Q$ & $\begin{array}{l}\text { DETECT } \\
\text { LIMIT }\end{array}$ \\
\hline 383МH1215.0 & $5 / 23 / 2004$ & soil & HASL300 & REG & $14331-83-0$ & Ac-228 & 1.9 & $\mathrm{PCl} / \mathrm{G}$ & G & 0.49 \\
\hline 383MH1215.0 & $5 / 23 / 2004$ & soil & EPA6010 & REG & 7440-38-2 & Arsenic & 3.2 & MG/KG & & 1.2 \\
\hline 383MH1215.0 & $5 / 23 / 2004$ & soil & EPA6010 & REG & 7440-39-3 & Barium & 110 & MG/KG & & 12 \\
\hline 383MH1215.0 & $5 / 23 / 2004$ & soil & EPA6010 & REG & $7440-41-7$ & Beryllium & 1.1 & MG/KG & & 0.58 \\
\hline 383МH1215.0 & $5 / 23 / 2004$ & soil & HASL300 & REG & 14733-03-0 & Bi-214 & 1.22 & $\mathrm{PCl} / \mathrm{G}$ & $\mathrm{G}, \mathrm{J}$ & 0.32 \\
\hline 383MH1215.0 & $5 / 23 / 2004$ & soil & EPA6010 & REG & $7440-47-3$ & Chromium & 0.98 & MG/KG & $B$ & 1.2 \\
\hline 383MH1215.0 & $5 / 23 / 2004$ & soil & HASL300 & REG & 10045-97-3 & Cs-137 & 23.5 & $\mathrm{PCl} / \mathrm{G}$ & G & 0.1 \\
\hline 383МH1215.0 & $5 / 23 / 2004$ & soil & HASL300 & REG & 13966-00-2 & $\mathrm{K}-40$ & 36.7 & $\mathrm{PCl} / \mathrm{G}$ & $\mathrm{G}$ & 1.3 \\
\hline 383MH1215.0 & $5 / 23 / 2004$ & soil & EPA6010 & REG & $7439-92-1$ & Lead & 12 & MG/KG & & 0.35 \\
\hline 383MH1215.0 & $5 / 23 / 2004$ & soil & EPA7470 & REG & $7439-97-6$ & Mercury & 0.011 & MG/KG & B & 0.12 \\
\hline 383MH1215.0 & $5 / 23 / 2004$ & soil & EPA8260 & REG & $75-09-2$ & Methylene Chloride & 0.88 & UG/KG & $J, B$ & 5.8 \\
\hline 383МH1215.0 & $5 / 23 / 2004$ & soil & HASL300 & REG & 15092-94-1 & $\mathrm{Pb}-212$ & 2.72 & $\mathrm{PCl} / \mathrm{G}$ & $\mathrm{J}$ & 0.33 \\
\hline 383MH1215.0 & $5 / 23 / 2004$ & soil & HASL300 & REG & $15067-28-4$ & $\mathrm{~Pb}-214$ & 1.26 & $\mathrm{PCl} / \mathrm{G}$ & $\mathrm{G}, \mathrm{J}$ & 0.35 \\
\hline 383MH1215.0 & $5 / 23 / 2004$ & soil & UGTAISOPU & REG & 13981-16-3 & Pu-238 & 0.072 & $\mathrm{PCl} / \mathrm{G}$ & & 0.009 \\
\hline 383MH1215.0 & $5 / 23 / 2004$ & soil & UGTAISOPU & REG & 15117-48-3 & Pu-239 & 0.74 & $\mathrm{PCl} / \mathrm{G}$ & $\mathrm{J}$ & 0.01 \\
\hline 383MH1215.0 & $5 / 23 / 2004$ & soil & EPA6010 & REG & $7782-49-2$ & Selenium & 0.78 & MG/KG & & 0.58 \\
\hline 383MH1215.0 & $5 / 23 / 2004$ & soil & HASL300 & REG & $14913-50-9$ & TI-208 & 0.68 & $\mathrm{PCl} / \mathrm{G}$ & G & 0.14 \\
\hline 383MH1220.5 & $5 / 23 / 2004$ & soil & EPA6010 & REG & 7440-38-2 & Arsenic & 1.5 & MG/KG & & 1.1 \\
\hline 383MH1220.5 & $5 / 23 / 2004$ & soil & EPA6010 & REG & 7440-39-3 & Barium & 8.7 & MG/KG & B & 11 \\
\hline 383MH1220.5 & $5 / 23 / 2004$ & soil & EPA6010 & REG & $7440-41-7$ & Beryllium & 0.19 & MG/KG & B & 0.53 \\
\hline 383МH1220.5 & $5 / 23 / 2004$ & soil & EPA6010 & REG & $7440-43-9$ & Cadmium & 1 & MG/KG & & 0.53 \\
\hline 383MH1220.5 & $5 / 23 / 2004$ & soil & EPA6010 & REG & $7440-47-3$ & Chromium & 1 & MG/KG & $B$ & 1.1 \\
\hline 383MH1220.5 & $5 / 23 / 2004$ & soil & EPA6010 & REG & $7439-92-1$ & Lead & 18 & MG/KG & & 1.6 \\
\hline 383МH1220.5 & $5 / 23 / 2004$ & soil & EPA7470 & REG & $7439-97-6$ & Mercury & 0.19 & MG/KG & & 0.11 \\
\hline 383MH1220.5 & $5 / 23 / 2004$ & soil & EPA8260 & REG & $75-09-2$ & Methylene Chloride & 0.83 & UG/KG & $\mathrm{J}, \mathrm{B}$ & 5.3 \\
\hline 383MH1220.5 & $5 / 23 / 2004$ & soil & EPA6010 & REG & $7440-22-4$ & Silver & 0.71 & MG/KG & B & 1.1 \\
\hline 383MH1306.5 & $5 / 25 / 2004$ & soil & HASL300 & REG & 14331-83-0 & Ac-228 & 1.82 & $\mathrm{PCl} / \mathrm{G}$ & $\mathrm{G}$ & 0.78 \\
\hline 383МH1306.5 & $5 / 25 / 2004$ & soil & EPA8260 & REG & 67-64-1 & Acetone & 7.3 & UG/KG & $\mathrm{J}$ & 24 \\
\hline 383MH1306.5 & $5 / 25 / 2004$ & soil & EPA6010 & REG & $7440-38-2$ & Arsenic & 1.8 & MG/KG & & 1.2 \\
\hline 383MH1306.5 & $5 / 25 / 2004$ & soil & EPA6010 & REG & 7440-39-3 & Barium & 68 & MG/KG & & 12 \\
\hline 383MH1306.5 & $5 / 25 / 2004$ & soil & EPA6010 & REG & $7440-41-7$ & Beryllium & 1.2 & MG/KG & & 0.61 \\
\hline 383MH1306.5 & $5 / 25 / 2004$ & soil & HASL300 & REG & $14733-03-0$ & $\mathrm{Bi}-214$ & 1.18 & $\mathrm{PCl} / \mathrm{G}$ & $\mathrm{G}, \mathrm{J}$ & 0.35 \\
\hline 383MH1306.5 & $5 / 25 / 2004$ & soil & EPA6010 & REG & $7440-47-3$ & Chromium & 0.49 & MG/KG & B & 1.2 \\
\hline 383MH1306.5 & $5 / 25 / 2004$ & soil & HASL300 & REG & $10045-97-3$ & Cs-137 & 0.57 & $\mathrm{PCl} / \mathrm{G}$ & G & 0.2 \\
\hline 383MH1306.5 & $5 / 25 / 2004$ & soil & HASL300 & REG & $13966-00-2$ & $\mathrm{~K}-40$ & 43.8 & $\mathrm{PCl} / \mathrm{G}$ & $\mathrm{G}$ & 2.7 \\
\hline
\end{tabular}




\begin{tabular}{|c|c|c|c|c|c|c|c|c|c|c|}
\hline SAMPLE \# & $\begin{array}{l}\text { SAMPLE } \\
\text { DATE }\end{array}$ & MATRIX & $\begin{array}{l}\text { USER TEST } \\
\text { PANEL }\end{array}$ & $\begin{array}{l}\text { SAMPLE } \\
\text { PURPOSE }\end{array}$ & CAS \# & PARAMETER & RESULT & UNITS & Q & $\begin{array}{l}\text { DETECT } \\
\text { LIMIT }\end{array}$ \\
\hline 383MH1306.5 & $5 / 25 / 2004$ & soil & EPA6010 & REG & $7439-92-1$ & Lead & 12 & MG/KG & & 0.36 \\
\hline 383MH1306.5 & $5 / 25 / 2004$ & soil & EPA7470 & REG & $7439-97-6$ & Mercury & 0.0018 & MG/KG & B & 0.12 \\
\hline 383MH1306.5 & $5 / 25 / 2004$ & soil & HASL300 & REG & 15092-94-1 & $\mathrm{Pb}-212$ & 1.91 & $\mathrm{PCl} / \mathrm{G}$ & $\mathrm{J}$ & 0.33 \\
\hline 383MH1306.5 & $5 / 25 / 2004$ & soil & HASL300 & REG & 15067-28-4 & $\mathrm{Pb}-214$ & 1.53 & $\mathrm{PCl} / \mathrm{G}$ & $\mathrm{G}, \mathrm{J}$ & 0.4 \\
\hline 383MH1306.5 & $5 / 25 / 2004$ & soil & HASL300 & REG & 14913-50-9 & TI-208 & 0.8 & $\mathrm{PCl} / \mathrm{G}$ & G & 0.22 \\
\hline 383MH1320.5 & $5 / 25 / 2004$ & soil & EPA8260 & REG & $67-64-1$ & Acetone & 10 & UG/KG & $\mathrm{J}$ & 20 \\
\hline 383MH1320.5 & $5 / 25 / 2004$ & soil & EPA6010 & REG & $7440-39-3$ & Barium & 3.1 & MG/KG & B & 10 \\
\hline 383MH1320.5 & $5 / 25 / 2004$ & soil & EPA6010 & REG & $7440-41-7$ & Beryllium & 0.15 & MG/KG & B & 0.5 \\
\hline 383MH1320.5 & $5 / 25 / 2004$ & soil & EPA6010 & REG & $7440-47-3$ & Chromium & 1.3 & MG/KG & & 1 \\
\hline 383MH1320.5 & $5 / 25 / 2004$ & soil & EPA7470 & REG & $7439-97-6$ & Mercury & 0.017 & MG/KG & B & 0.1 \\
\hline 383МH1320.5 & $5 / 25 / 2004$ & soil & EPA6010 & REG & $7440-22-4$ & Silver & 0.19 & MG/KG & B & 1 \\
\hline 383MH1407.5 & $5 / 20 / 2004$ & soil & HASL300 & REG & 14331-83-0 & Ac-228 & 1.84 & $\mathrm{PCl} / \mathrm{G}$ & G & 0.45 \\
\hline 383MH1407.5 & $5 / 20 / 2004$ & soil & EPA6010 & REG & $7440-38-2$ & Arsenic & 3 & MG/KG & & 1.1 \\
\hline 383МH1407.5 & $5 / 20 / 2004$ & soil & EPA6010 & REG & 7440-39-3 & Barium & 220 & MG/KG & & 11 \\
\hline 383MH1407.5 & $5 / 20 / 2004$ & soil & EPA6010 & REG & $7440-41-7$ & Beryllium & 1.1 & MG/KG & & 0.56 \\
\hline 383MH1407.5 & $5 / 20 / 2004$ & soil & HASL300 & REG & 14733-03-0 & $\mathrm{Bi}-214$ & 1.02 & $\mathrm{PCl} / \mathrm{G}$ & $\mathrm{G}, \mathrm{J}$ & 0.28 \\
\hline 383MH1407.5 & $5 / 20 / 2004$ & soil & EPA6010 & REG & $7440-47-3$ & Chromium & 0.99 & MG/KG & B & 1.1 \\
\hline 383MH1407.5 & $5 / 20 / 2004$ & soil & HASL300 & REG & $10045-97-3$ & Cs-137 & 10.7 & $\mathrm{PCl} / \mathrm{G}$ & G & 0.1 \\
\hline 383MH1407.5 & $5 / 20 / 2004$ & soil & EPA8015 & REG & $68334-30-5$ & Diesel Range Organics & 3.5 & MG/KG & $\mathrm{J}$ & 5.6 \\
\hline 383MH1407.5 & $5 / 20 / 2004$ & soil & HASL300 & REG & 13966-00-2 & $\mathrm{K}-40$ & 30.6 & $\mathrm{PCl} / \mathrm{G}$ & G & 1.5 \\
\hline 383MH1407.5 & $5 / 20 / 2004$ & soil & EPA6010 & REG & $7439-92-1$ & Lead & 12 & MG/KG & & 0.34 \\
\hline 383MH1407.5 & $5 / 20 / 2004$ & soil & EPA7470 & REG & $7439-97-6$ & Mercury & 0.068 & MG/KG & B & 0.11 \\
\hline 383MH1407.5 & $5 / 20 / 2004$ & soil & EPA8260 & REG & $75-09-2$ & Methylene Chloride & 1.1 & UG/KG & J,B & 5.6 \\
\hline 383MH1407.5 & $5 / 20 / 2004$ & soil & HASL300 & REG & 15092-94-1 & $\mathrm{Pb}-212$ & 2.35 & $\mathrm{PCl} / \mathrm{G}$ & $\mathrm{J}$ & 0.25 \\
\hline 383MH1407.5 & $5 / 20 / 2004$ & soil & HASL300 & REG & $15067-28-4$ & $\mathrm{~Pb}-214$ & 1.02 & $\mathrm{PCl} / \mathrm{G}$ & $\mathrm{G}, \mathrm{J}$ & 0.3 \\
\hline 383MH1407.5 & $5 / 20 / 2004$ & soil & UGTAISOPU & REG & 13981-16-3 & Pu-238 & 0.141 & $\mathrm{PCl} / \mathrm{G}$ & & 0.018 \\
\hline 383MH1407.5 & $5 / 20 / 2004$ & soil & UGTAISOPU & REG & $15117-48-3$ & Pu-239 & 1.86 & $\mathrm{PCl} / \mathrm{G}$ & $\mathrm{J}$ & 0.01 \\
\hline 383MH1407.5 & $5 / 20 / 2004$ & soil & SR7500 & REG & 10098-97-2 & Sr-90 & 0.67 & $\mathrm{PCl} / \mathrm{G}$ & & 0.26 \\
\hline 383MH1407.5 & $5 / 20 / 2004$ & soil & HASL300 & REG & 14913-50-9 & TI-208 & 0.61 & $\mathrm{PCl} / \mathrm{G}$ & G & 0.14 \\
\hline 383MH1422.0 & $5 / 20 / 2004$ & soil & EPA8260 & REG & 67-64-1 & Acetone & 18 & UG/KG & $\mathrm{J}$ & 20 \\
\hline 383MH1422.0 & $5 / 20 / 2004$ & soil & EPA6010 & REG & $7440-38-2$ & Arsenic & 1.1 & MG/KG & & 1 \\
\hline 383MH1422.0 & $5 / 20 / 2004$ & soil & EPA6010 & REG & 7440-39-3 & Barium & 14 & MG/KG & & 10 \\
\hline 383MH1422.0 & $5 / 20 / 2004$ & soil & EPA6010 & REG & $7440-41-7$ & Beryllium & 0.23 & MG/KG & B & 0.51 \\
\hline 383MH1422.0 & $5 / 20 / 2004$ & soil & EPA6010 & REG & $7440-43-9$ & Cadmium & 0.02 & MG/KG & B & 0.51 \\
\hline 383MH1422.0 & $5 / 20 / 2004$ & soil & EPA6010 & REG & 7440-47-3 & Chromium & 1.7 & MG/KG & & 1 \\
\hline
\end{tabular}




\begin{tabular}{|c|c|c|c|c|c|c|c|c|c|c|}
\hline SAMPLE \# & $\begin{array}{l}\text { SAMPLE } \\
\text { DATE }\end{array}$ & MATRIX & $\begin{array}{l}\text { USER TEST } \\
\text { PANEL }\end{array}$ & $\begin{array}{l}\text { SAMPLE } \\
\text { PURPOSE }\end{array}$ & CAS \# & PARAMETER & RESULT & UNITS & Q & $\begin{array}{l}\text { DETECT } \\
\text { LIMIT }\end{array}$ \\
\hline 383MH1422.0 & $5 / 20 / 2004$ & soil & EPA7470 & REG & $7439-97-6$ & Mercury & 0.023 & MG/KG & B & 0.1 \\
\hline 383MH1422.0 & $5 / 20 / 2004$ & soil & EPA8260 & REG & $75-09-2$ & Methylene Chloride & 0.91 & UG/KG & $\mathrm{J}, \mathrm{B}$ & 5.1 \\
\hline 383MH1422.0 & $5 / 20 / 2004$ & soil & HASL300 & REG & $15067-28-4$ & $\mathrm{~Pb}-214$ & 0.29 & $\mathrm{PCl} / \mathrm{G}$ & $\mathrm{J}$ & 0.14 \\
\hline 383МH1422.0 & $5 / 20 / 2004$ & soil & EPA6010 & REG & $7440-22-4$ & Silver & 0.11 & MG/KG & B & 1 \\
\hline 383МH1501.0 & $5 / 24 / 2004$ & soil & HASL300 & REG & $14331-83-0$ & Ac-228 & 2.02 & $\mathrm{PCl} / \mathrm{G}$ & G & 0.8 \\
\hline 383МH1501.0 & $5 / 24 / 2004$ & soil & EPA6010 & REG & $7440-38-2$ & Arsenic & 3.6 & MG/KG & & 1.1 \\
\hline 383МH1501.0 & $5 / 24 / 2004$ & soil & EPA6010 & REG & $7440-39-3$ & Barium & 520 & MG/KG & & 11 \\
\hline 383MH1501.0 & $5 / 24 / 2004$ & soil & EPA8270 & REG & $56-55-3$ & Benzo(A)Anthracene & 19 & UG/KG & $\mathrm{J}$ & 380 \\
\hline 383МH1501.0 & $5 / 24 / 2004$ & soil & EPA6010 & REG & $7440-41-7$ & Beryllium & 0.78 & MG/KG & & 0.57 \\
\hline 383МH1501.0 & $5 / 24 / 2004$ & soil & HASL300 & REG & 14733-03-0 & Bi-214 & 1.29 & $\mathrm{PCl} / \mathrm{G}$ & $\mathrm{G}, \mathrm{J}$ & 0.34 \\
\hline 383MH1501.0 & $5 / 24 / 2004$ & soil & EPA6010 & REG & $7440-47-3$ & Chromium & 4.9 & MG/KG & & 1.1 \\
\hline 383МH1501.0 & $5 / 24 / 2004$ & soil & HASL300 & REG & $10045-97-3$ & Cs-137 & 0.55 & $\mathrm{PCl} / \mathrm{G}$ & G & 0.18 \\
\hline 383МH1501.0 & $5 / 24 / 2004$ & soil & EPA8015 & REG & $68334-30-5$ & Diesel Range Organics & 52 & MG/KG & $\mathrm{M}, \mathrm{H}$ & 5.7 \\
\hline 383МH1501.0 & $5 / 24 / 2004$ & soil & HASL300 & REG & 13966-00-2 & $\mathrm{K}-40$ & 31.3 & $\mathrm{PCl} / \mathrm{G}$ & G & 2.1 \\
\hline 383МH1501.0 & $5 / 24 / 2004$ & soil & EPA6010 & REG & $7439-92-1$ & Lead & 27 & MG/KG & & 0.34 \\
\hline 383МH1501.0 & $5 / 24 / 2004$ & soil & EPA7470 & REG & $7439-97-6$ & Mercury & 0.015 & MG/KG & B & 0.11 \\
\hline 383МH1501.0 & $5 / 24 / 2004$ & soil & EPA8260 & REG & $75-09-2$ & Methylene Chloride & 1.7 & UG/KG & $\mathrm{J}, \mathrm{B}$ & 5.7 \\
\hline 383МH1501.0 & $5 / 24 / 2004$ & soil & HASL300 & REG & 15092-94-1 & $\mathrm{Pb}-212$ & 2.13 & $\mathrm{PCl} / \mathrm{G}$ & $\mathrm{J}$ & 0.24 \\
\hline 383МH1501.0 & $5 / 24 / 2004$ & soil & HASL300 & REG & $15067-28-4$ & $\mathrm{~Pb}-214$ & 1.17 & $\mathrm{PCl} / \mathrm{G}$ & $\mathrm{G}, \mathrm{J}$ & 0.31 \\
\hline 383МH1501.0 & $5 / 24 / 2004$ & soil & HASL300 & REG & $14913-50-9$ & TI-208 & 0.69 & $\mathrm{PCl} / \mathrm{G}$ & $\mathrm{G}$ & 0.16 \\
\hline 383МH1503.5 & $5 / 24 / 2004$ & soil & EPA8260 & REG & $67-64-1$ & Acetone & 8.2 & UG/KG & $\mathrm{J}$ & 20 \\
\hline 383MH1503.5 & $5 / 24 / 2004$ & soil & EPA6010 & REG & $7440-38-2$ & Arsenic & 0.46 & MG/KG & B & 1 \\
\hline 383MH1503.5 & $5 / 24 / 2004$ & soil & EPA6010 & REG & $7440-39-3$ & Barium & 3.2 & MG/KG & B & 10 \\
\hline 383МH1503.5 & $5 / 24 / 2004$ & soil & EPA6010 & REG & $7440-41-7$ & Beryllium & 0.16 & MG/KG & $B$ & 0.51 \\
\hline 383MH1503.5 & $5 / 24 / 2004$ & soil & HASL300 & REG & 14733-03-0 & Bi-214 & 0.41 & $\mathrm{PCl} / \mathrm{G}$ & $\mathrm{J}$ & 0.16 \\
\hline 383MH1503.5 & $5 / 24 / 2004$ & soil & EPA6010 & REG & $7440-47-3$ & Chromium & 0.91 & MG/KG & B & 1 \\
\hline 383MH1503.5 & $5 / 24 / 2004$ & soil & EPA8260 & REG & $87-68-3$ & Hexachlorobutadiene & 1.5 & UG/KG & $\mathrm{J}$ & 5.1 \\
\hline 383MH1503.5 & $5 / 24 / 2004$ & soil & EPA7470 & REG & $7439-97-6$ & Mercury & 0.11 & MG/KG & & 0.1 \\
\hline 383MH1503.5 & $5 / 24 / 2004$ & soil & EPA8260 & REG & $75-09-2$ & Methylene Chloride & 1.6 & UG/KG & $\mathrm{J}, \mathrm{B}$ & 5.1 \\
\hline 383МH1503.5 & $5 / 24 / 2004$ & soil & HASL300 & REG & $15067-28-4$ & $\mathrm{~Pb}-214$ & 0.46 & $\mathrm{PCl} / \mathrm{G}$ & $\mathrm{J}$ & 0.14 \\
\hline 383MH1503.5 & $5 / 24 / 2004$ & soil & EPA6010 & REG & $7782-49-2$ & Selenium & 0.29 & MG/KG & B & 0.51 \\
\hline 383MH1503.5X & $5 / 24 / 2004$ & soil & EPA8260 & FD & 67-64-1 & Acetone & 8.3 & UG/KG & $\mathrm{J}$ & 20 \\
\hline 383MH1503.5X & $5 / 24 / 2004$ & soil & EPA6010 & FD & $7440-38-2$ & Arsenic & 0.59 & MG/KG & $B$ & 1 \\
\hline 383MH1503.5X & $5 / 24 / 2004$ & soil & EPA6010 & FD & $7440-39-3$ & Barium & 3.1 & MG/KG & $B$ & 10 \\
\hline 383MH1503.5X & $5 / 24 / 2004$ & soil & EPA6010 & FD & $7440-41-7$ & Beryllium & 0.16 & MG/KG & $B$ & 0.51 \\
\hline
\end{tabular}




\begin{tabular}{|c|c|c|c|c|c|c|c|c|c|c|}
\hline SAMPLE \# & $\begin{array}{l}\text { SAMPLE } \\
\text { DATE }\end{array}$ & MATRIX & $\begin{array}{l}\text { USER TEST } \\
\text { PANEL }\end{array}$ & $\begin{array}{l}\text { SAMPLE } \\
\text { PURPOSE }\end{array}$ & CAS \# & PARAMETER & RESULT & UNITS & Q & $\begin{array}{l}\text { DETECT } \\
\text { LIMIT }\end{array}$ \\
\hline 383МH1503.5X & $5 / 24 / 2004$ & soil & HASL300 & FD & 14733-03-0 & $\mathrm{Bi}-214$ & 0.43 & $\mathrm{PCl} / \mathrm{G}$ & $\mathrm{J}$ & 0.13 \\
\hline 383МH1503.5X & $5 / 24 / 2004$ & soil & EPA6010 & FD & $7440-47-3$ & Chromium & 0.92 & MG/KG & $B$ & 1 \\
\hline 383MH1503.5X & $5 / 24 / 2004$ & soil & EPA7470 & FD & 7439-97-6 & Mercury & 0.13 & MG/KG & & 0.1 \\
\hline 383МH1503.5X & $5 / 24 / 2004$ & soil & EPA8260 & FD & $75-09-2$ & Methylene Chloride & 1.6 & UG/KG & J,B & 5.1 \\
\hline 383МH1503.5X & $5 / 24 / 2004$ & soil & HASL300 & FD & 15067-28-4 & $\mathrm{Pb}-214$ & 0.47 & $\mathrm{PCl} / \mathrm{G}$ & $\mathrm{J}$ & 0.11 \\
\hline 383MH1503.5X & $5 / 24 / 2004$ & soil & EPA6010 & FD & $7440-22-4$ & Silver & 0.099 & MG/KG & B & 1 \\
\hline 383МH1503.5X & $5 / 24 / 2004$ & soil & EPA8260 & FD & $100-42-5$ & Styrene & 0.89 & UG/KG & $\mathrm{J}$ & 5.1 \\
\hline 383МH1608.5 & $6 / 11 / 2004$ & soil & HASL300 & REG & 14331-83-0 & Ac-228 & 1.45 & $\mathrm{PCl} / \mathrm{G}$ & G & 0.59 \\
\hline 383MH1608.5 & $6 / 11 / 2004$ & soil & EPA8260 & REG & $67-64-1$ & Acetone & 7.5 & UG/KG & $\mathrm{J}$ & 22 \\
\hline 383MH1608.5 & $6 / 11 / 2004$ & soil & HASL300 & REG & 14596-10-2 & Am-241 & 0.52 & $\mathrm{PCl} / \mathrm{G}$ & G & 0.3 \\
\hline 383MH1608.5 & $6 / 11 / 2004$ & soil & EPA6010 & REG & $7440-38-2$ & Arsenic & 3.6 & MG/KG & & 1.1 \\
\hline 383MH1608.5 & $6 / 11 / 2004$ & soil & EPA6010 & REG & $7440-39-3$ & Barium & 64 & MG/KG & & 11 \\
\hline 383МH1608.5 & $6 / 11 / 2004$ & soil & EPA6010 & REG & $7440-41-7$ & Beryllium & 0.8 & MG/KG & & 0.55 \\
\hline 383МH1608.5 & $6 / 11 / 2004$ & soil & HASL300 & REG & 14913-49-6 & $\mathrm{Bi}-212$ & 2.2 & $\mathrm{PCl} / \mathrm{G}$ & G & 2 \\
\hline 383MH1608.5 & $6 / 11 / 2004$ & soil & HASL300 & REG & 14733-03-0 & Bi-214 & 0.8 & $\mathrm{PCl} / \mathrm{G}$ & $\mathrm{G}, \mathrm{J}$ & 0.28 \\
\hline 383МH1608.5 & $6 / 11 / 2004$ & soil & EPA6010 & REG & $7440-43-9$ & Cadmium & 0.091 & MG/KG & $B$ & 0.55 \\
\hline 383МH1608.5 & $6 / 11 / 2004$ & soil & EPA6010 & REG & $7440-47-3$ & Chromium & 2 & MG/KG & & 1.1 \\
\hline 383MH1608.5 & $6 / 11 / 2004$ & soil & HASL300 & REG & 10045-97-3 & Cs-137 & 2.67 & $\mathrm{PCl} / \mathrm{G}$ & G & 0.12 \\
\hline 383MH1608.5 & $6 / 11 / 2004$ & soil & HASL300 & REG & 13966-00-2 & $\mathrm{K}-40$ & 19.2 & $\mathrm{PCl} / \mathrm{G}$ & G & 1.5 \\
\hline 383МH1608.5 & $6 / 11 / 2004$ & soil & EPA6010 & REG & $7439-92-1$ & Lead & 15 & MG/KG & & 1.6 \\
\hline 383MH1608.5 & $6 / 11 / 2004$ & soil & EPA7470 & REG & 7439-97-6 & Mercury & 0.089 & MG/KG & $B$ & 0.11 \\
\hline 383MH1608.5 & $6 / 11 / 2004$ & soil & HASL300 & REG & 15092-94-1 & $\mathrm{Pb}-212$ & 1.44 & $\mathrm{PCl} / \mathrm{G}$ & G & 0.22 \\
\hline 383МH1608.5 & $6 / 11 / 2004$ & soil & HASL300 & REG & 15067-28-4 & $\mathrm{Pb}-214$ & 0.92 & $\mathrm{PCl} / \mathrm{G}$ & $\mathrm{G}, \mathrm{J}$ & 0.27 \\
\hline 383MH1608.5 & $6 / 11 / 2004$ & soil & UGTAISOPU & REG & 13981-16-3 & Pu-238 & 0.05 & $\mathrm{PCl} / \mathrm{G}$ & & 0.009 \\
\hline 383МH1608.5 & $6 / 11 / 2004$ & soil & UGTAISOPU & REG & 15117-48-3 & Pu-239 & 2.82 & $\mathrm{PCl} / \mathrm{G}$ & & 0.02 \\
\hline 383МH1608.5 & $6 / 11 / 2004$ & soil & SR7500 & REG & 10098-97-2 & Sr-90 & 0.21 & $\mathrm{PCl} / \mathrm{G}$ & LT & 0.18 \\
\hline 383МH1608.5 & $6 / 11 / 2004$ & soil & HASL300 & REG & 14913-50-9 & TI-208 & 0.46 & $\mathrm{PCl} / \mathrm{G}$ & $\mathrm{G}$ & 0.15 \\
\hline 383МH1609.5 & $6 / 11 / 2004$ & soil & HASL300 & REG & 14331-83-0 & Ac-228 & 2.13 & $\mathrm{PCl} / \mathrm{G}$ & G & 0.56 \\
\hline 383MH1609.5 & $6 / 11 / 2004$ & soil & EPA6010 & REG & $7440-38-2$ & Arsenic & 2.7 & MG/KG & & 1.1 \\
\hline 383МH1609.5 & $6 / 11 / 2004$ & soil & EPA6010 & REG & $7440-39-3$ & Barium & 58 & MG/KG & & 11 \\
\hline 383MH1609.5 & $6 / 11 / 2004$ & soil & EPA6010 & REG & $7440-41-7$ & Beryllium & 0.68 & MG/KG & & 0.56 \\
\hline 383MH1609.5 & $6 / 11 / 2004$ & soil & HASL300 & REG & 14913-49-6 & $\mathrm{Bi}-212$ & 3.7 & $\mathrm{PCl} / \mathrm{G}$ & G & 2.6 \\
\hline 383МH1609.5 & $6 / 11 / 2004$ & soil & HASL300 & REG & 14733-03-0 & Bi-214 & 1.02 & $\mathrm{PCl} / \mathrm{G}$ & $\mathrm{G}, \mathrm{J}$ & 0.4 \\
\hline 383МH1609.5 & $6 / 11 / 2004$ & soil & EPA6010 & REG & $7440-43-9$ & Cadmium & 0.041 & MG/KG & B & 0.56 \\
\hline 383MH1609.5 & $6 / 11 / 2004$ & soil & EPA6010 & REG & $7440-47-3$ & Chromium & 0.88 & MG/KG & B & 1.1 \\
\hline
\end{tabular}




\begin{tabular}{|c|c|c|c|c|c|c|c|c|c|c|}
\hline SAMPLE \# & $\begin{array}{l}\text { SAMPLE } \\
\text { DATE }\end{array}$ & MATRIX & $\begin{array}{l}\text { USER TEST } \\
\text { PANEL }\end{array}$ & $\begin{array}{l}\text { SAMPLE } \\
\text { PURPOSE }\end{array}$ & CAS \# & PARAMETER & RESULT & UNITS & Q & $\begin{array}{l}\text { DETECT } \\
\text { LIMIT }\end{array}$ \\
\hline 383МH1609.5 & $6 / 11 / 2004$ & soil & HASL300 & REG & $10045-97-3$ & Cs-137 & 34.5 & $\mathrm{PCl} / \mathrm{G}$ & G & 0.2 \\
\hline 383MH1609.5 & $6 / 11 / 2004$ & soil & HASL300 & REG & 13966-00-2 & $\mathrm{K}-40$ & 34 & $\mathrm{PCl} / \mathrm{G}$ & G & 1.3 \\
\hline 383MH1609.5 & $6 / 11 / 2004$ & soil & EPA6010 & REG & $7439-92-1$ & Lead & 9.5 & MG/KG & & 0.34 \\
\hline 383MH1609.5 & $6 / 11 / 2004$ & soil & EPA7470 & REG & $7439-97-6$ & Mercury & 0.012 & MG/KG & B & 0.11 \\
\hline 383МH1609.5 & $6 / 11 / 2004$ & soil & HASL300 & REG & 15092-94-1 & $\mathrm{Pb}-212$ & 2.26 & $\mathrm{PCl} / \mathrm{G}$ & G & 0.4 \\
\hline 383MH1609.5 & $6 / 11 / 2004$ & soil & HASL300 & REG & 15067-28-4 & $\mathrm{Pb}-214$ & 0.96 & $\mathrm{PCl} / \mathrm{G}$ & $\mathrm{G}, \mathrm{J}$ & 0.6 \\
\hline 383МH1609.5 & $6 / 11 / 2004$ & soil & UGTAISOPU & REG & 13981-16-3 & Pu-238 & 0.093 & $\mathrm{PCl} / \mathrm{G}$ & & 0.018 \\
\hline 383MH1609.5 & $6 / 11 / 2004$ & soil & UGTAISOPU & REG & $15117-48-3$ & Pu-239 & 0.5 & $\mathrm{PCl} / \mathrm{G}$ & & 0.01 \\
\hline 383MH1609.5 & $6 / 11 / 2004$ & soil & SR7500 & REG & 10098-97-2 & Sr-90 & 4.2 & $\mathrm{PCl} / \mathrm{G}$ & Y1 & 0.2 \\
\hline 383МH1609.5 & $6 / 11 / 2004$ & soil & HASL300 & REG & 14913-50-9 & TI-208 & 0.82 & $\mathrm{PCl} / \mathrm{G}$ & G & 0.2 \\
\hline 383МH1619.0 & $6 / 11 / 2004$ & soil & EPA8260 & REG & $67-64-1$ & Acetone & 7.7 & UG/KG & $\mathrm{J}$ & 20 \\
\hline 383MH1619.0 & $6 / 11 / 2004$ & soil & EPA6010 & REG & $7440-38-2$ & Arsenic & 1.6 & MG/KG & & 1 \\
\hline 383МH1619.0 & $6 / 11 / 2004$ & soil & EPA6010 & REG & $7440-39-3$ & Barium & 16 & MG/KG & & 10 \\
\hline 383МH1619.0 & $6 / 11 / 2004$ & soil & EPA6010 & REG & $7440-41-7$ & Beryllium & 0.27 & MG/KG & B & 0.51 \\
\hline 383МH1619.0 & $6 / 11 / 2004$ & soil & HASL300 & REG & 14733-03-0 & $\mathrm{Bi}-214$ & 0.35 & $\mathrm{PCl} / \mathrm{G}$ & $\mathrm{J}$ & 0.17 \\
\hline 383МH1619.0 & $6 / 11 / 2004$ & soil & EPA6010 & REG & $7440-43-9$ & Cadmium & 0.085 & MG/KG & B & 0.51 \\
\hline 383МH1619.0 & $6 / 11 / 2004$ & soil & EPA6010 & REG & $7440-47-3$ & Chromium & 2.4 & MG/KG & & 1 \\
\hline 383МH1619.0 & $6 / 11 / 2004$ & soil & HASL300 & REG & $10045-97-3$ & Cs-137 & 0.163 & $\mathrm{PCl} / \mathrm{G}$ & LT & 0.067 \\
\hline 383MH1619.0 & $6 / 11 / 2004$ & soil & HASL300 & REG & 13966-00-2 & $\mathrm{K}-40$ & 2.05 & $\mathrm{PCl} / \mathrm{G}$ & & 0.86 \\
\hline 383МH1619.0 & $6 / 11 / 2004$ & soil & EPA6010 & REG & $7439-92-1$ & Lead & 2.7 & MG/KG & & 1.5 \\
\hline 383МH1619.0 & $6 / 11 / 2004$ & soil & EPA7470 & REG & 7439-97-6 & Mercury & 0.057 & MG/KG & $B$ & 0.1 \\
\hline 383MH1619.0 & $6 / 11 / 2004$ & soil & HASL300 & REG & $15067-28-4$ & $\mathrm{~Pb}-214$ & 0.322 & $\mathrm{PCl} / \mathrm{G}$ & $\mathrm{J}$ & 0.134 \\
\hline 383МH1619.0 & $6 / 11 / 2004$ & soil & UGTAISOPU & REG & 13981-16-3 & Pu-238 & 0.068 & $\mathrm{PCl} / \mathrm{G}$ & & 0.009 \\
\hline 383МH1619.0 & $6 / 11 / 2004$ & soil & UGTAISOPU & REG & 15117-48-3 & Pu-239 & 0.338 & $\mathrm{PCl} / \mathrm{G}$ & & 0.009 \\
\hline 383МH1619.0 & $6 / 11 / 2004$ & soil & EPA6010 & REG & $7440-22-4$ & Silver & 0.1 & MG/KG & $B$ & 1 \\
\hline 383MH1716.5 & $5 / 22 / 2004$ & soil & HASL300 & REG & 14331-83-0 & Ac-228 & 2.24 & $\mathrm{PCl} / \mathrm{G}$ & G & 0.62 \\
\hline 383MH1716.5 & $5 / 22 / 2004$ & soil & EPA6010 & REG & $7440-38-2$ & Arsenic & 2.9 & MG/KG & & 1.1 \\
\hline 383MH1716.5 & $5 / 22 / 2004$ & soil & EPA6010 & REG & 7440-39-3 & Barium & 86 & MG/KG & & 11 \\
\hline 383MH1716.5 & $5 / 22 / 2004$ & soil & EPA8270 & REG & $56-55-3$ & Benzo(A)Anthracene & 21 & UG/KG & $\mathrm{J}$ & 380 \\
\hline 383МH1716.5 & $5 / 22 / 2004$ & soil & EPA8270 & REG & $205-99-2$ & Benzo(B)Fluoranthene & 18 & UG/KG & $\mathrm{J}$ & 380 \\
\hline 383MH1716.5 & $5 / 22 / 2004$ & soil & EPA6010 & REG & $7440-41-7$ & Beryllium & 1.3 & MG/KG & & 0.57 \\
\hline 383МH1716.5 & $5 / 22 / 2004$ & soil & HASL300 & REG & 14733-03-0 & Bi-214 & 1.49 & $\mathrm{PCl} / \mathrm{G}$ & $\mathrm{G}, \mathrm{J}$ & 0.33 \\
\hline 383MH1716.5 & $5 / 22 / 2004$ & soil & EPA6010 & REG & $7440-43-9$ & Cadmium & 0.031 & MG/KG & $B$ & 0.57 \\
\hline 383MH1716.5 & $5 / 22 / 2004$ & soil & EPA6010 & REG & $7440-47-3$ & Chromium & 0.65 & MG/KG & $B$ & 1.1 \\
\hline 383MH1716.5 & $5 / 22 / 2004$ & soil & EPA8270 & REG & $218-01-9$ & Chrysene & 21 & UG/KG & $\mathrm{J}$ & 380 \\
\hline
\end{tabular}




\begin{tabular}{|c|c|c|c|c|c|c|c|c|c|c|}
\hline SAMPLE \# & $\begin{array}{l}\text { SAMPLE } \\
\text { DATE }\end{array}$ & MATRIX & $\begin{array}{l}\text { USER TEST } \\
\text { PANEL }\end{array}$ & $\begin{array}{l}\text { SAMPLE } \\
\text { PURPOSE }\end{array}$ & CAS \# & PARAMETER & RESULT & UNITS & Q & $\begin{array}{l}\text { DETECT } \\
\text { LIMIT }\end{array}$ \\
\hline 383MH1716.5 & $5 / 22 / 2004$ & soil & HASL300 & REG & $10045-97-3$ & Cs-137 & 6.32 & $\mathrm{PCl} / \mathrm{G}$ & G & 0.15 \\
\hline 383MH1716.5 & $5 / 22 / 2004$ & soil & EPA8270 & REG & $206-44-0$ & Fluoranthene & 22 & UG/KG & $\mathrm{J}$ & 380 \\
\hline 383MH1716.5 & $5 / 22 / 2004$ & soil & HASL300 & REG & 13966-00-2 & $\mathrm{K}-40$ & 37.2 & $\mathrm{PCl} / \mathrm{G}$ & G & 1.4 \\
\hline 383MH1716.5 & $5 / 22 / 2004$ & soil & EPA6010 & REG & $7439-92-1$ & Lead & 13 & MG/KG & & 0.34 \\
\hline 383MH1716.5 & $5 / 22 / 2004$ & soil & EPA7470 & REG & 7439-97-6 & Mercury & 0.0029 & MG/KG & $B$ & 0.11 \\
\hline 383MH1716.5 & $5 / 22 / 2004$ & soil & EPA8260 & REG & $75-09-2$ & Methylene Chloride & 0.9 & UG/KG & J,B & 5.7 \\
\hline 383МH1716.5 & $5 / 22 / 2004$ & soil & HASL300 & REG & 15092-94-1 & $\mathrm{Pb}-212$ & 1.96 & $\mathrm{PCl} / \mathrm{G}$ & $\mathrm{J}$ & 0.29 \\
\hline 383MH1716.5 & $5 / 22 / 2004$ & soil & HASL300 & REG & 15067-28-4 & $\mathrm{Pb}-214$ & 1.43 & $\mathrm{PCl} / \mathrm{G}$ & $\mathrm{G}, \mathrm{J}$ & 0.31 \\
\hline 383MH1716.5 & $5 / 22 / 2004$ & soil & HASL300 & REG & 14913-50-9 & TI-208 & 0.71 & $\mathrm{PCl} / \mathrm{G}$ & $G$ & 0.16 \\
\hline 383MH1734.5 & $5 / 22 / 2004$ & soil & EPA8260 & REG & $67-64-1$ & Acetone & 6.1 & UG/KG & $\mathrm{J}$ & 21 \\
\hline 383MH1734.5 & $5 / 22 / 2004$ & soil & EPA6010 & REG & $7440-38-2$ & Arsenic & 1.8 & MG/KG & & 1 \\
\hline 383MH1734.5 & $5 / 22 / 2004$ & soil & EPA6010 & REG & 7440-39-3 & Barium & 26 & MG/KG & & 10 \\
\hline 383MH1734.5 & $5 / 22 / 2004$ & soil & EPA6010 & REG & $7440-41-7$ & Beryllium & 0.38 & MG/KG & $B$ & 0.52 \\
\hline 383МH1734.5 & $5 / 22 / 2004$ & soil & EPA6010 & REG & $7440-43-9$ & Cadmium & 0.94 & MG/KG & & 0.52 \\
\hline 383MH1734.5 & $5 / 22 / 2004$ & soil & EPA6010 & REG & $7440-47-3$ & Chromium & 3.5 & MG/KG & & 1 \\
\hline 383MH1734.5 & $5 / 22 / 2004$ & soil & EPA6010 & REG & $7439-92-1$ & Lead & 160 & MG/KG & & 1.6 \\
\hline 383МH1734.5 & $5 / 22 / 2004$ & soil & EPA7470 & REG & $7439-97-6$ & Mercury & 0.33 & MG/KG & & 0.1 \\
\hline 383MH1734.5 & $5 / 22 / 2004$ & soil & EPA8260 & REG & $75-09-2$ & Methylene Chloride & 0.9 & UG/KG & J,B & 5.2 \\
\hline 383MH1734.5 & $5 / 22 / 2004$ & soil & HASL300 & REG & 15067-28-4 & $\mathrm{Pb}-214$ & 0.3 & $\mathrm{PCl} / \mathrm{G}$ & $\mathrm{G}, \mathrm{J}$ & 0.15 \\
\hline 383MH1734.5 & $5 / 22 / 2004$ & soil & EPA6010 & REG & $7440-22-4$ & Silver & 2.8 & MG/KG & & 1 \\
\hline 383MH1803.5 & $5 / 23 / 2004$ & soil & HASL300 & REG & 14331-83-0 & Ac-228 & 0.92 & $\mathrm{PCl} / \mathrm{G}$ & G & 0.51 \\
\hline 383MH1803.5 & $5 / 23 / 2004$ & soil & EPA6010 & REG & $7440-38-2$ & Arsenic & 2.2 & MG/KG & & 1.1 \\
\hline 383MH1803.5 & $5 / 23 / 2004$ & soil & EPA6010 & REG & $7440-39-3$ & Barium & 75 & MG/KG & & 11 \\
\hline 383MH1803.5 & $5 / 23 / 2004$ & soil & EPA6010 & REG & $7440-41-7$ & Beryllium & 0.69 & MG/KG & & 0.56 \\
\hline 383MH1803.5 & $5 / 23 / 2004$ & soil & HASL300 & REG & 14733-03-0 & Bi-214 & 0.8 & $\mathrm{PCl} / \mathrm{G}$ & $\mathrm{G}, \mathrm{J}$ & 0.28 \\
\hline 383МH1803.5 & $5 / 23 / 2004$ & soil & EPA6010 & REG & $7440-43-9$ & Cadmium & 0.27 & MG/KG & $B$ & 0.56 \\
\hline 383MH1803.5 & $5 / 23 / 2004$ & soil & EPA6010 & REG & $7440-47-3$ & Chromium & 2 & MG/KG & & 1.1 \\
\hline 383МH1803.5 & $5 / 23 / 2004$ & soil & HASL300 & REG & $10045-97-3$ & Cs-137 & 5.14 & $\mathrm{PCl} / \mathrm{G}$ & G & 0.14 \\
\hline 383MH1803.5 & $5 / 23 / 2004$ & soil & EPA8015 & REG & $68334-30-5$ & Diesel Range Organics & 5.1 & MG/KG & $\mathrm{J}$ & 5.6 \\
\hline 383MH1803.5 & $5 / 23 / 2004$ & soil & HASL300 & REG & 13966-00-2 & $\mathrm{K}-40$ & 16.5 & $\mathrm{PCl} / \mathrm{G}$ & G & 1 \\
\hline 383MH1803.5 & $5 / 23 / 2004$ & soil & EPA6010 & REG & $7439-92-1$ & Lead & 25 & MG/KG & & 1.7 \\
\hline 383MH1803.5 & $5 / 23 / 2004$ & soil & EPA7470 & REG & $7439-97-6$ & Mercury & 0.15 & MG/KG & & 0.11 \\
\hline 383МH1803.5 & $5 / 23 / 2004$ & soil & EPA8260 & REG & $75-09-2$ & Methylene Chloride & 1.1 & UG/KG & J,B & 5.6 \\
\hline 383MH1803.5 & $5 / 23 / 2004$ & soil & HASL300 & REG & 15092-94-1 & $\mathrm{Pb}-212$ & 0.99 & $\mathrm{PCl} / \mathrm{G}$ & $\mathrm{J}$ & 0.22 \\
\hline 383MH1803.5 & $5 / 23 / 2004$ & soil & HASL300 & REG & 15067-28-4 & $\mathrm{Pb}-214$ & 0.65 & $\mathrm{PCl} / \mathrm{G}$ & $\mathrm{G}, \mathrm{J}$ & 0.32 \\
\hline
\end{tabular}




\begin{tabular}{|c|c|c|c|c|c|c|c|c|c|c|}
\hline SAMPLE \# & $\begin{array}{l}\text { SAMPLE } \\
\text { DATE }\end{array}$ & MATRIX & $\begin{array}{l}\text { USER TEST } \\
\text { PANEL }\end{array}$ & $\begin{array}{l}\text { SAMPLE } \\
\text { PURPOSE }\end{array}$ & CAS \# & PARAMETER & RESULT & UNITS & Q & $\begin{array}{l}\text { DETECT } \\
\text { LIMIT }\end{array}$ \\
\hline 383MH1803.5 & $5 / 23 / 2004$ & soil & UGTAISOPU & REG & $15117-48-3$ & Pu-239 & 0.112 & $\mathrm{PCl} / \mathrm{G}$ & $\mathrm{J}$ & 0.017 \\
\hline 383MH1803.5 & $5 / 23 / 2004$ & soil & EPA6010 & REG & $7440-22-4$ & Silver & 0.1 & MG/KG & B & 1.1 \\
\hline 383MH1803.5 & $5 / 23 / 2004$ & soil & HASL300 & REG & 14913-50-9 & TI-208 & 0.32 & $\mathrm{PCl} / \mathrm{G}$ & G & 0.12 \\
\hline 383MH1811.5 & $5 / 23 / 2004$ & soil & EPA8260 & REG & 67-64-1 & Acetone & 8.9 & UG/KG & $\mathrm{J}$ & 21 \\
\hline 383MH1811.5 & $5 / 23 / 2004$ & soil & EPA6010 & REG & $7440-38-2$ & Arsenic & 1.6 & MG/KG & & 1 \\
\hline 383MH1811.5 & $5 / 23 / 2004$ & soil & EPA6010 & REG & $7440-39-3$ & Barium & 34 & MG/KG & & 10 \\
\hline 383MH1811.5 & $5 / 23 / 2004$ & soil & EPA6010 & REG & $7440-41-7$ & Beryllium & 0.38 & MG/KG & B & 0.52 \\
\hline 383MH1811.5 & $5 / 23 / 2004$ & soil & HASL300 & REG & 14733-03-0 & Bi-214 & 0.53 & $\mathrm{PCl} / \mathrm{G}$ & $\mathrm{J}$ & 0.14 \\
\hline 383MH1811.5 & $5 / 23 / 2004$ & soil & EPA6010 & REG & $7440-43-9$ & Cadmium & 0.019 & MG/KG & B & 0.52 \\
\hline 383MH1811.5 & $5 / 23 / 2004$ & soil & EPA6010 & REG & $7440-47-3$ & Chromium & 3.5 & MG/KG & & 1 \\
\hline 383MH1811.5 & $5 / 23 / 2004$ & soil & HASL300 & REG & 13966-00-2 & $\mathrm{K}-40$ & 3.8 & $\mathrm{PCl} / \mathrm{G}$ & & 0.8 \\
\hline 383MH1811.5 & $5 / 23 / 2004$ & soil & EPA6010 & REG & $7439-92-1$ & Lead & 5.4 & MG/KG & & 1.5 \\
\hline 383MH1811.5 & $5 / 23 / 2004$ & soil & EPA7470 & REG & 7439-97-6 & Mercury & 0.13 & MG/KG & & 0.1 \\
\hline 383MH1811.5 & $5 / 23 / 2004$ & soil & EPA8260 & REG & $75-09-2$ & Methylene Chloride & 0.83 & UG/KG & J,B & 5.2 \\
\hline 383MH1811.5 & $5 / 23 / 2004$ & soil & HASL300 & REG & $15067-28-4$ & $\mathrm{~Pb}-214$ & 0.6 & $\mathrm{PCl} / \mathrm{G}$ & $\mathrm{J}$ & 0.14 \\
\hline 383MH1811.5 & $5 / 23 / 2004$ & soil & EPA6010 & REG & $7440-22-4$ & Silver & 0.17 & MG/KG & B & 1 \\
\hline 383MH1811.5 & $5 / 23 / 2004$ & soil & HASL300 & REG & 14913-50-9 & TI-208 & 0.125 & $\mathrm{PCl} / \mathrm{G}$ & & 0.066 \\
\hline 383MH2A08.0 & $6 / 8 / 2004$ & soil & HASL300 & REG & 14331-83-0 & Ac-228 & 2.16 & $\mathrm{PCl} / \mathrm{G}$ & G & 0.81 \\
\hline 383MH2A08.0 & $6 / 8 / 2004$ & soil & EPA6010 & REG & $7440-38-2$ & Arsenic & 3.4 & MG/KG & & 1.2 \\
\hline 383MH2A08.0 & $6 / 8 / 2004$ & soil & EPA6010 & REG & $7440-39-3$ & Barium & 140 & MG/KG & & 12 \\
\hline 383MH2A08.0 & $6 / 8 / 2004$ & soil & EPA6010 & REG & $7440-41-7$ & Beryllium & 1.1 & MG/KG & & 0.61 \\
\hline 383MH2A08.0 & $6 / 8 / 2004$ & soil & HASL300 & REG & 14733-03-0 & Bi-214 & 1.23 & $\mathrm{PCl} / \mathrm{G}$ & $\mathrm{G}, \mathrm{J}$ & 0.4 \\
\hline 383MH2A08.0 & $6 / 8 / 2004$ & soil & EPA8270 & REG & 117-81-7 & Bis(2-Ethylhexyl)Phthalate & 170 & UG/KG & $\mathrm{J}$ & 410 \\
\hline 383MH2A08.0 & $6 / 8 / 2004$ & soil & EPA6010 & REG & $7440-43-9$ & Cadmium & 0.09 & MG/KG & B & 0.61 \\
\hline 383MH2A08.0 & $6 / 8 / 2004$ & soil & EPA6010 & REG & 7440-47-3 & Chromium & 1.2 & MG/KG & B & 1.2 \\
\hline 383MH2A08.0 & $6 / 8 / 2004$ & soil & EPA8015 & REG & 68334-30-5 & Diesel Range Organics & 3.4 & MG/KG & $\mathrm{J}$ & 6.1 \\
\hline 383MH2A08.0 & $6 / 8 / 2004$ & soil & HASL300 & REG & 13966-00-2 & $\mathrm{K}-40$ & 36.7 & $\mathrm{PCl} / \mathrm{G}$ & G & 2 \\
\hline 383MH2A08.0 & $6 / 8 / 2004$ & soil & EPA6010 & REG & $7439-92-1$ & Lead & 15 & MG/KG & & 0.37 \\
\hline 383MH2A08.0 & $6 / 8 / 2004$ & soil & EPA7470 & REG & 7439-97-6 & Mercury & 0.0021 & MG/KG & $B$ & 0.12 \\
\hline 383MH2A08.0 & $6 / 8 / 2004$ & soil & HASL300 & REG & 15092-94-1 & $\mathrm{Pb}-212$ & 2.52 & $\mathrm{PCl} / \mathrm{G}$ & $\mathrm{J}$ & 0.27 \\
\hline 383MH2A08.0 & $6 / 8 / 2004$ & soil & HASL300 & REG & 15067-28-4 & $\mathrm{Pb}-214$ & 1.36 & $\mathrm{PCl} / \mathrm{G}$ & $\mathrm{G}, \mathrm{J}$ & 0.33 \\
\hline 383MH2A08.0 & $6 / 8 / 2004$ & soil & HASL300 & REG & 14913-50-9 & TI-208 & 0.66 & $\mathrm{PCl} / \mathrm{G}$ & G & 0.18 \\
\hline 383MH2A37.5 & $6 / 8 / 2004$ & soil & HASL300 & REG & 14331-83-0 & Ac-228 & 1.5 & $\mathrm{PCl} / \mathrm{G}$ & G & 0.66 \\
\hline 383MH2A37.5 & $6 / 8 / 2004$ & soil & HASL300 & REG & 14596-10-2 & Am-241 & 23.5 & $\mathrm{PCl} / \mathrm{G}$ & $\mathrm{J}$ & 2.3 \\
\hline 383MH2A37.5 & $6 / 8 / 2004$ & soil & EPA6010 & REG & 7440-38-2 & Arsenic & 3.5 & MG/KG & & 1.1 \\
\hline
\end{tabular}




\begin{tabular}{|c|c|c|c|c|c|c|c|c|c|c|}
\hline SAMPLE \# & $\begin{array}{l}\text { SAMPLE } \\
\text { DATE }\end{array}$ & MATRIX & $\begin{array}{l}\text { USER TEST } \\
\text { PANEL }\end{array}$ & $\begin{array}{l}\text { SAMPLE } \\
\text { PURPOSE }\end{array}$ & CAS \# & PARAMETER & RESULT & UNITS & Q & $\begin{array}{l}\text { DETECT } \\
\text { LIMIT }\end{array}$ \\
\hline 383MH2A37.5 & $6 / 8 / 2004$ & soil & EPA6010 & REG & $7440-39-3$ & Barium & 80 & MG/KG & & 11 \\
\hline 383MH2A37.5 & $6 / 8 / 2004$ & soil & EPA8270 & REG & $56-55-3$ & Benzo(A)Anthracene & 85 & UG/KG & $\mathrm{J}$ & 370 \\
\hline 383MH2A37.5 & $6 / 8 / 2004$ & soil & EPA8270 & REG & $50-32-8$ & Benzo(A)Pyrene & 60 & UG/KG & $\mathrm{J}$ & 370 \\
\hline 383MH2A37.5 & $6 / 8 / 2004$ & soil & EPA8270 & REG & $205-99-2$ & Benzo(B)Fluoranthene & 140 & UG/KG & $\mathrm{J}$ & 370 \\
\hline 383MH2A37.5 & $6 / 8 / 2004$ & soil & EPA8270 & REG & $207-08-9$ & Benzo(K)Fluoranthene & 34 & UG/KG & $\mathrm{J}$ & 370 \\
\hline 383MH2A37.5 & $6 / 8 / 2004$ & soil & EPA6010 & REG & $7440-41-7$ & Beryllium & 0.88 & MG/KG & & 0.56 \\
\hline 383MH2A37.5 & $6 / 8 / 2004$ & soil & EPA6010 & REG & $7440-43-9$ & Cadmium & 0.26 & MG/KG & B & 0.56 \\
\hline 383MH2A37.5 & $6 / 8 / 2004$ & soil & EPA6010 & REG & $7440-47-3$ & Chromium & 3.5 & MG/KG & & 1.1 \\
\hline 383MH2A37.5 & $6 / 8 / 2004$ & soil & EPA8270 & REG & 218-01-9 & Chrysene & 130 & UG/KG & $\mathrm{J}$ & 370 \\
\hline 383MH2A37.5 & $6 / 8 / 2004$ & soil & HASL300 & REG & $10045-97-3$ & Cs-137 & 218 & $\mathrm{PCl} / \mathrm{G}$ & G & 0 \\
\hline 383MH2A37.5 & $6 / 8 / 2004$ & soil & EPA8270 & REG & $206-44-0$ & Fluoranthene & 91 & UG/KG & $\mathrm{J}$ & 370 \\
\hline 383MH2A37.5 & $6 / 8 / 2004$ & soil & EPA8270 & REG & $193-39-5$ & Indeno(1,2,3-Cd)Pyrene & 27 & UG/KG & $\mathrm{J}$ & 370 \\
\hline 383MH2A37.5 & $6 / 8 / 2004$ & soil & HASL300 & REG & 13966-00-2 & $\mathrm{K}-40$ & 23.1 & $\mathrm{PCl} / \mathrm{G}$ & G & 1.3 \\
\hline 383MH2A37.5 & $6 / 8 / 2004$ & soil & EPA6010 & REG & $7439-92-1$ & Lead & 25 & MG/KG & & 1.7 \\
\hline 383MH2A37.5 & $6 / 8 / 2004$ & soil & EPA7470 & REG & $7439-97-6$ & Mercury & 0.1 & MG/KG & B & 0.11 \\
\hline 383MH2A37.5 & $6 / 8 / 2004$ & soil & HASL300 & REG & 15092-94-1 & $\mathrm{Pb}-212$ & 1.78 & $\mathrm{PCl} / \mathrm{G}$ & $\mathrm{J}$ & 0.68 \\
\hline 383MH2A37.5 & $6 / 8 / 2004$ & soil & EPA8270 & REG & $85-01-8$ & Phenanthrene & 34 & UG/KG & $\mathrm{J}$ & 370 \\
\hline 383MH2A37.5 & $6 / 8 / 2004$ & soil & UGTAISOPU & REG & 13981-16-3 & Pu-238 & 3.62 & $\mathrm{PCl} / \mathrm{G}$ & Y1 & 0.01 \\
\hline 383MH2A37.5 & $6 / 8 / 2004$ & soil & UGTAISOPU & REG & $15117-48-3$ & Pu-239 & 80 & $\mathrm{PCl} / \mathrm{G}$ & $\mathrm{J}$ & 0 \\
\hline 383MH2A37.5 & $6 / 8 / 2004$ & soil & EPA8270 & REG & $129-00-0$ & Pyrene & 93 & UG/KG & $\mathrm{J}$ & 370 \\
\hline 383MH2A37.5 & $6 / 8 / 2004$ & soil & EPA6010 & REG & $7440-22-4$ & Silver & 1.1 & MG/KG & B & 1.1 \\
\hline 383MH2A37.5 & $6 / 8 / 2004$ & soil & SR7500 & REG & 10098-97-2 & Sr-90 & 10.9 & $\mathrm{PCl} / \mathrm{G}$ & & 0.2 \\
\hline 383MH2A45.0 & $6 / 8 / 2004$ & soil & EPA8260 & REG & 67-64-1 & Acetone & 15 & UG/KG & $\mathrm{J}$ & 21 \\
\hline 383MH2A45.0 & $6 / 8 / 2004$ & soil & HASL300 & REG & 14596-10-2 & Am-241 & 1.1 & $\mathrm{PCl} / \mathrm{G}$ & & 0.25 \\
\hline 383MH2A45.0 & $6 / 8 / 2004$ & soil & EPA6010 & REG & $7440-38-2$ & Arsenic & 1.4 & MG/KG & & 1 \\
\hline 383MH2A45.0 & $6 / 8 / 2004$ & soil & EPA6010 & REG & $7440-39-3$ & Barium & 40 & MG/KG & & 10 \\
\hline 383MH2A45.0 & $6 / 8 / 2004$ & soil & EPA6010 & REG & $7440-41-7$ & Beryllium & 0.53 & MG/KG & & 0.52 \\
\hline 383MH2A45.0 & $6 / 8 / 2004$ & soil & HASL300 & REG & 14733-03-0 & Bi-214 & 0.62 & $\mathrm{PCl} / \mathrm{G}$ & $\mathrm{J}$ & 0.25 \\
\hline 383MH2A45.0 & $6 / 8 / 2004$ & soil & EPA6010 & REG & $7440-43-9$ & Cadmium & 0.16 & MG/KG & B & 0.52 \\
\hline 383MH2A45.0 & $6 / 8 / 2004$ & soil & EPA6010 & REG & $7440-47-3$ & Chromium & 4.3 & MG/KG & & 1 \\
\hline 383MH2A45.0 & 6/8/2004 & soil & HASL300 & REG & 10045-97-3 & Cs-137 & 9.8 & $\mathrm{PCl} / \mathrm{G}$ & & 0.1 \\
\hline 383MH2A45.0 & $6 / 8 / 2004$ & soil & HASL300 & REG & 13966-00-2 & $\mathrm{K}-40$ & 10.8 & $\mathrm{PCl} / \mathrm{G}$ & & 0.9 \\
\hline 383MH2A45.0 & $6 / 8 / 2004$ & soil & EPA6010 & REG & 7439-92-1 & Lead & 9.5 & MG/KG & & 1.6 \\
\hline 383MH2A45.0 & 6/8/2004 & soil & EPA7470 & REG & 7439-97-6 & Mercury & 0.043 & MG/KG & B & 0.1 \\
\hline 383MH2A45.0 & $6 / 8 / 2004$ & soil & HASL300 & REG & 15092-94-1 & $\mathrm{Pb}-212$ & 0.72 & $\mathrm{PCl} / \mathrm{G}$ & $\mathrm{J}$ & 0.21 \\
\hline
\end{tabular}




\begin{tabular}{|c|c|c|c|c|c|c|c|c|c|c|}
\hline SAMPLE \# & $\begin{array}{l}\text { SAMPLE } \\
\text { DATE }\end{array}$ & MATRIX & $\begin{array}{l}\text { USER TEST } \\
\text { PANEL }\end{array}$ & $\begin{array}{l}\text { SAMPLE } \\
\text { PURPOSE }\end{array}$ & CAS \# & PARAMETER & RESULT & UNITS & Q & $\begin{array}{l}\text { DETECT } \\
\text { LIMIT }\end{array}$ \\
\hline 383MH2A45.0 & $6 / 8 / 2004$ & soil & HASL300 & REG & $15067-28-4$ & $\mathrm{~Pb}-214$ & 0.45 & $\mathrm{PCl} / \mathrm{G}$ & $\mathrm{J}$ & 0.24 \\
\hline 383MH2A45.0 & $6 / 8 / 2004$ & soil & UGTAISOPU & REG & 13981-16-3 & Pu-238 & 0.213 & $\mathrm{PCl} / \mathrm{G}$ & & 0.025 \\
\hline 383MH2A45.0 & $6 / 8 / 2004$ & soil & UGTAISOPU & REG & $15117-48-3$ & Pu-239 & 4.55 & $\mathrm{PCl} / \mathrm{G}$ & $\mathrm{J}$ & 0.02 \\
\hline 383MH2A45.0 & $6 / 8 / 2004$ & soil & EPA6010 & REG & $7440-22-4$ & Silver & 0.45 & MG/KG & B & 1 \\
\hline 383MH2A45.0 & $6 / 8 / 2004$ & soil & HASL300 & REG & 14913-50-9 & TI-208 & 0.213 & $\mathrm{PCl} / \mathrm{G}$ & & 0.116 \\
\hline $3830 B 0100.5$ & $6 / 1 / 2004$ & soil & EPA8260 & REG & $78-93-3$ & 2-Butanone & 17 & UG/KG & $\mathrm{J}$ & 21 \\
\hline $3830 B 0100.5$ & $6 / 1 / 2004$ & soil & HASL300 & REG & 14331-83-0 & Ac-228 & 2.21 & $\mathrm{PCl} / \mathrm{G}$ & G & 0.61 \\
\hline $3830 B 0100.5$ & $6 / 1 / 2004$ & soil & EPA8260 & REG & $67-64-1$ & Acetone & 72 & UG/KG & & 21 \\
\hline 383OB0100.5 & $6 / 1 / 2004$ & soil & EPA6010 & REG & $7440-38-2$ & Arsenic & 2.9 & MG/KG & & 1 \\
\hline $3830 B 0100.5$ & $6 / 1 / 2004$ & soil & EPA6010 & REG & $7440-39-3$ & Barium & 180 & MG/KG & & 10 \\
\hline $3830 B 0100.5$ & $6 / 1 / 2004$ & soil & EPA8270 & REG & $56-55-3$ & Benzo(A)Anthracene & 260 & UG/KG & $\mathrm{J}$ & 1700 \\
\hline $3830 B 0100.5$ & $6 / 1 / 2004$ & soil & EPA6010 & REG & $7440-41-7$ & Beryllium & 0.64 & MG/KG & & 0.51 \\
\hline $3830 B 0100.5$ & $6 / 1 / 2004$ & soil & HASL300 & REG & 14733-03-0 & $\mathrm{Bi}-214$ & 0.58 & $\mathrm{PCl} / \mathrm{G}$ & $\mathrm{G}, \mathrm{J}$ & 0.29 \\
\hline $3830 B 0100.5$ & $6 / 1 / 2004$ & soil & EPA6010 & REG & $7440-43-9$ & Cadmium & 1.4 & MG/KG & & 0.51 \\
\hline 383OB0100.5 & $6 / 1 / 2004$ & soil & EPA6010 & REG & $7440-47-3$ & Chromium & 6.2 & MG/KG & & 1 \\
\hline $3830 B 0100.5$ & $6 / 1 / 2004$ & soil & EPA8270 & REG & $218-01-9$ & Chrysene & 590 & UG/KG & $\mathrm{J}$ & 1700 \\
\hline $3830 B 0100.5$ & $6 / 1 / 2004$ & soil & HASL300 & REG & $10045-97-3$ & Cs-137 & 1.34 & $\mathrm{PCl} / \mathrm{G}$ & G & 0.18 \\
\hline $3830 B 0100.5$ & $6 / 1 / 2004$ & soil & EPA8015 & REG & $68334-30-5$ & Diesel Range Organics & 3400 & MG/KG & $\mathrm{M}, \mathrm{H}$ & 26 \\
\hline $3830 B 0100.5$ & $6 / 1 / 2004$ & soil & EPA8270 & REG & $206-44-0$ & Fluoranthene & 170 & UG/KG & $\mathrm{J}$ & 1700 \\
\hline $3830 B 0100.5$ & $6 / 1 / 2004$ & soil & HASL300 & REG & 13966-00-2 & $\mathrm{K}-40$ & 21.7 & $\mathrm{PCl} / \mathrm{G}$ & G & 1.6 \\
\hline $3830 B 0100.5$ & $6 / 1 / 2004$ & soil & EPA6010 & REG & $7439-92-1$ & Lead & 30 & MG/KG & & 0.31 \\
\hline $3830 B 0100.5$ & $6 / 1 / 2004$ & soil & HASL300 & REG & 15092-94-1 & $\mathrm{Pb}-212$ & 2.25 & $\mathrm{PCl} / \mathrm{G}$ & $\mathrm{J}$ & 0.34 \\
\hline $3830 B 0100.5$ & $6 / 1 / 2004$ & soil & HASL300 & REG & $15067-28-4$ & $\mathrm{~Pb}-214$ & 0.86 & $\mathrm{PCl} / \mathrm{G}$ & $\mathrm{G}, \mathrm{J}$ & 0.31 \\
\hline $3830 B 0100.5$ & $6 / 1 / 2004$ & soil & EPA8270 & REG & $85-01-8$ & Phenanthrene & 220 & UG/KG & $\mathrm{J}$ & 1700 \\
\hline 383 OB0100.5 & $6 / 1 / 2004$ & soil & UGTAISOPU & REG & $15117-48-3$ & Pu-239 & 0.213 & $\mathrm{PCl} / \mathrm{G}$ & $\mathrm{J}$ & 0.017 \\
\hline $3830 B 0100.5$ & $6 / 1 / 2004$ & soil & EPA8270 & REG & $129-00-0$ & Pyrene & 2100 & UG/KG & $\mathrm{J}$ & 1700 \\
\hline $3830 B 0100.5$ & $6 / 1 / 2004$ & soil & HASL300 & REG & 14913-50-9 & TI-208 & 0.68 & $\mathrm{PCl} / \mathrm{G}$ & G & 0.16 \\
\hline 383OB0100.5RR1 & $6 / 1 / 2004$ & soil & EPA8270 & $\mathrm{RE}$ & $56-55-3$ & Benzo(A)Anthracene & 340 & UG/KG & $\mathrm{J}$ & 3400 \\
\hline 383OB0100.5RR1 & $6 / 1 / 2004$ & soil & EPA8270 & $\mathrm{RE}$ & $218-01-9$ & Chrysene & 570 & UG/KG & $\mathrm{J}$ & 3400 \\
\hline 383OB0100.5RR1 & $6 / 1 / 2004$ & soil & EPA8270 & $\mathrm{RE}$ & $206-44-0$ & Fluoranthene & 220 & UG/KG & $\mathrm{J}$ & 3400 \\
\hline 383OB0100.5RR1 & $6 / 1 / 2004$ & soil & EPA8270 & $\mathrm{RE}$ & $129-00-0$ & Pyrene & 1400 & UG/KG & $\mathrm{J}$ & 3400 \\
\hline $3830 B 0200.5$ & $6 / 1 / 2004$ & soil & EPA8260 & REG & $78-93-3$ & 2-Butanone & 11 & UG/KG & $\mathrm{J}$ & 21 \\
\hline 383 OB0200.5 & $6 / 1 / 2004$ & soil & HASL300 & REG & 14331-83-0 & Ac-228 & 1.99 & $\mathrm{PCl} / \mathrm{G}$ & G & 0.58 \\
\hline 383 OB0200.5 & $6 / 1 / 2004$ & soil & EPA8260 & REG & 67-64-1 & Acetone & 61 & UG/KG & & 21 \\
\hline 383 OB0200.5 & $6 / 1 / 2004$ & soil & EPA8270 & REG & $120-12-7$ & Anthracene & 86 & UG/KG & $\mathrm{J}$ & 1700 \\
\hline
\end{tabular}




\begin{tabular}{|c|c|c|c|c|c|c|c|c|c|c|}
\hline SAMPLE \# & $\begin{array}{l}\text { SAMPLE } \\
\text { DATE }\end{array}$ & MATRIX & $\begin{array}{l}\text { USER TEST } \\
\text { PANEL }\end{array}$ & $\begin{array}{l}\text { SAMPLE } \\
\text { PURPOSE }\end{array}$ & CAS \# & PARAMETER & RESULT & UNITS & Q & $\begin{array}{l}\text { DETECT } \\
\text { LIMIT }\end{array}$ \\
\hline 3830 OB0200.5 & $6 / 1 / 2004$ & soil & EPA6010 & REG & $7440-38-2$ & Arsenic & 4.9 & MG/KG & & 1 \\
\hline 3830 OB0200.5 & $6 / 1 / 2004$ & soil & EPA6010 & REG & $7440-39-3$ & Barium & 260 & MG/KG & & 10 \\
\hline $3830 B 0200.5$ & $6 / 1 / 2004$ & soil & EPA8270 & REG & $56-55-3$ & Benzo(A)Anthracene & 1400 & UG/KG & $\mathrm{J}$ & 1700 \\
\hline 383OB0200.5 & $6 / 1 / 2004$ & soil & EPA6010 & REG & $7440-41-7$ & Beryllium & 0.77 & MG/KG & & 0.51 \\
\hline 383 OB0200.5 & $6 / 1 / 2004$ & soil & HASL300 & REG & 14733-03-0 & $\mathrm{Bi}-214$ & 0.79 & $\mathrm{PCl} / \mathrm{G}$ & $\mathrm{G}, \mathrm{J}$ & 0.33 \\
\hline 383 OB0200.5 & $6 / 1 / 2004$ & soil & EPA6010 & REG & $7440-43-9$ & Cadmium & 1.4 & MG/KG & & 0.51 \\
\hline $3830 B 0200.5$ & $6 / 1 / 2004$ & soil & EPA8270 & REG & 86-74-8 & Carbazole & 160 & UG/KG & $\mathrm{J}$ & 1700 \\
\hline 383 OB0200.5 & $6 / 1 / 2004$ & soil & EPA6010 & REG & $7440-47-3$ & Chromium & 6.7 & MG/KG & & 1 \\
\hline $3830 B 0200.5$ & $6 / 1 / 2004$ & soil & EPA8270 & REG & 218-01-9 & Chrysene & 2500 & UG/KG & $\mathrm{J}$ & 1700 \\
\hline 383 OB0200.5 & $6 / 1 / 2004$ & soil & HASL300 & REG & $10045-97-3$ & Cs-137 & 4.1 & $\mathrm{PCl} / \mathrm{G}$ & G & 0.17 \\
\hline 383 OB0200.5 & $6 / 1 / 2004$ & soil & EPA8015 & REG & 68334-30-5 & Diesel Range Organics & 2500 & MG/KG & $\mathrm{M}, \mathrm{H}$ & 26 \\
\hline $3830 B 0200.5$ & $6 / 1 / 2004$ & soil & EPA8270 & REG & $206-44-0$ & Fluoranthene & 1000 & UG/KG & $\mathrm{J}$ & 1700 \\
\hline $3830 B 0200.5$ & $6 / 1 / 2004$ & soil & HASL300 & REG & 13966-00-2 & $\mathrm{K}-40$ & 24.2 & $\mathrm{PCl} / \mathrm{G}$ & G & 1.9 \\
\hline 383 OB0200.5 & $6 / 1 / 2004$ & soil & EPA6010 & REG & $7439-92-1$ & Lead & 30 & MG/KG & & 0.31 \\
\hline 383 OB0200.5 & $6 / 1 / 2004$ & soil & HASL300 & REG & 15092-94-1 & $\mathrm{Pb}-212$ & 2.13 & $\mathrm{PCl} / \mathrm{G}$ & $\mathrm{J}$ & 0.37 \\
\hline 383 OB0200.5 & $6 / 1 / 2004$ & soil & HASL300 & REG & $15067-28-4$ & $\mathrm{~Pb}-214$ & 0.88 & $\mathrm{PCl} / \mathrm{G}$ & $\mathrm{G}, \mathrm{J}$ & 0.39 \\
\hline 383 OB0200.5 & $6 / 1 / 2004$ & soil & EPA8270 & REG & $85-01-8$ & Phenanthrene & 1000 & UG/KG & $\mathrm{J}$ & 1700 \\
\hline 383 OB0200.5 & $6 / 1 / 2004$ & soil & EPA8270 & REG & $129-00-0$ & Pyrene & 25000 & UG/KG & $\mathrm{J}$ & 1700 \\
\hline 3830 OB0200.5 & $6 / 1 / 2004$ & soil & HASL300 & REG & 14913-50-9 & TI-208 & 0.88 & $\mathrm{PCl} / \mathrm{G}$ & G & 0.16 \\
\hline 383OB0200.5RR1 & $6 / 1 / 2004$ & soil & EPA8270 & $\mathrm{RE}$ & $56-55-3$ & Benzo(A)Anthracene & 1400 & UG/KG & $\mathrm{J}$ & 3400 \\
\hline 383OB0200.5RR1 & $6 / 1 / 2004$ & soil & EPA8270 & $\mathrm{RE}$ & $205-99-2$ & Benzo(B)Fluoranthene & 2800 & UG/KG & $\mathrm{J}$ & 3400 \\
\hline 383OB0200.5RR1 & $6 / 1 / 2004$ & soil & EPA8270 & $\mathrm{RE}$ & $207-08-9$ & Benzo(K)Fluoranthene & 1800 & UG/KG & $\mathrm{J}$ & 3400 \\
\hline 383OB0200.5RR1 & $6 / 1 / 2004$ & soil & EPA8270 & $\mathrm{RE}$ & 218-01-9 & Chrysene & 2400 & UG/KG & $\mathrm{J}$ & 3400 \\
\hline 383OB0200.5RR1 & $6 / 1 / 2004$ & soil & EPA8270 & $\mathrm{RE}$ & $206-44-0$ & Fluoranthene & 1900 & UG/KG & $\mathrm{J}$ & 3400 \\
\hline 383OB0200.5RR1 & 6/1/2004 & soil & EPA8270 & $\mathrm{RE}$ & $85-01-8$ & Phenanthrene & 1100 & UG/KG & $\mathrm{J}$ & 3400 \\
\hline 383OB0200.5RR1 & 6/1/2004 & soil & EPA8270 & $\mathrm{RE}$ & $129-00-0$ & Pyrene & 6400 & UG/KG & $\mathrm{J}$ & 3400 \\
\hline $3830 B 0300.5$ & $6 / 1 / 2004$ & soil & HASL300 & REG & 14331-83-0 & Ac-228 & 1.96 & $\mathrm{PCl} / \mathrm{G}$ & G & 0.57 \\
\hline $3830 B 0300.5$ & $6 / 1 / 2004$ & soil & EPA8260 & REG & $67-64-1$ & Acetone & 7.2 & UG/KG & $\mathrm{J}, \mathrm{B}$ & 21 \\
\hline 383 OB0300.5 & $6 / 1 / 2004$ & soil & EPA6010 & REG & $7440-38-2$ & Arsenic & 4.1 & MG/KG & & 1.1 \\
\hline 383 OB0300.5 & $6 / 1 / 2004$ & soil & EPA6010 & REG & 7440-39-3 & Barium & 180 & MG/KG & & 11 \\
\hline 383 OB0300.5 & $6 / 1 / 2004$ & soil & EPA8270 & REG & $56-55-3$ & Benzo(A)Anthracene & 120 & UG/KG & $\mathrm{J}$ & 350 \\
\hline 383 OB0300.5 & $6 / 1 / 2004$ & soil & EPA8270 & REG & $50-32-8$ & Benzo(A)Pyrene & 230 & UG/KG & $\mathrm{J}$ & 350 \\
\hline $3830 B 0300.5$ & $6 / 1 / 2004$ & soil & EPA8270 & REG & $205-99-2$ & Benzo(B)Fluoranthene & 510 & UG/KG & $\mathrm{J}$ & 350 \\
\hline 383 OB0300.5 & $6 / 1 / 2004$ & soil & EPA8270 & REG & $191-24-2$ & Benzo(G,H,I)Perylene & 120 & UG/KG & $\mathrm{J}$ & 350 \\
\hline $3830 B 0300.5$ & $6 / 1 / 2004$ & soil & EPA8270 & REG & 207-08-9 & Benzo(K)Fluoranthene & 220 & UG/KG & $\mathrm{J}$ & 350 \\
\hline
\end{tabular}




\begin{tabular}{|c|c|c|c|c|c|c|c|c|c|c|}
\hline SAMPLE \# & $\begin{array}{l}\text { SAMPLE } \\
\text { DATE }\end{array}$ & MATRIX & $\begin{array}{l}\text { USER TEST } \\
\text { PANEL }\end{array}$ & $\begin{array}{l}\text { SAMPLE } \\
\text { PURPOSE }\end{array}$ & CAS \# & PARAMETER & RESULT & UNITS & Q & $\begin{array}{l}\text { DETECT } \\
\text { LIMIT }\end{array}$ \\
\hline 3830 OB0300.5 & $6 / 1 / 2004$ & soil & EPA6010 & REG & $7440-41-7$ & Beryllium & 1.1 & MG/KG & & 0.53 \\
\hline $3830 B 0300.5$ & $6 / 1 / 2004$ & soil & HASL300 & REG & $14733-03-0$ & $\mathrm{Bi}-214$ & 1.01 & $\mathrm{PCl} / \mathrm{G}$ & $\mathrm{G}, \mathrm{J}$ & 0.29 \\
\hline $3830 B 0300.5$ & $6 / 1 / 2004$ & soil & EPA6010 & REG & $7440-47-3$ & Chromium & 3.9 & MG/KG & & 1.1 \\
\hline $3830 B 0300.5$ & $6 / 1 / 2004$ & soil & EPA8270 & REG & 218-01-9 & Chrysene & 340 & UG/KG & $\mathrm{J}$ & 350 \\
\hline $3830 B 0300.5$ & $6 / 1 / 2004$ & soil & HASL300 & REG & $10045-97-3$ & Cs-137 & 6.13 & $\mathrm{PCl} / \mathrm{G}$ & G & 0.13 \\
\hline $3830 B 0300.5$ & $6 / 1 / 2004$ & soil & EPA8015 & REG & $68334-30-5$ & Diesel Range Organics & 330 & MG/KG & $\mathrm{H}, \mathrm{Z}$ & 5.3 \\
\hline $3830 B 0300.5$ & $6 / 1 / 2004$ & soil & EPA8270 & REG & $206-44-0$ & Fluoranthene & 210 & UG/KG & $\mathrm{J}$ & 350 \\
\hline 3830 OB0300.5 & $6 / 1 / 2004$ & soil & HASL300 & REG & 13966-00-2 & $\mathrm{K}-40$ & 22 & $\mathrm{PCl} / \mathrm{G}$ & G & 1.5 \\
\hline $3830 B 0300.5$ & $6 / 1 / 2004$ & soil & EPA6010 & REG & $7439-92-1$ & Lead & 20 & MG/KG & & 0.32 \\
\hline $3830 B 0300.5$ & $6 / 1 / 2004$ & soil & EPA8270 & REG & $91-20-3$ & Naphthalene & 54 & UG/KG & $\mathrm{J}$ & 350 \\
\hline $3830 B 0300.5$ & $6 / 1 / 2004$ & soil & HASL300 & REG & 15092-94-1 & $\mathrm{Pb}-212$ & 2.13 & $\mathrm{PCl} / \mathrm{G}$ & $\mathrm{J}$ & 0.24 \\
\hline $3830 B 0300.5$ & $6 / 1 / 2004$ & soil & HASL300 & REG & 15067-28-4 & $\mathrm{Pb}-214$ & 0.97 & $\mathrm{PCl} / \mathrm{G}$ & $\mathrm{G}, \mathrm{J}$ & 0.28 \\
\hline $3830 B 0300.5$ & $6 / 1 / 2004$ & soil & EPA8270 & REG & $85-01-8$ & Phenanthrene & 65 & UG/KG & $\mathrm{J}$ & 350 \\
\hline $3830 B 0300.5$ & $6 / 1 / 2004$ & soil & UGTAISOPU & REG & $15117-48-3$ & Pu-239 & 0.59 & $\mathrm{PCl} / \mathrm{G}$ & $\mathrm{J}$ & 0.02 \\
\hline $3830 B 0300.5$ & $6 / 1 / 2004$ & soil & EPA8270 & REG & $129-00-0$ & Pyrene & 620 & UG/KG & $\mathrm{J}$ & 350 \\
\hline $3830 B 0300.5$ & $6 / 1 / 2004$ & soil & HASL300 & REG & 14913-50-9 & TI-208 & 0.69 & $\mathrm{PCl} / \mathrm{G}$ & G & 0.14 \\
\hline 3830 OB0400.5 & $6 / 1 / 2004$ & soil & HASL300 & REG & 14331-83-0 & Ac-228 & 2 & $\mathrm{PCl} / \mathrm{G}$ & G & 0.43 \\
\hline 3830 OB0400.5 & $6 / 1 / 2004$ & soil & EPA6010 & REG & $7440-38-2$ & Arsenic & 5.1 & MG/KG & & 1 \\
\hline $3830 B 0400.5$ & $6 / 1 / 2004$ & soil & EPA6010 & REG & $7440-39-3$ & Barium & 300 & MG/KG & & 10 \\
\hline $3830 B 0400.5$ & $6 / 1 / 2004$ & soil & EPA8270 & REG & $56-55-3$ & Benzo(A)Anthracene & 110 & UG/KG & $\mathrm{J}$ & 350 \\
\hline 3830 OB0400.5 & $6 / 1 / 2004$ & soil & EPA8270 & REG & $50-32-8$ & Benzo(A)Pyrene & 160 & UG/KG & $\mathrm{J}$ & 350 \\
\hline 3830 OB0400.5 & $6 / 1 / 2004$ & soil & EPA8270 & REG & $205-99-2$ & Benzo(B)Fluoranthene & 310 & UG/KG & $\mathrm{J}$ & 350 \\
\hline $3830 B 0400.5$ & $6 / 1 / 2004$ & soil & EPA8270 & REG & $191-24-2$ & Benzo(G,H,I)Perylene & 59 & UG/KG & $\mathrm{J}$ & 350 \\
\hline 3830 OB0400.5 & $6 / 1 / 2004$ & soil & EPA8270 & REG & $207-08-9$ & Benzo(K)Fluoranthene & 140 & UG/KG & $\mathrm{J}$ & 350 \\
\hline 3830 OB0400.5 & $6 / 1 / 2004$ & soil & EPA6010 & REG & $7440-41-7$ & Beryllium & 0.82 & MG/KG & & 0.52 \\
\hline $3830 B 0400.5$ & $6 / 1 / 2004$ & soil & HASL300 & REG & 14733-03-0 & $\mathrm{Bi}-214$ & 1.04 & $\mathrm{PCl} / \mathrm{G}$ & $\mathrm{G}, \mathrm{J}$ & 0.27 \\
\hline $3830 B 0400.5$ & $6 / 1 / 2004$ & soil & EPA6010 & REG & $7440-47-3$ & Chromium & 5.8 & MG/KG & & 1 \\
\hline 3830 OB0400.5 & $6 / 1 / 2004$ & soil & EPA8270 & REG & $218-01-9$ & Chrysene & 230 & UG/KG & $\mathrm{J}$ & 350 \\
\hline $3830 B 0400.5$ & $6 / 1 / 2004$ & soil & HASL300 & REG & $10045-97-3$ & Cs-137 & 5.97 & $\mathrm{PCl} / \mathrm{G}$ & G & 0.16 \\
\hline $3830 B 0400.5$ & $6 / 1 / 2004$ & soil & EPA8015 & REG & $68334-30-5$ & Diesel Range Organics & 63 & MG/KG & $\mathrm{M}, \mathrm{H}$ & 5.2 \\
\hline $3830 B 0400.5$ & $6 / 1 / 2004$ & soil & EPA8270 & REG & $206-44-0$ & Fluoranthene & 190 & UG/KG & $\mathrm{J}$ & 350 \\
\hline $3830 B 0400.5$ & $6 / 1 / 2004$ & soil & EPA8270 & REG & $193-39-5$ & Indeno(1,2,3-Cd)Pyrene & 54 & UG/KG & $\mathrm{J}$ & 350 \\
\hline 3830 OB0400.5 & $6 / 1 / 2004$ & soil & HASL300 & REG & 13966-00-2 & $\mathrm{K}-40$ & 25 & $\mathrm{PCl} / \mathrm{G}$ & G & 1.5 \\
\hline 3830 OB0400.5 & $6 / 1 / 2004$ & soil & EPA6010 & REG & $7439-92-1$ & Lead & 30 & MG/KG & & 0.31 \\
\hline $3830 B 0400.5$ & $6 / 1 / 2004$ & soil & HASL300 & REG & 15092-94-1 & $\mathrm{Pb}-212$ & 2.48 & $\mathrm{PCl} / \mathrm{G}$ & $\mathrm{J}$ & 0.25 \\
\hline
\end{tabular}




\begin{tabular}{|c|c|c|c|c|c|c|c|c|c|c|}
\hline SAMPLE \# & $\begin{array}{l}\text { SAMPLE } \\
\text { DATE }\end{array}$ & MATRIX & $\begin{array}{l}\text { USER TEST } \\
\text { PANEL }\end{array}$ & $\begin{array}{l}\text { SAMPLE } \\
\text { PURPOSE }\end{array}$ & CAS \# & PARAMETER & RESULT & UNITS & Q & $\begin{array}{l}\text { DETECT } \\
\text { LIMIT }\end{array}$ \\
\hline $383 \mathrm{OB} 0400.5$ & $6 / 1 / 2004$ & soil & HASL300 & REG & $15067-28-4$ & $\mathrm{~Pb}-214$ & 0.91 & $\mathrm{PCl} / \mathrm{G}$ & $\mathrm{G}, \mathrm{J}$ & 0.34 \\
\hline 3830 OB0400.5 & $6 / 1 / 2004$ & soil & EPA8270 & REG & $85-01-8$ & Phenanthrene & 55 & UG/KG & $\mathrm{J}$ & 350 \\
\hline $3830 B 0400.5$ & $6 / 1 / 2004$ & soil & UGTAISOPU & REG & 15117-48-3 & Pu-239 & 0.158 & $\mathrm{PCl} / \mathrm{G}$ & $\mathrm{J}$ & 0.01 \\
\hline $3830 B 0400.5$ & $6 / 1 / 2004$ & soil & EPA8270 & REG & $129-00-0$ & Pyrene & 220 & UG/KG & $\mathrm{J}$ & 350 \\
\hline $383 \mathrm{OB} 0400.5$ & $6 / 1 / 2004$ & soil & HASL300 & REG & 14913-50-9 & TI-208 & 0.59 & $\mathrm{PCl} / \mathrm{G}$ & $\mathrm{G}$ & 0.16 \\
\hline 3830 OB0500.5 & $6 / 1 / 2004$ & soil & HASL300 & REG & 14331-83-0 & Ac-228 & 2.2 & $\mathrm{PCl} / \mathrm{G}$ & $G$ & 0.66 \\
\hline $383 \mathrm{OB} 0500.5$ & $6 / 1 / 2004$ & soil & EPA6010 & REG & $7440-38-2$ & Arsenic & 3.8 & MG/KG & & 1.1 \\
\hline $3830 B 0500.5$ & $6 / 1 / 2004$ & soil & EPA6010 & REG & $7440-39-3$ & Barium & 140 & MG/KG & & 11 \\
\hline $383 \mathrm{OB} 0500.5$ & $6 / 1 / 2004$ & soil & EPA8270 & REG & $56-55-3$ & Benzo(A)Anthracene & 200 & UG/KG & $\mathrm{J}$ & 350 \\
\hline $383 \mathrm{OB} 0500.5$ & $6 / 1 / 2004$ & soil & EPA8270 & REG & $50-32-8$ & Benzo(A)Pyrene & 250 & UG/KG & $\mathrm{J}$ & 350 \\
\hline $383 \mathrm{OB} 0500.5$ & $6 / 1 / 2004$ & soil & EPA8270 & REG & $205-99-2$ & Benzo(B)Fluoranthene & 670 & UG/KG & $\mathrm{J}$ & 350 \\
\hline $3830 B 0500.5$ & $6 / 1 / 2004$ & soil & EPA8270 & REG & 207-08-9 & Benzo(K)Fluoranthene & 280 & UG/KG & $\mathrm{J}$ & 350 \\
\hline $3830 B 0500.5$ & $6 / 1 / 2004$ & soil & EPA6010 & REG & $7440-41-7$ & Beryllium & 1.2 & MG/KG & & 0.53 \\
\hline $383 \mathrm{OB} 0500.5$ & $6 / 1 / 2004$ & soil & HASL300 & REG & 14733-03-0 & Bi-214 & 1.01 & $\mathrm{PCl} / \mathrm{G}$ & $\mathrm{G}, \mathrm{J}$ & 0.34 \\
\hline $3830 B 0500.5$ & $6 / 1 / 2004$ & soil & EPA6010 & REG & $7440-47-3$ & Chromium & 2.5 & MG/KG & & 1.1 \\
\hline $3830 B 0500.5$ & $6 / 1 / 2004$ & soil & EPA8270 & REG & 218-01-9 & Chrysene & 410 & UG/KG & & 350 \\
\hline $383 \mathrm{OB} 0500.5$ & $6 / 1 / 2004$ & soil & HASL300 & REG & $10045-97-3$ & Cs-137 & 5.65 & $\mathrm{PCl} / \mathrm{G}$ & G & 0.24 \\
\hline 3830 O0500.5 & $6 / 1 / 2004$ & soil & EPA8270 & REG & 206-44-0 & Fluoranthene & 400 & UG/KG & & 350 \\
\hline 3830 OB0500.5 & $6 / 1 / 2004$ & soil & HASL300 & REG & 13966-00-2 & $\mathrm{K}-40$ & 24.6 & $\mathrm{PCl} / \mathrm{G}$ & $\mathrm{G}$ & 1.9 \\
\hline $383 \mathrm{OB} 0500.5$ & $6 / 1 / 2004$ & soil & EPA6010 & REG & 7439-92-1 & Lead & 17 & MG/KG & & 0.32 \\
\hline 3830 O0500.5 & $6 / 1 / 2004$ & soil & EPA8270 & REG & $91-20-3$ & Naphthalene & 15 & UG/KG & $\mathrm{J}$ & 350 \\
\hline $383 \mathrm{OB} 0500.5$ & $6 / 1 / 2004$ & soil & HASL300 & REG & 15092-94-1 & $\mathrm{Pb}-212$ & 3.1 & $\mathrm{PCl} / \mathrm{G}$ & $\mathrm{J}$ & 0.34 \\
\hline $383 \mathrm{OB} 0500.5$ & $6 / 1 / 2004$ & soil & HASL300 & REG & $15067-28-4$ & $\mathrm{~Pb}-214$ & 1.04 & $\mathrm{PCl} / \mathrm{G}$ & $\mathrm{G}, \mathrm{J}$ & 0.4 \\
\hline $383 \mathrm{OB} 0500.5$ & $6 / 1 / 2004$ & soil & EPA8270 & REG & $85-01-8$ & Phenanthrene & 140 & UG/KG & $\mathrm{J}$ & 350 \\
\hline $3830 B 0500.5$ & $6 / 1 / 2004$ & soil & UGTAISOPU & REG & 15117-48-3 & Pu-239 & 0.089 & $\mathrm{PCl} / \mathrm{G}$ & $\mathrm{J}$ & 0.025 \\
\hline $3830 B 0500.5$ & $6 / 1 / 2004$ & soil & EPA8270 & REG & $129-00-0$ & Pyrene & 460 & UG/KG & & 350 \\
\hline $3830 B 0500.5$ & $6 / 1 / 2004$ & soil & HASL300 & REG & 14913-50-9 & TI-208 & 0.97 & $\mathrm{PCl} / \mathrm{G}$ & G & 0.18 \\
\hline 383OB0500.5X & $6 / 1 / 2004$ & soil & HASL300 & FD & 14331-83-0 & Ac-228 & 2.41 & $\mathrm{PCl} / \mathrm{G}$ & $\mathrm{G}$ & 0.57 \\
\hline 3830B0500.5X & $6 / 1 / 2004$ & soil & EPA6010 & FD & $7440-38-2$ & Arsenic & 3.5 & MG/KG & & 1.1 \\
\hline 383OB0500.5X & $6 / 1 / 2004$ & soil & EPA6010 & FD & $7440-39-3$ & Barium & 130 & MG/KG & & 11 \\
\hline 383OB0500.5X & $6 / 1 / 2004$ & soil & EPA8270 & FD & $56-55-3$ & Benzo(A)Anthracene & 200 & UG/KG & $\mathrm{J}$ & 360 \\
\hline 3830B0500.5X & $6 / 1 / 2004$ & soil & EPA8270 & FD & $50-32-8$ & Benzo(A)Pyrene & 240 & UG/KG & $\mathrm{J}$ & 360 \\
\hline 383OB0500.5X & $6 / 1 / 2004$ & soil & EPA8270 & FD & $205-99-2$ & Benzo(B)Fluoranthene & 550 & UG/KG & $\mathrm{J}$ & 360 \\
\hline 383OB0500.5X & $6 / 1 / 2004$ & soil & EPA8270 & FD & $191-24-2$ & Benzo(G,H,I)Perylene & 81 & UG/KG & $\mathrm{J}$ & 360 \\
\hline 3830B0500.5X & $6 / 1 / 2004$ & soil & EPA8270 & FD & 207-08-9 & Benzo(K)Fluoranthene & 260 & UG/KG & $\mathrm{J}$ & 360 \\
\hline
\end{tabular}




\begin{tabular}{|c|c|c|c|c|c|c|c|c|c|c|}
\hline SAMPLE \# & $\begin{array}{l}\text { SAMPLE } \\
\text { DATE }\end{array}$ & MATRIX & $\begin{array}{l}\text { USER TEST } \\
\text { PANEL }\end{array}$ & $\begin{array}{l}\text { SAMPLE } \\
\text { PURPOSE }\end{array}$ & CAS \# & PARAMETER & RESULT & UNITS & Q & $\begin{array}{l}\text { DETECT } \\
\text { LIMIT }\end{array}$ \\
\hline 383OB0500.5X & $6 / 1 / 2004$ & soil & EPA6010 & FD & $7440-41-7$ & Beryllium & 1 & MG/KG & & 0.54 \\
\hline 383OB0500.5X & $6 / 1 / 2004$ & soil & HASL300 & FD & 14733-03-0 & $\mathrm{Bi}-214$ & 1.07 & $\mathrm{PCl} / \mathrm{G}$ & $\mathrm{G}, \mathrm{J}$ & 0.36 \\
\hline 383OB0500.5X & $6 / 1 / 2004$ & soil & EPA6010 & FD & $7440-47-3$ & Chromium & 2.5 & MG/KG & & 1.1 \\
\hline 383OB0500.5X & $6 / 1 / 2004$ & soil & EPA8270 & FD & $218-01-9$ & Chrysene & 410 & UG/KG & & 360 \\
\hline 383OB0500.5X & $6 / 1 / 2004$ & soil & HASL300 & FD & $10045-97-3$ & Cs-137 & 5 & $\mathrm{PCl} / \mathrm{G}$ & G & 0.19 \\
\hline 383OB0500.5X & $6 / 1 / 2004$ & soil & EPA8270 & FD & $206-44-0$ & Fluoranthene & 400 & UG/KG & & 360 \\
\hline 383OB0500.5X & $6 / 1 / 2004$ & soil & EPA8270 & FD & $193-39-5$ & Indeno(1,2,3-Cd)Pyrene & 77 & UG/KG & $\mathrm{J}$ & 360 \\
\hline 383OB0500.5X & $6 / 1 / 2004$ & soil & HASL300 & FD & $13966-00-2$ & $\mathrm{~K}-40$ & 24.1 & $\mathrm{PCl} / \mathrm{G}$ & G & 1.9 \\
\hline 383OB0500.5X & $6 / 1 / 2004$ & soil & EPA6010 & FD & $7439-92-1$ & Lead & 15 & MG/KG & & 0.32 \\
\hline 383OB0500.5X & $6 / 1 / 2004$ & soil & HASL300 & FD & 15092-94-1 & $\mathrm{Pb}-212$ & 2.97 & $\mathrm{PCl} / \mathrm{G}$ & $\mathrm{J}$ & 0.24 \\
\hline 383OB0500.5X & $6 / 1 / 2004$ & soil & HASL300 & FD & 15067-28-4 & $\mathrm{Pb}-214$ & 1.1 & $\mathrm{PCl} / \mathrm{G}$ & $\mathrm{G}, \mathrm{J}$ & 0.34 \\
\hline 3830B0500.5X & $6 / 1 / 2004$ & soil & EPA8270 & FD & $85-01-8$ & Phenanthrene & 140 & UG/KG & $\mathrm{J}$ & 360 \\
\hline 383OB0500.5X & $6 / 1 / 2004$ & soil & UGTAISOPU & FD & 15117-48-3 & Pu-239 & 0.157 & $\mathrm{PCl} / \mathrm{G}$ & $\mathrm{J}$ & 0.023 \\
\hline 3830B0500.5X & $6 / 1 / 2004$ & soil & EPA8270 & FD & $129-00-0$ & Pyrene & 510 & UG/KG & & 360 \\
\hline 383OB0500.5X & $6 / 1 / 2004$ & soil & HASL300 & FD & $14913-50-9$ & TI-208 & 0.81 & $\mathrm{PCl} / \mathrm{G}$ & G & 0.17 \\
\hline $3830 B 0600.5$ & $6 / 1 / 2004$ & soil & HASL300 & REG & 14331-83-0 & Ac-228 & 1.87 & $\mathrm{PCl} / \mathrm{G}$ & G & 0.56 \\
\hline $3830 B 0600.5$ & $6 / 1 / 2004$ & soil & EPA6010 & REG & $7440-38-2$ & Arsenic & 5.8 & MG/KG & & 1 \\
\hline 383 OB0600.5 & $6 / 1 / 2004$ & soil & EPA6010 & REG & $7440-39-3$ & Barium & 190 & MG/KG & & 10 \\
\hline $3830 B 0600.5$ & $6 / 1 / 2004$ & soil & EPA8270 & REG & $56-55-3$ & Benzo(A)Anthracene & 45 & UG/KG & $\mathrm{J}$ & 350 \\
\hline $3830 B 0600.5$ & $6 / 1 / 2004$ & soil & EPA8270 & REG & $50-32-8$ & Benzo(A)Pyrene & 64 & UG/KG & $\mathrm{J}$ & 350 \\
\hline $3830 B 0600.5$ & $6 / 1 / 2004$ & soil & EPA8270 & REG & $205-99-2$ & Benzo(B)Fluoranthene & 140 & UG/KG & $\mathrm{J}$ & 350 \\
\hline $3830 B 0600.5$ & $6 / 1 / 2004$ & soil & EPA8270 & REG & $207-08-9$ & Benzo(K)Fluoranthene & 45 & UG/KG & $\mathrm{J}$ & 350 \\
\hline $3830 B 0600.5$ & $6 / 1 / 2004$ & soil & EPA6010 & REG & $7440-41-7$ & Beryllium & 0.77 & MG/KG & & 0.52 \\
\hline $3830 B 0600.5$ & $6 / 1 / 2004$ & soil & HASL300 & REG & 14733-03-0 & Bi-214 & 0.98 & $\mathrm{PCl} / \mathrm{G}$ & $\mathrm{G}, \mathrm{J}$ & 0.27 \\
\hline 383 OB0600.5 & 6/1/2004 & soil & EPA6010 & REG & $7440-47-3$ & Chromium & 8.3 & MG/KG & & 1 \\
\hline 383 OB0600.5 & $6 / 1 / 2004$ & soil & EPA8270 & REG & $218-01-9$ & Chrysene & 110 & UG/KG & $\mathrm{J}$ & 350 \\
\hline $3830 B 0600.5$ & $6 / 1 / 2004$ & soil & HASL300 & REG & $10045-97-3$ & Cs-137 & 0.53 & $\mathrm{PCl} / \mathrm{G}$ & G & 0.12 \\
\hline 383 OB0600.5 & $6 / 1 / 2004$ & soil & EPA8270 & REG & $206-44-0$ & Fluoranthene & 93 & UG/KG & $\mathrm{J}$ & 350 \\
\hline 383 OB0600.5 & $6 / 1 / 2004$ & soil & HASL300 & REG & 13966-00-2 & $\mathrm{K}-40$ & 22.7 & $\mathrm{PCl} / \mathrm{G}$ & G & 1.5 \\
\hline 383 OB0600.5 & $6 / 1 / 2004$ & soil & EPA6010 & REG & $7439-92-1$ & Lead & 12 & MG/KG & & 0.31 \\
\hline 383 OB0600.5 & $6 / 1 / 2004$ & soil & HASL300 & REG & 15092-94-1 & $\mathrm{Pb}-212$ & 2.12 & $\mathrm{PCl} / \mathrm{G}$ & $\mathrm{J}$ & 0.2 \\
\hline 383 OB0600.5 & $6 / 1 / 2004$ & soil & HASL300 & REG & 15067-28-4 & $\mathrm{Pb}-214$ & 1.06 & $\mathrm{PCl} / \mathrm{G}$ & $\mathrm{G}, \mathrm{J}$ & 0.24 \\
\hline 383 OB0600.5 & $6 / 1 / 2004$ & soil & EPA8270 & REG & $85-01-8$ & Phenanthrene & 32 & UG/KG & $\mathrm{J}$ & 350 \\
\hline 383 OB0600.5 & 6/1/2004 & soil & EPA8270 & REG & $129-00-0$ & Pyrene & 75 & UG/KG & $\mathrm{J}$ & 350 \\
\hline 383 OB0600.5 & $6 / 1 / 2004$ & soil & HASL300 & REG & 14913-50-9 & TI-208 & 0.59 & $\mathrm{PCl} / \mathrm{G}$ & G & 0.13 \\
\hline
\end{tabular}




\begin{tabular}{|c|c|c|c|c|c|c|c|c|c|c|}
\hline SAMPLE \# & $\begin{array}{l}\text { SAMPLE } \\
\text { DATE }\end{array}$ & MATRIX & $\begin{array}{l}\text { USER TEST } \\
\text { PANEL }\end{array}$ & $\begin{array}{l}\text { SAMPLE } \\
\text { PURPOSE }\end{array}$ & CAS \# & PARAMETER & RESULT & UNITS & Q & $\begin{array}{l}\text { DETECT } \\
\text { LIMIT }\end{array}$ \\
\hline $3830 B 0700.5$ & $6 / 1 / 2004$ & soil & HASL300 & REG & 14331-83-0 & Ac-228 & 2.14 & $\mathrm{PCl} / \mathrm{G}$ & G & 0.54 \\
\hline $3830 B 0700.5$ & $6 / 1 / 2004$ & soil & EPA8270 & REG & $83-32-9$ & Acenaphthene & 39 & UG/KG & $\mathrm{J}$ & 700 \\
\hline $3830 B 0700.5$ & $6 / 1 / 2004$ & soil & EPA8270 & REG & $120-12-7$ & Anthracene & 150 & UG/KG & $\mathrm{J}$ & 700 \\
\hline $3830 B 0700.5$ & $6 / 1 / 2004$ & soil & EPA6010 & REG & $7440-38-2$ & Arsenic & 6.7 & MG/KG & & 1 \\
\hline $3830 B 0700.5$ & $6 / 1 / 2004$ & soil & EPA6010 & REG & $7440-39-3$ & Barium & 250 & MG/KG & & 10 \\
\hline $3830 B 0700.5$ & $6 / 1 / 2004$ & soil & EPA8270 & REG & $56-55-3$ & Benzo(A)Anthracene & 4300 & UG/KG & $\mathrm{J}$ & 700 \\
\hline $3830 B 0700.5$ & $6 / 1 / 2004$ & soil & EPA8270 & REG & $50-32-8$ & Benzo(A)Pyrene & 4300 & UG/KG & $\mathrm{J}$ & 700 \\
\hline $3830 B 0700.5$ & $6 / 1 / 2004$ & soil & EPA8270 & REG & $205-99-2$ & Benzo(B)Fluoranthene & 8200 & UG/KG & $\mathrm{J}$ & 700 \\
\hline $3830 B 0700.5$ & $6 / 1 / 2004$ & soil & EPA8270 & REG & $191-24-2$ & Benzo(G,H,I)Perylene & 7000 & UG/KG & $\mathrm{J}$ & 700 \\
\hline $3830 B 0700.5$ & $6 / 1 / 2004$ & soil & EPA8270 & REG & $207-08-9$ & Benzo(K)Fluoranthene & 3200 & UG/KG & $\mathrm{J}$ & 700 \\
\hline $3830 B 0700.5$ & $6 / 1 / 2004$ & soil & EPA6010 & REG & $7440-41-7$ & Beryllium & 1 & MG/KG & & 0.52 \\
\hline $3830 B 0700.5$ & $6 / 1 / 2004$ & soil & HASL300 & REG & $14733-03-0$ & $\mathrm{Bi}-214$ & 0.92 & $\mathrm{PCl} / \mathrm{G}$ & $\mathrm{G}, \mathrm{J}$ & 0.35 \\
\hline $3830 B 0700.5$ & $6 / 1 / 2004$ & soil & EPA8270 & REG & 117-81-7 & Bis(2-Ethylhexyl)Phthalate & 1000 & UG/KG & $\mathrm{J}$ & 700 \\
\hline 383 OB0700.5 & $6 / 1 / 2004$ & soil & EPA6010 & REG & $7440-43-9$ & Cadmium & 1.1 & MG/KG & & 1 \\
\hline $3830 B 0700.5$ & $6 / 1 / 2004$ & soil & EPA8270 & REG & 86-74-8 & Carbazole & 290 & UG/KG & $\mathrm{J}$ & 700 \\
\hline $3830 B 0700.5$ & $6 / 1 / 2004$ & soil & EPA6010 & REG & $7440-47-3$ & Chromium & 10 & MG/KG & & 1 \\
\hline $3830 B 0700.5$ & $6 / 1 / 2004$ & soil & EPA8270 & REG & 218-01-9 & Chrysene & 5300 & UG/KG & $\mathrm{J}$ & 700 \\
\hline $3830 B 0700.5$ & $6 / 1 / 2004$ & soil & HASL300 & REG & $10045-97-3$ & Cs-137 & 20.5 & $\mathrm{PCl} / \mathrm{G}$ & G & 0.2 \\
\hline $3830 B 0700.5$ & $6 / 1 / 2004$ & soil & EPA8270 & REG & $53-70-3$ & Dibenzo(A,H)Anthracene & 2100 & UG/KG & $\mathrm{J}$ & 700 \\
\hline $3830 B 0700.5$ & $6 / 1 / 2004$ & soil & EPA8015 & REG & $68334-30-5$ & Diesel Range Organics & 120 & MG/KG & $\mathrm{H}, \mathrm{Z}$ & 5.2 \\
\hline $3830 B 0700.5$ & $6 / 1 / 2004$ & soil & EPA8270 & REG & $206-44-0$ & Fluoranthene & 3800 & UG/KG & & 700 \\
\hline 383 OB0700.5 & $6 / 1 / 2004$ & soil & EPA8270 & REG & $86-73-7$ & Fluorene & 31 & UG/KG & $\mathrm{J}$ & 700 \\
\hline $3830 B 0700.5$ & $6 / 1 / 2004$ & soil & EPA8270 & REG & $193-39-5$ & Indeno(1,2,3-Cd)Pyrene & 5000 & UG/KG & $\mathrm{J}$ & 700 \\
\hline $3830 B 0700.5$ & $6 / 1 / 2004$ & soil & HASL300 & REG & $13966-00-2$ & $\mathrm{~K}-40$ & 19.8 & $\mathrm{PCl} / \mathrm{G}$ & G & 1.2 \\
\hline 383 OB0700.5 & 6/1/2004 & soil & EPA6010 & REG & $7439-92-1$ & Lead & 31 & MG/KG & & 0.63 \\
\hline $3830 B 0700.5$ & $6 / 1 / 2004$ & soil & EPA8270 & REG & $91-20-3$ & Naphthalene & 32 & UG/KG & $\mathrm{J}$ & 700 \\
\hline $3830 B 0700.5$ & $6 / 1 / 2004$ & soil & HASL300 & REG & 15092-94-1 & $\mathrm{Pb}-212$ & 2.32 & $\mathrm{PCl} / \mathrm{G}$ & $\mathrm{J}$ & 0.36 \\
\hline $3830 B 0700.5$ & $6 / 1 / 2004$ & soil & EPA8270 & REG & $85-01-8$ & Phenanthrene & 1200 & UG/KG & & 700 \\
\hline 383 OB0700.5 & $6 / 1 / 2004$ & soil & UGTAISOPU & REG & 15117-48-3 & Pu-239 & 0.051 & $\mathrm{PCl} / \mathrm{G}$ & $\mathrm{J}$ & 0.018 \\
\hline $3830 B 0700.5$ & $6 / 1 / 2004$ & soil & EPA8270 & REG & $129-00-0$ & Pyrene & 26000 & UG/KG & $\mathrm{J}$ & 700 \\
\hline 383 OB0700.5 & $6 / 1 / 2004$ & soil & HASL300 & REG & 14913-50-9 & TI-208 & 0.82 & $\mathrm{PCl} / \mathrm{G}$ & G & 0.19 \\
\hline 383OB0700.5RR1 & $6 / 1 / 2004$ & soil & EPA8270 & RE & $120-12-7$ & Anthracene & 160 & UG/KG & $\mathrm{J}$ & 2100 \\
\hline 383OB0700.5RR1 & $6 / 1 / 2004$ & soil & EPA8270 & $\mathrm{RE}$ & $56-55-3$ & Benzo(A)Anthracene & 4800 & UG/KG & $\mathrm{J}$ & 2100 \\
\hline 3830B0700.5RR1 & $6 / 1 / 2004$ & soil & EPA8270 & $\mathrm{RE}$ & $50-32-8$ & Benzo(A)Pyrene & 4800 & UG/KG & $\mathrm{J}$ & 2100 \\
\hline 383OB0700.5RR1 & $6 / 1 / 2004$ & soil & EPA8270 & $\mathrm{RE}$ & $205-99-2$ & Benzo(B)Fluoranthene & 9600 & UG/KG & $\mathrm{J}$ & 2100 \\
\hline
\end{tabular}




\begin{tabular}{|c|c|c|c|c|c|c|c|c|c|c|}
\hline SAMPLE \# & $\begin{array}{l}\text { SAMPLE } \\
\text { DATE }\end{array}$ & MATRIX & $\begin{array}{l}\text { USER TEST } \\
\text { PANEL }\end{array}$ & $\begin{array}{l}\text { SAMPLE } \\
\text { PURPOSE }\end{array}$ & CAS \# & PARAMETER & RESULT & UNITS & Q & $\begin{array}{l}\text { DETECT } \\
\text { LIMIT }\end{array}$ \\
\hline 383OB0700.5RR1 & $6 / 1 / 2004$ & soil & EPA8270 & $\mathrm{RE}$ & $191-24-2$ & Benzo(G,H,I)Perylene & 2100 & UG/KG & $\mathrm{J}$ & 2100 \\
\hline 383OB0700.5RR1 & $6 / 1 / 2004$ & soil & EPA8270 & $\mathrm{RE}$ & $207-08-9$ & Benzo(K)Fluoranthene & 5500 & UG/KG & $\mathrm{J}$ & 2100 \\
\hline 383OB0700.5RR1 & $6 / 1 / 2004$ & soil & EPA8270 & $\mathrm{RE}$ & $117-81-7$ & Bis(2-Ethylhexyl)Phthalate & 710 & UG/KG & $\mathrm{J}$ & 2100 \\
\hline 383OB0700.5RR1 & $6 / 1 / 2004$ & soil & EPA8270 & $\mathrm{RE}$ & 86-74-8 & Carbazole & 290 & UG/KG & $\mathrm{J}$ & 2100 \\
\hline 383OB0700.5RR1 & $6 / 1 / 2004$ & soil & EPA8270 & $\mathrm{RE}$ & $218-01-9$ & Chrysene & 5800 & UG/KG & $\mathrm{J}$ & 2100 \\
\hline 383OB0700.5RR1 & $6 / 1 / 2004$ & soil & EPA8270 & $\mathrm{RE}$ & $206-44-0$ & Fluoranthene & 5100 & UG/KG & & 2100 \\
\hline 383OB0700.5RR1 & $6 / 1 / 2004$ & soil & EPA8270 & $\mathrm{RE}$ & $193-39-5$ & Indeno(1,2,3-Cd)Pyrene & 1900 & UG/KG & $\mathrm{J}$ & 2100 \\
\hline 383OB0700.5RR1 & $6 / 1 / 2004$ & soil & EPA8270 & $\mathrm{RE}$ & $85-01-8$ & Phenanthrene & 1300 & UG/KG & $\mathrm{J}$ & 2100 \\
\hline 383OB0700.5RR1 & $6 / 1 / 2004$ & soil & EPA8270 & $\mathrm{RE}$ & $129-00-0$ & Pyrene & 15000 & UG/KG & $\mathrm{J}$ & 2100 \\
\hline $3830 B 0800.5$ & $6 / 1 / 2004$ & soil & HASL300 & REG & 14331-83-0 & Ac-228 & 1.27 & $\mathrm{PCl} / \mathrm{G}$ & G & 0.63 \\
\hline 383 OB0800.5 & $6 / 1 / 2004$ & soil & EPA8270 & REG & $83-32-9$ & Acenaphthene & 93 & UG/KG & $\mathrm{J}$ & 1400 \\
\hline $3830 B 0800.5$ & $6 / 1 / 2004$ & soil & EPA8270 & REG & $120-12-7$ & Anthracene & 470 & UG/KG & $\mathrm{J}$ & 1400 \\
\hline $3830 B 0800.5$ & $6 / 1 / 2004$ & soil & EPA6010 & REG & $7440-38-2$ & Arsenic & 7.1 & MG/KG & & 1 \\
\hline 383 OB0800.5 & $6 / 1 / 2004$ & soil & EPA6010 & REG & $7440-39-3$ & Barium & 220 & MG/KG & & 10 \\
\hline $3830 B 0800.5$ & $6 / 1 / 2004$ & soil & EPA8270 & REG & $56-55-3$ & Benzo(A)Anthracene & 17000 & UG/KG & $\mathrm{J}$ & 1400 \\
\hline $3830 B 0800.5$ & $6 / 1 / 2004$ & soil & EPA8270 & REG & $50-32-8$ & Benzo(A)Pyrene & 20000 & UG/KG & $\mathrm{J}$ & 1400 \\
\hline $3830 B 0800.5$ & $6 / 1 / 2004$ & soil & EPA8270 & REG & $205-99-2$ & Benzo(B)Fluoranthene & 37000 & UG/KG & $\mathrm{J}$ & 1400 \\
\hline 383 OB0800.5 & $6 / 1 / 2004$ & soil & EPA8270 & REG & $191-24-2$ & Benzo(G,H,I)Perylene & 32000 & UG/KG & $\mathrm{J}$ & 1400 \\
\hline $3830 B 0800.5$ & $6 / 1 / 2004$ & soil & EPA8270 & REG & $207-08-9$ & Benzo(K)Fluoranthene & 10000 & UG/KG & $\mathrm{J}$ & 1400 \\
\hline $3830 B 0800.5$ & $6 / 1 / 2004$ & soil & EPA6010 & REG & $7440-41-7$ & Beryllium & 0.77 & MG/KG & & 0.52 \\
\hline $3830 B 0800.5$ & $6 / 1 / 2004$ & soil & HASL300 & REG & 14733-03-0 & $\mathrm{Bi}-214$ & 1.1 & $\mathrm{PCl} / \mathrm{G}$ & $\mathrm{G}, \mathrm{J}$ & 0.28 \\
\hline $3830 B 0800.5$ & $6 / 1 / 2004$ & soil & EPA8270 & REG & 117-81-7 & Bis(2-Ethylhexyl)Phthalate & 4000 & UG/KG & $\mathrm{J}$ & 1400 \\
\hline $3830 B 0800.5$ & $6 / 1 / 2004$ & soil & EPA6010 & REG & $7440-43-9$ & Cadmium & 0.58 & MG/KG & & 0.52 \\
\hline $3830 B 0800.5$ & $6 / 1 / 2004$ & soil & EPA8270 & REG & 86-74-8 & Carbazole & 790 & UG/KG & $\mathrm{J}$ & 1400 \\
\hline 383 OB0800.5 & 6/1/2004 & soil & EPA6010 & REG & $7440-47-3$ & Chromium & 7.7 & MG/KG & & 1 \\
\hline 383 OB0800.5 & $6 / 1 / 2004$ & soil & EPA8270 & REG & $218-01-9$ & Chrysene & 21000 & UG/KG & $\mathrm{J}$ & 1400 \\
\hline $3830 B 0800.5$ & $6 / 1 / 2004$ & soil & HASL300 & REG & $10045-97-3$ & Cs-137 & 13.6 & $\mathrm{PCl} / \mathrm{G}$ & G & 0.2 \\
\hline $3830 B 0800.5$ & $6 / 1 / 2004$ & soil & EPA8270 & REG & $53-70-3$ & Dibenzo(A,H)Anthracene & 10000 & UG/KG & $\mathrm{J}$ & 1400 \\
\hline 383 OB0800.5 & $6 / 1 / 2004$ & soil & EPA8015 & REG & $68334-30-5$ & Diesel Range Organics & 490 & MG/KG & $\mathrm{H}, \mathrm{Z}$ & 5.2 \\
\hline 383 OB0800.5 & $6 / 1 / 2004$ & soil & EPA8270 & REG & $206-44-0$ & Fluoranthene & 10000 & UG/KG & & 1400 \\
\hline 383 OB0800.5 & $6 / 1 / 2004$ & soil & EPA8270 & REG & $86-73-7$ & Fluorene & 68 & UG/KG & $\mathrm{J}$ & 1400 \\
\hline $3830 B 0800.5$ & $6 / 1 / 2004$ & soil & EPA8270 & REG & $193-39-5$ & Indeno(1,2,3-Cd)Pyrene & 22000 & UG/KG & $\mathrm{J}$ & 1400 \\
\hline $3830 B 0800.5$ & $6 / 1 / 2004$ & soil & HASL300 & REG & 13966-00-2 & $\mathrm{K}-40$ & 22.4 & $\mathrm{PCl} / \mathrm{G}$ & G & 1.8 \\
\hline 383 OB0800.5 & $6 / 1 / 2004$ & soil & EPA6010 & REG & $7439-92-1$ & Lead & 25 & MG/KG & & 0.31 \\
\hline $3830 B 0800.5$ & $6 / 1 / 2004$ & soil & EPA8270 & REG & 91-20-3 & Naphthalene & 56 & UG/KG & $\mathrm{J}$ & 1400 \\
\hline
\end{tabular}




\begin{tabular}{|c|c|c|c|c|c|c|c|c|c|c|}
\hline SAMPLE \# & $\begin{array}{l}\text { SAMPLE } \\
\text { DATE }\end{array}$ & MATRIX & $\begin{array}{l}\text { USER TEST } \\
\text { PANEL }\end{array}$ & $\begin{array}{l}\text { SAMPLE } \\
\text { PURPOSE }\end{array}$ & CAS \# & PARAMETER & RESULT & UNITS & Q & $\begin{array}{l}\text { DETECT } \\
\text { LIMIT }\end{array}$ \\
\hline 383 OB0800.5 & $6 / 1 / 2004$ & soil & HASL300 & REG & 15092-94-1 & $\mathrm{Pb}-212$ & 1.83 & $\mathrm{PCl} / \mathrm{G}$ & $\mathrm{J}$ & 0.34 \\
\hline $3830 \mathrm{OB} 0800.5$ & $6 / 1 / 2004$ & soil & HASL300 & REG & 15067-28-4 & $\mathrm{Pb}-214$ & 0.9 & $\mathrm{PCl} / \mathrm{G}$ & $\mathrm{G}, \mathrm{J}$ & 0.41 \\
\hline 3830 OB0800.5 & 6/1/2004 & soil & EPA8270 & REG & $85-01-8$ & Phenanthrene & 2500 & UG/KG & & 1400 \\
\hline 383 OB0800.5 & $6 / 1 / 2004$ & soil & UGTAISOPU & REG & 15117-48-3 & Pu-239 & 1.04 & $\mathrm{PCl} / \mathrm{G}$ & $\mathrm{J}$ & 0.02 \\
\hline 383 OB0800.5 & $6 / 1 / 2004$ & soil & EPA8270 & REG & $129-00-0$ & Pyrene & 89000 & UG/KG & $\mathrm{J}$ & 1400 \\
\hline 383 OB0800.5 & $6 / 1 / 2004$ & soil & EPA6010 & REG & 7782-49-2 & Selenium & 1.2 & MG/KG & & 0.52 \\
\hline 383OB0800.5 & $6 / 1 / 2004$ & soil & HASL300 & REG & 14913-50-9 & TI-208 & 0.54 & $\mathrm{PCl} / \mathrm{G}$ & G & 0.18 \\
\hline 383OB0800.5RR1 & $6 / 1 / 2004$ & soil & EPA8270 & $\mathrm{RE}$ & $56-55-3$ & Benzo(A)Anthracene & 18000 & UG/KG & $\mathrm{J}$ & 6900 \\
\hline 383OB0800.5RR1 & $6 / 1 / 2004$ & soil & EPA8270 & $\mathrm{RE}$ & $50-32-8$ & Benzo(A)Pyrene & 23000 & UG/KG & $\mathrm{J}$ & 6900 \\
\hline 383OB0800.5RR1 & $6 / 1 / 2004$ & soil & EPA8270 & $\mathrm{RE}$ & $205-99-2$ & Benzo(B)Fluoranthene & 43000 & UG/KG & $\mathrm{J}$ & 6900 \\
\hline 383OB0800.5RR1 & $6 / 1 / 2004$ & soil & EPA8270 & $\mathrm{RE}$ & $191-24-2$ & Benzo(G,H,I)Perylene & 16000 & UG/KG & $\mathrm{J}$ & 6900 \\
\hline 383OB0800.5RR1 & $6 / 1 / 2004$ & soil & EPA8270 & $\mathrm{RE}$ & $207-08-9$ & Benzo(K)Fluoranthene & 19000 & UG/KG & $\mathrm{J}$ & 6900 \\
\hline 383OB0800.5RR1 & $6 / 1 / 2004$ & soil & EPA8270 & $\mathrm{RE}$ & 117-81-7 & Bis(2-Ethylhexyl)Phthalate & 3900 & UG/KG & $\mathrm{J}$ & 6900 \\
\hline 3830B0800.5RR1 & $6 / 1 / 2004$ & soil & EPA8270 & $\mathrm{RE}$ & $218-01-9$ & Chrysene & 23000 & UG/KG & $\mathrm{J}$ & 6900 \\
\hline 383OB0800.5RR1 & $6 / 1 / 2004$ & soil & EPA8270 & $\mathrm{RE}$ & $206-44-0$ & Fluoranthene & 13000 & UG/KG & $\mathrm{J}$ & 6900 \\
\hline 383OB0800.5RR1 & $6 / 1 / 2004$ & soil & EPA8270 & $\mathrm{RE}$ & $193-39-5$ & Indeno(1,2,3-Cd)Pyrene & 12000 & UG/KG & $\mathrm{J}$ & 6900 \\
\hline 3830B0800.5RR1 & $6 / 1 / 2004$ & soil & EPA8270 & $\mathrm{RE}$ & $129-00-0$ & Pyrene & 64000 & UG/KG & $\mathrm{J}$ & 6900 \\
\hline 383PB0102.0 & $6 / 10 / 2004$ & soil & EPA8260 & REG & $67-64-1$ & Acetone & 11 & UG/KG & $\mathrm{J}$ & 22 \\
\hline 383PB0102.0 & $6 / 10 / 2004$ & soil & EPA6010 & REG & $7440-38-2$ & Arsenic & 1 & MG/KG & $B$ & 1.1 \\
\hline 383PB0102.0 & $6 / 10 / 2004$ & soil & EPA6010 & REG & $7440-39-3$ & Barium & 4900 & MG/KG & & 110 \\
\hline 383PB0102.0 & $6 / 10 / 2004$ & soil & EPA6010 & REG & $7440-41-7$ & Beryllium & 0.19 & MG/KG & $B$ & 0.54 \\
\hline 383РВ0102.0 & $6 / 10 / 2004$ & soil & EPA6010 & REG & $7440-43-9$ & Cadmium & 0.16 & MG/KG & $B$ & 0.54 \\
\hline 383PB0102.0 & $6 / 10 / 2004$ & soil & EPA6010 & REG & $7440-47-3$ & Chromium & 4.2 & MG/KG & & 1.1 \\
\hline 383PB0102.0 & $6 / 10 / 2004$ & soil & HASL300 & REG & 13966-00-2 & $\mathrm{K}-40$ & 17.8 & $\mathrm{PCl} / \mathrm{G}$ & G & 1.3 \\
\hline 383PB0102.0 & $6 / 10 / 2004$ & soil & EPA6010 & REG & $7439-92-1$ & Lead & 5.3 & MG/KG & & 0.33 \\
\hline 383PB0102.0 & $6 / 10 / 2004$ & soil & EPA7470 & REG & $7439-97-6$ & Mercury & 0.0016 & MG/KG & $B$ & 0.11 \\
\hline 383PB0102.0 & $6 / 10 / 2004$ & soil & HASL300 & REG & 15092-94-1 & $\mathrm{Pb}-212$ & 0.27 & $\mathrm{PCl} / \mathrm{G}$ & G & 0.15 \\
\hline 383PB0102.0 & $6 / 10 / 2004$ & soil & EPA6010 & REG & $7440-22-4$ & Silver & 0.077 & MG/KG & B & 1.1 \\
\hline 383PB0104.5 & $6 / 10 / 2004$ & soil & HASL300 & REG & 14331-83-0 & Ac-228 & 1.67 & $\mathrm{PCl} / \mathrm{G}$ & G & 0.55 \\
\hline 383PB0104.5 & $6 / 10 / 2004$ & soil & EPA8260 & REG & $67-64-1$ & Acetone & 10 & UG/KG & $\mathrm{J}$ & 21 \\
\hline 383PB0104.5 & $6 / 10 / 2004$ & soil & EPA6010 & REG & $7440-38-2$ & Arsenic & 2.9 & MG/KG & & 1.1 \\
\hline 383PB0104.5 & $6 / 10 / 2004$ & soil & EPA6010 & REG & $7440-39-3$ & Barium & 120 & MG/KG & & 11 \\
\hline 383PB0104.5 & $6 / 10 / 2004$ & soil & EPA6010 & REG & $7440-41-7$ & Beryllium & 0.71 & MG/KG & & 0.53 \\
\hline 383PB0104.5 & $6 / 10 / 2004$ & soil & HASL300 & REG & 14733-03-0 & Bi-214 & 0.96 & $\mathrm{PCl} / \mathrm{G}$ & $\mathrm{G}, \mathrm{J}$ & 0.28 \\
\hline 383PB0104.5 & $6 / 10 / 2004$ & soil & EPA6010 & REG & $7440-43-9$ & Cadmium & 0.17 & MG/KG & $B$ & 0.53 \\
\hline
\end{tabular}




\begin{tabular}{|c|c|c|c|c|c|c|c|c|c|c|}
\hline SAMPLE \# & $\begin{array}{l}\text { SAMPLE } \\
\text { DATE }\end{array}$ & MATRIX & $\begin{array}{l}\text { USER TEST } \\
\text { PANEL }\end{array}$ & $\begin{array}{l}\text { SAMPLE } \\
\text { PURPOSE }\end{array}$ & CAS \# & PARAMETER & RESULT & UNITS & Q & $\begin{array}{l}\text { DETECT } \\
\text { LIMIT }\end{array}$ \\
\hline 383PB0104.5 & $6 / 10 / 2004$ & soil & EPA6010 & REG & $7440-47-3$ & Chromium & 2.7 & MG/KG & & 1.1 \\
\hline 383PB0104.5 & $6 / 10 / 2004$ & soil & HASL300 & REG & 13966-00-2 & $\mathrm{K}-40$ & 26.2 & $\mathrm{PCl} / \mathrm{G}$ & G & 1.2 \\
\hline 383PB0104.5 & $6 / 10 / 2004$ & soil & EPA6010 & REG & $7439-92-1$ & Lead & 5.7 & MG/KG & & 0.64 \\
\hline 383PB0104.5 & $6 / 10 / 2004$ & soil & EPA7470 & REG & $7439-97-6$ & Mercury & 0.022 & MG/KG & B & 0.11 \\
\hline 383PB0104.5 & $6 / 10 / 2004$ & soil & HASL300 & REG & 15092-94-1 & $\mathrm{Pb}-212$ & 1.87 & $\mathrm{PCl} / \mathrm{G}$ & $\mathrm{J}$ & 0.16 \\
\hline 383PB0104.5 & $6 / 10 / 2004$ & soil & HASL300 & REG & 15067-28-4 & $\mathrm{Pb}-214$ & 1.08 & $\mathrm{PCl} / \mathrm{G}$ & $\mathrm{G}, \mathrm{J}$ & 0.22 \\
\hline 383PB0104.5 & $6 / 10 / 2004$ & soil & HASL300 & REG & 14913-50-9 & TI-208 & 0.59 & $\mathrm{PCl} / \mathrm{G}$ & G & 0.11 \\
\hline 383PB0200.5 & $6 / 17 / 2004$ & soil & HASL300 & REG & 14331-83-0 & Ac-228 & 1.84 & $\mathrm{PCl} / \mathrm{G}$ & G & 0.54 \\
\hline 383PB0200.5 & $6 / 17 / 2004$ & soil & EPA6010 & REG & $7440-38-2$ & Arsenic & 3 & MG/KG & & 1.1 \\
\hline 383PB0200.5 & $6 / 17 / 2004$ & soil & EPA6010 & REG & 7440-39-3 & Barium & 5200 & MG/KG & & 550 \\
\hline 383PB0200.5 & $6 / 17 / 2004$ & soil & EPA6010 & REG & $7440-41-7$ & Beryllium & 1.3 & MG/KG & & 0.55 \\
\hline 383PB0200.5 & $6 / 17 / 2004$ & soil & HASL300 & REG & 14733-03-0 & Bi-214 & 1.58 & $\mathrm{PCl} / \mathrm{G}$ & $\mathrm{G}, \mathrm{J}$ & 0.66 \\
\hline 383PB0200.5 & $6 / 17 / 2004$ & soil & EPA6010 & REG & $7440-43-9$ & Cadmium & 0.16 & MG/KG & B & 0.55 \\
\hline 383PB0200.5 & $6 / 17 / 2004$ & soil & EPA6010 & REG & $7440-47-3$ & Chromium & 4.9 & MG/KG & & 1.1 \\
\hline 383РB0200.5 & $6 / 17 / 2004$ & soil & HASL300 & REG & $10045-97-3$ & Cs-137 & 91 & $\mathrm{PCl} / \mathrm{G}$ & G & 0 \\
\hline 383PB0200.5 & $6 / 17 / 2004$ & soil & EPA8015 & REG & 68334-30-5 & Diesel Range Organics & 5.8 & MG/KG & $\mathrm{Z}, \mathrm{H}$ & 5.5 \\
\hline 383PB0200.5 & $6 / 17 / 2004$ & soil & HASL300 & REG & 13966-00-2 & $\mathrm{K}-40$ & 22 & $\mathrm{PCl} / \mathrm{G}$ & G & 1.6 \\
\hline 383PB0200.5 & $6 / 17 / 2004$ & soil & EPA6010 & REG & $7439-92-1$ & Lead & 21 & MG/KG & & 0.33 \\
\hline 383PB0200.5 & $6 / 17 / 2004$ & soil & EPA7470 & REG & 7439-97-6 & Mercury & 0.047 & MG/KG & B & 0.11 \\
\hline 383PB0200.5 & $6 / 17 / 2004$ & soil & EPA8260 & REG & $75-09-2$ & Methylene Chloride & 0.97 & UG/KG & J,B & 5.5 \\
\hline 383PB0200.5 & $6 / 17 / 2004$ & soil & HASL300 & REG & 15092-94-1 & $\mathrm{Pb}-212$ & 2.18 & $\mathrm{PCl} / \mathrm{G}$ & G & 0.5 \\
\hline 383PB0200.5 & $6 / 17 / 2004$ & soil & HASL300 & REG & $15067-28-4$ & $\mathrm{~Pb}-214$ & 1.5 & $\mathrm{PCl} / \mathrm{G}$ & $\mathrm{G}, \mathrm{J}$ & 0.89 \\
\hline 383PB0200.5 & $6 / 17 / 2004$ & soil & UGTAISOPU & REG & 13981-16-3 & Pu-238 & 0.271 & $\mathrm{PCl} / \mathrm{G}$ & & 0.021 \\
\hline 383РB0200.5 & $6 / 17 / 2004$ & soil & UGTAISOPU & REG & 15117-48-3 & Pu-239 & 2.65 & $\mathrm{PCl} / \mathrm{G}$ & & 0.02 \\
\hline 383PB0200.5 & $6 / 17 / 2004$ & soil & SR7500 & REG & 10098-97-2 & Sr-90 & 1.38 & $\mathrm{PCl} / \mathrm{G}$ & Y1 & 0.24 \\
\hline 383PB0200.5 & $6 / 17 / 2004$ & soil & HASL300 & REG & 14913-50-9 & TI-208 & 0.57 & $\mathrm{PCl} / \mathrm{G}$ & G & 0.3 \\
\hline 383PB0300.5 & $6 / 17 / 2004$ & soil & HASL300 & REG & 14331-83-0 & Ac-228 & 1.98 & $\mathrm{PCl} / \mathrm{G}$ & G & 0.85 \\
\hline 383РВ0300.5 & $6 / 17 / 2004$ & soil & HASL300 & REG & 14596-10-2 & Am-241 & 0.72 & $\mathrm{PCl} / \mathrm{G}$ & G & 0.5 \\
\hline 383РВ0300.5 & $6 / 17 / 2004$ & soil & EPA6010 & REG & $7440-38-2$ & Arsenic & 3.6 & MG/KG & & 1.2 \\
\hline 383PB0300.5 & $6 / 17 / 2004$ & soil & EPA6010 & REG & 7440-39-3 & Barium & 6300 & MG/KG & & 590 \\
\hline 383РВ0300.5 & $6 / 17 / 2004$ & soil & EPA8270 & REG & $56-55-3$ & Benzo(A)Anthracene & 21 & UG/KG & $\mathrm{J}$ & 390 \\
\hline 383PB0300.5 & $6 / 17 / 2004$ & soil & EPA8270 & REG & $50-32-8$ & Benzo(A)Pyrene & 18 & UG/KG & $\mathrm{J}$ & 390 \\
\hline 383PB0300.5 & $6 / 17 / 2004$ & soil & EPA8270 & REG & $205-99-2$ & Benzo(B)Fluoranthene & 31 & UG/KG & $\mathrm{J}$ & 390 \\
\hline 383РB0300.5 & $6 / 17 / 2004$ & soil & EPA6010 & REG & $7440-41-7$ & Beryllium & 1.6 & MG/KG & & 0.59 \\
\hline 383РB0300.5 & $6 / 17 / 2004$ & soil & HASL300 & REG & 14733-03-0 & $\mathrm{Bi}-214$ & 1.1 & $\mathrm{PCl} / \mathrm{G}$ & $\mathrm{G}, \mathrm{J}$ & 0.72 \\
\hline
\end{tabular}




\begin{tabular}{|c|c|c|c|c|c|c|c|c|c|c|}
\hline SAMPLE \# & $\begin{array}{l}\text { SAMPLE } \\
\text { DATE }\end{array}$ & MATRIX & $\begin{array}{l}\text { USER TEST } \\
\text { PANEL }\end{array}$ & $\begin{array}{l}\text { SAMPLE } \\
\text { PURPOSE }\end{array}$ & CAS \# & PARAMETER & RESULT & UNITS & Q & $\begin{array}{l}\text { DETECT } \\
\text { LIMIT }\end{array}$ \\
\hline 383PB0300.5 & $6 / 17 / 2004$ & soil & EPA8270 & REG & 117-81-7 & Bis(2-Ethylhexyl)Phthalate & 85 & UG/KG & $\mathrm{J}$ & 390 \\
\hline 383PB0300.5 & $6 / 17 / 2004$ & soil & EPA6010 & REG & $7440-43-9$ & Cadmium & 0.34 & MG/KG & B & 0.59 \\
\hline 383PB0300.5 & $6 / 17 / 2004$ & soil & EPA6010 & REG & $7440-47-3$ & Chromium & 8.7 & MG/KG & & 1.2 \\
\hline 383PB0300.5 & $6 / 17 / 2004$ & soil & EPA8270 & REG & $218-01-9$ & Chrysene & 30 & UG/KG & $\mathrm{J}$ & 390 \\
\hline 383PB0300.5 & $6 / 17 / 2004$ & soil & HASL300 & REG & $10045-97-3$ & Cs-137 & 56.3 & $\mathrm{PCl} / \mathrm{G}$ & G & 0.3 \\
\hline 383PB0300.5 & $6 / 17 / 2004$ & soil & EPA8015 & REG & $68334-30-5$ & Diesel Range Organics & 17 & MG/KG & $\mathrm{Z}, \mathrm{H}$ & 5.9 \\
\hline 383PB0300.5 & $6 / 17 / 2004$ & soil & EPA8270 & REG & $206-44-0$ & Fluoranthene & 25 & UG/KG & $\mathrm{J}$ & 390 \\
\hline 383PB0300.5 & $6 / 17 / 2004$ & soil & HASL300 & REG & $13966-00-2$ & $\mathrm{~K}-40$ & 23.3 & $\mathrm{PCl} / \mathrm{G}$ & G & 2.6 \\
\hline 383PB0300.5 & $6 / 17 / 2004$ & soil & EPA6010 & REG & $7439-92-1$ & Lead & 38 & MG/KG & & 0.71 \\
\hline 383PB0300.5 & $6 / 17 / 2004$ & soil & EPA7470 & REG & $7439-97-6$ & Mercury & 0.048 & MG/KG & B & 0.12 \\
\hline 383PB0300.5 & $6 / 17 / 2004$ & soil & HASL300 & REG & 15092-94-1 & $\mathrm{Pb}-212$ & 1.4 & $\mathrm{PCl} / \mathrm{G}$ & G & 0.58 \\
\hline 383PB0300.5 & $6 / 17 / 2004$ & soil & HASL300 & REG & 15067-28-4 & $\mathrm{Pb}-214$ & 1.11 & $\mathrm{PCl} / \mathrm{G}$ & $\mathrm{G}, \mathrm{J}$ & 0.92 \\
\hline 383PB0300.5 & $6 / 17 / 2004$ & soil & UGTAISOPU & REG & 13981-16-3 & Pu-238 & 0.348 & $\mathrm{PCl} / \mathrm{G}$ & & 0.017 \\
\hline 383PB0300.5 & $6 / 17 / 2004$ & soil & UGTAISOPU & REG & 15117-48-3 & Pu-239 & 4.49 & $\mathrm{PCl} / \mathrm{G}$ & & 0.02 \\
\hline 383PB0300.5 & $6 / 17 / 2004$ & soil & EPA8270 & REG & $129-00-0$ & Pyrene & 50 & UG/KG & $\mathrm{J}$ & 390 \\
\hline 383PB0300.5 & $6 / 17 / 2004$ & soil & EPA6010 & REG & $7782-49-2$ & Selenium & 0.53 & MG/KG & B & 0.59 \\
\hline 383PB0300.5 & $6 / 17 / 2004$ & soil & SR7500 & REG & 10098-97-2 & Sr-90 & 3.58 & $\mathrm{PCl} / \mathrm{G}$ & & 0.24 \\
\hline 383PB0300.5 & $6 / 17 / 2004$ & soil & HASL300 & REG & 14913-50-9 & TI-208 & 0.63 & $\mathrm{PCl} / \mathrm{G}$ & G & 0.38 \\
\hline 383PB0300.5X & $6 / 17 / 2004$ & soil & HASL300 & FD & 14331-83-0 & Ac-228 & 1.88 & $\mathrm{PCl} / \mathrm{G}$ & G & 0.74 \\
\hline 383PB0300.5X & $6 / 17 / 2004$ & soil & EPA6010 & FD & $7440-38-2$ & Arsenic & 3.8 & MG/KG & & 1.2 \\
\hline 383PB0300.5X & $6 / 17 / 2004$ & soil & EPA6010 & FD & $7440-39-3$ & Barium & 6500 & MG/KG & & 580 \\
\hline 383PB0300.5X & $6 / 17 / 2004$ & soil & EPA8270 & FD & $56-55-3$ & Benzo(A)Anthracene & 21 & UG/KG & $\mathrm{J}$ & 380 \\
\hline 383PB0300.5X & $6 / 17 / 2004$ & soil & EPA8270 & FD & $50-32-8$ & Benzo(A)Pyrene & 19 & UG/KG & $\mathrm{J}$ & 380 \\
\hline 383PB0300.5X & $6 / 17 / 2004$ & soil & EPA8270 & FD & $205-99-2$ & Benzo(B)Fluoranthene & 26 & UG/KG & $\mathrm{J}$ & 380 \\
\hline 383PB0300.5X & $6 / 17 / 2004$ & soil & EPA6010 & FD & $7440-41-7$ & Beryllium & 1.5 & MG/KG & & 0.58 \\
\hline 383PB0300.5X & $6 / 17 / 2004$ & soil & HASL300 & FD & 14733-03-0 & $\mathrm{Bi}-214$ & 1.2 & $\mathrm{PCl} / \mathrm{G}$ & $\mathrm{G}, \mathrm{J}$ & 0.59 \\
\hline 383PB0300.5X & $6 / 17 / 2004$ & soil & EPA8270 & FD & $117-81-7$ & Bis(2-Ethylhexyl)Phthalate & 90 & UG/KG & $\mathrm{J}$ & 380 \\
\hline 383PB0300.5X & $6 / 17 / 2004$ & soil & EPA6010 & FD & $7440-43-9$ & Cadmium & 0.35 & MG/KG & B & 0.58 \\
\hline 383PB0300.5X & $6 / 17 / 2004$ & soil & EPA6010 & FD & $7440-47-3$ & Chromium & 8.5 & MG/KG & & 1.2 \\
\hline 383PB0300.5X & $6 / 17 / 2004$ & soil & EPA8270 & FD & $218-01-9$ & Chrysene & 29 & UG/KG & $\mathrm{J}$ & 380 \\
\hline 383PB0300.5X & $6 / 17 / 2004$ & soil & HASL300 & FD & $10045-97-3$ & Cs-137 & 60.9 & $\mathrm{PCl} / \mathrm{G}$ & G & 0.3 \\
\hline 383PB0300.5X & $6 / 17 / 2004$ & soil & EPA8015 & FD & $68334-30-5$ & Diesel Range Organics & 22 & MG/KG & $\mathrm{Z}, \mathrm{H}$ & 5.8 \\
\hline 383PB0300.5X & $6 / 17 / 2004$ & soil & EPA8270 & FD & $84-66-2$ & Diethyl Phthalate & 89 & UG/KG & $\mathrm{J}$ & 380 \\
\hline 383PB0300.5X & $6 / 17 / 2004$ & soil & EPA8270 & FD & $206-44-0$ & Fluoranthene & 23 & UG/KG & $\mathrm{J}$ & 380 \\
\hline 383PB0300.5X & $6 / 17 / 2004$ & soil & HASL300 & FD & 13966-00-2 & $\mathrm{K}-40$ & 22.8 & $\mathrm{PCl} / \mathrm{G}$ & G & 2.3 \\
\hline
\end{tabular}




\begin{tabular}{|c|c|c|c|c|c|c|c|c|c|c|}
\hline SAMPLE \# & $\begin{array}{l}\text { SAMPLE } \\
\text { DATE }\end{array}$ & MATRIX & $\begin{array}{l}\text { USER TEST } \\
\text { PANEL }\end{array}$ & $\begin{array}{l}\text { SAMPLE } \\
\text { PURPOSE }\end{array}$ & CAS \# & PARAMETER & RESULT & UNITS & Q & $\begin{array}{l}\text { DETECT } \\
\text { LIMIT }\end{array}$ \\
\hline 383PB0300.5X & $6 / 17 / 2004$ & soil & EPA6010 & FD & $7439-92-1$ & Lead & 38 & MG/KG & & 0.7 \\
\hline 383PB0300.5X & $6 / 17 / 2004$ & soil & EPA7470 & FD & 7439-97-6 & Mercury & 0.045 & MG/KG & $B$ & 0.12 \\
\hline 383PB0300.5X & $6 / 17 / 2004$ & soil & HASL300 & FD & 15092-94-1 & $\mathrm{Pb}-212$ & 2.03 & $\mathrm{PCl} / \mathrm{G}$ & $\mathrm{G}$ & 0.53 \\
\hline 383PB0300.5X & $6 / 17 / 2004$ & soil & HASL300 & FD & 15067-28-4 & $\mathrm{Pb}-214$ & 1.21 & $\mathrm{PCl} / \mathrm{G}$ & $\mathrm{G}, \mathrm{J}$ & 0.68 \\
\hline 383PB0300.5X & $6 / 17 / 2004$ & soil & EPA8270 & FD & $85-01-8$ & Phenanthrene & 18 & UG/KG & $\mathrm{J}$ & 380 \\
\hline 383PB0300.5X & $6 / 17 / 2004$ & soil & UGTAISOPU & FD & 13981-16-3 & Pu-238 & 0.361 & $\mathrm{PCl} / \mathrm{G}$ & & 0.017 \\
\hline 383PB0300.5X & $6 / 17 / 2004$ & soil & UGTAISOPU & FD & 15117-48-3 & Pu-239 & 4.48 & $\mathrm{PCl} / \mathrm{G}$ & & 0.01 \\
\hline 383PB0300.5X & $6 / 17 / 2004$ & soil & EPA8270 & FD & $129-00-0$ & Pyrene & 51 & UG/KG & $\mathrm{J}$ & 380 \\
\hline 383PB0300.5X & $6 / 17 / 2004$ & soil & SR7500 & FD & 10098-97-2 & Sr-90 & 3.62 & $\mathrm{PCl} / \mathrm{G}$ & Y1 & 0.23 \\
\hline 383PB0300.5X & $6 / 17 / 2004$ & soil & HASL300 & FD & 14913-50-9 & TI-208 & 0.6 & $\mathrm{PCl} / \mathrm{G}$ & G & 0.36 \\
\hline 383PB0400.5 & $6 / 16 / 2004$ & soil & HASL300 & REG & 14331-83-0 & Ac-228 & 1.48 & $\mathrm{PCl} / \mathrm{G}$ & G & 0.67 \\
\hline 383PB0400.5 & $6 / 16 / 2004$ & soil & EPA6010 & REG & 7440-38-2 & Arsenic & 2.5 & MG/KG & & 1 \\
\hline 383PB0400.5 & $6 / 16 / 2004$ & soil & EPA6010 & REG & 7440-39-3 & Barium & 4900 & MG/KG & & 520 \\
\hline 383PB0400.5 & $6 / 16 / 2004$ & soil & EPA6010 & REG & $7440-41-7$ & Beryllium & 1.6 & MG/KG & & 0.52 \\
\hline 383PB0400.5 & $6 / 16 / 2004$ & soil & HASL300 & REG & 14733-03-0 & $\mathrm{Bi}-214$ & 0.95 & $\mathrm{PCl} / \mathrm{G}$ & $\mathrm{G}, \mathrm{J}$ & 0.45 \\
\hline 383PB0400.5 & $6 / 16 / 2004$ & soil & EPA6010 & REG & $7440-43-9$ & Cadmium & 0.19 & MG/KG & $B$ & 0.52 \\
\hline 383PB0400.5 & $6 / 16 / 2004$ & soil & EPA6010 & REG & $7440-47-3$ & Chromium & 4 & MG/KG & & 1 \\
\hline 383PB0400.5 & $6 / 16 / 2004$ & soil & HASL300 & REG & 10045-97-3 & Cs-137 & 46.9 & $\mathrm{PCl} / \mathrm{G}$ & G & 0.2 \\
\hline 383PB0400.5 & $6 / 16 / 2004$ & soil & EPA8260 & REG & $100-41-4$ & Ethylbenzene & 1.6 & UG/KG & $\mathrm{J}$ & 5.2 \\
\hline 383PB0400.5 & $6 / 16 / 2004$ & soil & EPA8270 & REG & $206-44-0$ & Fluoranthene & 20 & UG/KG & $\mathrm{J}$ & 350 \\
\hline 383PB0400.5 & $6 / 16 / 2004$ & soil & HASL300 & REG & 13966-00-2 & $\mathrm{K}-40$ & 31.7 & $\mathrm{PCl} / \mathrm{G}$ & G & 1.7 \\
\hline 383PB0400.5 & $6 / 16 / 2004$ & soil & EPA6010 & REG & $7439-92-1$ & Lead & 30 & MG/KG & & 0.31 \\
\hline 383PB0400.5 & $6 / 16 / 2004$ & soil & EPA8260 & REG & $136777-61-2$ & M+P-Xylene & 8.3 & UG/KG & & 5.2 \\
\hline 383PB0400.5 & $6 / 16 / 2004$ & soil & EPA7470 & REG & 7439-97-6 & Mercury & 0.027 & MG/KG & $B$ & 0.1 \\
\hline 383PB0400.5 & $6 / 16 / 2004$ & soil & EPA8260 & REG & $75-09-2$ & Methylene Chloride & 1.2 & UG/KG & J,B & 5.2 \\
\hline 383PB0400.5 & $6 / 16 / 2004$ & soil & EPA8260 & REG & $95-47-6$ & O-Xylene & 3.3 & UG/KG & $\mathrm{J}$ & 5.2 \\
\hline 383PB0400.5 & $6 / 16 / 2004$ & soil & HASL300 & REG & 15092-94-1 & $\mathrm{Pb}-212$ & 2.04 & $\mathrm{PCl} / \mathrm{G}$ & $\mathrm{J}$ & 0.48 \\
\hline 383PB0400.5 & $6 / 16 / 2004$ & soil & HASL300 & REG & 15067-28-4 & $\mathrm{Pb}-214$ & 1.53 & $\mathrm{PCl} / \mathrm{G}$ & $\mathrm{G}, \mathrm{J}$ & 0.73 \\
\hline 383PB0400.5 & $6 / 16 / 2004$ & soil & UGTAISOPU & REG & 13981-16-3 & Pu-238 & 0.42 & $\mathrm{PCl} / \mathrm{G}$ & & 0.02 \\
\hline 383PB0400.5 & $6 / 16 / 2004$ & soil & UGTAISOPU & REG & 15117-48-3 & Pu-239 & 3.25 & $\mathrm{PCl} / \mathrm{G}$ & & 0.01 \\
\hline 383PB0400.5 & $6 / 16 / 2004$ & soil & SR7500 & REG & 10098-97-2 & Sr-90 & 0.76 & $\mathrm{PCl} / \mathrm{G}$ & & 0.29 \\
\hline 383PB0400.5 & $6 / 16 / 2004$ & soil & HASL300 & REG & $14913-50-9$ & TI-208 & 0.51 & $\mathrm{PCl} / \mathrm{G}$ & G & 0.23 \\
\hline 383PB0500.5 & $6 / 16 / 2004$ & soil & HASL300 & REG & 14331-83-0 & Ac-228 & 1.79 & $\mathrm{PCl} / \mathrm{G}$ & G & 0.46 \\
\hline 383PB0500.5 & $6 / 16 / 2004$ & soil & EPA6010 & REG & $7440-38-2$ & Arsenic & 2.1 & MG/KG & & 1 \\
\hline 383PB0500.5 & $6 / 16 / 2004$ & soil & EPA6010 & REG & $7440-39-3$ & Barium & 550 & MG/KG & & 10 \\
\hline
\end{tabular}




\begin{tabular}{|c|c|c|c|c|c|c|c|c|c|c|}
\hline SAMPLE \# & $\begin{array}{l}\text { SAMPLE } \\
\text { DATE }\end{array}$ & MATRIX & $\begin{array}{l}\text { USER TEST } \\
\text { PANEL }\end{array}$ & $\begin{array}{l}\text { SAMPLE } \\
\text { PURPOSE }\end{array}$ & CAS \# & PARAMETER & RESULT & UNITS & Q & $\begin{array}{l}\text { DETECT } \\
\text { LIMIT }\end{array}$ \\
\hline 383PB0500.5 & $6 / 16 / 2004$ & soil & EPA6010 & REG & $7440-41-7$ & Beryllium & 0.81 & MG/KG & & 0.51 \\
\hline 383PB0500.5 & $6 / 16 / 2004$ & soil & HASL300 & REG & $14733-03-0$ & $\mathrm{Bi}-214$ & 1.06 & $\mathrm{PCl} / \mathrm{G}$ & $\mathrm{G}, \mathrm{J}$ & 0.26 \\
\hline 383PB0500.5 & $6 / 16 / 2004$ & soil & EPA6010 & REG & $7440-43-9$ & Cadmium & 0.052 & MG/KG & B & 0.51 \\
\hline 383PB0500.5 & $6 / 16 / 2004$ & soil & EPA6010 & REG & $7440-47-3$ & Chromium & 2.5 & MG/KG & & 1 \\
\hline 383PB0500.5 & $6 / 16 / 2004$ & soil & HASL300 & REG & $10045-97-3$ & Cs-137 & 0.86 & $\mathrm{PCl} / \mathrm{G}$ & G & 0.13 \\
\hline 383PB0500.5 & $6 / 16 / 2004$ & soil & EPA8015 & REG & $68334-30-5$ & Diesel Range Organics & 100 & MG/KG & $\mathrm{Z}, \mathrm{H}$ & 5.1 \\
\hline 383PB0500.5 & $6 / 16 / 2004$ & soil & HASL300 & REG & 13966-00-2 & $\mathrm{K}-40$ & 30.5 & $\mathrm{PCl} / \mathrm{G}$ & G & 1.4 \\
\hline 383PB0500.5 & $6 / 16 / 2004$ & soil & EPA6010 & REG & $7439-92-1$ & Lead & 15 & MG/KG & & 0.31 \\
\hline 383PB0500.5 & $6 / 16 / 2004$ & soil & EPA7470 & REG & $7439-97-6$ & Mercury & 0.013 & MG/KG & B & 0.1 \\
\hline 383PB0500.5 & $6 / 16 / 2004$ & soil & EPA8260 & REG & $75-09-2$ & Methylene Chloride & 0.95 & UG/KG & $\mathrm{J}, \mathrm{B}$ & 5.1 \\
\hline 383PB0500.5 & $6 / 16 / 2004$ & soil & HASL300 & REG & 15092-94-1 & $\mathrm{Pb}-212$ & 2.21 & $\mathrm{PCl} / \mathrm{G}$ & $\mathrm{J}$ & 0.2 \\
\hline 383PB0500.5 & $6 / 16 / 2004$ & soil & HASL300 & REG & $15067-28-4$ & $\mathrm{~Pb}-214$ & 1.09 & $\mathrm{PCl} / \mathrm{G}$ & $\mathrm{G}, \mathrm{J}$ & 0.23 \\
\hline 383PB0500.5 & $6 / 16 / 2004$ & soil & UGTAISOPU & REG & $15117-48-3$ & Pu-239 & 0.318 & $\mathrm{PCl} / \mathrm{G}$ & & 0.011 \\
\hline 383PB0500.5 & $6 / 16 / 2004$ & soil & EPA6010 & REG & $7782-49-2$ & Selenium & 0.65 & MG/KG & & 0.51 \\
\hline 383PB0500.5 & $6 / 16 / 2004$ & soil & HASL300 & REG & $14913-50-9$ & TI-208 & 0.65 & $\mathrm{PCl} / \mathrm{G}$ & G & 0.12 \\
\hline 383РH0101.0 & $6 / 9 / 2004$ & soil & EPA8260 & REG & 67-64-1 & Acetone & 14 & UG/KG & $\mathrm{J}$ & 38 \\
\hline 383РН0101.0 & $6 / 9 / 2004$ & soil & EPA6010 & REG & $7440-38-2$ & Arsenic & 14 & MG/KG & & 1.9 \\
\hline 383РН0101.0 & 6/9/2004 & soil & EPA6010 & REG & $7440-39-3$ & Barium & 6300 & MG/KG & & 95 \\
\hline 383РH0101.0 & $6 / 9 / 2004$ & soil & EPA6010 & REG & $7440-41-7$ & Beryllium & 1.1 & MG/KG & & 0.95 \\
\hline 383РН0101.0 & $6 / 9 / 2004$ & soil & HASL300 & REG & 14733-03-0 & Bi-214 & 1 & $\mathrm{PCl} / \mathrm{G}$ & $\mathrm{G}, \mathrm{J}$ & 0.56 \\
\hline 383РН0101.0 & 6/9/2004 & soil & EPA6010 & REG & $7440-43-9$ & Cadmium & 0.39 & MG/KG & B & 0.95 \\
\hline 383РH0101.0 & $6 / 9 / 2004$ & soil & EPA6010 & REG & $7440-47-3$ & Chromium & 12 & MG/KG & & 1.9 \\
\hline 383РН0101.0 & $6 / 9 / 2004$ & soil & HASL300 & REG & $10045-97-3$ & Cs-137 & 19 & $\mathrm{PCl} / \mathrm{G}$ & G & 0.3 \\
\hline 383РН0101.0 & 6/9/2004 & soil & EPA8015 & REG & $68334-30-5$ & Diesel Range Organics & 21 & MG/KG & $\mathrm{Z}, \mathrm{H}$ & 9.5 \\
\hline 383РН0101.0 & $6 / 9 / 2004$ & soil & HASL300 & REG & 13966-00-2 & $\mathrm{K}-40$ & 8.1 & $\mathrm{PCl} / \mathrm{G}$ & G & 3.2 \\
\hline 383РH0101.0 & $6 / 9 / 2004$ & soil & EPA6010 & REG & $7439-92-1$ & Lead & 19 & MG/KG & & 2.8 \\
\hline 383РН0101.0 & $6 / 9 / 2004$ & soil & EPA7470 & REG & $7439-97-6$ & Mercury & 0.046 & MG/KG & B & 0.19 \\
\hline 383РH0101.0 & $6 / 9 / 2004$ & soil & HASL300 & REG & 15092-94-1 & $\mathrm{Pb}-212$ & 1.29 & $\mathrm{PCl} / \mathrm{G}$ & $\mathrm{J}$ & 0.49 \\
\hline 383РН0101.0 & 6/9/2004 & soil & HASL300 & REG & $15067-28-4$ & $\mathrm{~Pb}-214$ & 1.25 & $\mathrm{PCl} / \mathrm{G}$ & $\mathrm{G}, \mathrm{J}$ & 0.53 \\
\hline 383РН0101.0 & $6 / 9 / 2004$ & soil & UGTAISOPU & REG & $15117-48-3$ & Pu-239 & 0.305 & $\mathrm{PCl} / \mathrm{G}$ & $\mathrm{J}$ & 0.01 \\
\hline 383РН0101.0 & 6/9/2004 & soil & SR7500 & REG & 10098-97-2 & Sr-90 & 1.33 & $\mathrm{PCl} / \mathrm{G}$ & & 0.23 \\
\hline 383РН0101.0 & $6 / 9 / 2004$ & soil & HASL300 & REG & 14913-50-9 & TI-208 & 0.61 & $\mathrm{PCl} / \mathrm{G}$ & G & 0.27 \\
\hline 383РН0116.5 & 6/9/2004 & soil & HASL300 & REG & 14331-83-0 & Ac-228 & 2.4 & $\mathrm{PCl} / \mathrm{G}$ & G & 0.49 \\
\hline 383РН0116.5 & 6/9/2004 & soil & EPA8260 & REG & 67-64-1 & Acetone & 7.2 & UG/KG & $\mathrm{J}$ & 21 \\
\hline 383РН0116.5 & 6/9/2004 & soil & EPA6010 & REG & 7440-38-2 & Arsenic & 2 & MG/KG & & 1 \\
\hline
\end{tabular}




\begin{tabular}{|c|c|c|c|c|c|c|c|c|c|c|}
\hline SAMPLE \# & $\begin{array}{l}\text { SAMPLE } \\
\text { DATE }\end{array}$ & MATRIX & $\begin{array}{l}\text { USER TEST } \\
\text { PANEL }\end{array}$ & $\begin{array}{l}\text { SAMPLE } \\
\text { PURPOSE }\end{array}$ & CAS \# & PARAMETER & RESULT & UNITS & Q & $\begin{array}{l}\text { DETECT } \\
\text { LIMIT }\end{array}$ \\
\hline 383РH0116.5 & $6 / 9 / 2004$ & soil & EPA6010 & REG & $7440-39-3$ & Barium & 71 & MG/KG & & 10 \\
\hline 383РН0116.5 & $6 / 9 / 2004$ & soil & EPA6010 & REG & $7440-41-7$ & Beryllium & 0.65 & MG/KG & & 0.52 \\
\hline 383РH0116.5 & $6 / 9 / 2004$ & soil & HASL300 & REG & 14733-03-0 & Bi-214 & 1.46 & $\mathrm{PCl} / \mathrm{G}$ & $\mathrm{G}, \mathrm{J}$ & 0.24 \\
\hline 383РH0116.5 & $6 / 9 / 2004$ & soil & EPA6010 & REG & $7440-43-9$ & Cadmium & 0.11 & MG/KG & B & 0.52 \\
\hline 383РН0116.5 & $6 / 9 / 2004$ & soil & EPA6010 & REG & $7440-47-3$ & Chromium & 1.7 & MG/KG & & 1 \\
\hline 383РН0116.5 & $6 / 9 / 2004$ & soil & HASL300 & REG & 13966-00-2 & $\mathrm{K}-40$ & 29.9 & $\mathrm{PCl} / \mathrm{G}$ & G & 1.6 \\
\hline 383РH0116.5 & $6 / 9 / 2004$ & soil & EPA6010 & REG & $7439-92-1$ & Lead & 7.2 & MG/KG & & 0.31 \\
\hline 383РН0116.5 & $6 / 9 / 2004$ & soil & EPA7470 & REG & 7439-97-6 & Mercury & 0.015 & MG/KG & B & 0.1 \\
\hline 383РH0116.5 & $6 / 9 / 2004$ & soil & HASL300 & REG & 15092-94-1 & $\mathrm{Pb}-212$ & 2.51 & $\mathrm{PCl} / \mathrm{G}$ & $\mathrm{J}$ & 0.21 \\
\hline 383РH0116.5 & $6 / 9 / 2004$ & soil & HASL300 & REG & $15067-28-4$ & $\mathrm{~Pb}-214$ & 1.61 & $\mathrm{PCl} / \mathrm{G}$ & $\mathrm{G}, \mathrm{J}$ & 0.25 \\
\hline 383РH0116.5 & $6 / 9 / 2004$ & soil & HASL300 & REG & 14913-50-9 & TI-208 & 1.05 & $\mathrm{PCl} / \mathrm{G}$ & G & 0.12 \\
\hline 383PH0216.0 & $6 / 9 / 2004$ & soil & HASL300 & REG & 14331-83-0 & Ac-228 & 2.31 & $\mathrm{PCl} / \mathrm{G}$ & G & 0.72 \\
\hline 383PH0216.0 & $6 / 9 / 2004$ & soil & EPA6010 & REG & $7440-38-2$ & Arsenic & 2.3 & MG/KG & & 1.2 \\
\hline 383РH0216.0 & $6 / 9 / 2004$ & soil & EPA6010 & REG & $7440-39-3$ & Barium & 320 & MG/KG & & 12 \\
\hline 383РH0216.0 & $6 / 9 / 2004$ & soil & EPA8270 & REG & $56-55-3$ & Benzo(A)Anthracene & 26 & UG/KG & $\mathrm{J}$ & 390 \\
\hline 383PH0216.0 & $6 / 9 / 2004$ & soil & EPA8270 & REG & $50-32-8$ & Benzo(A)Pyrene & 28 & UG/KG & $\mathrm{J}$ & 390 \\
\hline 383PH0216.0 & $6 / 9 / 2004$ & soil & EPA8270 & REG & $205-99-2$ & Benzo(B)Fluoranthene & 59 & UG/KG & $\mathrm{J}$ & 390 \\
\hline 383PH0216.0 & $6 / 9 / 2004$ & soil & EPA8270 & REG & $207-08-9$ & Benzo(K)Fluoranthene & 31 & UG/KG & $\mathrm{J}$ & 390 \\
\hline 383PH0216.0 & $6 / 9 / 2004$ & soil & EPA6010 & REG & $7440-41-7$ & Beryllium & 1.3 & MG/KG & & 0.58 \\
\hline 383PH0216.0 & $6 / 9 / 2004$ & soil & HASL300 & REG & 14733-03-0 & Bi-214 & 1.16 & $\mathrm{PCl} / \mathrm{G}$ & $\mathrm{G}, \mathrm{J}$ & 0.59 \\
\hline 383PH0216.0 & $6 / 9 / 2004$ & soil & EPA8270 & REG & 117-81-7 & Bis(2-Ethylhexyl)Phthalate & 330 & UG/KG & $\mathrm{J}$ & 390 \\
\hline 383PH0216.0 & $6 / 9 / 2004$ & soil & EPA6010 & REG & $7440-43-9$ & Cadmium & 0.31 & MG/KG & B & 0.58 \\
\hline 383PH0216.0 & $6 / 9 / 2004$ & soil & EPA6010 & REG & $7440-47-3$ & Chromium & 1.4 & MG/KG & & 1.2 \\
\hline 383PH0216.0 & $6 / 9 / 2004$ & soil & EPA8270 & REG & $218-01-9$ & Chrysene & 66 & UG/KG & $\mathrm{J}$ & 390 \\
\hline 383РH0216.0 & $6 / 9 / 2004$ & soil & HASL300 & REG & $10045-97-3$ & Cs-137 & 142 & $\mathrm{PCl} / \mathrm{G}$ & G & 0 \\
\hline 383РH0216.0 & $6 / 9 / 2004$ & soil & EPA8015 & REG & $68334-30-5$ & Diesel Range Organics & 330 & MG/KG & M & 5.8 \\
\hline 383PH0216.0 & $6 / 9 / 2004$ & soil & EPA8270 & REG & $206-44-0$ & Fluoranthene & 31 & UG/KG & $\mathrm{J}$ & 390 \\
\hline 383PH0216.0 & $6 / 9 / 2004$ & soil & HASL300 & REG & 13966-00-2 & $\mathrm{K}-40$ & 33.7 & $\mathrm{PCl} / \mathrm{G}$ & G & 1.9 \\
\hline 383PH0216.0 & 6/9/2004 & soil & EPA6010 & REG & $7439-92-1$ & Lead & 12 & MG/KG & & 0.35 \\
\hline 383РH0216.0 & $6 / 9 / 2004$ & soil & EPA7470 & REG & 7439-97-6 & Mercury & 0.027 & MG/KG & B & 0.12 \\
\hline 383РH0216.0 & $6 / 9 / 2004$ & soil & HASL300 & REG & 15092-94-1 & $\mathrm{Pb}-212$ & 2.73 & $\mathrm{PCl} / \mathrm{G}$ & $\mathrm{J}$ & 0.62 \\
\hline 383РH0216.0 & 6/9/2004 & soil & UGTAISOPU & REG & 13981-16-3 & Pu-238 & 0.74 & $\mathrm{PCl} / \mathrm{G}$ & & 0.02 \\
\hline 383РH0216.0 & $6 / 9 / 2004$ & soil & UGTAISOPU & REG & 15117-48-3 & Pu-239 & 5.37 & $\mathrm{PCl} / \mathrm{G}$ & $\mathrm{J}$ & 0.01 \\
\hline 383РH0216.0 & $6 / 9 / 2004$ & soil & EPA8270 & REG & $129-00-0$ & Pyrene & 63 & UG/KG & $\mathrm{J}$ & 390 \\
\hline 383РH0216.0 & $6 / 9 / 2004$ & soil & EPA6010 & REG & 7782-49-2 & Selenium & 0.42 & MG/KG & $B$ & 0.58 \\
\hline
\end{tabular}




\begin{tabular}{|c|c|c|c|c|c|c|c|c|c|c|}
\hline SAMPLE \# & $\begin{array}{l}\text { SAMPLE } \\
\text { DATE }\end{array}$ & MATRIX & $\begin{array}{l}\text { USER TEST } \\
\text { PANEL }\end{array}$ & $\begin{array}{l}\text { SAMPLE } \\
\text { PURPOSE }\end{array}$ & CAS \# & PARAMETER & RESULT & UNITS & Q & $\begin{array}{l}\text { DETECT } \\
\text { LIMIT }\end{array}$ \\
\hline 383PH0216.0 & $6 / 9 / 2004$ & soil & SR7500 & REG & 10098-97-2 & Sr-90 & 2.84 & $\mathrm{PCl} / \mathrm{G}$ & & 0.25 \\
\hline 383РH0216.0 & $6 / 9 / 2004$ & soil & HASL300 & REG & $14913-50-9$ & TI-208 & 0.78 & $\mathrm{PCl} / \mathrm{G}$ & G & 0.33 \\
\hline 383РH0217.5 & $6 / 9 / 2004$ & soil & HASL300 & REG & 14331-83-0 & Ac-228 & 2.27 & $\mathrm{PCl} / \mathrm{G}$ & G & 0.6 \\
\hline 383PH0217.5 & $6 / 9 / 2004$ & soil & EPA8260 & REG & 67-64-1 & Acetone & 25 & UG/KG & & 21 \\
\hline 383РH0217.5 & $6 / 9 / 2004$ & soil & EPA6010 & REG & $7440-38-2$ & Arsenic & 1.7 & MG/KG & & 1.1 \\
\hline 383РH0217.5 & $6 / 9 / 2004$ & soil & EPA6010 & REG & $7440-39-3$ & Barium & 3200 & MG/KG & & 110 \\
\hline 383PH0217.5 & $6 / 9 / 2004$ & soil & EPA6010 & REG & $7440-41-7$ & Beryllium & 0.69 & MG/KG & & 0.53 \\
\hline 383РH0217.5 & 6/9/2004 & soil & HASL300 & REG & 14733-03-0 & Bi-214 & 1.49 & $\mathrm{PCl} / \mathrm{G}$ & $\mathrm{G}, \mathrm{J}$ & 0.31 \\
\hline 383РH0217.5 & $6 / 9 / 2004$ & soil & EPA6010 & REG & $7440-43-9$ & Cadmium & 0.12 & MG/KG & $\mathrm{B}$ & 0.53 \\
\hline 383РH0217.5 & $6 / 9 / 2004$ & soil & EPA6010 & REG & $7440-47-3$ & Chromium & 3.3 & MG/KG & & 1.1 \\
\hline 383PH0217.5 & $6 / 9 / 2004$ & soil & HASL300 & REG & 10045-97-3 & Cs-137 & 4.34 & $\mathrm{PCl} / \mathrm{G}$ & G & 0.17 \\
\hline 383РH0217.5 & $6 / 9 / 2004$ & soil & HASL300 & REG & 13966-00-2 & $\mathrm{K}-40$ & 33.2 & $\mathrm{PCl} / \mathrm{G}$ & G & 1.4 \\
\hline 383PH0217.5 & $6 / 9 / 2004$ & soil & EPA6010 & REG & $7439-92-1$ & Lead & 12 & MG/KG & & 0.32 \\
\hline 383РH0217.5 & $6 / 9 / 2004$ & soil & EPA7470 & REG & 7439-97-6 & Mercury & 0.011 & MG/KG & B & 0.11 \\
\hline 383РH0217.5 & $6 / 9 / 2004$ & soil & HASL300 & REG & 15092-94-1 & $\mathrm{Pb}-212$ & 2.4 & $\mathrm{PCl} / \mathrm{G}$ & $\mathrm{J}$ & 0.22 \\
\hline 383РH0217.5 & $6 / 9 / 2004$ & soil & HASL300 & REG & $15067-28-4$ & $\mathrm{~Pb}-214$ & 1.42 & $\mathrm{PCl} / \mathrm{G}$ & $\mathrm{G}, \mathrm{J}$ & 0.31 \\
\hline 383РH0217.5 & $6 / 9 / 2004$ & soil & UGTAISOPU & REG & $15117-48-3$ & Pu-239 & 0.154 & $\mathrm{PCl} / \mathrm{G}$ & $\mathrm{J}$ & 0.02 \\
\hline 383РН0217.5 & $6 / 9 / 2004$ & soil & EPA6010 & REG & $7440-22-4$ & Silver & 0.48 & MG/KG & B & 1.1 \\
\hline 383РH0217.5 & $6 / 9 / 2004$ & soil & HASL300 & REG & 14913-50-9 & TI-208 & 0.8 & $\mathrm{PCl} / \mathrm{G}$ & G & 0.16 \\
\hline 383PH0306.5 & $6 / 9 / 2004$ & soil & HASL300 & REG & 14331-83-0 & Ac-228 & 2.18 & $\mathrm{PCl} / \mathrm{G}$ & G & 0.58 \\
\hline 383РН0306.5 & 6/9/2004 & soil & EPA8260 & REG & 67-64-1 & Acetone & 7.2 & UG/KG & $\mathrm{J}$ & 23 \\
\hline 383РH0306.5 & $6 / 9 / 2004$ & soil & EPA6010 & REG & $7440-38-2$ & Arsenic & 2 & MG/KG & & 1.1 \\
\hline 383PH0306.5 & $6 / 9 / 2004$ & soil & EPA6010 & REG & $7440-39-3$ & Barium & 4000 & MG/KG & & 110 \\
\hline 383РH0306.5 & 6/9/2004 & soil & EPA6010 & REG & $7440-41-7$ & Beryllium & 2 & MG/KG & & 0.57 \\
\hline 383РН0306.5 & $6 / 9 / 2004$ & soil & HASL300 & REG & 14733-03-0 & $\mathrm{Bi}-214$ & 1.72 & $\mathrm{PCl} / \mathrm{G}$ & $\mathrm{G}, \mathrm{J}$ & 0.33 \\
\hline 383РH0306.5 & $6 / 9 / 2004$ & soil & EPA6010 & REG & $7440-43-9$ & Cadmium & 0.24 & MG/KG & B & 0.57 \\
\hline 383РН0306.5 & $6 / 9 / 2004$ & soil & EPA6010 & REG & $7440-47-3$ & Chromium & 3.4 & MG/KG & & 1.1 \\
\hline 383PH0306.5 & $6 / 9 / 2004$ & soil & HASL300 & REG & 10045-97-3 & Cs-137 & 1.33 & $\mathrm{PCl} / \mathrm{G}$ & G & 0.14 \\
\hline 383PH0306.5 & 6/9/2004 & soil & EPA8015 & REG & $68334-30-5$ & Diesel Range Organics & 1.9 & MG/KG & $\mathrm{J}$ & 5.7 \\
\hline 383PH0306.5 & $6 / 9 / 2004$ & soil & HASL300 & REG & 13966-00-2 & $\mathrm{K}-40$ & 29 & $\mathrm{PCl} / \mathrm{G}$ & G & 1.2 \\
\hline 383PH0306.5 & 6/9/2004 & soil & EPA6010 & REG & $7439-92-1$ & Lead & 22 & MG/KG & & 0.34 \\
\hline 383PH0306.5 & $6 / 9 / 2004$ & soil & EPA7470 & REG & 7439-97-6 & Mercury & 0.011 & MG/KG & B & 0.11 \\
\hline 383РH0306.5 & $6 / 9 / 2004$ & soil & HASL300 & REG & 15092-94-1 & $\mathrm{Pb}-212$ & 2.56 & $\mathrm{PCl} / \mathrm{G}$ & $\mathrm{J}$ & 0.25 \\
\hline 383PH0306.5 & $6 / 9 / 2004$ & soil & HASL300 & REG & $15067-28-4$ & $\mathrm{~Pb}-214$ & 1.74 & $\mathrm{PCl} / \mathrm{G}$ & $\mathrm{G}, \mathrm{J}$ & 0.3 \\
\hline 383PH0306.5 & 6/9/2004 & soil & EPA6010 & REG & 7782-49-2 & Selenium & 0.31 & MG/KG & $B$ & 0.57 \\
\hline
\end{tabular}




\begin{tabular}{|c|c|c|c|c|c|c|c|c|c|c|}
\hline SAMPLE \# & $\begin{array}{l}\text { SAMPLE } \\
\text { DATE }\end{array}$ & MATRIX & $\begin{array}{l}\text { USER TEST } \\
\text { PANEL }\end{array}$ & $\begin{array}{l}\text { SAMPLE } \\
\text { PURPOSE }\end{array}$ & CAS \# & PARAMETER & RESULT & UNITS & Q & $\begin{array}{l}\text { DETECT } \\
\text { LIMIT }\end{array}$ \\
\hline 383PH0306.5 & $6 / 9 / 2004$ & soil & HASL300 & REG & 14913-50-9 & TI-208 & 0.74 & $\mathrm{PCl} / \mathrm{G}$ & G & 0.12 \\
\hline 383РH0320.5 & $6 / 9 / 2004$ & soil & HASL300 & REG & 14331-83-0 & Ac-228 & 2.3 & $\mathrm{PCl} / \mathrm{G}$ & G & 0.51 \\
\hline 383PH0320.5 & $6 / 9 / 2004$ & soil & EPA8260 & REG & $67-64-1$ & Acetone & 7.9 & UG/KG & $\mathrm{J}$ & 22 \\
\hline 383PH0320.5 & $6 / 9 / 2004$ & soil & EPA6010 & REG & $7440-38-2$ & Arsenic & 1.8 & MG/KG & & 1.1 \\
\hline 383РH0320.5 & $6 / 9 / 2004$ & soil & EPA6010 & REG & $7440-39-3$ & Barium & 260 & MG/KG & & 11 \\
\hline 383PH0320.5 & $6 / 9 / 2004$ & soil & EPA6010 & REG & $7440-41-7$ & Beryllium & 1 & MG/KG & & 0.54 \\
\hline 383PH0320.5 & $6 / 9 / 2004$ & soil & HASL300 & REG & 14733-03-0 & Bi-214 & 1.36 & $\mathrm{PCl} / \mathrm{G}$ & $\mathrm{G}, \mathrm{J}$ & 0.28 \\
\hline 383PH0320.5 & 6/9/2004 & soil & EPA6010 & REG & $7440-43-9$ & Cadmium & 0.092 & MG/KG & B & 0.54 \\
\hline 383PH0320.5 & $6 / 9 / 2004$ & soil & EPA6010 & REG & $7440-47-3$ & Chromium & 3.4 & MG/KG & & 1.1 \\
\hline 383РH0320.5 & $6 / 9 / 2004$ & soil & HASL300 & REG & $10045-97-3$ & Cs-137 & 0.33 & $\mathrm{PCl} / \mathrm{G}$ & LT,G & 0.14 \\
\hline 383РH0320.5 & $6 / 9 / 2004$ & soil & HASL300 & REG & 13966-00-2 & $\mathrm{K}-40$ & 29.8 & $\mathrm{PCl} / \mathrm{G}$ & G & 1.5 \\
\hline 383РH0320.5 & $6 / 9 / 2004$ & soil & EPA6010 & REG & $7439-92-1$ & Lead & 10 & MG/KG & & 0.32 \\
\hline 383РH0320.5 & $6 / 9 / 2004$ & soil & EPA7470 & REG & 7439-97-6 & Mercury & 0.12 & MG/KG & & 0.11 \\
\hline 383РН0320.5 & 6/9/2004 & soil & HASL300 & REG & 15092-94-1 & $\mathrm{Pb}-212$ & 2.76 & $\mathrm{PCl} / \mathrm{G}$ & $\mathrm{J}$ & 0.22 \\
\hline 383PH0320.5 & 6/9/2004 & soil & HASL300 & REG & 15067-28-4 & $\mathrm{Pb}-214$ & 1.6 & $\mathrm{PCl} / \mathrm{G}$ & $\mathrm{G}, \mathrm{J}$ & 0.27 \\
\hline 383РH0320.5 & $6 / 9 / 2004$ & soil & HASL300 & REG & 14913-50-9 & TI-208 & 0.73 & $\mathrm{PCl} / \mathrm{G}$ & G & 0.14 \\
\hline 383РН0408.0 & $6 / 10 / 2004$ & soil & HASL300 & REG & 14331-83-0 & Ac-228 & 2.02 & $\mathrm{PCl} / \mathrm{G}$ & G & 0.55 \\
\hline 383PH0408.0 & $6 / 10 / 2004$ & soil & EPA6010 & REG & $7440-38-2$ & Arsenic & 2.4 & MG/KG & & 1.1 \\
\hline 383РН0408.0 & $6 / 10 / 2004$ & soil & EPA6010 & REG & $7440-39-3$ & Barium & 4900 & MG/KG & & 110 \\
\hline 383PH0408.0 & $6 / 10 / 2004$ & soil & EPA6010 & REG & $7440-41-7$ & Beryllium & 1.9 & MG/KG & & 0.55 \\
\hline 383PH0408.0 & $6 / 10 / 2004$ & soil & HASL300 & REG & $14733-03-0$ & $\mathrm{Bi}-214$ & 1.53 & $\mathrm{PCl} / \mathrm{G}$ & $\mathrm{G}, \mathrm{J}$ & 0.23 \\
\hline 383PH0408.0 & $6 / 10 / 2004$ & soil & EPA6010 & REG & $7440-43-9$ & Cadmium & 0.27 & MG/KG & B & 0.55 \\
\hline 383PH0408.0 & $6 / 10 / 2004$ & soil & EPA6010 & REG & $7440-47-3$ & Chromium & 3.4 & MG/KG & & 1.1 \\
\hline 383PH0408.0 & $6 / 10 / 2004$ & soil & HASL300 & REG & $10045-97-3$ & Cs-137 & 0.78 & $\mathrm{PCl} / \mathrm{G}$ & G & 0.15 \\
\hline 383PH0408.0 & $6 / 10 / 2004$ & soil & EPA8015 & REG & 68334-30-5 & Diesel Range Organics & 7.5 & MG/KG & $\mathrm{Z}, \mathrm{H}$ & 5.5 \\
\hline 383PH0408.0 & $6 / 10 / 2004$ & soil & HASL300 & REG & 13966-00-2 & $\mathrm{K}-40$ & 26.6 & $\mathrm{PCl} / \mathrm{G}$ & G & 1.3 \\
\hline 383PH0408.0 & $6 / 10 / 2004$ & soil & EPA6010 & REG & $7439-92-1$ & Lead & 24 & MG/KG & & 0.33 \\
\hline 383PH0408.0 & $6 / 10 / 2004$ & soil & EPA7470 & REG & 7439-97-6 & Mercury & 0.007 & MG/KG & $B$ & 0.11 \\
\hline 383РН0408.0 & $6 / 10 / 2004$ & soil & HASL300 & REG & 15092-94-1 & $\mathrm{Pb}-212$ & 2.42 & $\mathrm{PCl} / \mathrm{G}$ & $\mathrm{J}$ & 0.23 \\
\hline 383PH0408.0 & $6 / 10 / 2004$ & soil & HASL300 & REG & $15067-28-4$ & $\mathrm{~Pb}-214$ & 1.73 & $\mathrm{PCl} / \mathrm{G}$ & $\mathrm{G}, \mathrm{J}$ & 0.29 \\
\hline 383PH0408.0 & $6 / 10 / 2004$ & soil & HASL300 & REG & 14913-50-9 & TI-208 & 0.8 & $\mathrm{PCl} / \mathrm{G}$ & G & 0.15 \\
\hline 383РH0408.0X & $6 / 10 / 2004$ & soil & HASL300 & FD & 14331-83-0 & Ac-228 & 2.27 & $\mathrm{PCl} / \mathrm{G}$ & G & 0.57 \\
\hline 383РH0408.0X & $6 / 10 / 2004$ & soil & EPA6010 & FD & $7440-38-2$ & Arsenic & 2.3 & MG/KG & & 1.1 \\
\hline 383РН0408.0X & $6 / 10 / 2004$ & soil & EPA6010 & FD & $7440-39-3$ & Barium & 5200 & MG/KG & & 110 \\
\hline 383РH0408.0X & $6 / 10 / 2004$ & soil & EPA6010 & FD & $7440-41-7$ & Beryllium & 2 & MG/KG & & 0.55 \\
\hline
\end{tabular}




\begin{tabular}{|c|c|c|c|c|c|c|c|c|c|c|}
\hline SAMPLE \# & $\begin{array}{l}\text { SAMPLE } \\
\text { DATE }\end{array}$ & MATRIX & $\begin{array}{l}\text { USER TEST } \\
\text { PANEL }\end{array}$ & $\begin{array}{l}\text { SAMPLE } \\
\text { PURPOSE }\end{array}$ & CAS \# & PARAMETER & RESULT & UNITS & $Q$ & $\begin{array}{l}\text { DETECT } \\
\text { LIMIT }\end{array}$ \\
\hline 383РН0408.0X & $6 / 10 / 2004$ & soil & HASL300 & FD & $14733-03-0$ & $\mathrm{Bi}-214$ & 1.62 & $\mathrm{PCl} / \mathrm{G}$ & $\mathrm{G}, \mathrm{J}$ & 0.31 \\
\hline 383PH0408.0X & $6 / 10 / 2004$ & soil & EPA6010 & FD & $7440-43-9$ & Cadmium & 0.27 & MG/KG & B & 0.55 \\
\hline 383РH0408.0X & $6 / 10 / 2004$ & soil & EPA6010 & FD & $7440-47-3$ & Chromium & 3.6 & MG/KG & & 1.1 \\
\hline 383PH0408.0X & $6 / 10 / 2004$ & soil & HASL300 & FD & $10045-97-3$ & Cs-137 & 0.76 & $\mathrm{PCl} / \mathrm{G}$ & G & 0.15 \\
\hline 383PH0408.0X & $6 / 10 / 2004$ & soil & EPA8015 & FD & $68334-30-5$ & Diesel Range Organics & 4.7 & MG/KG & $\mathrm{J}$ & 5.5 \\
\hline 383PH0408.0X & $6 / 10 / 2004$ & soil & HASL300 & FD & $13966-00-2$ & $\mathrm{~K}-40$ & 28.5 & $\mathrm{PCl} / \mathrm{G}$ & $\mathrm{G}$ & 1.5 \\
\hline 383PH0408.0X & $6 / 10 / 2004$ & soil & EPA6010 & FD & $7439-92-1$ & Lead & 23 & MG/KG & & 0.33 \\
\hline 383РH0408.0X & $6 / 10 / 2004$ & soil & EPA7470 & FD & $7439-97-6$ & Mercury & 0.007 & MG/KG & B & 0.11 \\
\hline 383РH0408.0X & $6 / 10 / 2004$ & soil & HASL300 & FD & 15092-94-1 & $\mathrm{Pb}-212$ & 2.54 & $\mathrm{PCl} / \mathrm{G}$ & $\mathrm{J}$ & 0.22 \\
\hline 383РH0408.0X & $6 / 10 / 2004$ & soil & HASL300 & FD & $15067-28-4$ & $\mathrm{~Pb}-214$ & 1.81 & $\mathrm{PCl} / \mathrm{G}$ & $\mathrm{G}, \mathrm{J}$ & 0.28 \\
\hline 383РН0408.0X & $6 / 10 / 2004$ & soil & HASL300 & FD & 14913-50-9 & TI-208 & 0.87 & $\mathrm{PCl} / \mathrm{G}$ & G & 0.14 \\
\hline 383PH0418.5 & $6 / 10 / 2004$ & soil & HASL300 & REG & 14331-83-0 & Ac-228 & 2.19 & $\mathrm{PCl} / \mathrm{G}$ & $\mathrm{G}$ & 0.39 \\
\hline 383PH0418.5 & $6 / 10 / 2004$ & soil & EPA8260 & REG & 67-64-1 & Acetone & 6.7 & UG/KG & $\mathrm{J}$ & 21 \\
\hline 383PH0418.5 & $6 / 10 / 2004$ & soil & EPA6010 & REG & $7440-38-2$ & Arsenic & 2.1 & MG/KG & & 1.1 \\
\hline 383PH0418.5 & $6 / 10 / 2004$ & soil & EPA6010 & REG & $7440-39-3$ & Barium & 590 & MG/KG & & 11 \\
\hline 383PH0418.5 & $6 / 10 / 2004$ & soil & EPA6010 & REG & $7440-41-7$ & Beryllium & 0.74 & MG/KG & & 0.53 \\
\hline 383PH0418.5 & $6 / 10 / 2004$ & soil & HASL300 & REG & $14733-03-0$ & $\mathrm{Bi}-214$ & 1.4 & $\mathrm{PCl} / \mathrm{G}$ & G,J & 0.27 \\
\hline 383PH0418.5 & $6 / 10 / 2004$ & soil & EPA6010 & REG & $7440-43-9$ & Cadmium & 0.12 & MG/KG & $B$ & 0.53 \\
\hline 383PH0418.5 & $6 / 10 / 2004$ & soil & EPA6010 & REG & $7440-47-3$ & Chromium & 1.7 & MG/KG & & 1.1 \\
\hline 383PH0418.5 & $6 / 10 / 2004$ & soil & EPA8260 & REG & $87-68-3$ & Hexachlorobutadiene & 1.7 & UG/KG & $\mathrm{J}$ & 5.3 \\
\hline 383PH0418.5 & $6 / 10 / 2004$ & soil & HASL300 & REG & $13966-00-2$ & $\mathrm{~K}-40$ & 31.7 & $\mathrm{PCl} / \mathrm{G}$ & $\mathrm{G}$ & 1.4 \\
\hline 383PH0418.5 & $6 / 10 / 2004$ & soil & EPA6010 & REG & $7439-92-1$ & Lead & 6.4 & MG/KG & & 0.32 \\
\hline 383PH0418.5 & $6 / 10 / 2004$ & soil & EPA7470 & REG & $7439-97-6$ & Mercury & 0.017 & MG/KG & B & 0.11 \\
\hline 383PH0418.5 & $6 / 10 / 2004$ & soil & HASL300 & REG & 15092-94-1 & $\mathrm{Pb}-212$ & 2.68 & $\mathrm{PCl} / \mathrm{G}$ & $\mathrm{J}$ & 0.22 \\
\hline 383PH0418.5 & $6 / 10 / 2004$ & soil & HASL300 & REG & $15067-28-4$ & $\mathrm{~Pb}-214$ & 1.33 & $\mathrm{PCl} / \mathrm{G}$ & G,J & 0.3 \\
\hline 383PH0418.5 & $6 / 10 / 2004$ & soil & HASL300 & REG & $14913-50-9$ & TI-208 & 0.83 & $\mathrm{PCl} / \mathrm{G}$ & $\mathrm{G}$ & 0.14 \\
\hline 383RB0100.5 & 6/1/2004 & soil & HASL300 & REG & 14331-83-0 & Ac-228 & 2.9 & $\mathrm{PCl} / \mathrm{G}$ & $\mathrm{G}$ & 0.48 \\
\hline 383RB0100.5 & $6 / 1 / 2004$ & soil & EPA6010 & REG & $7440-38-2$ & Arsenic & 6.2 & MG/KG & & 1.1 \\
\hline 383RB0100.5 & $6 / 1 / 2004$ & soil & EPA6010 & REG & $7440-39-3$ & Barium & 140 & MG/KG & & 11 \\
\hline 383RB0100.5 & $6 / 1 / 2004$ & soil & EPA8270 & REG & $56-55-3$ & Benzo(A)Anthracene & 40 & UG/KG & $\mathrm{J}$ & 370 \\
\hline 383RB0100.5 & $6 / 1 / 2004$ & soil & EPA8270 & REG & $50-32-8$ & Benzo(A)Pyrene & 46 & UG/KG & $\mathrm{J}$ & 370 \\
\hline 383RB0100.5 & 6/1/2004 & soil & EPA8270 & REG & $205-99-2$ & Benzo(B)Fluoranthene & 96 & UG/KG & $\mathrm{J}$ & 370 \\
\hline 383RB0100.5 & $6 / 1 / 2004$ & soil & EPA8270 & REG & 207-08-9 & Benzo(K)Fluoranthene & 45 & UG/KG & $\mathrm{J}$ & 370 \\
\hline 383RB0100.5 & $6 / 1 / 2004$ & soil & EPA6010 & REG & $7440-41-7$ & Beryllium & 1.7 & MG/KG & & 0.55 \\
\hline 383RB0100.5 & $6 / 1 / 2004$ & soil & HASL300 & REG & $14913-49-6$ & $\mathrm{Bi}-212$ & 3.2 & $\mathrm{PCl} / \mathrm{G}$ & $\mathrm{G}$ & 1.9 \\
\hline
\end{tabular}




\begin{tabular}{|c|c|c|c|c|c|c|c|c|c|c|}
\hline SAMPLE \# & $\begin{array}{l}\text { SAMPLE } \\
\text { DATE }\end{array}$ & MATRIX & $\begin{array}{l}\text { USER TEST } \\
\text { PANEL }\end{array}$ & $\begin{array}{l}\text { SAMPLE } \\
\text { PURPOSE }\end{array}$ & CAS \# & PARAMETER & RESULT & UNITS & Q & $\begin{array}{l}\text { DETECT } \\
\text { LIMIT }\end{array}$ \\
\hline 383RB0100.5 & $6 / 1 / 2004$ & soil & EPA8270 & REG & 117-81-7 & Bis(2-Ethylhexyl)Phthalate & 260 & UG/KG & $\mathrm{J}$ & 370 \\
\hline 383RB0100.5 & $6 / 1 / 2004$ & soil & EPA8270 & REG & $85-68-7$ & Butyl Benzyl Phthalate & 780 & UG/KG & & 370 \\
\hline 383RB0100.5 & $6 / 1 / 2004$ & soil & EPA6010 & REG & $7440-43-9$ & Cadmium & 0.56 & MG/KG & & 0.55 \\
\hline 383RB0100.5 & $6 / 1 / 2004$ & soil & EPA6010 & REG & $7440-47-3$ & Chromium & 3.2 & MG/KG & & 1.1 \\
\hline 383RB0100.5 & $6 / 1 / 2004$ & soil & EPA8270 & REG & $218-01-9$ & Chrysene & 74 & UG/KG & $\mathrm{J}$ & 370 \\
\hline 383RB0100.5 & $6 / 1 / 2004$ & soil & HASL300 & REG & $10045-97-3$ & Cs-137 & 114 & $\mathrm{PCl} / \mathrm{G}$ & $G$ & 0 \\
\hline 383RB0100.5 & $6 / 1 / 2004$ & soil & EPA8015 & REG & $68334-30-5$ & Diesel Range Organics & 9.6 & MG/KG & Z,H & 5.5 \\
\hline 383RB0100.5 & $6 / 1 / 2004$ & soil & EPA8270 & REG & 84-74-2 & Di-N-Butyl Phthalate & 4500 & UG/KG & $E$ & 370 \\
\hline 383RB0100.5 & $6 / 1 / 2004$ & soil & EPA8270 & REG & $206-44-0$ & Fluoranthene & 91 & UG/KG & $\mathrm{J}$ & 370 \\
\hline 383RB0100.5 & $6 / 1 / 2004$ & soil & HASL300 & REG & 13966-00-2 & $\mathrm{K}-40$ & 27.8 & $\mathrm{PCl} / \mathrm{G}$ & $G$ & 1.4 \\
\hline 383RB0100.5 & $6 / 1 / 2004$ & soil & EPA6010 & REG & 7439-92-1 & Lead & 18 & MG/KG & & 0.33 \\
\hline 383RB0100.5 & $6 / 1 / 2004$ & soil & EPA7470 & REG & $7439-97-6$ & Mercury & 0.16 & MG/KG & & 0.11 \\
\hline 383RB0100.5 & $6 / 1 / 2004$ & soil & HASL300 & REG & 15092-94-1 & $\mathrm{Pb}-212$ & 2.96 & $\mathrm{PCl} / \mathrm{G}$ & $\mathrm{J}$ & 0.52 \\
\hline 383RB0100.5 & $6 / 1 / 2004$ & soil & EPA8270 & REG & $85-01-8$ & Phenanthrene & 42 & UG/KG & $J$ & 370 \\
\hline 383RB0100.5 & $6 / 1 / 2004$ & soil & UGTAISOPU & REG & 15117-48-3 & Pu-239 & 0.166 & $\mathrm{PCl} / \mathrm{G}$ & $\mathrm{J}$ & 0.018 \\
\hline 383RB0100.5 & $6 / 1 / 2004$ & soil & EPA8270 & REG & $129-00-0$ & Pyrene & 93 & UG/KG & $\mathrm{J}$ & 370 \\
\hline 383RB0100.5 & $6 / 1 / 2004$ & soil & HASL300 & REG & 15623-47-9 & Th-227 & 4.1 & $\mathrm{PCl} / \mathrm{G}$ & $\mathrm{J}$ & 2.3 \\
\hline 383RB0100.5 & $6 / 1 / 2004$ & soil & HASL300 & REG & $15065-10-8$ & Th-234 & 7.2 & $\mathrm{PCl} / \mathrm{G}$ & $\mathrm{G}, \mathrm{TI}$ & 4 \\
\hline 383RB0100.5 & $6 / 1 / 2004$ & soil & HASL300 & REG & 14913-50-9 & TI-208 & 0.85 & $\mathrm{PCl} / \mathrm{G}$ & G & 0.25 \\
\hline 383RB0100.5RR1 & $6 / 1 / 2004$ & soil & EPA8270 & $\mathrm{RE}$ & $56-55-3$ & Benzo(A)Anthracene & 60 & UG/KG & $\mathrm{J}$ & 740 \\
\hline 383RB0100.5RR1 & $6 / 1 / 2004$ & soil & EPA8270 & $\mathrm{RE}$ & $50-32-8$ & Benzo(A)Pyrene & 39 & UG/KG & $\mathrm{J}$ & 740 \\
\hline 383RB0100.5RR1 & $6 / 1 / 2004$ & soil & EPA8270 & $\mathrm{RE}$ & $205-99-2$ & Benzo(B)Fluoranthene & 84 & UG/KG & $\mathrm{J}$ & 740 \\
\hline 383RB0100.5RR1 & $6 / 1 / 2004$ & soil & EPA8270 & $\mathrm{RE}$ & 117-81-7 & Bis(2-Ethylhexyl)Phthalate & 230 & UG/KG & $\mathrm{J}$ & 740 \\
\hline 383RB0100.5RR1 & $6 / 1 / 2004$ & soil & EPA8270 & $\mathrm{RE}$ & $85-68-7$ & Butyl Benzyl Phthalate & 630 & UG/KG & $\mathrm{J}$ & 740 \\
\hline 383RB0100.5RR1 & $6 / 1 / 2004$ & soil & EPA8270 & $\mathrm{RE}$ & $218-01-9$ & Chrysene & 63 & UG/KG & $J$ & 740 \\
\hline 383RB0100.5RR1 & 6/1/2004 & soil & EPA8270 & $\mathrm{RE}$ & 84-74-2 & Di-N-Butyl Phthalate & 5100 & UG/KG & & 740 \\
\hline 383RB0100.5RR1 & $6 / 1 / 2004$ & soil & EPA8270 & $\mathrm{RE}$ & $206-44-0$ & Fluoranthene & 86 & UG/KG & $\mathrm{J}$ & 740 \\
\hline 383RB0100.5RR1 & $6 / 1 / 2004$ & soil & EPA8270 & $\mathrm{RE}$ & $85-01-8$ & Phenanthrene & 43 & UG/KG & $\mathrm{J}$ & 740 \\
\hline 383RB0200.5 & $6 / 1 / 2004$ & soil & HASL300 & REG & 14331-83-0 & Ac-228 & 1.64 & $\mathrm{PCl} / \mathrm{G}$ & G & 0.5 \\
\hline 383RB0200.5 & $6 / 1 / 2004$ & soil & EPA6010 & REG & $7440-38-2$ & Arsenic & 7.1 & MG/KG & & 1 \\
\hline 383RB0200.5 & $6 / 1 / 2004$ & soil & EPA6010 & REG & $7440-39-3$ & Barium & 180 & MG/KG & & 10 \\
\hline 383RB0200.5 & $6 / 1 / 2004$ & soil & EPA8270 & REG & $56-55-3$ & Benzo(A)Anthracene & 56 & UG/KG & $\mathrm{J}$ & 340 \\
\hline 383RB0200.5 & $6 / 1 / 2004$ & soil & EPA8270 & REG & $50-32-8$ & Benzo(A)Pyrene & 52 & UG/KG & $\mathrm{J}$ & 340 \\
\hline 383RB0200.5 & $6 / 1 / 2004$ & soil & EPA8270 & REG & $205-99-2$ & Benzo(B)Fluoranthene & 120 & UG/KG & $\mathrm{J}$ & 340 \\
\hline 383RB0200.5 & $6 / 1 / 2004$ & soil & EPA8270 & REG & 207-08-9 & Benzo(K)Fluoranthene & 41 & UG/KG & $\mathrm{J}$ & 340 \\
\hline
\end{tabular}




\begin{tabular}{|c|c|c|c|c|c|c|c|c|c|c|}
\hline SAMPLE \# & $\begin{array}{l}\text { SAMPLE } \\
\text { DATE }\end{array}$ & MATRIX & $\begin{array}{l}\text { USER TEST } \\
\text { PANEL }\end{array}$ & $\begin{array}{l}\text { SAMPLE } \\
\text { PURPOSE }\end{array}$ & CAS \# & PARAMETER & RESULT & UNITS & Q & $\begin{array}{l}\text { DETECT } \\
\text { LIMIT }\end{array}$ \\
\hline 383RB0200.5 & $6 / 1 / 2004$ & soil & EPA6010 & REG & $7440-41-7$ & Beryllium & 0.86 & MG/KG & & 0.52 \\
\hline 383RB0200.5 & $6 / 1 / 2004$ & soil & HASL300 & REG & $14733-03-0$ & $\mathrm{Bi}-214$ & 1.12 & $\mathrm{PCl} / \mathrm{G}$ & $\mathrm{G}, \mathrm{J}$ & 0.29 \\
\hline 383RB0200.5 & $6 / 1 / 2004$ & soil & EPA8270 & REG & 117-81-7 & Bis(2-Ethylhexyl)Phthalate & 540 & UG/KG & $\mathrm{J}$ & 340 \\
\hline 383RB0200.5 & $6 / 1 / 2004$ & soil & EPA6010 & REG & $7440-47-3$ & Chromium & 9.1 & MG/KG & & 1 \\
\hline 383RB0200.5 & $6 / 1 / 2004$ & soil & EPA8270 & REG & 218-01-9 & Chrysene & 84 & UG/KG & $\mathrm{J}$ & 340 \\
\hline 383RB0200.5 & $6 / 1 / 2004$ & soil & HASL300 & REG & $10045-97-3$ & Cs-137 & 7.63 & $\mathrm{PCl} / \mathrm{G}$ & G & 0.15 \\
\hline 383RB0200.5 & $6 / 1 / 2004$ & soil & EPA8270 & REG & 84-74-2 & Di-N-Butyl Phthalate & 81 & UG/KG & $\mathrm{J}$ & 340 \\
\hline 383RB0200.5 & $6 / 1 / 2004$ & soil & EPA8270 & REG & $206-44-0$ & Fluoranthene & 96 & UG/KG & $\mathrm{J}$ & 340 \\
\hline 383RB0200.5 & $6 / 1 / 2004$ & soil & HASL300 & REG & 13966-00-2 & $\mathrm{K}-40$ & 24.3 & $\mathrm{PCl} / \mathrm{G}$ & G & 1.6 \\
\hline 383RB0200.5 & $6 / 1 / 2004$ & soil & EPA6010 & REG & $7439-92-1$ & Lead & 18 & MG/KG & & 0.31 \\
\hline 383RB0200.5 & $6 / 1 / 2004$ & soil & HASL300 & REG & 15092-94-1 & $\mathrm{Pb}-212$ & 1.59 & $\mathrm{PCl} / \mathrm{G}$ & $\mathrm{J}$ & 0.24 \\
\hline 383RB0200.5 & $6 / 1 / 2004$ & soil & HASL300 & REG & $15067-28-4$ & $\mathrm{~Pb}-214$ & 0.96 & $\mathrm{PCl} / \mathrm{G}$ & $\mathrm{G}, \mathrm{J}$ & 0.33 \\
\hline 383RB0200.5 & $6 / 1 / 2004$ & soil & EPA8270 & REG & $85-01-8$ & Phenanthrene & 38 & UG/KG & $\mathrm{J}$ & 340 \\
\hline 383RB0200.5 & $6 / 1 / 2004$ & soil & UGTAISOPU & REG & 13981-16-3 & Pu-238 & 0.076 & $\mathrm{PCl} / \mathrm{G}$ & & 0.01 \\
\hline 383RB0200.5 & $6 / 1 / 2004$ & soil & UGTAISOPU & REG & $15117-48-3$ & Pu-239 & 2.09 & $\mathrm{PCl} / \mathrm{G}$ & $\mathrm{J}$ & 0.01 \\
\hline 383RB0200.5 & $6 / 1 / 2004$ & soil & EPA8270 & REG & $129-00-0$ & Pyrene & 73 & UG/KG & $\mathrm{J}$ & 340 \\
\hline 383RB0200.5 & $6 / 1 / 2004$ & soil & HASL300 & REG & 14913-50-9 & TI-208 & 0.61 & $\mathrm{PCl} / \mathrm{G}$ & G & 0.14 \\
\hline 383RB0300.5 & $6 / 1 / 2004$ & soil & HASL300 & REG & 14331-83-0 & Ac-228 & 1.26 & $\mathrm{PCl} / \mathrm{G}$ & G & 0.36 \\
\hline 383RB0300.5 & $6 / 1 / 2004$ & soil & EPA6010 & REG & $7440-38-2$ & Arsenic & 5.3 & MG/KG & & 1 \\
\hline 383RB0300.5 & $6 / 1 / 2004$ & soil & EPA6010 & REG & $7440-39-3$ & Barium & 260 & MG/KG & & 10 \\
\hline 383RB0300.5 & $6 / 1 / 2004$ & soil & EPA8270 & REG & $56-55-3$ & Benzo(A)Anthracene & 170 & UG/KG & $\mathrm{J}$ & 340 \\
\hline 383RB0300.5 & $6 / 1 / 2004$ & soil & EPA8270 & REG & $50-32-8$ & Benzo(A)Pyrene & 200 & UG/KG & $\mathrm{J}$ & 340 \\
\hline 383RB0300.5 & $6 / 1 / 2004$ & soil & EPA8270 & REG & $205-99-2$ & Benzo(B)Fluoranthene & 460 & UG/KG & $\mathrm{J}$ & 340 \\
\hline 383RB0300.5 & $6 / 1 / 2004$ & soil & EPA8270 & REG & $207-08-9$ & Benzo(K)Fluoranthene & 230 & UG/KG & $\mathrm{J}$ & 340 \\
\hline 383RB0300.5 & $6 / 1 / 2004$ & soil & EPA6010 & REG & $7440-41-7$ & Beryllium & 0.84 & MG/KG & & 0.52 \\
\hline 383RB0300.5 & $6 / 1 / 2004$ & soil & HASL300 & REG & 14733-03-0 & $\mathrm{Bi}-214$ & 0.87 & $\mathrm{PCl} / \mathrm{G}$ & $\mathrm{G}, \mathrm{J}$ & 0.29 \\
\hline 383RB0300.5 & $6 / 1 / 2004$ & soil & EPA8270 & REG & $117-81-7$ & Bis(2-Ethylhexyl)Phthalate & 1200 & UG/KG & $\mathrm{J}$ & 340 \\
\hline 383RB0300.5 & $6 / 1 / 2004$ & soil & EPA8270 & REG & $85-68-7$ & Butyl Benzyl Phthalate & 23 & UG/KG & $\mathrm{J}$ & 340 \\
\hline 383RB0300.5 & $6 / 1 / 2004$ & soil & EPA6010 & REG & $7440-43-9$ & Cadmium & 0.57 & MG/KG & & 0.52 \\
\hline 383RB0300.5 & $6 / 1 / 2004$ & soil & EPA6010 & REG & $7440-47-3$ & Chromium & 8.7 & MG/KG & & 1 \\
\hline 383RB0300.5 & $6 / 1 / 2004$ & soil & EPA8270 & REG & $218-01-9$ & Chrysene & 270 & UG/KG & $\mathrm{J}$ & 340 \\
\hline 383RB0300.5 & $6 / 1 / 2004$ & soil & HASL300 & REG & $10045-97-3$ & Cs-137 & 23.4 & $\mathrm{PCl} / \mathrm{G}$ & G & 0.1 \\
\hline 383RB0300.5 & $6 / 1 / 2004$ & soil & EPA8015 & REG & 68334-30-5 & Diesel Range Organics & 3 & MG/KG & $\mathrm{J}$ & 5.2 \\
\hline 383RB0300.5 & $6 / 1 / 2004$ & soil & EPA8270 & REG & 84-74-2 & Di-N-Butyl Phthalate & 79 & UG/KG & $\mathrm{J}$ & 340 \\
\hline 383RB0300.5 & $6 / 1 / 2004$ & soil & EPA8270 & REG & $206-44-0$ & Fluoranthene & 280 & UG/KG & $\mathrm{J}$ & 340 \\
\hline
\end{tabular}




\begin{tabular}{|c|c|c|c|c|c|c|c|c|c|c|}
\hline SAMPLE \# & $\begin{array}{l}\text { SAMPLE } \\
\text { DATE }\end{array}$ & MATRIX & $\begin{array}{l}\text { USER TEST } \\
\text { PANEL }\end{array}$ & $\begin{array}{l}\text { SAMPLE } \\
\text { PURPOSE }\end{array}$ & CAS \# & PARAMETER & RESULT & UNITS & Q & $\begin{array}{l}\text { DETECT } \\
\text { LIMIT }\end{array}$ \\
\hline 383RB0300.5 & $6 / 1 / 2004$ & soil & HASL300 & REG & 13966-00-2 & $\mathrm{K}-40$ & 21.4 & $\mathrm{PCl} / \mathrm{G}$ & G & 1.2 \\
\hline 383RB0300.5 & $6 / 1 / 2004$ & soil & EPA6010 & REG & $7439-92-1$ & Lead & 61 & MG/KG & & 0.31 \\
\hline 383RB0300.5 & $6 / 1 / 2004$ & soil & HASL300 & REG & 15092-94-1 & $\mathrm{Pb}-212$ & 1.52 & $\mathrm{PCl} / \mathrm{G}$ & $\mathrm{J}$ & 0.26 \\
\hline 383RB0300.5 & $6 / 1 / 2004$ & soil & HASL300 & REG & 15067-28-4 & $\mathrm{Pb}-214$ & 0.93 & $\mathrm{PCl} / \mathrm{G}$ & $\mathrm{G}, \mathrm{J}$ & 0.32 \\
\hline 383RB0300.5 & $6 / 1 / 2004$ & soil & EPA8270 & REG & $85-01-8$ & Phenanthrene & 79 & UG/KG & $\mathrm{J}$ & 340 \\
\hline 383RB0300.5 & $6 / 1 / 2004$ & soil & UGTAISOPU & REG & 13981-16-3 & Pu-238 & 0.151 & $\mathrm{PCl} / \mathrm{G}$ & & 0.023 \\
\hline 383RB0300.5 & $6 / 1 / 2004$ & soil & UGTAISOPU & REG & $15117-48-3$ & Pu-239 & 0.85 & $\mathrm{PCl} / \mathrm{G}$ & $\mathrm{J}$ & 0.02 \\
\hline 383RB0300.5 & $6 / 1 / 2004$ & soil & EPA8270 & REG & $129-00-0$ & Pyrene & 360 & UG/KG & & 340 \\
\hline 383RB0300.5 & $6 / 1 / 2004$ & soil & HASL300 & REG & $14913-50-9$ & TI-208 & 0.47 & $\mathrm{PCl} / \mathrm{G}$ & G & 0.14 \\
\hline 383RB0400.5 & $6 / 1 / 2004$ & soil & HASL300 & REG & 14331-83-0 & Ac-228 & 2.46 & $\mathrm{PCl} / \mathrm{G}$ & G & 0.43 \\
\hline 383RB0400.5 & $6 / 1 / 2004$ & soil & EPA6010 & REG & $7440-38-2$ & Arsenic & 4.5 & MG/KG & & 1.1 \\
\hline 383RB0400.5 & $6 / 1 / 2004$ & soil & EPA6010 & REG & $7440-39-3$ & Barium & 190 & MG/KG & & 11 \\
\hline 383RB0400.5 & $6 / 1 / 2004$ & soil & EPA8270 & REG & $56-55-3$ & Benzo(A)Anthracene & 140 & UG/KG & $\mathrm{J}$ & 360 \\
\hline 383RB0400.5 & $6 / 1 / 2004$ & soil & EPA8270 & REG & $50-32-8$ & Benzo(A)Pyrene & 230 & UG/KG & $\mathrm{J}$ & 360 \\
\hline 383RB0400.5 & $6 / 1 / 2004$ & soil & EPA8270 & REG & $205-99-2$ & Benzo(B)Fluoranthene & 620 & UG/KG & & 360 \\
\hline 383RB0400.5 & $6 / 1 / 2004$ & soil & EPA8270 & REG & $191-24-2$ & Benzo(G,H,I)Perylene & 90 & UG/KG & $\mathrm{J}$ & 360 \\
\hline 383RB0400.5 & $6 / 1 / 2004$ & soil & EPA8270 & REG & $207-08-9$ & Benzo(K)Fluoranthene & 170 & UG/KG & $\mathrm{J}$ & 360 \\
\hline 383RB0400.5 & $6 / 1 / 2004$ & soil & EPA6010 & REG & $7440-41-7$ & Beryllium & 1.1 & MG/KG & & 0.54 \\
\hline 383RB0400.5 & $6 / 1 / 2004$ & soil & HASL300 & REG & 14733-03-0 & $\mathrm{Bi}-214$ & 0.7 & $\mathrm{PCl} / \mathrm{G}$ & $\mathrm{G}, \mathrm{J}$ & 0.31 \\
\hline 383RB0400.5 & $6 / 1 / 2004$ & soil & EPA8270 & REG & $117-81-7$ & Bis(2-Ethylhexyl)Phthalate & 76 & UG/KG & $\mathrm{J}$ & 360 \\
\hline 383RB0400.5 & $6 / 1 / 2004$ & soil & EPA8270 & REG & $86-74-8$ & Carbazole & 23 & UG/KG & $\mathrm{J}$ & 360 \\
\hline 383RB0400.5 & $6 / 1 / 2004$ & soil & EPA6010 & REG & $7440-47-3$ & Chromium & 4.9 & MG/KG & & 1.1 \\
\hline 383RB0400.5 & $6 / 1 / 2004$ & soil & EPA8270 & REG & 218-01-9 & Chrysene & 480 & UG/KG & & 360 \\
\hline 383RB0400.5 & $6 / 1 / 2004$ & soil & HASL300 & REG & $10045-97-3$ & Cs-137 & 7.06 & $\mathrm{PCl} / \mathrm{G}$ & G & 0.13 \\
\hline 383RB0400.5 & $6 / 1 / 2004$ & soil & EPA8270 & REG & $53-70-3$ & Dibenzo(A,H)Anthracene & 27 & UG/KG & $\mathrm{J}$ & 360 \\
\hline 383RB0400.5 & $6 / 1 / 2004$ & soil & EPA8015 & REG & 68334-30-5 & Diesel Range Organics & 15 & MG/KG & $\mathrm{Z}, \mathrm{H}$ & 5.4 \\
\hline 383RB0400.5 & $6 / 1 / 2004$ & soil & EPA8270 & REG & 84-74-2 & Di-N-Butyl Phthalate & 45 & UG/KG & $\mathrm{J}$ & 360 \\
\hline 383RB0400.5 & $6 / 1 / 2004$ & soil & EPA8270 & REG & $206-44-0$ & Fluoranthene & 510 & UG/KG & & 360 \\
\hline 383RB0400.5 & $6 / 1 / 2004$ & soil & EPA8270 & REG & $193-39-5$ & Indeno(1,2,3-Cd)Pyrene & 80 & UG/KG & $\mathrm{J}$ & 360 \\
\hline 383RB0400.5 & $6 / 1 / 2004$ & soil & HASL300 & REG & 13966-00-2 & $\mathrm{K}-40$ & 27.3 & $\mathrm{PCl} / \mathrm{G}$ & G & 1.4 \\
\hline 383RB0400.5 & $6 / 1 / 2004$ & soil & EPA6010 & REG & $7439-92-1$ & Lead & 22 & MG/KG & & 0.32 \\
\hline 383RB0400.5 & $6 / 1 / 2004$ & soil & EPA8270 & REG & $91-20-3$ & Naphthalene & 18 & UG/KG & $\mathrm{J}$ & 360 \\
\hline 383RB0400.5 & $6 / 1 / 2004$ & soil & HASL300 & REG & 15092-94-1 & $\mathrm{Pb}-212$ & 2.44 & $\mathrm{PCl} / \mathrm{G}$ & $\mathrm{J}$ & 0.24 \\
\hline 383RB0400.5 & $6 / 1 / 2004$ & soil & HASL300 & REG & $15067-28-4$ & $\mathrm{~Pb}-214$ & 1.04 & $\mathrm{PCl} / \mathrm{G}$ & $\mathrm{G}, \mathrm{J}$ & 0.26 \\
\hline 383RB0400.5 & $6 / 1 / 2004$ & soil & EPA8270 & REG & $85-01-8$ & Phenanthrene & 210 & UG/KG & $\mathrm{J}$ & 360 \\
\hline
\end{tabular}




\begin{tabular}{|c|c|c|c|c|c|c|c|c|c|c|}
\hline SAMPLE \# & $\begin{array}{l}\text { SAMPLE } \\
\text { DATE }\end{array}$ & MATRIX & $\begin{array}{l}\text { USER TEST } \\
\text { PANEL }\end{array}$ & $\begin{array}{l}\text { SAMPLE } \\
\text { PURPOSE }\end{array}$ & CAS \# & PARAMETER & RESULT & UNITS & Q & $\begin{array}{l}\text { DETECT } \\
\text { LIMIT }\end{array}$ \\
\hline 383RB0400.5 & $6 / 1 / 2004$ & soil & UGTAISOPU & REG & 13981-16-3 & Pu-238 & 0.232 & $\mathrm{PCl} / \mathrm{G}$ & & 0.03 \\
\hline 383RB0400.5 & $6 / 1 / 2004$ & soil & UGTAISOPU & REG & $15117-48-3$ & Pu-239 & 1.15 & $\mathrm{PCl} / \mathrm{G}$ & $\mathrm{J}$ & 0.02 \\
\hline 383RB0400.5 & $6 / 1 / 2004$ & soil & EPA8270 & REG & $129-00-0$ & Pyrene & 430 & UG/KG & & 360 \\
\hline 383RB0400.5 & $6 / 1 / 2004$ & soil & HASL300 & REG & 14913-50-9 & TI-208 & 0.74 & $\mathrm{PCl} / \mathrm{G}$ & G & 0.12 \\
\hline 383TR10 & $6 / 3 / 2004$ & water & EPA8260 & TB & $75-09-2$ & Methylene Chloride & 0.97 & UG/L & J,B & 5 \\
\hline 383TR10 & $6 / 3 / 2004$ & water & EPA8260 & TB & $108-88-3$ & Toluene & 1.3 & UG/L & $\mathrm{J}$ & 5 \\
\hline 383TR11 & $6 / 7 / 2004$ & water & EPA8260 & TB & $75-09-2$ & Methylene Chloride & 0.87 & UG/L & J,B & 5 \\
\hline 383TR13 & $6 / 8 / 2004$ & water & EPA8260 & TB & $67-64-1$ & Acetone & 12 & UG/L & J,B & 20 \\
\hline 383TR13 & $6 / 8 / 2004$ & water & EPA8260 & TB & $75-09-2$ & Methylene Chloride & 0.85 & UG/L & J,B & 5 \\
\hline 383TR14 & $6 / 9 / 2004$ & water & EPA8260 & TB & $67-64-1$ & Acetone & 13 & UG/L & J,B & 20 \\
\hline 383TR14 & $6 / 9 / 2004$ & water & EPA8260 & TB & $75-09-2$ & Methylene Chloride & 5.8 & UG/L & B & 5 \\
\hline 383TR15 & $6 / 10 / 2004$ & water & EPA8260 & TB & $67-64-1$ & Acetone & 13 & UG/L & J,B & 20 \\
\hline 383TR15 & $6 / 10 / 2004$ & water & EPA8260 & TB & $75-09-2$ & Methylene Chloride & 15 & UG/L & $B$ & 5 \\
\hline 383TR16 & $6 / 11 / 2004$ & water & EPA8260 & TB & $75-09-2$ & Methylene Chloride & 2.3 & UG/L & $\mathrm{J}$ & 5 \\
\hline 383TR16 & $6 / 11 / 2004$ & water & EPA8260 & TB & $108-88-3$ & Toluene & 1.1 & UG/L & $\mathrm{J}$ & 5 \\
\hline 383TR17 & $6 / 12 / 2004$ & water & EPA8260 & TB & $75-09-2$ & Methylene Chloride & 0.96 & UG/L & $\mathrm{J}$ & 5 \\
\hline 383TR17 & $6 / 12 / 2004$ & water & EPA8260 & TB & $108-88-3$ & Toluene & 0.61 & UG/L & $\mathrm{J}$ & 5 \\
\hline 383TR18 & $6 / 13 / 2004$ & water & EPA8260 & TB & $75-09-2$ & Methylene Chloride & 2 & UG/L & $\mathrm{J}$ & 5 \\
\hline 383TR19 & $6 / 14 / 2004$ & water & EPA8260 & TB & $75-09-2$ & Methylene Chloride & 1.3 & UG/L & $\mathrm{J}$ & 5 \\
\hline 383TR19 & $6 / 14 / 2004$ & water & EPA8260 & TB & 108-88-3 & Toluene & 0.78 & UG/L & $\mathrm{J}$ & 5 \\
\hline 383TR20 & $6 / 15 / 2004$ & water & EPA8260 & TB & $75-09-2$ & Methylene Chloride & 1.5 & UG/L & $\mathrm{J}$ & 5 \\
\hline 383TR21 & $6 / 16 / 2004$ & water & EPA8260 & TB & $75-09-2$ & Methylene Chloride & 1.4 & UG/L & $\mathrm{J}$ & 5 \\
\hline 383TR22 & $6 / 17 / 2004$ & water & EPA8260 & TB & $75-09-2$ & Methylene Chloride & 1.3 & UG/L & $\mathrm{J}$ & 5 \\
\hline \multicolumn{11}{|c|}{ Note ; An X suffix indicates a duplicate sample. } \\
\hline An RR1 & ndiatos th & $\mathrm{con}$ & res- & cis at a & her dilutio & ighe & & & & \\
\hline
\end{tabular}


Appendix B

\section{Data Assessment}




\section{B.1.0 SW-846 Analysis}

\section{B.1.1 Introduction}

The SW-846 Chapter 9 Method is used to evaluate whether the site has been adequately characterized, as prescribed during the data quality objectives process (DTRA, 2003). The method is used to determine if the upper limit of the confidence interval in the concentration of a chemical (or in this case, radioactive) contaminant exceeds the regulatory threshold (RT). If the 90 -percent confidence level of the mean concentration of the suspected contaminant in the medium of interest exceeds the RT, the site is considered to be contaminated. For this analysis, radionuclide RTs are the NCRP Report No. 129 recommended screening limits for construction, commercial, and industrial land use scenario (NCRP, 1999) scaled from 25 to $15 \mathrm{mrem} / \mathrm{yr}$ dose and the generic guidelines for residual concentration of radionuclides in DOE Order 5400.5 (DOE, 1993) (PALs).

\section{B.1.2 Evaluation}

Because no soil sampling data were available for the E-Tunnel Sites, the cesium-137 results from characterization of the Muckpiles at CAUs 477 and 482 and the drainages at CAUs 482 and 504 were used to calculate the number of samples required to characterize the E-Tunnel Sites (DTRA, 2003). The CAIP (DTRA, 2003) committed to using the procedure described in Chapter 9 of the EPA SW-846 Method to confirm that sufficient samples were collected to characterize the site at the 90-percent confidence level (EPA, 1996). Only random samples will be used for the evaluation. The number of samples to collect is defined in equation (8) of Table 9-1 in SW 846, if the minimum number of samples to collect is less than one "1" will be used as the minimum.:

$$
n=t_{.20}^{2} \times s^{2} /(R T-\bar{x})^{2}
$$

where:

$\mathrm{n}=$ Minimum number of samples to ensure a 90-percent confidence level

$\mathrm{t}_{.20}{ }^{2}=$ The square of the " $\mathrm{t}$ " value in Table 99-2, SW-846, for a one-tailed 90-percent confidence interval

$\mathrm{s}^{2}=$ Variance in the concentration measured in the samples collected during characterization 
$\mathrm{RT}=$ Regulatory threshold; set to the PALs for each radionuclide

$0=$ The mean concentration of the COPC in the collected samples.

Cesium-137 was chosen for conducting the calculations because it was the most prevalent contaminant found during characterization of the other DTRA sites. The PALs screening level for $\mathrm{Cs}-137$ is 7.3 picocuries per gram $(\mathrm{pCi} / \mathrm{g})$.

Next, the concentration at the 90-percent confidence level was calculated using equation (6) of Table 9-1 in SW-846.

$$
C I=\bar{x} \pm t_{.20^{S}} \bar{x}
$$

The values were calculated and compared with the RT for Cs-137 of $7.3 \mathrm{pCi} / \mathrm{g}$. Table B.1-1 contains the values used in the SW-846 equations and the results of the calculations for the E-Tunnel Sites.

\section{B.1.3 Pond-Native Soils}

Native material samples were collected from under Pond One in four random and one biased locations. Looking only at the Cs-137 results from the four random muck samples, all were less than the RT. Using the Cs-137 results and the average Cs-137 detection limit for those sample results less than the detection limit, the SW-846 equation calculates that "1" sample is needed to achieve 90-percent confidence in the characterization of the Muckpile muck. More samples were collected than were required to determine that the muck is adequately characterized.

\section{B.1.4 Pond Sediment}

Pond sediment samples were collected from Ponds One, Two, and Three in four random and five biased locations. Looking at the Cs-137 results from the four random samples, two were less than the RT and two were more. Using the Cs-137 results from the random samples, the SW-846 equation calculates that 8.3 samples are needed to achieve 90 -percent confidence in the characterization of the pond sediments. Less samples were collected than were required to determine that the pond sediments are adequately characterized. 
Table B.1-1

Evaluation of the Number of Samples and Determination Whether Areas are Contaminated

\begin{tabular}{|c|c|c|c|c|c|}
\hline Variable & Pond Native & Pond Sediment & Drainage & Muckpile Native & Muckpile Muck \\
\hline $\mathrm{T}_{.20}$ & 1.638 & 1.638 & 1.307 & 1.333 & 1.323 \\
\hline $\mathrm{T}_{\cdot 20}{ }^{2}$ & 2.683 & 2.683 & 1.708 & 1.777 & 1.750 \\
\hline RT for Cs137 & 7.3 & 7.3 & 7.3 & 7.3 & 7.3 \\
\hline Avg (BarX) & 1.241 & 40.778 & 25.978 & 0.846 & 49.480 \\
\hline n collected & 4 & 4 & 35 & 18 & 22 \\
\hline n needed* & "1" & 8.305 & 1.970 & "1" & 11.098 \\
\hline Confidence Interval & 1.467 & 48.238 & 4.431 & 0.701 & 29.959 \\
\hline $90 \%$ Confidence & 2.707 & 89.015 & 30.409 & 1.547 & 79.439 \\
\hline $90 \%$ Confidence $>7.3 p \mathrm{pi} / \mathrm{g}$ & no & yes & yes & no & yes \\
\hline
\end{tabular}

Note: Only random samples were used in this evaluation.

* - Minimum samples needed is actually 0.234 and 0.212 respectively 
The 90-percent confidence level for Cs-137 concentrations in random samples from the ponds, $89 \mathrm{pCi} / \mathrm{g}$, exceeds the RT by a significant margin. By not meeting the 90 -percent confidence goal for characterization, there is an increased likelihood of a false positive decision error, but since the 90-percent confidence level exceeds the RT by over an order of magnitude, it is reasonable to assert that the pond sediments are contaminated above regulatory thresholds. This assertion is further supported by data from samples from biased locations, which also show concentrations of Cs-137 significantly in excess of the RT. If the determination is in error, the consequences are that resources may be expended that would not otherwise have needed to be, but risks to human health and the environment will not be increased if this error has occurred. Therefore, it is not necessary to collect additional samples to increase the confidence in the determination.

\section{B.1.5 Drainage}

Thirty-five random sample locations were selected and sampled. Twenty-nine Cs-137 sample results were more than the RT. Using the Cs-137 results the SW-846 equation calculates that 2.0 samples are needed to achieve 90-percent confidence in the characterization of the channel. More samples were collected than were required to determine that the drainage was adequately characterized.

\section{B.1.6 Muckpile-Native Soils}

Twenty-five native material samples were collected from under the Muckpile in 18 random and 7 biased locations. Looking at the Cs-137 results from the 18 random samples, 17 were less than the RT and 12 were less than the minimum detection limit. Using the Cs-137 results and the average Cs-137 detection limit for those sample results less than the detection limit, the SW-846 equation calculates that "1" sample is needed to achieve 90-percent confidence in the characterization of the native soils underneath the Muckpile. More samples were collected than were required to determine that the Muckpile native soils are adequately characterized.

\section{B.1.7 Muckpile-Muck}

Muck samples were collected from 22 random and 19 biased locations. Looking only at the Cs-137 results from the 22 random muck samples, 9 were more than the RT. Using the Cs-137 results the SW-846 equation calculates that 11.1 samples are needed to achieve 90 -percent confidence in the characterization of the Muckpile muck. More samples were collected than were required to determine that the muck is adequately characterized. 


\section{B.1.8 Oil Spill and Radioactive Material Area}

The SW-846 Chapter 9 Method is designed for using only random sample locations, but two of the areas in CAU $383 \mathrm{CAI}$, the oil spill and the RMA, were sampled using only biased locations. Therefore, to evaluate the oil spill and RMA with the SW-846 equations could give biased results, but if the equations are applied, both areas have an insufficient number of samples collected to meet the 90-percent confidence goal for characterization.

The 90-percent confidence level for Cs-137 concentrations in biased samples from the oil spill, $10.3 \mathrm{pCi} / \mathrm{g}$, exceeds the RT. By not meeting the 90-percent confidence goal for characterization, there is an increased likelihood of a false positive decision error, but since the 90-percent confidence level exceeds the RT, it is reasonable to assert that the Oil Spill is contaminated above regulatory thresholds. If the determination is in error, the consequences are that resources may be expended that would not otherwise have needed to be, but risks to human health and the environment will not be increased if this error has occurred.

The 90-percent confidence level for Cs-137 concentrations in biased samples from the RMA, $74.3 \mathrm{pCi} / \mathrm{g}$, exceeds the RT by a significant margin. By not meeting the 90 -percent confidence goal for characterization, there is an increased likelihood of a false positive decision error, but since the 90-percent confidence level exceeds the RT by an order of magnitude, it is reasonable to assert that the RMA is contaminated above regulatory thresholds. If the determination is in error, the consequences are that resources may be expended that would not otherwise have needed to be, but risks to human health and the environment will not be increased if this error has occurred. Therefore, it is not necessary to collect more samples to increase the confidence in the determination.

\section{B.1.9 SW-846 Summary}

The 90-percent confidence goal for characterization of areas randomly sampled was achieved in all areas but the pond sediments at CAU 383. The E-Tunnel Muckpile muck and drainage are contaminated, the Muckpile and pond native soils are not contaminated. Although insufficient samples were collected to achieve 90-percent confidence in the pond sediments, the oil spill and the RMA, and that the samples were biased in the oil spill and the RMA, enough samples were collected to decide that they are contaminated. This results in a possible error on the conservative side, ensuring protection of human health and environment. Therefore, in 
CAU 383 CADD

Appendix B

Revision: 0

Date: March 2005

Page B-6 of B-12

accordance with the CAIP (DTRA, 2003), the E-Tunnel Sites have been adequately characterized. 


\section{B.2.0 Completeness}

Completeness is defined as the acquisition of sufficient data of the appropriate quality to satisfy DQO decision data requirements. Table B.2-1 and Table B.2-2 provide the chemical and radiological completeness analysis results. In accordance with the CAU 383 CAIP (Table 6-1), 80 percent of measurements had valid results.

Table B.2-1

Chemical Completeness Measurements

\begin{tabular}{|c|c|c|c|c|c|}
\hline \multirow[b]{2}{*}{ Completeness Parameters } & \multicolumn{3}{|c|}{ ORGANICS } & \multicolumn{2}{|c|}{ INORGANICS } \\
\hline & VOCs & SVOCs & TPH-D & METALS* & MERCURY \\
\hline \multicolumn{6}{|c|}{ Sample Analysis Completeness } \\
\hline Total samples sent to laboratory & 170 & 149 & 149 & 157 & 153 \\
\hline Total samples analyzed & 170 & 149 & 149 & 157 & 153 \\
\hline $\begin{array}{l}\text { Total samples not analyzed by the } \\
\text { laboratory }\end{array}$ & 0 & 0 & 0 & 0 & 0 \\
\hline Percent Completeness & 100 & 100 & 100 & 100 & 100 \\
\hline \multicolumn{6}{|c|}{ Measurement Usability Completeness } \\
\hline Total measurements ** & 11,799 & 10,934 & 149 & 1,236 & 153 \\
\hline Total measurements rejected - Field & 0 & 0 & 0 & 0 & 0 \\
\hline Total measurements rejected - Lab/Matrix & 0 & 166 & 0 & 0 & 0 \\
\hline Percent Completeness & 100 & 98 & 100 & 100 & 100 \\
\hline
\end{tabular}

* Measurements include arsenic, barium, beryllium, cadmium, chromium, lead, selenium, silver, mercury

* Measurements include re-analyses

Table B.2-2

Radiological Completeness Measurements

\begin{tabular}{|c|c|c|c|}
\hline Completeness Parameters & $\begin{array}{c}\text { Gamma } \\
\text { Spectroscopy }\end{array}$ & $\begin{array}{c}\text { Isotopic } \\
\text { Plutonium }\end{array}$ & Strontium-90 \\
\hline \multicolumn{4}{|c|}{ Sample Analysis Completeness } \\
\hline Total samples sent to laboratory & 158 & 158 & 158 \\
\hline Total samples analyzed & 158 & 158 & 158 \\
\hline Total samples not analyzed by the laboratory & 0 & 0 & 0 \\
\hline Percent Completeness & 100 & 100 & 100 \\
\hline \multicolumn{4}{|c|}{ Measurement Usability Completeness } \\
\hline Total measurements * & 3,476 & 316 & 158 \\
\hline Total measurements rejected - Field & 0 & 0 & 0 \\
\hline Total measurements rejected - Lab/Matrix & 0 & 0 & 0 \\
\hline Percent Completeness & 100 & 100 & 100 \\
\hline
\end{tabular}

${ }^{*}$ Measurements include reanalyses 


\section{B.3.0 Precision of Laboratory Measurements}

Precision is a measure of agreement among a replicate set of measurements of the same property under similar conditions. This agreement is expressed as the relative percent difference (RPD) between duplicate measurements (EPA, 1996).

For the purpose of determining data precision of sample analyses, all water and soil samples, including field QC samples (i.e., trip blanks, equipment rinsate samples, field blanks, etc.) were evaluated and incorporated into the precision calculation.

\section{B.3.1 Precision for Chemical Analyses}

Table B.3-1 provides the chemical precision analysis results.

Table B.3-1

Chemical Precision Measurements

\begin{tabular}{|c|c|c|c|c|c|}
\hline & \multicolumn{3}{|c|}{ ORGANICS } & \multicolumn{2}{|c|}{ INORGANICS } \\
\hline & VOCs & SVOCs & TPH-D & METALS* & MERCURY \\
\hline \multicolumn{6}{|c|}{ Matrix Spike Duplicate (MSD) Precision } \\
\hline Total Number of MSD Measurements & 55 & 88 & 13 & 80 & 11 \\
\hline Total Number of RPDs within criteria & 55 & 88 & 13 & 80 & 11 \\
\hline MSD \% Precision & 100 & 100 & 100 & 100 & 100 \\
\hline \multicolumn{6}{|c|}{ Laboratory Control Sample Duplicate (LCSD) Precision } \\
\hline Total Number of LCSD Measurements & 90 & 165 & 15 & 129 & 17 \\
\hline Total Number of RPDs within criteria & 90 & 165 & 15 & 129 & 17 \\
\hline LCSD \% Precision & 100 & 100 & 100 & 100 & 100 \\
\hline \multicolumn{6}{|c|}{ "Field Sample Duplicate (FD) Precision } \\
\hline Total Number of FD Measurements & 483 & 497 & 7 & 64 & 8 \\
\hline Total Number of RPDs within criteria & 483 & 497 & 4 & 64 & 8 \\
\hline FD \% Precision & 100 & 100 & 57.1 & 100 & 100 \\
\hline \multicolumn{6}{|c|}{ Laboratory Sample Duplicate (Lab-Dup) Precision } \\
\hline Total Number of Lab-Dup Measurements & NA & NA & NA & 80 & 11 \\
\hline Total Number of RPDs within criteria & NA & NA & NA & 80 & 11 \\
\hline Lab-Dup \% Precision & NA & NA & NA & 100 & 100 \\
\hline
\end{tabular}

* Measurements include arsenic, barium, beryllium, cadmium, chromium, lead, selenium, silver, mercury NA = Not applicable

Field Sample Duplicate (FD) percent precision for DRO is 57.1 percent. Due to no FD criteria for organic analyses, no field sample results were qualified based on the RPD being outside the 
control limits. The low percentage of DRO RPD precision tests that are within criteria may be due to the in homogeneity of the DRO contamination on the soil samples.

\section{B.3.2 Precision for Radiological Analyses}

The duplicate precision is evaluated using the RPD or normalized difference (ND). The RPD is applicable when both the sample and its duplicate have concentrations of the target radionuclide exceeding five times their minimum detectable concentration. This excludes many measurements because the samples contain nondetectable or low levels of the target radionuclide. In situations where the RPD does not apply, duplicate results are evaluated using the ND.

Table B.3-2 provides the radiological precision analysis results. The low percentage of plutonium RPD precision tests that are within criteria may be due to the inhomogeneity of the plutonium contamination on the soil samples.

Table B.3-2

Radiological Precision Measurements

\begin{tabular}{|c|c|c|c|}
\hline & $\begin{array}{c}\text { Gamma } \\
\text { Spectroscopy }\end{array}$ & $\begin{array}{c}\text { Isotopic } \\
\text { Plutonium }\end{array}$ & Strontium-90 \\
\hline \multicolumn{4}{|c|}{ Laboratory Sample Duplicate (Lab-Dup) Precision } \\
\hline \multicolumn{4}{|c|}{ RPD } \\
\hline Total Number of Measurements & 36 & 21 & 6 \\
\hline Total Number of RPDs within criteria & 36 & 14 & 6 \\
\hline \% Precision & 100 & 66.7 & 100 \\
\hline \multicolumn{4}{|c|}{ Normalized Difference } \\
\hline Total Number of Measurements & 492 & 21 & 17 \\
\hline Total Number of NDs within criteria & 491 & 21 & 17 \\
\hline$\%$ Precision & 99.8 & 100 & 100 \\
\hline \multicolumn{4}{|c|}{ Field Sample Duplicate (FD) Precision } \\
\hline \multicolumn{4}{|c|}{ RPD } \\
\hline Total Number of Measurements & 18 & 6 & 1 \\
\hline Total Number of RPDs within criteria & 18 & 5 & 1 \\
\hline$\%$ Precision & 100 & 83.3 & 100 \\
\hline \multicolumn{4}{|c|}{ Normalized Difference } \\
\hline Total Number of Measurements & 158 & 10 & 7 \\
\hline Total Number of NDs within criteria & 158 & 10 & 6 \\
\hline$\%$ Precision & 100 & 100 & 85.7 \\
\hline
\end{tabular}

NA = Not applicable 


\section{B.4.0 Accuracy for Laboratory Measurements}

Accuracy is a measure of the closeness of an individual measurement or the average of a number of measurements to the true value. Accuracy includes a combination of random error (precision) and systematic error (bias) components that result from sampling and analytical operations. This closeness is expressed as percent recovery $(\% \mathrm{R})(\mathrm{EPA}, 1996)$.

For the purpose of determining data precision of sample analyses, all water and soil samples, including field QC samples (i.e. Trip blanks, equipment rinsate samples, field blanks, etc.) were evaluated and incorporated into the precision calculation.

\section{B.4.1 Accuracy for Chemical Analyses}

Table B.4-1 provides the chemical accuracy analysis results.

Table B.4-1

\section{Chemical Accuracy Measurements}

\begin{tabular}{|c|c|c|c|c|c|}
\hline & \multicolumn{3}{|c|}{ ORGANICS } & \multicolumn{2}{|c|}{ INORGANICS } \\
\hline & VOCs & SVOCs & TPH-D & METALS* & MERCURY \\
\hline \multicolumn{6}{|c|}{ Matrix Spike (MS) Accuracy } \\
\hline Total Number of MS measurements & 110 & 176 & 26 & 160 & 22 \\
\hline $\begin{array}{l}\text { Total Number of MS measurements within } \\
\text { criteria }\end{array}$ & 110 & 176 & 26 & 156 & 21 \\
\hline MS \% Accuracy & 100 & 100 & 100 & 97.5 & 95.4 \\
\hline \multicolumn{6}{|c|}{ Laboratory Control Sample (LCS) Accuracy } \\
\hline Total Number of LCS measurements & 180 & 330 & 30 & 258 & 34 \\
\hline $\begin{array}{l}\text { Total Number of LCS measurements within } \\
\text { criteria }\end{array}$ & 180 & 330 & 30 & 258 & 34 \\
\hline LCS \% Accuracy & 100 & 100 & 100 & 100 & 100 \\
\hline \multicolumn{6}{|c|}{ Surrogate Accuracy } \\
\hline Total Number of Measurements Analyzed & 11,799 & 10,934 & 149 & NA & NA \\
\hline $\begin{array}{l}\text { Total Number of measurements not affected } \\
\text { by Out-of-Control Surrogates }\end{array}$ & 11,786 & 10,721 & 149 & NA & NA \\
\hline Surrogate \%Accuracy & 99.9 & 98.1 & 100 & NA & NA \\
\hline
\end{tabular}

* Measurements include arsenic, barium, beryllium, cadmium, chromium, lead, selenium, silver NA = Not applicable 


\section{B.4.2 Accuracy for Radiological Analyses}

Table B.4-2 provides the accuracy results for the radiological analyses.

Table B.4-2

\section{Radiological Accuracy Measurements}

\begin{tabular}{|c|c|c|c|}
\hline & $\begin{array}{c}\text { Gamma } \\
\text { Spectroscopy }\end{array}$ & $\begin{array}{c}\text { Isotopic } \\
\text { Plutonium }\end{array}$ & Strontium-90 \\
\hline \hline Total Number of Measurements & 67 & 18 & 23 \\
\hline Total Number of \%RECs within criteria & 67 & 18 & 23 \\
\hline \% Accuracy & 100 & 100 & 100 \\
\hline
\end{tabular}




\section{B.5.0 References}

Defense Threat Reduction Agency. 2003. Corrective Action Investigation Plan for Corrective Action Unit 383: Area 12 E-Tunnel Sites, Nevada Test Site, Nevada, Rev. 0. Prepared by SNJV. Las Vegas, NV.

National Council on Radiation Protection and Measurements. 1999. Recommended Screening Limits for Contaminated Surface soil and Review of Factors Relevant to Site-Specific Studies. Report No. 129. Bethesda, MD.

U.S. Department of Energy. 1993. "Radiation Protection of the Public and Environment." DOE Order 5400.5 Change 2. January 7.

U.S. Environmental Protection Agency. 1996. Test Methods for Evaluating Solid Waste, Physical/Chemical Methods, SW-846 CD ROM PB97-501928GEI, which contains updates for 1986, 1992, 1994, and 1996. Washington, DC. 
Appendix C

Cost Estimates 


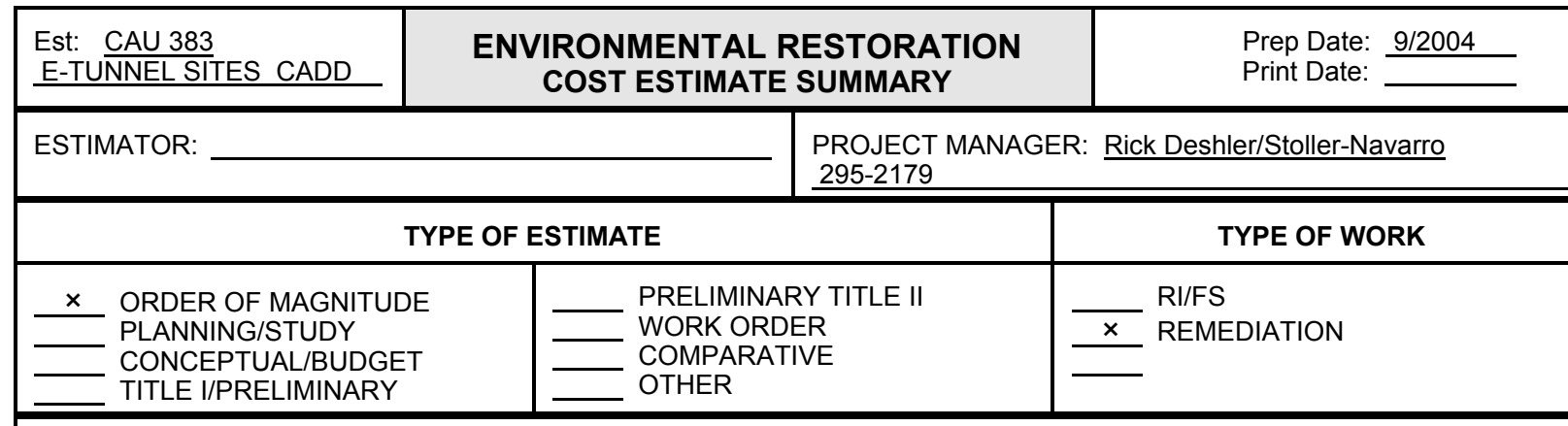

\section{STATEMENT OF WORK:}

This estimate has been prepared to provide remedial alternative costs for the closure of Corrective Action Unit (CAU) 383, Area 12: E-Tunnel Sites, an environmental restoration site listed in the FFACO. Three alternatives are evaluated for closure of the site. This estimate will be used to identify the most cost effective alternative for closure of the site while being protective of human health and the environment. Total estimated costs are intended for comparative analysis of remedial field work and field management only. Costs for long-term monitoring, project management, plan preparation, project support, or other overhead functions are not included.

\section{SCOPE:}

Provide site closure using one of the following alternatives:

Alternative 1 - No Action

Alternative 2 - Clean Closure by Excavation of the Oil Spill; Radioactive Material Area; Muckpile; Ponds One, Two, and Three; and Drainage Area.

Alternative 3 - Closure by Excavation of the Oil Spill and Radioactive Material Area, Grading and Capping of the Muckpile, Drainage Controls, and Administrative Controls

\section{GENERAL BASIS OF ESTIMATE/ASSUMPTIONS}

- These assumptions/basis of estimate apply to all the alternatives listed below.

- A bioassay program health physics support and RCT support will be required for work to be performed in hot areas. Assume PPE requirements to include levels $C$ and $D$.

- Twenty five percent contingency costs are considered in this estimate.

- Escalation is not included in this estimate. All costs are in FY2004 dollars.

\section{ALTERNATIVE SPECIFIC BASIS OF ESTIMATE/ASSUMPTIONS:}

\section{Alternative 1 - No Action}

- No associated costs.

Alternative 2 - Clean Closure by Excavation of the Oil Spill; Radioactive Material Area; Muckpile; Ponds One, Two, and

Three; and Drainage area.

- Total volume is approximately 390,000 cubic yards

- All areas will be excavated to native soil contact.

- Verification samples will be submitted for SVOC, TPH-d, and radionuclides.

- Excavated soil will be containerized, transported to and disposed of at the Area 3 RWMS

- Power supply lines in the area will be relocated to maintain power requirements of equipment in the areas adjacent to E-Tunnel Sites.

- Access roads through E-Tunnel Sites will be maintained and relocated as needed to provide access to the adjacent areas.

Alternative 3 - Closure by Excavation of the Oil Spill and Radioactive Material Area, Grading and Capping of the

Muckpile, Drainage Controls, and Administrative Controls

- Total volume to be excavated is approximately 2,000 cubic yards

- Excavated soils will be disposed of on the Muckpile or in the ponds.

- The Muckpile will be graded to a 3 to 1 slope away from the portal and across the drainage and ponds.

- The E-Tunnel Sites area (18 acres) will be capped with $2 \mathrm{ft}$ of clean soil, compacted, and graded per the NAC approved alternative cover for low risk municipal solid waste landfills.

- Signs prohibiting digging or intrusive work into the soils of the area shall be posted.

- Use restriction will be recorded with the appropriate authorities.

- Two earthen diversion dikes, both northeast of the cap (750 ft), one culvert under the cap (2,200 ft), and two cement drainage chutes across the top of the cap $(3,850 \mathrm{ft})$ will be constructed.

- Power supply lines in the area will be relocated to maintain power requirements of equipment in the areas adjacent to E-Tunnel Sites.

- Access roads through E-Tunnel Sites will be maintained and relocated as needed to provide access to the adjacent areas.

- Site inspections for a minimum of 5 years.

COST SUMMARY - TOTAL ESTIMATED COST PER REMEDIAL ALTERNATIVE:

- Alternative 1 - No Action

- Alternative 2 -Clean Closure by Excavation of the Oil Spill; Radioactive Material Area; Muckpile; Ponds One, Two, and Three; and Drainage Area.

$\$ \quad 0$

$\$ 108,508,000$

- Alternative 3 -Closure by Excavation of the Oil Spill and Radioactive Material Area Grading and Capping of the Muckpile, Drainage Controls, and Administrative Controls

$\$ 14,773,000$ 


\section{Appendix D}

\section{Assessment of Risk for Evaluated Alternatives CAU 383: E-Tunnel Sites, NTS}




\section{D.1.0 Evaluation of Risk Summary}

The purpose of this appendix is to provide a summary of the assessment for risk as discussed in Section 3.0 of the CADD. According to the Final Environmental Impact Statement for the Nevada Test Site and Off-Site Locations in the State of Nevada (DOE/NV, 1996) and as identified in this CADD, the E-Tunnel Sites current and future land use is identified as a Nuclear and High Explosive Test Zone.

Three alternatives for corrective action are presented in the CADD:

- Alternative 1 - No Action

- $\quad$ Alternative 2 - Clean Closure by Excavation of the Oil Spill; RMA; Muckpile; Ponds One, Two, and Three; and Drainage Area.

- Alternative 3 - Closure by Excavation of the Oil Spill and RMA, Grading and Capping of the Muckpile, Drainage Controls, and Administrative Controls

In Alternative 1, there is low risk of exposure to personnel conducting nonintrusive work at the surface of the ponds and channels because inadvertent intrusion would not be prevented. Alternative 2 poses a moderate risk to remediation workers, both from the potential for exposure and from the possibility of occupational injury during excavation and transportation of the soils. Alternative 3 also poses a low risk to NTS workers during transportation of the soils. Alternative 3 poses a lower risk to remediation workers than Alternative 2 because only the Oil Spill and RMA are excavated under Alternative 3. Alternative 3 poses low risk to future NTS workers because the contaminated soils are not transported outside of the CAU. Alternative 3 mitigates the potential for personnel exposure for future uses by capping the contaminated soils, installing signs, and placing use restrictions on the land. Because there are no activities planned at CAU 383 that would necessitate the excavation of soils, and because future activities will be restricted by the implementation of use restrictions as recommended in Alternative 3 , additional evaluation of the risk to NTS personnel is not required. 
The CAU is located in a government-controlled facility. Because the NTS is a restricted area that is guarded on a 24-hour, 365-day-per-year basis, unauthorized personnel cannot gain access to the facility.

All of the alternatives present low or minimal associated risk from future uses of the site based on the uses currently identified. Alternatives that restrict future use of the site, in combination with the control that already exists at the E-Tunnel Sites, afford the most certainty for continued minimal risk to future land users. 


\section{Appendix E}

\section{Project Organization}




\section{E.1.0 Project Organization}

Corrective Action Unit 383 is a joint DTRA and NNSA/NSO project. The DTRA point of contact is the Nevada Operations Office, Environmental Project Manager; currently Ms. Tiffany A. Lantow, her telephone number is (702) 295-7645. The NNSA/NSO point of contact is the Environmental Restoration, Industrial Sites Project Manager; currently Ms. Janet Appenzeller-Wing, her telephone number is (702) 295-0461.

The identification of the project Health and Safety Officer and the Quality Assurance Officer can be found in the appropriate DTRA or NNSA/NSO plan. However, personnel are subject to change, and it is suggested that the DTRA or NNSA/NSO Project Manager be contacted for further information. The Task Manager will be identified in the FFACO Monthly Activity Report prior to the start of field activities. 


\section{Library Distribution List}

U.S. Department of Energy

National Nuclear Security Administration

Nevada Site Office

Technical Library

P.O. Box 98518, M/S 505

Las Vegas, NV 89193-8518

U.S. Department of Energy

Office of Scientific and Technical Information

P.O. Box 62

Oak Ridge, TN 37831-0062

Southern Nevada Public Reading Facility

c/o Nuclear Testing Archive

P.O. Box 98521, M/S 400

Las Vegas, NV 89193-8521

Manager, Northern Nevada FFACO

Public Reading Facility

c/o Nevada State Library \& Archives

100 N. Stewart Street

Carson City, NV 89701-4285

\section{$\underline{\text { Copies }}$}

1 (Uncontrolled)
1 (Uncontrolled, electronic copy)

2 (Uncontrolled, electronic copy)

1 (Uncontrolled, electronic copy) 